\title{
Impact to Underground Sources of Drinking Water and Domestic Wells from Production Well Stimulation and Completion Practices in the Pavillion, WY Field
}

Dominic C. DiGiulio* ${ }^{* \dagger}$ and Robert B. Jackson ${ }^{\dagger, \ddagger}, \S$

${ }^{\dagger}$ Department of Earth System Science, ${ }^{\ddagger}$ Woods Institute for the Environment, and ${ }^{\S}$ Precourt Institute for Energy, Stanford University, Stanford, CA, 94305

*ddigiuli@stanford.edu

\section{Supporting Information}

\section{Table of Contents}

\section{Sections}

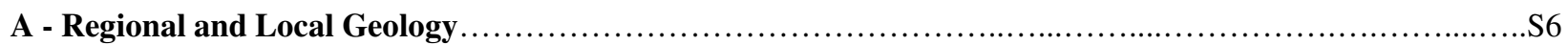

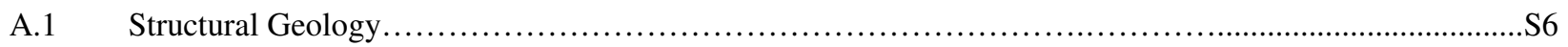

A.2 Identification of Source Rocks and Hydrocarbon Migration............................................. 88

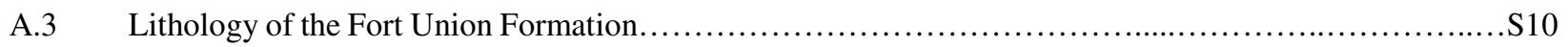

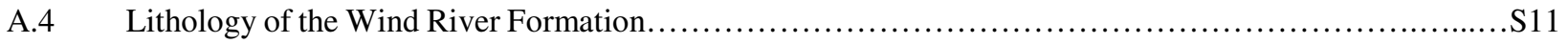

A.5 Lysite and Lost Cabin Members of the Wind River Formation..........................................

A.6 EPA Lithologic Cross-Section through Pavillion Field.................................................

B - Locations, Depths, and Major Ion Concentrations of Domestic Wells in and Around the Pavillion

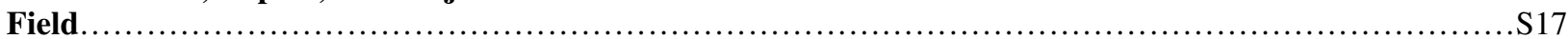

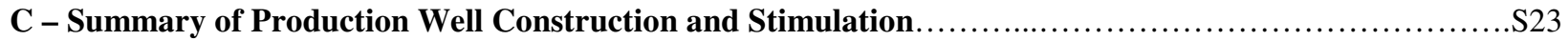

D - Extended Discussion on Impact to USDWs and Usable Water from Well Stimulation.....................S63

D.1 Examples of Potential Loss of Zonal Isolation (Data source: Well completion Reports and Sundry

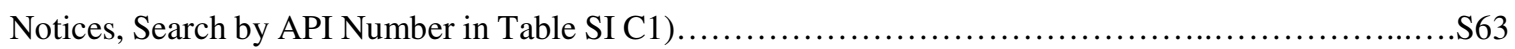

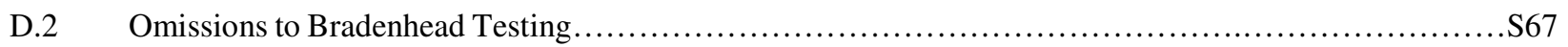

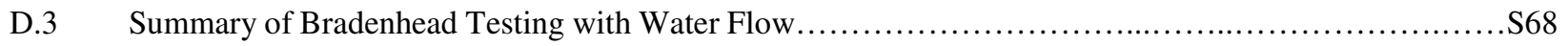

D.4 Summary of Major Ion Concentrations in Produced and Bradenhead Water.............................S70

D.5 Summary of Fixed Gases, Light Hydrocarbon, and BTEX Analyses in String and Bradenhead Gas.......S73

D.6 Summary of Organic Compounds Detected in Produced Water Samples.............................S74

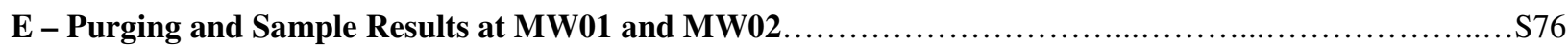

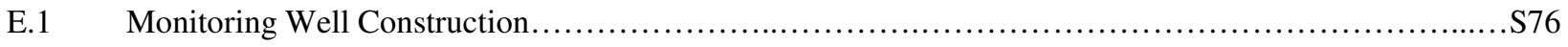

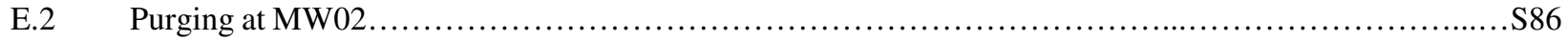

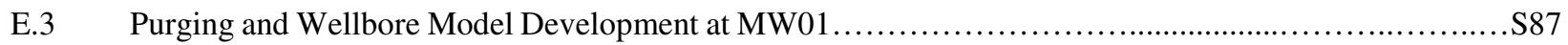

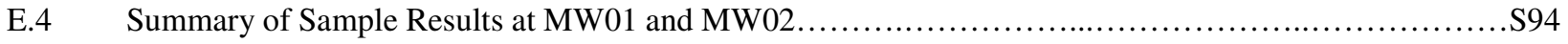




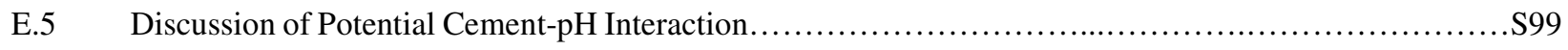

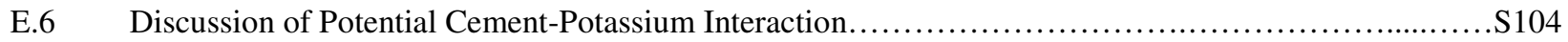

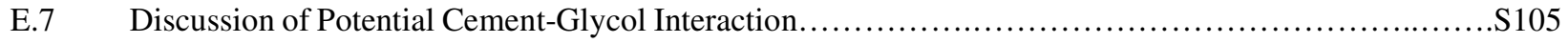

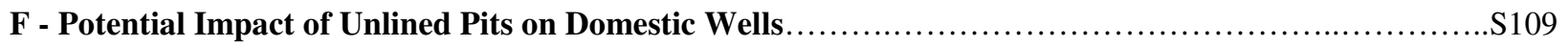

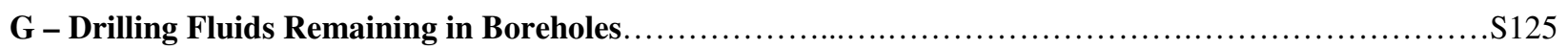

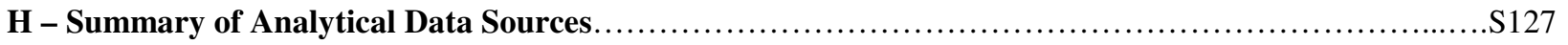

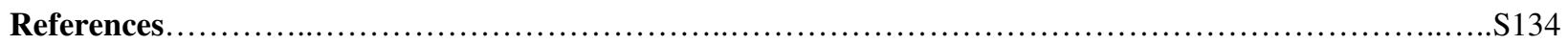

\section{Supporting Information Tables}

Table SI B1. Location and depth of domestic wells in and surrounding the Pavillion well field. State Engineers Office (SEO) permit provided when available. PGDW01 and PGDW02 are municipal wells PGPW01 and PGPW02. Information compiled from $\mathrm{EPA}^{27}$, $\mathrm{EPA}^{29,30}$, Wyoming Oil and Gas Conservation Commission

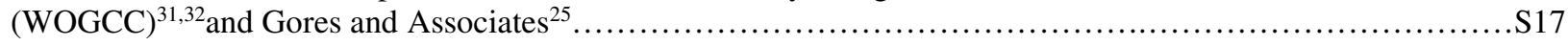

Table SI B2. Major ion concentrations in water sampled from domestic wells ..............................S19

Table SI C1. Production well construction summary (listed in order of completion date) Information retrieved from well completion reports using API number.

Table SI C2. Description of stimulation stages in the Pavillion well field. Information obtained from well completion reports using API search number.

Table SI C3. Summary of compounds used for well stimulation from Material Safety and Data Sheets provided to EPA from Encana ${ }^{35}$....

Table SI C4. Specified volumes of "gel" used with $\mathrm{CO}_{2}$ foam during hydraulic fracturing between 2001 and 2002 ( 1 barrel or $\mathrm{bbl}=42$ gallons or $159 \mathrm{~L}$ ).

Table SI C5. Specified volumes of "WF-125, WF-130, WF-135, and WF-12" polymer slurries with $\mathrm{CO}_{2}$ and $\mathrm{N}_{2}$ foam during hydraulic fracturing from 2001 to 2007 (1 barrel or bbl $=42$ gallons or $159 \mathrm{~L}$ )..................S60

Table SI C6. Volumes of slickwater used for hydraulic fracturing ( 1 barrel or bbl = 42 gallons or 159 L)..........S61

Table SI D1. Major Ions in Produced and Bradenhead Water. Data obtained using API number in Table SI C1

Table SI D2. String gas and bradenhead gas samples collected at production wells having sustained casing pressure. Concentrations in mole fractions x 100. Data obtained using API number in Table SI C1.

Table SI D3. Summary of organic compounds detected in produced water by EPA Region 8 and Zymax $\left(\right.$ contractor to $\left.\mathrm{EPA}^{30}\right)$. PGPPXX are EPA sample identification codes.

Table SI E1. Summary of products used for mud rotary drilling, mass ratios of product use, analyses of aqueous extracts of products, and maximum concentration of detection in MW01 and MW02.

Table SI E2. Well design and input parameters for calculation of casing, borehole, and screen exchange volumes during Phase V sampling event.

Table SI E3a. Summary of Organic Compound Detections in MW01 
Table SI E3b. Summary of Field Parameters, Major Ions, and Dissolved Metals at MW01.... S95

Table SI E4a. Summary of Organic Compound Detections in MW02.

Table SI E4b. Summary of Field Parameters, Major Ions, and Dissolved Metals at MW02.

Table SI E5. Light Hydrocarbon and Isotope Analysis of Production Wells and EPA Monitoring Wells.... S98

Table SI E6. Dissolved Inorganic Carbon, Water Isotopes, Tritium, SF 6 , and He in MW01 and MW02. ..S99

Table SI F1. Summary of disposal of drilling mud and production fluids in from production wells fluids. S109

Table SI F2. Summary of disposal of drilling mud and production fluids in pits, results of soil and surficial ground-water sampling, volumes of soil excavation, distance and direction of domestic wells with $600 \mathrm{~m}$ of pits, review of completion and stimulation record by WOGCC31 of production wells associated with unlined pits, and recommendations by $\mathrm{WOGCC}^{32}$ for further or no further investigation.

Table SI F3. Summary of detection of light hydrocarbons and organic compounds in domestic wells less than and greater than $600 \mathrm{~m}$ from unlined pits. Concentrations in $\mu \mathrm{g} / \mathrm{L}$ unless otherwise indicated.

Table SI G1. Domestic wells greater than intermediate depth and distance to nearest production well with invert Mud.....

Table SI H1. Major reports summarizing data and/or data quality.

Table SI H2. Summary of analytical methods used and sources of data and associated information on quality control and assurance.

\section{Supporting Information Figures}

Figure SI A1. Boundaries of the Wind River Basin, identification of major faults, and location of the Pavillion well field (red box). Modified was modified from Nelson and Kibler ${ }^{8}$....

Figure SI A2. Generalized stratigraphic columns and correlations of Pennsylvanian through Eocene strata in the Wind River Basin, Wyoming. The Pavillion Field is located in the western portion of the Wind River Basin. From Johnson et al. ${ }^{3}$

Figure SI A3. Map illustrating transect used to develop a lithologic cross section across the Pavillion Field. From $\mathrm{EPA}^{27}$

Figure SI A4. Lithologic cross-section along transect illustrating undifferentiated sandstone and shale units. From $\mathrm{EPA}^{27}$

Figure SI A5. Pavillion Field plot illustrating locations of production wells, domestic wells, EPA monitoring wells, and unlined pits. Domestic wells sampled by EPA identified by last two digits of PGDWXX series. Unsampled domestic wells having a Wyoming State Engineer's Office (SEO) permit number identified with the prefix "P." Unsampled domestic wells lacking SEO permits not identified. Depths of domestic well provided in Table SI B1. Full names of production wells, abbreviated here, are provided in Table SI C1. Locations of unlined pits are approximated by locations of associated production wells.

Figure SI C1. Elevation (m) Absolute Mean Seal Level (AMSL) of open intervals of domestic wells $<1 \mathrm{~km}$ from production wells, EPA monitoring wells, surface casing with invert mud, surface casing without invert mud, top of primary cement with invert mud, and top of cement without invert mud. Median land surface elevation = 1636 m AMSL. 
Figure SI C2. Instantaneous Shut-In Pressure (ISIP) values for well stimulation with depth....

Figure SI D1. (a) Potassium concentration as a function of calcium concentration and (b) total dissolved solids (TDS) concentration as a function of sulfate concentration for the Wind River Indian Reservation (Daddow 1992) ${ }^{5}$, Fremont County (Plafcan et al. 1995) ${ }^{26}$, domestic wells sampled by the Wyoming Water Development Commission (WWDC) greater than $1 \mathrm{~km}$ from production wells, domestic wells (PGDWXX) less than and greater than $1 \mathrm{~km}$ from production wells, EPA monitoring wells MW01 and MW02, produced water samples, and bradenhead water samples. Mean values are represented for domestic well locations sampled more than once. Produced water and bradenhead locations were sampled once. Measurement at MW01 and MW02 represent samples collected during Phase III, IV, and V sample events to illustrate variability. TDS values for PGDWXX wells estimated from Daddow $(1995)$ TDS $(\mathrm{mg} / \mathrm{L})=0.785 *$ specific conductance $(\mu \mathrm{S} / \mathrm{cm})-130\left(\mathrm{n}=151, \mathrm{r}^{2}=0.979\right)$

Figure SI D2. (a) Ratio of potassium to calcium and (b) chloride to sulfate concentrations for the Wind River IndianReservation (Daddow 1992) , Fremont County (Plafcan et al. 1995) ${ }^{26}$, domestic wells (PGDWXX) less than and greater than $1 \mathrm{~km}$ from production wells, EPA monitoring wells MW01 and MW02, produced water samples, and bradenhead water samples. Mean values are represented for domestic well locations sampled more than once. Produced water and bradenhead locations were sampled once. Measurement at MW01 and MW02 represent samples collected during Phase III, IV, and V sample events to illustrate variability.

Figure SI D3. Organic compounds (volatile and semivolatile analyses) detected by EPA ${ }^{30}$ in produced water at Tribal Pavillion 14-10 (PGPP01), Tribal Pavillion 33-10 (PGPP05) and Tribal Pavillion 14-02 (PGPP06) and product present with water at Tribal Pavillion 24-02 (PGPP04P). Triethylene glycol was a tentatively identified compound (gas chromatography area at least $10 \%$ as large as the area of the nearest internal standard and a mass

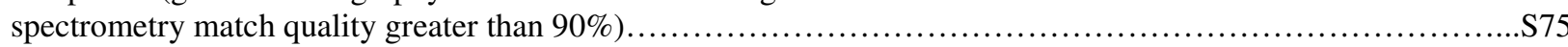

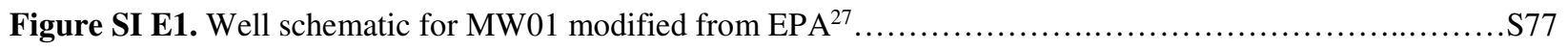

Figure SI E2. Well schematic for MW02 modified from EPA ${ }^{27}$. S78

Figure SI E3. Illustration of purging and sampling sequence (date and times provided) at MW02 for Phase V sampling event. Vertical scale reflects relative lengths of screen, casing, and water levels: (a) Pre-purge condition. Dark gray color denotes water stored in submersible pump casing remaining from the Phase IV sampling event. Mustard colored water denotes water in casing from recovery following the Phase IV sampling event and targeted for removal during the first sample set. Light gray colored water denotes stored casing water from recovery during Phase III and IV sampling events. (b) Water is removed (170L or $45 \mathrm{gal})$ from the submersible pump casing, replaced with water from above the pump inlet, and sampled. (c) Water above pump inlet is removed (964 L or $255 \mathrm{gal})$. (d) Recovery occurs (132 L or 35 gallons). Light blue color indicates mixing of water initially present in the screened interval with incoming formation water. (e) Water above pump inlet removed ( $491 \mathrm{~L}$ or 130 gal) from casing. (f) Recovery occurs (167 L or 44 gallons) with further mixing of water in the screened interval. (g) Water above pump inlet removed ( $215 \mathrm{~L}$ or $57 \mathrm{gal})$. (h) Recovery occurs $(189 \mathrm{~L}$ or $50 \mathrm{gal})$ with further mixing of water in the screened interval. (i) Water in submersible pump casing is removed ( $165 \mathrm{~L}$ or $45 \mathrm{gal})$ and replaced with mixed casing/formation water. (j) Mixed casing/formation water is sampled. Total volume of water removed during the Phase V sampling event prior to collection of the second sample was $2011 \mathrm{~L}$ or 532 gal equivalent to 1.04 borehole volumes.

Figure SI E4. Plug flow - screen mixing model to support evaluation of purging: (a) Prior to purging, the concentration of a solute in the screened interval (and surrounding borehole) and overlying casing is equal to an initial value $\mathrm{C}_{0}$ and a constant concentration $\mathrm{C}_{\mathrm{C}}$ respectively. (b) During drawdown at commencement of purging, both downward and upward plug flow is assumed in casing above and below a pump inlet, respectively. Radial flow occurs from the surrounding formation with subsequent mixing of formation water and water initially in the borehole and screen. (c) As drawdown stabilizes, all of the water entering the pump inlet is directly from the formation. (d) Water level recovery is allowed to occur to ensure that all water entering the inlet is directly from the formation in the event of slight water level perturbation during pumping .................................. 89 
Figure SI E5. Trends in $\mathrm{pH}$ and specific conductance during purging at MW01 during Phase III, IV, and V sampling events as a function of purge volume and borehole volumes (1 borehole volume $\sim 1500 \mathrm{~L}$ ). Times of sample collection illustrated by straight bars

Figure SI E6. (a) Observed decrease in $\mathrm{pH}$ (in mmoles/L) and specific conductance during purging as a function of time during the Phase V sampling event in MW01. Increase in casing volumes (up to 3.8), borehole volumes (up to 3.4), and screen exchanges (up to 26), and EPA sample collection times (approximately 30 minutes in duration) illustrated. Simulation of fraction of store casing water in sample train ( $0.003 \%$ at first sample collection), sample train $\left(\mathrm{C}_{\mathrm{P}}\right)$ / formation $\left(\mathrm{C}_{\mathrm{F}}\right)$ concentration ratios as a function of initial screen $\mathrm{C}_{0}$ and casing $\mathrm{C}_{\mathrm{C}} /$ formation $\left(\mathrm{C}_{\mathrm{F}}\right)$ concentration ratios using a casing plug flow - screen mixing wellbore process model illustrated. (b) Rise in water level in well casing as a result of reduced pumping rate in MW01 during Phase V sampling.... S101

Figure SI E7. (a) Box and whisker plots of minimum, quartiles, median (line), mean (cross), and maximum values of $\mathrm{pH}$ of domestic wells (PGDWXX) greater than $1 \mathrm{~km}$ from a production well, domestic wells less than $1 \mathrm{~km}$ from a production wells, MW01, MW02, produced water, and Bradenhead water samples. Mean values are represented for domestic well locations sampled more than once. Produced water and bradenhead locations were sampled once. Measurement at MW01 and MW02 represent samples collected during Phase III, IV, and V sample events to illustrate variability. (b) $\mathrm{pH}$ levels in domestic wells (PGDWXX) less than and greater than $1 \mathrm{~km}$ of a production well (red and blue respectively) and monitoring wells as a function of absolute mean seal level (AMSL). All data points are illustrated for locations sampled more than once.

Figure SI E8. (a) Box and whisker plots of minimum, quartiles, median (line), mean (cross), and maximum values of Ca of domestic wells (PGDWXX) greater than $1 \mathrm{~km}$ from a production well, domestic wells less than $1 \mathrm{~km}$ from a production wells, MW01, MW02, produced water, and Bradenhead water samples. Mean values are represented for domestic well locations sampled more than once. Produced water and bradenhead locations were sampled once. Measurement at MW01 and MW02 represent samples collected during Phase III, IV, and V sample events to illustrate variability. (b) Ca levels in domestic wells (PGDWXX) less than and greater than $1 \mathrm{~km}$ of a production well (red and blue respectively) and monitoring wells as a function of absolute mean seal level (AMSL). All data points are illustrated for locations sampled more than once....

Figure SI E9. Photograph of foam in YSI flow cell during purging at MW01

Figure SI E10. (a) Potassium concentration and (b) potassium/calcium concentration ratios for domestic wells (PGDWXX) less than and greater than $1 \mathrm{~km}$ of a production well, monitoring wells MW01 and MW02, production wells, and bradenhead samples as a function of absolute mean sea level (AMSL). Domestic wells are identified when sampled more than once. S105

Figure SI E11. Concentration variation of a) potassium $(\mathrm{K})$, calcium $(\mathrm{Ca})$, silicon $(\mathrm{Si})$, dissolved inorganic carbon (DIC), chloride (Cl), and fluoride (F); (b) sodium (Na) and sulfate (SO4); and (c) Strontium (Sr), magnesium (Mg), boron (B), and aluminum (Al) during purging at MW01 as a function of borehole volume during Phase III, IV, and $\mathrm{V}$ sampling events.... .S106

Figure SI E12. Concentration of diethylene glycol (DEG) and triethylene glycol (TEG), as a function of borehole volume during Phase III and IV sampling events and while purging during the Phase V sampling event. Concentrations of gasoline range organics (GRO), diesel range organics (DRO), phenol, and isopropanol included for comparison S108

Figure SI F1. Chromatograms of DRO analysis at (a) PGDW05 during Phase V sampling event, and (b) PGDW30 during Phase I sampling event. Handwritten note, "Early Diesel”, by EPA Region 8 chemist....

Figure SI F2. Chromatograms of DRO analysis at PGDW20 during Phase I sampling event (a) aqueous sample and (b) carbon trap sample. Handwritten note, "Back End of Crude Oil" by EPA Region 8 analytical chemist. During the Phase (I) sampling event, water was circulated through a carbon trap for 24 hours (cumulative water approximately 2,950 L) with detection of DRO at $377 \mu \mathrm{g} / \mathrm{L}$ (EPA 2009) 


\section{A - Regional and Local Geology}

\section{A.1 Structural Geology}

The Pavillion Field is located in the west-central portion of the Wind River Basin (WRB) (Figure SI A1). The WRB is one of many structural and sedimentary basins that formed in the Rocky Mountain foreland (an elongate north-south structural depression flooded by a broad epicontinental sea referred to as the Western Interior Seaway) during the Laramide orogeny ${ }^{1,2}$ (Late Cretaceous through early Eocene). The WRB is fault-bounded by Laramide uplifts with Washakie Range, Owl Creek Mountains, and southern Bighorn Mountains to the north, the Wind River Range to the west, the Granite Mountains to the south, and Casper arch to the east ${ }^{1-4}$ (Figure SI A1).

Igneous and metamorphic rocks of Precambrian age comprise the core of the mountain ranges and underlie sedimentary rocks within the basin. Rocks from all geologic periods except Silurian age are present in the basin ${ }^{5,6}$. Sedimentary strata dip $10^{\circ}-20^{\circ}$ along the south and west margins of the WRB and are commonly vertical to overturned in the north and east margins of the WRB resulting in marked asymmetry with the deepest portion of the of the basin on the north and eastern margins ${ }^{3}$. The center part of the basin is filled with nearly horizontal fluvial and lacustrine Quaternary and Cenozoic Tertiary age sediment, overlying Paleozoic and Mesozoic age rocks. Surface deposits in the basin interior, where the Pavillion field lies, consists of Quaternary alluvium and coalluvum and lower Eocene rocks of the Wind River Formation ${ }^{3}$ in which over 1520 m of basin-fill strata was removed by post-lower Eocene erosion ${ }^{4}$. In the Pavillion area, the thicknesses of colluvial-alluvial deposits can be $6 \mathrm{~m}$ or more ${ }^{7}$

Oil and gas wells in the WRB are generally associated with anticlines or more subtle closures, however, controls on confinement cannot be discerned for some basin-centered gas accumulations ${ }^{8}$. The Pavillion Field is situated on a structural closure that lies on the hanging wall of Circle Ridge/Maverick Springs thrust fault - one of three major northwest-trending thrust fault systems transecting the $\mathrm{WRB}^{3,9}$ (Figure SI A1). There is no publicly available information on the precise location of this fault in the Pavillion field. However, it appears to lie along the western portion of the field ${ }^{10}$. 


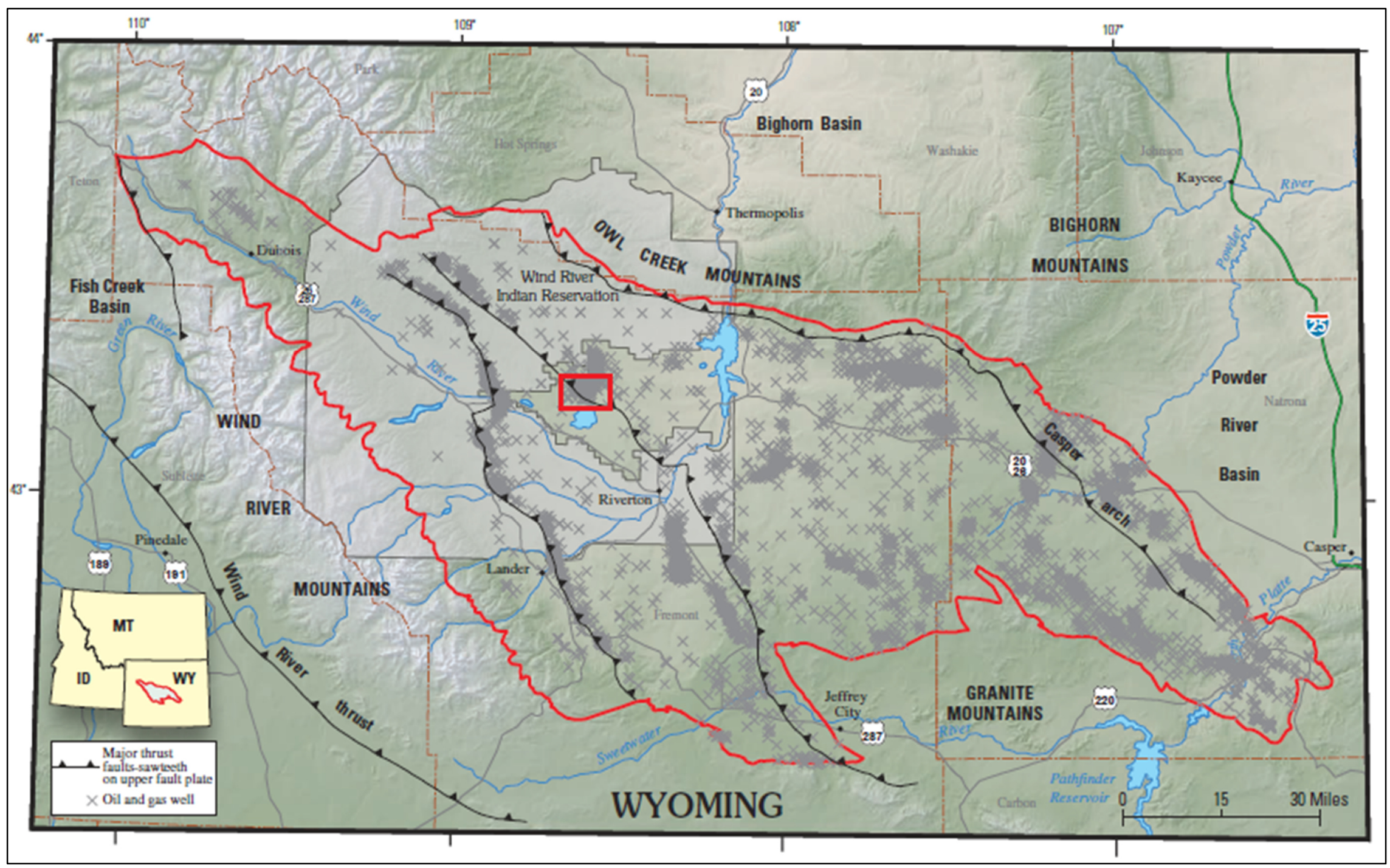

Figure SI A1. Boundaries of the Wind River Basin, identification of major faults, and the approximate location of the Pavillion Field (red square). Figure was modified from Nelson and Kibler ${ }^{8}$. 


\section{A.2 Identification of Source Rocks and Hydrocarbon Migration}

Hydrocarbon production from the Pavillion Field is primarily gas from the Paleocene Fort Union and overlying Early Eocene Wind River Formation. However, oil has also been produced from a number of production wells in these formations, especially in western portion of the field in proximity to the suspected location of a fault. Source rocks for gas generation consist of marine rocks deposited from Early to Late Cretaceous time ( 105-80 Ma) including the Thermopolis Shale, Mowry Shale, Belle Fourche Member of the Frontier Formation, and Cody Shale and non-marine source rocks deposited from Late Cretaceous to Paleocene time ( 82-55 Ma) including coal and carbonaceous shale from the Mesaverde, Meeteetse, and lower member of the Fort Union Formation ${ }^{3}$ (Figure SI A2).

The source rock for oil production in the Pavillion Field is not explicitly identified in the literature. Oil was generated by source rocks in the Mowry Shale and lower shaly member of the Cody Shale throughout most of the deep basin but present day thermal maturities are too high for oil to be preserved in these source rocks except in marginal areas of the basin ${ }^{3}$. Oil generated by these source rocks may have migrated into shallow reservoirs but evidence is lacking ${ }^{3}$. The Waltman Shale Member of the Fort Union Formation is oil prone but absent at the Pavillion field ${ }^{4,8}$ or at its western boundary ${ }^{11}$.

Light hydrocarbons from the Fort Union and Wind River Formations are isotopically similar and "dry." For example, methane $\left(\mathrm{C}_{1}\right), \delta^{13} \mathrm{C}_{1}=-38.04$ to $-39.24 \%$ o in Fort Union formation and $\delta^{13} \mathrm{C}_{1}=-39.24$ to $-40.20 \%$ in Wind River Formation. The ratio of methane/methane to pentane $\left(\mathrm{C}_{1} / \mathrm{C}_{1-5}\right)=0.95$ to 0.96 in Fort Union Formation and $\mathrm{C}_{1} / \mathrm{C}_{1-5}=0.95$ and 0.96 in the Wind River Formation ${ }^{12,13}$. The high level of thermal maturity of light hydrocarbons is incompatible with gas generation from these formations indicating extensive upward vertical migration from underlying mature and post-mature Upper Cretaceous source rocks ${ }^{13}$. Thermal maturities of coal beds in Fort Union are low indicating that any gas present is either of biogenic origin or is migrated thermogenic gas $^{3}$.

In the Pavillion Field, light hydrocarbons are believed to have migrated and commingled in faulted and highly fractured zones along anticlines and structural noses ${ }^{3,13-15}$. Light hydrocarbons migrated to the base of the Waltman Shale Member ${ }^{3}$ east of the Pavillion Field, and to Eocene Wind River Formation west of the pinchout of the Waltman Shale ${ }^{12.13}$ where the Pavillion field is present. As uplift and erosion proceeded in the Wind River Basin, ground water produced an alteration zone where microbial activity generated biogenic methane from source rocks and degraded ethane in preexisting gas accumulations $^{3}$. Biodegradation of ethane has occurred at the Pavillion and East Riverton dome fields ${ }^{14}$. 


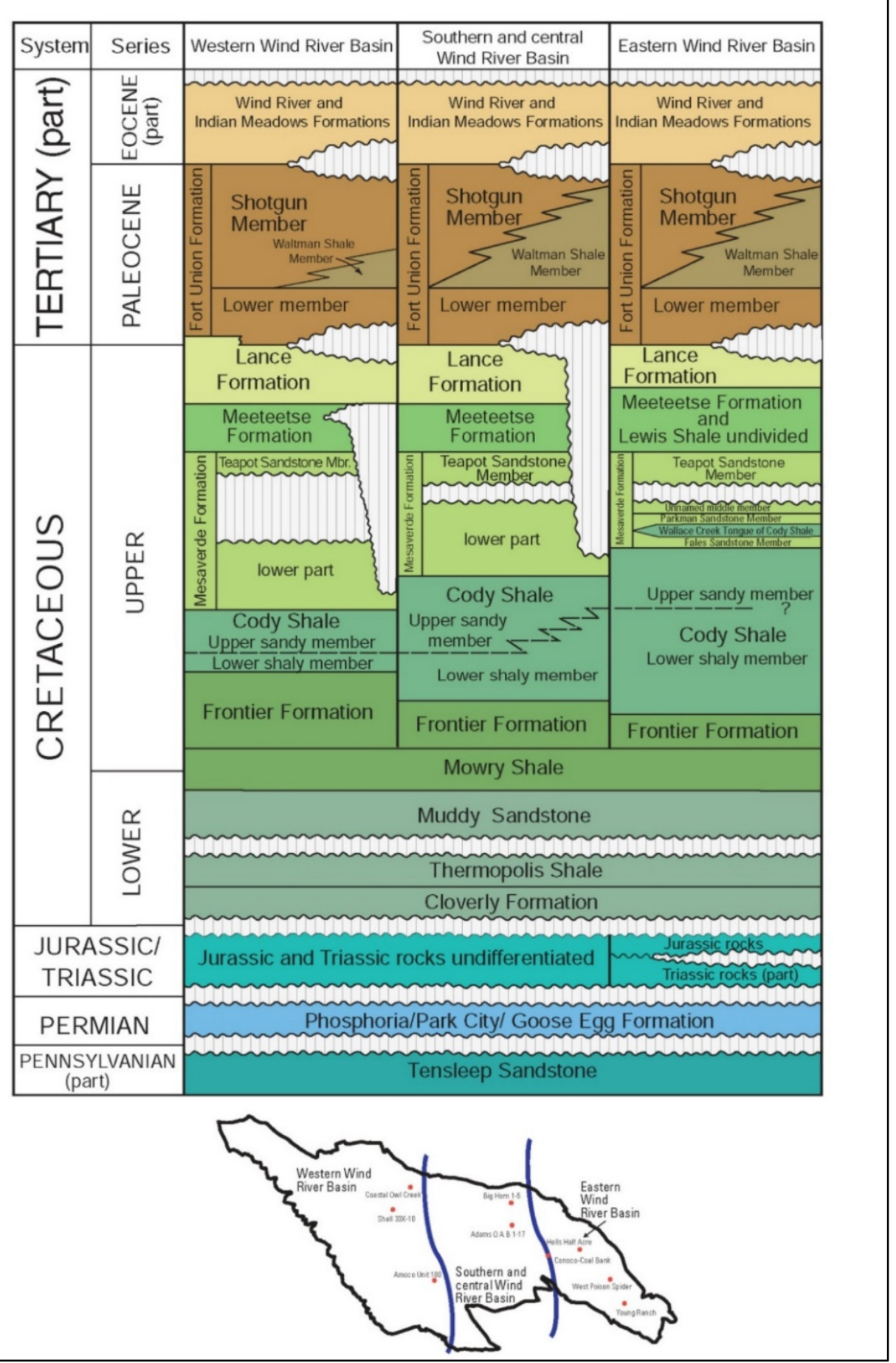

Figure SI A2. Generalized stratigraphic columns and correlations of Pennsylvanian through Eocene strata in the Wind River Basin, Wyoming. The Pavillion Field is located in the western portion of the Wind River Basin. From Johnson et al. ${ }^{3}$ 
Gas trapping in the Wind River and Fort Union Formations in the Pavillion field occurs in localized stratigraphic sandstone pinchouts on the crest and along flanks of a broad structural dome ${ }^{9,10,16}$. A decrease in fracturing away from the crest of a fold rather than loss of gas saturation largely may control economic gas production ${ }^{3}$. During early development of the Pavillion field, Upper Cretaceous and Tertiary rocks were normally pressured (hydrostatic gradient) and had a low temperature gradient of $1.2^{\circ} \mathrm{F} / 100 \mathrm{ft}^{8}$. Cumulative water to gas ratios, measured in barrels (bbls) $(1 \mathrm{bbl}=42$ gallons $=159$ liters $)$ to million cubic feet (MMCF), for targeted production intervals in the Wind River and Fort Union are considered relatively low at $0.9 \mathrm{bbl} / \mathrm{MMCF}(\mathrm{n}=84)$ and $2.4 \mathrm{bbl} / \mathrm{MMCF}(\mathrm{n}=66)$, respectively ${ }^{8}$.

\section{A.3 Lithology of the Fort Union Formation}

The Fort Union Formation is divided into two general lithologic units. The lower unnamed member is characterized by conglomerates, white to gray fine- to coarse-grained, massive to cross-bedded standstone, interbedded with dark gray to black shale, claystone, and siltstone deposited under various fluvial depositional systems $s^{3,17-19}$. The upper unit is divided into two laterally equivalent members - the Waltman Shale and the Shotgun members ${ }^{20}$. The Waltman Shale is a lacustrine deposit in the central portion of the WRB that formed from an extensive body of water that developed in the basin during late Paleocene time ${ }^{11}$. The Waltman Shale is absent or at its western boundary at the Pavillion field ${ }^{4,8,11}$.

The Shotgun Member is a marginal lacustrine deposit that formed in fluvial and shoreline areas that expanded during the late Paleocene ${ }^{20}$ and is dominated by siltstones, mudstones, carbonaceous shales, coals, and subordinated sandstones ${ }^{18}$. The Shotgun Member directly overlies and lithologically merges with the lower member in areas where the Waltman shale is absent ${ }^{11,19}$. The thickness of the Fort Union is relatively unaffected by upwarping and downwarping indicating that downfolding occurred after deposition of the formation. Conglomerates and sandstones of the lower member of the Fort Union were initially targeted as producing zones in the Pavillion Field ${ }^{18}$.

Based on gamma ray, resistivity, and combined gamma ray-resistivity logs, Flores and Keighin ${ }^{18}$ identified four fluvial depositional systems in the lower member of the Fort Union Formation in the Pavillion Field. Type I reservoirs were characterized as stacked sandstone (up to $\sim 18 \mathrm{~m}$ thick) with internal scours marked by lag conglomerates formed by low sinuosity side and mid-channel bars. Type II reservoirs were characterized as multistory sandstones ( $\sim 4$ to $\sim 9$ m thick) separated by siltstone and mudstone seal rocks ( $\sim 1$ to $\sim 6 \mathrm{~m}$ thick) formed by low sinuosity cut and fill channels. Type III reservoirs were characterized as multistory sandstones ( $\sim 2$ to $\sim 14 \mathrm{~m}$ thick) interbedded with siltstones and mudstones ( $\sim 2$ to $\sim 12 \mathrm{~m}$ thick) formed by high sinuosity meander channels. Type IV reservoirs were 
characterized as sandstones (up to $\sim 6 \mathrm{~m}$ thick) within mudstones and siltstones formed by crevasse splays.

\section{A.4 Lithology of the Wind River Formation}

Fluvial deposition of the Wind River Formation occurred during a time of intense folding and uplift during the later stages of the Laramide orogeny $y^{6,11,20,21}$ resulting in considerable lithological variation of the formation throughout the $\mathrm{WRB}^{20}$. Thickness of the Wind River Formation in the basin varies from 0 to about $1524 \mathrm{~m}^{6}$. Information from well completion reports and drilling logs indicates that in the Pavillion Field, thickness of the Wind River Formation ranges from 853 to $1228 \mathrm{~m}$. The underlying Paleocene Fort Union Formation ranges in thickness from 762 to $914 \mathrm{~m}$ in the area ${ }^{18}$.

Keefer ${ }^{19,20}$ stated that, in general, two facies predominate in the Wind River Formation throughout the WRB - a coarse boulder facies representative of deposition along mountain slopes and a fine-grained commonly brightly varicolored facies representative of deposition farther out in the basin with gradation of these two facies in overlapping mountain and basin areas. However, depending on the transport power of ancient streams, coarse grained sandstone and conglomerate extend several miles in to the basin in channel deposits.

McGreevy et al. ${ }^{6}$ stated that in the Wind River Indian Reservation (WRIR) (an area directly west of the Pavillion Field), oil well logs and test drilling indicated: (1) an upper fine-grained sequence at the surface in most of the eastern part of the reservation consisting primarily of gray and green siltstone, shale, and sandstone with numerous sheet and channel deposits of brown siltstone and sandstone with a maximum thickness of about $244 \mathrm{~m}$ in most of the area; (2) a sequence containing many coarse-grained, well sorted, loosely cemented, very porous, largely arkostic sandstone and conglomerated beds of thickness of $\sim 305 \mathrm{~m}$ but thinning to the northeast part of the WRIR; and (3) a lower sequence consisting of fine-grained brown, maroon, red, and gray siltstone and shale with some sandstone of thickness of several thousand feet which intertongues with the coarse-grained sequence toward the south end of the WRIR.

A continuous sandstone unit, termed the Basal Wind River unit, lies at the base of the Wind River Formation in the Pavillion Field ${ }^{9,10}$. This unit appears frequently in drilling and well completion reports. The continuity of this unit contrasts with substantial vertical and lateral stratigraphic variation in sandstone units over short distances in the Pavillion area ${ }^{9,10}$ and throughout the Wind River Formation in general $^{18,22}$ precluding mapping of these units using conventional borehole log techniques. Sandstone units are generally characterized as lenticular, discontinuous, and separated by confining shale, mudstone, and siltstone units ${ }^{6}$. 
Sandstone lenses in the Wind River Formation in the Pavillion field are reported to be generally fine to very fine grained, well sorted, have porosity in the range of 4 to 28 percent and permeability in the range of 0.1 to 300 millidarcies (md) ${ }^{9}$. Referring to development in 14 gas wells and 8 dry holes in the Wind River and Fort Union Formations, Single ${ }^{9}$ stated that on average 75 sandstone units (varying in thickness between $\sim 1$ to $21 \mathrm{~m}$ ) were penetrated in each well of which 6 to 20 units were gas productive and the remaining had limited porosity and/or permeability, or were "water bearing". Drilling in the Pavillion field is complicated by the presence of water sensitive shales and petrophysical evaluation is complicated due to the presence of fresh water and pore-filling clays 9 .

The central part of the basin, is characterized by stream-valley fill and broad flood plains and that the presence of large quantities of overbank mudstones and near linear channel-sandstone ridges suggest that meandering streams of low sinuosity were dominant during deposition of the Wind River Formation $^{21}$. The Eocene Wind River appears to have flowed directly through an area that is now the Pavillion Field in the vicinity of production well Tribal Unit 14X-1121. Maximum intervals of continuous sandstone deposits are greatest near the course of the Eocene Wind River where channel sandstone bodies can be as much as 40 meters thick, but most are much thinner ${ }^{21}$.

\section{A.5 Lysite and Lost Cabin Members of the Wind River Formation}

In the eastern and northeastern margins of the WRB (north and east of the Pavillion field), the Wind River Formation has been divided into the Lysite and overlying Lost Cabin Member based on lithological and faunal differences ${ }^{19,20}$. Faunal distinction was largely based on the presence of the genus Lambdotherium in the Lost Cabin beds and its absence in Lysite beds ${ }^{20}$. In the northeastern portion of the WRB, the Lysite Member consists of yellow to brown sandstone and conglomerate interbedded with red, gray, and greenish-gray sandy claystone and siltstone ${ }^{20}$. The Lost Cabin Member consists of gray, yellow, and brown sandstone with locally prominent beds of conglomerate alternating with violet, red, purple, gray, and green sandy clayey sandstone ${ }^{20}$. Both members contain an abundance of fine-grained strata ${ }^{19}$.

One of the primary differences between these two members is the composition of conglomerates. Conglomerate in the Lost Cabin Member contains abundant Precambrian rock fragments of granite and gneiss $^{19}$. Conglomerate in the Lysite Member is derived almost exclusively from Paleozoic formations ${ }^{19}$ and contains an abundance of limestone, dolomite, and chert fragments ${ }^{20}$. In the central part of the WRB (where the Pavillion Field is located), there is no sample or electric log basis to separate the Wind River Formation into Lysite and Lost Cabin Members ${ }^{20}$. Throughout the WRB, the name Wind River Formation is applied to all lower Eocene rocks, including the Indian Meadows Formation and the Lost Cabin and Lysite Members of the Wind River Formation because there are no viable criteria for distinguishing individual members of the Wind River Formation from sample and well log data ${ }^{11,19,20}$. 
Stephens ${ }^{23}$ and Itasca Denver Inc. ${ }^{24}$ state that the Wind River Formation in the Pavillion field can be divided into the Lost Cabin member and underlying Lysite member and that the former is a source of potable water while the later has total dissolved solids (TDS) levels exceeding 10,000 $\mathrm{mg} / \mathrm{L}$. They state that EPA's monitoring well MW01 was completed in the Lower Lost Cabin member, and MW02 was completed in the Upper Lysite Member where elevated chloride was detected. However, neither Stephens ${ }^{23}$ nor Itasca Denver Inc. ${ }^{24}$ provide any justification (e.g., faunal or lithologic) for identification of these units outside established geographic areas in the WRB.

In available lithologic logs in the Pavillion Field, limestone and dolomite fragments characteristic of the Lysite member were not identified at Tribal 1-21, Tribal 1-22, Taylor Patented 1, Shell 33X-10, Mae H. Rhodes 1, Runners Herford 1, Roland Patented 34-13, Ocean Lake Tribal 1-15, John K. Coolidge 1-4, Unit 12-3, Govt Ocean Lake 1, Doles Unit 44-15, Tribal 21-9, and Tribal Pavillion 14-2 at or below the depth of MW02. Chert fragments were identified at $856 \mathrm{~m}$ below ground surface (bgs) at Shell 33X10 and at $1036 \mathrm{~m}$ bgs at Taylor Patented 1.

Also, neither Stephens ${ }^{23}$ nor Itasca Denver Inc. ${ }^{24}$ provide references or documentation that, even where present, the Lysite member contains TDS levels exceeding 10,000 mg/L. In an evaluation of alternative water supply options for residents in the Pavillion field, Gores and Associates ${ }^{25}$ identified three domestic wells of similar depth of MW02 in the immediate vicinity of the Pavillion field at 274, 305, and $322 \mathrm{~m}$ bgs with associated TDS concentrations of 631, 590 (775 duplicate), and $607 \mathrm{mg} / \mathrm{L}$ TDS respectively. Also, USGS reports (McGreevy et al. ${ }^{6}$, Plafcan et al. ${ }^{26}$, and Daddow et al. ${ }^{5}$ ) relevant to the Pavillion area indicate no instance of TDS levels exceeding 10,000 mg/L in the Wind River Formation. Plafcan et al. ${ }^{26}$ identified a water supply well at $674 \mathrm{~m}$ bgs having a TDS concentration of $1190 \mathrm{mg} / \mathrm{L}$. A.6 EPA Lithologic Cross-Section through Pavillion Field $\mathrm{EPA}^{27}$ used borehole geophysical logs from 32 production wells to develop a lithologic crosssection in the vicinity of MW01 and MW02. Depending upon the specific production well, various combinations of natural gamma, resistivity, self-potential, density, and neutron porosity logs were utilized. No gradational/intermediate values between shale and sandstone (e.g. $80 \%$ shale, $20 \%$ sandstone) were used although they are known to exist throughout the Wind River Formation. This designation was maintained for consistency for near surface deposits where fine-, medium-, and coarsegrained sandstones could be differentiated from driller's logs. It was generally clear from the various logs whether a specific interval contained sandstone or shale, but not where each layer started or ended. Thus, assignment of boundaries was subjective. Each sandstone or shale layer was represented as having at least one meter in thickness since log resolution was insufficient to discern individual layers less than this resolution. 
Data was used to populate a three-dimensional lithology model using a commercial software package Rockware15. A lithologic cross-section (Figure SI A4) developed along a transect (Figure SI A3) represents a slice taken through this 3D model. In general, lithology was highly variable and difficult to correlate from borehole to borehole, even for boreholes in close proximity to one another consistent with the experience of others (e.g., Osiensky et al. ${ }^{22}$ ). However, it is apparent that there are no laterally continuous confining layers above depths of well stimulation (Figure SI A4). Since the majority of geophysical logs from the oil and gas wells were run after installation of surface casing, the shallow lithology structure (upper 150-200 meters) in the model was dominated by information from the two EPA installed monitoring wells. Thus, the apparent existence of continuous shale units near the surface may be a result of insufficient data rather than physical reality.

The aspect ratio (length versus width) of sandstone lenses cannot be discerned from available data. Itasca Denver Inc. ${ }^{24}$ used exposures from Upper Jurassic Salt Wash Sandstone Member of the Morrison Formation in the Henry Mountains area of southern Utah investigated by Robinson and McCabea $^{28}$ to define the aspect (length versus width) ratios of sandstone bodies in the Paleocene Fort Union and Eocene Wind River Formations. Justification for this comparison was not provided.

A plot of the entire Pavillion Field is provided in Figure SI A5. 


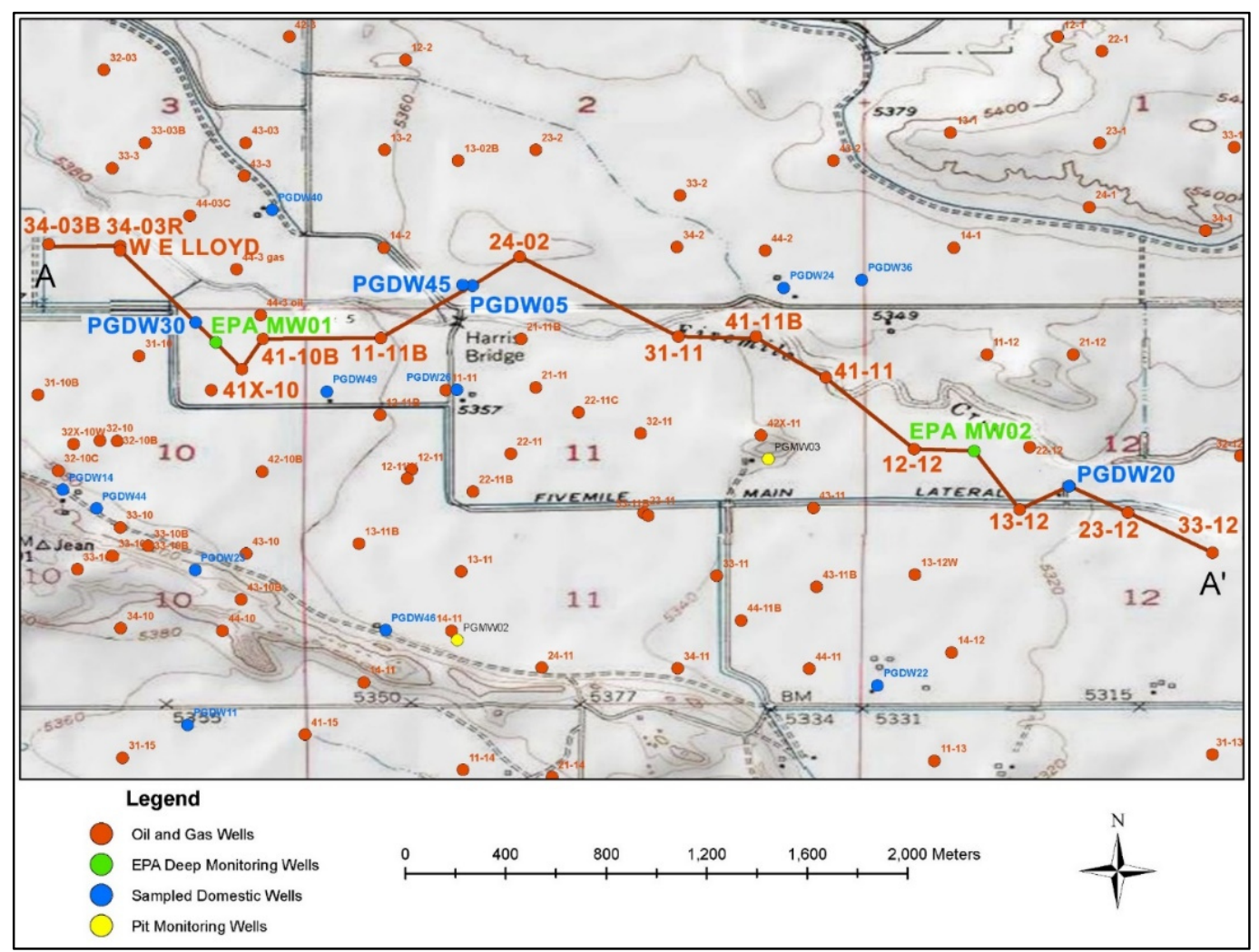

Figure SI A3. Map illustrating transect used to develop a lithologic cross section across the Pavillion Field. From $\mathrm{EPA}^{27}$

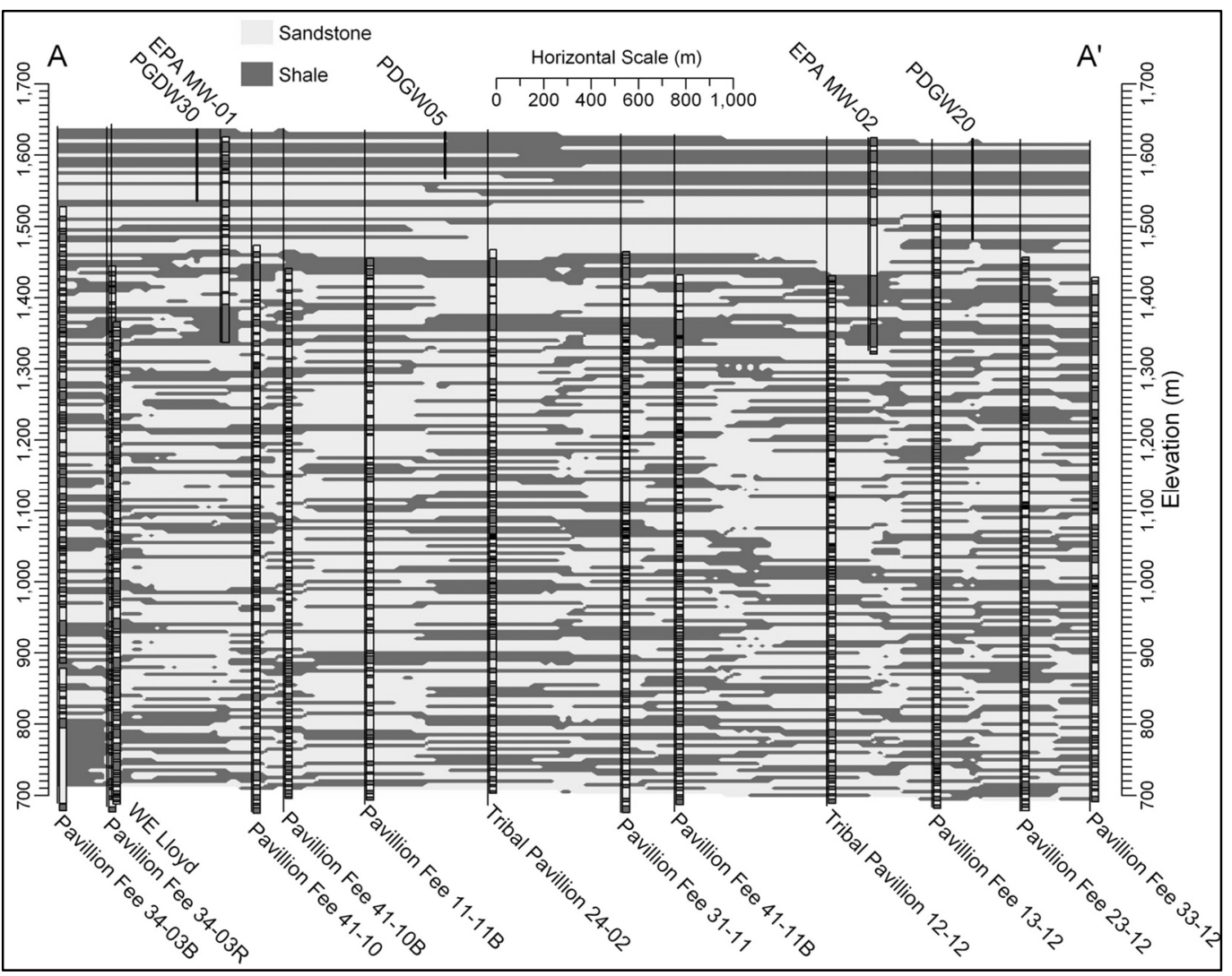

Figure SI A4. Lithologic cross-section along transect illustrating undifferentiated sandstone and shale units. From $\mathrm{EPA}^{27}$ 


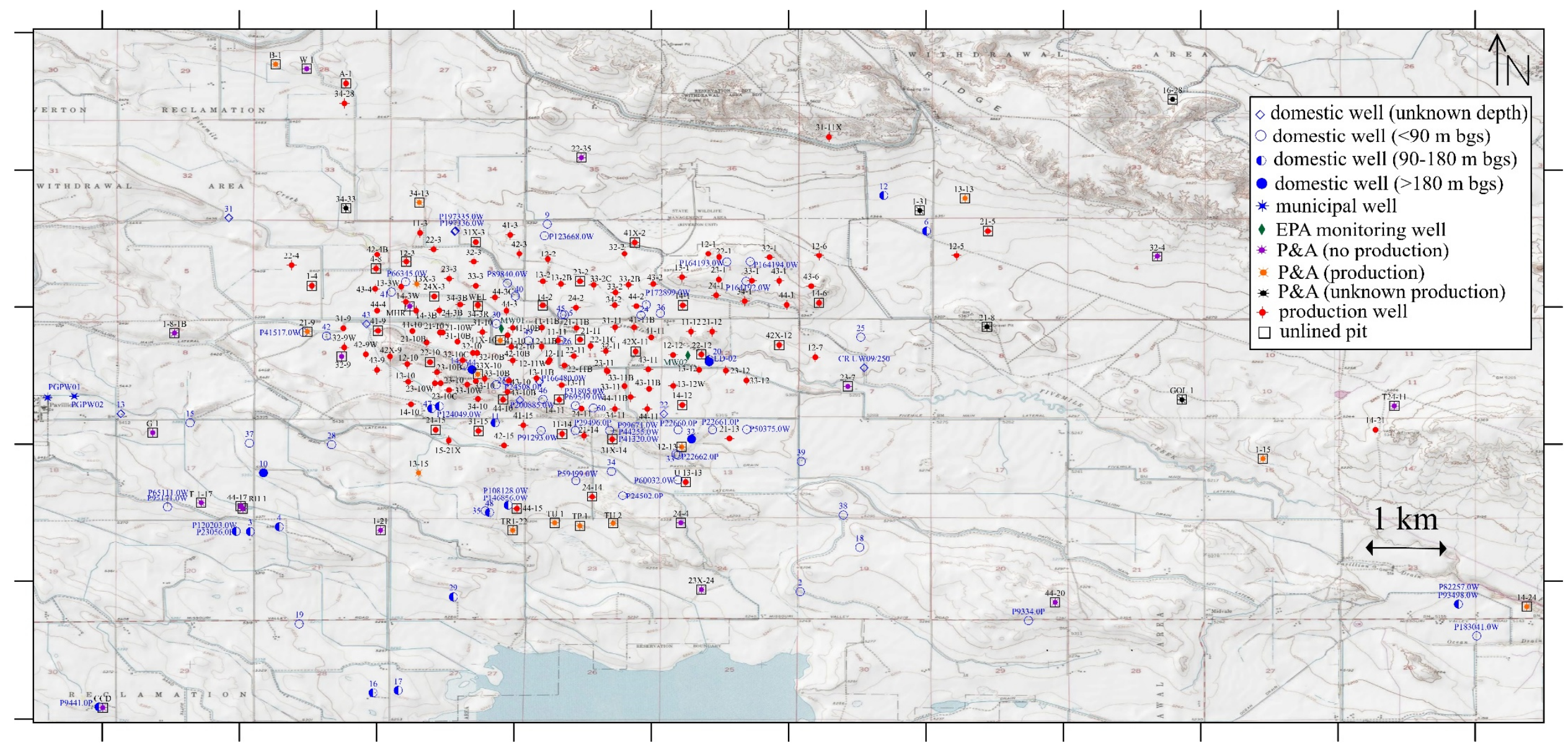

Figure SI A5. Pavillion Field plot illustrating locations of production wells, domestic wells, EPA monitoring wells (MW01 and MW02), and unlined pits. Domestic wells sampled by EPA identified by last two digits of PGDWXX series. Unsampled domestic wells having a Wyoming State Engineer's Office (SEO) permit number identified with the prefix "P." Unsampled domestic wells lacking SEO permits not identified. Depths of domestic well provided in Table SI B1. Full names of production wells, abbreviated here, are provided in Table SI C1. Locations of unlined pits are approximated by locations of associated production wells. 


\section{B - Locations, Depths, and Major Ion Concentrations of Domestic Wells in and Around the Pavillion Field}

Table SI B1. Location and depth of domestic wells in and surrounding the Pavillion well field. Domestic wells with prefix PGDWXX were sampled by EPA. State Engineers Office (SEO) permit provided when available. PGDW01 and PGDW02 are municipal wells PGPW01 and PGPW02. Information compiled from EPA ${ }^{27}$, EPA ${ }^{29,30}$, Wyoming Oil and Gas Conservation Commission (WOGCC) (W1,32 and Gores and Associates $^{25}$

\begin{tabular}{|c|c|c|c|c|c|c|}
\hline 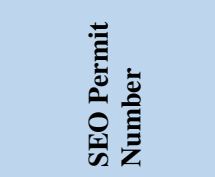 & = & : & 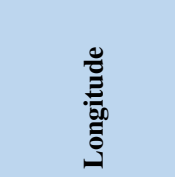 & 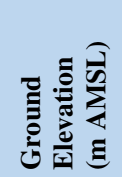 & 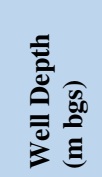 & 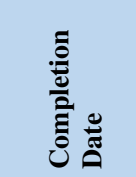 \\
\hline$?$ & PGPW01/PGDW07 & 43.24678802 & -108.6879349 & 1666.0 & 154.2 & $?$ \\
\hline$?$ & PGPW02/PGDW08 & 43.24697113 & -108.6840515 & 1641.0 & 157.0 & $?$ \\
\hline$?$ & PGDW01 & 43.2185 & -108.5783 & 1610.0 & $?$ & $?$ \\
\hline$?$ & PGDW02 & 43.21848912 & -108.5783117 & 1629.8 & 15.2 & $?$ \\
\hline$?$ & PGDW03 & 43.22721318 & -108.6584107 & 1629.8 & 137.2 & $?$ \\
\hline$?$ & PGDW04 & 43.22790909 & -108.6541901 & 1638.3 & 134.1 & $?$ \\
\hline P32161.0W & PGDW05 & 43.25884628 & -108.6126556 & 1632.2 & 64.0 & $02 / 02 / 1976$ \\
\hline$?$ & PGDW06 & 43.27110813 & -108.5599211 & 1663.9 & 115.8 & $?$ \\
\hline$?$ & PGDW09 & 43.27211644 & -108.615144 & 1635.6 & 9.1 & $?$ \\
\hline$?$ & PGDW10 & 43.2358284 & -108.6565018 & 1633.7 & 225.6 & $?$ \\
\hline$?$ & PGDW11 & 43.24312049 & -108.6228628 & 1636.8 & & $?$ \\
\hline$?$ & PGDW12 & 43.27628927 & -108.5661502 & 1652.9 & 115.8 & $?$ \\
\hline$?$ & PGDW13 & 43.2444467 & -108.6772771 & 1642.6 & $?$ & $?$ \\
\hline No permit on file & PGDW14 & 43.25154027 & -108.6273311 & 1642.3 & 57.9 & $?$ \\
\hline$?$ & PGDW15 & 43.24312129 & -108.6671791 & 1606.9 & 30.5 & $?$ \\
\hline$?$ & PGDW16 & 43.20381363 & -108.6405183 & 1610.9 & 164.6 & $?$ \\
\hline$?$ & PGDW17 & 43.20416653 & -108.6368713 & 1615.1 & 152.4 & $?$ \\
\hline$?$ & PGDW18 & 43.22491388 & -108.569651 & 1612.7 & 67.1 & $?$ \\
\hline$?$ & PGDW19 & 43.21382469 & -108.651274 & 1622.1 & 19.8 & $?$ \\
\hline No permit on file & PGDW20 & 43.25230026 & -108.5915756 & 1624.0 & 140.2 & $?$ \\
\hline No permit on file & LD-02 & 43.25437 & -108.58919 & 1623.0 & 185.9 & $?$ \\
\hline$?$ & PGDW22 & 43.2444191 & -108.598175 & 1627.0 & $?$ & $?$ \\
\hline P24508.0P & PGDW23 & 43.2486496 & -108.6225891 & 1656.9 & 53.3 & $12 / 31 / 1964$ \\
\hline$?$ & PGDW24 & 43.25877211 & -108.6015059 & 1622.1 & 30.5 & $?$ \\
\hline$?$ & PGDW25 & 43.25558722 & -108.5694867 & 1643.8 & 24.4 & $?$ \\
\hline$?$ & PGDW26 & 43.25512275 & -108.6132115 & 1649.9 & 19.8 & $?$ \\
\hline$?$ & PGDW28 & 43.23993143 & -108.6465688 & 1612.7 & 25.9 & $?$ \\
\hline$?$ & PGDW29 & 43.21773909 & -108.6288449 & 1634.9 & 121.9 & $?$ \\
\hline No permit on file & PGDW30 & 43.25753 & -108.62258 & 1637.1 & 79.2 & $?$ \\
\hline$?$ & PGDW31 & 43.27302485 & -108.6615302 & 1624.9 & $?$ & $?$ \\
\hline P64110.0W & PGDW32 & 43.24074936 & -108.5941391 & 1624.9 & 205.7 & $5 / 24 / 1983$ \\
\hline P22662.0P & PGDW33 & 43.23855522 & -108.5964146 & 1626.1 & 9.1 & $12 / 31 / 1934$ \\
\hline$?$ & PGDW34 & 43.23605297 & -108.6058086 & 1634.0 & 30.5 & $?$ \\
\hline$?$ & PGDW35 & 43.23021564 & -108.6241763 & 1630.1 & 88.4 & $?$ \\
\hline$?$ & PGDW36 & 43.25905726 & -108.5987059 & 1641.0 & 30.5 & $?$ \\
\hline$?$ & PGDW37 & 43.24016136 & -108.6585376 & 1615.4 & 24.4 & $?$ \\
\hline$?$ & PGDW38 & 43.2296203 & -108.572037 & 1610.9 & 48.8 & $?$ \\
\hline$?$ & PGDW39 & 43.23750687 & -108.5781708 & 1615.4 & 17.4 & $?$ \\
\hline$?$ & PGDW40 & 43.26156616 & -108.6198273 & 1638.0 & 67.1 & $?$ \\
\hline P66345.0W & PGDW41 & 43.262146 & -108.6378479 & 1645.0 & 21.3 & $1 / 31 / 1984$ \\
\hline P41517.0W & PGDW42 & 43.25574493 & -108.647316 & 1645.9 & 61.0 & $11 / 29 / 1977$ \\
\hline$?$ & PGDW43 & 43.25749207 & -108.64151 & 1645.0 & $?$ & $?$ \\
\hline P24506.0P & PGDW44 & 43.25086975 & -108.6261292 & 1645.6 & 228.6 & $12 / 31 / 1932$ \\
\hline No permit on file & PGDW45 & 43.25888062 & -108.6130142 & 1632.5 & 30.5 & $?$ \\
\hline$?$ & PGDW46 & 43.24651337 & -108.6157684 & 1638.9 & 14.6 & $?$ \\
\hline$?$ & PGDW47 & 43.24520493 & -108.6319885 & 1641.3 & 147.5 & $?$ \\
\hline$?$ & PGDW48 & 43.2299881 & -108.6235733 & 1633.1 & 115.8 & $?$ \\
\hline No permit on file & PGDW49 & 43.25505829 & -108.6178741 & 1637.7 & 15.2 & $?$ \\
\hline P166481.0W & PGDW50 & 43.2453 & -108.6085 & 1633.1 & 61.0 & $4 / 7 / 2005$ \\
\hline CR UW09/250 & None & 43.25114 & -108.56899 & 1614.4 & $?$ & $8 / 19 / 1977$ \\
\hline P108128.0W & None & 43.23104 & -108.62085 & 1626.1 & 115.5 & $11 / 5 / 1997$ \\
\hline P120203.0W & None & 43.22725 & -108.66048 & 1628.2 & 137.2 & $11 / 8 / 1999$ \\
\hline P123668.0W & None & 43.27042 & -108.61556 & 1638.9 & 18.3 & $3 / 6 / 2000$ \\
\hline P124049.0W & None & 43.24552 & -108.63087 & 1639.2 & 147.5 & $3 / 6 / 2000$ \\
\hline P146856.0W & None & 43.23104 & -108.62085 & 1626.1 & 115.8 & $9 / 5 / 2002$ \\
\hline P164192.0W & None & 43.26372 & -108.5862 & 1649.0 & 24.4 & $12 / 6 / 2004$ \\
\hline P164193.0W & None & 43.26653 & -108.589 & 1654.1 & 30.5 & $12 / 6 / 2004$ \\
\hline
\end{tabular}




\begin{tabular}{|c|c|c|c|c|c|c|}
\hline 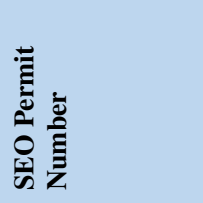 & 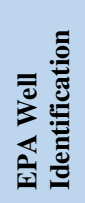 & 芵 & 总 & 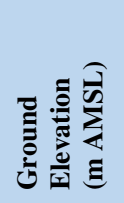 & 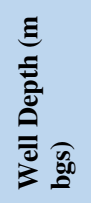 & 总 \\
\hline P164194.0W & None & 43.26658 & -108.5856 & 1654.1 & 31.2 & $12 / 6 / 2004$ \\
\hline P166480.0W & None & 43.24909 & -108.6163 & 1633.1 & $?$ & $4 / 7 / 2005$ \\
\hline P172899.0W & None & 43.26029 & -108.60075 & 1631.0 & 30.5 & $10 / 19 / 2005$ \\
\hline P183041.0W & None & 43.21207 & -108.47977 & 1567.9 & 73.2 & $8 / 1 / 2007$ \\
\hline P197335.0W & None & 43.27103 & -108.62861 & 1645.9 & $?$ & $1 / 27 / 2012$ \\
\hline P197336.0W & None & 43.27115 & -108.62854 & 1645.9 & $?$ & $1 / 27 / 2012$ \\
\hline P200885.0W & None & 43.24642 & -108.61915 & 1657.0 & $?$ & $8 / 16 / 2013$ \\
\hline $\mathrm{P} 22660.0 \mathrm{P}$ & None & 43.24208 & -108.59609 & 1624.9 & 53.3 & $9 / 30 / 1938$ \\
\hline P22661.0P & None & 43.24212 & -108.5911 & 1622.1 & 14.6 & $8 / 31 / 1947$ \\
\hline P22662.0P & None & 43.23844 & -108.59611 & 1625.5 & 9.1 & $12 / 31 / 1934$ \\
\hline P23056.0P & None & 43.22725 & -108.66048 & 1628.2 & 19.8 & $1 / 4 / 1960$ \\
\hline P24502.0P & None & 43.23248 & -108.60418 & 1628.9 & 54.9 & $12 / 31 / 1942$ \\
\hline P24506.0P & None & 43.24918 & -108.62593 & 1658.4 & 228.6 & $12 / 31 / 1932$ \\
\hline P24508.0P & None & 43.2492 & -108.62099 & 1637.1 & 53.3 & $12 / 31 / 1964$ \\
\hline P29496.0P & None & 43.24198 & -108.61107 & 1633.1 & 39.6 & $3 / 31 / 1975$ \\
\hline P30217.0W & None & 43.24311 & -108.62275 & 1633.1 & 106.7 & $6 / 18 / 1975$ \\
\hline P31805.0W & None & 43.24562 & -108.61107 & 1633.1 & 30.5 & $1 / 20 / 1976$ \\
\hline P34936.0W & None & 43.24311 & -108.62275 & 1633.1 & 106.7 & $9 / 14 / 1976$ \\
\hline P41320.0W & None & 43.24201 & -108.60608 & 1642.9 & 30.5 & $12 / 19 / 1977$ \\
\hline P41517.0W & None & 43.25628 & -108.6507 & 1644.1 & 61.0 & $11 / 29 / 1977$ \\
\hline P44255.0W & None & 43.24201 & -108.60608 & 1642.9 & 68.6 & $7 / 18 / 1978$ \\
\hline P50375.0W & None & 43.24216 & -108.58612 & 1618.0 & 30.5 & $10 / 15 / 1979$ \\
\hline P59499.0W & None & 43.23471 & -108.611 & 1622.1 & 33.5 & $2 / 3 / 1982$ \\
\hline P60032.0W & None & 43.2348 & -108.59614 & 1618.0 & 26.2 & $3 / 23 / 1982$ \\
\hline P65111.0W & None & 43.23078 & -108.67048 & 1645.0 & 27.4 & $8 / 3 / 1983$ \\
\hline P66345.0W & None & 43.26365 & -108.6358 & 1642.9 & 21.3 & $1 / 31 / 1984$ \\
\hline P69549.0W & None & 43.24562 & -108.61107 & 1633.1 & 30.5 & $2 / 25 / 1985$ \\
\hline P82257.0W & None & 43.21668 & -108.48244 & 1571.8 & 91.4 & $4 / 20 / 1990$ \\
\hline P89840.0W & None & 43.26343 & -108.62097 & 1639.5 & 65.5 & 9/17/1992 \\
\hline P91293.0W & None & 43.24195 & -108.61606 & 1631.0 & 3.0 & $4 / 5 / 1993$ \\
\hline $\mathrm{P} 9334.0 \mathrm{P}$ & None & 43.21429 & -108.54506 & 1625.5 & 6.1 & $12 / 31 / 1935$ \\
\hline P93498.0W & None & 43.21668 & -108.48244 & 1571.8 & 69.5 & $11 / 15 / 1993$ \\
\hline P9441.0P & None & 43.20178 & -108.68044 & 1634.9 & 177.4 & $1 / 1 / 1944$ \\
\hline P95171.0W & None & 43.23078 & -108.67048 & 1645.0 & 25.9 & $5 / 9 / 1994$ \\
\hline P99671.0W & None & 43.24201 & -108.60608 & 1643.0 & 16.7 & 6/28/1995 \\
\hline P14914P & None & ? & ? & ? & 39.6 & ? \\
\hline P98757W & None & $?$ & $?$ & 1670.0 & 156.1 & $?$ \\
\hline P58929W & None & $?$ & $?$ & 1652.0 & 17.4 & $?$ \\
\hline P34345W & None & $?$ & $?$ & 1667.9 & 150.9 & $?$ \\
\hline P59104W & None & $?$ & $?$ & 1667.9 & 152.4 & $?$ \\
\hline P24507P & None & $?$ & $?$ & 1647.1 & 228.6 & $?$ \\
\hline P97501W & None & $?$ & $?$ & 1624.0 & $?$ & $?$ \\
\hline P30217W & None & $?$ & $?$ & $?$ & 106.7 & $?$ \\
\hline P182983W & None & $?$ & $?$ & 1638.6 & 231.6 & $?$ \\
\hline P46362W & None & $?$ & $?$ & 1642.3 & 54.9 & $?$ \\
\hline P62641W & None & $?$ & $?$ & 1662.7 & 208.8 & $?$ \\
\hline P53567W & None & $?$ & $?$ & 1652.0 & 42.7 & $?$ \\
\hline P25636W & None & $?$ & $?$ & 1633.7 & 12.5 & $?$ \\
\hline P110443 & None & $?$ & $?$ & 1633.7 & 127.1 & $?$ \\
\hline P28496W & None & $?$ & $?$ & 1613.3 & 11.0 & $?$ \\
\hline P26200W & None & $?$ & $?$ & 1603.2 & 88.4 & $?$ \\
\hline $\mathrm{P} 40603 \mathrm{~W}$ & None & $?$ & $?$ & 1619.1 & 12.2 & $?$ \\
\hline P76475W & None & $?$ & $?$ & 1621.5 & 97.5 & $?$ \\
\hline P14548P & None & $?$ & $?$ & 1615.4 & 18.3 & $?$ \\
\hline P30162W & None & $?$ & $?$ & 1645.9 & 61.0 & $?$ \\
\hline P32163W & None & $?$ & $?$ & 1639.8 & 114.3 & $?$ \\
\hline P9941P & None & $?$ & $?$ & 1637.1 & 177.4 & $?$ \\
\hline P116598W & None & $?$ & $?$ & 1629.8 & 143.3 & $?$ \\
\hline $\mathrm{P} 25011 \mathrm{~W}$ & None & $?$ & $?$ & 1627.6 & 88.4 & $?$ \\
\hline P177246W & None & $?$ & $?$ & 1611.5 & 304.8 & $?$ \\
\hline P190223W & None & $?$ & $?$ & 1618.2 & 321.6 & $?$ \\
\hline P191733W & None & $?$ & $?$ & 1606.9 & 274.3 & $?$ \\
\hline $\begin{array}{l}\text { Unidentified (16 } \\
\text { domestic wells) }\end{array}$ & None & $?$ & $?$ & $?$ & $?$ & $?$ \\
\hline
\end{tabular}


Table SI B2. Major ion concentrations in water sampled from domestic wells.

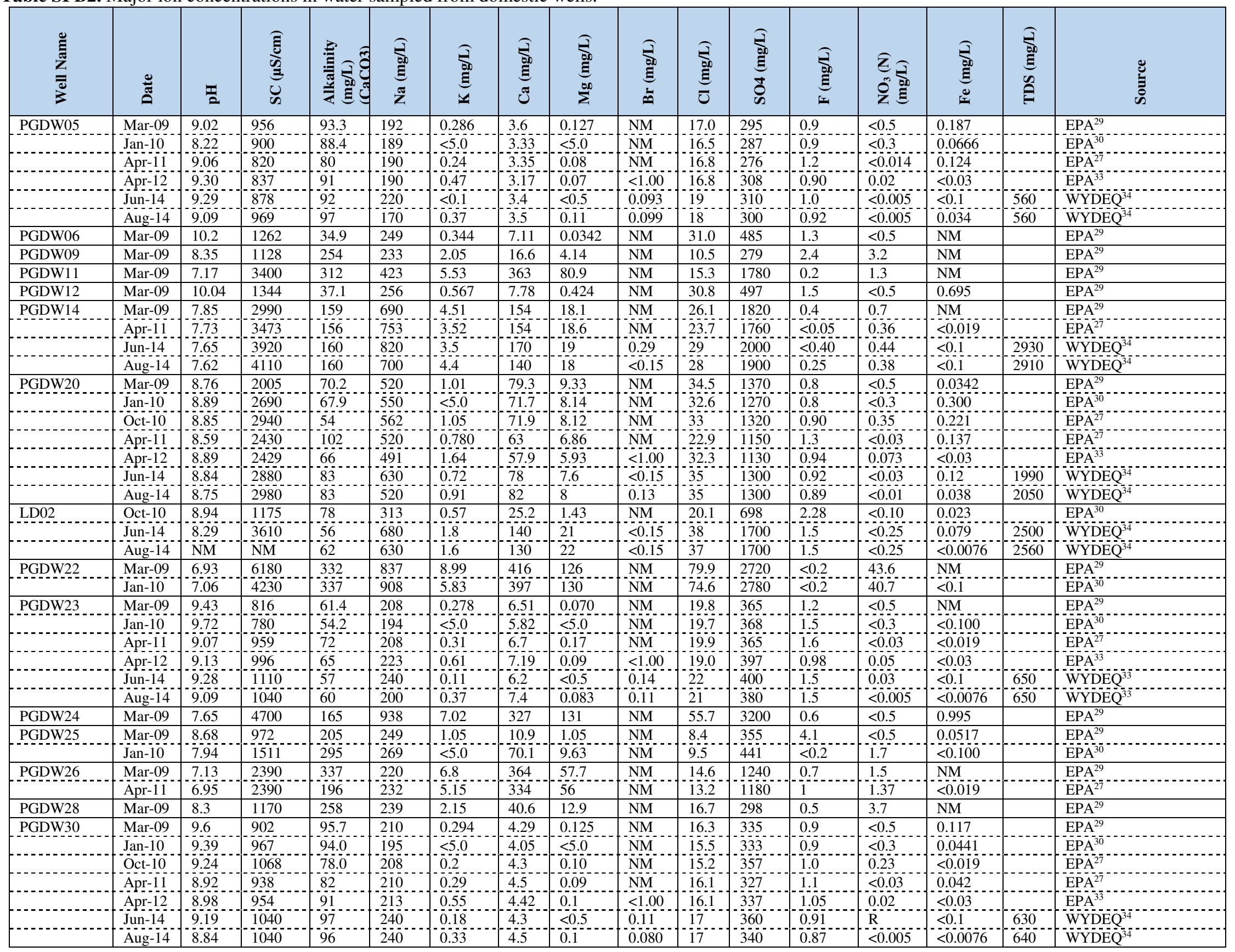




\begin{tabular}{|c|c|c|c|c|c|c|c|c|c|c|c|c|c|c|c|c|}
\hline 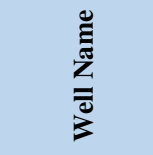 & 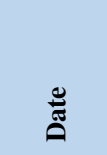 & 贾 & 氞 & 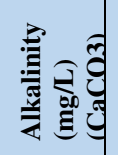 & 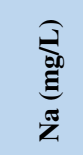 & 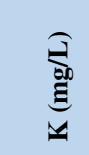 & שَ & 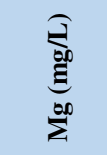 & & 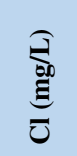 & 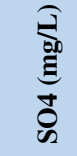 & 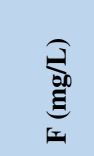 & 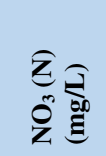 & 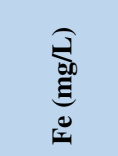 & 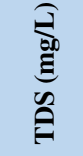 & 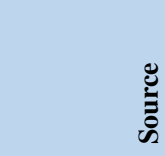 \\
\hline PGDW31 & Mar-09 & 8.6 & 2006 & 82.5 & 435 & 0.901 & 31.2 & 0.843 & NM & 13.3 & 1030 & 0.4 & 0.5 & NM & & $\mathrm{EPA}^{29}$ \\
\hline \multirow[t]{5}{*}{ PGDW32 } & Mar-09 & 10.47 & 908 & 34.1 & 199 & 0.267 & 7.16 & 0.017 & NM & 21.8 & 373 & 2.3 & $<0.5$ & 0.412 & & $\mathrm{EPA}^{29}$ \\
\hline & Jan-10 & 9.87 & 1018 & 31.5 & $193^{-}$ & $<5.0$ & 6.89 & $<5.0$ & NM & 21.4 & 368 & 2.4 & $<0.3$ & 0.125 & & $\mathrm{EPA}^{30}$ \\
\hline & Apr-11 & 9.3 & 885 & 46 & $198^{\circ}$ & 0.09 & 7.2 & 0.03 & $\mathrm{NM}$ & 18.8 & 361 & $2-$ & $<0.03$ & 0.024 & & $\mathrm{EPA}^{27}$ \\
\hline & Jun-14 & 9.97 & 1110 & 30 & $230^{\circ}$ & $<0.1$ & 7.4 & $<0.02$ & $<0.03$ & 24 & $400^{\circ}$ & $2.5^{-}$ & $<0.005$ & $<0.1$ & $620^{\circ}$ & WYDEQ ${ }^{34}$ \\
\hline & Aug-14 & $9.88^{-}$ & 1120 & 34 & $190^{-}$ & 0.42 & 8 & 0.038 & 0.086 & 22 & $380^{-}$ & 2.3 & $<0.005$ & $<0.0076^{-}$ & 640 & $\mathrm{WYDEQ}^{34}$ \\
\hline \multirow[t]{3}{*}{ PGDW33 } & Mar-09 & 7.77 & 1662 & 276 & 178 & 4.99 & 228 & 40.9 & NM & 23.0 & 2690 & 0.2 & 2.1 & NM & & $\mathrm{EPA}^{29}$ \\
\hline & Jun-14 & 7.05 & 1860 & 310 & $150^{\circ}$ & 3.8 & 200 & 39 & $<0.03$ & 20 & 700 & 0.65 & 3.2 & 0.087 & 1350 & WYDEQ $^{3 \overline{4}}$ \\
\hline & Aug-14 & $7.21^{-}$ & 1700 & 260 & $160^{\circ}$ & 3 & 150 & 27 & 0.043 & 18 & 590 & 0.98 & 2.0 & $<0.0076$ & 1180 & $\mathrm{WYDEQ}^{34}$ \\
\hline PGDW34 & Mar-09 & 7.87 & 4480 & 373 & 786 & 7.4 & 325 & 113 & NM & 28 & 670 & 0.5 & 3.5 & NM & & $\mathrm{EPA}^{29}$ \\
\hline PGDW35 & Mar-09 & 8.63 & 2810 & 84.0 & 587 & 1.09 & 118 & 1.1 & NM & 24.1 & 1610 & 0.3 & 0.5 & 1.10 & & $\mathrm{EPA}^{29}$ \\
\hline PGDW36 & Mar-09 & 7.62 & 649 & 232 & 41.7 & 2.58 & 89.5 & 28.9 & NM & 3.2 & 195 & 1.0 & 1.2 & NM & & $\mathrm{EPA}^{29}$ \\
\hline PGDW37 & Mar-09 & 8.14 & 819 & 342 & 187 & 0.887 & 12.1 & 1.3 & NM & 8.7 & 89.9 & 0.9 & 1.2 & NM & & $\mathrm{EPA}^{29}$ \\
\hline PGDW39 & Jan-10 & 7.79 & 6410 & 129 & 1110 & 5.28 & 389 & 147 & NM & 52.9 & 3640 & 0.3 & $<0.3$ & 0.33 & & $\mathrm{EPA}^{30}$ \\
\hline PGDW40 & Jan-10 & 9.06 & 1229 & 86.3 & 244 & $<5.0$ & 6.57 & $<<5.0$ & NM & 13.1 & 426 & $<0.2$ & $<0.3$ & 1.26 & & $\mathrm{EPA}^{30}$ \\
\hline \multirow[t]{2}{*}{ PGDW41A } & Jun-14 & 7.22 & 7680 & 320 & 1400 & 6.2 & 670 & 56 & $<0.30$ & 710 & 3200 & 0.15 & 21 & $<0.1$ & 5840 & WYDEQ $^{34}$ \\
\hline & Aug-14 & $7.07^{-}$ & 8080 & 340 & $1300^{-}$ & 5.4 & 580 & 54 & $<0.30$ & 850 & 3100 & 0.17 & 17 & 0.0090 & 6350 & WYDEQ \\
\hline \multirow[t]{4}{*}{ PGDW41B } & Jan-10 & 7.63 & 4470 & 108 & 1030 & 2.68 & 270 & 57.5 & NM & 31.4 & 2670 & 0.5 & $<0.3$ & 1.88 & & $\mathrm{EPA}^{30}$ \\
\hline & Apr-11 & $7.05^{-}$ & 4866 & 112 & 896 & 3.18 & 452 & 46.9 & NM & 97.6 & $2640^{\circ}$ & $<0.05$ & 17.5 & $<0.019$ & & $\mathrm{EPA}^{27}$ \\
\hline & Jun-14 & 7.07 & 5240 & 130 & $980^{-}$ & $4.3^{-1}$ & 290 & 55 & $<0.15$ & 35 & $2800^{-}$ & $0.44^{-}$ & $<0.03$ & 0.56 & 4170 & $\mathrm{WY}^{-3 Q^{33}}$ \\
\hline & Aug-14 & $7.61^{-}$ & 5270 & 140 & $920^{-}$ & 3.4 & $270^{-}$ & 61 & $<0.30$ & 34 & $2700^{-}$ & 0.39 & 0.18 & 0.55 & 4220 & $\mathrm{WYDEQ}^{33}$ \\
\hline PGDW42 & Jan-10 & 9.18 & 888 & 88.5 & 181 & $<5.0$ & 5.06 & $<5.0$ & NM & 13.2 & 311 & 1.0 & $<0.3$ & 0.0966 & & $\mathrm{EPA}^{30}$ \\
\hline PGDW43 & Jan-10 & 8.19 & 4410 & 113 & 911 & $<5.0$ & 208 & 13.7 & NM & 38.4 & 2470 & 0.4 & $<0.3$ & 0.403 & & $\mathrm{EPA}^{30}$ \\
\hline \multirow[t]{4}{*}{ PGDW44 } & Jan-10 & 8.13 & 4080 & 100 & 994 & $<5.0$ & 259 & 28.3 & NM & 39.5 & 2880 & 0.3 & $<0.3$ & 2.07 & & $\mathrm{EPA}^{30}$ \\
\hline & Apr-11 & $8.17^{-}$ & 4730 & 94 & $1060^{-}$ & 2.09 & 259 & $19.2^{-}$ & $\mathrm{NM}$ & 32.1 & $2900^{-}$ & $<0.05$ & $<0.03$ & 2.06 & & $\mathrm{EPA}^{27}$ \\
\hline & Jun-14 & 8.11 & 5680 & 81 & $1100^{-}$ & 2.2 & 290 & 20 & $<0.15$ & 45 & 3100 & 0.34 & $<0.03$ & 2.4 & 4420 & $\mathrm{WYDEQ}^{3 \overline{4}}$ \\
\hline & Âug-14 & 8.28 & 5900 & $80^{-}$ & $1000^{-}$ & 2.8 & 250 & 21 & $<0.15$ & $44^{--}$ & $2900^{-}$ & 0.22 & $<0.03$ & 3 & 4440 & $\mathrm{WYDEQ}^{34}$ \\
\hline \multirow[t]{4}{*}{ PGDW45 } & Jan-10 & 7.63 & 1103 & 379 & 59.4. & 2.61 & 138 & 31.2 & NM & 14.5 & 213 & 1.9 & 0.3 & $<0.100$ & & $\mathrm{EPA}^{30}$ \\
\hline & Apr-11 & 6.85 & 1085 & 364 & 61.6 & 2.81 & 159 & 34.5 & NM & 18.4 & 251 & 1.7 & 0.64 & $<0.019$ & & $\mathrm{EPA}^{27}$ \\
\hline & Jun-14 & 7.17 & 1310 & 350 & 97 & 2.7 & 160 & 35 & 0.19 & 31 & 330 & 2.0 & 1.4 & $<0.0076$ & 820 & $\mathrm{WYDEQ}^{34}$ \\
\hline & Aug-14 & 7.01 & 1150 & 360 & $79^{--}$ & 3 & 120 & 32 & 0.24 & 36 & $250^{\circ}$ & $1.7^{-}$ & 0.98 & $<0.0076$ & 740 & $\mathrm{WYDEQ}^{34}$ \\
\hline PGDW46 & Jan-10 & 7.79 & 855 & 329 & 91.1 & 1.81 & 90.3 & 9.89 & NM & 8.4 & 126 & 0.5 & 2.3 & $<0.100$ & & $\mathrm{EPA}^{30}$ \\
\hline PGDW47 & Jan-10 & 9.52 & 970 & 44.1 & 183 & $<5.0$ & 6.87 & $<5.0$ & NM & 21.6 & 330 & 1.5 & $<0.3$ & $<0.100$ & & $\mathrm{EPA}^{30}$ \\
\hline PGDW48 & Jan-10 & 8.21 & 3550 & 89.8 & 725 & $<5.0$ & 147 & 4.35 & NM & 24.1 & 1840 & 0.3 & $<0.3$ & 0.0491 & & $\mathrm{EPA}^{30}$ \\
\hline \multirow[t]{4}{*}{ PGDW49 } & Jan-10 & 7.66 & 5470 & 243 & 1210. & 11.4 & 486 & 153 & NM & 64.3 & 3160 & 0.4 & 7.7 & 11.4 & & $\mathrm{EPA}^{30}$ \\
\hline & Apr-11 & 7.34 & 5333 & 296 & $982^{-}$ & 966 & 417 & 127 & NM & 54.3 & $3200^{\circ}$ & $<0.05$ & 8.75 & 2.41 & & $\mathrm{EPA}^{27}$ \\
\hline & Jun-14 & $7.31^{-}$ & 5840 & 330 & $990^{-}$ & 10 & 430 & 140 & 0.20 & 68 & $3500^{-}$ & 0.58 & 6.0 & 0.084 & 5340 & $\mathrm{WY}^{3} \mathrm{Q}^{34}$ \\
\hline & Aug-14 & 7.09 & 6190 & 310 & $960^{\circ}$ & $11^{-}$ & $420^{\circ}$ & 130 & 0.19 & $61^{-1}$ & $3300^{-}$ & 0.46 & 4.4 & 0.18 & 5220 & WYDEQ $^{34}$ \\
\hline PGDW50 & Apr-12 & 8.04 & 5922 & 33 & 1290 & 3.04 & 314 & 12.8 & $<1.42$ & 57.8 & 3470 & $<0.20$ & $<0.008$ & $<0.03$ & & $\mathrm{EPA}^{33}$ \\
\hline \multirow[t]{2}{*}{ PGPW01 } & Mar-09 & 8.85 & 1016 & 60.6 & 213 & 0.287 & 8.85 & 0.0847 & NM & 15.7 & 390 & 1.2 & $<0.5$ & 0.0793 & & EPA $^{29}$ \\
\hline & Jan-10 & & & 74.7 & $173^{\circ}$ & $<5.0$ & $5.7^{-1}$ & $<5.0$ & $\mathrm{NM}$ & 15.3 & 300 & $1.2^{-}$ & $<0.3$ & 0.112 & & $\mathrm{EPA}^{30}$ \\
\hline \multirow[t]{3}{*}{ PGPW02 } & Mar-09. & 8.57 & 1883 & 82.9 & 390. & 0.643 & 36.7 & 0.240 & NM & 8.9 & 857 & 0.5 & $<0.5$ & 0.283 & & $\mathrm{EPA}^{29}$ \\
\hline & Jan-10 & & & 82.8 & 393 & $<5.0$ & 34.4 & $<5.0$ & $\mathrm{NM}$ & 8.5 & 847 & 0.5 & $<0.3$ & 0.255 & & $\mathrm{EPA}^{30}$ \\
\hline & Apr-12 & $8.46^{-}$ & 1856 & $77^{-1}$ & $41 \overline{4}^{-}$ & 0.92 & $34.7^{-}$ & $0.24^{-}$ & $<1.00$ & 8.51 & 886 & $<0.20$ & 0.14 & 0.11 & & $\mathrm{EPA}^{33}$ \\
\hline PGDW01 & Mar-09 & & & 234 & 808 & 6.15 & 398 & 93.6 & NM & 34.3 & 1860 & 0.4 & 6.2 & NM & & $\mathrm{EPA}^{29}$ \\
\hline
\end{tabular}




\begin{tabular}{|c|c|c|c|c|c|c|c|c|c|c|c|c|c|c|c|c|}
\hline 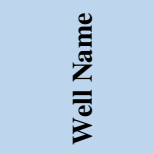 & 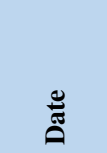 & 를 & 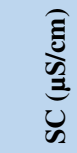 & 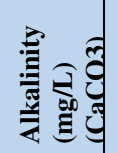 & 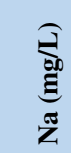 & 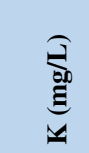 & త్రే & 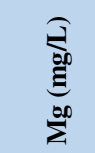 & 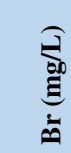 & 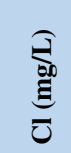 & 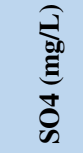 & 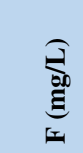 & 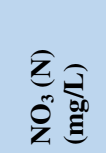 & 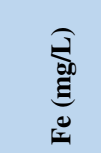 & 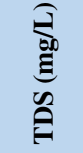 & : \\
\hline PGDW02 & Mar-09 & 8.11 & 551 & 108 & 85.8 & 1.8 & 34.8 & 5.32 & NM & 2.6 & 175 & 0.7 & $<0.5$ & NM & & $\mathrm{EPA}^{29}$ \\
\hline PGDW03 & Mar-09 & 9.37 & 1333 & 39.5 & 272 & 0.403 & 16.3 & 0.266 & NM & 25.1 & 549 & 0.9 & $<0.5$ & 0.0147 & & $\mathrm{EPA}^{29}$ \\
\hline & Jan-10 & 8.71 & 1390 & 28.0 & $251^{-}$ & $<5.0$ & $16.3^{-}$ & $<5.0$ & $\mathrm{NM}$ & 20.7 & $570^{-1}$ & 0.8 & $<0.3$ & $<0.100^{-}$ & & $\mathrm{EPA}^{30-}$ \\
\hline PGDW04 & Mar-09 & 9.17 & 1370 & 28.7 & 270 & 0.384 & 18 & 0.12 & NM & 21.6 & 551 & 0.9 & $<0.5$ & NM & & $\mathrm{EPA}^{29}$ \\
\hline & Jan-10 & 9.07 & 1388 & 38.3 & 265 & $<5.0$ & $15.5^{-}$ & $<5.0$ & $\mathrm{NM}$ & 23.3 & $532^{-}$ & 0.9 & $<0.3$ & $<0.100^{\circ}$ & & $\overline{\mathrm{E}} \mathrm{A}^{30}$ \\
\hline PGDW10 & Mar-09 & 8.95 & 948 & 147 & 204 & 0.394 & 6.13 & 0.133 & NM & 8.0 & 293 & 0.9 & $<0.5$ & NM & & $\mathrm{EPA}^{29}$ \\
\hline & Mar-09 & 8.62 & 985 & $147^{\cdots}$ & $204^{-1}$ & 0.400 & 6.10 & 0.132 & NM & 8.0 & $289^{\circ}$ & 0.9 & $<0.3$ & $\mathrm{NM}$ & & $\mathrm{EPA}^{29}$ \\
\hline & Jan-10 & 8.62 & 147 & $147^{\circ}$ & $195^{-}$ & $<5.0$ & 5.76 & $<5.0$ & $\mathrm{NM}$ & 7.5 & 293 & 0.9 & $<0.3$ & $<0.100^{-}$ & & $\mathrm{EPA}^{30}$ \\
\hline PGDW13 & Mar-09 & 6.89 & 1155 & 303 & 196 & 1.85 & 61 & 19.9 & NM & 6.2 & 343 & 0.7 & 1.0 & NM & & $\mathrm{EPA}^{29}$ \\
\hline PGDW15 & Mar-09 & 7.48 & 1728 & 277 & 269 & 1.24 & 72.2 & 10.2 & NM & 9.9 & 520 & 0.6 & 1.8 & 0.274 & & $\mathrm{EPA}^{29}$ \\
\hline PGDW16 & Mar-09 & 9.3 & 1011 & 145 & 188 & 0.312 & 6.42 & 0.0815 & NM & 13.4 & 258 & 0.8 & $<0.5$ & NM & & $\mathrm{EPA}^{29}$ \\
\hline PGDW17 & Mar-09 & 9.61 & 1490 & 21.2 & 278 & 0.418 & 21.2 & 0.527 & NM & 49.5 & 583 & 2.0 & $<0.5$ & 0.0204 & & $\mathrm{EPA}^{29}$ \\
\hline PGDW18 & Mar-09 & 8.87 & 2002 & 20.5 & 509 & 0.827 & 84.5 & 0.279 & NM & 27.0 & 1380 & 1.8 & 0.5 & NM & & $\mathrm{EPA}^{29^{-}}$ \\
\hline PGDW19 & Mar-09 & 7.75 & 707 & 291 & 194 & 1.36 & 29 & 3.24 & NM & 6.9 & 196 & 0.9 & 2.6 & NM & & $\mathrm{EPA}^{29}$ \\
\hline PGDW29 & Mar-09 & 9.72 & 1442 & 52.3 & 298 & 0.417 & 19.7 & 0.46 & NM & 24.6 & 596 & 0.9 & $<0.5$ & NM & & $\mathrm{EPA}^{29}$ \\
\hline PGDW38 & Mar-09 & 8.68 & 2030 & 46.9 & 373 & 2.28 & 70 & 2.3 & NM & 46.9 & 908 & 1.3 & 5.9 & 0.018 & & $\mathrm{EPA}^{29}$ \\
\hline unidentified & & & 1340 & & 210 & & & & & & 860 & & & & 884 & Gores \& Assoc. ${ }^{25}$ \\
\hline unidentified & & & & & & & & & & & 300 & & & & 580 & Gores \& Assoc. ${ }^{25}$ \\
\hline unidentified & & & 2180 & & 459 & & & & & & 990 & & & & 1540 & Gores \& Assoc. ${ }^{25}$ \\
\hline P14914P & & & 2810 & & 362 & & & & & & 945 & & & & 2150 & Gores \& Assoc. ${ }^{25}$ \\
\hline P98757W & & & 1261 & & 255 & & & & & & 439 & & & & 813 & Gores \& Assoc. ${ }^{25}$ \\
\hline unidentified & & & 1400 & & 203 & & & & & & 480 & & & & 994 & Gores \& Assoc. ${ }^{25}$ \\
\hline P58929W & & & 1250 & & & & & & & & & & & & 825 & Gores \& Assoc. ${ }^{25}$ \\
\hline P34345W & & & & & 190 & & & & & & 400 & & & & 680 & Gores \& Assoc. ${ }^{25}$ \\
\hline P59104W & & & & & 210 & & & & & & 460 & & & & 644 & Gores \& Assoc. ${ }^{25}$ \\
\hline unidentified & & & 974 & & 210 & & & & & & 345 & & & & 647 & Gores \& Assoc. ${ }^{25}$ \\
\hline P24507P & & & & & & & & & & & 2900 & & & & 4250 & Gores \& Assoc. ${ }^{25}$ \\
\hline unidentified & & & 913 & & 174 & & & & & & 320 & & & & 570 & Gores \& Assoc. ${ }^{25}$ \\
\hline unidentified & & & & & 447 & & & & & & 1110 & & & & 1750 & Gores \& Assoc. ${ }^{25}$ \\
\hline P97501W & & & & & 555 & & & & & & 1161 & & & & 4010 & Gores \& Assoc. ${ }^{25}$ \\
\hline unidentified & & & 457 & & 38 & & & & & & 67 & & & & 302 & Gores \& Assoc. ${ }^{25}$ \\
\hline P30217W & & & & & & & & & & & 2700 & & & & 4180 & Gores \& Assoc. ${ }^{25}$ \\
\hline unidentified & & & & & & & & & & & 827 & & & & 1290 & Gores \& Assoc. ${ }^{25}$ \\
\hline P182983W & & & 886 & & & & & & & & & & & & 585 & Gores \& Assoc. ${ }^{25}$ \\
\hline unidentified & & & 1140 & & & & & & & & & & & & 752 & Gores \& Assoc. ${ }^{25}$ \\
\hline P46362W & & & & & 140 & & & & & & 1100 & & & & 1550 & Gores \& Assoc. ${ }^{25}$ \\
\hline P62641W & & & & & 970 & & & & & & 2200 & & & & 3550 & Gores \& Assoc. ${ }^{25}$ \\
\hline unidentified & & & & & & & & & & & 2140 & & & & 3100 & Gores \& Assoc. ${ }^{25}$ \\
\hline unidentified & & & & & & & & & & & 642 & & & & 1300 & Gores \& Assoc. ${ }^{25}$ \\
\hline P53567W & & & & & 175 & & & & & & 84 & & & & 384 & Gores \& Assoc. ${ }^{25}$ \\
\hline P25636W & & & & & & & & & & & 750 & & & & 2070 & Gores \& Assoc. ${ }^{25}$ \\
\hline P110443 & & & 1539 & & 298 & & & & & & 570 & & & & 1010 & Gores \& Assoc. ${ }^{25}$ \\
\hline unidentified & & & & & 454 & & & & & & 620 & & & & 1800 & Gores \& Assoc. ${ }^{25}$ \\
\hline P28496W & & & & & & & & & & & 29 & & & & 288 & Gores \& Assoc. ${ }^{25}$ \\
\hline P26200W & & & & & & & & & & & 2610 & & & & 3880 & Gores \& Assoc. ${ }^{25}$ \\
\hline
\end{tabular}




\begin{tabular}{|c|c|c|c|c|c|c|c|c|c|c|c|c|c|c|c|c|}
\hline $\begin{array}{l}\text { Ẽ } \\
\text { ż } \\
\bar{\Xi} \\
z\end{array}$ & 气ี๊ & $\frac{\pi}{2}$ & 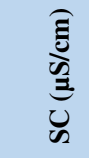 & 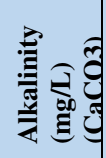 & 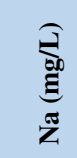 & 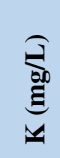 & 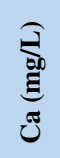 & 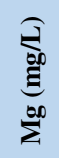 & 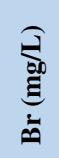 & 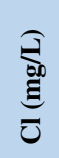 & 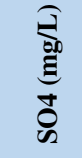 & 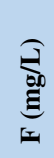 & 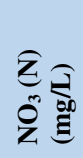 & 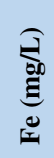 & 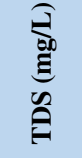 & 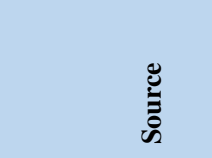 \\
\hline unidentified & & & 2160 & & 445 & & & & & & 988 & & & & 1530 & Gores \& Assoc. $^{25}$ \\
\hline unidentified & & & 3790 & & 339 & & & & & & 2310 & & & & 3510 & Gores \& Assoc. $^{25}$ \\
\hline $\mathrm{P} 40603 \mathrm{~W}$ & & & & & & & & & & & 210 & & & & 710 & Gores \& Assoc. ${ }^{25}$ \\
\hline P76475W & & & 1320 & & 260 & & & & & & 540 & & & & 808 & Gores \& Assoc. ${ }^{25}$ \\
\hline $\mathrm{P} 14548 \mathrm{P}$ & & & & & & & & & & & 1049 & & & & 1690 & Gores \& Assoc. $^{25}$ \\
\hline P30162W & & & & & & & & & & & 169 & & & & & Gores \& Assoc. $^{25}$ \\
\hline P32163W & & & & & & & & & & & 690 & & & & 1130 & Gores \& Assoc. $^{25}$ \\
\hline P9941P & & & 2720 & & 579 & & & & & & 1290 & & & & 2040 & Gores \& Assoc. $^{25}$ \\
\hline P116598W & & & & & 229 & & & & & & 119 & & & & 376 & Gores \& Assoc. ${ }^{25}$ \\
\hline unidentified & & & & & & & & & & & 296 & & & & 930 & Gores \& Assoc. ${ }^{25}$ \\
\hline $\mathrm{P} 25011 \mathrm{~W}$ & & & & & & & & & & & 2400 & & & & 3560 & Gores \& Assoc. $^{25}$ \\
\hline P177246W & & & 1180 & & 248 & & & & & & 457 & & & & 775 & Gores \& Assoc. ${ }^{25}$ \\
\hline & & & $1180^{-}$ & & $126^{-}$ & & & & & & 237 & & & & 590 & Gores \& Assoc. $^{25}$ \\
\hline P190223W & & & 920 & & & & & & & & & & & & 607 & Gores \& Assoc. ${ }^{25}$ \\
\hline P191733W & & & 956 & & & & & & & & & & & & 631 & Gores \& Assoc. ${ }^{25}$ \\
\hline
\end{tabular}

NM - not measured 


\section{C - Summary of Production Well Construction and Stimulation}

Table SI C1. Production well construction summary (listed in order of completion date). Information retrieved from well completion reports using API number

\begin{tabular}{|c|c|c|c|c|c|c|c|c|c|c|c|c|c|}
\hline 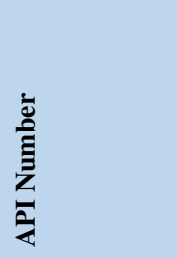 & $\begin{array}{l}\stackrel{\mathscr{E}}{\bar{E}} \\
\underline{Z} \\
\bar{\Xi} \\
z\end{array}$ & 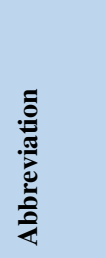 & 苞 & 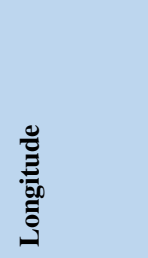 & 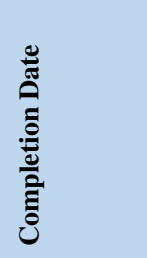 & 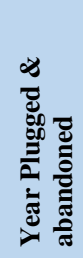 & 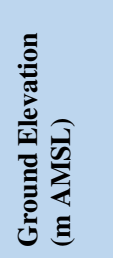 & 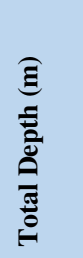 & 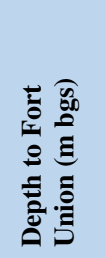 & 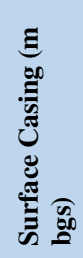 & 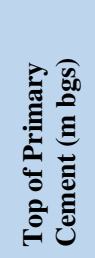 & 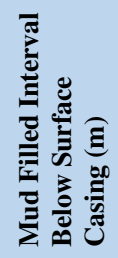 & 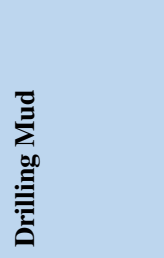 \\
\hline 49-013-06388 & Mae H. Rhodes 1 & MHR 1 & 43.26009 & -108.63519 & $12 / 31 / 1953$ & 1953 & 1642.0 & 3,353 & 1041.8 & 156.7 & 541 & 2812 & "Chem Gel" \\
\hline 49-013-06357 & Ora Wells 14-12 & $14-12$ & 43.24571 & -108.59549 & $8 / 7 / 1960$ & --- & 1622.8 & 1,983 & 1048.5 & 190.5 & 191 & 1 & Invert \\
\hline 49-013-06359 & Govt 23-7 & $23-7$ & 43.24841 & -108.57139 & $5 / 11 / 1961$ & 1961 & $1612.7^{\mathrm{G}}$ & 2,450 & $?$ & 176.2 & 1960 & 1784 & Q-Broxin Gel \\
\hline 49-013-06474 & Wirth 1 & W 1 & 43.29472 & -108.65019 & $10 / 22 / 1961$ & 1961 & 1680.7 & 2,899 & 984.5 & 200.9 & None $^{\mathrm{B}}$ & 2698 & "Gel" \\
\hline 49-013-06395 & Tribal Pavillion 23-2 & $23-2$ & 43.26371 & -108.61039 & $12 / 3 / 1962$ & --- & 1631.9 & 1,585 & 1027.5 & 195.1 & 195 & 0 & Invert \\
\hline 49-013-06451 & Shell Govt 22-35 & $22-35$ & 43.28179 & -108.61019 & $2 / 23 / 1963$ & 1963 & $1657.5^{\mathrm{G}}$ & 1,676 & ? & 189.6 & None $^{B}$ & 1486 & Invert \\
\hline $49-013-06358$ & Govt-Ocean Lake 1 & GOL 1 & 43.24649 & -108.52271 & $6 / 11 / 1963$ & 1974 & $1599.0^{\mathrm{G}}$ & 1,829 & 1219.2 & 177.7 & 533 & 355 & Invert \\
\hline 49-013-06389 & Tribal Pavillion 14-1 & $14-1$ & 43.26021 & -108.59541 & $6 / 18 / 1963$ & --- & 1631.6 & 1,254 & 1141.2 & 205.4 & 222 & 17 & Invert \\
\hline 49-013-06376 & Govt 21-8 & $21-8$ & 43.25711 & -108.55111 & $7 / 11 / 1963$ & $?$ & $1629.5^{\mathrm{G}}$ & 1,372 & 1096.7 & 189.3 & $?^{\mathrm{E}}$ & $?$ & Invert \\
\hline 49-013-06413 & $21-5$ & $21-5$ & 43.27111 & -108.55099 & $7 / 13 / 1963$ & --- & 1626.7 & 1,524 & 1125 & 192.3 & $655^{\mathrm{R}}$ & 463 & Invert \\
\hline 49-013-06341 & Unit 13-13 & U 13-13 & 43.23449 & -108.595 & $7 / 16 / 1963$ & --- & $1617.9^{\mathrm{G}}$ & 1,411 & ? & 190.2 & $?^{\mathrm{E}}$ & $?$ & Invert \\
\hline 49-013-06392 & Tribal Pavillion 14-6 & $14-6$ & 43.26056 & -108.57556 & $7 / 16 / 1963$ & --- & $1641.0^{\mathrm{G}}$ & 1,219 & $?$ & 184.7 & $?^{\mathrm{E}}$ & $?$ & Invert \\
\hline 49-013-06402 & Govt 32-4 & $32-4$ & 43.26739 & -108.52629 & $7 / 18 / 1963$ & 1963 & 1627.0 & 1,524 & $?$ & 188.1 & None $^{\mathrm{B}}$ & 1336 & Invert \\
\hline 49-013-06336 & Unit 24-14 & $24-14$ & 43.23231 & -108.60861 & $11 / 22 / 1963$ & ---- & 1617.0 & 1,159 & 1121.7 & 186.8 & 396 & 209 & Invert \\
\hline 49-013-06273 & Govt $44-20$ & $44-20$ & 43.21694 & -108.54119 & $3 / 16 / 1964$ & 1964 & $1642.2^{\mathrm{G}}$ & 1524 & $?$ & 187.4 & None $^{\mathrm{B}}$ & 1337 & Invert \\
\hline 49-013-06281 & Woodring 23X-24 & $23 X-24$ & 43.21879 & -108.59271 & $4 / 25 / 1964$ & 1964 & 1598.1 & 1,250 & ? & 184.4 & None $^{B}$ & 1066 & Invert \\
\hline 49-013-08017 & Runner Herfords 44-17 & $44-17$ & 43.23091 & -108.65981 & $5 / 23 / 1964$ & 1964 & 1631.9 & 1,292 & $?$ & 183.8 & None $^{B}$ & 1108 & Invert \\
\hline $49-013-06424$ & Govt 34-33 & $34-33$ & 43.27444 & -108.64449 & $5 / 27 / 1964$ & $?$ & $1652.9^{\mathrm{G}}$ & 1,558 & $?$ & 183.8 & $?^{\mathrm{E}}$ & $?$ & Invert \\
\hline $49-013-06363$ & Govt Tribal 33X-10 & $33 \mathrm{X}-10$ & 43.25019 & -108.62528 & $12 / 26 / 1964$ & 1983 & 1643.2 & 5,863 & 1025.7 & 67.4 & 4023 & $2787^{1}$ & Invert \\
\hline 49-013-06387 & Tribal Pavillion 24X-3 & $24 X-3$ & 43.26151 & -108.63161 & $11 / 30 / 1965$ & --- & 1641.7 & 1,492 & 1002.2 & 188.1 & $947^{\mathrm{R}}$ & 759 & Invert \\
\hline $49-013-06355$ & $14-11$ & $14-11$ & 43.24649 & -108.61341 & $12 / 10 / 1965$ & ---- & $1633.1^{\mathrm{G}}$ & 1,503 & $?$ & 185.3 & $?^{\mathrm{E}}$ & $?$ & Invert \\
\hline 49-013-06917 & IND 13-13 & $13-13$ & 43.27589 & -108.55431 & $1 / 20 / 1966$ & $?$ & $1637.1^{\mathrm{G}}$ & 1,177 & $?$ & 186.8 & $?^{\mathrm{R}}$ & $?$ & "Chem Gel" \\
\hline $49-013-20062$ & Tribal Pavillion 31-15 & $31-15$ & 43.24194 & -108.62519 & $2 / 26 / 1968$ & --- & 1632.2 & 1,050 & $?$ & 205.7 & $?^{\mathrm{N}}$ & $?$ & Invert \\
\hline 49-013-20084 & Maxson 32-9 & $32-9$ & 43.25278 & -108.64511 & $5 / 27 / 1968$ & 1968 & 1642.9 & 1,070 & 1057 & 187.8 & None $^{B}$ & 882 & Invert \\
\hline $49-013-20298$ & W. E. Lloyd & WEL & 43.26011 & -108.62528 & $2 / 23 / 1972$ & 1981 & 1639.2 & 4,747 & 1025.7 & 277.7 & $?^{\mathrm{N}}$ & $?$ & "Chem Gel" \\
\hline 49-013-20414 & Unit $41 \mathrm{X}-10$ & $41 \mathrm{X}-10$ & 43.25511 & -108.62201 & $1 / 29 / 1973$ & 1981 & 1635.6 & 1,538 & 1024.1 & 188.7 & $418^{\mathrm{P}}$ & 229 & Invert \\
\hline 49-013-20454 & IND 14-20-258 41X-2 & $41 X-2$ & 43.26944 & -108.60241 & $11 / 29 / 1973$ & --- & 1640.1 & 1,526 & 1072.9 & 185.0 & $?^{\mathrm{A}}$ & $?$ & Invert \\
\hline 49-013-20457 & Tribal Pavillion 31X-3 & $31 \mathrm{X}-3$ & 43.26949 & -108.62556 & $12 / 27 / 1973$ & --- & 1644.1 & 1,569 & 1028.4 & 188.4 & 35 & 0 & Invert \\
\hline 49-013-20442 & W.H. Paul Patent 42X-11 & $42 X-11$ & 43.25349 & -108.60231 & $1 / 19 / 1974$ & --- & 1627.9 & 1,533 & 1016.5 & 183.2 & $?^{\mathrm{A}}$ & $?$ & Invert \\
\hline $49-013-20443$ & Unit 42X-12 & $42 X-12$ & 43.25444 & -108.58139 & $1 / 19 / 1974$ & --- & 1631.0 & 1,509 & 1054.6 & 184.4 & $?^{\mathrm{A}}$ & $?$ & Invert \\
\hline 49-013-20456 & Unit $31 \mathrm{X}-14$ & $31 X-14$ & 43.24071 & -108.60569 & $1 / 19 / 1974$ & --- & 1629.2 & 1,569 & 1058 & 185.0 & $?^{\mathrm{A}}$ & $?$ & Invert \\
\hline 49-013-0491 & Clair C Day & CCD & 43.20167 & -108.67991 & $5 / 16 / 1974$ & 1974 & 1634.9 & 2445 & 1030.2 & 194.5 & None $^{B}$ & 2251 & Invert \\
\hline 49-013-20581 & TR1-22 & TR1-22 & 43.22741 & -108.62019 & $2 / 7 / 1976$ & 1986 & 1622.8 & 1,280 & 1104 & 186.5 & 793 & 607 & Invert \\
\hline 49-013-20586 & Tribal 1-21 & $1-21$ & 43.22741 & -108.63944 & $3 / 17 / 1976$ & 1976 & 1627.0 & 1,209 & 1173.5 & 190.5 & None $^{B}$ & 1019 & Invert \\
\hline 49-013-20598 & Taylor Patented 1 & TP 1 & 43.22806 & -108.61041 & $5 / 14 / 1976$ & $?$ & 1610.0 & 1,222 & ? & 127.4 & $?^{\mathrm{N}}$ & $?$ & Invert \\
\hline $49-013-20602$ & Tribal 1-31 & $1-31$ & 43.27409 & -108.56089 & $7 / 23 / 1976$ & 1977 & 1631.6 & 1,174 & 1138.1 & 125.3 & $?^{\mathrm{N}}$ & $?$ & Invert \\
\hline 49-013-20654 & Tribal Unit 1 & TU 1 & 43.22849 & -108.61409 & $11 / 3 / 1976$ & 1982 & 1613.3 & 1,119 & 1082.3 & 200.3 & $<549$ & $<349$ & Invert \\
\hline $49-013-20668$ & Blankenship Fee 4-8 & $4-8$ & 43.26556 & -108.64009 & $3 / 25 / 1977$ & --- & 1647.1 & 1,586 & $?$ & 135.3 & $564^{R, P}$ & 429 & Invert \\
\hline 49-013-20700 & Roland Patented 34-13 & $34-13$ & 43.27528 & -108.63379 & $8 / 31 / 1977$ & ? & 1615.4 & 1,981 & 1228.3 & 147.2 & $?^{\mathrm{N}}$ & $?$ & Invert \\
\hline 49-013-20748 & Shoshone-Arapahoe 24-11 & T24-11 & 43.24556 & -108.49179 & $5 / 13 / 1978$ & 1978 & $1608.1^{\mathrm{G}}$ & 4,564 & $?$ & 457.8 & None $^{\mathrm{B}}$ & 4106 & Invert \\
\hline
\end{tabular}




\begin{tabular}{|c|c|c|c|c|c|c|c|c|c|c|c|c|c|}
\hline 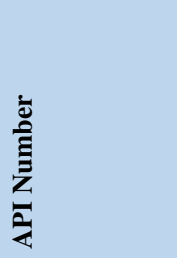 & $\begin{array}{l}\stackrel{\Xi}{\Xi} \\
\stackrel{\Xi}{\Xi} \\
\bar{\Xi}\end{array}$ & 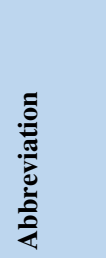 & : & 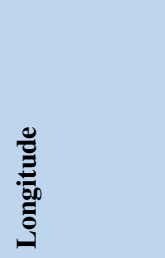 & 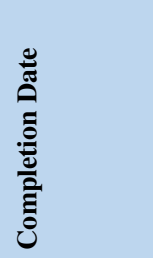 & 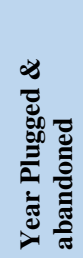 & 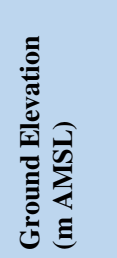 & 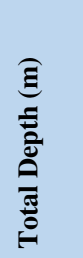 & 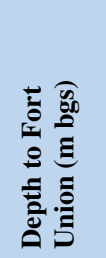 & 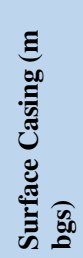 & 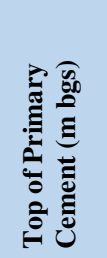 & 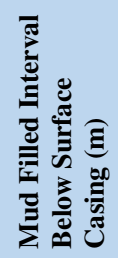 & 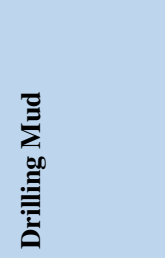 \\
\hline 49-013-20775 & Tribal 24-4 & $24-4$ & 43.22849 & -108.59569 & $5 / 22 / 1978$ & 1978 & 1622.1 & 1,216 & 1099.7 & 120.7 & None $^{B}$ & 1095 & Invert \\
\hline 49-013-20764 & Doles Unit 44-15 & $44-15$ & 43.23056 & -108.61959 & $6 / 2 / 1978$ & --- & 1629.5 & 1,638 & 1123.5 & 189.6 & 198 & 8 & Invert \\
\hline 49-013-20854 & Unit 21-11 & $21-11$ & 43.25521 & -108.61039 & $3 / 14 / 1979$ & --- & 1632.2 & 1,555 & 1021.1 & 187.5 & 466 & 279 & Invert \\
\hline 49-013-20855 & Tribal Pavillion 12-13 & $12-13$ & 43.239720 & -108.595768 & $5 / 12 / 1979$ & 2001 & 1624.0 & 1,646 & 1090.3 & 175.6 & 290 & 114 & Invert \\
\hline 49-013-20875 & USA Tribal $25841-9$ & $41-9$ & 43.25651 & -108.63981 & $8 / 24 / 1979$ & --- & 1641.3 & 1,585 & $?$ & 184.1 & 274 & 90 & Invert \\
\hline $49-013-08274$ & Tribal Unit 2 & TU 2 & 43.22841 & -108.60556 & $8 / 24 / 1979$ & 1979 & 1611.5 & 1,309 & $?$ & 199.6 & 838 & 638 & Invert \\
\hline 49-013-20876 & USA Tribal $25822-10$ & $22-10$ & 43.25194 & -108.63222 & 10/13/1979 & ---- & 1648.4 & 1,570 & 1024.1 & 185.3 & 550 & 365 & Invert \\
\hline $49-013-20965$ & Garrett 1 & G 1 & 43.24167 & -108.67261 & $10 / 31 / 1979$ & 1979 & 1644.1 & 1,675 & $?$ & 200.9 & None $^{B}$ & 1474 & Invert \\
\hline 49-013-20889 & USA Tribal 258 21-15 & $21-15$ & 43.24209 & -108.63139 & $12 / 6 / 1979$ & --- & 1632.8 & 1,585 & 1058 & 188.7 & $?^{A, R, P}$ & $?$ & Invert \\
\hline 49-013-20879 & Unit 44-10 & $44-10$ & 43.24649 & -108.62161 & $12 / 17 / 1979$ & --- & 1642.6 & 1,585 & 1027.2 & 184.4 & 585 & 401 & Invert \\
\hline $49-013-20878$ & Unit 22-12 & $22-12$ & 43.253096 & -108.593332 & $1 / 17 / 1980$ & --- & 1622.8 & 1,585 & 1033.6 & 178.6 & $?^{\mathrm{A}}$ & $?$ & Invert \\
\hline 49-013-20857 & Tribal Unit 238 11-14 & $11-14$ & 43.24151 & -108.61299 & $2 / 5 / 1980$ & --- & 1628.5 & 1,600 & 1031.1 & 184.1 & $?^{\mathrm{N}}$ & $?$ & Invert \\
\hline $49-013-21026$ & Tribal Pavillion 16-28 & $16-28$ & 43.29028 & -108.52409 & $7 / 16 / 1980$ & $?$ & $1612.7^{\mathrm{G}}$ & 1,386 & $?$ & 163.1 & $?^{\mathrm{N}}$ & $?$ & $\mathrm{KCl}$ polymer \\
\hline 49-013-21086 & Finayson 1-17 & F 1-17 & 43.23139 & -108.66556 & 9/7/1980 & 1980 & 1645.0 & 1,710 & 1121.7 & 190.5 & None $^{B}$ & 1520 & Invert \\
\hline $49-013-21128$ & Tribal Pavillion 14-2 & $14-2$ & 43.26021 & -108.61583 & $5 / 28 / 1981$ & --- & $1634.9^{\mathrm{G}}$ & 1,577 & 1007.1 & 182.6 & $677^{\mathrm{R}, \mathrm{P}}$ & 494 & Invert \\
\hline $49-013-21088$ & West Pavillion 1-8-1B & $1-8-1 \mathrm{~B}$ & 43.25619 & -108.66944 & $6 / 19 / 1981$ & 1981 & $1673.0^{\mathrm{G}}$ & 1,587 & $?$ & 183.5 & None $^{B}$ & 1404 & Invert \\
\hline 49-013-21130 & Tribal 21-9 & $21-9$ & 43.25639 & -108.65011 & $6 / 24 / 1981$ & 1992 & 1642.9 & 1,617 & 1007.7 & 248.4 & 318 & 70 & Invert \\
\hline $49-013-21129$ & Unit $12-3$ & $12-3$ & 43.26659 & -108.63569 & $7 / 8 / 1981$ & $-\ldots$ & $1644.4^{\mathrm{G}}$ & 1,590 & 1043.3 & 185.9 & $?^{\mathrm{A}}$ & $?$ & Invert \\
\hline 49-013-21157 & Runner Herfords 1 & RH 1 & 43.23056 & -108.65949 & $10 / 7 / 1981$ & 1981 & 1632.8 & 1,221 & 1118.6 & 185.9 & None $^{B}$ & 1035 & Invert \\
\hline 49-013-21087 & John K. Coolidge 1-4 & $1-4$ & 43.26306 & -108.64944 & $5 / 20 / 1982$ & ---- & 1647.7 & 1,143 & 1072.3 & 200.6 & $<396$ & $<195$ & Invert \\
\hline 49-013-21302 & Tribal 14-24 & $14-24$ & 43.21631 & -108.4725 & $9 / 22 / 1982$ & 1982 & 1575.8 & 2,698 & 1592.6 & 315.5 & $<579$ & $<264$ & $\mathrm{KCl}$ polymer \\
\hline 49-013-21346 & Fike Tribal A-1 & A-1 & 43.29259 & -108.64449 & $4 / 20 / 1983$ & --- & 1677.6 & 1,635 & 1010.1 & 184.7 & 515 & 330 & Invert \\
\hline $49-013-21312$ & Ocean Lake Tribal 1-15 & $1-15$ & 43.23789 & -108.51091 & $4 / 24 / 1983$ & 1990 & 1583.4 & 4,807 & 1143 & 304.8 & $<1189$ & $<884$ & Invert \\
\hline 49-013-21421 & Tribal B-1 & B-1 & 43.29539 & -108.65472 & $9 / 19 / 1984$ & 1984 & 1671.5 & 1,674 & $?$ & 181.4 & $?^{\mathrm{A}}$ & $?$ & Invert \\
\hline 49-013-21670 & Pavillion Fee 33-11 & $33-11$ & 43.24846 & -108.6039 & 9/15/1993 & ---- & 1627.6 & 1,539 & 1051.6 & 190.8 & $<91$ & 0 & $\mathrm{KCl}$ polymer \\
\hline $49-013-21669$ & Pavillion Fee 12-11 & $12-11$ & 43.25227 & -108.61483 & $10 / 19 / 1993$ & --- & 1632.8 & 1,976 & 1005.8 & 191.1 & 268 & 77 & $\mathrm{KCl}$ polymer \\
\hline 49-013-21676 & Tribal Pavillion 11-10 & $11-10$ & 43.25648 & -108.6348 & $12 / 29 / 1993$ & --- & 1642.0 & 2,463 & 1044.5 & 190.5 & 792 & 602 & $\mathrm{KCl}$ polymer \\
\hline $49-013-21696$ & Tribal Pavillion 42-10 & $42-10$ & 43.254231 & -108.620931 & $6 / 15 / 1994$ & --- & 1636.2 & 1,827 & 1009.5 & 190.8 & $789^{\mathrm{R}(3)}$ & 598 & $\mathrm{KCl}$ polymer \\
\hline 49-013-21691 & Tribal Pavillion 31-10 & $31-10$ & 43.256365 & -108.625138 & $6 / 25 / 1994$ & --- & 1637.4 & 1,820 & 1008.9 & 182.3 & 644 & 462 & $\mathrm{KCl}$ polymer \\
\hline 49-013-21692 & Tribal Pavillion 23-10 & $23-10$ & 43.24889 & -108.63068 & $7 / 15 / 1994$ & --- & 1647.7 & 1,829 & 1042.4 & 184.4 & $?^{\mathrm{T}}$ & $?$ & $\mathrm{KCl}$ polymer \\
\hline 49-013-21704 & Tribal Pavillion 43-10 & $43-10$ & 43.249234 & -108.621267 & $9 / 21 / 1994$ & --- & 1639.2 & 1,815 & 1013.8 & 195.4 & $?^{\mathrm{A}}$ & $?$ & $\mathrm{KCl}$ polymer \\
\hline 49-013-21693 & Tribal Pavillion 33-2 & $33-2$ & 43.26208 & -108.60522 & $11 / 8 / 1994$ & --- & 1631.6 & 1,676 & 1026.6 & 192.0 & 61 & 0 & $\mathrm{KCl}$ polymer \\
\hline 49-013-21697 & Tribal Pavillion 23-1 & $23-1$ & 43.26396 & -108.59019 & 11/11/1994 & $-\ldots$ & 1651.7 & 1,617 & 1077.8 & 182.9 & 594 & 411 & $\mathrm{KCl}$ polymer \\
\hline $49-013-21695$ & Tribal Pavillion 41-15 & $41-15$ & 43.24277 & -108.61865 & $11 / 23 / 1994$ & --- & 1631.0 & 1,989 & 1038.1 & 185.0 & $?^{\mathrm{N}}$ & $?$ & $\mathrm{KCl}$ polymer \\
\hline 49-013-21720 & Tribal-Pavillion 43-6 & $43-6$ & 43.26299 & -108.5767 & $5 / 26 / 1995$ & --- & 1621.2 & 1,219 & 1108.3 & 128.0 & $?^{\mathrm{A}}$ & $?$ & $\mathrm{KCl}$ polymer \\
\hline 49-013-21806 & Tribal Pavillion 23-11 & $23-11$ & 43.25071 & -108.60653 & $2 / 9 / 1998$ & --- & 1631.0 & 1,689 & 1013.2 & $\begin{array}{l}120.0 \\
167.0\end{array}$ & $<152$ & 0 & KCl polymer \\
\hline $49-013-21834$ & Tribal NP 31-11X & $31-11 \mathrm{X}$ & 43.2848 & -108.57413 & $11 / 19 / 1998$ & --- & 1677.6 & 4,359 & 1158.2 & 705.9 & $?^{\mathrm{E}}$ & $?$ & $\mathrm{KCl}$ polymer \\
\hline 49-013-21904 & Pavillion 13X-3 & $13 X-3$ & 43.26333 & -108.63417 & $1 / 18 / 1999$ & 1999 & 1630.7 & 1,842 & 1038.1 & 192.0 & $?^{\mathrm{T}}$ & $?$ & PHPA/LSND \\
\hline 49-013-21905 & Pavillion Fee 42X-9 & $42 X-9$ & 43.25278 & -108.63806 & $2 / 11 / 1999$ & --- & 1647.1 & 1,554 & 1055.8 & 185.9 & 518 & 332 & LSND \\
\hline 49-013-21866 & Pavillion Fee 41-11 & $41-11$ & 43.25556 & -108.6000 & $2 / 12 / 1999$ & ---- & 1627.3 & 1,554 & 1032.7 & 169.2 & 76 & 0 & LSND \\
\hline $49-013-21862$ & Tribal Pavillion 33-10 & $33-10$ & 43.248612 & -108.626454 & $5 / 6 / 1999$ & ---- & 1667.3 & 1,820 & 1055.2 & 156.7 & 207 & 50 & PHPA/LSND \\
\hline 49-013-21907 & Tribal Pavillion 33-3 & $33-3$ & 43.26306 & -108.62556 & $5 / 25 / 1999$ & ---- & 1642.0 & 1,190 & 1022.3 & 154.5 & $?^{\mathrm{T}}$ & $?$ & PHPA \\
\hline $49-013-21906$ & Tribal Pavillion 44-3 & $44-3$ & 43.25944 & -108.62111 & $6 / 29 / 1999$ & ---- & 1638.9 & 1,832 & 1010.4 & 171.6 & $671^{\mathrm{R}}$ & 499 & PHPA/LSND \\
\hline $49-013-21840$ & Tribal Pavillion 15-21X & $15-21 X$ & 43.24055 & -108.62952 & $7 / 2 / 1999$ & ---- & 1638.3 & 4,919 & 1066.8 & 244.8 & $?^{\mathrm{T}}$ & $?$ & Invert \\
\hline 49-013-21968 & Tribal Pavillion 32-10 & $32-10$ & 43.253238 & -108.630100 & $5 / 10 / 2000$ & ---- & 1643.2 & 1,707 & 1005.8 & 190.8 & 610 & 419 & LSND \\
\hline
\end{tabular}




\begin{tabular}{|c|c|c|c|c|c|c|c|c|c|c|c|c|c|}
\hline 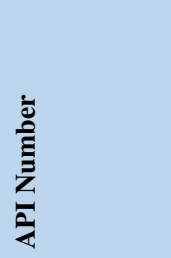 & 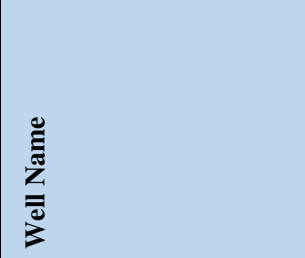 & 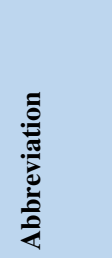 & 芯 & 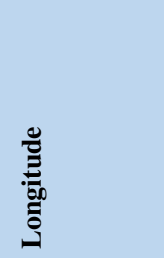 & 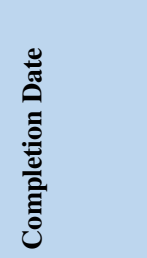 & 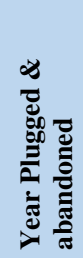 & 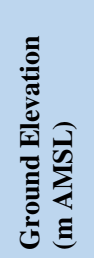 & 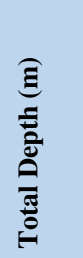 & 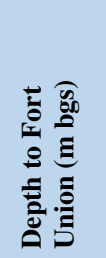 & 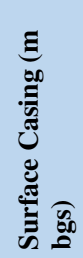 & 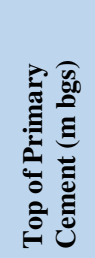 & 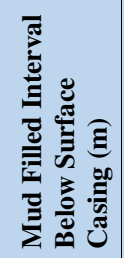 & 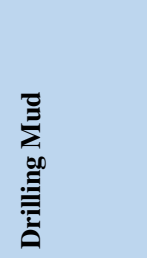 \\
\hline $49-013-22058$ & Pavillion Fee 13-11 & $13-11$ & 43.24861 & -108.61306 & $2 / 23 / 2001$ & ---- & 1630.1 & 1,768 & 1005.2 & 186.5 & $<244$ & $<58$ & PHPA \\
\hline 49-013-22057 & Pavillion Fee 21-13 & $21-13$ & 43.24083 & -108.58861 & $2 / 23 / 2001$ & ---- & 1620.0 & 1,067 & ? & 134.7 & $?^{\mathrm{N}}$ & ? & DeepDrill ${ }^{\circ}$ \\
\hline 49-013-22068 & \begin{tabular}{|c|} 
Tribal Pavillion 43-2 \\
\end{tabular} & $43-2$ & 43.26333 & -108.59972 & $3 / 1 / 2001$ & ---- & 1634.6 & 1,036 & $?$ & 109.7 & $<107$ & 0 & Unknown $^{R}$ \\
\hline 49-013-22070 & \begin{tabular}{|l} 
Tribal-Pavillion 13-2 \\
\end{tabular} & $13-2$ & 43.26371 & -108.6158 & $3 / 8 / 2001$ & ---- & 1633.7 & 1,036 & ? & 123.1 & 198 & 75 & DeepDrill ${ }^{\circ}$ \\
\hline 49-013-22101 & Tribal-Pavillion 24-1 & $24-1$ & 43.261667 & -108.59056 & $3 / 23 / 2001$ & ---- & 1637.7 & 1,036 & $?$ & 125.0 & 122 & 0 & LSND \\
\hline 49-013-22026 & Tribal-Pavillion 32-1 & $32-1$ & 43.26722 & -108.58278 & $3 / 23 / 2001$ & ---- & 1652.6 & 1,030 & $?$ & 109.7 & 182.9 & 73 & LSND \\
\hline 49-013-22072 & Tribal Pavillion 34-2 & $34-2$ & 43.26023 & -108.60533 & $3 / 24 / 2001$ & ---- & 1632.2 & 1,036 & 1022.9 & 118.0 & 15 & 0 & LSND \\
\hline 49-013-22069 & Tribal Pavillion 12-6 & $12-6$ & 43.2675 & -108.57555 & $3 / 29 / 2001$ & ---- & 1620.0 & 1,097 & 1085.7 & 120.4 & $?^{\mathrm{N}}$ & $?$ & DeepDrill \\
\hline $49-013-22027$ & Tribal Pavillion 44-1 & $44-1$ & 43.26027 & -108.58027 & $3 / 31 / 2001$ & --- & 1638.0 & 1,067 & $?$ & 111.9 & $<122$ & $<10$ & LSND \\
\hline 49-013-22099 & Tribal Pavillion 14-10 & $14-10$ & 43.245833 & -108.635 & $4 / 13 / 2001$ & --- & 1640.7 & 1,067 & 974.75 & 110.3 & 442 & 332 & DeepDrill ${ }^{\circ}$ \\
\hline 49-013-22104 & Pavillion Fee 13-15 & $13-15$ & 43.23583 & -108.63389 & $4 / 14 / 2001$ & 2006 & 1632.5 & 1,113 & $?$ & 132.9 & $?^{\mathrm{N}}$ & $?$ & PHPA \\
\hline 49-013-22102 & Pavillion Fee $12-11 \mathrm{~W}$ & $12-11 \mathrm{~W}$ & 43.25194 & -108.615 & $5 / 3 / 2001$ & --- & 1632.5 & 991 & $?$ & 131.1 & $594^{\mathrm{R}}$ & 463 & LSND \\
\hline 49-013-22087 & Pavillion Fee 34-3R & $34-3 \mathrm{R}$ & 43.26028 & -108.62528 & $5 / 4 / 2001$ & --- & 1638.6 & 1,097 & 1015.0 & 110.3 & 668 & 558 & PHPA \\
\hline $49-013-22059$ & Pavillion Fee 11-11 & $11-11$ & 43.255122 & -108.613706 & $5 / 17 / 2001$ & --- & 1632.2 & 1,782 & 1001.6 & 161.8 & $<91^{\mathrm{R}}$ & 0 & PHPA \\
\hline 49-013-22061 & Tribal Pavillion 12-5 & $12-5$ & 43.2675 & -108.55555 & $5 / 23 / 2001$ & --- & 1621.5 & 1,058 & $?$ & 112.2 & 494 & 382 & PHPA \\
\hline $49-013-22060$ & Pavillion Fee 13-12 & $13-12$ & 43.250891 & -108.593728 & $6 / 1 / 2001$ & $-\ldots$ & 1622.5 & 998 & $?$ & 99.7 & 280 & 180 & LSND \\
\hline 49-013-22103 & Pavillion Fee 34-11 & $34-11$ & 43.24514 & -108.60531 & $6 / 13 / 2001$ & --- & 1628.5 & 1,024 & $?$ & 161.5 & $?^{\mathrm{A}}$ & $?$ & "gel" \\
\hline $49-013-22128$ & Tribal Pavillion 13-1 & $13-1$ & 43.26433 & -108.59553 & $6 / 28 / 2001$ & --- & 1652.3 & 1,071 & $?$ & 163.1 & $?^{\mathrm{N}}$ & $?$ & LSND \\
\hline 49-013-22105 & Tribal Pavillion 11-12 & $11-12$ & 43.25639 & -108.59422 & $8 / 3 / 2001$ & --- & 1626.7 & 970 & $?$ & 164.3 & 122 & 0 & LSND \\
\hline 49-013-22125 & Pavillion Fee 21-10 & $21-10$ & 43.25625 & -108.63078 & $8 / 10 / 2001$ & --- & 1641.0 & 1,807 & 1015 & 201.2 & 692 & 491 & LSND \\
\hline $49-013-22145$ & Tribal Pavillion 43-1 & $43-1$ & 43.26378 & -108.58139 & $9 / 1 / 2001$ & --- & 1648.7 & 1,128 & $?$ & 162.2 & 15 & 0 & PHPA \\
\hline 49-013-22144 & \begin{tabular}{|l} 
Tribal Pavillion 33-1 \\
\end{tabular} & $33-1$ & 43.26381 & -108.58536 & $9 / 6 / 2001$ & --- & 1646.8 & 1,059 & $?$ & 163.1 & 15 & 0 & LSND \\
\hline $49-013-22126$ & Tribal Pavillion 12-7 & $12-7$ & 43.25265 & -108.57613 & $9 / 8 / 2001$ & --- & 1621.8 & 1,021 & $?$ & 164.0 & $?^{\mathrm{N}}$ & $?$ & LSND \\
\hline $49-013-22188$ & Pavillion Fee 21-10W & $21-10 \mathrm{~W}$ & 43.25625 & -108.63039 & $9 / 15 / 2001$ & --- & 1640.4 & 1,341 & 1011.9 & 189.0 & 442 & 253 & LSND \\
\hline 49-013-22186 & Pavillion Fee 23-12 & $23-12$ & 43.250699 & -108.589188 & 9/19/2001 & --- & 1621.5 & 994 & $?$ & 162.8 & 15 & 0 & LSND \\
\hline 49-013-22147 & Tribal Pavillion 44-2 & $44-2$ & 43.26011 & -108.60217 & $9 / 22 / 2001$ & --- & 1634.3 & 1,250 & 1021.1 & 162.8 & $?^{\mathrm{A}}$ & $?$ & $?$ \\
\hline $49-013-22172$ & Pavillion Fee 31-9 & $31-9$ & 43.25686 & -108.64486 & $9 / 28 / 2001$ & --- & 1645.6 & 1,050 & $?$ & 162.8 & 61 & 0 & LSND \\
\hline 49-013-22149 & Tribal Pavillion 34-10 & $34-10$ & 43.24658 & -108.62526 & $10 / 6 / 2001$ & --- & 1646.5 & 1,747 & 1046.4 & 197.5 & $<188$ & 0 & LSND \\
\hline 49-013-22146 & \begin{tabular}{|l} 
Tribal-Pavillion 34-1 \\
\end{tabular} & $34-1$ & 43.26083 & -108.58639 & $10 / 13 / 2001$ & --- & 1639.8 & 1,020 & $?$ & 162.8 & $?^{\mathrm{A}}$ & $?$ & LSND \\
\hline $49-013-22150$ & Tribal Pavillion 21-12 & $21-12$ & 43.25639 & -108.59114 & $10 / 20 / 2001$ & --- & 1629.2 & 1,128 & $?$ & 107.3 & 159 & 52 & LSND \\
\hline 49-013-22141 & Tribal Pavillion 12-10 & $12-10$ & 43.25167 & -108.63556 & $11 / 7 / 2001$ & --- & 1645.0 & 1,496 & $?$ & 189.6 & 31 & 0 & LSND \\
\hline 49-013-22181 & \begin{tabular}{|l} 
Tribal Pavillion 12-1 \\
\end{tabular} & $12-1$ & 43.26778 & -108.59169 & $11 / 10 / 2001$ & --- & 1642.0 & 1,128 & ? & 162.8 & 73 & 0 & LSND \\
\hline 49-013-22191 & Tribal Pavillion 42-3 & $42-3$ & 43.26778 & -108.61922 & $11 / 16 / 2001$ & --- & 1641.0 & 1,227 & 1035.4 & 162.8 & $<61$ & 0 & LSND \\
\hline $49-013-22180$ & Tribal-Pavillion 22-1 & $22-1$ & 43.26725 & -108.59011 & $11 / 21 / 2001$ & --- & 1657.2 & 1,128 & $?$ & 162.5 & 46 & 0 & LSND \\
\hline 49-013-22195 & Tribal Pavillion 33-10W & $33-10 \mathrm{~W}$ & 43.248702 & -108.626805 & $12 / 6 / 2001$ & --- & 1668.2 & 1,545 & 1054.6 & 160.9 & 204 & 43 & LSND \\
\hline $49-013-22153$ & \begin{tabular}{|l|} 
Tribal Pavillion 41-3 \\
\end{tabular} & $41-3$ & 43.27056 & -108.62056 & $12 / 7 / 2001$ & --- & 1643.8 & 1,228 & 1037.2 & 157.6 & $?^{\mathrm{A}}$ & $?$ & LSND \\
\hline 49-013-22215 & Pavillion Fee 22-11 & $22-11$ & 43.25283 & -108.61128 & $12 / 15 / 2001$ & --- & 1632.2 & 972 & $?$ & 189.0 & $?^{\mathrm{A}}$ & $?$ & LSND \\
\hline $49-013-22200$ & Pavillion Fee 43-11 & $43-11$ & 43.25089 & -108.60044 & $12 / 22 / 2001$ & --- & 1627.0 & 1,219 & 1026.3 & 162.5 & $<122$ & 0 & LSND \\
\hline 49-013-22212 & Pavillion Fee 11-3 & $11-3$ & 43.27083 & -108.63369 & $1 / 9 / 2002$ & --- & 1647.7 & 1,216 & 1032.4 & 160.9 & $?^{\mathrm{N}}$ & ? & LSND \\
\hline 49-013-22198 & Pavillion Fee 41-10 & $41-10$ & 43.255851 & -108.620913 & $1 / 23 / 2002$ & --- & 1637.4 & 969 & ? & 162.8 & 165 & 2 & LSND \\
\hline $49-013-22214$ & Pavillion Fee 13-12W & $13-12 \mathrm{~W}$ & 43.2485 & -108.59681 & $1 / 26 / 2002$ & --- & 1624.3 & 1,227 & 1044.9 & 159.7 & 0 & 0 & PHPA \\
\hline 49-013-22229 & Pavillion Fee 24-3B & $24-3 B$ & 43.25947 & -108.63089 & $2 / 8 / 2002$ & --- & 1641.3 & 1,731 & 1012.9 & 200.9 & $<30$ & 0 & LSND \\
\hline $49-013-22220$ & Pavillion Fee 11-11B & 11-11B & 43.25697 & -108.61594 & $2 / 16 / 2002$ & --- & 1635.3 & 1,457 & 1004.3 & 170.7 & $<152$ & 0 & LSND \\
\hline $49-013-22106$ & Pavillion Fee 31-11 & $31-11$ & 43.25703 & -108.60528 & $2 / 22 / 2002$ & --- & 1629.5 & 1,207 & 1019.6 & 165.8 & $<15$ & 0 & LSND \\
\hline 49-013-22207 & Pavillion Fee 23-3 & $23-3$ & 43.26411 & -108.62944 & $2 / 27 / 2002$ & --- & 1644.4 & 1,890 & 1022 & 189.0 & $?^{\mathrm{A}}$ & $?$ & $?$ \\
\hline
\end{tabular}




\begin{tabular}{|c|c|c|c|c|c|c|c|c|c|c|c|c|c|}
\hline 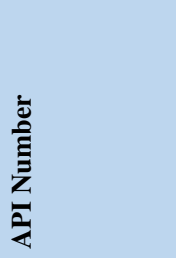 & $\begin{array}{l}\stackrel{\Xi}{\Xi} \\
\text { ż } \\
\text { z }\end{array}$ & 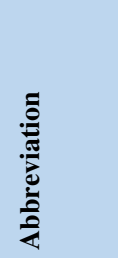 & 菢 & 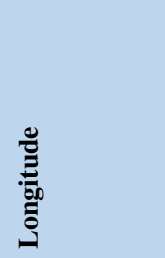 & 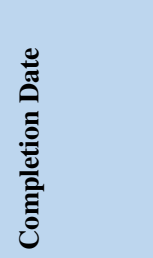 & 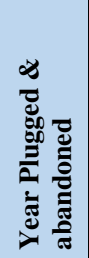 & 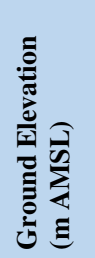 & 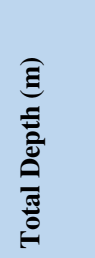 & 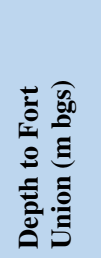 & 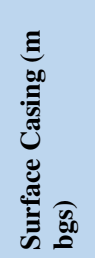 & 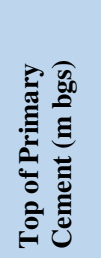 & 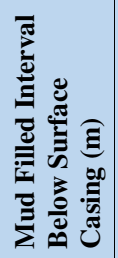 & 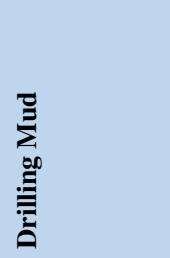 \\
\hline $49-013-22223$ & Pavillion Fee 32-11 & $32-11$ & 43.25356 & -108.60664 & $3 / 2 / 2002$ & --- & 1630.7 & 1,219 & 1016.8 & 175.0 & 0 & 0 & LSND \\
\hline 49-013-22199 & Pavillion Fee 44-11 & $44-11$ & 43.24513 & -108.60059 & $3 / 6 / 2002$ & --- & 1627.0 & 1,241 & 1036.3 & 162.2 & $?^{\mathrm{A}}$ & $?$ & LSND \\
\hline $49-013-22228$ & Pavillion Fee 42-9W & $42-9 \mathrm{~W}$ & 43.25308 & -108.64158 & $3 / 21 / 2002$ & --- & 1642.9 & 1,042 & $?$ & 161.8 & 0 & 0 & LSND \\
\hline 49-013-22227 & Pavillion Fee 32-9W & $32-9 \mathrm{~W}$ & 43.25408 & -108.64472 & $3 / 22 / 2002$ & --- & 1645.6 & 1,055 & $?$ & 162.2 & 0 & 0 & LSND \\
\hline 49-013-22224 & Tribal Pavillion 32-10B & $32-10 \mathrm{~B}$ & 43.25328 & -108.62539 & $3 / 28 / 2002$ & --- & 1638.0 & 1,783 & 1012.9 & 189.6 & 55 & 0 & LSND \\
\hline $49-013-22240$ & Pavillion Fee 13-11B & 13-11B & 43.24961 & -108.61672 & $4 / 9 / 2002$ & --- & 1633.7 & 1,725 & 1016.2 & 188.1 & $625^{\mathrm{R}(4)}$ & 437 & LSND \\
\hline 49-013-22219 & Pavillion Fee 14-3W & $14-3 \mathrm{~W}$ & 43.26072 & -108.63575 & $4 / 13 / 2002$ & --- & 1644.7 & 1,737 & 1027.5 & 189.0 & 0 & 0 & LSND \\
\hline $49-013-22182$ & Tribal-Pavillion $12-2$ & $12-2$ & 43.26694 & -108.61506 & $12 / 1 / 2002$ & --- & 1634.9 & 1,128 & 1036.3 & 159.1 & 30.5 & 0 & LSND \\
\hline 49-013-22217 & Tribal-Pavillion 23-10W & $23-10 \mathrm{~W}$ & 43.24886 & -108.63153 & $12 / 19 / 2002$ & --- & 1648.4 & 1,399 & 1041.8 & 162.8 & 0 & 0 & LSND \\
\hline 49-013-22418 & Tribal Pavillion 23-10C & $23-10 \mathrm{C}$ & 43.24789 & -108.62947 & $10 / 13 / 2004$ & $-\ldots$ & 1656.1 & 1,747 & $?$ & 175.6 & $?$ & $?$ & LSND \\
\hline $49-013-22270$ & Pavillion Fee 34-3B & $34-3 B$ & 43.26033 & -108.62783 & $10 / 24 / 2004$ & --- & 1640.1 & 1,829 & 1005.8 & 196.6 & $838^{\mathrm{R}}$ & 641 & LSND \\
\hline 49-013-22236 & Tribal Pavillion 24-2 & $24-2$ & 43.259882 & -108.610983 & $11 / 4 / 2004$ & --- & 1636.2 & 1,202 & 1020.8 & 171.3 & $213^{\mathrm{R}}$ & 42 & LSND \\
\hline $49-013-22417$ & Tribal Pavillion 23-10B & $23-10 \mathrm{~B}$ & 43.25047 & -108.63122 & $11 / 5 / 2004$ & --- & 1649.0 & 1,721 & 1036.3 & 195.4 & 299 & 104 & LSND \\
\hline 49-013-22274 & Tribal Pavillion 33-10B & $33-10 \mathrm{~B}$ & 43.249535 & -108.624267 & $12 / 14 / 2004$ & --- & 1638.0 & 1,710 & 1024.1 & 194.8 & 61 & 0 & LSND \\
\hline 49-013-22272 & Pavillion Fee 12-11B & 12-11B & 43.254237 & -108.615986 & $1 / 15 / 2005$ & --- & 1634.3 & 1,177 & 1001.9 & 167.9 & $311^{\mathrm{R}}$ & 143 & LSND \\
\hline $49-013-22420$ & Tribal Pavillion 43-10B & 43-10B & 43.247613 & -108.620928 & $1 / 20 / 2005$ & --- & 1657.8 & 1,756 & 1036.3 & 193.9 & $357^{\mathrm{R}, \mathrm{P}}$ & 163 & LSND \\
\hline 49-013-22222 & Tribal Pavillion 24-11 & $24-11$ & 43.24517 & -108.61017 & $1 / 20 / 2005$ & --- & 1630.7 & 1,197 & 1028.7 & 160.9 & 177 & 16 & LSND \\
\hline $49-013-22268$ & Pavillion Fee 31-10B & $31-10 \mathrm{~B}$ & 43.25494 & -108.62822 & $1 / 23 / 2005$ & --- & 1639.2 & 1,693 & 1011.3 & 192.0 & $411^{\mathrm{R}}$ & 219 & LSND \\
\hline 49-013-22313 & Pavillion Fee 33-11B & 33-11B & 43.25061 & -108.60636 & $1 / 24 / 2005$ & --- & 1629.8 & 1,212 & $?$ & 155.8 & 146 & 0 & "water based" \\
\hline 49-013-22599 & Pavillion Fee 44-11B & 44-11B & 43.24686 & -108.60303 & $1 / 26 / 2005$ & --- & 1627.6 & 1,203 & 1033.3 & 193.5 & 183 & 0 & LSND \\
\hline $49-013-22216$ & Tribal Pavillion 21-14 & $21-14$ & 43.24125 & -108.60981 & $1 / 28 / 2005$ & --- & 1632.2 & 1,707 & 1050 & 192.0 & $?^{\mathrm{T}}$ & $?$ & LSND \\
\hline $49-013-22243$ & Pavillion Fee 13-10 & $13-10$ & 43.24911 & -108.63539 & $2 / 3 / 2005$ & --- & 1642.6 & 1,739 & 1045.5 & 193.2 & $?^{\mathrm{N}}$ & $?$ & LSND \\
\hline $49-013-22269$ & Pavillion Fee 21-10B & $21-10 B$ & 43.25481 & -108.63272 & $2 / 3 / 2005$ & --- & 1640.4 & 1,715 & 1013.2 & 193.2 & $1265^{\mathrm{R}}$ & 1072 & "water based" \\
\hline $49-013-22600$ & Pavillion Fee 43-11B & $43-11 \mathrm{~B}$ & 43.24806 & -108.60033 & $2 / 11 / 2005$ & --- & 1626.1 & 1,203 & 1031.7 & 192.9 & 0 & 0 & "water based" \\
\hline 49-013-22324 & Tribal Pavillion 42-10B & $42-10 \mathrm{~B}$ & 43.25219 & -108.62019 & $2 / 16 / 2005$ & --- & 1635.6 & 1,708 & 1009.2 & 189.3 & 0 & 0 & LSND \\
\hline $49-013-22271$ & Pavillion Fee 22-11B & $22-11 \mathrm{~B}$ & 43.25147 & -108.61264 & $2 / 16 / 2005$ & --- & 1632.5 & 1,372 & 1010.7 & 192.3 & 0 & 0 & "water mud" \\
\hline 49-013-22419 & Tribal Pavillion 32-10C & $32-10 \mathrm{C}$ & 43.252211 & -108.627491 & $2 / 18 / 2005$ & --- & 1640.1 & 1,225 & $?$ & 190.8 & 31 & 0 & LSND \\
\hline $49-013-22314$ & Pavillion Fee $22-11 \mathrm{C}$ & $22-11 \mathrm{C}$ & 43.25431 & -108.60886 & $2 / 23 / 2005$ & --- & 1631.6 & 1,173 & 955.55 & 193.5 & $?^{\mathrm{N}}$ & $?$ & LSND \\
\hline $49-013-22634$ & Pavillion Fee 44-4 & $44-4$ & 43.25944 & -108.63994 & $3 / 15 / 2005$ & --- & 1644.1 & 1,737 & ? & 186.2 & SBL & & LSND \\
\hline $41-013-22255$ & Tribal Pavillion 12-12 & $12-12$ & 43.253 & -108.59683 & $3 / 17 / 2005$ & --- & 1625.8 & 1,205 & 1049.1 & 193.5 & $290^{\mathrm{R}}$ & 97 & "water based" \\
\hline 49-013-22617 & Tribal Pavillion 33-2C & $33-2 \mathrm{C}$ & 43.26319 & -108.60839 & $3 / 22 / 2005$ & --- & 1632.2 & 1,131 & 1027.2 & 194.8 & 15 & 0 & LSND/PHPA \\
\hline $49-013-22627$ & Tribal Pavillion 13-2B & 13-2B & 43.26333 & -108.61317 & $3 / 29 / 2005$ & --- & 1633.7 & 1,210 & 1026 & 196.6 & 15 & 0 & $?$ \\
\hline 49-013-22633 & Pavillion Fee 43-4 & $43-4$ & 43.262618 & -108.640212 & $4 / 2 / 2005$ & --- & 1645.6 & 1,446 & 1035.1 & 182.9 & 15 & 0 & LSND \\
\hline $49-013-22625$ & Tribal Pavillion 33-2B & $33-2 \mathrm{~B}$ & 43.26322 & -108.60333 & $4 / 5 / 2005$ & --- & 1633.1 & 1,129 & 1034.8 & 196.6 & 15 & 0 & LSND \\
\hline $49-013-22623$ & Pavillion Fee 14-3B & 14-3B & 43.25944 & -108.63425 & $4 / 5 / 2005$ & --- & 1642.6 & 1,199 & 1028.7 & 189.0 & 15 & 0 & DeepDrill@ \\
\hline $49-013-22601$ & Pavillion Fee 41-11B & $41-11 \mathrm{~B}$ & 43.25703 & -108.6025 & $4 / 14 / 2005$ & --- & 1628.5 & 1,189 & 1017.1 & 196.0 & 274 & 78 & $?$ \\
\hline $49-013-22635$ & Pavillion Fee 42-4B & $42-4 B$ & 43.26764 & -108.63994 & $4 / 16 / 2005$ & --- & 1649.3 & 1,209 & 1046.1 & 196.6 & 198 & 1 & LSND \\
\hline 49-013-22586 & Tribal Pavillion 21-11B & 21-11B & 43.256953 & -108.610928 & $4 / 19 / 2005$ & --- & 1632.2 & 1,181 & 988.47 & 195.7 & 15 & 0 & LSND \\
\hline $49-013-22246$ & Pavillion Fee 13-3W & $13-3 \mathrm{~W}$ & 43.262919 & -108.636420 & $4 / 23 / 2005$ & --- & 1645.6 & 1,403 & 1026 & 185.3 & 198 & 13 & LSND \\
\hline $49-013-22624$ & Pavillion Fee 41-10B & 41-10B & 43.256943 & -108.620175 & $5 / 7 / 2005$ & --- & 1637.1 & 1,171 & 996.39 & 195.1 & 152 & 0 & LSND \\
\hline $49-013-22642$ & NW Pavillion Fee 34-28 & $34-28$ & 43.28969 & -108.64469 & $5 / 24 / 2005$ & --- & 1666.6 & 1,721 & 1018.9 & 196.0 & $?^{\mathrm{N}}$ & $?$ & DeepDrill $®$ \\
\hline $49-013-22259$ & Tribal Pavillion 32-3 & $32-3$ & 43.26658 & -108.62586 & $6 / 12 / 2005$ & --- & 1643.5 & 1,664 & 1013.8 & 196.3 & $?^{\mathrm{A}}$ & $?$ & LSND \\
\hline 49-013-22171 & Pavillion Fee 43-9 & $43-9$ & 43.25081 & -108.63997 & $6 / 16 / 2005$ & --- & 1642.0 & 1,728 & 1055.2 & 196.0 & 31 & 0 & DeepDrill@ \\
\hline $49-013-22258$ & Tribal Pavillion 22-3 & $22-3$ & 43.26844 & -108.63169 & $7 / 16 / 2005$ & --- & 1645.9 & 1,676 & 1029.6 & 196.0 & $<183$ & 0 & "water based" \\
\hline 49-013-22721 & Tribal Pavillion 44-3C & $44-3 \mathrm{C}$ & 43.26136 & -108.62278 & $7 / 19 / 2005$ & --- & 1640.7 & 1,190 & 1004.9 & 196.0 & 46 & 0 & $?$ \\
\hline
\end{tabular}




\begin{tabular}{|c|c|c|c|c|c|c|c|c|c|c|c|c|c|}
\hline 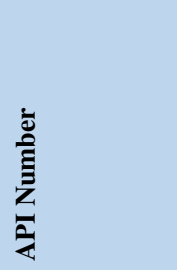 & 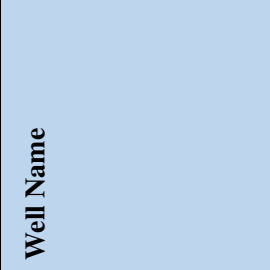 & 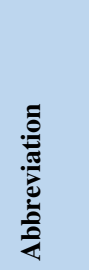 & 菢 & 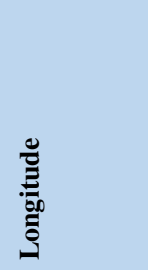 & 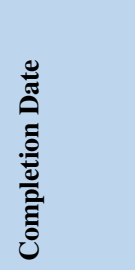 & 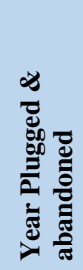 & 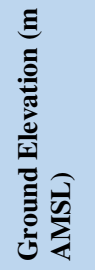 & 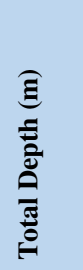 & 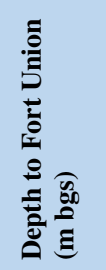 & 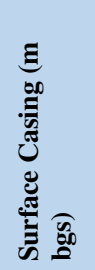 & 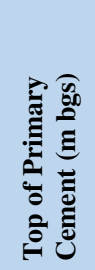 & 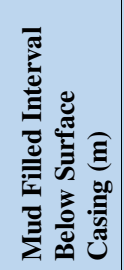 & 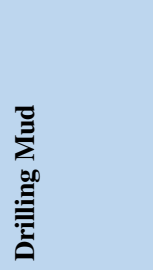 \\
\hline $49-013-22245$ & Tribal Pavillion 32-2 & $32-2$ & 43.26949 & -108.6039 & $3 / 30 / 2006$ & --- & 1640.1 & 1,609 & 1052.8 & 195.1 & 0 & 0 & "Gel-Chem" \\
\hline $49-013-22824$ & Pavillion Fee 33-12 & $33-12$ & 43.24928 & -108.58614 & $4 / 1 / 2006$ & --- & 1619.1 & 1,682 & 1060.7 & 193.9 & 61 & 0 & "Gel-Chem" \\
\hline $49-013-22819$ & Haymaker 14-21 & $14-21$ & 43.24208 & -108.49453 & $5 / 16 / 2006$ & --- & 1606.0 & 3,597 & 1349.0 & 766.3 & $?^{\mathrm{E}}$ & $?$ & $\mathrm{KCl}$ polymer \\
\hline $49-013-22825$ & Pavillion Fee 22-4 & $22-4$ & 43.26606 & -108.65239 & $4 / 3 / 2007$ & --- & 1651.4 & 1,674 & 1074.7 & 158.5 & 61 & 0 & PHPA \\
\hline $49-013-22100$ & Tribal Pavillion 42-15 & $42-15$ & 43.23981 & -108.62144 & $4 / 12 / 2007$ & --- & 1634.6 & 1,659 & 1033 & 157.6 & $?^{\mathrm{A}}$ & ? & PHPA \\
\hline $49-013-23068$ & Leonhardt 41-26 & $41-26$ & 43.1249 & -108.28517 & $6 / 5 / 2007$ & 2007 & 1557.2 & 2,286 & 1207 & 202.7 & None $^{B}$ & 2083 & Water based \\
\hline
\end{tabular}

Coordinates for Tribal Pavillion 32-2 incorrect in well file -approximate coordinates provided.

\section{Abbreviations}

AMSL - Absolute mean sea level

bgs - Below ground surface

PHPA - partially hydrolized polyacrylamides

DeepDrill@ is a product of Newpark Drilling Fluids and is described as a buffered blend of polyhydroxyl alcohols. No MSDS on this product was provided to EPA.

$\underline{\text { Superscripts }}$

A - Cement bond $\log (\mathrm{CBL})$ conducted at completion but not available

B - No apparent use of production casing in borehole

E - Either cement bond log not conducted at completion or not available

G - Ground elevation estimated from coordinates using "GPS Visualizer" located at: http://www.gpsvisualizer.com/elevation.

I - Invert mud below intermediate casing at $1236 \mathrm{~m}$ bgs at $33 \mathrm{X}-10$

$\mathrm{N}$ - Well completion report indicates cement bond or temperature log not conducted at completion

P - Parted casing, leak in casing, or casing failure

$\mathrm{R}$ - Remedial cement squeeze(s) following primary cement. Number of CBLs in parenthesis

$\mathrm{T}$ - Top of cement difficult to discern - high amplitude readings in cement bond log 
Table SI C2. Description of stimulation stages in the Pavillion Field. Information obtained from well completion reports using API search number

\begin{tabular}{|c|c|c|c|c|c|c|c|c|c|c|c|c|c|c|c|c|}
\hline 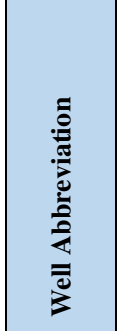 & 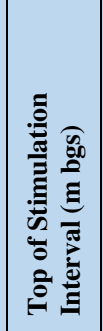 & 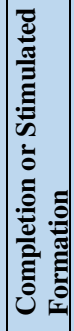 & 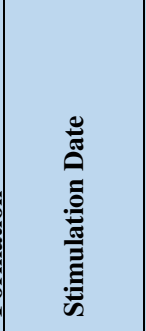 & 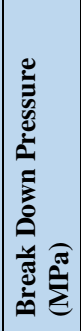 & 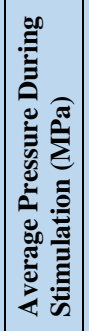 & 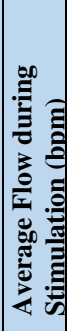 & 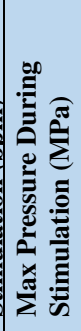 & 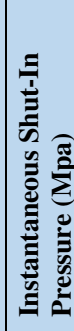 & 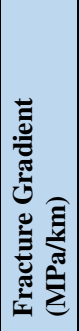 & 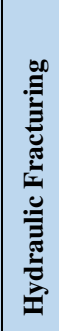 & 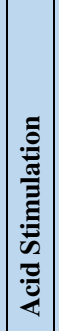 & 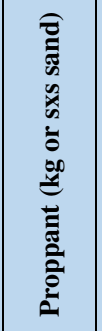 & 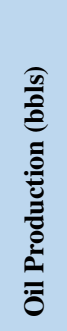 & 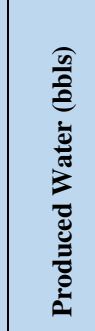 & 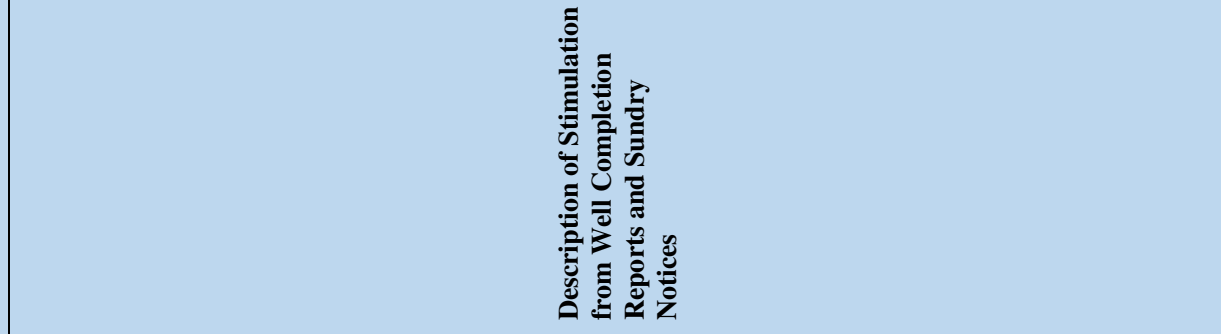 & 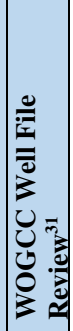 \\
\hline MHR 1 & & MV & & & & & & & & & & & & & $\begin{array}{l}\text { P\&A after completion in 1953, “Water sands" at } 5780^{\prime}-5810^{\prime}, 7145^{\prime}-7250^{\prime}, 7970 \text { '-8040', 9000'-9100', } \\
\text { Filled with "heavy mud” from 2,141'-11,000'. Cement plug from } 2017 \text { '-2141' Intermediate casing cut at } \\
1700 \text { ' and pulled. No production or stimulation. }\end{array}$ & Yes \\
\hline $14-12$ & 2634.6. & TER_. & $112 / 20 / 1999$ & 8.76 & 99.46. & 9 & 10.93 & 8.36 & & $\bar{X}$ & & 15,150 & & 1,134 & "Pumped 25\# linear gel w/75\% CO2, 157 bbls fluid, 85 tons CO2" & $\overline{\mid N o}$ \\
\hline & $94 \overline{1}-1$. & W.-1. & 121111999 & $15.93-$ & & & & & & $\mathrm{X}^{-}$ & & 314 sxs & & & $137 \mathrm{~b} 1 \mathrm{~s} 75 \% \mathrm{CO} 2 \mathrm{fam}$ & \\
\hline & $10 \overline{0} \overline{4} . \overline{0}$ & WR & $11 / 3 / 1964$ & & $10.3 \overline{4}$ & & $10.69^{-1}$ & 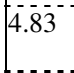 & & $\mathrm{X}^{-}$ & & 5,103 & & & 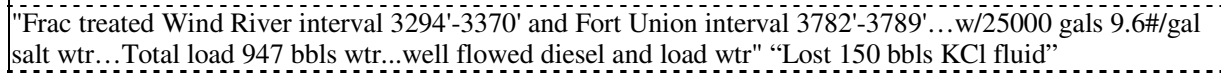 & \\
\hline & 1152.8 & $\mathrm{Fu}^{-1}$ & $4 / 17 / 1980$ & $6.89^{-}$ & & & & & & & $\mathrm{x}$ & & & & 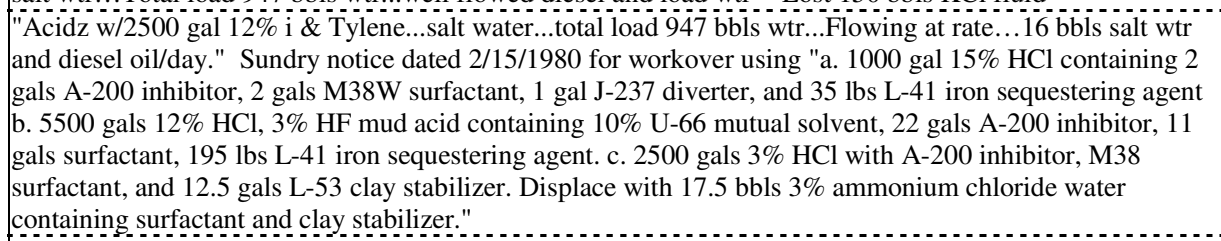 & \\
\hline & 1618.5 & Fi'- & $8 / 5 / 1960$ & & & & & & & & $\mathrm{x}$ & & & & "250 gallons of "breakdown acid followed by cement squeeze. & \\
\hline & 1630.1 & $\mathrm{FU}-1$ & $8 / 5 / 1960$ & & & & & & & & $\mathrm{X}$ & & & & 250 gal breakdown acid followed by cement squeeze & \\
\hline & 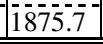 & $\mathrm{F} \overline{\mathrm{Fu}}^{-1}$ & $8 / 3 / 1960^{-1}$ & & & & & & & & $\mathrm{X}$ & & & & "250 gal breakdown acid" followed by cement squeeze & \\
\hline $23-7$ & & $\mathrm{~L}$ & & & & & & & & & & & & & P\&A after completion in 1961.Well history missing from well completion report. & No \\
\hline W 1 & & MT & & & & & & & & & & & & & P\&A after completion in 1961. No apparent production well casing. No stimulation or production. & No \\
\hline $23-2$ & 501.7 & WR & $6 / 16 / 2001$ & 9.38 & 7.58 & 30 & & & & $\bar{X}$ & & 13,605 & $\overline{0}$ & 130 & "70QCO2 foam". & |No. \\
\hline & $536-4$ & 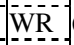 & $6016-12001$ & $17.93-$ & 12.07 & 35 & & & & $\bar{X}$ & & 19210 & & & "70QQ $\mathrm{CO} 2$ foam" & \\
\hline & 679.1 & WR. & $6 / 15 / 2001$ & & 9.65 & 25 & & & & & & 10,725 & & & "70Q CO2 foam" & \\
\hline & $7720^{-0.5-1}-$ & Win- & $601 / 5 / 2001$ & & 12.76 & & & & & $\mathrm{X}$ & & 21955 & & & "70Q $\mathrm{CO} 2$ foam" & \\
\hline & $89 \overline{3}-1$ & W. & $2 / 1 / 1965$ & & & & & & & 1 & & & & & "Frac ali zones" & \\
\hline & $10 \overline{3} . \overline{4}$ & $\mathrm{Fu} \overline{F u}^{-1}$ & $2 / 1 / 1965^{-1}$ & & & & & & & & & & & & "Frac all zones" & \\
\hline $22-35$ & & $\mathrm{FU}$ & & & & & & & & & & & & & $\begin{array}{l}\text { P\&A after completion in 1963. No apparent production well casing. Well history missing from well } \\
\text { completion report. No record of production or stimulation. }\end{array}$ & No \\
\hline$\overline{\mathrm{GOL}} 1$ & & FU & & & & & & & & & & & & & $\begin{array}{l}\text { Completed in 1963. Perforated in three intervals in WR Formation. Put into production on 6/11/1963. P\&A } \\
\text { in 1974. No information on stimulation. }\end{array}$ & No \\
\hline$\overline{14-1}$ & 322.5 . & WR. & $11 / 5 / 2000$ & & 4.76. & & 6.96 & & & $\bar{X}$ & & $217 \mathrm{sks}$ & & 2,735 & "Totals pumped 54 tons CO2 4360 gals w/methanol.". & No. \\
\hline & 6618.4 & WR & 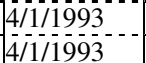 & & & & & & & & $x$ & & & & 1500 gal $15 \%$ & \\
\hline & $925.4-$ & $\bar{W}-$ & $10 / 16 / 1964$ & $22.75^{-}$ & 16.55 & $4 \overline{3}^{-}$ & 17.24 & 10.34 & & $\bar{X}$ & & & & & $\begin{array}{l}\text { "Injected } 12,000 \text { gallons } \\
3 / 25 / 1993 \text { plan to "plug back water bearing perforation in the Fort Union at } 3744-3780 \text {." }\end{array}$ & \\
\hline $21-8$ & & WR & & & & & & & & & & & & & $\begin{array}{l}\text { Perforated in WR Formation. Completed in } 1963 \text { as a shut-in well and P\&A unknown time later. Well } \\
\text { history and completion information missing. }\end{array}$ & No \\
\hline $21-5$ & 352.0 & WR & $12 / 8 / 1999$ & & & & & & & $\mathrm{X}$ & & & $\overline{0}$ & 898 & $\begin{array}{l}\text { Recompletion in } 1999 \text {. "HES } 70 \mathrm{Q} \text { foam, } 4 \mathrm{gal} / \mathrm{M} \mathrm{HgClean,} 4 \% \mathrm{KCl} \text { on } 12 / 8 / 1999 \text {. Numerous perforated } \\
\text { and squeezed intervals. No documented production or stimulation prior to recompletion. }\end{array}$ & No \\
\hline U 13-13 & & FU & & & & & & & & & & & $\overline{2}$ & 1,130 & $\begin{array}{l}\text { Production from Fort Union Formation. Information on completion and possible stimulation missing from } \\
\text { well completion report. }\end{array}$ & No \\
\hline
\end{tabular}




\begin{tabular}{|c|c|c|c|c|c|c|c|c|c|c|c|c|c|c|c|c|}
\hline 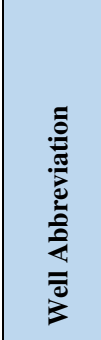 & 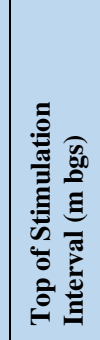 & 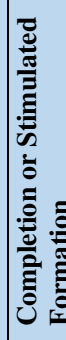 & 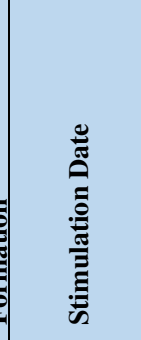 & 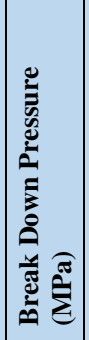 & 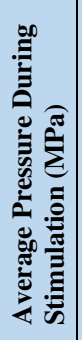 & 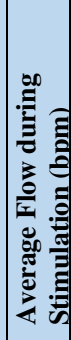 & 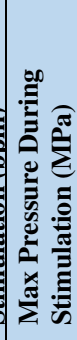 & 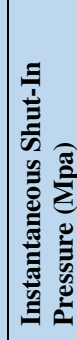 & 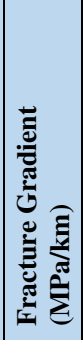 & 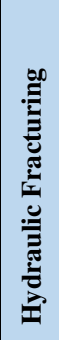 & 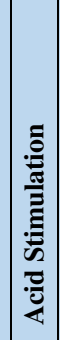 & 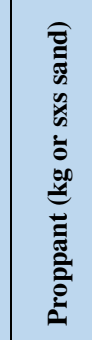 & 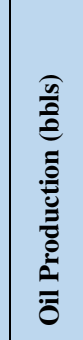 & 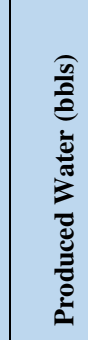 & 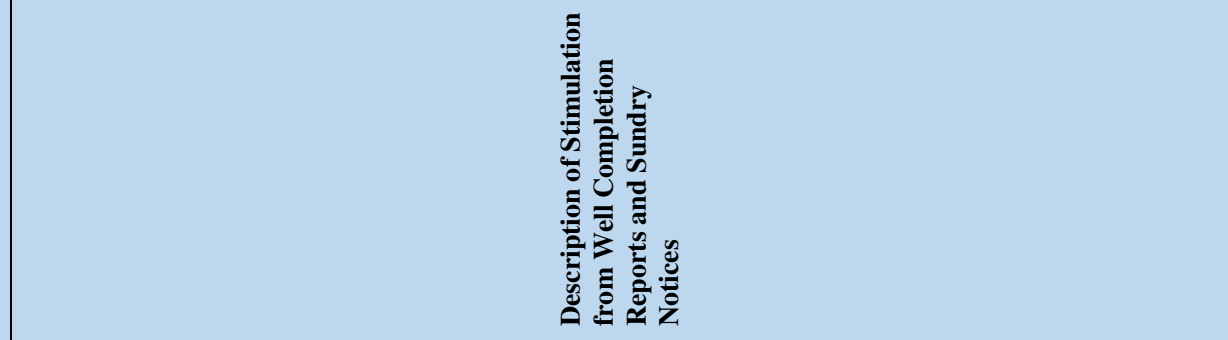 & 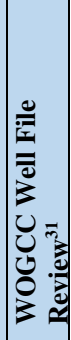 \\
\hline $14-6$ & & WR & & & & & & & & & & & 0 & 27 & $\begin{array}{l}\text { Production from Wind River Formation. Information on completion and possible stimulation missing from } \\
\text { well completion report. }\end{array}$ & No \\
\hline $32-4$ & & FU & & & & & & & & & & & & & $\begin{array}{l}\text { P\&A after completion in 1963. Information on well history missing from completion report. No apparent } \\
\text { production well casing. No stimulation or production. }\end{array}$ & No \\
\hline $24-14$ & & FU & & & & & & & & & & & 2 & 1,834 & $\begin{array}{l}\text { Production from base of Wind River Formation. Five perforated intervals between 3443'-3515' without } \\
\text { stimulation. }\end{array}$ & No \\
\hline $44-20$ & & FU & & & & & & & & & & & & & $\begin{array}{l}\text { P\&A after completion in 1964. No apparent production well casing or production. Drilling report missing } \\
\text { from well completion report. }\end{array}$ & No \\
\hline $23 \mathrm{X}-24$ & & FU & & & & & & & & & & & & & $\begin{array}{l}\text { P\&A after completion in 1964. No apparent production well casing or production. Cement plugs at surface, } \\
522^{\prime}-675^{\prime}, 2035^{\prime}-2200^{\prime} \text {, and } 3765^{\prime}-3930^{\prime} \text {, }\end{array}$ & No \\
\hline $4 \overline{44-17}$ & & FU & & & & & & & & & & & & & P\&A after completion in 1964. No apparent production well casing or production. & No \\
\hline $34-33$ & & FU & & & & & & & & & & & & & $\begin{array}{l}\text { Perforated at } 2566 \text { '-2588' in WR Formation. No information on stimulation or production. Well history } \\
\text { missing from well completion report. P\&A date unknown }\end{array}$ & No \\
\hline $33 \mathrm{X}-10$ & 986.3 & WR & $2 / 22 / 1965$ & 21.36 & & 16.5 & & 6.89 & & $\mathrm{X}$ & & $5,5,897$ & 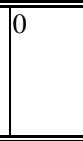 & 0 & $\begin{array}{l}\text { In 1964, acidized in Frontier Formation with " } 15,000 \text { gals MCA.” In 1964, "sptd } 500 \text { gals } 15 \% \text { HCl on perfs } \\
\text { [in WR Formation] \& broke formation down w/1400 psi TP (600 psi CP)...total load } 532 \text { bbls. "Frac treated } \\
\text { Wind River pers...15,000 gals 9.6\#/gal salt water containing fluid loss additives...Total load } 933 \text { bbls" } \\
\text { Completed in 1964. P\&A in 1983. Drilled to Madison Formation. }\end{array}$ & Yes \\
\hline $24 \mathrm{X}-3$ & 1359.4 & FU & $4 / 28 / 1966$ & & & 1.0 & 8.27 & & & & $\bar{X}$ & & 2 & 9,653 & "Treated...w/1000 gal P-12 solvent (Dow) containing 10 gal free-flo 'C'" & No \\
\hline $14-11$ & & WR & & & & & & & & & & & 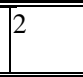 & 1,426 & $\begin{array}{l}\text { Producing from Wind River Formation. Perforations from 3192'-3864'. Well completion report is not } \\
\text { available. }\end{array}$ & No \\
\hline $13-13$ & 1018.0 & WR & $9 / 7 / 1965$ & 16.55 & & 23.0 & 24.82 & 19.31 & & $\mathrm{X}$ & & 2,268 & & & $\begin{array}{l}\text { "Breakdown with... salt water...spearhead frac with } 500 \text { gal. } 15 \% \text { HCl. Frac with ... } 5000 \text { gal } 4 \% \text { salt } \\
\text { water." }\end{array}$ & \\
\hline & 1099.7 & FE'- & $9 / 6 / 1965$ & 14.48 & & 28.5 & & 14.48 & & $\bar{X}$ & & 5,443 & & & Breakdown with 750 gal salt water. Inj 500 gal $15 \%$ HCl. Frac with. 12,000 gal $4 \%$ salt water," & \\
\hline & 1099.7 & $\mathrm{FU}^{-}$ & $11 / 6 / 1965$ & & & 21.6 & 28.96 & 27.58 & & $\mathrm{X}$ & & 5,443 & & & "Diesel frac with 24000 gal diesel." & \\
\hline $32-9$ & & FU & & & & & & & & & & & & & $\begin{array}{l}\text { P\&A after completion in 1968. No apparent production well casing or production. Cemented intervals 567- } \\
667^{\prime}, 1200-1300^{\prime}, 3200-3300^{\prime} \text { during P\&A. }\end{array}$ & Yes \\
\hline WEL & 4789.3 & $\mathrm{~F}$ & $6 / 14 / 1971$ & & & & & & & & & & & & $\begin{array}{l}\text { Drilled to Mowry Formation. Acidized in Frontier formation with 50,000 gal 7-1/2\% } \mathrm{HCl} \text { and } 1000 \mathrm{gal} \mathrm{KCl.} \\
\text { 1979. P\&A in 1981. }\end{array}$ & Yes \\
\hline $41 \mathrm{X}-10$ & 551.4 & WR & $2 / 7 / 1973$ & & & & 19.31 & 13.79 & & $\mathrm{X}$ & & & & & 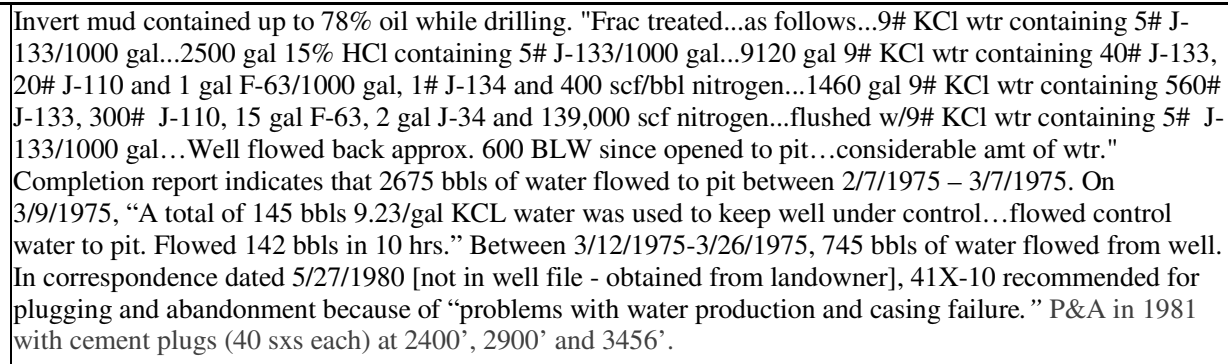 & Yes \\
\hline
\end{tabular}




\begin{tabular}{|c|c|c|c|c|c|c|c|c|c|c|c|c|c|c|c|c|}
\hline 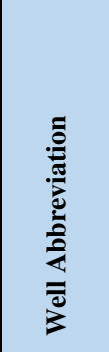 & 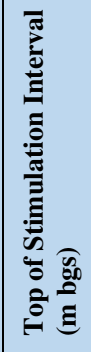 & 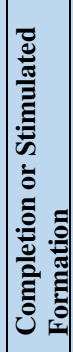 & 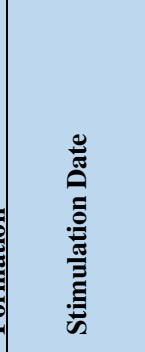 & 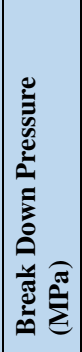 & 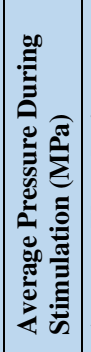 & 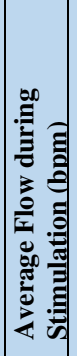 & 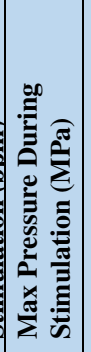 & 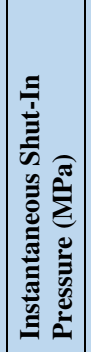 & 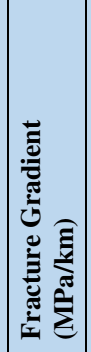 & 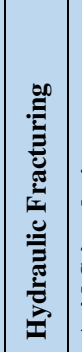 & & 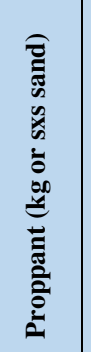 & 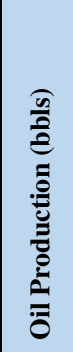 & 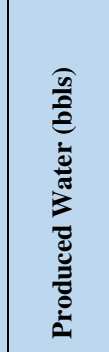 & 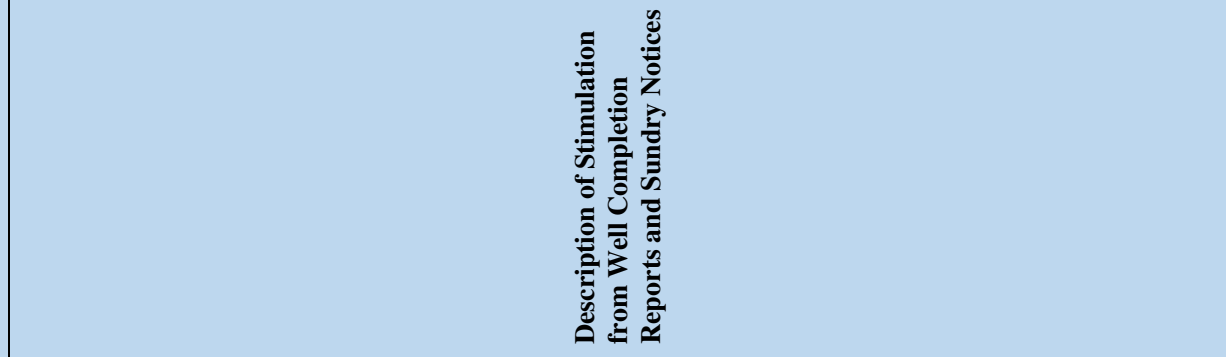 & 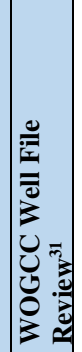 \\
\hline & 1232.6 & $\mathrm{FU}$ & $2 / 8 / 1979$ & & & 6.0 & 44.82 & 22.75 & & $\mathrm{x}$ & & 77,174 & & & 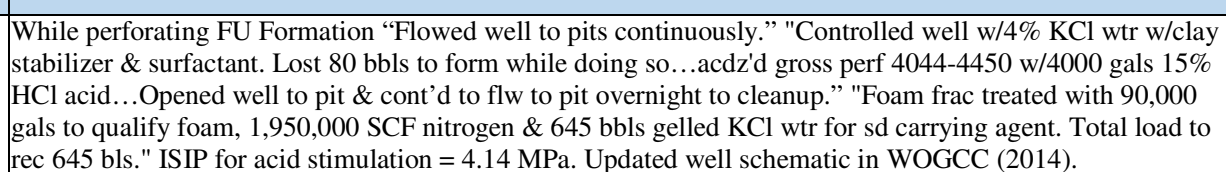 & \\
\hline $41 \mathrm{A1X}-2$ & & WR & $\mid 11 / 28 / 1973$ & & & & & & & & & & & & $\begin{array}{l}17 \text { perforations from 1554'-4398' with diesel oil to control well. No documented stimulation or gas } \\
\text { production }\end{array}$ & No \\
\hline $31 X-3$ & 395.6 & WR & $8 / 30 / 2001$ & & & & & & & $\mathrm{X}$ & & & 0 & 1,215 & $\begin{array}{l}\text { Completed in Dec } 1973 \text { with } 27 \text { holes from } 1665^{\prime}-4988^{\prime} \text { and } 8 \text { holes } 3154^{\prime}-3161^{\prime} \text { - no apparent stimulation. } \\
\text { Stimulated in Aug 2001, "Frac } 1298-1309^{2}\end{array}$ & No \\
\hline$\overline{42 X-11}$ & & $\overline{\mathrm{TWR}}$ & & & & & & & & & & & 0 & $\frac{222}{222}$ & \begin{tabular}{|l}
30 perforations from $2014^{\prime}-4741^{\prime}$ and 3 perforated intervals from $3773^{\prime}$ and $37933^{\prime}$ during initial completion \\
in 1974 . No documented stimulation fluids. 20 perforated intervals added during recompletion in 2001 . No \\
documented stimulation.
\end{tabular} & $\overline{\mathrm{No}}$ \\
\hline 4 & 1149.7 & $\overline{F U}$ & $\overline{\mid 1 / 19 / 1974}$ & & & & & & & & & & $\overline{0}$ & 2295 & "400 gal 15\% HCl" & $\overline{\mid N o}$ \\
\hline 31X-14 & & WR & & & & & & & & & & & 2 & 7,691 & $\begin{array}{l}\text { Producing from Wind River Formation. Perforations in Wind River and Fort Union Formations during initial } \\
\text { completion in } 1974 \text { - no apparent stimulation. }\end{array}$ & 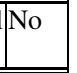 \\
\hline CCD & & MV & & & & & & & & & & & & & $\begin{array}{l}\text { P\&A after completion in 1974. No apparent production well casing. No stimulation or production. Water at } \\
2715^{\circ} \text {. Converted to water well. }\end{array}$ & No \\
\hline $\begin{array}{lll}\text { TR1-22 } \\
\end{array}$ & 961.6 & TRR & $277 / 1980$ & & & & & & & $\overline{\bar{X}}$ & & & 0 & $\overline{5,281}$ & 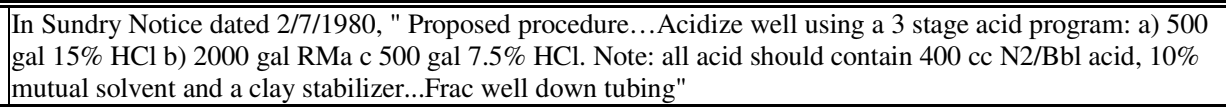 & $\overline{\mathrm{No}}$ \\
\hline $11-21$ & & FU & & & & & & & & & & & & & 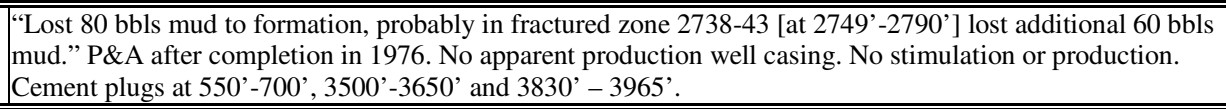 & No \\
\hline TP 1 & & WR & & & & & & & & & & & 0 & 5,607 & 77 perforated intervals between $3406-3572$. No record of stimulation. & No \\
\hline $1-31$ & & $\mathrm{WR}$ & & & & & & & & & & & & & 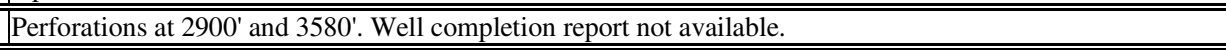 & \\
\hline TU 1 & & $\mathrm{WR}$ & & & & & & & & & & & 0 & 220 & 537! . No record of stimulation. & No \\
\hline 4-8. & 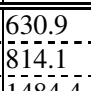 & 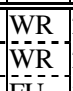 & $\begin{array}{l}2 / 12 / 2005 \\
218 / 2005 \\
2-2005\end{array}$ & $\begin{array}{ll}3.10 \\
8.27 \\
-27\end{array}$ & 26.05 & 18.0. & $\begin{array}{ll}39.38 \\
38.24 \\
-3.24\end{array}$ & 45 & & & & $\begin{array}{ll}0,160 \\
5,254 \\
-2,25\end{array}$ & 365 & $63,110$. & 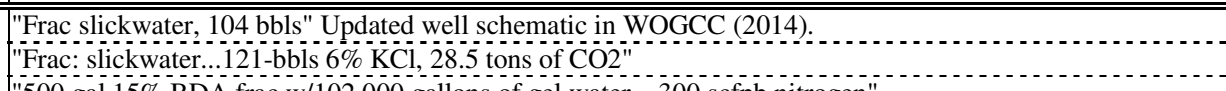 & YYes \\
\hline & 1484.4 & $\mathrm{Fu}-1$ & $3 / 26 / 1977^{-1}$ & & & & & & & & & 61,235 & & & 7500 gal $15 \%$ BDA frac w/102,000 gallons of gel water.. 300 sctpi nitrogen & \\
\hline $34-13$ & & FU & & & & & & & & & & & 0 & 290 & Perforations from 2664-2676. Information on well completion missing. & No \\
\hline $\mid 224-11$ & & & & & & & & & & & & & & & P\&A after completion in 1978. No apparent production well casing. No stimulation or production. & No \\
\hline $24-4$ & & IWR & & & & & & & & & & & & & P\&A after completion in 1978. No apparent production well casing. No stimulation or production. & 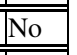 \\
\hline $44-15$ & $\frac{1286.3}{1376.3}$ & $\mathrm{~F}$ & 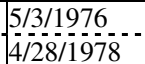 & 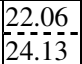 & |.655. & & 84.13 & $\frac{4.83}{6.21}$ & & & & & & 1,348 & 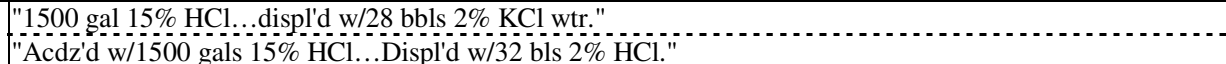 & Noo. \\
\hline & 1530.1 & Fu & 40 & 5.86 & 13.79 & & & 10.34 & & & & & & & 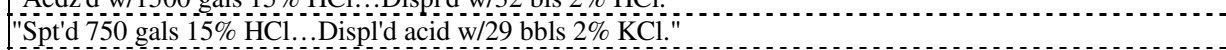 & \\
\hline & 1530.4 & Fu & $41 / 241978$ & 20.68 & 13.79 & & & 6.55 & & & & & & & Pump 1500 gal $15 \%$ HCI displacing acid w w0 0 bils KCI.:" & $\cdots$. \\
\hline & 1530.4 & FU & 4 & 33.09- & 10.34 & $4.0^{-1}$ & & 6.55 & & & 管- & & & & Pmp' 500 gals $7-1 / 2 \% \mathrm{HCl} \ldots \mathrm{Displaced}$ acid w/38 bbls $2 \% \mathrm{KCl}$ & \\
\hline $21-11$ & 958.3 & BW & $3 / 12 / 1979$ & 13.79 & 6.89 & & & $\overline{4.83}$ & & $\overline{\bar{X}}$ & & 8,897 & 0 & 335 & 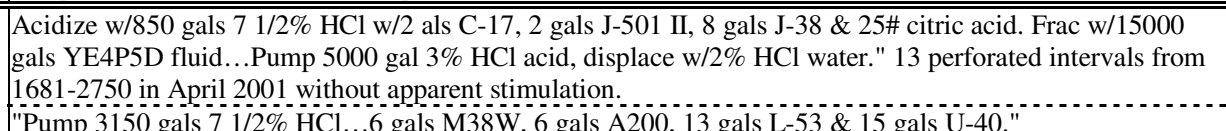 & No \\
\hline
\end{tabular}




\begin{tabular}{|c|c|c|c|c|c|c|c|c|c|c|c|c|c|c|c|c|}
\hline \multirow[t]{2}{*}{ 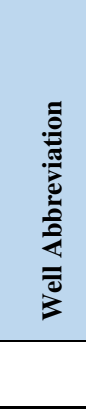 } & 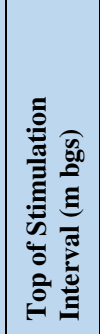 & 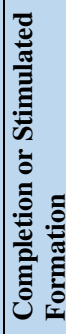 & 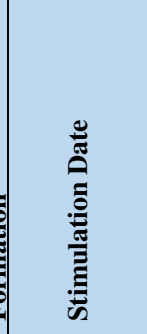 & 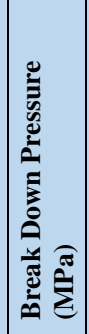 & 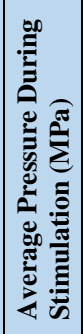 & 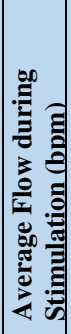 & 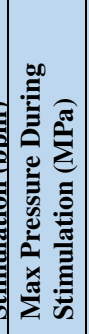 & 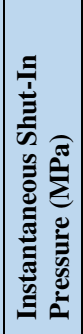 & 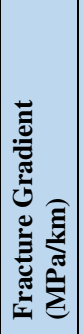 & 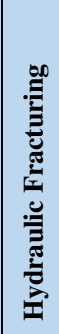 & 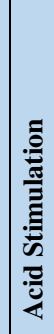 & 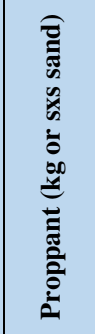 & $\begin{array}{l}\frac{0}{0} \\
\frac{0}{0} \\
\tilde{0} \\
\frac{0}{0} \\
\frac{\Xi}{0} \\
\frac{0}{0} \\
\overline{0}\end{array}$ & 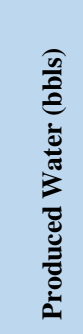 & 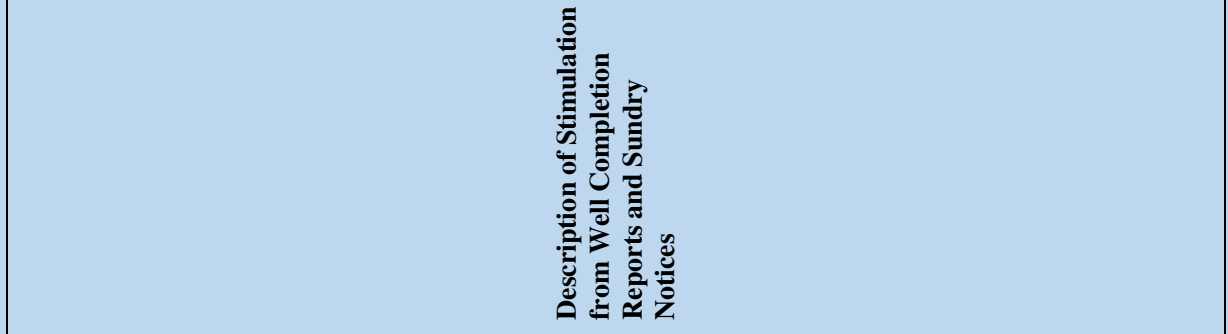 & 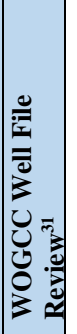 \\
\hline & 1471.6 & $\mathrm{FU}$ & $2 / 21 / 1979$ & & 13.79 & 4.5 & 22.06 & 6.89 & & & $\mathrm{X}$ & & & & $\begin{array}{l}\text { "Breakdown w/1100 gals } 7 \text { 1/2\% HCl w/2 gal M38W, } 2 \text { gal A200, } 4 \text { gal L-53 \& 25/L-41. Displace w/9 bbls } \\
\text { L-53 water foll'd } 9 \text { bbls fresh water." }\end{array}$ & \\
\hline \multirow[t]{4}{*}{12} & 578.5 & WR. & $5 / 16 / 2001$ & & & & & & & $\underline{X}$ & & 10,259 & & 18 & "CT [coiled tube] frac 256 bbls, 177 mcf N2". Updated well schematic in WOGCC (2014). & s. \\
\hline & 1032.7 & $\mathrm{WR}$ & $2 / 5 / 1981^{-}$ & & & & & & & $\mathrm{X}^{-}$ & & 26,762 & & & "Acidize w/4000 gals $71 / 2 \%$..Frac BWR ...versa gel" & \\
\hline & 1134.2 & Fî & $3 / 22 / 1979$ & 24.82 & $11.72-$ & & & & & $\mathrm{X}_{-}^{-}$ & & & & & "Frac [with] 4 bbls L $53+15$ bbls L 53 wtr+6bbls acid." "Lost thousands of bbls KCl to Basal wind river." & \\
\hline & 1296.0 & $\overline{F U}^{-1}$ & $3 / 19 / 1979$ & & & & & & & & $\mathrm{x}^{-}$ & & & & "Acidize w/6000 gals wtr." $4252-4580$ "WET" & \\
\hline \multirow[t]{9}{*}{ 41-9. } & 560.5 & WR. & $10 / 1 / 2004$ & & & & & & & X & & $5,443$. & & 15,593 & "74 bbls $6 \% \mathrm{KCl}, 16.3$ tons $\mathrm{CO} 2 \mathrm{Frac}$ down casing" & No. \\
\hline & 634.0 & WR- & $10 / 1 / 2004$ & & & & & & & $\mathrm{X}^{-}$ & & 5,443 & & & "78 bbls $6 \%$ KCl, 16.3 tons CO2...$F r a c$ down casing" & \\
\hline & 688.2 & WR. & $10 / 1 / 2004$ & & & & & & & $\mathrm{X}$ & & 5,443 & & & $" 82$ bbls $6 \%$ KCl, 16.3 tons CO2 2 Frac down casing" & \\
\hline & 788.2 & Wi- & $10 / 1 / 2004$ & & & & & & & $\mathrm{X}_{-}^{-}$ & & 5,443 & & & "87 bbils $6 \%$ KCl, 16.3 tons CO2" & \\
\hline & 826.6 & $\mathrm{WR}$ & $10 / 1 / 2004$ & & & & & & & X- & & 6,804 & & & $" 100$ bbls $6 \%$ KCl, 20.4 tons CO2" & \\
\hline & 876.0 & $\mathrm{WR}$ & $10 / 1 / 2004^{--}$ & & & & & & & $\mathrm{X}$ & & $6,804^{-}$ & & & $" 103 \mathrm{bbls} 6 \% \mathrm{KCl}, 20.4$ tons $\mathrm{CO} 2 "$ & \\
\hline & 1129.6 & $\mathrm{FU}^{-1}$ & $10 / 1 / 2004^{-1}$ & & & 35.0 & 16.87 & & & $\bar{X}$ & & $8,165^{-}$ & & & $\begin{array}{l}128 \text { bls } 6 \% \mathrm{KC}, 24.4 \text { tons } \mathrm{CO} 2,18,843 \text { gal } 70 \mathrm{Q} \text { CO2 WF } 125 \text {..Frac down casing." In proposed fracturing } \\
\text { schedule "Pumping schedule to achieve a propped fracture half-length (Xf) of } 82.9 \mathrm{ft} \text { with an average } \\
\text { conductivity of (Kfw) of } 3383 \text { md.ft...gel conc } 7.5 \mathrm{lb} / \mathrm{mgal} \text {. }\end{array}$ & \\
\hline & 1163.1 & $\overline{F U}$ & $10 / 1 / 2004$ & & $17.39^{-}$ & 35.0 & 17.51 & & & $\mathrm{X}^{-}$ & & 27,216 & & & 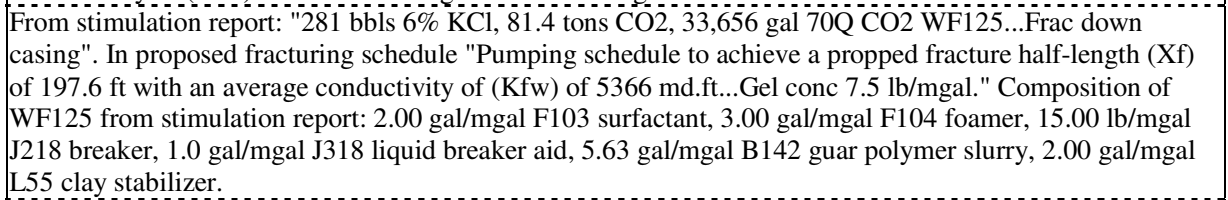 & \\
\hline & 1472.2 & $\mathrm{FU}$ & $8 / 1 / 1979$ & 15.17 & 11.03 & 4.0 & & 4.14 & & & $\mathrm{x}^{-}$ & & & & $\begin{array}{l}\text { "Lost approx. } 50 \text { bbls mud in } 24 \text { hrs." [invert mud while drilling] "At'd gross Ft. Union perfs } 4830-5097 \\
\text { with } 4000 \text { gal } 7.5 \% \mathrm{HCl} \text { containing } 2 \text { gals A-200, } 2 \text { gals M38W, } 4 \text { gals L-53 and } 35 \% \text { L-31 per } 1000 \text { gal } \\
\text { acid. Press csing to } 1200 \text { psi with invert oil. Pumped } 1000 \text { gal acid...spaced...ball sealers in next } 2500 \text { gal } \\
\text { acid. Followed with } 500 \text { gal acid without ball sealers. Flushed with } 20 \text { bbls } 2 \% \text { KCl water containing } 6 \text { gal } \\
\text { L-53 clay stabilizer." "A total of } 65 \text { bbls invert oil has been pmp'd into well" [to control well]. }\end{array}$ & \\
\hline \multirow[t]{2}{*}{ TUL2. } & 1069.5 & WR & $8 / 24 / 1979$ & & & & & & & & $\underline{X}$ & & & & 2500 gal 7-1/2\% HCl" Cement squeeze after stimulation & No \\
\hline & $10 \overline{7} \overline{4} . \overline{4}$ & $\mathrm{WR}^{-1}$ & $8 / 24 / 1979$ & & & & & & & & $\mathrm{X}$ & & & & $" 500$ gal $15 \% \mathrm{HCl}^{-}$ & \\
\hline \multirow[t]{10}{*}{$22-10$} & 607.8 & WR & $12 / 14 / 2004$ & 13.00 & & & 18.58 & 8.14 & & $\bar{X}$ & & $6,6,554$ & & (363. & "89 bbls of clean fluid, 22 ton CO2" & 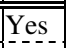 \\
\hline & 660.8 & WR & $12 / 14 / 2004$ & 22.34 & & & 22.37 & 8.45 & & X-1. & & $9,085^{-}$ & & & "129 bbls of clean fluid..29 tons of CO2" & \\
\hline & 734.3 & WR & $12 / 14 / 2004$ & 29.65 & & & 29.65 & 8.95 & & X- & & 6,559 & & & "270 bbls of clean fluid...17 tons CO $2 "$ & \\
\hline & 937.6 & WR & $12 / 14 / 2004$ & 28.96 & & & 28.96 & 9.51 & & X- & & 10,841 & & & "165 bbls of clean fluid...27 ton CO2" & \\
\hline & 994.3 & $\mathrm{WR}$ & $12 / 14 / 2004$ & & & & 11.76 & 0.69 & & X-1. & & 10,886 & & & "Frac: pump 92 bbls of $6 \% \mathrm{KCl}$..didn't see a break...clean fluid 242 bbl...34 tons cO2" & \\
\hline & 997.9 & $\overline{F U}^{-1}$ & $10 / 6 / 1979$ & 7.58 & & 4.0 & & 1.17 & & & $\mathrm{X}^{-}$ & 0 & & & $\begin{array}{l}\text { "A.T. perfs } 3274-94 \text { w/1000 gals } 15 \% \text { HCl } \& 48 \text { frac balls...acid containing } 2 \text { gals inhibitor, } 2 \text { gals } \\
\text { surfactant, } 4 \text { gals clay stabilizer \& } 3-5 \# \text { iron sequestration per } 1000 \text { gals acid. Formation broke ...Flushed } 20 \\
\text { bbls of clay stabilizer water." }\end{array}$ & \\
\hline & 1384.1 & FE'- & $12 / 11 / 2004$ & 31.21 & & & 46.68 & 32.08 & & X-1. & & 7,824 & & & "Frac 10 bbls to catch pressure...clean fluid $119 \mathrm{bbls} . .19$ ton CO2" & \\
\hline & 1396.3 & $\overline{\mathrm{Fu}}$ & $12 / 9 / 2004$ & $31.90^{-1}$ & & & 49.29 & 1.94 & & $\mathrm{X}^{-}$ & & 24,176 & & & "260 bbi clean fluid...55 tons $\mathrm{CO} 2 "-$ & \\
\hline & 1468.8 & $\mathrm{FU}$ & $9 / 12 / 1979$ & & & & & 5.17 & & & $\mathrm{x}^{-}$ & & & & $\begin{array}{l}\text { Pmp'd } 57 \text { bbls } 9 \# / g a l ~ K C l ~ w / .5 \text { gal per bbl brine saver, } .2 \text { surfactant } \& .5 \text { clay stablizer...pmp'd } 5 \text { bbls KCl } \\
\text { into formation...pmp'd } 40 \text { bbls } 9 \# / \text { gal } \mathrm{KCl} @ 3 \text { BPM } 600 \# \text { into formation... acidized ...w/3400 gal } 15 \% \\
\text { HCl...Flushed w/25 bbls of fresh water containing clay stablizer. }\end{array}$ & \\
\hline & 1530.1 & $\overline{F U}^{-1}$ & $9 / 12 / 1979$ & & & & & & & & $\mathrm{x}^{--}$ & & & & "Acid treat w/3400 gal $15 \% \mathrm{HCl}^{-}$ & \\
\hline
\end{tabular}




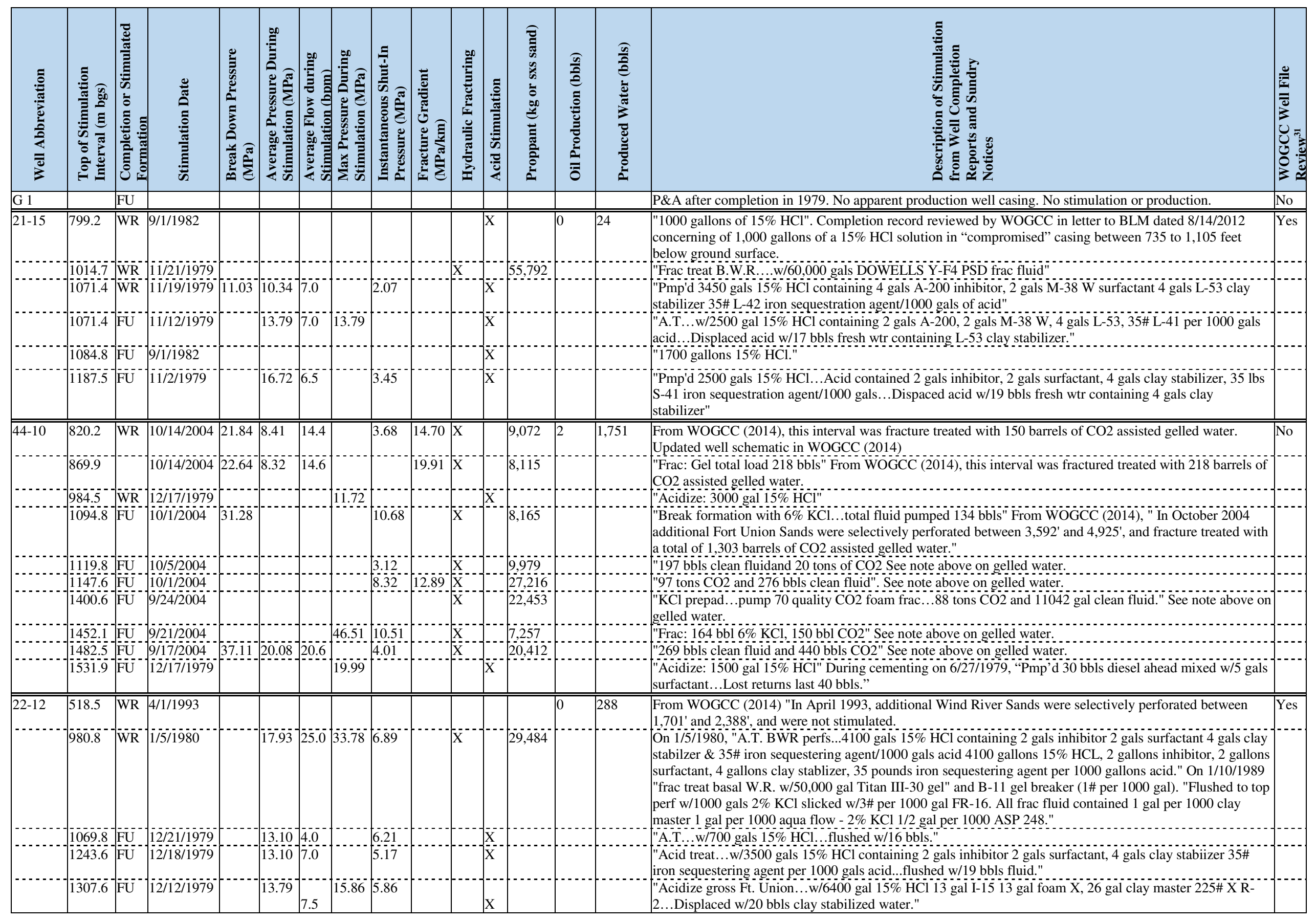




\begin{tabular}{|c|c|c|c|c|c|c|c|c|c|c|c|c|c|c|c|c|}
\hline 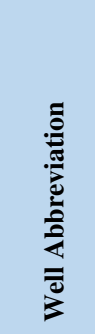 & 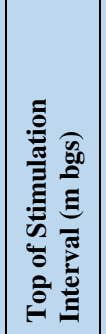 & 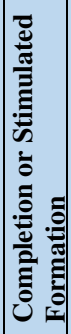 & 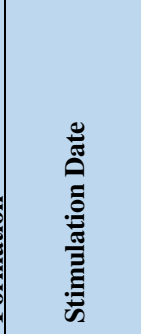 & 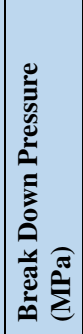 & 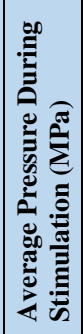 & 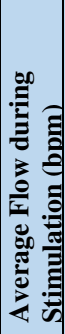 & 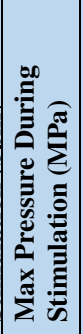 & 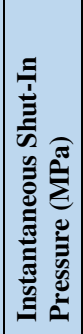 & 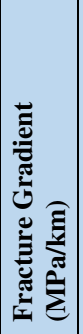 & 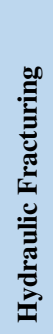 & 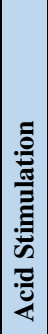 & 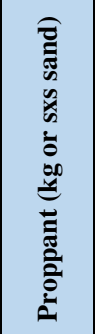 & 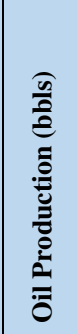 &  & 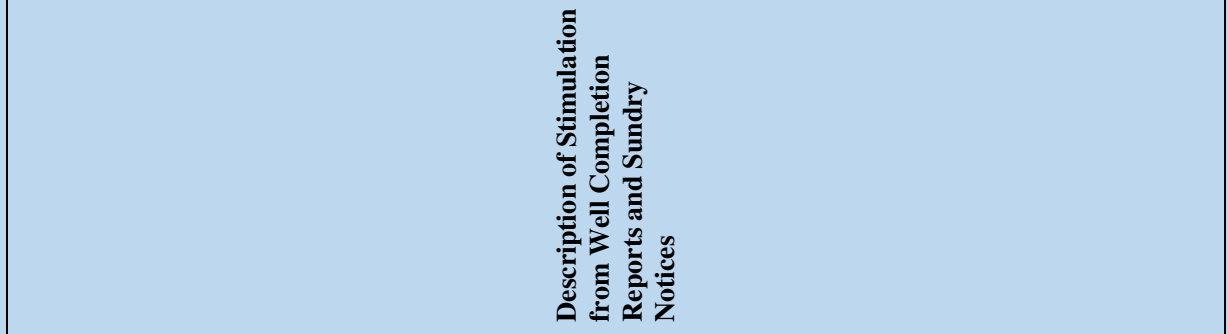 & 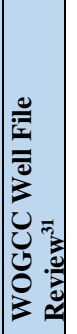 \\
\hline & 1432.6 & $\mathrm{FU}$ & $12 / 10 / 1979$ & & 15.17 & & 17.24 & 7.93 & & & $\mathrm{X}$ & & & & $\begin{array}{l}\text { "Acidized FT. Union...w/4000 gal } 15 \% \text { HCL cont. } 8 \text { gal A-200 inhibitor, } 8 \text { gal M-38W surfactant, } 16 \text { gal L- } \\
53 \text { clay stabilizer, } 140 \text { \# L-41 iron sequestering agent...Displaced w/31 bbls fresh water containing } 10 \text { gal L- } \\
53 \text { clay stabilizer." }\end{array}$ & \\
\hline & 1500.2 & FU'- & $12 / 10 / 1979$ & & & & & & & & $\underline{X}-$ & & & & "14000 & \\
\hline & 1511.8 & $\overline{F U}^{-1}$ & $12 / 10 / 1979$ & & & & & & & & $\mathrm{X}^{--}$ & & & & "4000 gal HCl" & \\
\hline $11-14$. & 991.2 & WR & $2 / 5 / 1980$ & & & & & & & & $\mathrm{X}$ & & 5. & 12,550 & "3600 gal HCl". & No. \\
\hline & 1544.1 & FU'-- & $2 / 5 / 1980$ & & & & & & & & $\underline{X-1}$ & & & & 2500 gal HCl". & \\
\hline & 1564.2 & $\mathrm{FU}^{-}$ & $2 / 5 / 1980$ & & & & & & & & $\mathrm{X}$ & & & & "2500 gal HCl" & \\
\hline $16-28$ & & & & & & & & & & & & & & & Perforations from 3714'-3736'. Well completion report not available. & No \\
\hline $1-17$ & & & & & & & & & & & & & & & P\&A after completion in 1980. No apparent production well casing. No stimulation or production. & No \\
\hline $14-2$ & 481.9 & WR & $8 / 27 / 1982$ & & & & 0.69 & & & & $\mathrm{X}$ & & 0 & 44 & 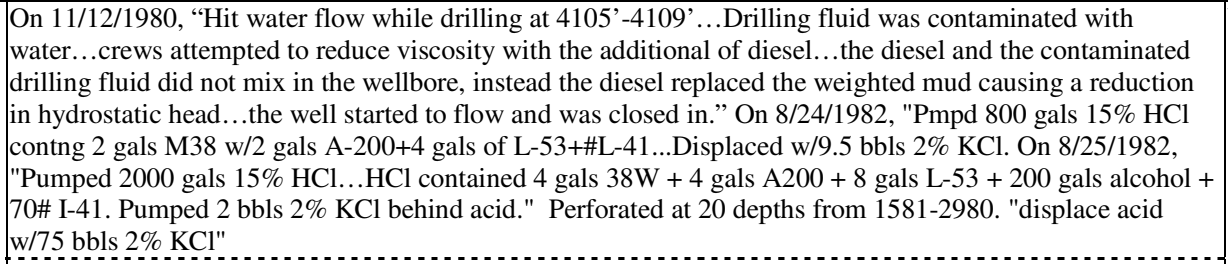 & Yes \\
\hline & 664.5 & WR & $4 / 1 / 1981$ & & & & & & & & $\underline{X-1}$ & & & & "800 gal $15 \%$ HCl containing 2 gals M38 W/2 gal A $200+4$ gals L $-53+\mathrm{HL}-41,95 \mathrm{bb} \mathrm{s} \mathrm{KCl}$ & \\
\hline & 1039.1 & $\overline{\mathrm{FU}}$ & $4 / 14 / 1981$ & & & & $8.62^{-1}$ & & & & $\mathrm{X}^{--}$ & & & & 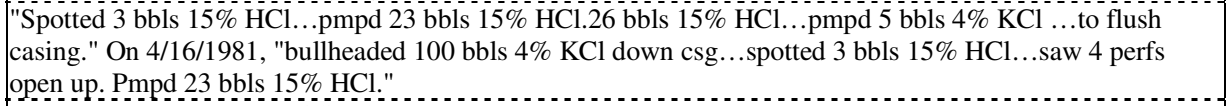 & \\
\hline & $11 \overline{4} 8.2$ & $\overline{\mathrm{FU}}$ & $4 / 9 / 1981$ & & & & 7.93 & & & & $\mathrm{X}$ & & & & From WOGGCC (2014) "acidized" & \\
\hline & 1189.3 & FE'-- & $4 / 3 / 1981$ & 17.93 & & & 17.93 & 3.10 & & & $\underline{X}$ & & & & "Spotted 21 bbls HCl took 2 hrs to pmp 10 bbls into form...pmpd 22 bbls flush" & \\
\hline & 1370.1 & $\mathrm{FU}$ & $3 / 27 / 1981$ & & 26.20 & 17.0 & 27.58 & & & $\mathrm{X}$ & & 23,587 & & & $\begin{array}{l}\text { "Pmp'd } 20 \text { bbls } 15 \% \text { HCl w/additives...pumped } 30 \text { bbls acid...flushed backside w/5 bbls KCl. On } \\
3 / 30 / 1981 \text {, "frac as follows...143 bbls Apollo } 30 \text { pad...24 bbls gel...48 bbls gel...72 bbls gel...72 bbls } \\
\text { gel...21 bbls 4\% KCl flush." }\end{array}$ & \\
\hline & 1405.7 & $\mathrm{FU}$ & $3 / 27 / 1981$ & & 24.13 & 17.0 & 26.20 & $7.58^{-}$ & & $\mathrm{X}$ & & & & & $\begin{array}{l}\text { Pmp'd } 26 \text { bbls acid...followed w/24 bbls flush ..flushed backside } w / 5 \text { bbls KCl wtr...Frac...as follow-100 } \\
\text { bbls Apollo } 30 \text { pad...40 bbls Pad...24 bbls gel...48 bbls gel...72 bbls gel...72 bbls gel...flushed w/21 bbls } \\
4 \% \text { KCl." }\end{array}$ & \\
\hline & 1481.3 & $\overline{\mathrm{FU}}$ & $3 / 20 / 1981^{-}$ & $37.23^{-}$ & $22.75^{-}$ & 17.0 & 27.58 & 8.27 & & $\mathrm{X}^{-}$ & & $9,072^{--}$ & & & $\begin{array}{l}\text { "Pmpd } 12 \text { bbls acid...pumped } 12 \text { bbls acid...pmpd } 24 \text { bbls acid...pmpd } 12 \text { bbls acid...Displaced acid w/26 } \\
\text { bbls KCl wtr...Pumped } 48 \text { bbls YF4PSD pad. } 24 \text { bbls gelled wtr." then } 180 \text { bbls YF4PSD. Materials charged } \\
\text { for in frac include } 700 \text { lbs J-347 gelling agent, } 48 \text { lbs J-218 breaker, } 16 \text { gal J-318 breaker aid, } 32 \text { gal L-53 } \\
\text { clay stabilizer, } 5 \text { gal D-47, } 5 \text { gal MW38 surfactant, A-200 inhibitor, U42" }\end{array}$ & \\
\hline $1-8-1 \mathrm{~B}$ & & & & & & & & & & & & & & & P\&A after completion in 1981. No apparent production well casing. No stimulation or production. & No \\
\hline $21-9$ & 657.8 & WR & $6 / 15 / 1981$ & & & & & & & & & & 0 & 11 & $\begin{array}{l}\text { Information missing from well completion report but reviewed by WOGCC (2014). Well schematic in } \\
\text { WOGCC }(2014)\end{array}$ & Yes \\
\hline & 725.4 & Wi- & $6 / 8 / 1981$ & & & & 6.89 & & & & $\underline{X}$ & & & & "Acidized" from WóGCC $(2014)$ & \\
\hline & 796.7 & WR- & $6 / 6 / 1981$ & & & & 6.89 & & & & $\underline{X-1}$ & & & & "Acidized" from WoGGCC $(2014)$ & \\
\hline & 1022.9 & FU'-1 & $5 / 28 / 1981$ & & & & 23.44 & & & $\mathrm{X}$ & & 11,754 & & & WOOGCC (2014) indicates that this interval was stimulated with "357 barrels of gelled water." & \\
\hline & 1129.3 & FU.-. & $5 / 26 / 1981$ & & & & 11.72 & & & & $\underline{X-1}$ & & & & "Acidized" from WOGGCC $(2014)$ & \\
\hline & 1209.1 & $\overline{\mathrm{FU}}$ & $5 / 21 / 1981$ & & & & $11.72^{-1}$ & & & & $\mathrm{X}$ & & & & "Acidized" from WOGCC (2014) & \\
\hline & 1295.1 & $\mathrm{FU}$ & $5 / 20 / 1981^{-}$ & & & & 11.72 & & & & $\mathrm{X}$ & & & & "Acidized" from WÖGCC (2014) & \\
\hline
\end{tabular}




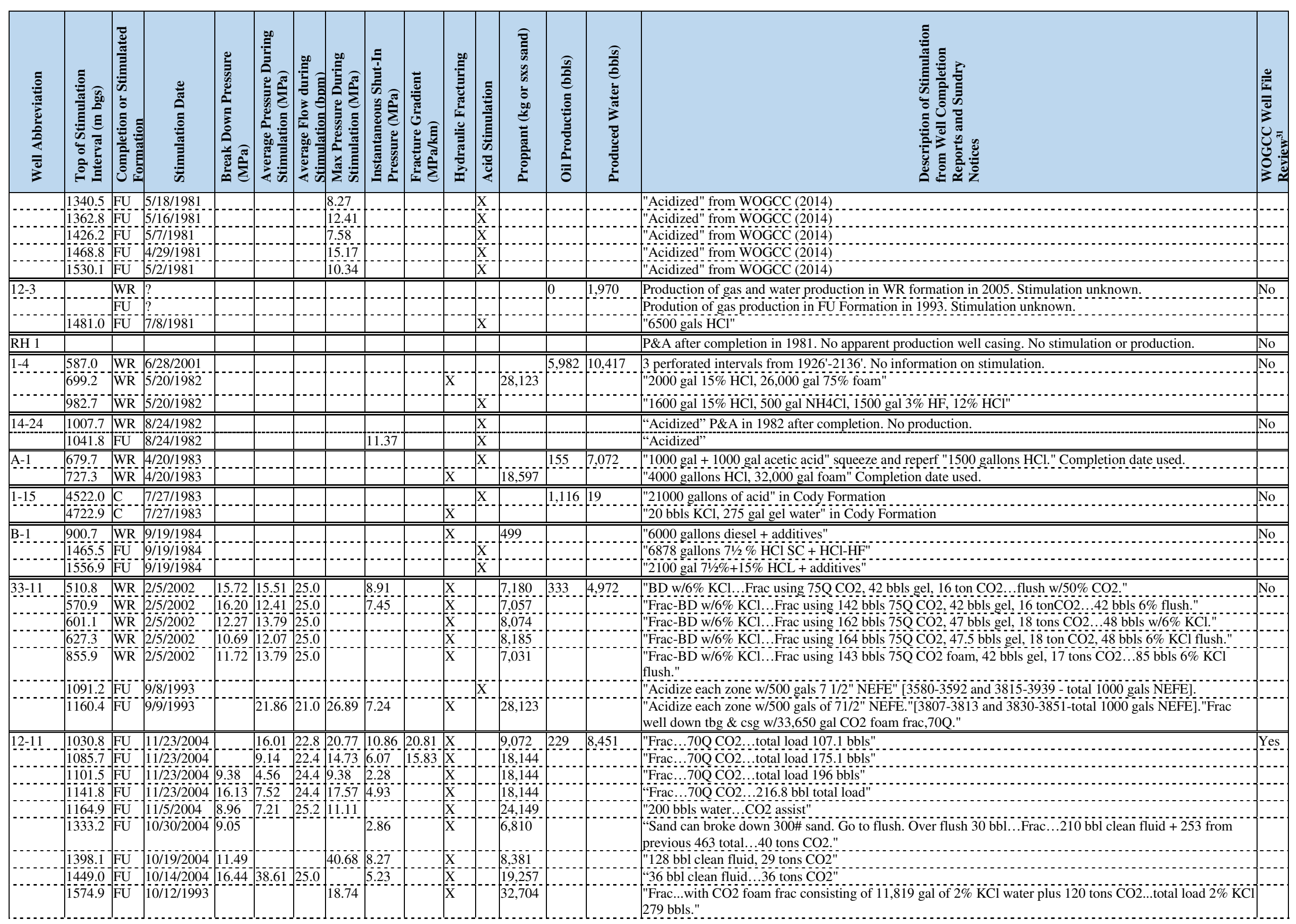




\begin{tabular}{|c|c|c|c|c|c|c|c|c|c|c|c|c|c|c|c|c|}
\hline 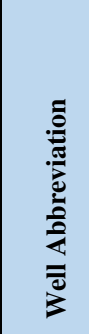 & 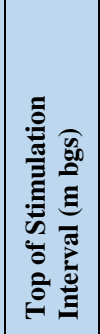 & 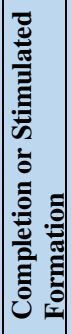 & 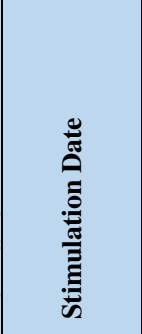 & 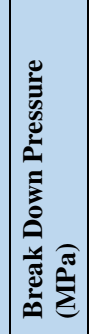 & 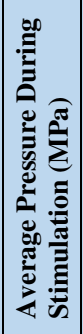 & 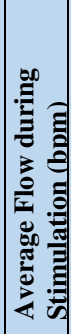 & 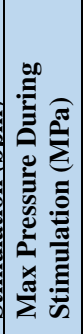 & 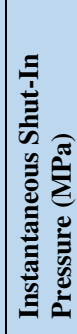 & 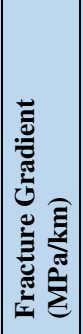 & 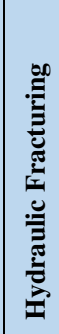 & 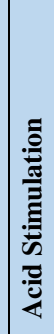 & 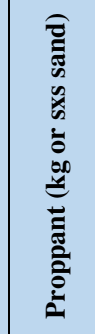 & 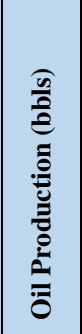 & 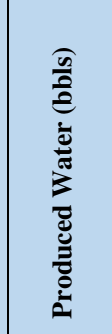 & 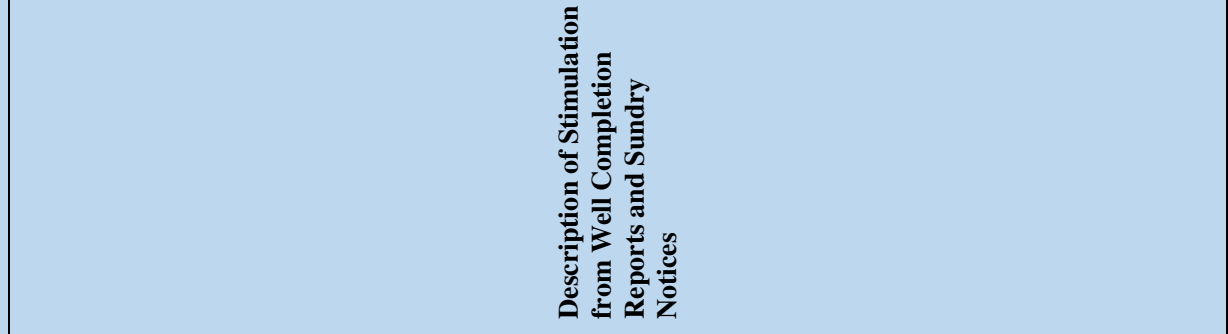 & 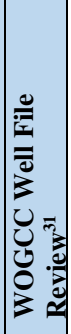 \\
\hline & 1624.9 & FU & $10 / 12 / 2004$ & & 37.23 & 25.0 & & 10.25 & & $\mathrm{X}$ & & 14,197 & & & "Frac...39 tons CO2 and 208 bbl clean fluid" & \\
\hline \multirow[t]{10}{*}{$11-10}$. & 800.7 & WR & $10 / 15 / 2004$ & 11.18 & 9.56 & 21.4 & & 6.98. & & $\mathrm{X}$ & & 8,165 & 1,238 & 21,643 & Information on fluids used for stimulation not available. & No \\
\hline & $8 \overline{18.7}$ & WR'-1 & $10 / 15 / 2004$ & $5.70^{--}$ & $6 . \overline{4} 9^{-}$ & $15 . \overline{4}$ & & 7.02 & $18 . \overline{5}$ & $\mathrm{X}^{-}$ & & 8,165 & & & Information on fluids used for stimulation not available. & \\
\hline & 858.0 & $\mathrm{WR}$ & $10 / 14 / 2004$ & 18.96 & 10.92 & 18.6 & & & & $\mathrm{X}$ & & $6,804^{-}$ & & & Information on fluids used for stimulation not available. & \\
\hline & 897.9 & WR & $10 / 14 / 2004$ & $5.35^{-1}$ & 10.71 & 16.6 & & & 20.3 & $\mathrm{X}^{-}$ & & 6,804 & & & Information on fluids used for stimulation not available & \\
\hline & 920.5 & WR & $10 / 8 / 2004$ & 9.38 & 15.17 & 35.0 & & 10.38 & & $\mathrm{X}^{-}$ & & 8,165 & & & "frac... 129 bbls clean fluid, 29 tons CO2" & \\
\hline & 971.7 & WR & $10 / 8 / 2004^{-}$ & 12.71 & 22.75 & 32.0 & & 10.55 & 20.81 & $\mathrm{X}^{-}$ & & 5,443 & & & 1444 bbis clean fluid, 19 tons CO22" & \\
\hline & 1049.4 & FE'- & $6 / 20 / 2005$ & 16.29 & 22.06 & 30.0 & & 11.20 & & $\mathrm{X}^{-}$ & & 13,608 & & & "frac.. 232 bbl clean fluid, 39 tons CO $2 "$ & \\
\hline & 1358.2 & FU & $10 / 8 / 2004$ & 7.05 & 8.96 & 30.0 & & 4.24 & & $\mathrm{X}$ & & 19,051 & & & "frac...359 clean fluid, 54 ton $\mathrm{CO} 2$ " & \\
\hline & 1462.7 & FU & $9 / 3 / 2004$ & & 20.17 & 24.2 & 38.74 & 1.78 & {$[11.54$} & $\mathrm{X}$ & & 24,494 & & & "CO 2 frac 226 bbs total load...recovered 37 bbls of 226 bbl loa." & \\
\hline & 1528.9 & $\mathrm{FU}$ & $9 / 2 / 2004$ & & 21.52 & 18.5 & 50.81 & & 15.16 & $\mathrm{X}$ & & 14,969 & & & $\begin{array}{l}\text { "fraced down...12,887 gal CO2...7OQ ...pad volume } 3952.6 \text { gal total load } 195 \text { bbls...blew rupture disk on } \\
\# 1 \text { CO2 pump...recoverd } 41 \text { bbls of } 195 \text { bbl load." }\end{array}$ & \\
\hline \multirow[t]{13}{*}{$42-10$} & 762.6 & WR & $12 / 20 / 2004$ & 9.11 & 8.46. & & 12.67 & 8.32 & 21.04 & X. & & 6,804 & 595 & $14,503$. & "Total load 86.1 bbls." Treatment report available for 2004 stimulation events. & Yes. \\
\hline & 807.1 & $\mathrm{WR}$ & $12 / 15 / 2004$ & $9.74^{-}$ & $8.27^{-}$ & & 13.31 & 7.35 & & $\mathrm{X}^{-}$ & & 6,871 & & & 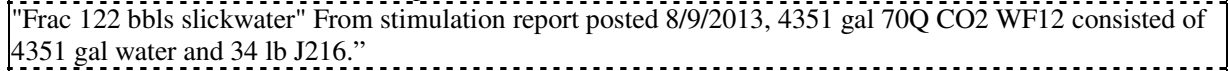 & \\
\hline & 1005 & WR-1. & $6 / 24 / 2001^{-}$ & 12.82 & 12.07 & 25.0 & 12.41 & $7.70^{-1}$ & & $\mathrm{X}_{-}^{-}$ & & $9,072^{-}$ & & & frac...Flush w/50Q CO2 foam" & \\
\hline & 1034 & FE'- & $6 / 23 / 2001$ & 9.51 & 15.51 & 30.0 & 15.51 & & & X- & & 5,859 & & & "frac" No information on stimulation fluids. & \\
\hline & 1068 & FE'-- & $6 / 23 / 2001^{-}$ & $6.16^{-}$ & 10.69 & 35.0 & 11.89 & 6.48 & & $x_{-}^{-}$ & & 9,072 & & & "frac" No information on stimulation fluids. & \\
\hline & 1202 & FE'- & $6 / 22 / 2001$ & 10.16 & 13.51 & 35.0 & 13.44 & 7.36 & & X- & & 11,340 & & & "frac..70Q CO2 foam total clean fluid 267 bbls." & \\
\hline & 1239 & FU & $6 / 22 / 2001$ & 10.07 & 10.82 & 30.0 & 11.55 & 9.27 & & $\mathrm{X}$ & & 11,340 & & & "frac...clean fluid $197 \mathrm{bbls"}$ & \\
\hline & 1256 & FE'- & $6 / 22 / 2001$ & 6.52 & 13.44 & 36.0 & 10.00 & 6.83 & & X- & & 7,525 & & & "frac...pumped 84 bbls clean fluid" & \\
\hline & 1380 & FU & $6 / 22 / 2001$ & 5.80 & 10.51 & 35.0 & 10.51 & 6.14 & & X & & 11,340 & & & ffrac total clean fluid 302 bbls, 89.5 tons CO2 & \\
\hline & 1448 & $\mathrm{FU}^{-1}$ & $9 / 16 / 1994$ & & & & & & & & $\mathrm{x}$ & & & & $\begin{array}{l}\text { In sundry notice dated } 5 / 27 / 2005 \text {, "Acidize w/2000 gallons } 15 \% \text { NEFE, } 230 \overline{0} \text { rock salt". WOGCC (2014) } \\
\text { states "no treatment" at this depth. }\end{array}$ & \\
\hline & $1620^{-1}$ & $\mathrm{FU}^{-1}$ & $6 / 13 / 1994^{-1}$ & & & & & & & & $\mathrm{X}$ & & & & $\begin{array}{l}\text { Perf } 5315-39,5389-54033^{2}, 5476-86 \ldots \text { acidize w/1250 gal } 71 / 2 \% \text { HCl acid }(6 / 13 / 94) .0 \mathrm{On} 6 / 12 / 2001 \\
\text { "Acidize - Stage 3 (5315-5339') Pump 750 gal } 15 \% \text { HCl...750 gal } 15 \% \text { drop } 48 \text { balls, } 700 \text { gal \& flush." }\end{array}$ & \\
\hline & 1643 & FU & $6 / 12 / 2001$ & & & & & & & & $\mathrm{X}$ & & & & $\begin{array}{l}\text { "Stage } 2(5389-5403 \text { ') Pump } 700 \text { gal } 15 \% \mathrm{HCl}, 250 \mathrm{gal} 6 \% \mathrm{KCl} \text { w/28 frac balls, } 700 \mathrm{gal} 15 \% \mathrm{HCl} \\
\text { Stimulation at this interval not reported by WOGCC }(2014)\end{array}$ & \\
\hline & 1669 & $\overline{F U}$ & $6 / 12 / 2001$ & & & & & & & & $\mathrm{x}^{-}$ & & & & $\begin{array}{l}\text { Stage } 1 \text { (5476-5486) Pump } 900 \text { gal } 15 \% \text { HCl, } 10 \text { bbls } 6 \% \text { KCl, w/30 frac balls, } 500 \text { gal } 15 \% " \text { Stimulation at } \\
\text { this interval not reported by WOGCC (2014). Modified wellbore schematic available in WOGCC (2014). }\end{array}$ & \\
\hline \multirow[t]{5}{*}{ 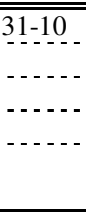 } & -1017 & FU & $1 / 16 / 2002$ & 11.30 & 15.17 & 30.0 & & 6.14 & & $\bar{X}$ & & 8,211 & $1,1,412$ & 15,504 & "Break w 6\% kcl... start 70Q foam 242 bbl, 80 bbl fluid, 29 ton co2 ...77 bbl 40Q flush" & Yes \\
\hline & $1095^{-1}$ & FE'-1. & $1 / 16 / 2002-$ & 10.69 & 14.48 & 35.0 & & 8.27 & & $\bar{X}_{-}^{-}$ & & 12,527 & & & Break w $6 \%$ kcl .. start $70 \mathrm{Q}$ co2 foam, $405 \mathrm{bbl}, 151.5 \mathrm{bbl}$ fluid, 39 ton co2".flush w $84.5 \mathrm{bbl} 6 \% \mathrm{kcl}$ & \\
\hline & 1377 & FE'- & $1 / 15 / 2002-$ & 12.07 & 15.86 & 35.0 & & 6.03 & & X- & & 20,550 & & & Break w $6 \%$ kcl...start $70 \mathrm{Q}$ co2, 695 bbl foam, 232 fluid, 74 ton co2 .. flushed $50 \%$ co2 bbl.". & \\
\hline & 1411 & FU & $1 / 15 / 2002$ & 14.13 & 16.89 & 35.0 & & 8.80 & & $\mathrm{X}$ & & 10,704 & & & Break w $6 \% \mathrm{kcl}$ start $70 \mathrm{Q}$ co 2 foam, $254 \mathrm{bbls}$ fluid, 36 ton co 2 flush was $109.5 \mathrm{bbl} 6 \% \mathrm{kcl}$ & \\
\hline & 1362 & $\mathrm{FU}$ & $6 / 22 / 1994$ & & & & & & & & $\mathrm{x}$ & & & & $\begin{array}{l}\text { In sundry notice dated } 5 / 28 / 2002 \text {, "Acid frac...each \& all sets of perfs w/600 gals } 71 / 2 \% \mathrm{HCl} \text { acid each } \\
47700^{\prime}-5478 \text {." }\end{array}$ & \\
\hline \multirow[t]{3}{*}{$23-10$} & 1400 & FU & $7 / 8 / 2000$ & 9.10 & 6.03 & 30.0 & & 7.03 & & $\mathrm{X}$ & & 22,389 & 7 & 112,463 & $\begin{array}{l}\text { "Pump 70Q CO2 foam frac...3000 gals pad, } 480 \text { gals...540 gals...640 gals...812 gals...1200 gals...3696 } \\
\text { gals 50Q flush ...pumped 226 bbls KCL, 47 ton CO2." }\end{array}$ & Yes \\
\hline & 1476 & FU & $6 / 29 / 2000$ & 14.92 & 8.27 & 28.5 & & 4.27 & & $\mathrm{X}$ & & 12,519 & & & Pumped $70 \mathrm{Q}$ CO2 foam frac 3000 gal pad, 1700 gal..1840 gals..2843 gals $50 \mathrm{Q}$ flush." & \\
\hline & $1620^{-}$ & $\mathrm{FU}^{-1}$ & $8 / 8 / 1994^{-1}$ & & & & & & & $\bar{X}$ & & 14,742 & & & $1 " 500$ gal $15 \%$ HCl" "Frac w/16500 gal plus $700 \mathrm{~N} 2$ foam." & \\
\hline 43-10 & 1389 & FU & $2 / 6 / 2002$ & 11.86 & 19.31 & 35.0 & & 5.28 & & $\mathrm{X}$ & & 21,896 & 606 & 28,467 & $\begin{array}{l}\text { "Frac break w/6\% KCL...Frac using } 547 \text { bbls 70Q CO2 foam, } 125 \text { bbls gel, } 63 \text { ton CO2...flush w/30\% } \\
\text { CO2." }\end{array}$ & Yes \\
\hline
\end{tabular}




\begin{tabular}{|c|c|c|c|c|c|c|c|c|c|c|c|c|c|c|c|c|}
\hline 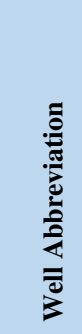 & 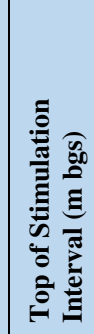 & 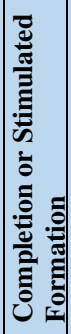 & 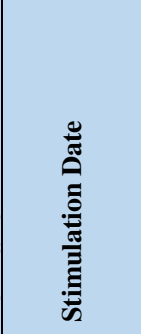 & 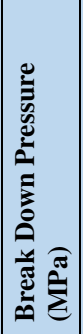 & 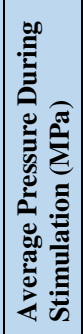 & 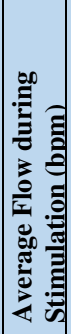 & 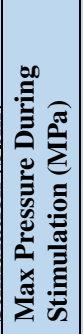 & 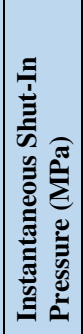 & 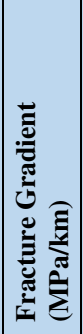 & 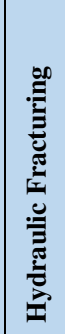 & 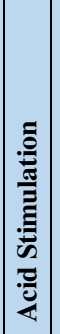 & 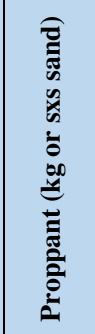 & 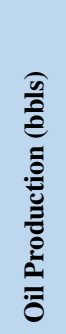 & 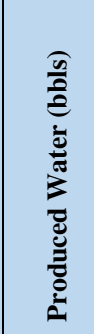 & 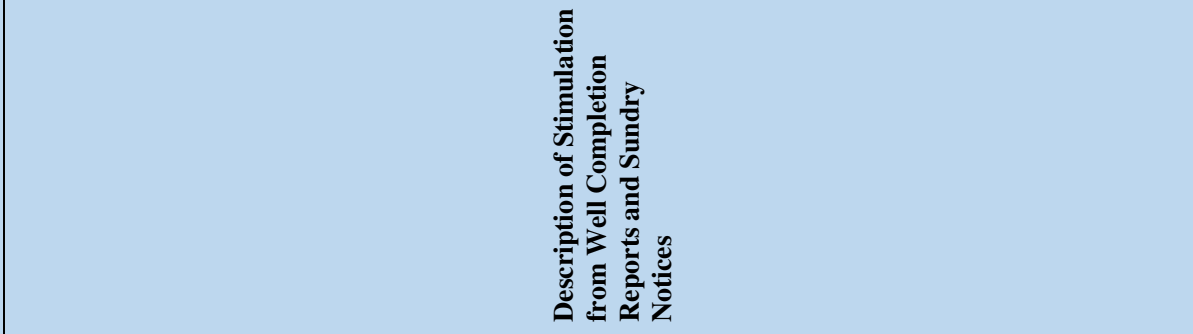 & 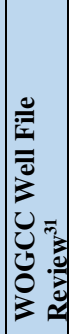 \\
\hline & 1467 & $\mathrm{FU}$ & $2 / 6 / 2002$ & 11.24 & 17.24 & 35.0 & & 5.16 & & $\mathrm{X}$ & & 28,931 & & & $\begin{array}{l}\text { "Frac-Break w/6\% KCL...Frac using } 837 \text { bbls 70Q CO2, } 235 \text { bbls gel, } 95 \text { tons CO2...112 bbls 6\% KCl } \\
\text { flush." }\end{array}$ & \\
\hline & 1574 & FE'- & $2 / 6 / 2002$ & & 20.34 & 34.0 & & & & $\mathrm{X}$ & & 15,941 & & & "Frac using $457 \mathrm{bbls} 70 \mathrm{Q}$ foam, 130 bbls gel, 52 ton CO2 flush $\mathrm{w} / 120$ bbls $6 \% \mathrm{KCl}$ & \\
\hline & 1597 & FU'-1 & $2 / 6 / 2002$ & 20.48 & 24.13 & 32.0 & & 14.89 & & $\mathrm{X}$ & & $19,595^{-}$ & & & "Frac using 545 bbls $70 \mathrm{COO} 2 \ldots 156$ bbls gel, 61 ton $\mathrm{CO} 2$ flush w/124 bbl $6 \% \mathrm{KCl}$ & \\
\hline & 1623 & $\mathrm{FU}$ & $9 / 20 / 1994^{--}$ & & $19.31^{\circ}$ & & & 19.54 & & & $\mathrm{X}$ & & & & $\begin{array}{l}\text { Sundry notice dated } 2 / 19 / 2002 \text {, "Picke tbg w/600 gal } 15 \% \text { acid. Spot } 250 \mathrm{~g} 15 \% \text { NEFE acid...press tbg to } \\
5300 \text { psi w/N2...Pump } 20 \text { mcf N2 @ } 2100 \text { scfm @ } 2800 \text { psi" WOGCC (2014) indicates no stimulation at } \\
\text { this depth. }\end{array}$ & \\
\hline & $1678^{-}$ & $\overline{F U}^{-}$ & $1 / 26 / 2002$ & & 16.41 & 50.0 & & $7.93^{-}$ & & $\mathrm{X}$ & & $30,551^{-}$ & & & "Frac-total pumped 317 bbils KCl, 598 b bils COC2" & \\
\hline 33-2 & 432.8 & WR & $10 / 18 / 2000$ & & & & & & & $\bar{X}$ & & 8,245 & (ب. & $1,263$. & "Coil frac...104 bbls clean fluid, 41300 scf of N2.". & No. \\
\hline & $5 \overline{5} 2.3^{-1}$ & Win & $10 / 18 / 2000$ & & & & & & & $\bar{X}$ & & 4,954 & & & "Coil frac...93 bbils clean fluid, 43300 scf of $\mathrm{N} 2$. & \\
\hline & $605.6-$ & WR'-1- & $10 / 18 / 2000$ & & & & & & & $\bar{X}$ & & $10,870^{-}$ & & & "Coil frac...177 bbils clean fluid, 961000 scf of $\mathrm{N} 2 "$ & \\
\hline & 624.5 & WR & $10 / 18 / 2000$ & & & & & & & $\bar{X}$ & & 13,525 & & & "Coil frac...139 bbis clean fluid, 77100 scf of N2" & \\
\hline & 644 & $\mathrm{WR}$ & $10 / 18 / 2000$ & & & & & & & $\mathrm{X}$ & & 15,037 & & & "Coil frac...206 bbls clean fluid, 124200 scf of $\mathrm{N}^{2}$ & \\
\hline & 680.6 & WR & $10 / 18 / 2000$ & & & & & & & $\mathrm{X}$ & & 8,698 & & & "Coil frac... 126 bbls clean fluid, 78000 scf of $\mathrm{N} 2 "$ & \\
\hline & 715.1 & Whe-1 & $10 / 18 / 2000$ & & & & & & & $\mathrm{X}$ & & 12,701 & & & "Coil frac...183 bbls clean fluid, 115800 scf of N2" & \\
\hline & 750.1 & WER-1 & $10 / 18 / 2000$ & & & & & & & X- & & 17,049 & & & "Coil frac...254 bbls clean fluid, 162300 scf of N2" & \\
\hline & 776.6 & WR- & $10 / 18 / 2000$ & & & & & & & X- & & 12,927 & & & "Coil frac 317 bbls clean fluid, 83800 scf of $\mathrm{N2}$ & \\
\hline & 849.2 & WR & $10 / 21 / 1994$ & & & & & & & & $\mathrm{X}$ & & & & "Acidize...W/300 gals $15 \%$ NEFE" & \\
\hline & $862^{-1}$ & WR & $10 / 21 / 1994$ & & & & & & & & X & & & & Acidize...W/300 gals $15 \%$ NEFE" & \\
\hline & $904.3^{-1}$ & WR & $10 / 21 / 1994$ & & & & & & & & $\mathrm{X}$ & & & & "Acidize...w/300 gals $15 \%$ NEFE" & \\
\hline $23-1$ & 810.5 & WR & $10 / 13 / 1994$ & & 11.40 & 5.0 & 12.34 & 5.38 & & & $\mathrm{X}$ & & 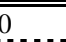 & 480 & "Spot \& pump 400 gal 15\% NEFE. . gained 30.48 bbls in 72 hrs 15,000 ppm chl." & No \\
\hline & 857.7 & WhR-1 & $10 / 13 / 1994$ & & 12.25 & 5.0 & 14.13 & 5.38 & & & X- & & & & "Spot \& pump 400 gal 15\% NEFE" & \\
\hline & 907.1 & WR. & $10 / 13 / 1994$ & & 11.07 & & 16.76 & 7.93 & & & X & & & & "Spot \& pump 400 gals 15\% NEFE" & \\
\hline & 1393 & $\overline{F U}^{-}$ & $8 / 13 / 1994^{--}$ & & $17 . \overline{3}$ & & $18.60^{\circ}$ & $6.23^{-}$ & & & $\mathrm{X}$ & & & & "Pump 500 gals $15 \%$ NEFE" & \\
\hline & 1526 & $\mathrm{FU}^{-1}$ & $8 / 13 / 1994$ & & 21.28 & & 23.39 & 7.96 & & & $\mathrm{X}$ & & & & "Spot \& pump 500 gals $15 \%$ NEFE." & \\
\hline 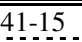 & 1650 & $\overline{F U}$ & $11 / 2 / 1994$ & & 10.34 & 3.0 & 39.09 & 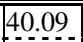 & & $\bar{X}$ & 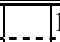 & $14,742$. & 355 & $64,192$. & "1500 gal 15\% NEFE-PB", "frac w/16,600 gal 65Q N2 foam.". & $\overline{\mathrm{No}}$ \\
\hline & $1897^{-}$ & $\mathrm{FU}$ & $11 / 2 / 1994^{-}$ & & & & & & & & $\bar{X}$ & & & & "2000 gal $15 \%$ NEFE-PB ...gained $3.34 \mathrm{bbl}$ in $1 \mathrm{hr} . .(11,000 \mathrm{ppm}$ chl $) "$ & \\
\hline $43-6$ & 287.7 & WR & $8 / 10 / 1999$ & & 6.62 & 6.0 & & 1.57 & & & $\mathrm{X}$ & & 0 & 1,062 & 300 gal "7.5\% HL w/mutual solvent, $\mathrm{HC} 2$ surfactant, flush $4 \% \mathrm{KCl}$ & No. \\
\hline & 359.1 & WR & $8 / 10 / 1999$ & & 8.55 & 6.0 & & 4.24 & & & $\mathrm{X}$ & & & & $400 \mathrm{gal}$ "7.5\% HL w/mutual solvent, $\mathrm{HC} 2$ surfactant, flush $4 \% \mathrm{KCl}$ & \\
\hline & 379.2 & WR- & $8 / 10 / 1999$ & & 13.79 & 6.5 & & 4.92 & & & X & & & & 150 gal "7.5\% HL w/mutual solvent, $\mathrm{HC} 2$ surfactant, flush $4 \% \mathrm{KCl}$ & \\
\hline & 476.1 & WR & $8 / 10 / 1999$ & & 12.07 & 6.6 & & 5.54 & & & $\mathrm{X}$ & & & & 250 gal $7.5 \% \mathrm{HL}$ w/mutual solvent, $\mathrm{HC} 2$ surfactant, flush $4 \% \mathrm{KCl}$ & \\
\hline & 575.5 & Wi- & $8 / 10 / 1999$ & & 12.76 & 6.0 & & 4.59 & & & $\mathrm{X}$ & & & & 1000 gal $7.5 \%$ HL w/mutual solvent, $\mathrm{HC} 2$ surfactant, flush $4 \% \mathrm{KCl}$ & \\
\hline & 723.6 & WR & $8 / 4 / 1999^{-1}$ & & & & & $\overline{7} . \overline{3} 8^{-}$ & & $\mathrm{X}$ & & 21,387 & & & "clear frac" & \\
\hline & 751.6 & WER-1 & $8 / 4 / 1999$ & & & & & $8.61^{-}$ & & $x^{-}$ & & 18,079 & & & "clear frac" & \\
\hline & $1055^{-}$ & $\mathrm{WR}$ & $5 / 23 / 1995$ & & & & & & & & $\mathrm{X}$ & & & & "Pmp 100 gals $15 \%$ HCl acid $\& 95,180$ SCF/N2" & \\
\hline $23-11$ & 507.2 & WR & $10 / 14 / 2000$ & & & & & & & $\mathrm{X}$ & & $4,975$. & 88 & 208 & "Coil frac...81 bbls clean fluid, 34600 scf N2" & No \\
\hline & 522.1 & $\mathrm{WR}$ & $10 / 14 / 2000$ & & & & & & & $\mathrm{X}$ & & 20,936 & & & "Coil frac...282 bbls clean fluid, 141000 scf N2" & \\
\hline & 554.1 & WR & $10 / 142000$ & & & & & & & $\mathrm{X}$ & & 7,967 & & & "Coil frac. 119.5 bbls clean fluid, 59400 scf N2" & \\
\hline & 607.5 & WR & $10 / 14 / 2000$ & & & & & & & $\mathrm{X}$ & & 7,976 & & & ["Coil frac..117 bbls clean fluid, 64100 scf N2" & \\
\hline & 739.7 & WR- & $10 / 14 / 2000$ & & & & & & & $\mathrm{X}$ & & 5,993 & & & "Coil frac...96 bbbls clean fluid, 62100 scf N $2 "$ & \\
\hline & 792.2 & [WR & $10 / 12 / 2000$ & & & & & & & & & {$[9,617]$} & & & Coil frac..136 bbls clean fluid, 10700 scf N2" & \\
\hline
\end{tabular}




\begin{tabular}{|c|c|c|c|c|c|c|c|c|c|c|c|c|c|c|c|c|}
\hline 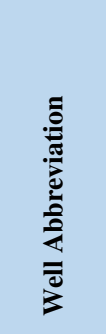 & 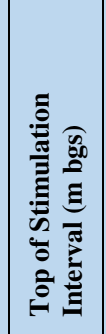 & 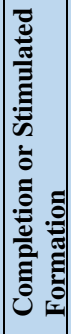 & 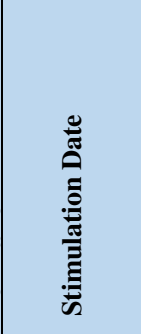 & 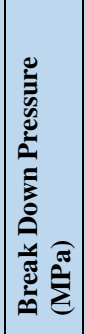 & 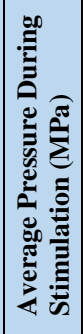 & 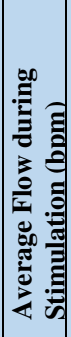 & 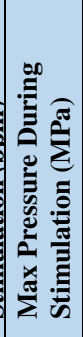 & 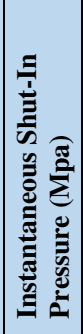 & 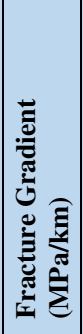 & 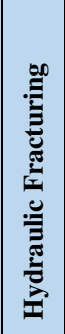 & 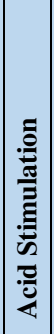 & 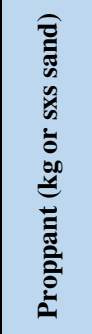 & 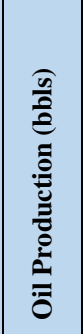 & 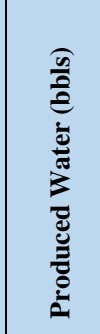 & 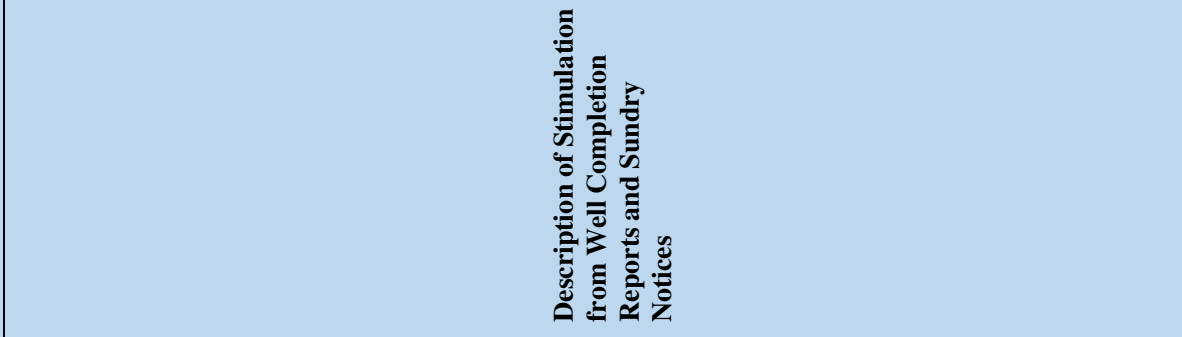 & 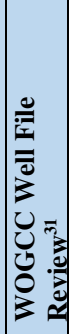 \\
\hline & 843.1 & $\mathrm{WR}$ & $10 / 12 / 2000$ & & & & & & & $\mathrm{X}$ & & 12,226 & & & "Coil frac..198.6 bbls clean fluid, 168,000 scf N2" & \\
\hline & $866.2^{-}$ & WR & $10 / 12 / 2000$ & & & & & & & & & $4,652^{--}$ & & & Coil frac...86.2 bbls clean fluid, 69000 scf $\mathrm{N} 2$ & \\
\hline & 1054 & FE'- & $3 / 5 / 2005$ & 7.78 & & & & $8.42^{--}$ & & $\mathrm{X}_{-}^{-}$ & & $8,649^{-}$ & & & "Frac: $4172 \mathrm{~b} b \mathrm{~b} \mathrm{~s} 70 \mathrm{Q}^{\prime \prime}$ & \\
\hline & 1103 & $\mathrm{FU}^{-1}$ & $3 / 2 / 2005$ & 4.94 & & & & 3.25 & & $\mathrm{X}$ & & 13,166 & & & "Frac: $181 \mathrm{bbls} 70 \mathrm{Q}^{\prime}$ & \\
\hline & 1159 & FE'- & $2 / 23 / 2005$ & 11.03 & & & & 3.72 & & $\mathrm{X}$ & & 13,114 & & & "Frac: 9348 bbls $60 \mathrm{Q}$ & \\
\hline & 1358 & FU'- & $3 / 6 / 1998$ & & & & & & & & $\mathrm{X}$ & & & & "Acidize w/300 gals 7.5\% $\mathrm{HCl}$ & \\
\hline & 1388 & FE'- & $1 / 19 / 1998$ & & & & & & & & X & & & & "Acidize w/1000 gallons $15 \%$ HCl". & \\
\hline & 1403 & FU'-- & $12 / 17 / 1997$ & & & & & & & & X & & & & "Acidize w/500 gal $15 \%$ HCl, flush w/brine" & \\
\hline & 1458 & FU. & $12 / 4 / 1997^{-}$ & & & & & & & $\mathrm{X}_{-}^{-}$ & -.- & & & & "Acidize w/500 gal $15 \%$ HCl...Frac-Delta frac-30\% N2 used except flush. Total $742.3 \mathrm{bbbs}$ & \\
\hline & 1653 & $\overline{\mathrm{FU}}$ & $11 / 21 / 1997$ & & & & & & & & $\mathrm{x}$ & & & & "Acidize w/500 gal $15 \% \mathrm{HCl}$ & \\
\hline $31-11 X$ & 2828 & $\mathrm{C}$ & $10 / 22 / 1998$ & & & & & & & & & & 1,666 & 8,797 & $\begin{array}{l}\text { Hydraulic fracturing in Lance, Meetesse, Mesaverde, and Cody Formations. Acidize with 18,500 gallons } \\
15 \% \text { HCl. Frac w/1015 bbls CO2 foam }\end{array}$ & No \\
\hline $13 \mathrm{X}-3$. & 410.9 & WR & $2 / 18 / 1999$ & & 7.58 & 1.5 & & 5.40 & & & $\mathrm{X}$ & & 0. & 0 & "Acd...w/400 gal 15\% $\mathrm{HCl}$ & \\
\hline & 527.3 & WR & $2 / 18 / 1999$ & & 3.79 & 1.5 & & 3.25 & & & $\mathrm{x}$ & & & & "500 gal HCl" & \\
\hline & 584 & WR & $2 / 18 / 1999$ & & 924 & 1.6 & & 7.65 & & & $\mathrm{x}$ & & & & "Acd ...w/300 gal HCl" & \\
\hline & 635.2 & WR & $2 / 18 / 1999$ & & 6.21 & 1.5 & & 5.10 & & & $\mathrm{X}$ & & & & "Acd...w/250 gal HCl" & \\
\hline & 640.4 & WR & $2 / 18 / 1999$ & & & & & & & & $\mathrm{X}$ & & & & "Acd ..pmpd 73 gal HCl" & \\
\hline & 676.7 & WR & $2 / 18 / 1999$ & & & & & & & & X & & & & Acd...w/250 gal" & \\
\hline & 689.2 & WR & $2 / 18 / 1999$ & & 5.52 & 1.5 & & 4.73 & & & $\mathrm{X}$ & & & & "Acidize w/250 gal $15 \% \mathrm{HCl}$ & \\
\hline & 11799 & FU'- & $2 / 15 / 1999$ & & & 4.0 & & 0.26 & & & X- & & & & "Acidize...w/1000 gal 15\% HCl1..disp w/2\% KCl...Fluid pmp 41.5 bbl, fluid recurd 12.6." & \\
\hline & 1381 & FU'- & $2 / 9 / 1999$ & & & & & 0.06 & & & $\mathrm{X}$ & & & & "500 gal HCl". & \\
\hline & 1489 & FU'- & $2 / 9 / 1999$ & & & & & & & & X & & & & "500 gal HCl"- & \\
\hline & 1510 & $\mathrm{FU}$ & $2 / 9 / 1999$ & & & & & 6.35 & & & X- & & & & 500 gal HCl" & \\
\hline & 1558 & $\overline{\mathrm{FU}}$ & $2 / 9 / 1999^{--}$ & & & & & $2.95^{-1}$ & & & $\mathrm{X}$ & & & & "500 gal HCl" & \\
\hline $42 \mathrm{X}-9$ & & $\mathrm{FU}$ & & & & & & & & & & & 100 & 10,083 & $\begin{array}{l}\text { Completed in Fort Union Formation. Information on perforations and stimulation missing from well } \\
\text { completion report. }\end{array}$ & No \\
\hline $\mid 41-11$. & -602.3. & WR & $2 / 1 / 1999$ & & & & & & & & $\mathrm{X}$ & & 0. & 207 & Acidize... w 1100 gallons 15\% HCl". & $\{$ No \\
\hline & 622.1 & WR & $2 / 1 / 1999$ & & & & 6.41 & 1.61 & & & $\mathrm{X}$ & & & & ["Acidize ...W 300 gallons $15 \%$ HCl each [perforated interval] (600 gals total)" & \\
\hline & 1033 & FU & $1 / 21 / 1999$ & & 12.41 & 4.1 & & 0.85 & & & $\mathrm{X}$ & & & & "Acidize...w/500 gallons $15 \% \mathrm{HCl}$ & \\
\hline & 1054 & FU'- & $1 / 21 / 1999$ & & 11.79 & 4.1 & & 4.63 & & & $\mathrm{X}$ & & & & "Acidize. w/500 gallons $15 \% \mathrm{HCl}$ & \\
\hline & 1183 & FU'- & $1 / 21 / 1999$ & & 7.58 & 4.0 & & 0.99 & & & $\mathrm{X}$ & & & & Acidize... w/1000 gallons $15 \% \mathrm{HCl}$ & \\
\hline & 1384 & $\mathrm{FE}-$ & $1 / 19 / 1999$ & 18.89 & 15.86 & 4.5 & & 7.04 & & & $\mathrm{X}$ & & & & "Acidize... w/1000 gallons $15 \% \mathrm{HCl}$ & \\
\hline & 1527 & $\mathrm{FU}$ & $1 / 11 / 1999$ & $12.62^{-}$ & 12.27 & 3.1 & & $7.29^{-}$ & & & $\mathrm{X}$ & & & & "Acidiz...w/500 gallons $\mathrm{HLC} "[\mathrm{HCl}]$ & \\
\hline $33-10$ & 640.4 & WR & $1 / 5 / 2005$ & 111.29 & 13.13 & 24 & & & & 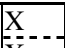 & & 9,525 & 2. & 2,547 & "28 tons CO2" & Yes \\
\hline & 740.1 & $\mathrm{WR}$ & $1 / 5 / 2005$ & 6.58 & $7.43^{-1}$ & 16.0 & & 6.96 & & $\mathrm{X}^{-}$ & & 8,165 & & & "24 tons" & \\
\hline & 756.5 & $\mathrm{~W} R$ & $1 / 4 / 2005^{-}$ & $13.29^{-}$ & 10.74 & 23.5 & & 8.69 & & $\mathrm{X}_{-}^{-}$ & & 10,886 & & & "32.6 tons CO22, 148 bbls $6 \%$ KCl" & \\
\hline & 1146 & {$\left[\bar{F} \bar{U}^{-}\right]$} & $1 / 4 / 2005$ & 12.40 & 8.05 & & & 3.99 & & $\mathrm{x}^{-}$ & & 9,525 & & & 170 bbls $6 \% \mathrm{KCl}, 286$ tons CO2" & \\
\hline
\end{tabular}




\begin{tabular}{|c|c|c|c|c|c|c|c|c|c|c|c|c|c|c|c|c|}
\hline 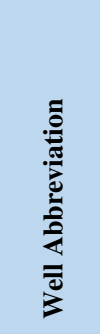 & 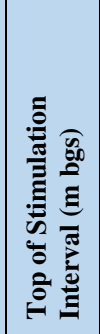 & 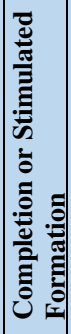 & 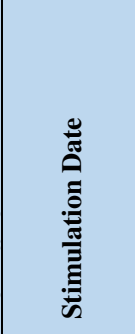 & 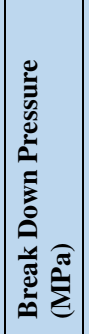 & 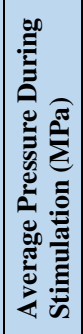 & 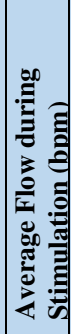 & 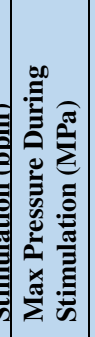 & 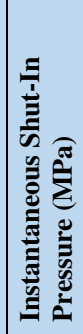 & 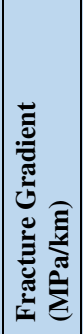 & 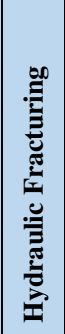 & 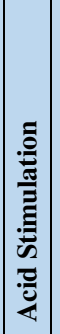 & 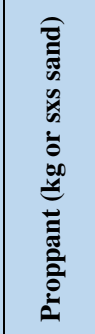 & 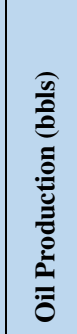 & 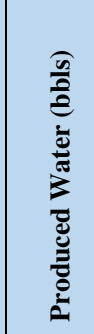 & 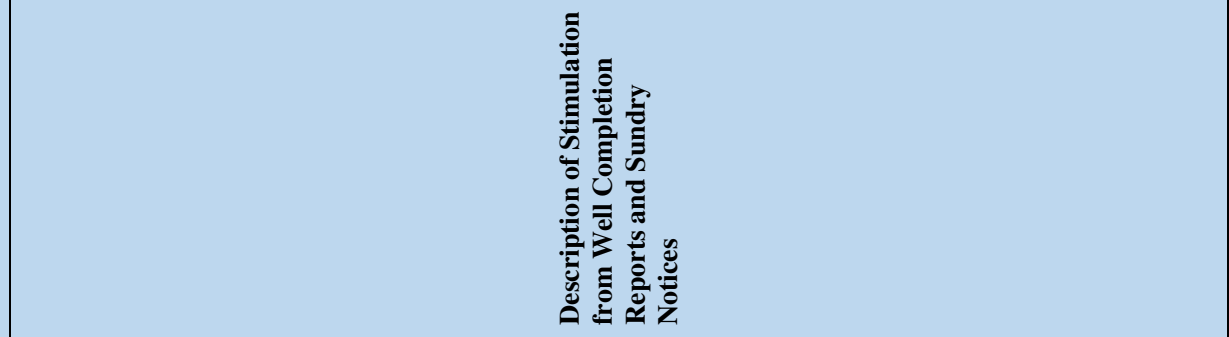 & 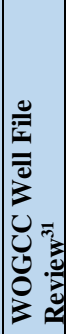 \\
\hline & 1562 & $\bar{F} \dot{U}^{-1}$ & $4 / 20 / 1999$ & & 12.14 & 33.0 & 15.55 & 10.84 & & $\mathrm{X}$ & & & & & "Frac w/35\# liner [linear] gel \& $70 \%$ CO 2 foam. Total fluid pmped 10,235 gals \& 76.5 tons CO 2. & \\
\hline & 1637 & $F U^{-1}$ & $4 / 12 / 1999$ & & 13.55 & 25.0 & & 10.45 & & $\mathrm{X}$ & & 24,902 & & & $\begin{array}{l}\text { "Acidize w/1500 gal } 15 \% \text { HCl. Frac...35\# linear gel w/70\% CO2 } 2 \text {. total fluid pmped } 13,754 \text { gal. Total CO } 22 \\
68 \text { tons." }\end{array}$ & \\
\hline $33-3$ & 1372 & $\overline{F U}$ & & & & & & & & & & & 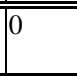 & 337 & $\begin{array}{l}\text { Perforated intervals in Wind River and Fort Union Formations. Production from Wind River Formation. } \\
\text { Information on stimulation missing. }\end{array}$ & No \\
\hline $44-3$ & 213.1 & WR & $4 / 8 / 1999$ & & 7.58 & & & 2.30 & & & $\mathrm{X}$ & & & 1,663 & "Acidize $\ldots$ w/500 gals $15 \% \mathrm{HCl}$ " & Yes \\
\hline & 505.4 & $\mathrm{WR}$ & $4 / 8 / 1999$ & & 9.65 & 15.0 & 12.41 & $8.84^{-}$ & & $\bar{x}$ & & 46,090 & & & $\begin{array}{l}\text { On } 4 / 8 / 1999 \text {, "Acidize ... w/500 gals } 15 \% \text { HCl" On } 5 / 26 / 1999 \text {, "frac...w } / 273 \text { bbl clear frac" ISIP for acid } \\
\text { stimulation was } 3.63 \mathrm{MPa} \text {. }\end{array}$ & \\
\hline & 634.3 & WR'-1. & $4 / 8 / 1999$ & & 10.34 & 5.0 & & 6.72 & & & X & & & & $\begin{array}{l}\text { On } 4 / 8 / 1999 \text {, "Acidize... w/500 gals } 15 \% \text { HCl" On } 6 / 2 / 1999 \text {, "frac...w } / 243 \text { bbl clear frac". ISIP for acid } \\
\text { stimulation was } 1.03 \text { Mpa. }\end{array}$ & \\
\hline & 679.7 & Whe- & $4 / 8 / 1999$ & & 6.82 & 3.0 & & 4.92 & & & $\mathrm{X}$ & & & & "Acidizew $/ 200$ gals $15 \%$ HCl" & \\
\hline & 838.8 & WR- & $4 / 8 / 1999$ & & 11.20 & 5.3 & & 3.06 & & & X- & & & & Acidize...w/500 gals $15 \% \mathrm{HCl}$ & \\
\hline & 849.8 & WR- & $4 / 8 / 1999$ & & 11.38 & 5.0 & & $3.45^{\circ}$ & & & $\mathrm{X}$ & & & & "Acidize w/300 gals $15 \%$ HCl" & \\
\hline & $1070^{-}$ & $\overline{F U}$ & $3 / 23 / 1999$ & & & & & 5.69 & & & $\mathrm{X}$ & & & & $\begin{array}{l}\text { Pmp } 7 \text { bbl } 15 \% \text { HCl and } 5 \text { bbl } 2 \% \text { KCl ..displ acid w/10 bbl } 2 \% \text { KCl...reset pkr, pmp } 2 \% \text { KCl...at } 1000 \\
\text { psi...oad pmpd } 38 \text { bbl" }\end{array}$ & \\
\hline & 1080 & FU'-- & $3 / 23 / 1999$ & & & & & & & & X- & & & & "Acidize & \\
\hline & 1183 & FE- & $3 / 16 / 1999$ & 6.49 & 13.79 & $6.0-1$ & & 3.03 & & & X- & & & & "Acidize...w/500 gals $15 \%$ HCl" & \\
\hline & 1214 & FE'- & $3 / 9 / 1999$ & 6.34 & 10.34 & 6.0 & & 3.56 & & & X & & & & "Acidize $. . \mathrm{w} / 500$ gals $15 \% \mathrm{HCl}$ & \\
\hline & $13222_{-1}^{-1}$ & FË & $3 / 5 / 1999$ & & & 3.0 & & 3.85 & & & X- & & & & "Acidize...w/950 gal 15\% HCl" - & \\
\hline & 1361 & $\overline{F U}$ & $3 / 5 / 1999$ & & & 5.0 & & 0.15 & & & $\mathrm{X}$ & & & & "pmp 420 gal $15 \%$ HCl" & \\
\hline & $1451^{-}$ & $\overline{\mathrm{FU}}$ & $2 / 25 / 1999$ & & & 4.0 & & $4.83-$ & & & $\mathrm{X}$ & & & & "Breakdown on perf w/1250 gal $15 \% \mathrm{HCl}$ & \\
\hline $15-21 X$ & 1196 & FU & $2 / 9 / 2001$ & 6.21 & 4.83. & 4.0 & & & & X & & & 161 & $1,276$. & "Pmp 20 bbls 6\% KCl ..pump 20 bbls 6\% KCl" & No \\
\hline & 1617 & FË & $2 / 7 / 2001$ & 10.34 & $3.45^{-}$ & 2.0 & & & & $\mathrm{X}_{-}^{\mathrm{X}}$ & & & & & Pmp 10 bbls $6 \% \mathrm{KCl}$ & \\
\hline & 1652 & FE & $2 / 2 / 2001$ & 10.34 & 4.14 & 2.0 & & 2.41 & & X-. & & & & & Pump 10 bbls..Acidize w/15 bbls 7 1/2\% acid, flush w/52 bbls KCl water ...no flow back". & \\
\hline & 2711 & M-. & $12 / 5 / 2000$ & & & & & & & & & & & & Acidize w/41 bbls prod wtr. pump 5.6 bbls more/pump 11.7 bbls $15 \%$ HCl $\&$ over flush perf & \\
\hline & 4659 & F & $1 / 27 / 1999$ & & & & & & & & & & & & Pmp perfs w/3000 gals diesel \& 1000 gal pad acid" & \\
\hline & 4709 & $\mathrm{~F}$ & $1 / 6 / 1999$ & & & & & & & & & & & & "Suspected casing collapse @ 15273...Breakdown w/4000 gal diesel w/FE 1A Hyflo IV, Musol Ā" & \\
\hline & 4868 & $\mathrm{~F}$ & $12 / 6 / 1998$ & & & & & & & & & & & & $\begin{array}{l}\text { "Breakdn w/2000 gals diesel w/20 gal Hyflo IV, } 100 \text { gal Musol A...No circulation before or during cement } \\
\text { job; lost } 1100 \text { bbls mud [invert] before job" }\end{array}$ & \\
\hline 32-10 & 1617 & FU & $5 / 12 / 2000$ & & 7.58 & 25.0 & & 4.62 & & $\underline{\underline{X}}$ & & & 29 & 2,200 & "Frac w/24,096 gal CO2 foam" & Yes \\
\hline $13-11$ & 764.4 & WR & $2 / 21 / 2001$ & & 16.55 & 26.0 & & 6.62 & & $\bar{X}$ & & & 0 & 431 & $\begin{array}{l}\text { "Frac... using } 231 \text { bbls wf } 135 \text { in } 6 \% \mathrm{KCl} \text { water w/10\% methanol + adds, } 59 \text { tons CO2...573 bbls } 70 \mathrm{Q} \text { CO2 } \\
\text { foam. Flush } \mathrm{w} / 37 \mathrm{bbls} \mathrm{KCl} \mathrm{w} / 10 \% "\end{array}$ & No \\
\hline & 807.1 & $\mathrm{WR}$ & $2 / 21 / 2001$ & & 15.86 & 25.0 & & 8.58 & & $\mathrm{X}^{-}$ & & & & & $\begin{array}{l}\text { "Frac...using } 100 \text { bbls wf } 135 \text { in } 6 \% \mathrm{kcl} \text { w/10\% methanol + adds, } 24 \text { tons } \mathrm{CO} 2 \ldots 258 \text { bbls } 70 \mathrm{Q} \text { CO2 foam. } \\
\text { Flush w/40 bbls } 6 \% \mathrm{kcl} \text { w } / 10 \% \text { methanol." }\end{array}$ & \\
\hline & 849.8 & $\mathrm{WR}$ & $2 / 20 / 2001$ & & $16.55^{-}$ & 30.0 & & $9.24^{\circ}$ & & $\mathrm{X}^{-}$ & & & & & $\begin{array}{l}\text { "Frac...using } 130 \text { bbls wf } 135 \text { in } 6 \% \text { KCl water w/10\% methanol + adds, } 35 \text { ton } \mathrm{CO} 2 \ldots 359 \text { bbis } 70 \mathrm{Q} \mathrm{CO}^{2} \\
\text { foam. Flush w/42 bbls } 6 \% \text { kcl water w/10\% methanol." }\end{array}$ & \\
\hline & 1103 & $\mathrm{FU}$ & $2 / 20 / 2001$ & & 7.58 & 25.0 & & 7.48 & & $\mathrm{X}^{-}$ & & & & & $\begin{array}{l}\text { "Frac...using } 276.2 \text { bbls wf } 135 \text { in } 6 \% \text { kcl water w/10\% methanol + adds, } 90 \text { tons CO2 ...849 bbls } 70 \mathrm{Q} \text { CO2 } \\
\text { foam. Flush w/55 bbls } 6 \% \mathrm{kcl} \text { wtr w/10\% methanol." }\end{array}$ & \\
\hline
\end{tabular}




\begin{tabular}{|c|c|c|c|c|c|c|c|c|c|c|c|c|c|c|c|c|}
\hline \multirow[t]{3}{*}{ 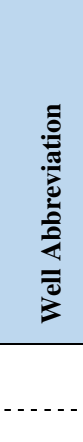 } & 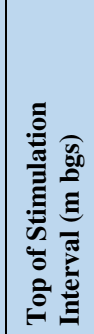 & 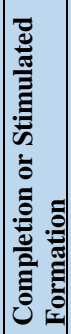 & 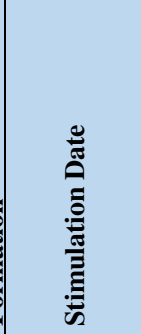 & 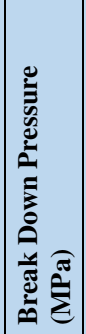 & 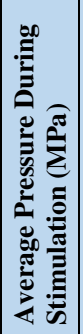 & 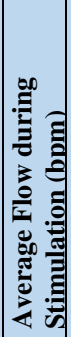 & 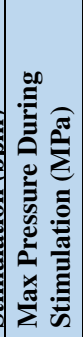 & 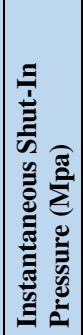 & 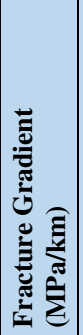 & 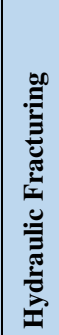 & 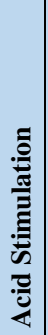 & 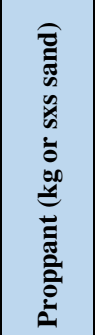 & 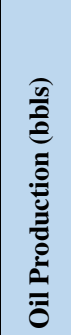 & 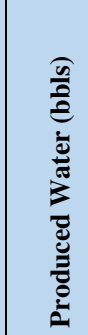 & 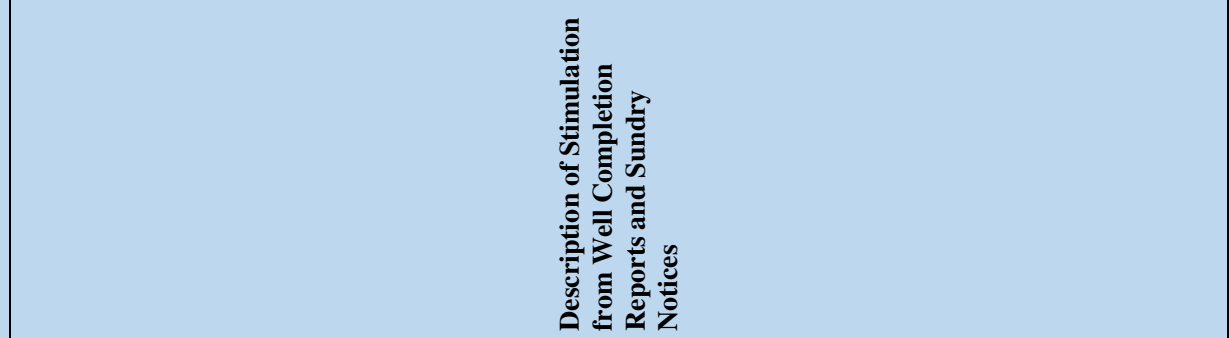 & 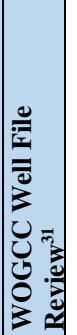 \\
\hline & 1390 & FU & $2 / 20 / 2001$ & & 20.68 & 33.0 & & 7.38 & & $\mathrm{X}$ & & & & & $\begin{array}{l}\text { "Frac...using } 174.5 \text { bbls wf } 135 \text { in } 6 \% \mathrm{KCl} \text { wtr w/10\% methanol + adds, } 40 \text { tons } \mathrm{CO} 2 . .433 \text { bbls } 70 \mathrm{Q} \text { CO2 } \\
\text { foam. Flush w/ } 69 \mathrm{bbls} 6 \% \mathrm{kcl} \text { wtr w/10\% methanol" }\end{array}$ & \\
\hline & $15 \overline{4} 4^{-}$ & $\overline{F U}^{-1}$ & $2 / 15 / 2001$ & $11.72^{-}$ & $9.65^{-}$ & 2.5 & & & & $\overline{\mathrm{X}}^{-}$ & & & & & & \\
\hline \multirow[t]{3}{*}{$21-13$} & 504.1 & WR & $2 / 17 / 2001$ & 11.38 & 11.72 & 30.0 & & 8.27 & & $\bar{X}$ & & 10,886 & 2 & 1,422 & $\begin{array}{l}\text { "Frac... using } 98 \text { bbls wf } 135 \text { in } 6 \% \mathrm{kcl} \text { water w/10\% methanol + adds, } 28 \text { tons CO2...275 bbl 70Q CO2 } \\
\text { foam. Flush w/33 bbls } 6 \% \mathrm{kcl} w / 10 \% \text { methanol." }\end{array}$ & \\
\hline & 651.1 & $\overline{W R}$ & $2 / 17 / 2001$ & 18.62 & 17.93 & 30.0 & & $8.96^{-1}$ & & $\bar{X}^{-}$ & & $19,504^{-1}$ & & & 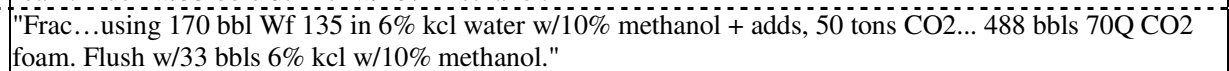 & \\
\hline & 804.7 & $\mathrm{WR}$ & $2 / 17 / 2001$ & $15.17^{-}$ & 15.17 & 30.0 & & 11.58 & & $\mathrm{X}^{-}$ & & $19,051^{-}$ & & & $\begin{array}{l}\text { "Frac...using } 174 \text { bbl wf } 135 \text { in } 6 \% \text { KCl water w/10\% methanol + adds, } 51 \text { tons CO2 } 2 . .264 \text { bbls } 70 \mathrm{Q} \text { CO } 2 \\
\text { foam. Flush w/41 bbls } 6 \% \mathrm{kcl} \text { wtr w/10\% methanol." }\end{array}$ & \\
\hline \multirow[t]{4}{*}{$43-2$} & 502.9 & WR & $2 / 24 / 2001$ & & 0.97 & 35.0 & & 6.83 & & $\mathrm{X}$ & & & 0 & 97 & $\begin{array}{l}\text { "Frac } \ldots \text { using } 298 \text { bbls wf } 135 \text { in } 6 \% \mathrm{KCl} \text { water w/10\% methanol }+ \text { adds, } 106 \text { tons } \mathrm{CO} 2 \ldots 1000 \text { bbls } 70 \mathrm{Q} \\
\mathrm{CO} 2 \text { foam. Flush w/25 bbls } 6 \% \mathrm{kcl} \text { w/10\% methanol."Frac... using } 174 \mathrm{bbl} \text { wf } 135 \text { in } 6 \% \mathrm{KCl} \text { water w/10\% } \\
\text { methanol + adds, } 51 \text { tons } \mathrm{CO} 2 \ldots 264 \text { bbls 70Q CO2 foam. Flush w/41 bbls } 6 \% \text { kcl wtr w/10 } \% \text { methanol." }\end{array}$ & No \\
\hline & 603.5 & $\mathrm{WR}$ & $2 / 23 / 2001$ & & 12.82 & 27.0 & & $7.79^{-}$ & & $\mathrm{X}^{-}$ & & & & & $\begin{array}{l}\text { Frac... } 159 \text { bbls wf } 135 \text { in } 6 \% \text { KCl water w/10\% methanol + adds, } 50 \text { tons } \mathrm{CO} 2 \ldots 496 \text { bbls } 70 \mathrm{Q} \text { CO } 2 \text { foam. } \\
\text { Flush w/30.5 bbls } 6 \% \text { kcl water w/10\% methanol." }\end{array}$ & \\
\hline & 748.6 & $\mathrm{WR}$ & $2 / 23 / 2001$ & & 11.03 & 21.0 & & 8.14 & & $\mathrm{X}^{-}$ & & & & & $\begin{array}{l}\text { "Frac...using } 156 \text { bbls wf } 135 \text { in } 6 \% \text { methanol [typo } 6 \% \mathrm{kcl} \text { water w } / 10 \% \text { methanol?] + adds, } 43 \text { tons } \\
\mathrm{CO} 2 . .452 \text { bbls } 70 \mathrm{Q} \text { CO2 foam. Flush w/38 bbls } 6 \% \mathrm{KCl} \text { w } 10 \% \text { methanol." }\end{array}$ & \\
\hline & $902.2^{-}$ & $\overline{W R}$ & $2 / 23 / 2001^{--}$ & & $14.24^{-}$ & 30.0 & & 10.76 & & $\bar{x}^{-}$ & & & & & $\begin{array}{l}\text { "Frac... using } 209 \text { bbls wf } 135 \text { in } 6 \% \text { kcl wtr w/10\% methanol + adds, } 67 \text { tons CO } 2 \ldots 661 \text { bbis } 70 \mathrm{Q} \text { CO } 2 \\
\text { foam. Flush } / 46 \text { bbls } 6 \% \mathrm{kcl} w / 10 \% \text { methanol." }\end{array}$ & \\
\hline \multirow[t]{7}{*}{$13-2$} & 500.2 & WR & $1 / 11 / 2002$ & 13.55 & 12.55 & 30.0 & & 6.21 & & $\bar{X}$ & & & 0 & 1,333 & "Frac...totals pumped 104 bbls KCl, 153 bbls CO2" & vis \\
\hline & 569.7 & WR & $1 / 10 / 2002$ & 23.84 & $22.75^{-}$ & 32.0 & & 8.36 & & $\mathrm{X}^{-}$ & & & & & "Frac..totals pumped 121 bbls KCl, 245 bbls CO2" & \\
\hline & 671.2 & Win- & $1 / 10 / 2002-$ & 23.69 & 22.75 & 29.0 & & 7.84 & & $\mathrm{X}_{-}^{-}$ & & & & & Frac...totals pumped $122 \mathrm{bb} \mathrm{bls}, 192 \mathrm{bbls} \mathrm{CO} 2 "$ & \\
\hline & 766.6 & WR- & $1 / 10 / 2002$ & 2482 & 19.99 & & & 7.10 & & $\mathrm{X}^{-}$ & & & & & "Frac...totals pumped $160 \mathrm{bbls} \mathrm{KCl}, 280 \mathrm{bb} \mathrm{ls} \mathrm{CO} 2 "$ & \\
\hline & 805.9 & WR- & $1 / 10 / 2002$ & 24.13 & 15.17 & 25.0 & & 9.38 & & $\mathrm{X}$ & & & & & "Frac ...totals pumped $95.6 \mathrm{bbls} \mathrm{CO} 2,136.6 \mathrm{bbls} \mathrm{KCl"}$ & \\
\hline & 856.8 & WR & $1 / 9 / 2002$ & 21.68 & 17.93 & 30.0 & & $9.97^{-1}$ & & $\mathrm{X}$ & & & & & "Frac using $75 \mathrm{Q}$ foam...totals pumped $158 \mathrm{bbls} \mathrm{CO} 2,131 \mathrm{bbls} \mathrm{KCl}$ & \\
\hline & $9 \overline{2} 3.5^{-1}$ & $\mathrm{WR}$ & $1 / 8 / 2002^{2}$ & 20.48 & 47.57 & 20.0 & & $9.84^{-1}$ & & $\mathrm{X}^{-}$ & & & & & $\begin{array}{l}\text { "Frac-breakdown w/6.3 bbls KCl...Pumped } 70 \mathrm{Q} \text { foam pad } 50 \mathrm{Q} \text { san stages...Totals pumped } 125 \mathrm{bb} \mathrm{b} \text { KC̄, } \\
120 \text { bbls CO2" }\end{array}$ & \\
\hline $24-1$ & 432.2 & WR & $3 / 23 / 2001$ & & & & & & & & & & 0 & 817 & $\begin{array}{l}\text { Producing in Wind River Formation. Six perforated intervals from } 1418 \text { to } 2964 \text { '. No information provided } \\
\text { on stimulation. }\end{array}$ & No \\
\hline \multirow[t]{5}{*}{$32-1$} & 371.9 & WR & $3 / 23 / 2001$ & & 9.26 & 22.0 & & 5.45 & & $\mathrm{X}$ & & & 0 & 102 & $\begin{array}{l}\text { "Frac... using } 165 \text { bbls wf } 130 \text { in } 6 \% \mathrm{kcl} \text { water w/10\% methanol + adds, } 39 \text { tons CO2...458 bbls 70Q CO2 } \\
\text { foam. Flush w/18 bbls } 6 \% \mathrm{kcl} \text { water w/10\% methanol." }\end{array}$ & \\
\hline & 535.8 & $\mathrm{WR}$ & $3 / 23 / 2001$ & & 9.65 & 21.4 & & 5.86 & & $\mathrm{X}^{-}$ & & & & & $\begin{array}{l}\text { Frac...using } 153 \text { bbls wf } 130 \text { in } 6 \% \text { Kkcl water w/10\% methanol + adds, } 35 \text { tons } \mathrm{CO} 2 . .398 \text { bbls } 70 \mathrm{Q} \text { CO } 2 \\
\text { foam. Flush w/27 bbls } 6 \% \text { kcl water w/10\% methanol." }\end{array}$ & \\
\hline & $648^{-}$ & $\mathrm{WR}^{-1}$ & $3 / 23 / 2001^{-}$ & & 12.89 & 21.9 & & 8.14 & & $\mathrm{X}^{-}$ & & & & & $\begin{array}{l}\text { "Frac...using } 105 \text { bbls wf } 130 \text { in } 6 \% \text { kcl w/10\% methanol + adds, } 21 \text { tons CO } 2 . . .232 \text { bbls } 70 \mathrm{Q} \text { CO } 2 \text { foam. } \\
\text { Flush w/32 bbl } 6 \% \text { kcl water w/10\% methanol." }\end{array}$ & \\
\hline & 856.5 & $\mathrm{~W} \bar{R}^{-1}$ & $3 / 22 / 2001$ & & 8.69 & 21.0 & & $7.29^{-1}$ & & $\mathrm{X}^{-}$ & & & & & $\begin{array}{l}\text { Frac...using } 144.4 \text { bbls wf } 130 \text { in } 6 \% \text { KCl water w/10\% methanol + adds, } 29 \text { tons } \mathrm{CO} 2 . .333 \text { bbls } 70 \mathrm{Q} \text { CO2 } \\
\text { foam. Flush w/43.8 bbl kcl water w/10\% methanol." }\end{array}$ & \\
\hline & $91 \overline{7} .4$ & $\mathrm{~W} \mathrm{R}^{-1}$ & $3 / 20 / 2001$ & & 15.72 & 30.0 & & $9.47^{-}$ & & $\mathrm{X}$ & & & & & $\begin{array}{l}\text { "Frac...using } 177.3 \text { bbls wf } 130 \text { in } 6 \% \text { kcl w/10\% methanol + adds, } 38 \text { tons CO2...332 bbls } 70 \mathrm{Q} \text { CO2 foam. } \\
\text { Flush w/46 bbls } 6 \% \mathrm{kcl} \mathrm{w} / 10 \% \text { methanol." }\end{array}$ & \\
\hline \multirow[t]{2}{*}{$34-2$} & 385.3 & WR & $3 / 20 / 2001$ & & 8.46 & 18.0 & & 6.00 & & $\mathrm{X}$ & & & 0 & 14 & $\begin{array}{l}\text { "Frac ... using } 92.1 \text { bbls wf } 130 \text { in } 6 \% \mathrm{kcl} \mathrm{w} / 10 \% \text { methanol, } 20 \text { ton } \mathrm{CO} 2 \ldots .228 \text { bbls } 70 \mathrm{Q} \text { CO2 foam. Flush } \\
\text { w/18.5 bbls } 6 \% \mathrm{kcl} \text { w/10\% methanol. }\end{array}$ & No \\
\hline & 469.4 & $\bar{W} \bar{R}^{-1}$ & $3 / 17 / 2001^{-}$ & & $10.85^{-}$ & 19.9 & & $7.58^{\circ}$ & & $\mathrm{X}^{-}$ & & & & & 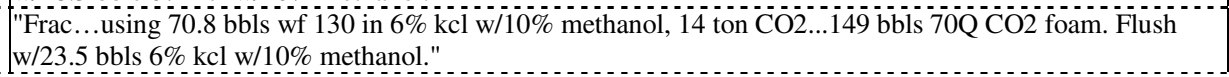 & \\
\hline
\end{tabular}




\begin{tabular}{|c|c|c|c|c|c|c|c|c|c|c|c|c|c|c|c|c|}
\hline 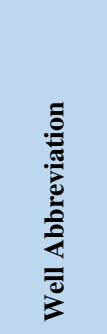 & 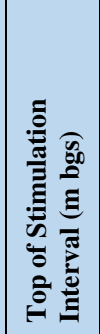 & 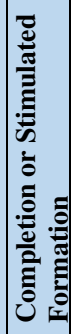 & 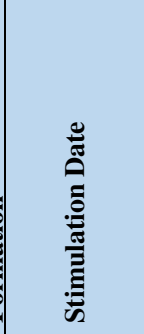 & 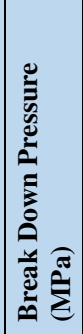 & 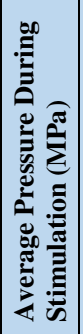 & 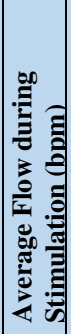 & 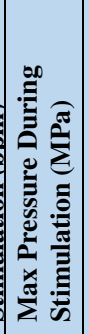 & 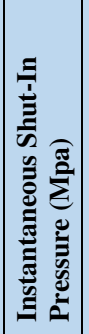 & 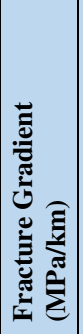 & 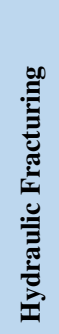 & 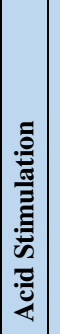 & 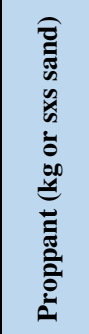 & 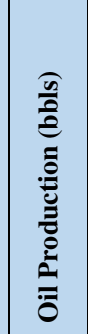 & 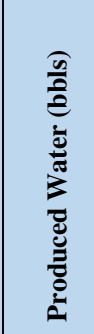 & 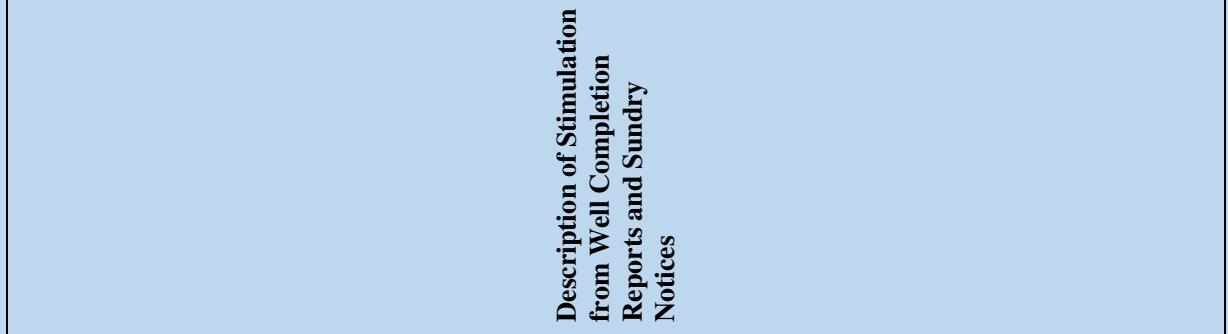 & 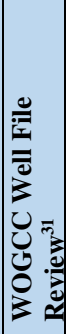 \\
\hline & 497.4 & WR & $3 / 17 / 2001$ & & 7.47 & 18.0 & & 5.10 & & $\mathrm{X}$ & & & & & $\begin{array}{l}\text { "Frac...using } 75.9 \mathrm{bbls} \text { wf } 130 \text { in } 6 \% \mathrm{kcl} \text { w/10\% methanol + adds, } 14 \text { ton CO2...160 bbls } 70 \mathrm{Q} \text { CO2 foam. } \\
\text { Flush w/24 bbls } 6 \% \mathrm{kcl} \text { water w/10\% methanol." }\end{array}$ & \\
\hline & 554.7 & $\mathrm{~W} \mathrm{~W}^{-1}$ & $3 / 17 / 2001$ & & 10.98 & 19.0 & & $7.58^{-}$ & & $\bar{X}^{-}$ & & & & & $\begin{array}{l}\text { "Frac... using } 85.2 \text { bbls wf } 130 \text { in } 6 \% \text { kcl water w/10\% methanol + adds, } 19 \text { ton } \mathrm{CO} 2 . .180 \mathrm{bbls} 70 \mathrm{Q} \mathrm{CO} 2 \\
\text { foam. Flush w/28 bbls } 6 \% \mathrm{kcl} \text { water w/10\% methanol." }\end{array}$ & \\
\hline & 609.9 & $\mathrm{WR}$ & $3 / 17 / 2001$ & & 10.81 & 21.0 & & $8.02^{-}$ & & $\mathrm{X}^{-}$ & & & & & $\begin{array}{l}\text { "Frac...using } 175.3 \text { bbls wf } 130 \text { in } 6 \% \text { kcl w/10\% methanol }+ \text { adds, } 47.7 \text { ton } \mathrm{CO} 2 \ldots . .460 \text { bbls } 70 \mathrm{Q} \text { CO } 2 \text { foam. } \\
\text { Flush w/31 bbls } 6 \% \text { kcl water w/10\% methanol." }\end{array}$ & \\
\hline & 648.6 & $\mathrm{WR}$ & $3 / 17 / 2001$ & & $7.11^{-}$ & 22.0 & & $6.57^{-}$ & & $\mathrm{X}^{-}$ & & & & & 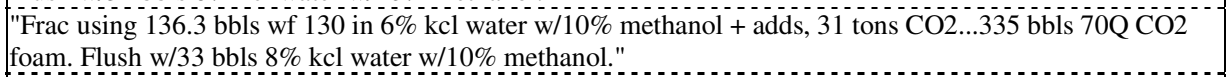 & \\
\hline & $714.5^{-}$ & $\mathrm{WR}$ & $3 / 16 / 2001$ & & $7.08^{-}$ & 22.0 & & $5.72^{-1}$ & & $\mathrm{X}^{-}$ & & & & & $\begin{array}{l}\text { "Frac...using } 190 \text { bbls wf } 130 \text { in } 6 \% \text { kcl w/10\% methanol + adds, } 51.2 \text { ton } \mathrm{CO} 2 \ldots 498 \text { bbls } 70 \mathrm{Q} \text { CO } 2 \text { foam. } \\
\text { Flush w/36 bbls } 6 \% \mathrm{kcl} \text { w/10\% methanol." }\end{array}$ & \\
\hline & 793.1 & $\mathrm{WR}$ & $3 / 15 / 2001$ & & $13.44^{-}$ & 20.0 & & $8.89^{-}$ & & $\mathrm{X}^{-}$ & & & & & $\begin{array}{l}\text { "Frac...using } 134 \text { bbls wf } 130 \text { in } 6 \% \text { kcl w/ } / 10 \% \text { methanol + adds, } 21.5 \text { ton } \mathrm{CO} 2 . . .242 \text { bbls } 70 \mathrm{Q} \text { CO } 2 \text { foam. } \\
\text { Flush w/40 bbls } 6 \% \mathrm{kcl} \text { w/10\% methanol." }\end{array}$ & \\
\hline & 843.1 & $\mathrm{WR}$ & $3 / 15 / 2001$ & & 10.24 & 22.0 & & $7.89^{-1}$ & & $\mathrm{X}^{-}$ & & & & & $\begin{array}{l}\text { "Frac...using } 86 \text { bbls wf } 130 \text { in } 6 \% \mathrm{kcl} \text { w/10\% methanol + adds, } 17 \text { ton } \mathrm{CO} 2 . .157 \text { bbls } 70 \mathrm{Q} \text { CO2 foam. } \\
\text { Flush w/43 bbls } 6 \% \text { kcl water w/10\% methanol.". }\end{array}$ & \\
\hline & 900.4 & $\mathrm{WR}$ & $3 / 15 / 2001$ & & 12.35 & 23.7 & & 8.20 & & $\mathrm{X}^{-}$ & & & & & $\begin{array}{l}\text { "Frac...using } 128.4 \mathrm{bbls} \text { wf } 130 \text { in } 6 \% \mathrm{kcl} \text { w/10\% methanol + adds, } 31 \text { ton } \mathrm{CO} 2 . . .283 \text { bbls } 70 \mathrm{Q} \mathrm{CO} 2 \text { foam. } \\
\text { Flush w/46 bbls } 6 \% \mathrm{kcl} \text { w/10\% methanol." }\end{array}$ & \\
\hline & 986.6 & $\mathrm{WR}$ & $3 / 15 / 2001$ & & 10.81 & 30.0 & & 7.54 & & $\mathrm{X}^{-}$ & & & & & $\begin{array}{l}\text { "Frac...using } 105 \text { bbls wf } 130 \text { in } 6 \% \text { kcl w/10\% methanol + adds, } 20 \text { ton CO2...192 bbls } 70 \mathrm{Q} \text { CO } 2 \text { foam. } \\
\text { Flush w/50 bbls } 6 \% \mathrm{kcl} \text { water w/10\% methanol." }\end{array}$ & \\
\hline $12-6$ & & & & & & & & & & & & & 0 & 1,686 & $\begin{array}{l}\text { Producing from Wind River Formation. Information on perforation and stimulation missing from well } \\
\text { completion report }\end{array}$ & $\overline{\text { No }}$ \\
\hline $44-1$ & & WR & & & & & & & & & & & 0 & 1,375 & $\begin{array}{l}\text { Producing from Wind River Formations (8 perforated intervals). Information on stimulation missing from } \\
\text { well completion report. }\end{array}$ & No \\
\hline \multirow[t]{4}{*}{$14-10$} & 639.5 & WR & $4 / 11 / 2001$ & & 14.09 & 21.9 & & 8.27 & & $\mathrm{X}$ & & 10,565 & 3,678 & 3,225 & $\begin{array}{l}\text { "Frac... using } 102.8 \text { bbls wf } 130 \text { in } 6 \% \mathrm{KCl} \text { w/10\% methanol + adds, } 24 \text { tons } \mathrm{CO} 2 \ldots 219 \text { bbls } 70 \mathrm{Q} \mathrm{CO} 2 \\
\text { foam. Flush w/31 bbls } 6 \% \mathrm{kcl} \text { water w/10\% methanol." }\end{array}$ & No \\
\hline & 662 & $\mathrm{WR}$ & $4 / 11 / 2001$ & & 10.81 & 20.4 & & $6.89^{-1}$ & & $\mathrm{X}^{-}$ & & $11,115^{-}$ & & & $\begin{array}{l}\text { "Frac...using } 112.6 \text { bbbls wf } 130 \text { in } 6 \% \text { KCl w/10\% methanol + adds, } 25 \text { tons } \mathrm{CO} 2 . .219 \text { bbi } 70 \mathrm{Q} \mathrm{CO} 2 \\
\text { foam. Flush w/42.2 bbls } 6 \% \text { kcl water w/10\% methanol." }\end{array}$ & \\
\hline & 872.3 & $\mathrm{WR}$ & $4 / 11 / 2001$ & & $9.83^{-}$ & 20.5 & & $7.31^{-}$ & & $\mathrm{X}^{-}$ & & 20,342 & & & $\begin{array}{l}\text { "Frac...using } 171.6 \text { bbls wf } 130 \text { in } 6 \% \text { KCl water w/10\% methanol + adds, } 42 \text { tons } \mathrm{CO} 22 . .397 \text { bbls } 70 \mathrm{Q} \text { CO } 2 \\
\text { foam. Flush w/44.5 bbls } 6 \% \mathrm{kcl} \text { water w/10\% methanol." }\end{array}$ & \\
\hline & 931.5 & $\mathrm{WR}$ & $4 / 11 / 2001$ & & 17.03 & 20.0 & & 8.62 & & $\mathrm{X}^{-}$ & & 10,454 & & & $\begin{array}{l}\text { "Frac...using } 121.6 \text { bbls wf } 130 \text { in } 6 \% \text { kcl water w/ } 10 \% \text { methanol + adds, } 24 \text { tons } \mathrm{CO} 2 \ldots 224 \text { bbls } 70 Q \mathrm{CO} 2 \\
\text { foam. Flush w/47 bbls } 6 \% \mathrm{kcl} \text { water w/10\% methanol." }\end{array}$ & \\
\hline \multirow[t]{4}{*}{$13-15$} & 657.1 & WR & $5 / 17 / 2001$ & 15.86 & 9.91 & 25.8 & & 8.76 & & $\mathrm{X}$ & & 13,213 & 0 & 0 & $\begin{array}{l}\text { "Frac... using } 120.2 \text { bbls Wf125 in } 8 \% \mathrm{KCl} \text { with } 10 \% \text { methanol, } 38 \text { tons CO2...277 bbls } 80 \mathrm{Q}, 75 \mathrm{Q}, 70 \mathrm{Q} \text {, and } \\
60 \mathrm{Q} \text { CO2 foam. Flush w/49 bbls 6\% KCL w/10\% methanol" }\end{array}$ & No \\
\hline & 754.7 & $\mathrm{WR}$ & $5 / 17 / 2001$ & $5.60^{-}$ & $7.74^{-1}$ & 23.8 & & $4.48^{-}$ & & $\mathrm{X}$ & & $16,442^{-}$ & & & $\begin{array}{l}\text { "Frac...using } 140.3 \text { bbls Wf } 125 \text { in } 8 \% \text { KCl with } 10 \% \text { methanol, } 45 \text { tons CO2...325 bbls } 80 \mathrm{Q}, 75 \mathrm{Q}, 70 \mathrm{Q} \text {, } \\
\text { and 60Q CO2 foam. Flush w/56.5 bbls } 8 \% \mathrm{KCl} \text { w/10\% methanol" }\end{array}$ & \\
\hline & & $\mathrm{W} \mathrm{W}^{-1}$ & $5 / 17 / 2001^{-}$ & 896 & 8.51 & 26.8 & & $7.97^{-1}$ & & $\mathrm{X}^{-}$ & & $13,099^{-}$ & & & $\begin{array}{l}\text { "Frac...using } 138.9 \text { bbls Wf } 125 \text { in } 6 \% \mathrm{KCl} \text { with } 10 \% \text { methanol, } 36 \text { tons } \mathrm{CO} 2 . .270 \text { bुbls } 80 \mathrm{Q}, 75 \mathrm{Q}, 70 \mathrm{Q}, \mathrm{\&}^{-} \\
60 \mathrm{CO} 2 \text { foam. Flush w/67 bbls } 6 \% \mathrm{KCl} \text { w } 10 \% \text { methanol" }\end{array}$ & \\
\hline & 1048 & $\mathrm{WR}$ & $5 / 16 / 2001$ & $7.76^{-1}$ & $6.47^{-}$ & 27.0 & & $4.83^{-1}$ & & $\mathrm{X}^{-}$ & & 13,297 & & & $\begin{array}{l}\text { "Frac...using } 153.6 \text { bbls Wf } 125 \text { in } 6 \% \mathrm{KCl} \text { w/10\% methanol, } 38 \text { tons } \mathrm{CO} 2 . .277 \text { bbls } 80 \mathrm{Q}, 75 \mathrm{Q}, 70 \mathrm{Q} \text {, and } \\
60 \mathrm{CO} 2 \text { foam. Flush w/79 bbls } 8 \% \mathrm{KCl} \text { w/10\% methanol" }\end{array}$ & \\
\hline \multirow[t]{2}{*}{$12-11 \mathrm{~W}$} & $\overline{7770.8}$ & WR & $5 / 3 / 2001$ & 11.86 & 9.07 & 24.7 & & 6.87 & & $\mathrm{X}$ & & & 0 & 460 & $\begin{array}{l}\text { "Frac... using } 144.2 \text { bbls wf } 125 \text { in } 6 \% \mathrm{KCl} \text { water }+ \text { adds, } 37 \text { tons } \mathrm{CO} 2 \ldots 290 \text { bbls } 70 \mathrm{Q} \text { CO2 foam. Flush w/5 } \\
\text { bbls } 6 \% \mathrm{KCl} \text { w/10\% methanol" }\end{array}$ & Yes \\
\hline & 843.4 & $\mathrm{WR}$ & $5 / 3 / 2001$ & 2.76 & 7.51 & 21.5 & & 6.62 & & $\mathrm{X}^{-}$ & & & & & $\begin{array}{l}\text { "Frac... using } 120.4 \text { bbls wf } 130 \text { in } 6 \% \mathrm{kcl} \text { water w/10\% methanol + adds, } 25 \text { tons } \mathrm{CO} 2 \ldots 180 \mathrm{bb} 170 \mathrm{Q} \mathrm{CO} 2 \\
\text { foam. Flush w/63 bbls } 6 \% \mathrm{KCl} \text { water w/10\% methanol." }\end{array}$ & \\
\hline
\end{tabular}




\begin{tabular}{|c|c|c|c|c|c|c|c|c|c|c|c|c|c|c|c|c|}
\hline \multirow[t]{4}{*}{ 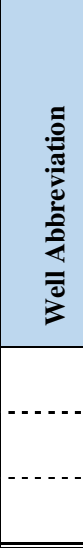 } & 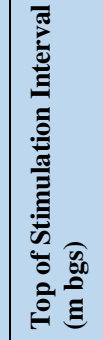 & 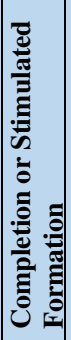 & 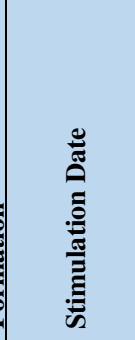 & 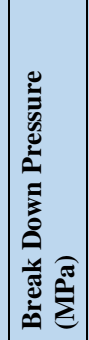 & 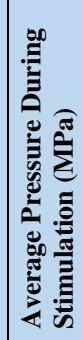 & 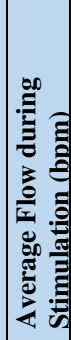 & 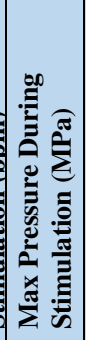 & 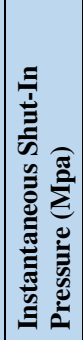 & 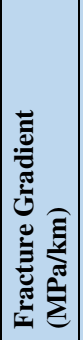 & 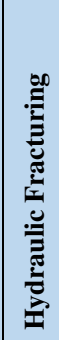 & 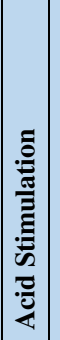 & 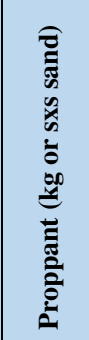 & 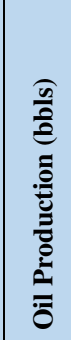 & 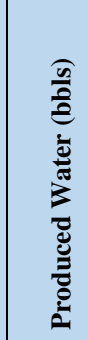 & 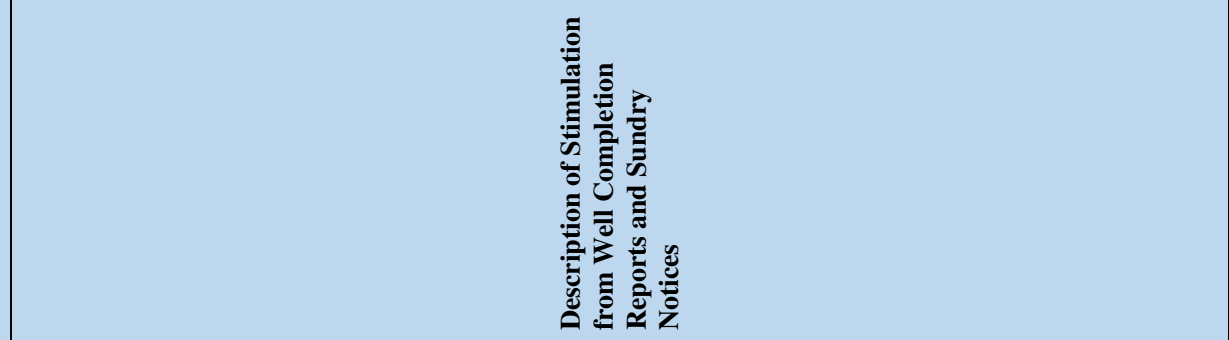 & 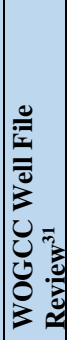 \\
\hline & 864.1 & WR & $5 / 2 / 2001$ & 15.17 & 10.14 & 22.4 & & 7.69 & & $\mathrm{X}$ & & & & & $\begin{array}{l}\text { "Frac... using } 116.7 \text { bbls wf } 130 \text { in } 6 \% \mathrm{KCl} \text { water w/10\% methanol + adds, } 22 \text { tons } \mathrm{CO} 2 \ldots 170 \text { bbls } 70 \mathrm{Q} \\
\text { CO2 foam. Flush w/65 bbls } 6 \% \mathrm{KCl} \text { w/10\% methanol." }\end{array}$ & \\
\hline & 883.3 & $\mathrm{WR}$ & $5 / 2 / 2001$ & 9.79 & 14.11 & 23.0 & & $7.98^{-}$ & & $\mathrm{X}^{-}$ & & & & & $\begin{array}{l}\text { "Frac...using } 131.2 \text { bbls wf } 130 \text { in } 6 \% \text { KCl w/10\% methanol + adds, } 24 \text { tons } \mathrm{CO} 2 . .223 \text { bbis } 70 \mathrm{Q} \text { CO } 2 \\
\text { foam. Flush w/66 bbls } 6 \% \mathrm{KCl} \text { water w/10\% methanol." }\end{array}$ & \\
\hline & 923.2 & WR & $5 / 2 / 2001$ & $7.24^{-}$ & 10.99 & 20.0 & & $7.72^{-1}$ & & $\overline{\mathrm{X}}^{-}$ & & & & & $\begin{array}{l}\text { "Frac... } \\
\text { foam. Flush w/ } 69 \text { bbls KCl water w/10\% methanol." }\end{array}$ & \\
\hline \multirow[t]{5}{*}{ 34-3R } & 726.3 & WR & $5 / 4 / 2001$ & & 10.72 & 21.2 & & 8.96 & & $\bar{X}$ & & & 2 & 1,153 & $\begin{array}{l}\text { Start frac } \& \text { pump } 86 \mathrm{bbl} 80 \mathrm{Q} \text { CO2 foam....Leak on wellhead. Flush w/37 bbl kcl water...Start to frac } \\
\text { again...Leak on wellhead again...Frac using } 67.7 \mathrm{bbl} \text { wf } 125 \text { in } 6 \% \mathrm{kcl} \text { water w/10\% methanol + adds, } 16 \\
\text { tons CO2..119 bbl 80Q CO2 foam. Flush } 6 / 37 \mathrm{bbl} 6 \% \mathrm{kcl} \text { water w/10\% methanol.". }\end{array}$ & Yes \\
\hline & 773.6 & $\mathrm{WR}$ & $5 / 4 / 2001$ & & 11.13 & 22.6 & & $7.93^{-}$ & & $\mathrm{X}^{-}$ & & & & & 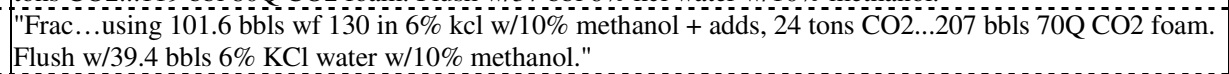 & \\
\hline & 809.2 & $\bar{W} R^{-1}$ & $5 / 4 / 2001$ & 16.84 & 13.48 & 27.2 & & $8.55^{-}$ & & $\bar{X}$ & & & & & $\begin{array}{l}\text { "Frac.. } \\
\text { foam. Flush w/41.3 bbls } 6 \% \mathrm{KCl} \text { water w/10\% methanol" }\end{array}$ & \\
\hline & 842.5 & $\mathrm{WR}^{-1}$ & $5 / 4 / 2001^{-}$ & $13.93^{-}$ & 13.33 & 24.0 & & 8.69 & & $\bar{X}^{-}$ & & & & & $\begin{array}{l}\text { Frac. ussing } 108.8 \mathrm{bbl} \text { wf } 130 \text { in } 6 \% \mathrm{KCl} \text { water w/10\% methanol }+ \text { adds, } 29 \text { tons } \mathrm{CO} 2 . .26 \text { bbls } 70 \mathrm{Q} \mathrm{CO} 2 \\
\text { foam. Flush } \mathrm{w} / 43 \mathrm{bbls} 6 \% \mathrm{KCl} \text { water } \mathrm{w} / 10 \% \text { methanol." }\end{array}$ & \\
\hline & 942.7 & $\mathrm{WR}$ & $5 / 4 / 2001$ & & 11.84 & 21.5 & & $9.65^{\circ}$ & & $\mathrm{X}$ & & & & & $\begin{array}{l}\text { "Frac...using } 103.6 \text { bbls WF } 130 \text { in } 6 \% \text { KCl water w/10\% methanol + adds, } 22 \text { tons CO2 } 2 . .170 \text { bb1 } 70 \mathrm{Q} \text { CO2 } \\
\text { foam. Flush w/48 bbls } 6 \% \mathrm{KCl} \text { water w/10\% methanol" }\end{array}$ & \\
\hline \multirow[t]{7}{*}{$11-11$} & 1024 & $\mathrm{FU}$ & $5 / 31 / 2001$ & 14.13 & 19.85 & 31.3 & & 9.45 & & $\mathrm{X}$ & & 20,818 & 0 & 9,051 & $\begin{array}{l}\text { "Frac... using } 161.2 \text { bbls Wf } 125 \text { in } 6 \% \mathrm{KCl} \text { water w/10\% methanol, } 45 \text { tons } \mathrm{CO} 2 \ldots 441 \text { bbls } 70 \mathrm{Q} \& \text { 60Q } \\
\mathrm{CO} 2 \text { foam. Flush w/50 bbls } 50 \% \mathrm{CO} 2 \& 50 \%-6 \% \mathrm{KCl} \text { water w/10\% methanol." }\end{array}$ & Yes \\
\hline & $11169^{-}$ & $\overline{\mathrm{FU}}$ & $5 / 31 / 2001$ & & 20.68 & 28.7 & & $5.17^{-}$ & & $\mathrm{X}^{-}$ & & $21,881^{-}$ & & & 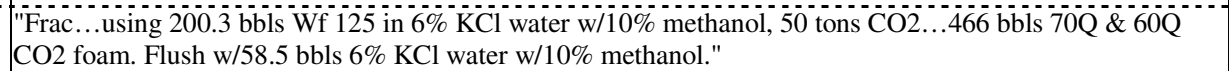 & \\
\hline & 1238 & $\overline{F U}$ & $5 / 30 / 2001$ & 24.82 & 18.73 & $2 \overline{2} .2$ & & 13.44 & & $\bar{X}$ & & 15,708 & & & $\begin{array}{l}\text { "Frac...u using } 178.5 \text { bbls Wf } 125 \text { in } 6 \% \mathrm{KCl} \text { water w/ } 10 \% \text { methanol, } 41 \text { tons } \mathrm{CO} 2 \ldots 372 \text { bbls } 70 \mathrm{Q} \& 60 \mathrm{Q} \\
\mathrm{CO} 2 \text { foam. Flush } / 62.5 \text { bbls } 6 \% \mathrm{KCl} \text { water w/10\% methanol." }\end{array}$ & \\
\hline & 1358 & $\mathrm{FU}$ & $5 / 30 / 2001$ & 9.31 & 15.32 & 22.3 & & 10.82 & & $\mathrm{X}$ & & 12,146 & & & $\begin{array}{l}\text { Frac...using } 153.1 \text { bbls Wf } 125 \text { in } 6 \% \text { KCl water w/10\% methanol, } 28 \text { tons } \mathrm{CO} 2 \ldots 262 \text { bbls } 70 \mathrm{Q} \& 60 \mathrm{Q} \\
\mathrm{CO} 2 \text { foam. Flush w/68 bbls } 6 \% \mathrm{KCl} \text { water w/10\% methanol." }\end{array}$ & \\
\hline & 1380 & $\mathrm{FU}$ & $5 / 11 / 2001$ & 24.34 & 21.35 & 24.0 & & & & $\bar{X}$ & & 16,594 & & & $\begin{array}{l}\text { "Frac...using } 85.2 \text { bbls Wf } 125 \text { in } 6 \% \text { KCl water w/10\% methanol + adds, } 45 \text { tons CO2...334.8 [bbls] 80Q, } \\
75 \mathrm{Q}, 70 \mathrm{Q} \& 60 \mathrm{Q} \text { CO2 foam. Screened out." }\end{array}$ & \\
\hline & 1394 & $\overline{F U}^{-}$ & $5 / 11 / 2001$ & 24.61 & 15.49 & $25 . \overline{4}$ & & $9.85^{-}$ & & $\bar{X}$ & & 12,207 & & & $\begin{array}{l}\text { "Frac...using } 136.2 \text { bbls Wf } 125 \text { in } 6 \% \mathrm{KCl} \text { water w/10\% methanol + adds, } 38 \text { tons } \mathrm{CO} 2 . .258 \mathrm{bbls} 80 \mathrm{Q} \text {, } \\
75 \mathrm{Q}, 70 \mathrm{Q} \& 60 \mathrm{C} \text { foam. Flush w/70 bbls } 6 \% \mathrm{KCl} \text { water w/10\% methanol." }\end{array}$ & \\
\hline & $1467^{-}$ & $\overline{\mathrm{FU}}$ & $5 / 9 / 2001$ & $20.51^{-}$ & 15.55 & 28.5 & & $8.41^{\circ}$ & & $\mathrm{X}$ & & 18,033 & & & $\begin{array}{l}\text { "Frac... using } 178 \text { bbls Wf } 125 \text { in } 6 \% \text { KCl water w/10\% methanol + adds, } 58 \text { tons CO2 } 2 . .417 \text { bbls } 80 \mathrm{Q}, 75 \mathrm{Q} \text {, } \\
70 \mathrm{Q} \& 60 \mathrm{Q} \text { CO2 foam. Flush w/70 bbls } 6 \% \mathrm{KCl} \text { water w/10\% methanol." }\end{array}$ & \\
\hline \multirow[t]{5}{*}{$12-5$} & 571.2 & WR & $5 / 22 / 2001$ & & 12.41 & 18.0 & & 6.50 & & X & & & 0 & $\overline{2402}$ & "Frac...using 45.5 bbls $6 \% \mathrm{KCl}$ w/10\% methanol + 40 bbls CO2" & No \\
\hline & 609.6 & $\mathrm{WR}$ & $5 / 22 / 2001$ & & 13.48 & 31.0 & & 763 & & $\overline{\mathrm{v}}$ & & 15,291 & & & 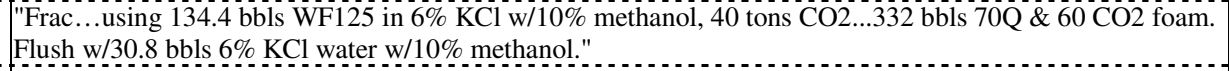 & \\
\hline & 665.4 & $\mathrm{WR}$ & $5 / 22 / 2001$ & & 15.55 & 29.4 & & $7.72^{-}$ & & $\mathrm{X}$ & & $12, \overline{783}$ & & & $\begin{array}{l}\text { "Frac...using } 119 \text { bbls WFi } 25 \text { in } 6 \% \text { KCl w/ } 10 \% \text { methanol, } 32 \text { tons } \mathrm{CO} 2 \ldots 267 \text { bbls } 70 \mathrm{Q} \& \mathrm{6} 6 \mathrm{Q} \text { CO } 2 \text { foam. } \\
\text { Flush w/33.7 bbls } 6 \% \mathrm{KCl} \text { w/10\% methanol." }\end{array}$ & \\
\hline & 748.9 & $\mathrm{WR}$ & $5 / 22 / 2001$ & & 15.50 & 28.3 & & $7.93^{-1}$ & & $\mathrm{X}$ & & 10,389 & & & 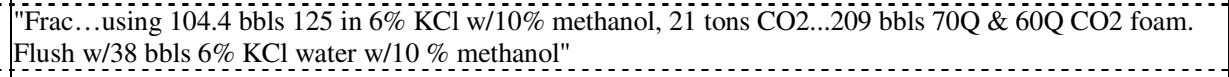 & \\
\hline & 829.1 & $\mathrm{WR}$ & $2 / 21 / 2001$ & & 9.65 & 3.0 & & 6.34 & & $\bar{X}$ & & & & & $\begin{array}{l}\text { "Breakdown perfs w/6\% KCL, } 35 \text { bbls...start ball sealers. Pump ... } 48 \text { bbls } 6 \% \text { KCL...Flush w/17 bbls } 6 \% \\
\text { KCl...No gas." }\end{array}$ & \\
\hline$\overline{13-1}$ & 661.4 & WR & $\overline{1 / 2001}$ & & 11.64 & 21.7 & 12.27 & 8.41 & & $\bar{X}$ & & 8,504 & $\overline{0}$ & 21 & $\begin{array}{l}\text { "Frac... using } 87.9 \text { bbls wf } 125 \text { in } 6 \% \mathrm{KCl} \text { water w/10\% methanol, } 21 \text { tons } \mathrm{CO} 2 \ldots 170 \text { bbls } 70 \mathrm{Q} \& 60 \mathrm{Q} \mathrm{CO} 2 \\
\text { foam. Flush w/33 bbls } 6 \% \mathrm{KCl} \text { water } \mathrm{w} / 10 \% \text { methanol" }\end{array}$ & Yes \\
\hline
\end{tabular}




\begin{tabular}{|c|c|c|c|c|c|c|c|c|c|c|c|c|c|c|c|c|}
\hline \multirow[t]{6}{*}{ 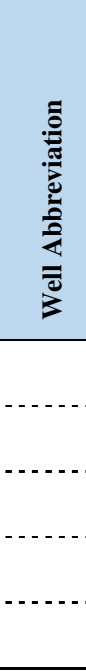 } & 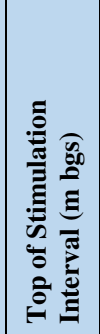 & 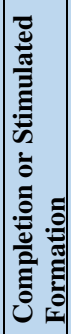 & 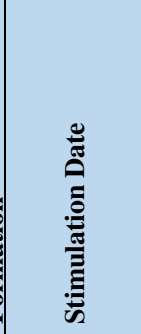 & 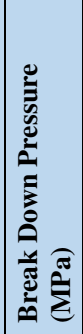 & 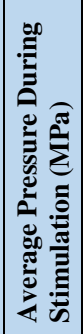 & 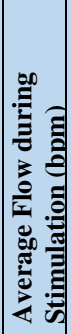 & 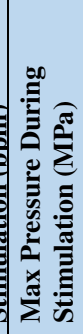 & 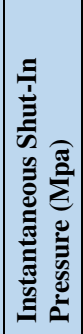 & 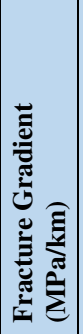 & 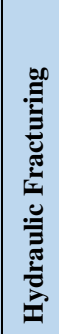 & 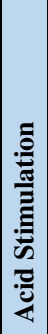 & 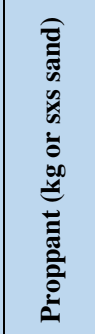 & 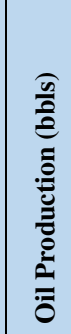 & 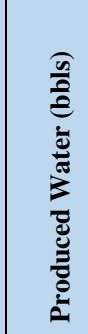 & 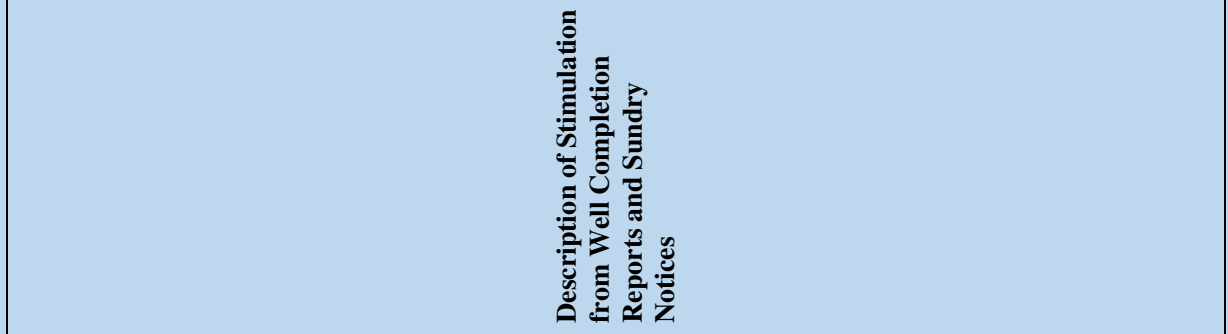 & 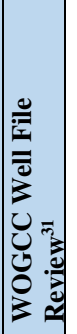 \\
\hline & 706.2 & WR & $6 / 1 / 2001$ & & 10.92 & 26.4 & 18.96 & 7.27 & & $\mathrm{X}$ & & 14,989 & & & $\begin{array}{l}\text { "Frac ... using } 135.2 \text { bbls wf } 125 \text { in } 6 \% \mathrm{KCl} \text { water w/10\% methanol, } 35 \text { tons } \mathrm{CO} 2 \ldots 323 \text { bbls } 70 \mathrm{Q} \& 60 \mathrm{Q} \\
\mathrm{CO} 2 \text { foam. Flush w/36 bbls } 6 \% \mathrm{KCl} \text { water w/10\% methanol" }\end{array}$ & \\
\hline & $7 \overline{55}-$ & $\bar{W} R^{-1}$ & $6 / 1 / 2001$ & & $13.72^{-}$ & 17.9 & 19.22 & $9.50^{\circ}$ & & $\overline{\mathrm{X}}^{-}$ & & $5,377^{-}$ & & & 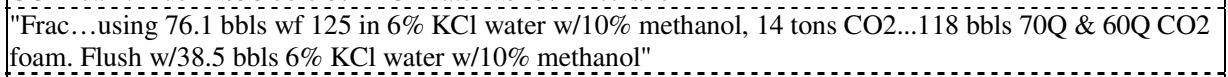 & \\
\hline & 841.6 & $\mathrm{WR}$ & $6 / 1 / 2001$ & & $16.89^{-}$ & 26.3 & 17.58 & $7.87^{-1}$ & & $\mathrm{X}^{-}$ & & 12,313 & & & $\begin{array}{l}\text { "Frac...using } 125.4 \text { bbls wf } 125 \text { in } 6 \% \text { KCl water w/10\% methanol, } 24 \text { tons } \mathrm{CO} 22 . .258 \text { bbls } 70 \mathrm{Q} \& 60 \mathrm{Q} \\
\mathrm{CO} 2 \text { foam. Flsuh } / 43 \text { bbls } 6 \% \mathrm{KCl} \text { water w/10\% methanol" }\end{array}$ & \\
\hline & 878.7 & $\mathrm{WR}$ & $6 / 1 / 2001$ & & 14.15 & 20.8 & 18.13 & 10.07 & & $\mathrm{X}^{-}$ & & 8,335 & & & $\begin{array}{l}\text { "Frac...using } 102.6 \text { bbls wf } 125 \text { in } 6 \% \text { KCl water w/10\% methanol, } 21 \text { tons } \mathrm{CO} 2 \ldots 180 \text { bbls } 70 \mathrm{Q} \& 60 \mathrm{Q} \\
\mathrm{CO} 2 \text { foam. Flush } \mathrm{w} / 45 \text { bbls } 6 \% \mathrm{KCl} \text { water w/10\% methanol" }\end{array}$ & \\
\hline & 905.6 & WR'-1 & $5 / 31 / 2001$ & & 17.33 & 23.4 & $17 \overline{58}$ & $10.10^{-1}$ & & $\mathrm{X}^{-}$ & & 14,715 & & & $\begin{array}{l}\text { "Frac...using } 145.4 \mathrm{bbls} \text { wf } 125 \text { in } 6 \% \mathrm{KCl} \text { water w/10\% methanol, } 35 \text { tons } \mathrm{CO} 2 . .320 \text { bbls } 70 \mathrm{Q} \& 60 \mathrm{Q} \\
\mathrm{CO} 2 \text { foam. Flush w/46.5 bbls } 6 \% \mathrm{KCl} \text { water w/10\% methanol" }\end{array}$ & \\
\hline 34-11 & & WR & & & & & & & & & & & 2 & 1,227 & $\begin{array}{l}\begin{array}{l}\text { Producing from Wind River Formation with } 9 \text { perforated intervals. Information on stimulation missing from } \\
\text { well completion report. }\end{array} \\
\end{array}$ & Vo \\
\hline $13-1$ & & WR & & & & & & & & & & & 0 & 309 & $\begin{array}{l}\text { Produced in Wind River Formation (7 perforated intervals). Information on stimulation missing from well } \\
\text { completion report. }\end{array}$ & $\overline{\text { No }}$ \\
\hline \multirow[t]{5}{*}{$11-12}$. & 571.8 & WR & $8 / 3 / 2001$ & & 12.07 & 25.0 & & & & X & & $9,245$. & 0 & 63 & "Frac...70Q CO2 foam...68 bbls KCl, 113 bbls CO2". & No \\
\hline & 655.9 & WR- & $8 / 3 / 2001$ & & 12.07 & 25.0 & & $7.45^{\circ}$ & & $\mathrm{X}$ & & 10,175 & & & "Frac...70Q CO2 foam...87 bbls KCl, 117 bbls CO2" & \\
\hline & 689.5 & WR & $8 / 2 / 2001$ & & 10.72 & 25.0 & & 7.13 & & $\mathrm{X}$ & & 11,837 & & & "Frac-pump $70 \mathrm{Q}$ CO2 foam 88 bbls KCl, $152 \mathrm{bbls}$ CO2 & \\
\hline & $7 \overline{8} \overline{5} .2$ & WR'- & $8 / 2 / 2001$ & & 16.24 & 30.0 & & $8.87^{-}$ & & $\mathrm{X}^{-}$ & & 15,515 & & & "Frac-pump $70 \mathrm{Q}$ CO2 2 foam...121 bbls $\mathrm{KCl}, 183$ bbls CO2" & \\
\hline & 922.9 & WR'-1 & $8 / 1 / 2001^{-}$ & & 17.24 & & & $9.13^{-1}$ & & $\mathrm{X}^{-}$ & & $8,233^{-}$ & & & "Frac-pump $70 \mathrm{Q}$ foam...92 bbls KCl, 99 bbls CO2" & \\
\hline \multirow[t]{8}{*}{$21-10$} & 1350 & FU & $8 / 8 / 2001$ & 27.58 & 24.13 & 30.0 & & 9.10 & & $\underline{X}$ & & 14,893 & 6. & 9,066 & "Frac-Breakdown w/8 bbls KCl...Pumped 70Q CO2 foam frac using...145 bbls KCl, 22 & No \\
\hline & 1394 & $\mathrm{FU}^{-1}$ & $8 / 8 / 2001$ & 23.44 & 25.86 & 32.0 & & 6.88 & & $\mathrm{X}$ & & 23,970 & & & "Frac-Breakdown w/4 bbls KCl ..Pumped $70 \mathrm{Q} C \mathrm{CO} 2$ foam frac using 226 bbls KCl, $316 \mathrm{bbls}$ CO2". & \\
\hline & $14 \overline{4} 7$ & FU'- & $8 / 8 / 2001$ & $7.54^{-1}$ & 17.93 & 30.0 & & 2.59 & & $\mathrm{X}^{-}$ & & 10,971 & & & "Frac-Breakdown w/3 bls KCl...Pumped $70 Q \mathrm{CO} 2$ foam frac uisng...144 bbls KCl, 139 bbls CO2" & \\
\hline & 1471 & $\bar{F} \bar{U}^{-1}$ & $8 / 8 / 2001$ & 20.45 & 26.20 & 35.0 & & 3.48 & & $\mathrm{X}$ & & 13,757 & & & "Frac-Breakdown w/ 60 bbls KCl...Pump with $70 \mathrm{Q}$ CO 2 foam frac using ... 162 bbls KCl, 172 bls CO2" & \\
\hline & 1539 & FU'-1 & $6 / 1 / 2001$ & $19.04^{-}$ & & & & & & $\mathrm{X}$ & & $6,832^{-}$ & & & "Frac-Breakdown w/29 bbls $6 \%$ KCl...Pumped $70 \mathrm{Q}$ CO 2 frac using...141 bbls KCl, 146 bbls CO $2 "$ & \\
\hline & $1570^{-}$ & $\overline{F U}-1$ & $7 / 31 / 2001$ & $18.37^{-}$ & & & & & & $\mathrm{X}^{-}$ & & $9,596^{-1}$ & & & $\begin{array}{l}\text { Frac-Breakdown w/12 bbls } 6 \% \text { KCl...Pumped } 70 \mathrm{Q} \text { CO } 2 \text { foam frac using } 74 \text { bbls } 6 \% \text { KCl...126 bbls } \\
\text { CO2...screened out w/ } 6 \text { bbls of the } 4 \text { PPA \& } 32 \text { bbls of } 6 \text { PPA left in csg. Pumped } 70.4 \text { bbls } 6 \% \mathrm{KCl} \& \\
\text { screened out again". }\end{array}$ & \\
\hline & 1666 & Fi'- & $7 / 31 / 2001$ & $10 . \overline{5} 8^{-}$ & & & & & & $\mathrm{X}$ & & 20,160 & & & "Frac-Breakdown w/ 16 bbls $6 \%$ KCl... Pumped $70 \mathrm{Q}$ CO2 foam using 204 bbls $6 \%$ KCl...242 bbls CO2" & \\
\hline & $1743^{-}$ & $\mathrm{FU}$ & $7 / 26 / 2001$ & 8.96 & & & & 3.45 & & $\mathrm{X}^{-}$ & & 0 & & & "Pump 10 bbls $8 \%$ KCl into formation" & \\
\hline \multirow[t]{5}{*}{$43-1$} & 622.7 & WR & $9 / 1 / 2001$ & 11.72 & 13.84 & 20.2 & & 7.58 & & $\mathrm{X}$ & & 6,976 & 12 & 8,500 & $\begin{array}{l}\text { "Frac...using } 69.2 \text { bbls WF } 125 \text { in } 6 \% \mathrm{KCl} \text { water, } 15 \text { tons } \mathrm{CO} 2 \ldots 147 \text { bbls } 70 \mathrm{Q} \text { and } 60 \mathrm{Q} \mathrm{CO} 2 \text { foam. Flush } \\
\text { w/31 bbls } 6 \% \mathrm{KCL} \text { water" }\end{array}$ & No \\
\hline & 662.6 & WR & $9 / 1 / 2001$ & & 12.87 & 19.8 & & 7.33 & & $\mathrm{X}^{-}$ & & 8,264 & & & $\begin{array}{l}\text { "Frac...using } 79.4 \text { bbls WF } 125 \text { in } 6 \% \text { KCl water, } 20 \text { tons } \mathrm{CO} 2 \ldots 174 \text { bbls } 70 \mathrm{Q} \text { and } 60 \mathrm{Q} \mathrm{CO} 2 \text { foam. Flush } \\
\text { w/33.5 bbls } 6 \% \text { KCL water" }\end{array}$ & \\
\hline & 689.2 & WR & $8 / 31 / 2001$ & & 14.21 & 24.2 & & 7.28 & & $\bar{X}$ & & $9,508^{-}$ & & & $\begin{array}{l}\text { "Frac...using } 89.1 \text { bbls WF } 125 \text { in } 6 \% \text { KCl water, } 23 \text { tons } \mathrm{CO} 2 \ldots 222 \text { bbls } 70 \mathrm{Q} \text { and } 60 \mathrm{Q} \mathrm{CO} 2 \text { foam. Flush } \\
\text { w/35 bbls } 6 \% \text { KCL water" }\end{array}$ & \\
\hline & 760.8 & $\mathrm{WR}$ & $8 / 31 / 2001$ & 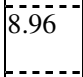 & 17.21 & 27.7 & & $8.69^{-}$ & & $\bar{x}$ & & 16,480 & & & $\begin{array}{l}\text { "Frac...using } 124.8 \text { bbls WF } 125 \text { in } 6 \% \mathrm{KC} \text { water, } 41 \text { tons } \mathrm{CO} 2 . .357 \text { bbls } 70 \mathrm{Q} \text { and } 60 \mathrm{Q} \text { CO } 2 \text { foam. Flush } \\
\text { w/39 bbls } 6 \% \text { KCL water" }\end{array}$ & \\
\hline & $8 \overline{8} \overline{5} . \overline{7}$ & $\bar{W} \bar{R}^{-1}$ & $8 / 29 / 2001^{-}$ & $13.65^{-}$ & 15.32 & $24 . \overline{4}$ & & 11.45 & & $\overline{\mathrm{X}}^{-}$ & & 14,427 & & & $\begin{array}{l}\text { "Frac...using } 112.1 \text { bbls WF } 125 \text { in } 6 \% \text { KCl water, } 32 \text { tons CO2...28 } 29 \text { bls } 70 \mathrm{Q} \text { and } 60 \mathrm{Q} \text { CO } 2 \text { foam. Flush } \\
\text { w/45 bbls } 6 \% \text { KCL water" }\end{array}$ & \\
\hline 33-1 & & & & & & & & & & & & & 0 & 3,176 & $\begin{array}{l}\text { Producing in Wind River Formation (10 perforated intervals). Information on stimulaton missing from well } \\
\text { completion report. }\end{array}$ & No \\
\hline
\end{tabular}




\begin{tabular}{|c|c|c|c|c|c|c|c|c|c|c|c|c|c|c|c|c|}
\hline 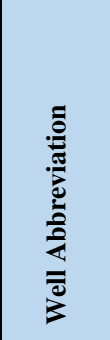 & 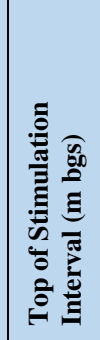 & 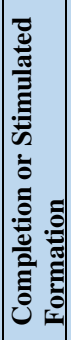 & 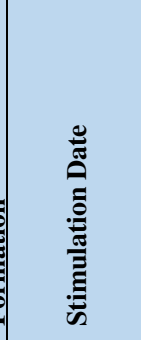 & 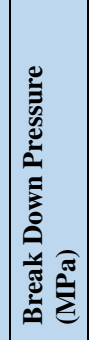 & 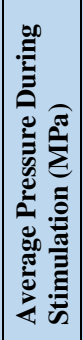 & 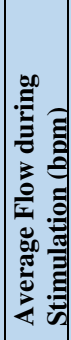 & 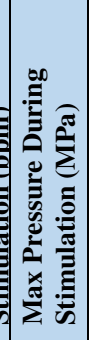 & 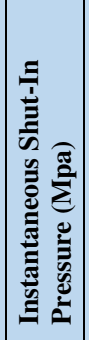 & 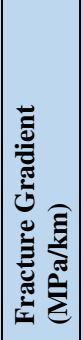 & 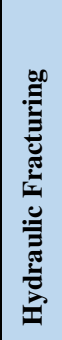 & 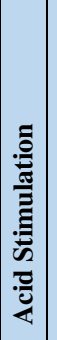 & 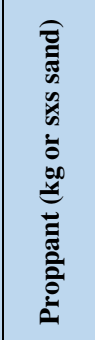 & 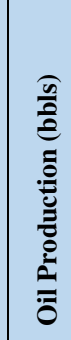 & 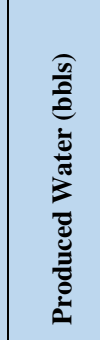 & 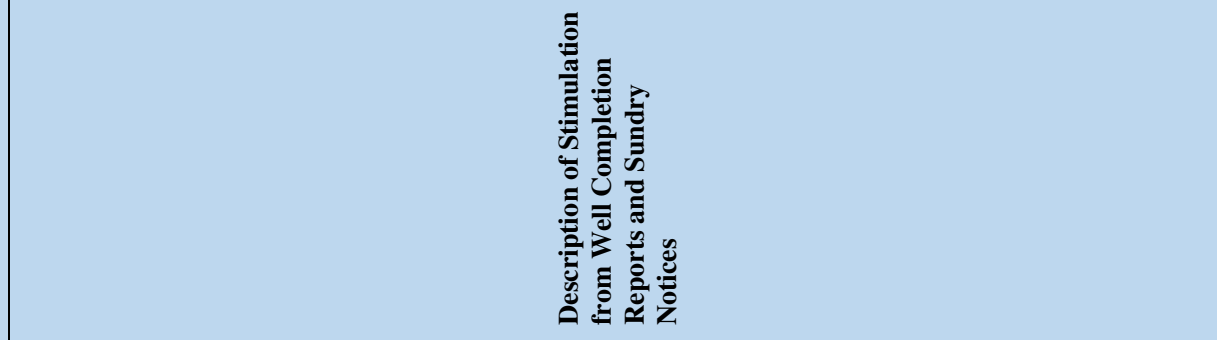 & 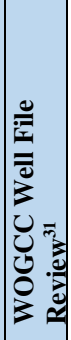 \\
\hline $12-7$ & & & & & & & & & & & & & 0 & 118 & $\begin{array}{l}\text { Producing in Wind River Formation (6 perforated intervals). Information on stimulation missing from well } \\
\text { completion report. }\end{array}$ & No \\
\hline \multirow[t]{8}{*}{ 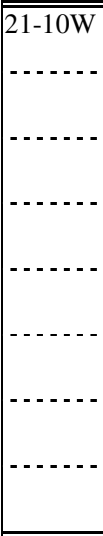 } & 585.5 & WR & $9 / 15 / 2001$ & & 18.02 & 19.1 & & 8.80 & & $\bar{X}$ & & 16,245 & 0 & 21,102 & $\begin{array}{l}\text { "Frac... using } 108.6 \text { bbls YF } 125 \text { LG in } 6 \% \text { kcl wtr, } 30 \text { tons CO2...287 bbls 70Q \& 60Q CO2 foam. Flush } \\
\text { w/28 bbls 50/50 6\% kcl wtr \& CO2". }\end{array}$ & No \\
\hline & 645.9 & $\mathrm{WR}$ & $9 / 15 / 2001$ & & 15.44 & 28.0 & & $8.62^{-1}$ & & $\mathrm{X}^{-}$ & & $12,674^{-}$ & & & $\begin{array}{l}\text { "Frac...using } 101.4 \text { bbls YF } 125 \text { LG in } 6 \% \text { kcl wtr, } 22 \text { tons } \mathrm{CO} 2 \ldots 241 \text { bbls } 70 \mathrm{Q} \& 60 \mathrm{Q} \text { CO } 2 \text { foam. Flush } \\
\text { w/32 bbls } 6 \% \text { kcl wtr." }\end{array}$ & \\
\hline & 760.8 & $\mathrm{WR}$ & $9 / 15 / 2001$ & & 16.24 & 26.9 & & 7.67 & & $\mathrm{X}^{-}$ & & $18,493^{-}$ & & & $\begin{array}{l}\text { "Frac...using } 139.5 \text { bbls YF } 125 \text { LG in } 6 \% \text { kcl wtr, } 38 \text { tons } \mathrm{CO} 2 \ldots 342 \text { bbls } 70 \mathrm{Q} \& 60 \mathrm{Q} \text { CO2 foam. Flush } \\
\text { w/38 bbls } 6 \% \text { kcl wtr". }\end{array}$ & \\
\hline & 850.1 & $\mathrm{WR}$ & $9 / 15 / 2001$ & & 10.64 & 21.2 & & 7.29 & & $\mathrm{X}^{-}$ & & $7,439^{--}$ & & & $\begin{array}{l}\text { "Frac...using } 91.9 \text { bbls wf } 125 \text { in } 6 \% \text { KCl wtr, } 22 \text { tons } \mathrm{CO} 2 \ldots 176 \text { bbls } 70 \mathrm{Q} \& 60 \mathrm{Q} \text { foam. Flush w/42.3 bbls } \\
6 \% \text { kcl wtr" }\end{array}$ & \\
\hline & 914.4 & $\mathrm{WR}$ & $9 / 14 / 2001$ & & $20.39^{-}$ & $12 . \overline{4}$ & & & & $\mathrm{X}^{-}$ & & $8,900^{-}$ & & & $\begin{array}{l}\text { "Frac...using } 88.5 \text { bbls wf } 125 \text { in } 6 \% \text { KCl wtr, } 20 \text { tons } \mathrm{CO} 2 \ldots 166 \text { bbls } 70 \mathrm{Q} \& 60 \mathrm{Q} \text { foam. Flush w/43 bbls } \\
6 \% \mathrm{KCl} \text { water" }\end{array}$ & \\
\hline & $1062^{-}$ & $\overline{\mathrm{FU}} \overline{\mathrm{H}}^{-}$ & $9 / 14 / 2001$ & & $16.80^{-}$ & 25.8 & & 9.45 & & $\mathrm{X}^{-}$ & & $14,369^{-}$ & & & $\begin{array}{l}\text { "Frac...132.4 bbls wf } 125 \text { in } 6 \% \text { kcl wtr, } 36 \text { tons } \mathrm{CO} 2 . .289 \mathrm{bbls} 70 \mathrm{Q} \& 60 \mathrm{Q} \text { foam. Flush w/53 bbls } 6 \% \text { kcl } \\
\text { wtr" }\end{array}$ & \\
\hline & 1134 & $\overline{\mathrm{FU}} \mathrm{U}^{-}$ & $9 / 14 / 2001$ & & 14.53 & 30.9 & & $5.45^{-1}$ & & $\mathrm{X}^{-}$ & & $28,038^{-}$ & & & $\begin{array}{l}\text { "Frac ...using } 214.9 \text { bblswf } 125 \text { in } 6 \% \text { kcl wtr, } 72 \text { tons } \mathrm{CO} 2 \ldots 615 \text { bbls } 70 \mathrm{Q} \& 60 \mathrm{Q} \text { foam. Flush w/57 bbls } \\
6 \% \text { kcl wtr" }\end{array}$ & \\
\hline & $1291^{-}$ & $\mathrm{FU}^{-}$ & $9 / 14 / 2001$ & & 17.11 & 27.6 & & 7.98 & & $\mathrm{x}^{-}$ & & $21,811^{-}$ & & & $\begin{array}{l}\text { "Frac...uisng } 184.3 \text { bbls wf } 125 \text { in } 6 \% \text { kcl wtr, } 59 \text { tons } \mathrm{CO} 2 . .536 \text { bbls } 70 \mathrm{Q} \& 60 \mathrm{Q} \text { CO } 2 \text { foam. Flush w/65 } \\
\text { bbls } 6 \% \text { kcl wtr." }\end{array}$ & \\
\hline \multirow[t]{5}{*}{$23-12$} & 527.3 & WR & $9 / 19 / 2001$ & 11.48 & 17.93 & 35.0 & & 7.45 & & X. & & 17,037 & 0. & 139 & "totals pumped 112 bbls KCl, 218 bbls CO2" & Yes \\
\hline & 679.1 & WR & $9 / 19 / 2001$ & 17.17 & 15.51 & 30.0 & & 8.83 & & X- & & 15,072 & & & totals pumped 118 bbls KCl, 180 bbls CO2" & \\
\hline & $7 \overline{12.6}$ & WR & $9 / 19 / 2001$ & 16.89 & 15.17 & 25.0 & & 8.07 & & $\mathrm{X}^{-}$ & & $7,459^{-}$ & & & "totals pumped 78 bbls $\mathrm{KCl}, 83$ bbls $\mathrm{CO} 2 "$ & \\
\hline & 878.1 & Win & $9 / 19 / 2001$ & 13.51 & 20.68 & 26.0 & & 8.68 & & $\mathrm{X}^{-}$ & & 11,050 & & & totals pumped $106 \mathrm{~b} \mathrm{~b} \mathrm{l} s \mathrm{KC} \mathrm{l}, 13 \overline{\mathrm{b}} \mathrm{b} \mathrm{ls} \mathrm{CO} 2 "$ & \\
\hline & 1009 & $\mathrm{WR}$ & $9 / 19 / 2001$ & 20.12 & 17.24 & 30.0 & & 0.00 & & $\mathrm{X}^{-}$ & & 11,016 & & & "totals pumped $111 \mathrm{bb} \mathrm{ls} \mathrm{KCl}, 134.6 \mathrm{bb} \mathrm{ls} \mathrm{CO} 2 "$ & \\
\hline \multirow[t]{6}{*}{$44-2$} & 609 & WR & $9 / 21 / 2001$ & 18.85 & 13.04 & 20.9 & & 8.20 & & $\underline{X}$ & & 7,042 & 0 & 198 & "Totals pumped $58 \mathrm{KCl}, 88 \mathrm{CO} 2 "$. & No. \\
\hline & 648 & WR & $9 / 21 / 2001$ & 15.51 & 10.63 & 25.3 & & 6.76 & & $\mathrm{X}$ & & 14,223 & & & "Totals pumped $107 \mathrm{KCl}, 162 \mathrm{CO} 2 "$ & \\
\hline & $7 \overline{5} \overline{3} . \overline{5}$ & $\mathrm{WR}$ & $9 / 21 / 2001$ & 24.82 & 11.91 & 27.0 & & $6.89^{-1}$ & & $\mathrm{X}$ & & 15,162 & & & "Totals pumped $119 \mathrm{bbls} \mathrm{KCl}, 180 \mathrm{CO} 2 "$ & \\
\hline & $783^{-1}$ & $\mathrm{WR}$ & $9 / 21 / 2001$ & 21.57 & 17.20 & 28.3 & & 8.00 & & $\mathrm{X}$ & & 23,336 & & & "Totals pumped 164 bbls KCl, 272 CO2" & \\
\hline & 921.7 & WR & $9 / 21 / 2001$ & 22.75 & 13.93 & 22.0 & & 8.62 & & $\mathrm{X}^{-}$ & & $8,091^{-}$ & & & $\begin{array}{l}\text { Frac-pumped WF } 12570 Q \\
105 \text { bbls } \mathrm{KCl}, 116 \text { bbls CO2" }\end{array}$ & \\
\hline & 1132 & $\overline{\mathrm{FU}}^{-}$ & $9 / 20 / 2001$ & 21.68 & 19.31 & 34.0 & & $6 . \overline{5} 5^{-}$ & & $\mathrm{X}^{-}$ & & $13,180^{-}$ & & & "Frac-pumped WF $12570 \mathrm{Q}$...Totals pumped $131 \mathrm{bbls} \mathrm{KCl}, 152 \mathrm{bbls} \mathrm{CO} 2 "$ & \\
\hline \multirow[t]{5}{*}{$31-9$} & 705 & WR. & $10 / 18 / 2001$ & 26.10 & 13.44 & 23.0 & & 10.55 & & $\underline{X}$ & & $7,382$. & 2. & $1,346$. & "66 bbl clean fluid, 92.4 bbls CO2. 422 bbl total load". & Yes \\
\hline & 802.2 & Win- & $10 / 18 / 2001$ & & 15.31 & 26.0 & & 9.33 & & X- & & 11,660 & & & 92 bbls clean fluid, 150.7 bbi co2" & \\
\hline & 829.7 & $\mathrm{WR}$ & $10 / 18 / 2001$ & 28.19 & 14.51 & 22.7 & & 9.86 & & $\mathrm{X}_{-}^{-}$ & & 6,924 & & & 82 bbls clean fluid, 81.6 bbi có2" & \\
\hline & 892.1 & WR & $10 / 18 / 2001$ & 24.71 & 13.29 & 23.0 & & 12.09 & & X- & & $7,022^{-1}$ & & & Total clean fluid 95 bbls, 108.1 bbls co2" & \\
\hline & 1008 & WR & $10 / 18 / 2001$ & 17.11 & 15.81 & 20.0 & & 26.09 & & $\mathrm{X}$ & & $7,154^{-}$ & & & "Total clean fluid $87 \mathrm{bbls}$ and $89.4 \mathrm{bbl} \mathrm{CO} 2 "$ & \\
\hline \multirow[t]{6}{*}{$34-10$} & 1407 & FU & $10 / 6 / 2001$ & 19.51 & 20.12 & 28.4 & 28.91 & 8.38 & & $\mathrm{X}$ & & 17,553 & 5 & 4,816 & "Total pumped 143 bbls KCl, 219 bbls CO2" & Yes \\
\hline & 1447 & FU & $10 / 5 / 2001$ & 20.20 & 16.15 & 18.9 & 26.89 & 19.10 & & X- & & 8,437 & & & Total pumped 120 bbls KCl,167 bbls CO2" & \\
\hline & 1538 & $\mathrm{FU}$ & $10 / 4 / 2001$ & 25.51 & 19.44 & 28.4 & 26.45 & 9.16 & & X- & & 13,738 & & & Total pumped 136 bbls KCl, 206 bbls CO2" & \\
\hline & $1590^{-1}$ & FE'- & $10 / 3 / 2001$ & 15.03 & 19.65 & 27.4 & 19.71 & 9.65 & & X- & & 16,320 & & & Total pumped 156 bbls KCl, 242 bbls CO2" & \\
\hline & $1639^{-1}$ & $\overline{\mathrm{FU}} \overline{\mathrm{U}}^{-1}$ & $10 / 2 / 2001^{-1}$ & $14 . \overline{4} 8^{-}$ & $2 \overline{1} .02$ & 33.0 & 21.02 & $6.39^{--}$ & & $\mathrm{X}^{-}$ & & $16,035^{-}$ & & & "Total pumped 145 bbls KCl, 230 bbls CO2" & \\
\hline & $1682^{-}$ & $\mathrm{FU}^{-1}$ & $9 / 26 / 2001$ & 10.64 & 16.97 & 2.0 & 16.97 & & & $\mathrm{X}^{-}$ & & $8,440^{-}$ & & & "Screened out with $8458 \#$ in $12 / 20$ sand in csg...Total pump $83.6 \mathrm{KCl}, 108 \mathrm{bbls} \mathrm{CO} 2 "$ & \\
\hline 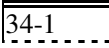 & 564.8. & [WR & ] $10 / 11 / 2001$ & 12.37 & $\mid 10.51$ & 21.0 & 13.13 & 7.45 & & $\underline{\underline{X}}$ & & 8,145 & 0. & 930. & "Total pumped 82 bbls KCl, 95 bbls CO2". & No. \\
\hline
\end{tabular}




\begin{tabular}{|c|c|c|c|c|c|c|c|c|c|c|c|c|c|c|c|c|}
\hline 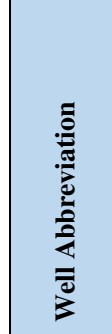 & 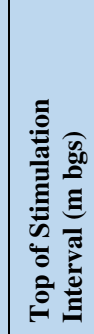 & 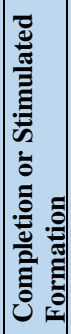 & 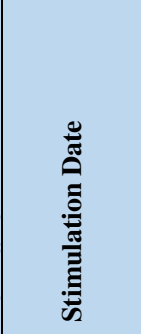 & 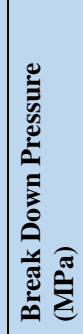 & 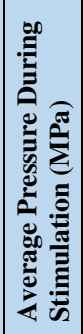 & 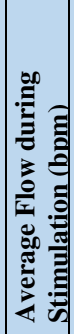 & 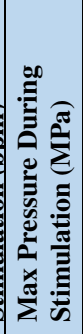 & 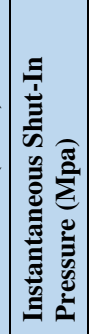 & 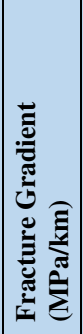 & 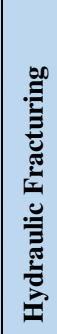 & 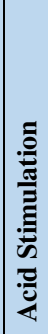 & 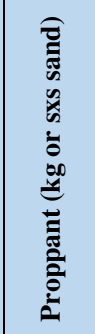 & 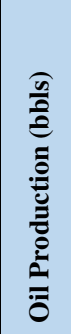 & 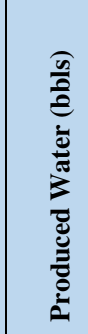 & 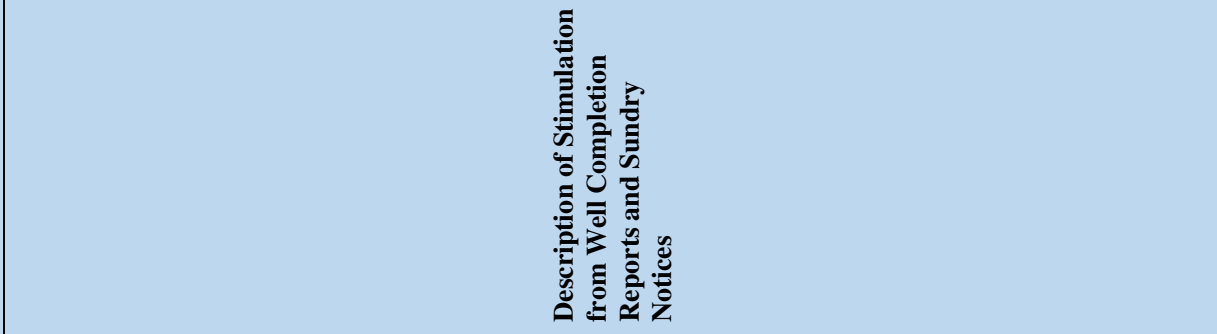 & 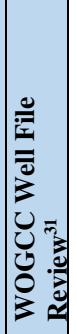 \\
\hline & 785.2 & WR- & $10 / 11 / 2001$ & 11.89 & 13.79 & 30.0 & 15.51 & 7.03 & & $\mathrm{X}$ & & $10,864^{-}$ & & & "Totals pumped 98 bbls KCl, 124 bbis CO2" & \\
\hline & 947.3 & WR- & $10 / 11 / 2001$ & 15.58 & 11.72 & 25.0 & 13.70 & 8.48 & & $\mathrm{X}$ & & 7,584 & & & "Frac-WF $12570 \mathrm{Q} \ldots$ Totals pumped $92 \mathrm{bbls} \mathrm{KCl}$, & \\
\hline $21-12$ & .506 & WR & $10 / 27 / 2001$ & 7.58 & 18.96 & 35.0 & 24.82 & 5.86 & & $\bar{X}$ & & 18,001 & 0 & 14 & "Totals pumped 112 bbls KCl, 223 bbls CO2" & $\overline{\mathrm{No}}$ \\
\hline & $563.3^{-}$ & WR & $10 / 27 / 2001$ & 22.93 & 18.96 & $35^{--1}$ & 23.79 & $7.70^{-}$ & & $\bar{X}$ & & 15,142 & & & "Totals pumped $107 \mathrm{bbls} \mathrm{KCl}, 180$ & \\
\hline & 823.9 & WR- & $10 / 26 / 2001$ & 12.74 & 14.13 & 25 & 26.20 & 6.89 & & $\mathrm{X}$ & & 7,232 & & & "Totals pumped $67 \mathrm{bbls}$ KCl, $104 \mathrm{bbls}$ CO 2 " & \\
\hline & 963.8 & WR & $10 / 28 / 2001$ & $19.99^{\circ}$ & 20.68 & $27^{-1}$ & 25.51 & 11.20 & & $\mathrm{X}$ & & $11,377^{-}$ & & & "Totals pumped $105 \mathrm{bb} \mathrm{b}_{\mathrm{s}} \mathrm{KCl}, 134 \mathrm{bb} \mathrm{ls} \mathrm{CO} 22^{-}$ & \\
\hline $12-10$ & 726.6 & WR & $10 / 26 / 2001$ & 7.58 & 17.24 & 35.0 & 18.62 & 9.09 & & $\bar{X}$ & & 13,778 & 2 & $1,1,465$ & "Totals pumped 102 bbls KCL, 196 bbls CO2". & No \\
\hline & $790^{-1}$ & $\mathrm{WR}$ & $10 / 25 / 2001$ & 14.48 & 17.93 & 25.0 & 27.21 & & & $\mathrm{X}$ & & 7,244 & & & "Frac-WF $12570 \mathrm{Q}$ CO 2 foam frac...screened out w/6032\#...To & \\
\hline $\mid 12-1$ & 606.6 & WR & $11 / 10 / 2001$ & 18.24 & 13.79 & 25.0 & & 9.10 & & $\mathrm{X}$ & & 9,066 & 0 & 653 & "Totals pumped 70 bbls $\mathrm{KCl}, 111 \mathrm{bbls} \mathrm{CO} 2$ & No. \\
\hline & 929.9 & $\mathrm{WR}$ & $11 / 9 / 2001$ & 21.05 & 21.72 & 28.0 & & $9.40^{-}$ & & $\mathrm{X}$ & & 10,928 & & & "Totals pumped 88 bbls KCl, 153 bbls CO $2 "$ & \\
\hline & 1024 & WR & $11 / 9 / 2001^{-}$ & 19.82 & $19.99^{\circ}$ & 25.0 & & 13.51 & & $\mathrm{X}$ & & 7,448 & & & "Totals pumped 93 bbls KCl, 83 bbis CO2" & \\
\hline$\overline{42-3}$ & 4338.9 & WR & $1 / 29 / 2005$ & 12.69 & & & & 7.43 & 27.14 & $\mathrm{X}$ & & 6,978 & 0 & 1,675 & "Frac w/64 bbls slickwater" & No \\
\hline & 5078 & WR & $1 / 29 / 2005$ & 11.36 & & & & 6.92 & 23.75 & $\mathrm{X}$ & & 6,812 & & & "Frac w/100 bbls slickwater" & \\
\hline & $687^{-}$ & WR & $1 / 26 / 2005$ & 14.34 & & & & 7.51 & 21.04 & $\mathrm{X}$ & & 6,573 & & & "Frac w/93 bbls slickwater" & \\
\hline & 727.6 & WR-1 & $11 / 14 / 2001$ & & & & & 9.14 & & $\mathrm{X}$ & & 8,718 & & & "Frac w/68 bbls KCl, 103 bbls CO2" & \\
\hline & 774.8 & WR & $11 / 13 / 2001$ & & & & & 12.20 & & $\mathrm{X}$ & & $7,944^{-}$ & & & "Frac w/67 bbls KCl, 1100 bbls CO2" & \\
\hline & 850.4 & WR & $11 / 8 / 2001$ & & & & & 9.51 & & $\mathrm{X}$ & & 12,775 & & & "Frac w/95 bbls KCl, 163 bbls CO2" & \\
\hline & 1109 & $\overline{F U}^{-1}$ & $11 / 2 / 2001$ & & & & & 17.53 & & $\mathrm{X}$ & & $7,127^{-}$ & & & "Frac w/72 bbis KCil, 110 bbls CO $2 "$ & \\
\hline $22-1$ & 626.7 & WR & $11 / 1 / 2001$ & & & & & & & $\mathrm{X}$ & & 6,898 & 0 & 892 & "Frac w/16 & No \\
\hline & 687.6 & WR & $11 / 1 / 2001$ & & & & & & & $\mathrm{X}$ & & $10,005^{-}$ & & & "Frac w/197 bbl $75 \mathrm{Q}$ CO2 foam" & \\
\hline 33-10W & 1064 & FU & $12 / 1 / 2001$ & 24.13 & & & & 12.34 & & $\mathrm{X}$ & & 9,929 & 2 & 8,228 & $\begin{array}{l}\text { Frac-BD w/6\% KCl...Pmp } 230 \mathrm{bbl} \text { 70Q WF-125 CO2 foam...Total fluid 104, CO2 tons. Stimulation report } \\
\text { indicates that 70Q CO2 WF-125 foam and fluids used contained Biocide B69, Surfactant EZEFLO F103, } \\
\text { Foaming Agent F104, Breaker J218, Breaker Aid Liquid J318, Slurry PSG Polymer J877, Clay Stabilizer } \\
\text { L55. }\end{array}$ & Yes \\
\hline & $1 \overline{1} \overline{4}$ & $\bar{F}$ & $11 / 30 / 2001$ & & & & & $4.30^{-}$ & & $\bar{X}$ & & $12,623^{-}$ & & & "Start $70 \mathrm{Q} \mathrm{CO} 2,310 \mathrm{bbl} "$ & \\
\hline & $1 \overline{170-}$ & $\overline{\mathrm{FU}}$ & $12 / 10 / 2004$ & & & & & & & & & 0 & & & "Breakdown w Linear gel" & \\
\hline & $1191^{-}$ & FË & $12 / 9 / 2004$ & & & & & & & & & 0 & & & "Breakdown w Linear gel" & \\
\hline & 1368 & $\overline{\mathrm{FU}}$ & $11 / 16 / 2001$ & 7.93 & 21.03 & 30.0 & & 10.96 & & $\mathrm{X}$ & & $11,337^{-}$ & & & "Total pumped $113 \mathrm{bbls} \mathrm{KCl}, 163$ & \\
\hline & 534.9 & WR & $12 / 6 / 2001$ & 19.39 & 14.48 & 25.0 & & 8.48 & & X & & 5,126 & & & "Frac-BD w/6\% KCl...Start 70Q CO2 143 bbls & \\
\hline & 571.2 & $\mathrm{WR}$ & $2 / 1 / 2005$ & 25.07 & & & & 7.52 & $23.30^{-}$ & $\mathrm{X}$ & & 6,905 & & & "Frac w/86 bbl slickwater" & \\
\hline & 595 & $\overline{W R}$ & $1 / 28 / 2005$ & 26.10 & & & & 8.79 & 25.11 & $\mathrm{X}$ & & 6,657 & & & "Frac w/93 bbl slickwater" & \\
\hline & 613.9 & WR & $1 / 26 / 2005$ & 17.29 & & & & 8.55 & 23.98 & $\mathrm{X}$ & & $7,799^{-}$ & & & "Frac w/108 bbl slickwater" & \\
\hline & 731.8 & $\mathrm{WR}$ & $1 / 22 / 2005^{\circ}$ & 28.28 & & & & 10.74 & $24.88^{-}$ & $\mathrm{X}$ & & 8,196 & & & "Frac w/104 bbl slickwater" & \\
\hline & 801.6 & WhR-1 & $12 / 5 / 2001$ & 27.85 & 17.93 & 25.0 & & 10.55 & & $\mathrm{X}_{-}^{-}$ & & $8,657^{-1}$ & & & "Frac-BD w/6\% KCl ...Start $70 \mathrm{Q} \mathrm{CO} 2170$ bb1...22 ton CO2." & \\
\hline & 851 & WR & $12 / 5 / 2001$ & 18.53 & 20.68 & 30.0 & & 8.72 & & $\bar{X}_{-}^{-}$ & & 9,571 & & & "Frac-BD w/6\% KCl ..Start $70 \mathrm{Q} C \mathrm{C} 2211 \mathrm{bb} 1 \mathrm{~s} .109 \mathrm{bbl}$ fluid \& 20 ton CO2" & \\
\hline & 1065 & FE. & $12 / 4 / 2001$ & 25.86 & 20.68 & {$[30.0]$} & & 10.82 & & $\mathrm{X}^{-}$ & & 8,008 & & & Frac-BD w/6\% KCl..Pump $23370 \mathrm{Q}$ CO2 foam...24 ton CO2, 129 bbi fluid: & \\
\hline
\end{tabular}




\begin{tabular}{|c|c|c|c|c|c|c|c|c|c|c|c|c|c|c|c|c|}
\hline \multirow[t]{3}{*}{ 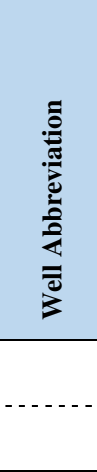 } & 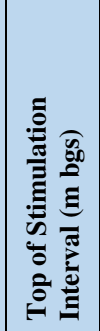 & 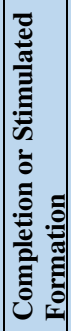 & 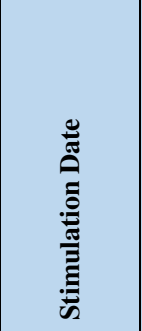 & 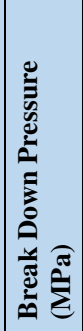 & 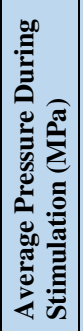 & 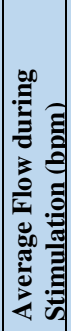 & 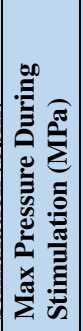 & 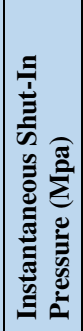 & 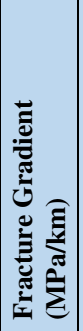 & 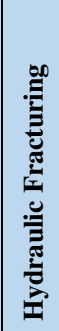 & 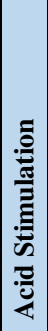 & 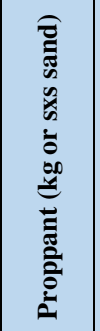 & 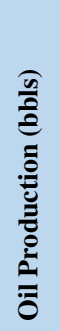 & 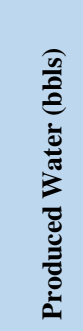 & 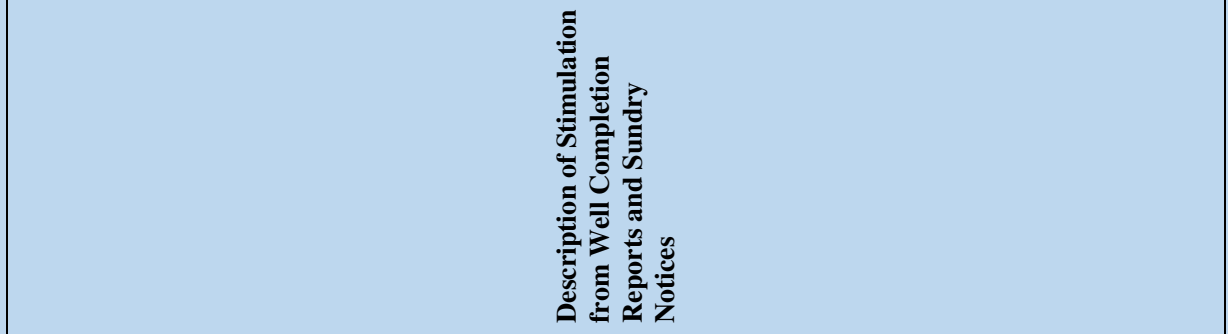 & 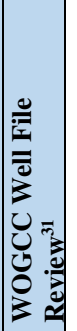 \\
\hline & 1120 & $\mathrm{FU}$ & $11 / 29 / 2001$ & 19.99 & 19.65 & 30.0 & & & & $\mathrm{X}$ & & 5,139 & & & $\begin{array}{l}\text { "Frac-Breakdown } 29 \mathrm{bbl} 6 \% \mathrm{KCl} . . \text { Start 70Q CO2 foam } 162 \mathrm{bbls} . .57 \text { bbls fluid \& } 19 \text { ton } \mathrm{CO} 2 \ldots .21 \text { bbls } \\
\text { flush screened out w/4410\# } 6 \# \text { sand in formation." }\end{array}$ & \\
\hline & $11 \overline{8} 3$ & $\overline{F U}^{-}$ & $11 / 27 / 2001$ & & 18.27 & 30.0 & & 10.55 & & $\overline{\mathrm{X}}^{-}$ & & $11,141^{-}$ & & & 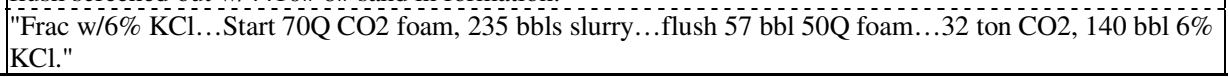 & \\
\hline \multirow[t]{6}{*}{$22-11$} & 568.1 & WR & $12 / 15 / 2001$ & 12.83 & 12.07 & 30.0 & & 7.38 & & $\bar{X}$ & & 9,885 & $\overline{1}$ & 306 & "Frac-Start 75Q foam... pump 197 bbls foam...25 tons CO2, 67 bbls fluid" & \\
\hline & 690.1 & WR & $12 / 15 / 2001$ & $20.20^{-}$ & 13.79 & 35.0 & & $6.83^{-}$ & & $\mathrm{X}$ & & 12,391 & & & "Frac-Breakdown w/6\% KCl...Start $75 \mathrm{Q} \mathrm{CO} 2$ foam 28 & \\
\hline & 730.9 & WR & $12 / 14 / 2001$ & 26.54 & 13.79 & 30.0 & & 8.08 & & $\bar{x}-$ & & 10,146 & & & "Frac-Breakdown...Totals pumped 93 bbls KCl, 134 bbls CO2 & \\
\hline & $84 \overline{5} . \overline{5}$ & WR & $12 / 14 / 2001$ & $16.20^{-}$ & & & 30.15 & 0.00 & & $\mathrm{X}^{-}$ & & 0 & & & $\begin{array}{l}\text { "Frac...psi increasded to } 4373 \text {-bled well down, psi continued to climb to } 4007 \text { shut down job. Pumped } 87 \\
\text { bbls KCl \& } 96 \text { bls CO2" }\end{array}$ & \\
\hline & $9 \overline{0} \overline{5} .6$ & $\overline{\mathrm{W}} \mathrm{N}^{-1}$ & $12 / 14 / 2001$ & $24.15^{-}$ & 22.06 & 33.0 & 23.44 & $10.59^{-}$ & & $\bar{X}^{-}$ & & $8,303^{-1}$ & & & "Frac...Totals pumped 146 b̄bls KCl, 149 b̄bls cö2" & \\
\hline & 951.6 & WR'-1 & $12 / 13 / 2001$ & & 18.62 & 25.0 & 29.58 & & & $\overline{\mathrm{X}}^{-}$ & & $5,645^{-}$ & & & "Frac-Pump 41 bbls KCl, 85.7 bbls CO2...Screened out w/32 27 \# in formation, $9219 \#$ in csg" & \\
\hline \multirow[t]{7}{*}{ 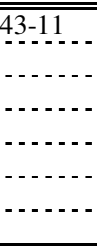 } & 507.2 & WR & $1 / 4 / 2002$ & 11.50 & 19.31 & 37.0 & & 8.23. & & $\underline{X}$ & & & ). & 140 & "Frac-Breakdown w/6\% KCl .. Start 75Q CO2, 411 bbls foam, 123 bbls fluid, 44 tons CO2" & \\
\hline & 589.5 & WR- & $1 / 4 / 2002$ & 10.59 & 16.55 & 30.0 & & 9.17 & & $\mathrm{X}$ & & $8,059^{-}$ & & & "Frac-Breakdown w/6\% KCl & \\
\hline & 734.9 & Whe-1 & $1 / 4 / 2002$ & $14.82^{-}$ & 20.68 & 35.0 & & 6.89 & & $\mathrm{X}^{-}$ & & $9,684^{-}$ & & & "Frac-Breakdown w/6\% KCl 2 Start $75 \mathrm{Q}$ CO2, 243 bbls, 97 bbls fluid, 23 tons CO2" & \\
\hline & 766.9 & $\mathrm{WR}$ & $1 / 4 / 2002$ & $1.31^{--}$ & 21.37 & 35.0 & & $8.80^{-}$ & & $\mathrm{X}$ & & 12,222 & & & "Frac-Breakdwon w/ $6 \%$ KCl...Start $75 \mathrm{Q}$ CO2 foam, 143 bbls fluid, 26 tons CO2" & \\
\hline & 1032 & $\overline{F U}^{-1}$ & $1 / 3 / 2002$ & $16.26^{-}$ & 21.37 & 32.0 & & 8.91 & & $\mathrm{X}_{-}^{-}$ & & $6,967^{-1}$ & & & Frac-Break down w/6\% KCl Start $70 \mathrm{Q} C \mathrm{CO} 2$ frac, $299 \mathrm{bbls}, 136$ fluid, 28 tons CO2 & \\
\hline & 1106 & $F \bar{H}^{-1}$ & $12 / 19 / 2001$ & 17.23 & 22.41 & 32.5 & & 4.90 & & $\mathrm{X}$ & & 7,586 & & & "Frac-Break down w/6\% KCl . Start $70 \mathrm{Q}$ foam $306 \mathrm{bbls}, 157 \mathrm{bb}$ & \\
\hline & $1160^{-1}$ & $\overline{F U}^{-1}$ & $12 / 18 / 2001$ & $16.10^{-}$ & 19.99 & 38.0 & & $5.91^{\circ}$ & & $\mathrm{X}$ & & 27,318 & & & "Frac-Break w/6\% KCl...Start $70 \mathrm{Q}$ foam $881 \mathrm{bbls}, 275$ bbls gel, 97 tons $\mathrm{CO} 2 "$ & \\
\hline \multirow[t]{4}{*}{$11-3$} & 702.9 & WR & $12 / 12 / 2001$ & 26.89 & 15.86 & 25.0 & & 7.45 & & $\bar{X}$ & & 5,648 & $=$ & 1,895 & "Frac-Break w/6\% KCK...Start w/168 bbls 75Q CO2 foam, 17 ton CO2, 60 bbls of fluid" & No \\
\hline & 804.7 & WR- & $12 / 12 / 2001$ & 18.68 & 15.17 & 25.0 & & 8.66 & & X- & & 7,983 & & & "Frac-Break w/6\% KCl . Start w/180 bbls 75Q CO2, 19 ton CO2, 96 bbls wtr" & \\
\hline & 899.8 & WR & $12 / 12 / 2001$ & 19.08 & 18.62 & 25.0 & & 8.63 & & $\mathrm{X}$ & & 7,888 & & & "Frac-Break w/6\% KCl ..Start w/169 bbls $75 \mathrm{Q}$ CO 2 foam, 51 bbl gel, 19 ton $\mathrm{CO} 2 "$ & \\
\hline & $1167^{-1}$ & $\overline{F U}$ & $12 / 6 / 2001^{--1}$ & $29.30^{-}$ & 24.13 & 27.0 & & & & $\mathrm{X}^{-}$ & & $7,121^{-1}$ & & & $\begin{array}{l}\text { Frac-Break down w/6\% KCL ..Start } 70 \mathrm{Q} \mathrm{CO} 2185 \mathrm{bbls} . .20 \text { tons } \mathrm{CO} 2,55 \mathrm{bbl} \text { gel...Screened out } 4161 \text { in } \\
\text { formation, } 11540 \text { in pipe... Re-frac break w/6\% KCl } 50 \mathrm{bbls} \text {. Start } 337 \mathrm{bbl} 75 \mathrm{Q} \text { foam, } 36 \text { ton } \mathrm{CO} 2,147 \mathrm{bbl} \\
\text { fluid...screened out w/12331 in formation, } 5867 \# \text { in pipe" }\end{array}$ & \\
\hline \multirow[t]{7}{*}{$41-10$} & 4993.2 & WR & $1 / 20 / 2002$ & 15.72 & 14.48 & 35.0 & & 6.89 & & $\bar{P}$ & & 11,832 & & 157 & "Frac-Break w/6\% KCL...Start 75Q CO2, 70 bbls fluid, 28 ton CO2...flush 24 bbls 50/50" & r \\
\hline & 661.7 & WR- & $1 / 20 / 2002$ & $19.27^{-}$ & 13.79 & 35.0 & & 7.86 & & $\mathrm{X}^{-}$ & & 11,104 & & & "Frac-Break down w/6\% KCLL..Start $75 \mathrm{Q}$ foam, 96 bbls gel, 27 ton CO2 232 bbls $6 \%$ KCl flush" & \\
\hline & 766.9 & WR & $1 / 19 / 2002$ & $17.65^{-}$ & 14.48 & 30.0 & & 8.48 & & $\mathrm{X}^{-}$ & & $9,525^{-1}$ & & & $\begin{array}{l}\text { "Frac-Break down w/6\% KCLL...Start } 75 \mathrm{Q} 208 \mathrm{bbls} \text {, foam } 63.5 \mathrm{bbls}, 26 \text { ton CO } 2 \text {...flush } 37 \mathrm{bbls} 50 / 50^{\circ} \\
\text { flush" }\end{array}$ & \\
\hline & 789.1 & $\mathrm{WR}^{-1}$ & $1 / 19 / 2002$ & $11.89^{-}$ & 15.86 & 35.0 & & $7.14^{-}$ & & $\bar{X}$ & & 11,118 & & & 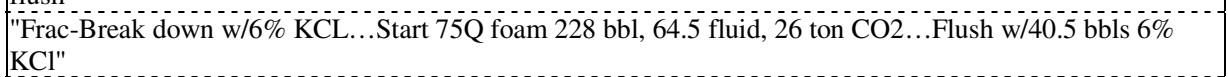 & \\
\hline & 841.2 & WR'-1 & $1 / 29 / 2002$ & $11.03^{-}$ & 15.86 & 35.0 & & $6.41^{\circ}$ & & $\mathrm{X}$ & & $10,559^{-}$ & & & 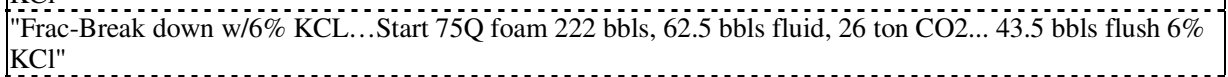 & \\
\hline & 863.8 & WR & $1 / 19 / 2002$ & 18.62 & 13.79 & 25.0 & & 8.96 & & $\mathrm{X}$ & & $6,562^{-}$ & & & "Frac-Break w/6\% KCL ..Start $75 \mathrm{Q}$ foam $144 \mathrm{bbls}, 44$ bbls fluid, 16 ton CO 2 fflush $44.5 \mathrm{bbls} 6 \% "$ & \\
\hline & 959.5 & WR & $1 / 19 / 2002$ & 16.13 & 14.48 & 25.0 & & & & $\overline{\mathrm{X}}-$ & & 7,228 & & & 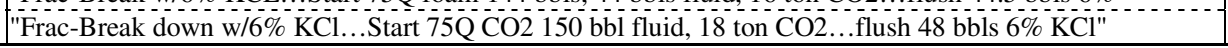 & \\
\hline \multirow[t]{5}{*}{$13-12 \mathrm{~W}$} & 780.9 & WR & $1 / 23 / 2002$ & 18.09 & 14.48 & |30.0. & & & & 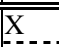 & & 11,819 & & 322 & "Frac-Break down w/6\% $\mathrm{KCl} \ldots$..Start 75Q foam, 72 bbls fluid, 30 tons $\mathrm{CO} 2 \ldots .37$ bbl 50/50". & 0 \\
\hline & 823.6 & WER-1 & $1 / 23 / 2002$ & $15.49^{-}$ & 14.48 & 30.0 & & 9.10 & & $\underline{X}$ & & 6,214 & & & "Frac-Break down w/6\% KCl..Start $75 \mathrm{Q}$ CO2 foam, 175 bbls, 78 bbl fluid, 16 ton CO2" & \\
\hline & $9 \overline{8} \overline{2.4}$ & $\overline{\mathrm{WR}}-1$ & $1 / 22 / 2002$ & $19.05^{-}$ & 18.62 & 25.0 & 29.33 & & & $\mathrm{X}$ & & $6,456^{-1}$ & & & $\begin{array}{l}\text { "Break w/ } 6 \% \text { KCl...Start } 75 \mathrm{Q} \text { foam, } 146 \mathrm{bb} \mathrm{s}, 43 \mathrm{bbl} \text { fluid, } 17 \text { tons } \mathrm{CO} 2 \ldots \text { screened out w/7900\# in } \\
\text { formation, } 6340 \# \text { in pipe" }\end{array}$ & \\
\hline & 1052 & $\overline{\mathrm{FU}}$ & $1 / 22 / 2002$ & $20.82^{-}$ & 18.62 & 30.0 & & $8 . \overline{5} 6$ & & $\mathrm{X}^{-}$ & & 6,917 & & & "Frac-Break w $6 \%$ KCl...Start $70 \mathrm{Q}$ foam $258 \mathrm{bbls}, 81$ bbl fluid, 24 ton $\mathrm{CO} 2 \ldots 52 \mathrm{bbl} 6 \%$ flush" & \\
\hline & $11125^{-}$ & $\overline{F U}-1$ & $1 / 22 / 2001$ & $16.55^{-}$ & $19.99^{-}$ & 35.0 & & $6.00^{-}$ & & $\mathrm{X}^{-}$ & & 16,565 & & & $\begin{array}{l}\text { "Frac-Break w/ } 6 \% \text { KCl...Start } 70 \mathrm{Q} \text { CO } 2 \text { foam, } 482 \text { bbis, } 142 \text { bbls fluid, } 55 \text { ton CO } 2 \ldots 55 \text { bbl flush } 6 \%{ }^{-} \\
\text {KCL" }\end{array}$ & \\
\hline
\end{tabular}




\begin{tabular}{|c|c|c|c|c|c|c|c|c|c|c|c|c|c|c|c|c|}
\hline 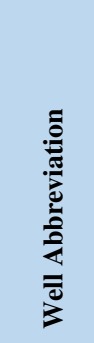 & 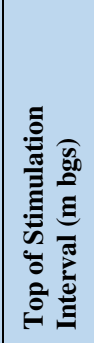 & 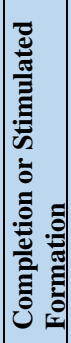 & 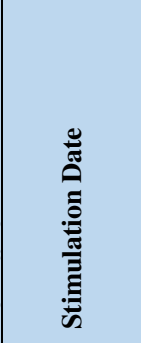 & 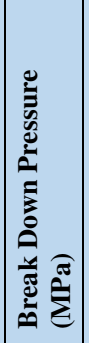 & 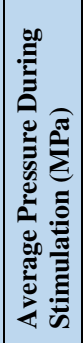 & 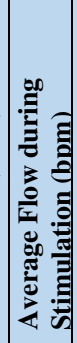 & 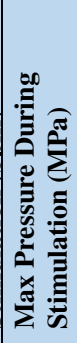 & 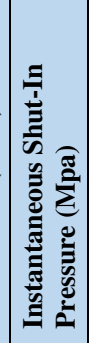 & 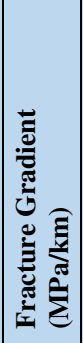 & 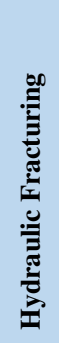 & 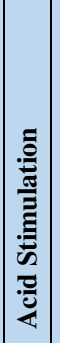 & 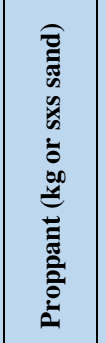 & 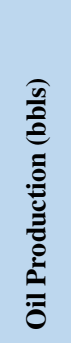 & 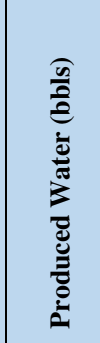 & 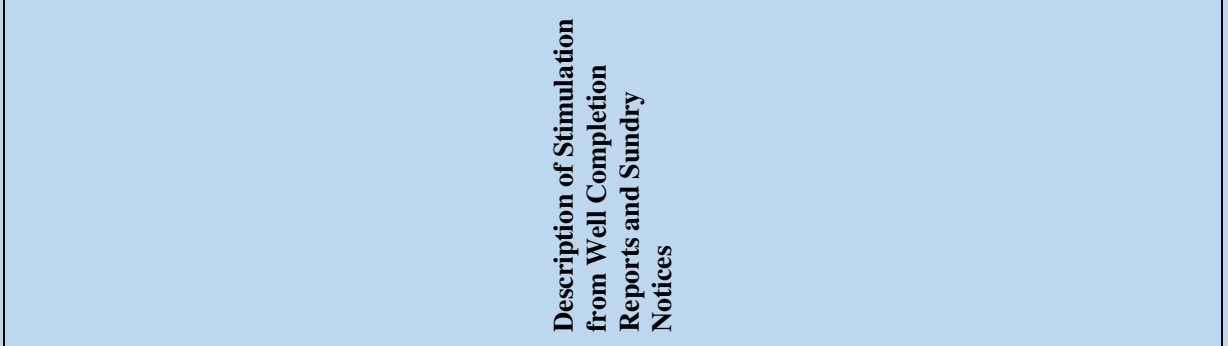 & 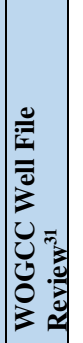 \\
\hline \multirow{4}{*}{ 24-3B } & $1401^{-}$ & $\bar{F}$ & $2 / 6 / 2002^{-}$ & 25.51 & & & 27.58 & 7.36 & & $\bar{X}^{-}$ & & 17,156 & & & "Frac-Breakdown...totals pumped 184 bbls KCl, 337 bbls $\mathrm{CO} 2$... $\mathrm{CO} 2$ hose leaking" & \\
\hline & 1464 & $\bar{F} \bar{U}^{-1}$ & $1 / 30 / 2002$ & $11.72^{-}$ & 21.72 & $3 \overline{5} .0$ & 23.24 & 6.17 & & $\mathrm{X}$ & & 15,161 & & & "Frac...Breakdown...Totals pumped 161 bbls KCl, 297 bbls CO2" & \\
\hline & 1470 & FU'-1 & $1 / 30 / 2002$ & 12.09 & 21.37 & 35.0 & 22.41 & 3.74 & & $\mathrm{X}_{-}^{-}$ & & 15,663 & & & "Frac-Breakdown . Total pumped $207 \mathrm{~b} b \mathrm{ls} \mathrm{KC} \mathrm{l}, 282$ bbls CO $2 "$ & \\
\hline & 1534 & $F U^{-1}$ & $1 / 29 / 2002$ & 12.41 & 25.51 & 35.0 & $27.92^{-}$ & 9.62 & & $\mathrm{X}^{-}$ & & 13,287 & & & "Frac...Breakdown...total pumped 190 böls KCl, 254 bubis CO $2 "$ & \\
\hline \multirow[t]{12}{*}{$111-11 \mathrm{~B}$} & 462.1 & WR & $2 / 18 / 2005$ & 12.31 & & 33.9 & 19.37 & 6.48 & & $\mathrm{X}_{-}$ & & 7,221 & & 13,056 & "Frac...18 ton of CO2, 63 bbl of clean fluid" & YYes \\
\hline & $524^{-1}$ & WR'- & $2 / 18 / 2005$ & $12.79^{-}$ & & 33.9 & 20.29 & 10.32 & & $\mathrm{X}^{-}$ & & 6,550 & & & "Frac... 15 tons $\mathrm{CO} 2$ and 78 bbls clean fluid" & \\
\hline & 566.3 & $\bar{W}-1$ & $2 / 18 / 2005$ & 10.02 & & 35.0 & 17.01 & 7.09 & & $\mathrm{X}^{-}$ & & 9,254 & & & "Frac...23 ton $\mathrm{CO} 2$ and 95 bbis clean fluid" & \\
\hline & 659 & WR & $2 / 18 / 2005$ & 26.23 & & 35.6 & 21.13 & 7.04 & & $\mathrm{X}^{-}$ & & 8,315 & & & "Frac...19 tons of $\mathrm{CO} 2$ and 91 bbls clean fluid" & \\
\hline & 1027 & $\overline{F U}$ & $2 / 14 / 2002$ & 21.93 & $16.55^{-}$ & 35.0 & & & & $\bar{x}$ & & 4,923 & & & $\begin{array}{l}\text { "Frac..Break w/6\% KCl...Frac using } 197 \text { bbis } 70 \mathrm{Q} \text { CO } 2 \text { foam, } 64 \text { bbls gel, } 22 \text { ton CO } 2 . . . \text { Flush w/ } / 6 \% \text { KCl } \\
\& 50 / 50 \text { CO2" }\end{array}$ & \\
\hline & $10 \overline{4}$ & $\overline{\mathrm{Fu}}$ & $2 / 15 / 20005$ & $18.73^{-1}$ & & & & 10.02 & & $\mathrm{X}$ & & 16,084 & & & "Frac...211 bbl clean fluid and 42 ton $\mathrm{CO} 2$ & \\
\hline & 1078 & $\mathrm{FU}-1$ & $2 / 14 / 2002$ & 9.17 & $18.62^{-}$ & 35.0 & & & & $\mathrm{X}$ & & $10,778^{-}$ & & & $\begin{array}{l}\text { "Frac-Break w/6\% KCl...Frac uisng } 386 \text { bbi } 70 \mathrm{Q} \text { CO } 2 \text { foam, } 140.5 \text { bbls gel, } 37 \text { tons } \mathrm{CO} 2 . .53 .5 \text { bbis } 6 \%{ }^{-1} \\
\text { flush" }\end{array}$ & \\
\hline & 1158 & $\overline{\mathrm{FU}}$ & $2 / 13 / 2002$ & $15.76^{-}$ & 19.31 & 35.0 & & & & $\mathrm{X}$ & & 10,600 & & & $\begin{array}{l}\text { "Frac-Break w/6\% KCl...Frac using } 346 \text { bbls } 70 \mathrm{Q} \text { CO2, } 138 \text { bbls gel, } 33 \text { tons CO2. Flush w/56 bbis } 50 / 50 \\
\mathrm{CO} 2 "\end{array}$ & \\
\hline & 1316 & $\overrightarrow{F U}$ & $2 / 12 / 2002$ & 17.58 & 21.72 & $3 \overline{3} .0$ & & & & $\bar{X}$ & & 11,547 & & & "Frac: Break w/6\% KCl..Frac using $70 \mathrm{Q}$ CO2 foam, $157 \mathrm{bbls}$ gel, 37 tons CO2 64 bbl flush $50 / 50$ & \\
\hline & 1375 & FU'- & $2 / 9 / 2002$ & 19.62 & 19.31 & 35.0 & & 7.61 & & $\mathrm{X}$ & & 15,569 & & & "Frac: Break w/ $6 \%$ KCl. Frac w/ 438 bbls $70 \mathrm{Q}$ foam, 145 bbls gel, 50 tons CO 2 flush $60 \%$ CO 2. & \\
\hline & $1404^{-1}$ & $\overline{F U}^{-1}$ & $2 / 12 / 2005$ & 24.13 & & & & 7.03 & & $\mathrm{X}$ & & $20,118^{-}$ & & & "Frac...pumped 356 bbl clean fluid....and 33 tons $\mathrm{CO} 2 "$ & \\
\hline & 1442 & $\mathrm{FU}^{-1}$ & $2 / 9 / 2002^{-1}$ & 22.85 & $21.37^{-}$ & 35.0 & & & & $\mathrm{X}$ & & 9,515 & & & "Frac: Break w/6\% KCl...Frac w/2 80 bbls $70 \mathrm{Q}$ CO2 foam, 83 bbls gel, 28 tons CO $2 "$ & \\
\hline \multirow[t]{8}{*}{$31-11$} & 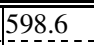 & WR & $2 / 19 / 2002$ & 10.67 & 18.96 & 35.0 & & 7.10 & & $\mathrm{X}$ & & 12,955 & & 207 & "Frac-BD w/6\% KCl...Frac using 75Q foam, 63 bbls gel, 25 tons CO2...27 bbls 30Q f & No \\
\hline & 678.2 & WR- & $2 / 20 / 2002$ & $7.79^{-1}$ & 12.07 & 25.0 & & 6.14 & & $\mathrm{X}$ & & 6,781 & & & "Frac-BD w/6\% KCl..Frac using 140 bbls $75 \mathrm{Q}$ foam, 41.5 bbls gel, 17 ton CO 2 flush 3 & \\
\hline & $730^{--}$ & W'TR'- & $2 / 19 / 2002^{-}$ & $21.92^{-}$ & $1 \overline{3} . \overline{79}$ & $2 \overline{5} . \overline{0}$ & & $7 . \overline{5} 8^{-}$ & & $\mathrm{X}$ & & 6,715 & & & "Frac-BD w/6\% KCl...Frac using 142 bbls $75 \mathrm{Q}$ CO 2 foam, 41 bbls gel, 16 tons CO2 .. 36 bbls $6 \%$ KCl" & \\
\hline & $77 \overline{4} .5$ & Wh & $2 / 19 / 2002$ & 13.65 & 17.93 & 30.0 & & 8.20 & & $\mathrm{X}$ & & 9,167 & & & Frac-BD w/6\% KCl...Frac us & \\
\hline & 808.9 & WRR-1. & $2 / 16 / 2002$ & 7.01 & 19.31 & 30.0 & & 8.52 & & $\mathrm{X}$ & & 10,883 & & & "Frac-BD w/6\% KCl ...Frac using 750 foam, 63 bbls gel, 24 ton CO $2 \ldots$ flush w/40 & \\
\hline & 1034 & FU'-- & $2 / 16 / 2002$ & $16.75^{-1}$ & 25.51 & 35.0 & & & & $\mathrm{X}^{-}$ & & 12,244 & & & "Frac-BD w/6\% KCl ..Frac uisng $75 \mathrm{Q} \mathrm{CO} 2,123 \mathrm{bbls}$ gel, 41 tons $\mathrm{CO} 2$ & \\
\hline & 1109 & $\overline{\mathrm{FU}}$ & $2 / 16 / 2002$ & 20.93 & 18.62 & $3 \overline{5} .0$ & & 4.96 & & $\mathrm{X}^{-}$ & & $11,494^{-}$ & & & $\begin{array}{l}\text { "Frac-BD w/6\% KCl...Frac using } 380 \text { bbls } 70 \mathrm{Q} \text { CO } 2 \text { foam, } 112 \text { bbls gel, } 45 \text { ton CO } 2 . .54 \text { bbils } 6 \% \text { KCl } \\
\text { flush" }\end{array}$ & \\
\hline & 1180 & $\overline{F U}$ & $2 / 14 / 2002$ & $27.30^{-}$ & 16.20 & 35.0 & & $4.34^{-}$ & & $\mathrm{X}^{-}$ & & 12,280 & & & $\begin{array}{l}\text { "Frac-BD w/6\% KCl...Frac uisng } 346 \text { bbls } 70 Q \mathrm{CO} 2 \text { foam, } 104 \text { bbls gel, } 39 \text { ton CO2 ..flush w/4 } 6 \text { bbis } \\
50 / 50+10 \text { bbls } 6 \% \mathrm{KCl} . .111 \text { of } 190 \text { recovered" }\end{array}$ & \\
\hline \multirow{5}{*}{ 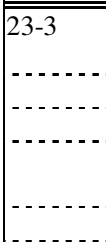 } & $\overline{510.2}$ & WR & $2 / 21 / 2002$ & 16.55 & 12.07 & 25.0 & & 7.93 & & $\mathrm{X}$ & & 8,741 & 2 & 202 & $\begin{array}{l}\text { "Frac-BD w/6\% KCl...Frac using } 176 \text { bbls 75Q CO2, } 44 \text { bbls gel, } 20 \text { ton CO2...23 bbls 6\% KCl + CO2 } \\
\text { flush" }\end{array}$ & No \\
\hline & 641.9 & WR & $2 / 21 / 2002$ & & 15.17 & 25.0 & & 8.89 & & $\mathrm{X}^{-}$ & & $8,482^{-}$ & & & "Frac-BD w/6\% KCl..Frac using 176 bbls $75 \mathrm{Q}$ CO2, 52 bbls gel, 20 ton CO2 230 bbls $6 \%$ flush" & \\
\hline & $7 \overline{7} \overline{5} . \overline{4}$ & WR'- & $2 / 21 / 2002^{--}$ & $11.65^{-}$ & 19.31 & 32.0 & & $7 . \overline{5} 8^{-}$ & & $\mathrm{X}^{-}$ & & $11,113^{-}$ & & & "Frac-BD w $6 \%$ KCl..Frac using $75 \mathrm{Q}$ CO2, 109 bbls gel, 26 ton $\mathrm{CO} 2 \ldots 36.5$ bbls $6 \%$ KCl flush" & \\
\hline & $823^{-}$ & $\mathrm{WR}$ & $2 / 20 / 2002$ & 22.41 & 15.17 & 27.0 & & 9.89 & & $\mathrm{X}^{-}$ & & 9,843 & & & $\begin{array}{l}\text { "Frac-BD w/6\% KCl..Frac using } 75 \mathrm{Q} \text { CO } 2,64 \text { bbls gel, } 19 \text { ton CO } 2 . .39 \text { bbls } 50 / 50 \text { flush...made recover } \\
122 \text { bbls of } 629 "\end{array}$ & \\
\hline & 844.9 & Wi- & $2 / 20 / 2002$ & 12.03 & 15.86 & {$\left[\begin{array}{lll}27 & -1 \\
-2 & 0\end{array}\right]$} & & 10.17 & & $\bar{x}$ & & 10,985 & & & "Frac-BD w/6\% KCl...Frac using $75 \mathrm{Q}$ CO2, 65.5 bbls gel, 27 ton CO2...42.5 bbls $6 \%$ KCl flush" & \\
\hline
\end{tabular}




\begin{tabular}{|c|c|c|c|c|c|c|c|c|c|c|c|c|c|c|c|c|}
\hline 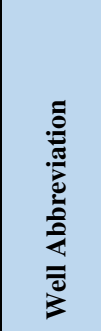 & 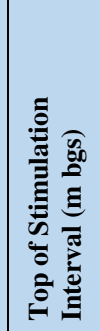 & 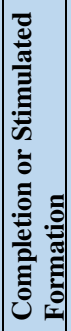 & 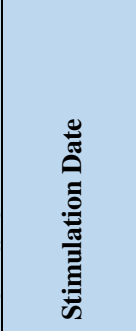 & 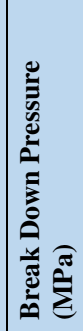 & 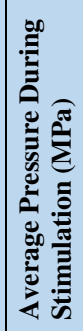 & 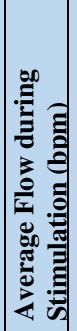 & 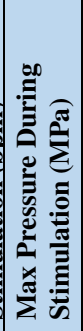 & 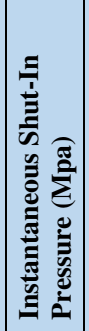 & 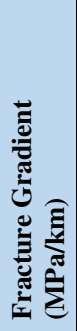 & 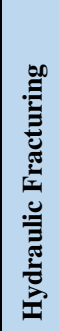 & 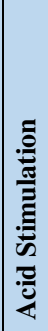 & 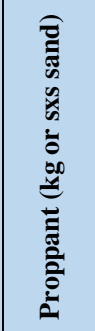 & 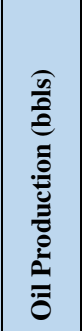 & 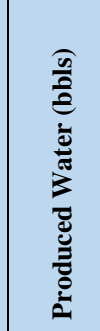 & 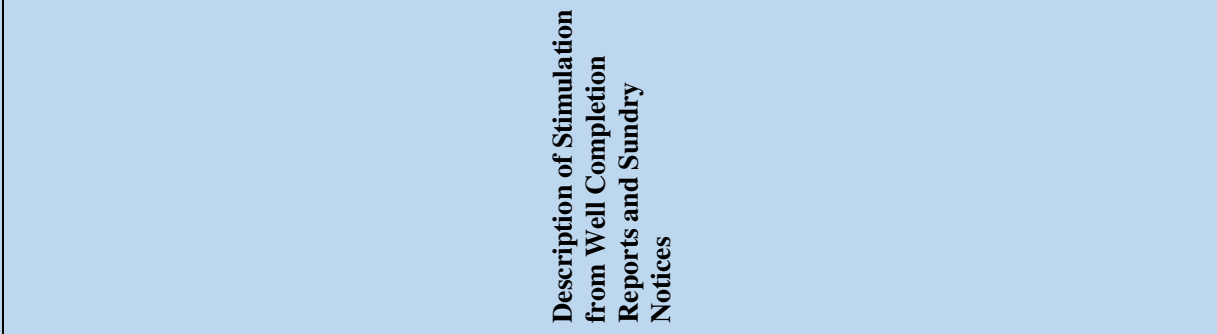 & 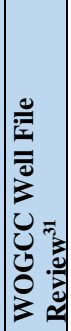 \\
\hline & 1055 & FU & $2 / 20 / 2002$ & 20.77 & 18.27 & 30.0 & & 9.17 & & $\mathrm{X}$ & & 6,668 & & & "Frac-BD w/6\% KCl...Frac using 206 bbls $70 \mathrm{Q} \mathrm{CO} 2,65$ bbls gel, 24 ton $\mathrm{CO} 2 \ldots .52$ bbls $6 \% \mathrm{KCl}$ flush" & \\
\hline & $1155^{-1}$ & $\mathrm{FU}^{-1}$ & $2 / 20 / 2002$ & 8.27 & 18.96 & 35.0 & & 4.07 & & $\mathrm{X}^{-}$ & & $14,878^{-}$ & & & Frac-BD w/ $6 \%$ KCl...Frac using 492 bbls $70 \mathrm{COO} 2,184$ bbls gel, 50 ton CO $2 \ldots 56$ bbls $6 \%$ KCl flush" & \\
\hline & $1372^{-1}$ & $\overline{F U}^{-}$ & $2 / 19 / 2002$ & 15.38 & 16.89 & 35.0 & & 5.38 & & $\mathrm{X}^{-}$ & & 14,945 & & & "BD w/6\% KCl...Frac using $70 \mathrm{Q}$ CO2, 144 bbls gel, 53 ton CO2 ...flush w/67 bbls $50 \%$ CO2" & \\
\hline \multirow[t]{9}{*}{$32-11$} & 532.8 & WR & $2 / 26 / 2002$ & 12.55 & 13.79 & 25.0 & & 7.83 & & $\bar{X}$ & & 8,056 & 2 & 1,027 & $\begin{array}{l}\text { "Frac-BD w/6\% KCl...Frac using } 161 \text { bbls 75Q CO2 foam, } 45 \text { bbls gel, } 18 \text { ton CO [CO2]...24 bbls KCl } \\
\text { flush" }\end{array}$ & No \\
\hline & 603.2 & $\mathrm{~W} \mathrm{WR}^{-1}$ & $2 / 26 / 2002$ & $17.45^{-}$ & 15.86 & 30.0 & & 5.52 & & $\mathrm{X}^{-}$ & & $9,764^{-}$ & & & "Frac-BD w/ $6 \%$ KCl ..Frac using 201 bbls $75 \mathrm{Q} \mathrm{CO} 2,56$ bbls gel, 23 ton $\mathrm{CO} 2 \ldots 28.5$ bbls $6 \%$ flush" & \\
\hline & 721.2 & $\mathrm{WR}$ & $2 / 26 / 2002$ & 19.31 & 13.79 & $30^{-}$ & & $6 . \overline{7} \overline{9}^{-}$ & & $\mathrm{X}^{-}$ & & 9,906 & & & "Frac-BD w $/ 6 \%$ KCl...Frac using 206 bbls $75 \mathrm{Q}$ CO2, 60 bbls gel, 23 tons CO2..34 bbls $8 \%$ flush" & \\
\hline & $7 \overline{7} 7.1$. & WR- & $2 / 26 / 2002$ & $13.15^{-}$ & 20.68 & 35 & & 6.21 & & $\mathrm{X}$ & & 12,079 & & & "Frac-BD w/6\% KCl...Frac uisng 273 bbls $70 \mathrm{Q}$ CO22, 110 bbls gel, 28 ton CO2 37.5 bbls $6 \%$ flush" & \\
\hline & 963.8 & $\begin{array}{ll}\mathrm{B} W \overline{\mathrm{W}} \\
\mathrm{R}\end{array}$ & $2 / 23 / 2002$ & $15.44^{-}$ & 12.07 & $35^{-1}$ & & 3.35 & & $\mathrm{X}^{-}$ & & 13,816 & & & "Frac-BD w/ $6 \%$ KCl...Frac using 296 bbis $75 \mathrm{Q}$ CO 2,87 gel, 35 ton CO $2 \ldots 46$ bbls $50 \%$ CO 2 flush" & \\
\hline & $1035^{-1}$ & $F \bar{F}^{-}$ & $2 / 22 / 2002$ & $30.67^{-}$ & $26.20^{-}$ & 28 & & & & $\overline{\mathrm{X}}$ & & 6,218 & & & $\begin{array}{l}\text { "Frac-BD w/6\% KCl...Frac using } 207 \text { bbls } 70 \mathrm{Q} \text { CO2} 2,72 \text { bbls gel, } 22 \text { tons } \mathrm{CO} 2 \ldots \text { screened out w/ } 6570 \# \text { in } \\
\text { formation, } 7136 \text { in pipe ...recover } 122 \text { of } 609 "\end{array}$ & \\
\hline & $1102^{-}$ & $\mathrm{FU}$ & $2 / 22 / 2002$ & 20.34 & 18.96 & 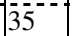 & & 5.52 & & $\mathrm{X}$ & & 12,428 & & & "Frac-BD w $8 \%$ KCl ..Frac using 382 bbls $70 \mathrm{Q}$ CO2, 121 bbls gel, 43 ton $\mathrm{CO} 2 \ldots 54$ bbls $6 \%$ flush" - & \\
\hline & 1133 & FU'-1 & $2 / 22 / 2002$ & $26.20^{-}$ & 17.24 & 33 & & 4.69 & & $\mathrm{X}$ & & 17,565 & & & "Frac-BD w/6\% KCl...Frac using $70 \mathrm{Q}$ foam, 164 bbls gel, 59 ton CO2 257 bbls $6 \%$ flush" & \\
\hline & 1161 & $\overline{F U}$ & $2 / 21 / 2002$ & $24.82^{-}$ & $17.93^{-}$ & 40.0 & & 2.34 & & $\mathrm{X}^{-}$ & & & & & $\begin{array}{l}\text { "Frac-BD w/6\% KCl...Frac using } 50 \text { bbis } 75 \mathrm{Q} \text { foam, flush w/50Q foam...Total fluid } 53 \mathrm{bbls}, 12 \text { ton } \mathrm{CO} 2 \text {, } \\
\text { flush } 60 \text { bls } 50 \mathrm{Q} \text { foam" }\end{array}$ & \\
\hline \multirow[t]{6}{*}{$\overline{444-11}$} & 536.4 & WR & $2 / 28 / 2002$ & 19.55 & 13.79 & 25.0 & & 6.62 & & $\mathrm{X}$ & & 7,248 & 2 & 11,643 & $\begin{array}{l}\text { "Frac-BD w/6\% KCl...Frac using } 150 \text { bbls 75Q CO2 foam, } 47 \text { bbls gel, } 16 \text { tons } \mathrm{CO} 2 \ldots \text { Flush w/24.5 bbls } \\
50 \% \text { CO2" }\end{array}$ & No \\
\hline & 642.8 & $\mathrm{~W} \mathrm{R}^{-1}$ & $2 / 28 / 2002$ & 14.60 & 14.48 & 25.0 & & & & $\bar{X}$ & & $6,940^{-}$ & & & "Frac-BD w/6\% KCl ..Frac using $147 \mathrm{bbls} 75 \mathrm{Q}$ CO2 foam, 44 bbls gel, 17 ton $\mathrm{CO} 2 \ldots 30$ bbls $6 \%$ flush" & \\
\hline & 872.9 & WR-1 & $2 / 28 / 2002$ & 20.11 & 15.86 & 35.0 & & 11.17 & & $\mathrm{X}_{-}^{-}$ & & 6,917 & & & "Frac-BD w/6\% KCl...Frac using 152 bbis $75 \mathrm{Q}$ CO2 faom [foam], $47 \mathrm{bbls}$ gel, 17 ton CO2" & \\
\hline & 996.7 & $\mathrm{BW}$ & $2 / 28 / 2002$ & 19.17 & 19.17 & 35.0 & & 1.52 & & $\mathrm{X}$ & & 13,653 & & & "Frac-BD w/6\% KCl...Frac using 303 bbls $75 \mathrm{Q}$ CO2 foam, 83 bbls gel, 35 ton CO2" & \\
\hline & $1 \overline{1} 109^{-1}$ & $\overline{F U}$ & $2 / 28 / 2002$ & $29.65^{-}$ & $13.10^{-1}$ & 30.0 & & $5.17^{-}$ & & $\mathrm{X}^{-}$ & & $8, \overline{2} \overline{5} \overline{5}^{-}$ & & & 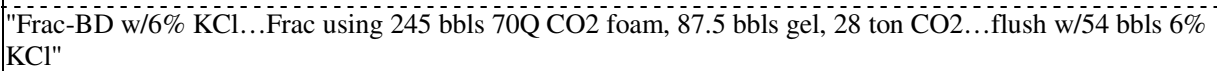 & \\
\hline & $1172^{-}$ & $\bar{F}$ & $2 / 26 / 2002$ & 13.13 & 20.68 & $34^{--}$ & & 6.96 & & $\mathrm{X}$ & & 21,469 & & & $\begin{array}{l}\text { "Frac-Breakdown w/ } 6 \% \text { KCl...Frac using } 620 \text { bbls } 70 \mathrm{Q} \text { CO2 foam, } 182 \text { bbls gel, } 69 \text { tons CO } 2 \text {...flush w/57 } \\
\text { bbls } 50 \% \text { CO2" }\end{array}$ & \\
\hline $42-9 \mathrm{~W}$ & & & & & & & & & & & & & $\mid \overline{5,051}$ & 25,303 & Production in Wind River Formation. 8 perforated intervals from 1776-3138.' No information on stimulation & No \\
\hline \multirow[t]{4}{*}{ 32-9W } & 779.7 & WR & $3 / 19 / 2002$ & 17.42 & 18.27 & 32.0 & & 6.83 & & $\mathrm{X}$ & & 13,420 & 2. & 1,380 & "Frac-Break...Pump pad...28+2 tons CO2, 132.6 load". & Yes \\
\hline & 832.1 & WR-1 & $3 / 19 / 2002$ & 17.24 & 13.10 & 25.0 & & 8.76 & & $\mathrm{X}_{-}^{-}$ & & $7,487^{-1}$ & & & "Frac-Break formation...Pump pad...17+1 CO2, 105 bbls load" & \\
\hline & 894.9 & WR & $3 / 19 / 2002$ & 22.23 & 18.62 & 30.0 & & 10.34 & & $\mathrm{X}$ & & 9,784 & & & "Frac-Break formation...Pump pad...128 bbls load, $22+1$ tons $\mathrm{CO}^{2}$ & \\
\hline & $1001^{-}$ & $\mathrm{WR}$ & $3 / 19 / 2002$ & 23.92 & 26.89 & 28.0 & & 10.55 & & $\mathrm{X}^{-}$ & & $9,502^{-}$ & & & "Frac-Break formation...Pump pad...103.6 bbls clean fluid, $22+2$ tons $\mathrm{CO}^{2}$ & \\
\hline \multirow[t]{7}{*}{$32-10 \mathrm{~B}$} & 1378 & FU & $3 / 22 / 2002$ & 10.62 & 19.31 & 35.0 & & & & $\underline{X}$ & & 27,373 & 14 & 4,727 & "Frac-Break...CO2 hose ruptured during pad/replace. Extend pad 53 bbls...97 tons CO2...296.4 bbls" & Yes \\
\hline & $14 \overline{4} 2^{-}$ & $\mathrm{FU}$ & $3 / 33 / 2002$ & 12.41 & 19.31 & 35.0 & & 3.45 & & $\mathrm{X}$ & & 19,395 & & & "Frac-fill hole 57 bbls, break ...Frac using 62 tons $\mathrm{CO} 2 \ldots 275$ bbls" & \\
\hline & 1476 & FE'- & $3 / 21 / 2002$ & 18.13 & 26.54 & 34.0 & & 3.79 & & $\underline{X}$ & & 18,277 & & & "Frac-Break formation...Frac using .. 67 tons CO2, 198 bbls load" & \\
\hline & 1569 & FE'- & $3 / 21 / 2002$ & 13.79 & 26.89 & 28.0 & & 10.14 & & $\mathrm{X}_{-}^{-}$ & & 18,286 & & & "Frac-Break ...Frac using ...63 tons cô2, 314 bbils load" & \\
\hline & $1614^{-1}$ & $\overline{\mathrm{FU}}^{-}$ & $3 / 20 / 2002$ & $17.25^{-}$ & 22.06 & 35.0 & & $6.69^{-1}$ & & $\mathrm{X}^{-}$ & & $14,080^{-}$ & & & "Frac-Break ...Frac using ...50+2 ton $\mathrm{CO} 2,318$ bbls kcl" & \\
\hline & 1663 & Fí- & $3 / 20 / 2002$ & 18.96 & 25.51 & 32.0 & & 3.52 & & $\mathrm{X}^{-}$ & & 17,780 & & & "Frac-Break...Frac-pump pad... $56+2$ ton $\mathrm{CO} 2,234 \mathrm{kcl}$ & \\
\hline & 1716 & $\overline{F U}^{-1}$ & $3 / 20 / 2002$ & $17.93^{-}$ & 24.13 & 30.0 & & 14.65 & & $\mathrm{X}^{-}$ & & $7,167^{-}$ & & & "Frac-Break formation...Frac using ...204 bbls $6 \% \mathrm{kcl}, 23+2$ tons $\mathrm{CO} 2 "$ & \\
\hline \multirow[t]{3}{*}{ 13-11B } & 1102 & FU & $4 / 6 / 2002$ & & 19.65 & 35.0 & & & & $\mathrm{X}$ & & 14,787 & 0 & 7,087 & $\begin{array}{l}\text { "Frac-Breakdown w/6\% KCl...Frac using 70Q CO2 foam, } 125 \text { bbls gel, } 39 \text { tons } \mathrm{CO} 2 . .53 \text { bbls } 50 \% \text { CO2 } \\
\text { flush" }\end{array}$ & No \\
\hline & $11 \overline{1} 5^{-}$ & FU'-. & $4 / 6 / 2002$ & $111.72-$ & 24.13 & 42.0 & & 5.07 & & $\mathbb{X}_{-}^{-}$ & & 30,552 & & & Frac-Break w/6\% kcl .Frac using $70 Q \mathrm{CO} 2,896 \mathrm{bls}, 283$ bbls gel, 97 ton CO2 57 bbls $30 \%$ CO2 flush" & \\
\hline & 1385 & FE'- & $3 / 23 / 2002$ & 10.58 & & & & 6.83 & & & & 19,758 & & & "Frac-Break 67 tons CO2+209 bbis load"67 tons CO22, 209 bbls "load". & \\
\hline
\end{tabular}




\begin{tabular}{|c|c|c|c|c|c|c|c|c|c|c|c|c|c|c|c|c|}
\hline 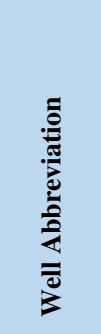 & 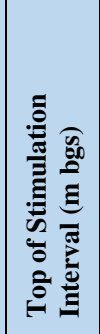 & 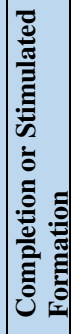 & 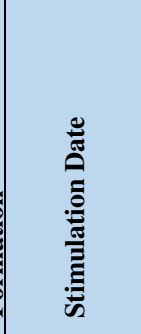 & 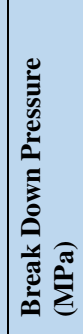 & 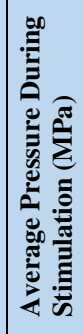 & 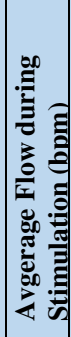 & 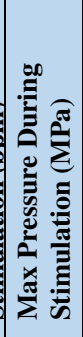 & 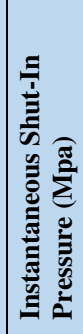 & 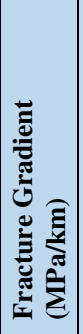 & 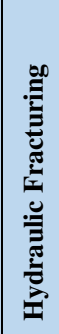 & 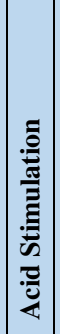 & 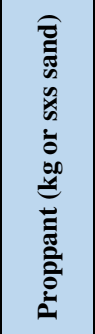 & 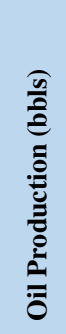 & 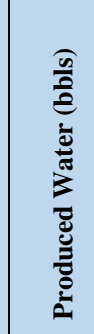 & 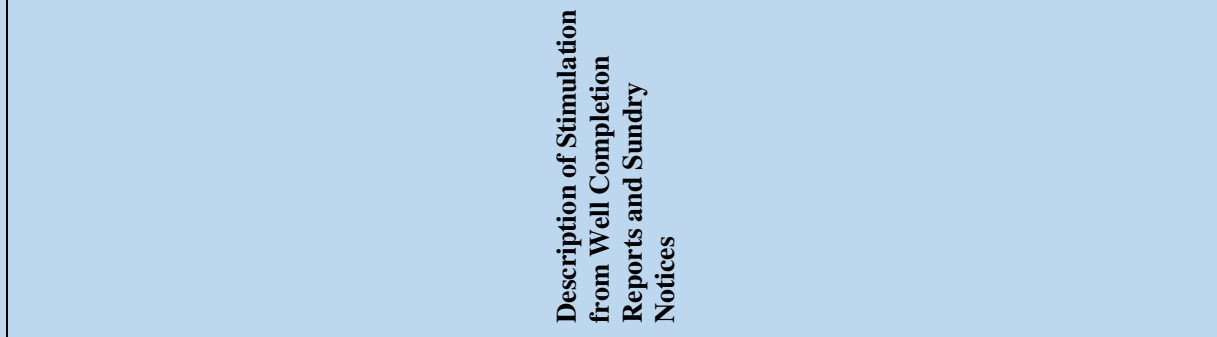 & 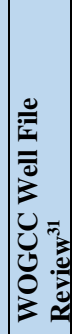 \\
\hline & 1452 & FU. & $3 / 23 / 2002$ & & 20.68 & 35.0 & & 8.27 & & $\underline{X}$ & & 10,851 & & & "Frac-Break formation... 167 bbls, 34 tons CO2"167 bbls of ? 34 tons CO2 & \\
\hline & $1572^{-}$ & $\overline{F U}$ & $3 / 23 / 2002$ & & 16.55 & 35.0 & & 10.48 & & $\mathrm{X}^{-}$ & & 9,568 & & & "Frac-Break formation... 35 ton $\mathrm{CO} 2 \& 175$ bbls fluid" 35 tons $\mathrm{CO} 2,175$ bbls "fluid" & \\
\hline \multirow[t]{5}{*}{$14-3 \mathrm{~W}$} & 1359 & FU & $4 / 9 / 2002$ & 8.19 & 22.75 & 37.0 & & & & $\mathrm{X}$ & & 23,224 & 3 & 5,695 & $\begin{array}{l}\text { "Frac-Break down w/6\% KCl...Frac using 70Q CO2. } 650 \text { bbls, } 183 \text { bbls gel, } 70 \text { tons } \mathrm{CO} 2 \ldots 86 \text { bbls } 50 \% \\
\text { CO2 flush" }\end{array}$ & Yes \\
\hline & $1408^{-}$ & $\overline{\mathrm{FU}} \overline{\mathrm{C}}^{-1}$ & $4 / 9 / 2002^{--}$ & 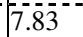 & 22.75 & 36.0 & & $4.48^{--}-$ & & $\mathrm{X}$ & & $22,290^{-}$ & & & "Breakdown w $6 \%$ KCl...Frac using $70 \mathrm{Q}$ CO2 foam $630 \mathrm{bbls}, 174$ bbls gel, 71 ton CO2...72 bbls $6 \%$ flush" & \\
\hline & 1538 & FU'- & $4 / 9 / 2002$ & 14.20 & 22.75 & 33.0 & & 7.58 & & $\mathrm{X}^{-}$ & & 11,857 & & & "Break down w/6\% KCl...Frac using $70 \mathrm{Q} \mathrm{CO} 2$ faom [foam], $338 \mathrm{bbls}, 39$ ton $\mathrm{CO} 2 \ldots 76 \mathrm{bbls} 6 \%$ flush & \\
\hline & 1578 & $\mathrm{FU}$ & $4 / 6 / 2002$ & 15.86 & 26.20 & 27.0 & & & & $\mathrm{X}$ & & 7,145 & & & $\begin{array}{l}\text { Break down w/6\% KCl...Frac using } 70 \mathrm{Q} \text { CO2 } 209 \text { bbls, } 22 \text { ton CO2 ...screened out w/12411\# in formation, } \\
3342 \# \text { in pipe" }\end{array}$ & \\
\hline & 1608 & $\overline{\mathrm{FU}}$ & $4 / 5 / 2002$ & 17.13 & 27.58 & 27.0 & & & & $\mathrm{X}$ & & 7,108 & & & "Break down w/6\% KCl..Frac using 206 bbls gel, 20 ton CO2" & \\
\hline \multirow[t]{4}{*}{$12-2$} & 683.4 & WR & $11 / 29 / 2001$ & 15.51 & & & & 9.79 & & $\mathrm{X}_{-}$ & & 8,763 & 0 & 0 & "BD w/6\% KCl...171 bbls 70Q CO2...54 bbls 6\% KCL, 22 ton CO2, 32 bbls 50Q foam" & No \\
\hline & $8 \overline{5} 1-$ & W- & $11 / 29 / 2001$ & 21.51 & 15.17 & 25.0. & & 9.24 & & X-. & & 7,385 & & & "Frac BD w/6\% KCl . start 70Q CO2 foam 177 bbls..41.5 bbls KCi flush" & \\
\hline & 941.2 & $\mathrm{WR}$ & $11 / 29 / 2001$ & 28.96 & 14.82 & & & $9.97^{-}$ & & $\bar{X}$ & & 8,432 & & & $\begin{array}{l}\text { "Frac-BD w/6\% KCl...Start } 70 \mathrm{Q} \text { CO2 foam. } 174 \text { bbls... } 81 \text { bbls } 6 \% \text { KCl, } 22 \text { ton CO2. Flush w/45 bbis } 50 \mathrm{Q} \\
\text { CO2" }\end{array}$ & \\
\hline & 1066 & $\mathrm{FU}$ & $11 / 27 / 2001$ & 27.58 & & & & & & $\mathrm{X}$ & & 7,879 & & & $\begin{array}{l}\text { "Break w/6\% KCl...Start 70Q CO2 foam, } 164 \text { bbls slurry pumped...17370\# 12/20 sand screened out } \\
\text { w/11278\# in pipe, } 6083 \# \text { in formation. Total } 51 \text { bbls no flush pumped" }\end{array}$ & \\
\hline \multirow[t]{6}{*}{ 23-10W } & 651.1 & WR & $12 / 14 / 2002$ & 21.50 & 24.13 & 32.0 & & 10.98 & & X. & & 15,987 & & 5,892 & "Frac-Pumped 114 bbls KCl, 219 bbls CO2" & No \\
\hline & 793.1 & $\mathrm{~W} \mathrm{R}$ & $12 / 14 / 2002$ & 26.24 & 17.93 & 35.0 & & 10.62 & & $\mathrm{X}$ & & 17,251 & & & "Frac-Pumped 136 bbls KCl, 220 bbls CO2" & \\
\hline & 881.8 & $\mathrm{~W}$ & $12 / 14 / 2002$ & 23.12 & 16.89 & 25.0 & & & & $\mathrm{X}_{-}^{-}$ & & $6,130^{\circ}$ & & & "Frac-Pumped 111 bbls KCCl, 138 bbis có2" & \\
\hline & 1138 & $\overline{\mathrm{FU}}$ & $12 / 13 / 2002$ & 12.94 & 16.55 & 30.0 & & 7.27 & & $\mathrm{X}$ & & $7,644^{-}$ & & & "Frac-Pumped 105 bbls KCl, 178 bbls CO2" & \\
\hline & 1161 & FU & $12 / 13 / 2002$ & 7.64 & 20.68 & 35.0 & & 4.14 & & $\mathrm{X}$ & & {$[23,873$} & & & "Frac-Pumped 258 bbls KCL, 442 bls CO2" & \\
\hline & $1310^{-}$ & $\overline{\mathrm{F}}$ & $12 / 12 / 2002$ & 16.33 & 22.41 & 35.0 & & $9.65^{\circ}$ & & $\mathrm{X}$ & & 14,436 & & & "Frac-Pumped 192 bbls KCl, 260 bbls CO2" & \\
\hline \multirow[t]{15}{*}{$23-10 \mathrm{C}$} & 539.8 & WR & $10 / 13 / 2004$ & & & & & & & $X_{-2}$ & & $7,741$. & 6. & 4,554 & "Flush w/22 bbls. Screen out w/2 bbls let on flush" & Yes \\
\hline & 557.2 & $\mathrm{WR}$ & $10 / 13 / 2004$ & 7.36 & & & & 7.62 & & $\mathrm{X}$ & & 6,705 & & & "Flush w/28 bbls water" & \\
\hline & $701^{-}$ & $\mathrm{WR}$ & $10 / 13 / 2004$ & 13.10 & & & & $7.79^{-}$ & & $\mathrm{X}$ & & 10,550 & & & "Flush w/34 bbls water" & \\
\hline & 730.9 & WR & $10 / 13 / 2004$ & 8.57 & & & & 7.43 & & $\mathrm{X}$ & & 7,928 & & & "Flush w/36 bbls" & \\
\hline & 812 & $\mathrm{~W} R$ & $10 / 13 / 2004$ & 14.94 & & & & & & X- & & 6,895 & & & "Start flush 20 bbls". & \\
\hline & 864.1 & WR & $10 / 13 / 2004$ & 11.38 & & & & 7.45 & & $\underline{X}$ & & 8,342 & & & "Flush w/43.5bbbls water" & \\
\hline & 927.5 & WR & $10 / 13 / 2004$ & 18.34 & & & & 7.98 & & X-1. & & 6,581 & & & "Flush to top perf w/ 46 bbls water" & \\
\hline & 949.8 & WR & $10 / 13 / 2004$ & 9.17 & & & & 7.58 & & X- & & 7,032 & & & "Flush to top perf w/48 bbls water" & \\
\hline & 1077 & $\bar{F} \bar{H}_{--1}^{-1}$ & $10 / 4 / 2004$ & 10.74 & & & & & & $\mathrm{X}_{-}^{-}$ & & 0 & & & "Start frac when 26 bbils flush away" & \\
\hline & 1143 & FE'- & $10 / 4 / 2004^{-1}$ & 7.58 & 15.51 & 35.0 & & 6.21 & & X-1. & & 9,295 & & & "131 bbi clean fluid and 30 ton cô2" & \\
\hline & 1411 & FE'- & $10 / 4 / 2004$ & & 15.17 & 35.0 & & 6.48 & & X- & & 9,388 & & & 28 ton CO2" & \\
\hline & 1544 & FU & $10 / 4 / 2004$ & 10.30 & 22.06 & 35.0 & & 7.14 & & $\mathrm{X}$ & & 7,711 & & & "166 bbl "clean fluid", 24 ton CO2" & \\
\hline & 1594 & FU & $10 / 4 / 2004$ & 8.73 & 16.55 & 35.0 & & 5.81 & & X- & & 10,216 & & & "182 bbil clean fluid and 28 ton CO2 2 & \\
\hline & 1646 & $\mathrm{FU}$ & $10 / 4 / 2004$ & $8.73^{--}$ & 22.06 & 35.0 & & $6.71^{\circ}$ & & $\mathrm{X}$ & & 13,017 & & & "225 bbl "clean fluid" and 39 tons CO2" & \\
\hline & $1690^{-}$ & $\overline{\mathrm{FU}}$ & $10 / 4 / 2004$ & 11.88 & 24.13 & 35.0 & & $6.71^{\circ}$ & & $\mathrm{X}$ & & 12,987 & & & " 268 bbl "clean fluid" and 39 tons CO2" & \\
\hline \multirow[t]{5}{*}{ 34-3B. } & 951.3 & WR & $4 / 19 / 2005$ & 15.86 & & & 19.24 & 7.24 & & $\mathrm{X}$. & & 7,511 & 2. & 9,501 & "Frac 139 bbls slickwater". & No. \\
\hline & 1039 & FE'- & $11 / 9 / 2004$ & & & & & & & X- & & 13,608 & & & "Frac w/70Q CO2 foam." & \\
\hline & 1070 & FU & $11 / 9 / 2004$ & & & & & & & $\mathrm{X}$ & & 13,608 & & & "Frac w/70QQ CO2 foam" & \\
\hline & $1134^{-1}$ & $\bar{F} \bar{H}^{-}$ & $11 / 9 / 2004$ & & & & & & & $\mathrm{X}$ & & $9, \overline{525}-$ & & & "Frac w/0Q CO2 foam" & \\
\hline & 1401 & $\bar{F} \bar{U}^{-1}$ & $11 / 9 / 2004$ & & & & & & & $\mathrm{X}$ & & 13,608 & & & "Frac w70Q CO2 foam" & \\
\hline
\end{tabular}




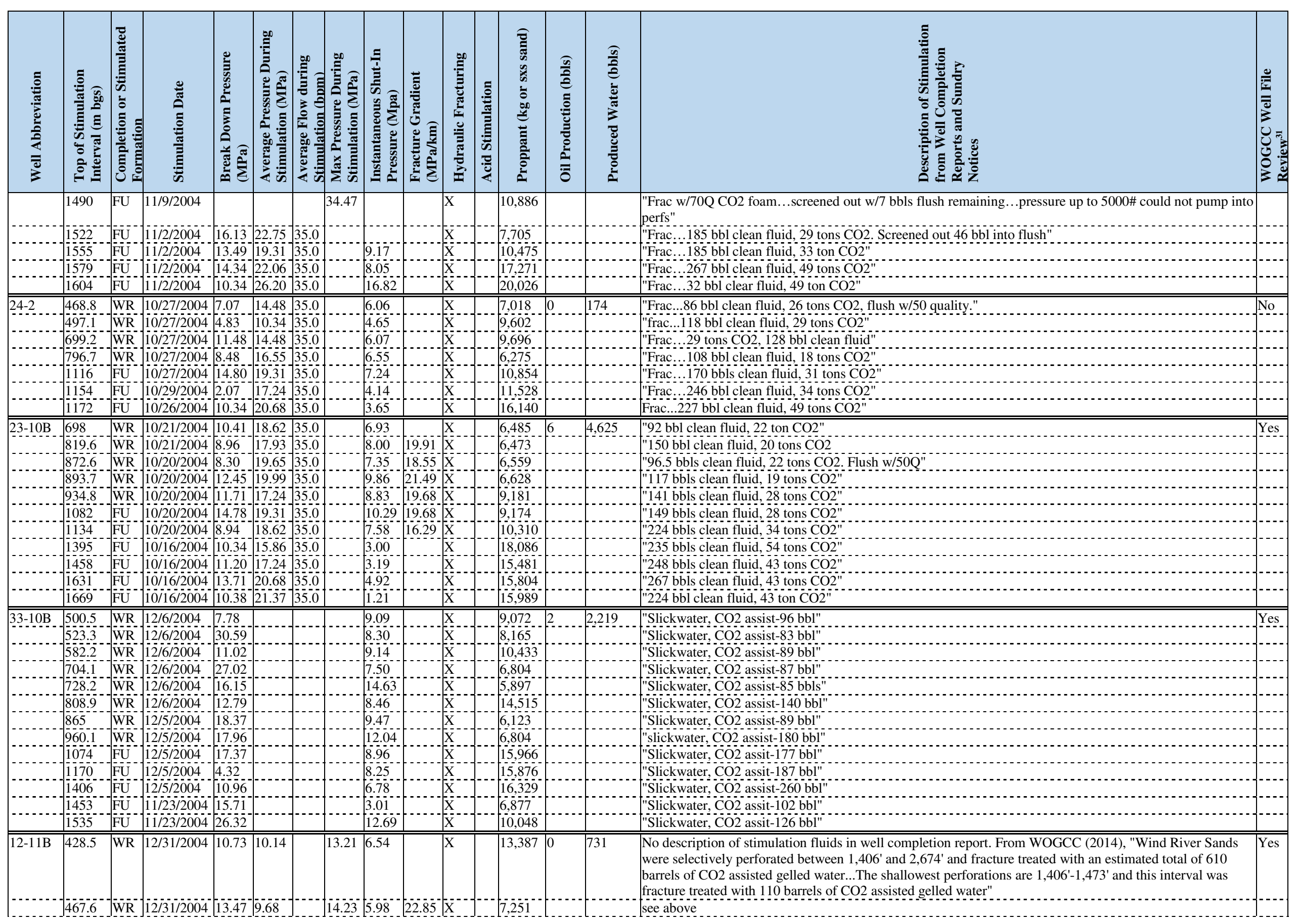




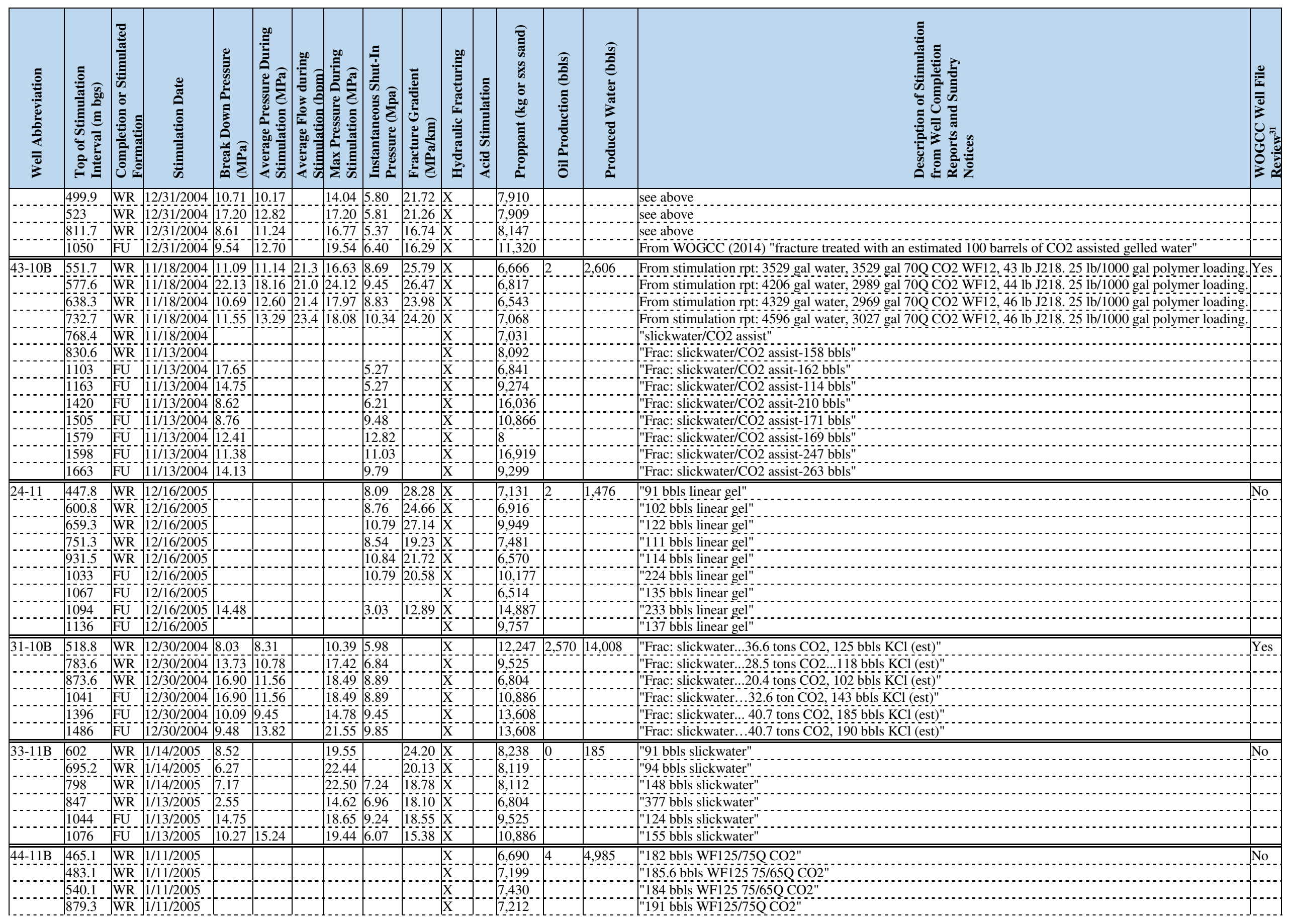




\begin{tabular}{|c|c|c|c|c|c|c|c|c|c|c|c|c|c|c|c|c|}
\hline 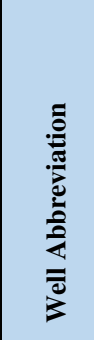 & 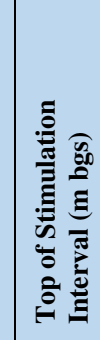 & 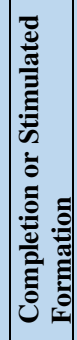 & 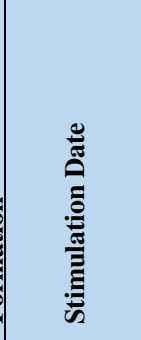 & 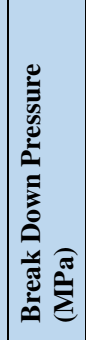 & 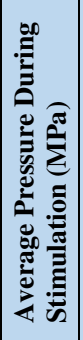 & 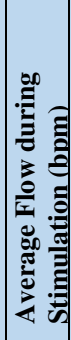 & 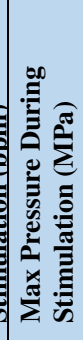 & 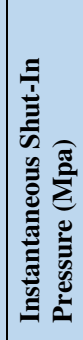 & 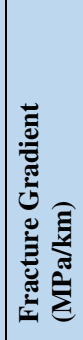 & 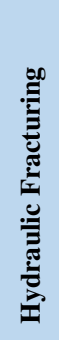 & 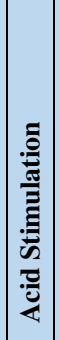 & 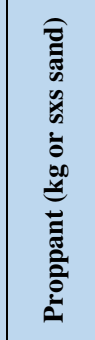 & 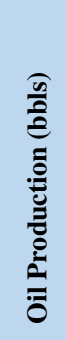 & 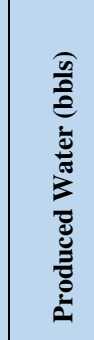 & 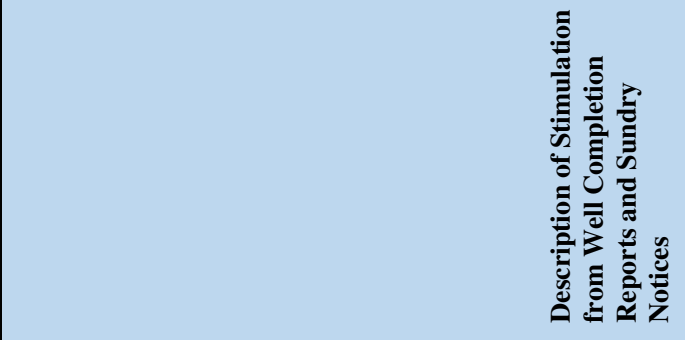 & 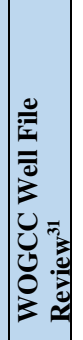 \\
\hline & 974.1 & WR & $1 / 11 / 2005$ & & & & & & & $\underline{X}$ & & 7,044 & & & "288 bbls WF125/70Q CO2". & \\
\hline & 1041 & Fü-1. & $1 / 11 / 2005$ & & & & & & & $\mathrm{X}_{-}^{-}$ & & 11,412 & & & 325 bbls WF125/70Q CO2". & \\
\hline & 1074 & $\mathrm{Fu}$ & $1 / 11 / 2005$ & & & & & & & $\mathrm{X}^{-}$ & & $8,831^{-1}$ & & & "298.5 bbls WF125/70Q CO2" & \\
\hline \multirow[t]{10}{*}{$21-14$} & 619 & TER & $12 / 17 / 2004$ & & & & & 8.61 & & $\bar{X}$ & & 6,532 & 3 & 1,886 & "Slickwater, 92 bbl....CO2 assist" & No \\
\hline & 684 & WR & $12 / 17 / 2004$ & $16.82^{-}$ & & & & & & $\underline{-}$ & & 7,121 & & & Slickwater, screen-out, 65 bbl..CO2 assist" & \\
\hline & 728.5 & WR-1 & $12 / 17 / 2004$ & 16.83 & & & & $7.41^{-1}$ & & $\mathrm{X}_{-}^{-}$ & & $6,827^{\circ}$ & & & "Slickwater, 93 bbi...CO2 assist" & \\
\hline & $768.1-$ & $\mathrm{WR}$ & $12 / 17 / 2004$ & 12.69 & & & & 8.27 & & $\frac{1}{2}-$ & & $7,072^{-1}$ & & & "Slickwater, 115 bb1..co2 assist" & \\
\hline & 1077 & FU & $12 / 16 / 2004$ & 12.13 & & & & & & $\mathrm{X}$ & & 6,808 & & & Slickwater, screen-out, 72 bbl..CO2 assist" & \\
\hline & 1401 & FE'-1. & $12 / 16 / 2004$ & 10.68 & & & & 4.85 & & X- & & 10,845 & & & Slickwater, 161 bb1 .CO2 assist" & - \\
\hline & 1419 & FU & $12 / 16 / 2004$ & 8.94 & & & & 4.43 & & $\underline{X}$ & & 10,587 & & & Slickwater, 163 bbls...cO2 assist" & \\
\hline & 1473 & FU'-1 & $12 / 16 / 2004$ & 11.86 & & & & 7.03 & & $\mathrm{X}$ & & 8,119 & & & "Slickwater, 155 bb1 ..co2 assist" & \\
\hline & 1558 & FU' & $12 / 16 / 2004$ & $11.17^{-}$ & & & & 7.33 & & $\mathrm{X}$ & & 10,791 & & & "Slickwater, 175 bbi co2 assist" & \\
\hline & $1643^{-1}$ & $\overline{F U^{-1}}$ & $12 / 16 / 2004$ & $9.51^{--}$ & & & & 8.62 & & $\mathrm{X}^{-}$ & & $11,939^{-}$ & & & "Slickwater, 190 b̄bil...CO2 2 assist" & \\
\hline \multirow{14}{*}{$\begin{array}{l}13-10 \\
\end{array}$} & 670 & WR & $12 / 7 / 2004$ & 10.56 & & & & 9.80 & & $\bar{X}$ & & 6,804 & 2 & 7,329 & "Slickwater/CO2 assist $\ldots 20$ tons CO2", & Noo \\
\hline & 761.1 & $\mathrm{WR}$ & $12 / 7 / 2004$ & 12.05 & & & & & & $\mathrm{X}$ & & 6,804 & & & "Slickwater/CO2 assist-95 bbls...20 otns CO2" & \\
\hline & 956.5 & WR-1 & $12 / 7 / 2004$ & 15.61 & & & & & & X- & & 6,804 & & & "Slickwater/CO2 assit-106 bbl...20 tons COC2" & \\
\hline & 982.1 & WR & $12 / 7 / 2004$ & 11.05 & & & & & & $\underline{X}$ & & 60.804 & & & SSlickwater/CO2 assist-108 bbls..20 tons CO2" & \\
\hline & $1003^{-1}$ & WR-1 & $12 / 7 / 2004$ & 7.33 & & & & & & $\underline{X}$ & & 8,165 & & & SSlickwater/CO2 assist-120 bbls..243 tons CO2" & \\
\hline & 1100 & FU' & $12 / 7 / 2004$ & 9.83 & & 28.0 & & & & X- & & 6,804 & & & slickwater/CO2 assist-114 bbls 20 tons" & \\
\hline & 1184 & FU' & $12 / 7 / 2004$ & 7.71 & & 29.0 & & & & X- & & 6,804 & & & Slickwater/CO2 assist-119 bbls..20 tons" & \\
\hline & 1211 & FU'-1 & $12 / 7 / 2004$ & 7.14 & & 29.0 & & & & $\mathrm{X}$ & & 8,165 & & & "Slickwater/CO2 assist-131 bbls...24 tons" & 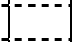 \\
\hline & 1377 & FU'- & $12 / 7 / 2004$ & 10.56 & & 31.0 & & & & $\mathrm{X}$ & & 10,886 & & & "Slickwater/CO2 assist-164 bbls...20 tons" & \\
\hline & 1407 & FU' & $12 / 7 / 2004$ & 10.19 & & 32.0 & & & & $\mathrm{X}$ & & $6,804^{-}$ & & & "Slickwater/CO2 assist-130 bbls...32 tons" & \\
\hline & $14755^{-}$ & FU'-1 & $12 / 7 / 2004$ & 11.03 & & 30.0 & & & & $\mathrm{X}$ & & 10,886 & & & "Slickwater/CO2 assist-168 bbls...32 tons" & \\
\hline & 1505 & FE'-1 & $12 / 7 / 2004$ & 11.80 & & 28.0 & & & & $\mathrm{X}$ & & $8,165^{-}$ & & & "Slickwater/COO2 assist-148 bbls...24 tons" & - \\
\hline & 1571 & FU'--1 & $12 / 7 / 2004$ & 10.97 & & 29.0 & & & & $\mathrm{X}$ & & 8,165 & & & "Slickwater/CO2 assist-151 bbls...24 tons" & \\
\hline & 1639 & $\mathrm{Fu}$ & $12 / 7 / 2004$ & 7.45 & & 29.0 & & 5.55 & & $\mathrm{X}$ & & 10,886 & & & "Slickwater/CO2 assist- 177 bbls...32.2 tons CO2" & \\
\hline \multirow[t]{3}{*}{$21-10 \mathrm{~B}$} & 1018 & FU & $1 / 24 / 2005$ & 12.27 & & & & 7.58 & & $\mathrm{X}_{-}$ & & 6,957 & 8 & 14,747 & "100 bbls clean fluid" & No \\
\hline & 1079 & FE'-1. & $1 / 24 / 2005$ & 13.97 & & & & 8.62 & & X- & & 9,458 & & & 147 bbils clean fluid". & \\
\hline & 1292 & $\mathrm{FU}^{-1}$ & $1 / 12 / 2005$ & 14.48 & & & & 8.96 & & $\mathrm{X}$ & & 9,056 & & & "168 bbl clean fluid" & \\
\hline \multirow{6}{*}{$43-11 \mathrm{~B}$} & 517.2 & WR- & $1 / 7 / 2005$ & & & & & & & X- & & 5,418 & & & 180 bbls WF125 75Q CO2" & \\
\hline & 563.6 & $\mathrm{WR}$ & $1 / 27 / 2005$ & & & & & & & $\mathrm{X}$ & & 6,319 & & & "191 bbis WF125 75Q CO2" & \\
\hline & 862 & WR- & $1 / 27 / 2005$ & & & & & & & $\mathrm{X}$ & & 7,702 & & & $" 231 \mathrm{bb} \mathrm{b}$ WF125 75Q CO2" & \\
\hline & $933^{-1}$ & WR & $1 / 27 / 2005$ & & & & & & & X- & & 8,581 & & & $278 \mathrm{bbls}$ WF125 75Q CO2" & \\
\hline & 961 & WR & $1 / 27 / 2005$ & & & & & & & $\mathrm{X}$ & & 8,383 & & & ["306 bbls WF12575Q CO2" & \\
\hline & 1098 & $\overline{F U^{-1}}$ & $1 / 27 / 2005$ & & & & & & & $\mathrm{X}$ & & 11,638 & & & $" 338 \mathrm{bb} 1 \mathrm{~s} \mathrm{WF} 12575 \mathrm{Q} \mathrm{CO} 2^{-"}$ & \\
\hline \multirow[t]{3}{*}{$42-10 \mathrm{~B}$} & 576.1 & WR & $2 / 3 / 2005$ & & & & & & & $\bar{X}$ & & 5,118 & 0 & 2,882 & "175 bbls WF $12575 \mathrm{Q} "$ & Tyes \\
\hline & $660.2-$ & WR & $2 / 3 / 2005$ & & & & & & & X & & 6,998 & & & 200 bbis WF $12575 \mathrm{Q}$ & \\
\hline & 752.2 & WR & $2 / 3 / 2005$ & & & & & & & 8 & & 9,656 & & & $" 262 \mathrm{bbls} \mathrm{WF} 12575 \mathrm{Q} "$ & \\
\hline
\end{tabular}




\begin{tabular}{|c|c|c|c|c|c|c|c|c|c|c|c|c|c|c|c|}
\hline 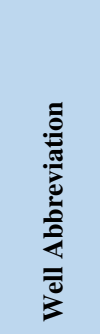 & 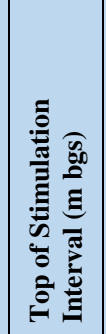 & 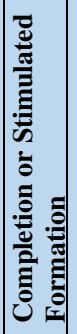 & 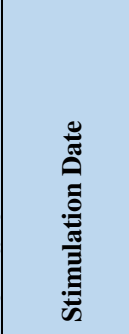 & 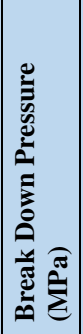 & 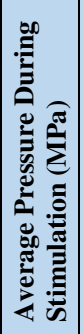 & 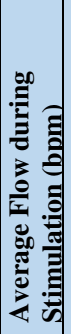 & 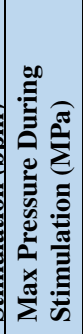 & 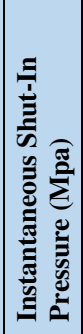 & 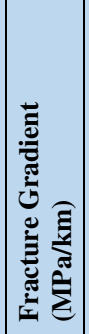 & 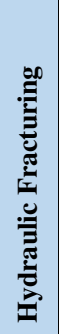 & 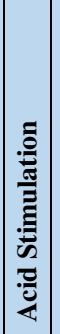 & 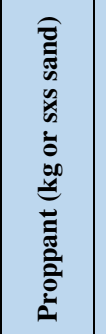 & 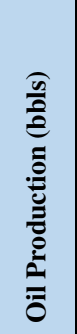 & 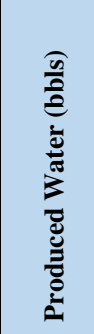 & 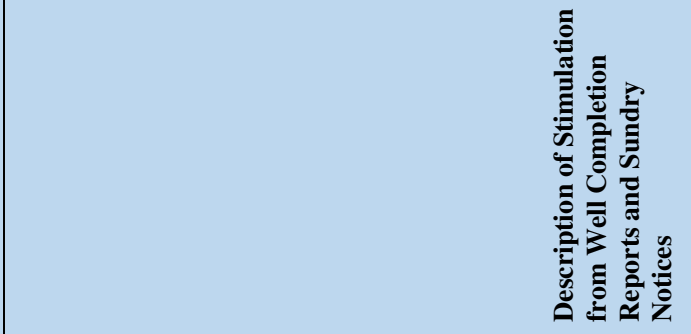 \\
\hline & 909.5 & $\mathrm{WR}$ & $2 / 3 / 2005$ & & & & & & & $\bar{x}-$ & & 6,854 & & & $" 208$ bbls WF $12575 \mathrm{Q} "$ \\
\hline & 939.4 & $\mathrm{WR}$ & $2 / 3 / 2005$ & & & & & & & $\mathrm{X}^{-}$ & & 6,804 & & & $" \mathrm{WF} 12575 \mathrm{Q} "$ \\
\hline & 1005 & $\mathrm{WR}$ & $2 / 2 / 2005$ & & & & & & & $\mathrm{X}^{-}$ & & $6,855^{-1}$ & & & $" 240 \mathrm{bbls} \mathrm{WF} 12575 \mathrm{Q}^{-}$ \\
\hline & 1062 & $\mathrm{FU}-12$ & $2 / 2 / 2005$ & & & & & & & $\bar{X}^{-}$ & & 8,165 & & & "WF $12570 Q^{\prime \prime}$ \\
\hline & 1081 & $\mathrm{FE}$ & $2 / 2 / 2005$ & & & & & & & $\mathrm{X}_{-}^{-}$ & & 9,329 & & & $" 329 \mathrm{bb}$ ls WF $12570 \mathrm{Q}$ \\
\hline & 1377 & $\mathrm{FE}$ & $2 / 2 / 2005$ & & & & & & & $X_{-}^{-}$ & & 9,935 & & & "339 bbls WF $12570 \mathrm{Q}^{\circ}$ \\
\hline & $1467^{-1}$ & $\mathrm{FU}^{-1}$ & $2 / 2 / 2005^{--}$ & & & & & & & $\mathrm{X}^{-}$ & & 14,077 & & & $" 422$ bbls WF $12570 \mathrm{Q}^{-}$ \\
\hline $22-11 \mathrm{~B}$ & .680 .9 & WR 2 & $2 / 7 / 2005$ & & & & & & & $\bar{X}$ & & $6,804.6$ & & 817 & "WF 125 70Q CO2", \\
\hline & 730.3 & $\mathrm{WR}$ & $2 / 7 / 2005$ & & & & & & & $X_{-}^{-}$ & & $8,165^{-}$ & & & $" \mathrm{WF} 12570 \mathrm{Q} C \mathrm{CO} "$ \\
\hline & 1127 & $\mathrm{FU}$ & $277 / 2005$ & & & & & & & $x^{-}$ & & 6,595 & & & "285 bbls WF $12570 \mathrm{Q} C \mathrm{CO}$ \\
\hline & 1162 & $\mathrm{FU}^{-1}$ & $2 / 4 / 2005^{-1}$ & & & & & & & $\mathrm{X}^{-}$ & & $11,393^{-}$ & & & "340 bbis WF $12570 \mathrm{Q}^{-1}$ \\
\hline $32-10 \mathrm{C}$ & 585.2 & WR 2 & $2 / 10 / 2005$ & & & & & & & $\bar{X}$ & & $13,944.8$ & & 7,099 & "347 bbls WF 125 75Q". \\
\hline & 649.2 & $\mathrm{WR}$ & $2 / 9 / 2005$ & & & & & & & $X_{-}^{-}$ & & 6,459 & & & 190.5bbls WF $12575 \mathrm{Q}$ CO2" \\
\hline & 756.5 & $\mathrm{WR}$ & $2 / 9 / 2005$ & & & & & & & $\mathrm{X}^{-}$ & & 3,239 & & & $" 163$ bbls WF $12575 \mathrm{Q} "$ \\
\hline & $790^{-1}$ & $\mathrm{WR}$ & $2 / 9 / 2005$ & & & & & & & $x_{-}^{-}$ & & 8,190 & & & "237 bbls WF $12575 \mathrm{Q} C \mathrm{C} 2 "$ \\
\hline & 867.5 & $\mathrm{WR}$ & $2 / 9 / 2005$ & & & & & & & $X_{-}^{-}$ & & 6,329 & & & "204.5 bbls WF $12575 \mathrm{Q}$ CO2" \\
\hline & 1031 & $\mathrm{WR}$ & $2 / 9 / 2005$ & & & & & & & $x^{-}$ & & $9,371^{-}$ & & & $" 342 \mathrm{bbls} \mathrm{WF} 125$ 70Q CO2" \\
\hline & 1059 & $\overline{\mathrm{WR}}$ & $2 / 9 / 2005$ & & & & & & & $\mathrm{X}^{-}$ & & 6,768 & & & $" 270.5 \mathrm{bbls}$ WF $12570 \mathrm{Q}$ CO2" \\
\hline $22-11 \mathrm{C}$ & -449.3. & WR 2 & $2 / 16 / 2005$ & & & & & & & $\underline{X}$ & & $5,979$. & & 8,725 & "179 bbls WF 125, 75Q CO2". \\
\hline & 620 & $\mathrm{WR}$ & $2 / 16 / 2005$ & & & & & & & $X^{-}$ & & 6,566 & & & $" 194 \mathrm{bbls} \mathrm{WF} 125,75 \mathrm{Q}$ CO2" \\
\hline & 759.6 & $\mathrm{WR}$ & $2 / 16 / 2005$ & & & & & & & $x^{-}$ & & 8,375 & & & $" 229$ bbls WF $125,75 \mathrm{Q}$ CO2 $"$ \\
\hline & 857.7 & $\overline{\mathrm{W}} \mathrm{R}^{-}$ & $2 / 16 / 2005$ & & & & & & & $\mathrm{X}^{-}$ & & 6,838 & & & $=204 \mathrm{~b} b \mathrm{l} \mathrm{s} \mathrm{WF} 125,75 \mathrm{Q}$ CO2 \\
\hline & $880^{-}$ & $\mathrm{WR}$ & $2 / 16 / 2005$ & & & & & & & $X_{-}^{-}$ & & 6,878 & & & "207.5 bbls WF $125,75 \mathrm{Q} C \mathrm{CO}$ \\
\hline & 926.3 & $\mathrm{WR}$ & $2 / 16 / 2005$ & & & & & & & $\mathrm{X}^{-}$ & & $6,688^{-}$ & & & $" 202.5 \mathrm{bbls} \mathrm{WF} 125,75 \mathrm{Q}$ CO2" \\
\hline & 1074 & $\mathrm{FU}^{-1}$ & $2 / 16 / 2005$ & & & & & & & $\mathrm{X}^{-}$ & & $6,709^{-}$ & & & $" 230 \mathrm{bbls} \mathrm{WF} 125,70 \mathrm{Q} \mathrm{CO} 2 "$ \\
\hline 44-4 & 1153 & FU & $3 / 8 / 2005$ & & & & & 6.09 & 15.38 & $\bar{X}$ & & $16,451 \quad 1$ & 1,265 & 26,707 & "160 bbls slickwater" \\
\hline & 1369 & $\mathrm{FE}$ & $3 / 8 / 2005$ & & & & & 6.69 & 14.93 & $X_{-}^{-}$ & & 20,428 & & & "178 bbls slickwater" \\
\hline & 1411 & $\mathrm{FU}$ & $3 / 8 / 2005$ & & & & & 8.80 & 16.29 & $X_{-}^{-}$ & & 15,947 & & & "175 bbls slickwater" \\
\hline & 11111 & $\mathrm{FU}^{-1}$ & $3 / 3 / 2005^{-}$ & $7.26^{-1}$ & & & & $6.12^{-2}$ & 15.61 & $x_{-}^{-}$ & & 11,423 & & & "109 bbls slickwater" \\
\hline & $1151^{-}$ & $\mathrm{F} \mathrm{EU}^{-1}$ & $3 / 3 / 2005$ & 11.42 & & & & 6.23 & 15.38 & $x^{-}$ & & 20,732 & & & "183 bbls slickwater" \\
\hline $33-2 \mathrm{C}$ & 568.5 & WR 2 & $2 / 24 / 2005$ & 15.76 & & & & 9.17 & & $\bar{X}$ & & $6,844 \ldots$ & 0 & 0 & "2912 bbls 75Q slickwater" \\
\hline & 695.9 & $\mathrm{WR}$ & $2 / 24 / 2005$ & 15.44 & & & & 8.98 & & $X_{-}^{-}$ & & $6,133^{-}$ & & & "4790 bbls 75Q slickwater" \\
\hline & 759 & WR & $2 / 24 / 2005$ & 12.51 & & & & & & $x^{-}$ & & 7,304 & & & "2111 bls 75Q slickwater". \\
\hline & 789.1 & $\mathrm{WR}$ & $2 / 24 / 2005$ & 13.11 & & & & 8.69 & & $X_{-}^{-}$ & & 6,542 & & & "3846 bbls $75 \mathrm{Q}$ slickwater" \\
\hline & 812 & $\mathrm{WR}$ & $2 / 24 / 2005$ & 14.62 & & & & $7.72^{-}$ & & $X_{-}^{-}$ & & 10,825 & & & "5054 bbls $75 \mathrm{Q}$ slickwater" \\
\hline & 835.2 & $\mathrm{WR}$ & $2 / 24 / 2005$ & 17.07 & & & & $7 . \overline{58}$ & & $X^{-}$ & & 6,758 & & & "4002 bbls 75Q slickwater" \\
\hline
\end{tabular}




\begin{tabular}{|c|c|c|c|c|c|c|c|c|c|c|c|c|c|c|c|c|}
\hline 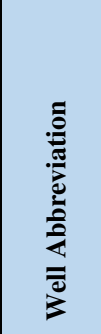 & 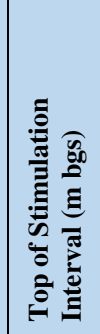 & 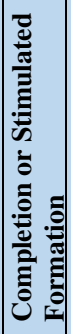 & 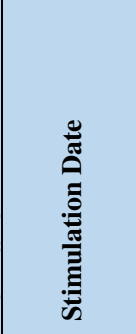 & 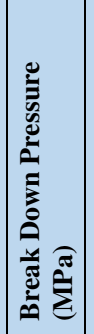 & 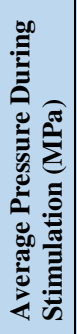 & 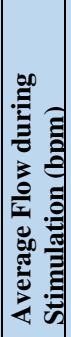 & 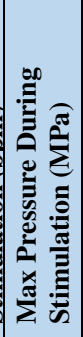 & 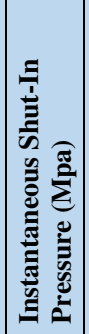 & 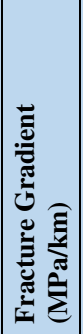 & 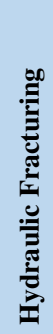 & 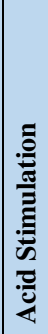 & 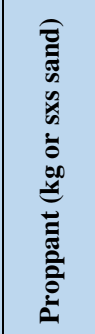 & 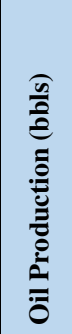 & 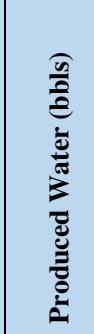 & 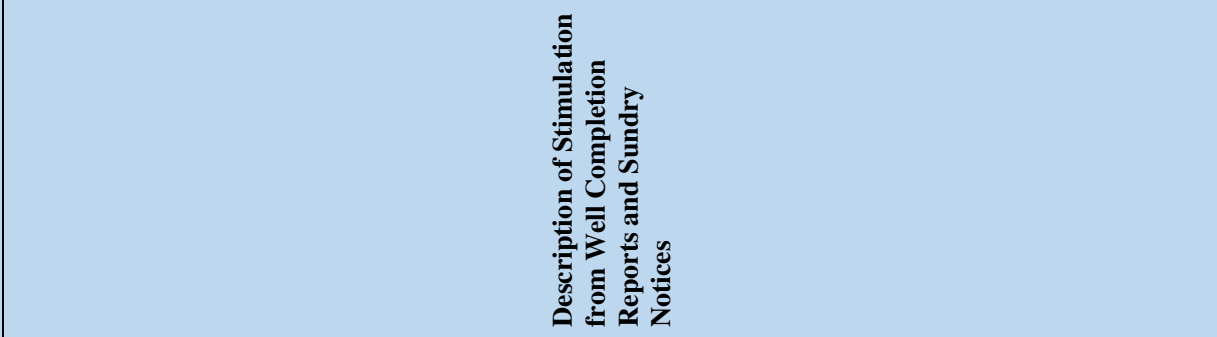 & 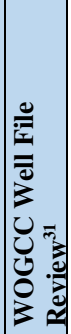 \\
\hline & 1102 & FU & $2 / 24 / 2005$ & 14.75 & & & & 7.93 & & $X$ & & 12,390 & & & "7158 bbls 75Q slickwater" & \\
\hline \multirow{9}{*}{$13-2 \mathrm{~B}$} & 4429.5 & WR & $3 / 19 / 2005$ & & & & & 6.00 & 23.98 & $\overline{\mathrm{X}}$ & & 6,720 & 0 & 3,944 & "59 bbls slickwater" & No \\
\hline & 480.4 & WR & $3 / 19 / 2005$ & & & & & 6.55 & 23.75 & $\bar{x}$ & & 9,807 & & & "70 büls slickwater" & \\
\hline & 536.8 & WR & $3 / 19 / 2005$ & & & & & 7.07 & 23.30 & $\bar{x}-$ & & $6,720^{\circ}$ & & & "67 bubls slickwater" & \\
\hline & 586.4 & WR & $3 / 19 / 2005$ & & & & & 7.89 & 23.53 & $x^{-}$ & & 9,730 & & & "67 bbls slickwater" & \\
\hline & 670 & WR & $3 / 19 / 2005$ & & & & & 5.58 & 18.55 & $\bar{x}$ & & 9,625 & & & "70 büls slickwater" & \\
\hline & 800.1 & $\mathrm{WR}$ & $3 / 19 / 2005$ & & & & & 8.27 & 20.36 & $\mathrm{X}_{-}^{-}$ & & 13,706 & & & "128 bubls slickwater" - & \\
\hline & $1063^{-1}$ & $\overline{\mathrm{FU}}^{-}$ & $3 / 18 / 2005$ & & & & & $9.10^{-1}$ & $18.78^{-}$ & $\mathrm{X}^{-}$ & & $7,253^{--}$ & & & "68 bobls slickwater" & \\
\hline & 1107 & $\overline{F u}^{-}$ & $3 / 18 / 2005$ & & & & & & & $\bar{X}-$ & & 6,536 & & & "66 bbls slickwater" & \\
\hline & 1128 & $\mathrm{FU}^{-}$ & $3 / 18 / 2005$ & & & & & 7.17 & $16.51^{-1}$ & $\mathrm{X}$ & & $6,720^{-}$ & & & "92 bbls slickwater" & \\
\hline \multirow[t]{7}{*}{ 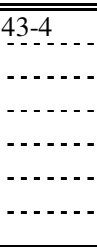 } & 524.3 & WR & $3 / 16 / 2005$ & 8.55 & & & & 7.83 & 10.18 & $\mathrm{X}$ & & 10,300 & 44 & 19,681 & "3573 bbls 70Q x-linked gel...reservoir pressure 963 psi" & Yes \\
\hline & $64 \overline{5} .3$ & $\mathrm{~W} \mathrm{WR}^{-1}$ & $3 / 16 / 2005$ & $14.60^{-}$ & & & & 7.21 & 10.18 & $\mathrm{X}^{-}$ & & $7,165^{-}$ & & & "2555 bbls $70 \mathrm{Q}$ x-linked gel...reservoir pressure $1167 \mathrm{psi}$ & \\
\hline & 808.3 & WR & $3 / 16 / 2005$ & 12.78 & & & & 5.99 & 10.18 & $\overline{-}$ & & 9,306 & & & 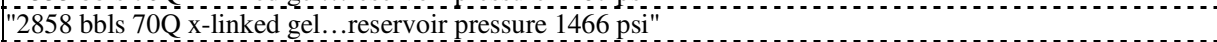 & \\
\hline & 954.3 & $\mathrm{WR}$ & $3 / 16 / 2005$ & 10.00 & & & & 10.39 & 10.18 & $\mathrm{X}$ & & 9,525 & & & "3074 bbls $70 \mathrm{Q}$ x-linked gel .reservoir pressure $1736 \mathrm{psi}$. & \\
\hline & 1053 & $\mathrm{FU}-$ & $3 / 16 / 2005$ & 16.64 & & & & & 10.18 & $\mathrm{X}$ & & 7,719 & & & (3424 bbls $70 \mathrm{Q}$ x-linked gel...reservoir pressure $1904 \mathrm{psi}$ & \\
\hline & 1156 & $\bar{F} \bar{U}^{-}$ & $3 / 16 / 2005$ & 15.49 & & & & 5.56 & 10.18 & $\bar{x}$ & & 20,518 & & & $" 8293$ bbis $70 \mathrm{Q}$ x-linked gel...reservoir pressure $2107 "$ & \\
\hline & 1402 & $\bar{F} \dot{H}^{-1}$ & $3 / 16 / 2005$ & 13.70 & & & & & 10.18 & $\bar{x}$ & & 6,823 & & & "3077 bbils $70 \mathrm{Q}$ x-linked gel...reservor pressure $2531 \mathrm{psi"}$ & \\
\hline \multirow[t]{6}{*}{$33-2 \mathrm{~B}$} & - 430.4 & WR & $3 / 23 / 2005$ & & & & & & & $\overline{\mathrm{X}}$ & & $7,121$. & 31 & 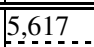 & "56 bbls slickwater". & No \\
\hline & 549.2 & $\mathrm{WR}$ & $3 / 23 / 2005$ & 10.65 & & & & 5.80 & 20.58 & $\mathrm{X}$ & & 8,258 & & & "62 bbls slickwater" & \\
\hline & 565.7 & WR & $3 / 23 / 2005$ & $12 . \overline{4}$ & & & & 7.34 & 23.07 & $\mathrm{X}$ & & 20,318 & & & "144 bubl slickwater" & \\
\hline & 676.7 & WR & $3 / 23 / 2005$ & 13.80 & & & & 6.30 & 19.45 & $\bar{x}$ & & $6,985^{-}$ & & & "60 bubls slickwater" & \\
\hline & 824.2 & WR & $3 / 23 / 2005$ & 14.13 & & & & & 20.13 & $\bar{x}-$ & & 13,574 & & & "162 bubls slickwater" & \\
\hline & 863.5 & $\mathrm{WR}$ & $3 / 12 / 2005$ & 10.97 & & & & 9.80 & 21.49 & $x^{-}$ & & $6,439^{-}$ & & & "1442 bbils slickwater" & \\
\hline \multirow[t]{3}{*}{$14-3 \mathrm{~B}$} & 887.3 & WR & $4 / 2 / 2005$ & 20.64 & & & & 8.97 & 10.18 & $\mathrm{X}$ & & 6,748 & 5 & 1,901 & "54 bbls slickwater" & Yes \\
\hline & 941.8 & WR & $4 / 2 / 2005$ & 14.94 & & & & 10.25 & $21.04^{-}$ & $\bar{x}$ & & 11,520 & & & "111 büls slickwater" & \\
\hline & $1146^{-}$ & $\overline{\mathrm{Fu}}$ & $4 / 2 / 2005$ & & & & & & & $\mathrm{x}$ & & 10,810 & & & "1215 bubis slickwater" & \\
\hline \multirow[t]{7}{*}{ 41-11B } & 4498.3 & WR & $4 / 6 / 2005$ & 3.00 & & & & 4.54 & 19.45 & $\overline{\mathrm{X}}$ & & 3,835 & 12 & 640 & "53 bbls slickwater...Flush with 23 bbl 6\% KCL w/50\% CO2"1 & No \\
\hline & 679.7 & WR & $4 / 8 / 2005$ & 4.37 & & & & 6.10 & 19.23 & $\mathrm{X}-$ & & 7,380 & & & $" 60$ bbls slickwater...Flush with $33.5 \mathrm{bbl} 6 \% \mathrm{KCl}^{\circ}$ & \\
\hline & 713.5 & WR- & $4 / 5 / 2005$ & 0.00 & & & & & & $\bar{X}$ & & 7,213 & & & " 55 bbils slickwater...Flush with 34 bbl $6 \%$ KCL and $60 \%$ CO2" & \\
\hline & $840^{-1}$ & WR & $4 / 5 / 2005$ & 12.47 & & & & 7.02 & $18.55^{-1}$ & $\mathrm{X}$ & & 10,851 & & & "80 bbls slickwater...Flush with 40 bbls $6 \%$ KCL" & \\
\hline & $8 \overline{8} 6.1$ & $\mathrm{~W} \mathrm{WR}^{-1}$ & $4 / 5 / 2005$ & $11.09^{-1}$ & & & & 9.22 & 20.58 & $\mathrm{X}^{-}$ & & 7,536 & & & "61 bbls slickwater...Flush with 45 bbl $6 \%$ KCL" & \\
\hline & $1077^{-1}$ & $\overline{\mathrm{Fu}}$ & $4 / 5 / 2005$ & 15.36 & & & & 7.35 & 17.19 & $\mathrm{X}^{-}$ & & 8,275 & & & $" 105$ bbls slickwater...Flush with 54 bbi $6 \% \mathrm{KCl} "$ & \\
\hline & $11 \overline{158}$ & $\mathrm{FU}^{-1}$ & $4 / 5 / 2005$ & & & & & $4.01^{-1}$ & 13.80 & $\mathrm{X}^{-}$ & & 12,432 & & & $" 170$ bbis slickwater...Flush with 58 bbls $6 \% \mathrm{KCl}^{-}$ & \\
\hline \multirow[t]{7}{*}{ 42-4B } & 526.4 & WR & $3 / 29 / 2005$ & 9.63 & & & & 8.16 & 26.01 & $\bar{X}$ & & 13,608 & 0. & $8,857$. & "Flush with $17 \mathrm{bbl} 6 \% \mathrm{KCL}$ and $7 \mathrm{bbl} \mathrm{CO2".}$ & No \\
\hline & 730 & WR & $3 / 29 / 2005$ & 16.11 & & & & 9.64 & 23.53 & $\mathrm{X}-$ & & 6,804 & & & "Flush with 36 bbl $6 \%$ KCL" & \\
\hline & 802.5 & WR & $3 / 29 / 2005$ & 9.14 & & & & 10.18 & 22.85 & $\bar{x}-$ & & 9,525 & & & "Flushed with 40 bbl $6 \%$ KCL" & \\
\hline & $8 \overline{4} 2 . \overline{2}$ & $\mathrm{WR}^{-1}$ & $3 / 29 / 2005$ & $10.07^{-}$ & & & & & & $\bar{x}^{-}-$ & & 4,667 & & & $\begin{array}{l}\text { "Screen out } 22.4 \text { bbl into flush, } 19.9 \text { bbi shy of full flush...Pumped } 81 \text { bbl of clean fluid and } 16 \text { tons of } \mathrm{CO} 2 \\
+ \text { sand" }\end{array}$ & \\
\hline & 864.7 & WR & $3 / 29 / 2005$ & 15.48 & & & 19.84 & 9.96 & 21.72 & $\bar{X}^{-}$ & & 11,366 & & & $" 126$ bbl clean fluid and 27 ton $\mathrm{CO} 2 "$ & \\
\hline & 1136 & $\overline{\mathrm{FU}}$ & $3 / 29 / 2005$ & 12.15 & & & & 10.46 & 19.45 & $x^{-}$ & & 14,063 & & & $" 180$ bbl clean fluid and 39 ton CO2" & \\
\hline & $1169^{-}$ & $\mathrm{Fu}^{-}$ & $3 / 29 / 2005$ & 16.33 & & & 22.39 & 10.68 & 19.45 & $\bar{x}-$ & & 10,978 & & & $" 165 \mathrm{bb} \mathrm{l}$ clean fluid and 30 ton $\mathrm{CO} 2 "$ & \\
\hline $21-11 \mathrm{~B}$ & I440.1 & WLR & $4 / 12 / 2005$ & 7.18 & & & & 5.16 & 10.18 & $\overline{\mathrm{X}}$ & & $6,811 \ldots$ & 18 & 1,560 & "54 bbls slickwater". & Yes \\
\hline
\end{tabular}




\begin{tabular}{|c|c|c|c|c|c|c|c|c|c|c|c|c|c|c|c|c|}
\hline 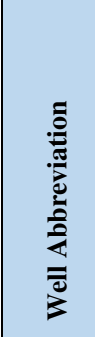 & 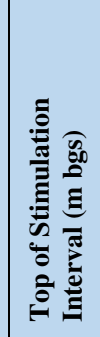 & 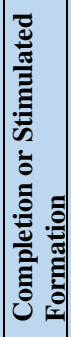 & 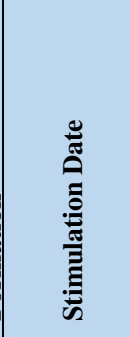 & 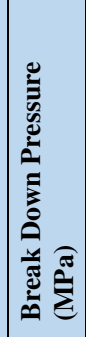 & 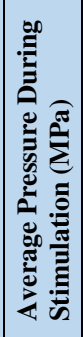 & 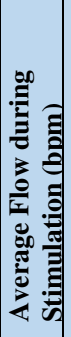 & 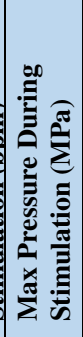 & 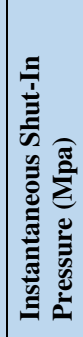 & 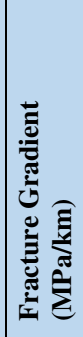 & 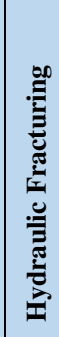 & 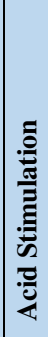 & 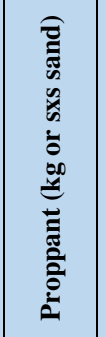 & 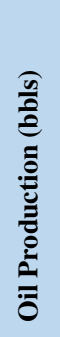 & 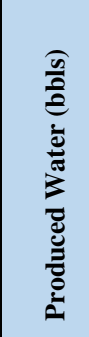 & 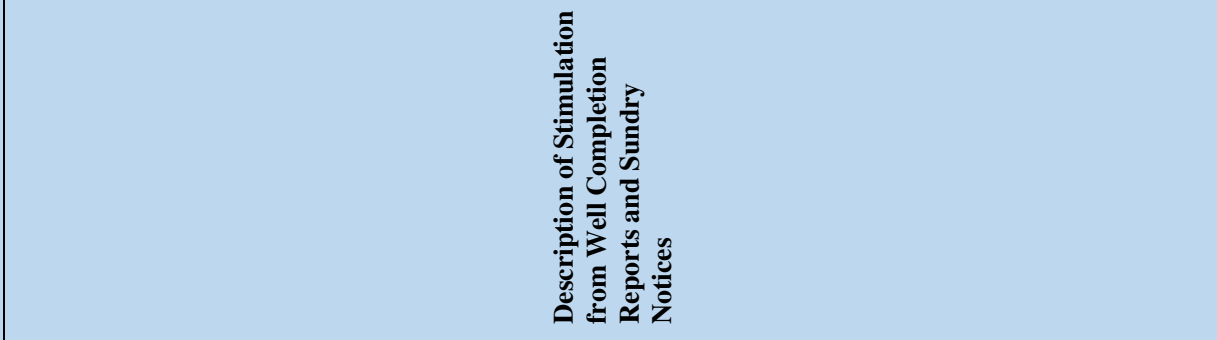 & 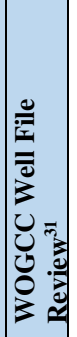 \\
\hline & 626.4 & WR & $4 / 12 / 2005$ & 7.17 & & & & 6.49 & 10.18 & $\mathrm{X}$ & & $10,906$. & & & "77 bbls slickwater". & \\
\hline & -695.2 & WR & $4 / 12 / 2005$ & 7.57 & & & & 6.83 & $10.18^{-}$ & $\mathrm{X}^{-}$ & & 6,803 & & & "55 bbils slickwater" & \\
\hline & $7 \overline{4} 1.6$ & $\mathrm{WR}$ & $4 / 12 / 2005$ & 10.27 & & & & 7.27 & $10.18^{-}$ & $\mathrm{X}$ & & $7,511^{-}$ & & & "58 bbiss slickwater" & \\
\hline & 919.6 & $\mathrm{~W} \mathrm{R}$ & $4 / 12 / 2005$ & 17.58 & & & & 8.43 & $10.18^{-}$ & $\mathrm{X}$ & & $6,826^{-}$ & & & "63 bbls slickwater" & \\
\hline & 1027 & FE & $4 / 12 / 2005$ & 9.19 & & & & 6.32 & $10.18^{-}$ & X & & $9,740^{-}$ & & & "89 bbls slickwater" & \\
\hline & 1066 & FU & $4 / 12 / 2005$ & 14.16 & & & & 6.59 & $10.18^{-}$ & $\mathrm{X}$ & & $6,891^{-}$ & & & "74 bbls slickwater" & \\
\hline & 1102 & $\mathrm{FU}$ & $4 / 12 / 2005$ & 11.09 & & & & 6.14 & $13.80^{-}$ & $\mathrm{X}$ & & 7,018 & & & "87 bbls slickwater" & \\
\hline $13-3 \mathrm{~W}$ & 456 & WR & $4 / 16 / 2005$ & 17.07 & & & & 7.58 & 26.69 & $\bar{X}$ & & 6,483 & & 1,340 & "60 bbls slickwater" & Yes \\
\hline & 708.4 & $\mathrm{WR}$ & $4 / 16 / 2005$ & 17.07 & & & & 7.58 & 20.81 & X & & $6,582^{-}$ & & & "74 bbls slickwater" & \\
\hline & 739.4 & $\mathrm{WR}$ & $4 / 16 / 2005$ & 17.07 & & & & 7.58 & 20.36 & X & & 6,104 & & & "66 bbis slickwater" & \\
\hline & 820.2 & $\mathrm{WR}$ & $4 / 16 / 2005$ & 17.07 & & & & 7.58 & 19.23 & $\mathrm{X}$ & & 11,132 & & & "93 bbls slickwater" & \\
\hline & 860.1 & $\mathrm{WR}$ & $4 / 16 / 2005$ & 17.07 & & & & 7.58 & 19.00 & X & & 2,417 & & & "68 bbls slickwater" & \\
\hline & 963.5 & $\mathrm{WR}$ & $4 / 16 / 2005$ & 17.07 & & & & 7.58 & $17.87^{-}$ & $\mathrm{X}^{-}$ & & 7,071 & & & "100 bbls slickwater" & \\
\hline & 1363 & $\overline{F U}$ & $4 / 16 / 2005^{-}$ & $17.07^{-}$ & & & & $7.58^{-}$ & $13.35^{\circ}$ & $\mathrm{X}$ & & $6,720^{-}$ & & & "134 b̄bls slickwater" & \\
\hline 41-10B & 546.2. & WR & $4 / 30 / 2005$ & 11.58 & & & & 7.14 & 23.30 & $\bar{X}$ & & 5,285 & & 293 & "48 bbls slickwater". & \\
\hline & 679.1 & $\mathrm{WR}$ & $4 / 30 / 2005$ & 12.01 & & & & 8.09 & 21.94 & X & & 11,864 & & & "82 bbls slickwater" & \\
\hline & 919.3 & $\mathrm{~W} R$ & $4 / 30 / 2005$ & 23.32 & & & & 9.34 & 20.36 & $\mathrm{X}$ & & 6,393 & & & "53 bbls slickwater" & \\
\hline & 1081 & $\overline{\mathrm{Fu}}$ & $4 / 30 / 2005$ & & & & & 0.00 & $17.64^{-}$ & $\mathrm{X}$ & & $13,494^{-}$ & & & Information on stimulation missing from completion report. From WOGCC (2014) "CO2 assist" & \\
\hline $34-28$ & 605 & WR & $5 / 20 / 2005$ & 113.61 & 13.56 & & 18.61 & 8.62 & & $\mathrm{X}$ & & $8,165 . .1$ & & 0 & "97 bbls 6\% kcl 24.4 tons co2". & Io. \\
\hline & 816.3 & WR & $5 / 20 / 2005$ & 7.89 & 14.08 & & 22.83 & 8.09 & & X- & & $8,165^{-}$ & & & $" 109$-bbls $6 \%$ kcl 24.4 tons co2" & \\
\hline & 927.2 & $\mathrm{~W} \mathrm{~W}$ & $5 / 16 / 2005$ & 15.58 & 19.78 & 17.9 & 32.96 & $13.93^{-}$ & & $\mathrm{X}$ & & $8,165^{-}$ & & & 115 bbls $6 \%$ kcl 24.4 tons co 2 & \\
\hline & 951.6 & $\mathrm{WR}$ & $5 / 10 / 2005$ & 19.43 & 13.21 & $21.1]$ & 24.11 & 111.76 & & X & & $8,811^{-}$ & & & $" 172.2$ bbls total co $2-27$ tons" & \\
\hline & $1285^{-}$ & $\bar{F}$ & $4 / 29 / 2005$ & 19.03 & 19.03 & & 23.17 & $5.85^{-}$ & & & - & 0 & & & "Acidize w/750 gal KCl, $7.5 \%$ acid" & \\
\hline & 1367 & $\mathrm{FU}$ & $4 / 14 / 2005$ & 14.26 & & & & 7.89 & 15.83 & $\mathrm{X}$ & & 11,197 & & & $\begin{array}{l}\text { "Total fluid pumped was } 145.6 \text { bbl, total slurry was } 172 \mathrm{bbl} \text {, total } \mathrm{CO} 2 \text { was } 33 \text { tons...Left }+/-10,000 \text { pounds } \\
\text { lbs sand in casing" }\end{array}$ & \\
\hline & 1392 & FE'- & $4 / 8 / 2005$ & 15.17 & & & & 4.14 & & & $\mathrm{X}$ & & & & "Acidize w/23 bbls kcl, $7.5 \%$ acid...Flush w/18 bbls kcl" & \\
\hline & 1562 & $\mathrm{FU}^{-1}$ & $3 / 26 / 2005$ & 17.93 & & & & 10.03 & $16.51^{\circ}$ & $\mathrm{X}$ & & 10,886 & & & "WF 125 Slickwater...Flush with 78 bbl $6 \%$ KCL and $50 \%$ CO2" & \\
\hline $32-3$ & 575.2 & WR & $5 / 25 / 2005$ & 17.06 & 18.62 & & 20.68 & 8.40 & & $\mathrm{X}$ & & 6,133 & 1 & 50,290 & "85-bbld 6\% kcl 20.4 tons co2" & No \\
\hline & 705.3 & WR & $5 / 25 / 2005$ & 17.31 & 20.68 & & 22.06 & 8.74 & & X & & 11,037 & & & $125-b b 1 s 6 \% \mathrm{kcl} 32.6$ tons co $2 "$ & \\
\hline & 910.7 & WR & $5 / 25 / 2005$ & 12.25 & 20.68 & & 23.44 & 9.47 & & X & & 7,021 & & & $104-b b i s 6 \% \mathrm{kcl} 20.4$ tons co 2 & \\
\hline & 933.9 & WR & $5 / 25 / 2005$ & 16.51 & 22.75 & & 26.54 & 8.48 & & $\mathrm{X}$ & & 9,370 & & & $127-\mathrm{bbls} 6 \% \mathrm{kcl} 28.5$ tons co2" & \\
\hline & 1427 & FU' & $5 / 25 / 2005$ & 20.04 & 20.68 & & 27.58 & 7.07 & & X & & $6,931^{-}$ & & & 133 -bbls $6 \% \mathrm{kcl} 20.4$ tons co 2 & \\
\hline & 1492 & $\mathrm{Fu}^{-1}$ & $5 / 25 / 2005$ & 22.51 & $22.75^{-}$ & & 25.51 & 6.62 & & $\mathrm{X}$ & & 20,412 & & & "245 bbls $6 \%$ kcl 61.1 tons co $2 "$ & \\
\hline $43-9$ & 1546 & FU. & $5 / 27 / 2005$ & 9.63 & 24.13 & & & 5.41 & & $\underline{X}$ & & $8,066 \ldots$ & & $20,928$. & "161-bbls 6\% kcl 28.5 tons co2" & No. \\
\hline & 1618 & $\mathrm{FU}$ & $5 / 27 / 2005$ & 14.80 & 26.20 & & & & & $\mathrm{X}$ & & 7,157 & & & 143 bbls $6 \%$ kcl 20.4 tons co 2 & \\
\hline & 1649 & FU & $5 / 27 / 2005$ & 14.39 & 24.13 & & 27.72 & 6.32 & & X & & 13,641 & & & "200-bis $6 \%$ kcl 40.7 tns co2" & \\
\hline & $1697^{-}$ & $\mathrm{FU}^{-1}$ & $5 / 27 / 2005$ & 20.26 & 24.13 & & $29.99^{-}$ & $8.55^{\circ}$ & & $\mathrm{X}$ & & $17, \overline{5} 5 \overline{7}$ & & & "180-bbis $6 \%$ kcl 32.6 tons co $2 "$ & \\
\hline $22-3$ & 775.4 & WWR. & 7/9/2005 & 16.20 & & & & 7.65 & & $\mathrm{X}$ & & 10,210 & & 19,153 & "100 bbls fluid". & No. \\
\hline & 842.5 & WR & $7 / 9 / 2005$ & 13.54 & & & & 9.48 & & $\mathrm{X}$ & & 7,354 & & & "108 bbis fluid" & \\
\hline & 912 & $\mathrm{~W} R$ & $7 / 9 / 2005$ & 13.38 & & & & 8.93 & & $\mathrm{X}$ & & 7,208 & & & "185 bbbls fluid" & \\
\hline & 970.8 & WR & $7 / 9 / 2005$ & 15.69 & & & & 10.34 & & $\mathrm{X}$ & & 11,387 & & & "132 bbis fluid" & \\
\hline & 1166 & FE & $179 / 2005$ & 27.03 & & & & 6.41 & & & & 13,950 & & & 206 bbls fluid". & \\
\hline
\end{tabular}




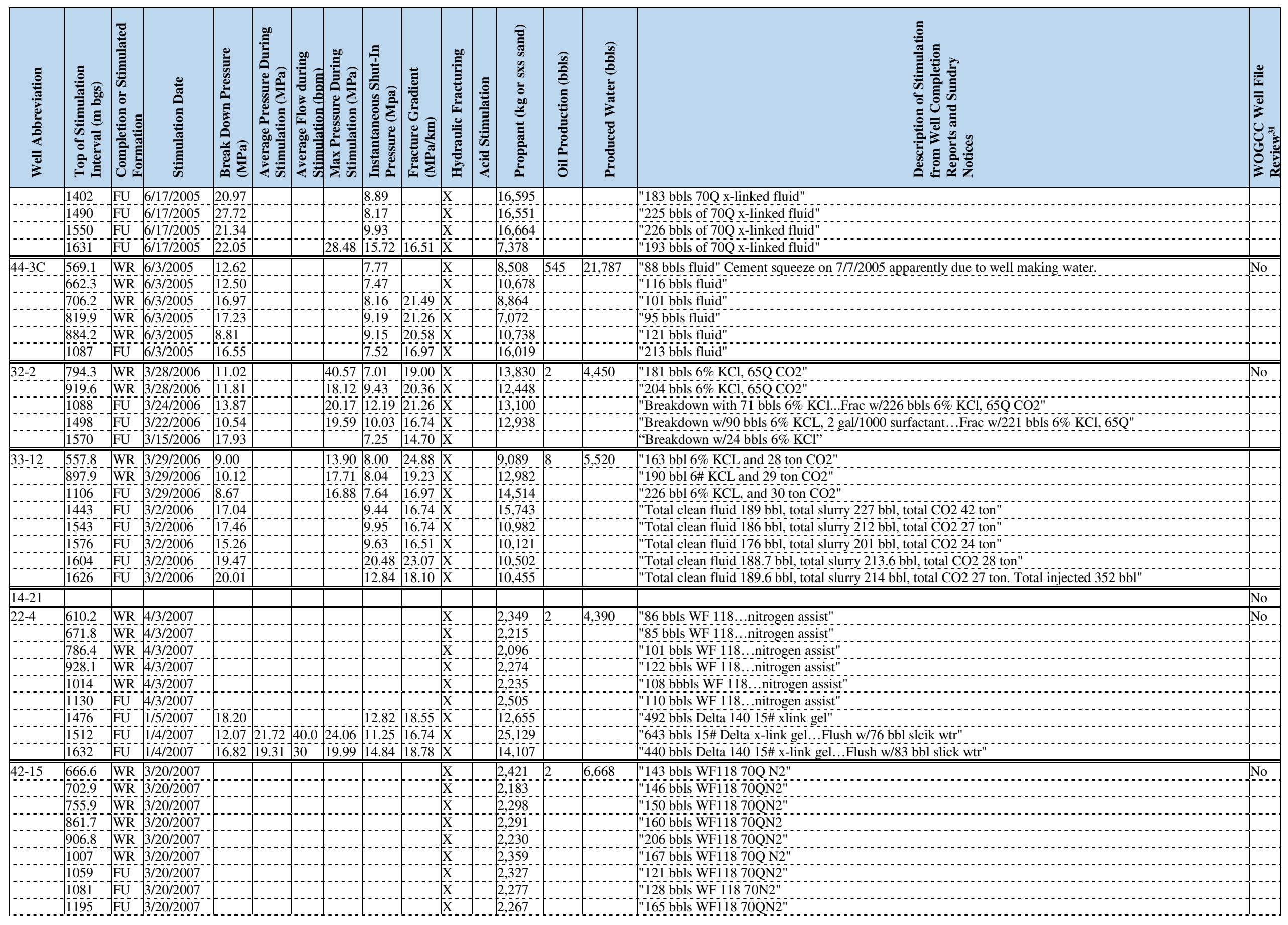




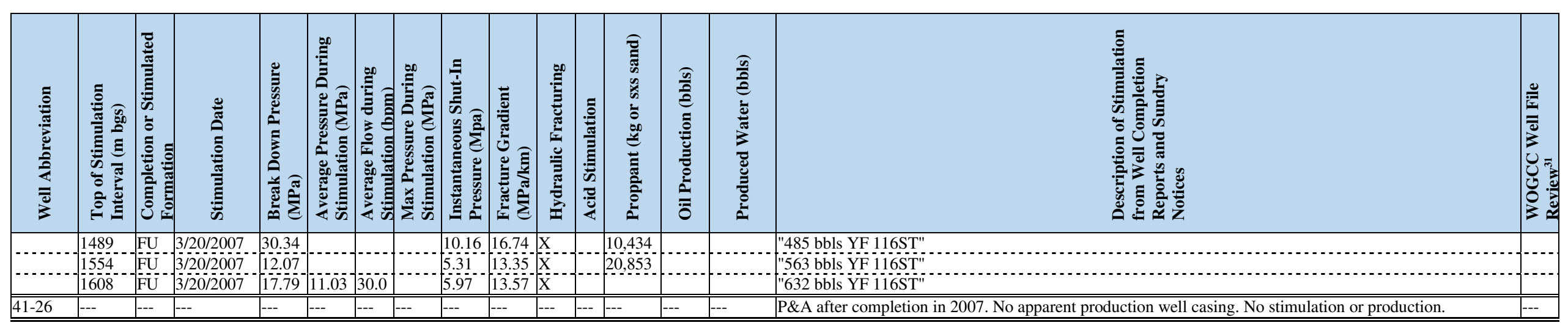

BWR - Basal Wind River Formation

WR - Wind River Formation

FU - Fort Union Formation

L - Lance Formation

MT - Meeteetse Formation

F- Frontier Formation

C- - Cody Formation

MV - Mesaverde Formation

$\mathrm{bbm}=$ barrels per minute

bbls - barrels

sxs $=$ sacks 
Table SI C3. Summary of compounds used for well stimulation. Information from Material Safety and Data Sheets provided to EPA from Encana ${ }^{35}$

\begin{tabular}{|c|c|c|c|c|c|}
\hline Compound & Cas No. & Product & Manufacturer & $\begin{array}{l}\text { Percent } \\
\text { Composition }\end{array}$ & Specified Product Use \\
\hline acetic acid & \begin{tabular}{|l}
$64-19-7$ \\
\end{tabular} & FE-1A Acidizing Composition & Halliburton & $30-60$ & additive \\
\hline & & BA-20 Buffering Agent & Halliburton & $10-30$ & buffer \\
\hline acetic anhydride & \begin{tabular}{|l|}
$108-24-7$ \\
\end{tabular} & FE-1A Acidizing Composition & Halliburton & $60-100 \%$ & additive \\
\hline acetone & $67-64-1$ & MC-B-8630 & Multi-Chem Group & $15-40$ & biocide \\
\hline alcohol (proprietary) & not provided & WFT 9527 & Weatherford & $1-5$ & flow enhancer \\
\hline alcohol (oxylated) & not provided & Musol A Solvent & Halliburton & $10-30$ & solvent \\
\hline $\begin{array}{l}\text { alcohols, C6-10, ethoxylated, } \\
\text { sulfate, ammonium salts }\end{array}$ & $68037-05-8$ & MC FA-4013 & Multi-Chem Group & $<8$ & foaming agent/scale \& corrosion inhibitor combination \\
\hline alkylated quaternary chloride & not provided & Clayfix-II Material & Halliburton & not provided & clay stabilizer \\
\hline alkylamine salts & not provided & EC6106A Biocide & Nalco/Exxon Energy Chemicals & $40-70$ & biocide \\
\hline alkyl hexanol & not provided & HYFLO IV & Halliburton & $5-10$ & surfactant \\
\hline ammonium acetate & $631-61-8$ & BA-20 Buffering Agent & Halliburton & $60-100$ & buffer \\
\hline ammonium bisulfite & $10192-30-0$ & EC1385A & Nalco/Exxon Energy Chemicals & $10-30$ & corrosion inhibitor \\
\hline ammonium chloride & $12125-02-9$ & CL-12 & Halliburton & $1-27$ & crosslinker \\
\hline anionic surfactants & not provided & MC FA-4013 & Multi-Chem Group & $<20$ & foaming agent/scale \& corrosion inhibitor combination \\
\hline aromatic solvent & $64741-68-0$ & MC DF-7120 & Multi-Chem Group & $<75$ & defoamer/emulsion breaker \\
\hline 2-bromo-2-nitro-1,3-propanediol & $52-51-7$ & BE-6 Microbiocide & Halliburton & $60-100$ & biocide \\
\hline 2-butoxyethanol & $111-76-2$ & F104 & Schlumberger & $10-30$ & foaming agent \\
\hline $4-2-2-2-1$ & & AQQF-2 Foaming Agent & Halliburton & $10-30$ & foaming agent \\
\hline “. & & MCFA-4500 & Multi-Chem Group. & $1-10$ & foaming agent \\
\hline$"$ & $\because$ & $M C F A-4295$ & Multi-Chem Group & $<10$ & foaming agent \\
\hline .. & $\therefore$ & MCFA-4001 & Multi-Chem Group. & $<11$ & foaming agent/scale \& corrosion inhibitor combination \\
\hline “. & $\cdots$ & MCFA-4013 & Multi-Chem Group. & $<12$ & foaming agent/scale \& corrosion inhibitor combination \\
\hline & & Fio3 & Schlumberger & $10-30$ & surfactant \\
\hline “. & - & Musol Solvent & Halliburton & $60-100$ & solvent \\
\hline "“ & $\because-$ & Musol ÁSolvent & Halliburton & $60-100$ & solvent \\
\hline “- & 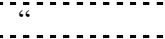 & WSP $9999^{-}$ & Weatherford & not provided & solvent \\
\hline (-- & & WSP 92011 & Weatherford & $15-40$ & not provided \\
\hline$" \cdots$ & $\because \cdots$ & Transfoam C & Weatherford & $5-10$ & drilling foam \\
\hline cationic polymer & not provided & L55 & Schlumberger & $10-30$ & clay stabilizer \\
\hline copper iodide & 7681-65-4 & HAI-85M Acid Inhibitor & Halliburton & $1-5$ & corrosion inhibitor \\
\hline diammonium peroxidisulphate & $7727-54-0$ & $\mathrm{~J} 218$ & Schlumberger & $60-100$ & breaker \\
\hline $\begin{array}{l}\text { 2,2-dibromo-3- } \\
\text { nitrilopropionamide }\end{array}$ & $10222-01-2$ & BE-3S Bactericide & Halliburton & $60-100$ & biocide \\
\hline “- & "“ & B069 & Baker Hughes & $60-100$ & biocide \\
\hline diethylene glycol & $111-46-6$ & AQF-2 Foaming Agent & Halliburton & $2-10$ & foaming agent \\
\hline “ & “" & Triethylene glycol & Shell Chemicals & $0.01-5.0$ & not provided \\
\hline diesel fuel \#2 & $68476-34-6$ & LGC-VI & Halliburton & $30-60$ & liquid gel concentrate \\
\hline “" & -“- & LGC-8 & Halliburton & $30-60$ & liquid gel concentrate \\
\hline '; & " & $\mathrm{J} 877$ & Schlumberger & $30-60$ & guar polymer slurry \\
\hline dimethyl formamide & \begin{tabular}{|l|}
$68-12-3$ \\
\end{tabular} & HAI-85M Acid Inhibitor & Halliburton & $10-30$ & corrosion inhibitor \\
\hline d-limonene & $5989-27-5$ & WFT 9527 & Weatherford & $3-7$ & flow enhancer \\
\hline EDTA/copper chelate & not provided & CAT-3 Activator & Halliburton & $10-30$ & activator \\
\hline ester salt & not provided & F104 & Schlumberger & $30-60$ & foaming agent \\
\hline ethanol & $64-17-5$ & LOSURF-300M & Halliburton & $30-60$ & surfactant \\
\hline "“ & "“ & F104 & Schlumberger & $10-30$ & foaming agent \\
\hline
\end{tabular}




\begin{tabular}{|c|c|c|c|c|c|}
\hline Compound & Cas No. & Product & Manufacturer & $\begin{array}{l}\text { Percent } \\
\text { Composition }\end{array}$ & Specified Product Use \\
\hline ethoxylated alcohol & $9016-45-9$ & WFT 9785 & Weatherford & $1-3$ & foaming agent \\
\hline ethoxylated alcohol linear (1) & not provided & F103 & Schlumberger & $5-10$ & surfactant \\
\hline ethoxylated alcohol linear (2) & not provided & F103 & Schlumberger & $5-10$ & surfactant \\
\hline ethoxylated alcohol linear (3) & not provided & F103 & Schlumberger & $5-10$ & surfactant \\
\hline ethylene glycol & $107-21-1$ & WSP 9030 & Weatherford & $40-70$ & hydrate inhibitor \\
\hline " & " & MC FA-4001 & Mult-Chem Group & $54-58$ & foaming agent/scale \& corrosion inhibitor combination \\
\hline “" & “ & MC FA-4500 & Mult-Chem Group & $<33$ & foaming agent \\
\hline “ & “ & MC FA-4295 & Mult-Chem Group & $1-5$ & foaming agent \\
\hline " & " & MC WC-7549 & Mult-Chem Group & $<70$ & water clarifier \\
\hline " & “ & Artictherm E-50 & Quadra & $47-53$ & heat transfer fluid \\
\hline 2-ethyl hexanol & $104-76-7$ & Klean-Break & Weatherford & $10-30$ & not provided \\
\hline ethyl octynol & $5877-42--9$ & HAI-85M Acid Inhibitor & Halliburton & $1-5$ & corrosion inhibitor \\
\hline glutaraldehyde & $111-30-8$ & MC B-8630 & Multi-Chem Group & $10-30$ & biocide \\
\hline $\begin{array}{l}\text { heavy aromatic petroleum } \\
\text { naptha }\end{array}$ & $64742-94-5$ & HYFLO IV & Halliburton & $30-60$ & surfactant \\
\hline “ & “- & Pad acid with aromatic naphtha & Halliburton & $10-50$ & pad acid \\
\hline “" & "“- & LOSURF-300M & Halliburton & $10-30$ & surfactant \\
\hline “" & “- & LOSURF-259 & Halliburton & $5-10$ & surfactant \\
\hline hydrochloric acid & $7647-01-0$ & Pad acid with aromatic naphtha & Halliburton & $10-30$ & pad acid \\
\hline “ & & Hydrochloric acid & Halliburton & $30-60$ & solvent \\
\hline $\begin{array}{l}\text { hydrotreated light petroleum } \\
\text { distillates }\end{array}$ & $64742-47-8$ & $\mathrm{~B} 221 \mathrm{~B}$ & Schlumberger & $40-60$ & polymer slurry \\
\hline inner salt of alkyl amines & & HC-2 & Halliburton & $10-30$ & additive \\
\hline isooctanol & $26952-21-6$ & HYFLO IV & Halliburton & $5-10$ & surfactant \\
\hline isopropanol & $67-63-0$ & LOSURF-259 & Halliburton & $30-60$ & surfactant \\
\hline “- & “- & F103 & Schlumberger & $10-30$ & surfactant \\
\hline “" & “- & MC DF-7120 & Multi-Chem Group & not provided & defoamer/emulsion breaker \\
\hline “" & "- & MC FA-4295 & Multi-Chem Group & $1-5$ & foaming agent \\
\hline “- & “- & MC FA-4001 & Multi-Chem Group & $<8$ & foaming agent/scale \& corrosion inhibitor combination \\
\hline "“ & "“ & MC FA-4013 & Multi-Chem Group & $<3$ & foaming agent/scale $\&$ corrosion inhibitor combination \\
\hline "“ & "- & MC EB- 1790 & Mult-Chem Group & $<1$ & emulsion breaker \\
\hline 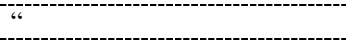 & -" & Klean-Break & Weatherford & $10-30$ & not provided \\
\hline “" & "- & HAI-85M Acid Inhibitor & Halliburton & $10-30$ & corrosion inhibitor \\
\hline "“ & "- & EC1385A & Nalco/Exxon Energy Chemicals & $10-30$ & corrosion inhibitor \\
\hline “ & - & Transfoam C & Weatherford & $10-30$ & drilling foam \\
\hline$"$ & "“ & WFT $97112 \mathrm{~W}$ & Weatherford & $1-3$ & mixture \\
\hline “ & "“- & EC6106A Biocide & Nalco/Exxon Energy Chemicals & $20-40$ & Biocide \\
\hline methanol & $67-56-1$ & Klean-Break & Weatherford & $7-13 \%$ & not provided \\
\hline 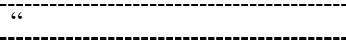 & - & $\mathrm{L} 55$ & Schlumberger & not provided & clay stabilizer \\
\hline 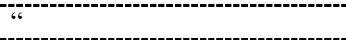 & $\cdots$ & WSP 9030 & Weatherford & $40-70$ & hydrate inhibitor \\
\hline "“ & "- & WSP 92011 & Weatherford & $15-40$ & not provided \\
\hline "“ & "“ & WFT $97112 \mathrm{~W}$ & Weatherford & $15-40$ & mixture \\
\hline "“ & - & WFT 9785 & Weatherford & $10-30$ & foaming agent \\
\hline “ & “- & MC FA-4295 & Multi-Chem Group & $1-25$ & foaming agent \\
\hline “- & “- & MC FA-2013 & Multi-Chem Group & $<25$ & foaming agent/scale \& corrosion combination \\
\hline 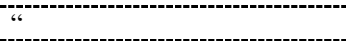 & $\cdots$ & EC9037a Surfactant & Nalco/Exxon Energy Chemicals & $20-40$ & surfactant \\
\hline “- & "- & Methanol & Murex N.A. & 99.8 & methanol \\
\hline
\end{tabular}




\begin{tabular}{|c|c|c|c|c|c|}
\hline Compound & Cas No. & Product & Manufacturer & $\begin{array}{l}\text { Percent } \\
\text { Composition }\end{array}$ & Specified Product Use \\
\hline methyl isobutyl ketone & $108-10-1$ & WFT 9527 & Weatherford & $1-5$ & flow enhancer \\
\hline $\begin{array}{l}\text { 2- monobromo-3- } \\
\text { nitrilopropionamide }\end{array}$ & $1113-55-9$ & BE-3S Bactericide & Halliburton & $1-5$ & biocide \\
\hline naphthalene & $91-20-3$ & HYFLO IV & Halliburton & $5-10$ & surfactant \\
\hline “" & "“- & LOSURF-300M & Halliburton & $0-1$ & surfactant \\
\hline 2,2',2-nitrilotriethanol & $102-71-6$ & $\mathrm{~J} 318$ & Schlumberger & $60-100$ & breaker \\
\hline organic salt & not provided & FE-3A & Halliburton & $60-100 \%$ & iron control agent \\
\hline petroleum raffinates & not provided & Klean-Break & Weatherford & $30-60$ & not provided \\
\hline $\begin{array}{l}\text { polyepichlorohydrin, } \\
\text { trimethylamine quaternized }\end{array}$ & $51838-31-4$ & Cla-Sta XP Additive & Halliburton & $30-60 \%$ & clay stabilizer \\
\hline polyether & not provided & EC9037A & Nalco/Exxon Energy Chemicals & $20-40$ & surfactant \\
\hline potassium hydroxide & $1310-58-3$ & CL-31 & Halliburton & $<5$ & crosslinker \\
\hline potassium metaborate & $13709-94-9$ & CL-31 & Halliburton & $30-60$ & crosslinker \\
\hline “ & “ & CL-31/Water Blend & Halliburton & $5-10$ & crosslinker \\
\hline propargyl alcohol & $107-19-7$ & HAI-85M Acid Inhibitor & Halliburton & $5-10$ & corrosion inhibitor \\
\hline proprietary blend & not provided & MC FA-4001 & Multi-Chem Group & $<8$ & foaming agent/scale \& corrosion inhibitor combination \\
\hline proprietary blend & not provided & MC FA-4013 & Multi-Chem Group & $<12$ & foaming agent/scale \& corrosion inhibitor combination \\
\hline quaternary ammonium salts & not provided & HAI-85M Acid Inhibitor & Halliburton & $10-30$ & corrosion inhibitor \\
\hline sodium chloride & $7647-14-5$ & HC-2 & Halliburton & $5-10$ & additive \\
\hline sodium persulfate & $7775-27-1$ & SP Breaker & Halliburton & $60-100$ & breaker \\
\hline toluene & $108-88-3$ & WFT 9527 & Weatherford & $3-7$ & flow enhancer \\
\hline triethylamine hydrochloride & $593-81-7$ & EC1385A & Nalco/Exxon Energy Chemicals & $1-5$ & corrosion inhibitor \\
\hline triethylene glycol & $112-27-6$ & \begin{tabular}{|l|} 
Triethylene glycol \\
\end{tabular} & Shell Chemicals & $95.0-99.9$ & not provided \\
\hline 1,2,4-trimethylbenzene & $95-63-6$ & LOSURF-300M & Halliburton & $0-1$ & surfactant \\
\hline xylene & $1330-20-7$ & WFT 9527 & Weatherford & $40-70$ & flow enhancer \\
\hline " & " & MC DF-7120 & Multi-Chem Group & not provided & defoamer/emulsion breaker \\
\hline zirconium complex & not provided & CL-12 & Halliburton & $30-60$ & crosslinker \\
\hline
\end{tabular}


Table SI C4. Specified volumes of "gel" used with $\mathrm{CO}_{2}$ foam during hydraulic fracturing between 2001 and 2002 $(1$ barrel or bbl $=42$ gallons or $159 \mathrm{~L}$ )

\begin{tabular}{|c|c|c|}
\hline Year & Production Well & Volume \\
\hline 2001 & Pavillion Fee 11-3 & $51 \mathrm{bbls}$ \\
\hline$"$ & Pavillion Fee 43-11 & $157 \mathrm{bbls}$ \\
\hline 2002 & Pavillion Fee 41-10 & 96 bbls \\
\hline$"$ & Pavillion Fee 33-11 & $221 \mathrm{bbls}$ \\
\hline "“ & Tribal Pavillion 43-10 & 646 bbls \\
\hline " & Pavillion Fee 24-3B & $169 \mathrm{bbls}$ \\
\hline$" “$ & Pavillion Fee 11-11B & 728 bbls \\
\hline "“ & Pavillion Fee 31-11 & 528 bbls \\
\hline$"$ & Pavillion Fee 23-3 & 92 bbls \\
\hline " & Pavillion Fee 23-3 & 584 bbls \\
\hline " & Pavillion Fee 32-11 & 715 bbls \\
\hline " & Pavillion Fee 44-11 & $491 \mathrm{bbls}$ \\
\hline “" & Pavillion Fee 14-3W & 206 bbls \\
\hline " & Pavillion Fee 13-11B & 363 bbls \\
\hline$"$ & Pavillion Fee 14-3W & $357 \mathrm{bbls}$ \\
\hline
\end{tabular}

Table SI C5. Specified volumes of "WF-125, WF-130, WF-135, and WF-12" polymer slurries with $\mathrm{CO}_{2}$ and $\mathrm{N}_{2}$ foam during hydraulic fracturing from 2001 to 2007 ( 1 barrel or bbl $=42$ gallons or $159 \mathrm{~L}$ )

\begin{tabular}{|c|c|c|c|c|c|c|}
\hline Year & Production Well & $\begin{array}{l}\text { Polymer } \\
\text { Slurry }\end{array}$ & $\begin{array}{l}\text { Use of } 6 \% \\
\text { KCl } \\
\text { solution }\end{array}$ & $\begin{array}{l}\text { Use of } 10 \% \\
\text { Methanol } \\
\text { Solution }\end{array}$ & Foam & Volume of Polymer Slurry (per well) \\
\hline 2001 & Tribal Pavillion 43-1 & $W F-125$ & Yes & No & $\mathrm{CO}_{2}$ & $658 \mathrm{bbls}$ \\
\hline “" & Pavillion Fee 21-10W & $W F-125$ & Yes & No & $\mathrm{CO}_{2}$ & $1420 \mathrm{bbls}$ \\
\hline “" & Tribal Pavillion $12-5$ & $W F-125$ & Yes & Yes & $\mathrm{CO}_{2}$ & 506 bbls \\
\hline "“ & Pavillion Fee 11-11 & $W F-125$ & Yes & Yes & $\mathrm{CO}_{2}$ & $1,471 \mathrm{bbls}$ \\
\hline " & Pavillion Fee 13-15 & $W F-125$ & Yes & Yes & $\mathrm{CO}_{2}$ & 805 bbls \\
\hline "“ & Tribal Pavillion 34-2 & $W F-130$ & Yes & Yes & $\mathrm{CO}_{2}$ & $1,652 \mathrm{bbls}$ \\
\hline " & Tribal Pavillion 32-1 & $W F-130$ & Yes & Yes & $\mathrm{CO}_{2}$ & 912 bbs \\
\hline " & Tribal Pavillion 14-10 & $W F-130$ & Yes & Yes & $\mathrm{CO}_{2}$ & 673 bbls \\
\hline " & Pavillion Fee 34-3R & $W F-130$ & Yes & Yes & $\mathrm{CO}_{2}$ & 718 bbls \\
\hline " & Pavillion Fee 12-11W & $\begin{array}{l}W F-125, \\
W F-130\end{array}$ & Yes & Yes & $\mathrm{CO}_{2}$ & 927 bbls \\
\hline “" & Pavillion Fee 13-11 & $W F-135$ & Yes & Yes & $\mathrm{CO}_{2}$ & $1,155 \mathrm{bbls}$ \\
\hline " & Tribal Pavillion 43-2 & $W F-135$ & Yes & Yes & $\mathrm{CO}_{2}$ & $1,177 \mathrm{bbls}$ \\
\hline 2004 & Tribal Pavillion 43-10B & $W F-12$ & & No & $\mathrm{CO}_{2}$ & $\sim 286$ bbls \\
\hline 2005 & Pavillion Fee 43-11B & $W F-125$ & $?$ & No & $\mathrm{CO}_{2}$ & 362 bbls \\
\hline “ & Pavillion Fee 44-11B & $W F-125$ & $?$ & No & $\mathrm{CO}_{2}$ & 1,472 bbls \\
\hline " & Pavillion Fee 43-11B & $W F-125$ & $?$ & No & $\mathrm{CO}_{2}$ & $1,543 \mathrm{bbls}$ \\
\hline “" & Tribal Pavillion 42-10B & $W F-125$ & $?$ & No & $\mathrm{CO}_{2}$ & $2,141 \mathrm{bbls}$ \\
\hline "“ & Pavillion Fee 22-11B & $W F-125$ & $?$ & No & $\mathrm{CO}_{2}$ & 897 bbls \\
\hline “ & Tribal Pavillion 32-10C & $W F-125$ & $?$ & No & $\mathrm{CO}_{2}$ & 1,564 bbls \\
\hline “" & Pavillion Fee 22-11C & $W F-125$ & $?$ & No & $\mathrm{CO}_{2}$ & $1,446 \mathrm{bbls}$ \\
\hline 2007 & Pavillion Fee 22-4 & $W F-118$ & $?$ & No & $\mathrm{N}_{2}$ & 612 bbls \\
\hline "“ & Tribal Pavillion 42-15 & $\begin{array}{l}W F-118, \\
Y F 116 S T\end{array}$ & $?$ & No & $\mathrm{N}_{2}$ & $\begin{array}{l}1,386 \text { bbls of WF-118 } \\
\text { and } 1,680 \text { bbls of YF116ST }\end{array}$ \\
\hline
\end{tabular}


Table SI C6. Volumes of slickwater used for hydraulic fracturing (1 barrel or bbl = 42 gallons or $159 \mathrm{~L}$ )

\begin{tabular}{|l|l|l|l|}
\hline Year & Production Well & Foam & Volume (per well) \\
\hline 2004 & Tribal Pavillion 43-10B & $\mathrm{CO}_{2}$ & $1,494 \mathrm{bbls}$ \\
\hline & Tribal Pavillion 33-10B & $\mathrm{CO}_{2}$ & $3,432 \mathrm{bbls}$ \\
\hline & Tribal Pavillion 42-10 & $\mathrm{CO}_{2}$ & $122 \mathrm{bbls}$ \\
\hline & Pavillion Fee 13-10 & $\mathrm{CO}_{2}$ & $1,731 \mathrm{bbls}$ \\
\hline & Tribal Pavillion 21-14 & $\mathrm{CO}_{2}$ & $1,353 \mathrm{bbls}$ \\
\hline & Pavillion Fee 31-10B & $\mathrm{CO}_{2}$ & $863 \mathrm{bbls}$ \\
\hline & Pavillion Fee 33-11B & None & $949 \mathrm{bbls}$ \\
\hline & Tribal Pavillion 41-3 & None & $524 \mathrm{bbls}$ \\
\hline & Tribal Pavillion 33-2C & $?$ & $29,873 \mathrm{bbls}$ \\
\hline & Tribal Pavillion 42-3 & None & $201 \mathrm{bbls}$ \\
\hline & Blankebship Fee 4-8 & None & $121 \mathrm{bbls}$ \\
\hline & Tribal Pavillion 12-12 & None & $388 \mathrm{bbls}$ \\
\hline & Pavillion Fee 44-4 & None & $620 \mathrm{bbls}$ \\
\hline & Tribal Pavillion 33-2B & None & $1,442 \mathrm{bbls}$ \\
\hline & Tribal Pavillion 13-2B & None & $1,249 \mathrm{bbls}$ \\
\hline & Pavillion Fee 14-3B & None & $1,380 \mathrm{bbls}$ \\
\hline & Pavillion Fee 41-11B & None & $584 \mathrm{bbls}$ \\
\hline & Tribal Pavillion 21-11B & None & $557 \mathrm{bbls}$ \\
\hline & Pavillion Fee 13-3W & None & $595 \mathrm{bbls}$ \\
\hline & Pavillion Fee 34-3B & None & $139 \mathrm{bbls}$ \\
\hline & Pavillion Fee 41-10B & None & $183 \mathrm{bbls}$ \\
\hline
\end{tabular}

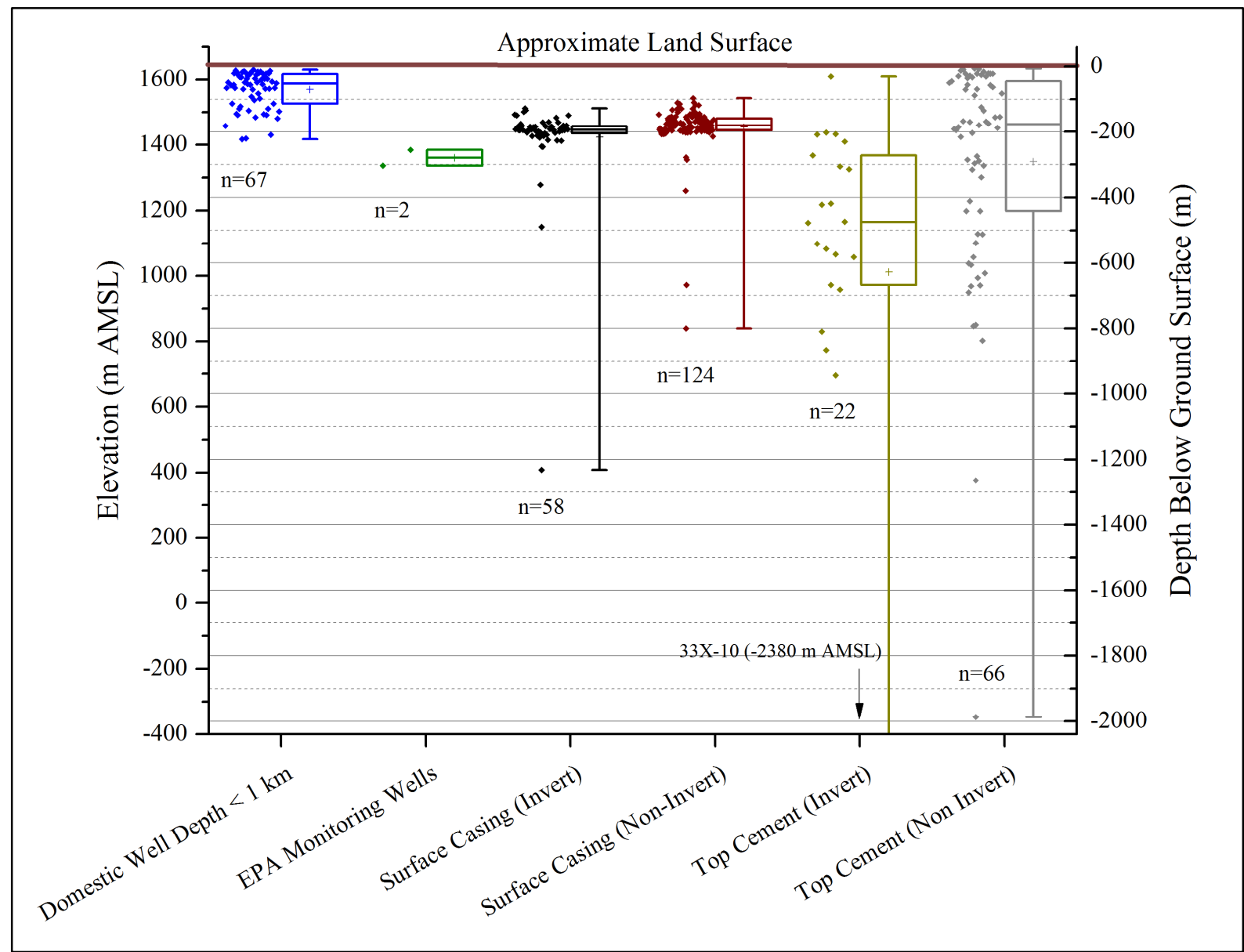

Figure SI C1. Elevation (m) Absolute Mean Sea Level (AMSL) of open intervals of domestic wells $<1 \mathrm{~km}$ from production wells, EPA monitoring wells, surface casing with invert mud, surface casing without invert mud, top of primary cement with invert mud, and top of cement without invert mud. Median land surface elevation $=1636 \mathrm{~m}$ AMSL. 


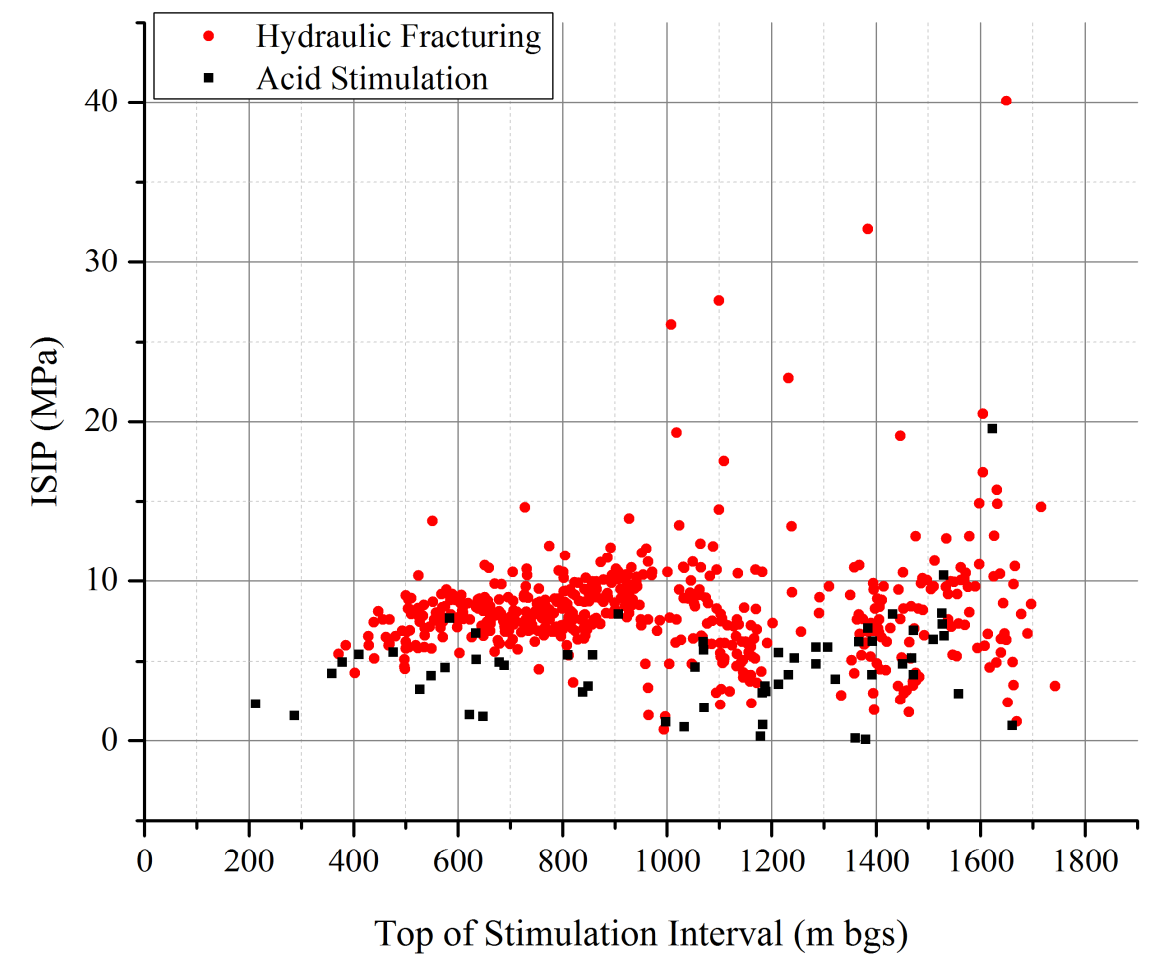

Figure SI C2. Instantaneous Shut-In Pressure (ISIP) values for well stimulation with depth 


\section{D - Extended Discussion on Impact to USDWs and Usable Water from Well Stimulation}

\section{D.1 Examples of Potential Loss of Zonal Isolation (Data Source: Well Completion Reports and Sundry Notices, Seach by API Number in Table SI C1)}

\section{Parted Casing and Cement Squeezes}

Casing failure occurred at five production wells following well stimulation resulting in potential loss of zonal isolation.

\section{$\underline{\text { Blankenship 4-8 }}$}

Blankenship 4-8 was completed in March 1977. The base of surface casing is at $134.4 \mathrm{~m}$ bgs. On 3/26/1977, hydraulic fracturing was conducted at a depth of $1484 \mathrm{~m}$ bgs using "500 gal 15\% BDA frac w/102,000 gallons of gel water...300 scfpb nitrogen." A cement bond log/variable density log (CBL/VDL) conducted at $2.76 \mathrm{MPa}$ (400 psig) on 1/05/2005 indicated top of primary cement at $564 \mathrm{~m}$ bgs with high amplitude to $625 \mathrm{~m}$ bgs. During recompletion activities in January 2005, "a hole” in casing was noted between 312 to $322 \mathrm{~m}(1025-1062 \mathrm{ft}) \mathrm{bgs}$. The time in which the hole in casing formed over a 28-year period between 1977 and 2005 is unknown. Since the base of surface casing is at $134.4 \mathrm{~m} \mathrm{bgs,}$ there was $429 \mathrm{~m}$ of uncemented production casing below surface casing and no cement behind casing at depth of the hole. The potential for migration of stimulation fluid during hydraulic fracturing in 1977 was not assessed. The 1977 well completion report indicates tubing pressure at $4.41 \mathrm{MPa}(640 \mathrm{psig})$ and casing pressure at $9.48 \mathrm{MPa}(1375 \mathrm{psig})$ - well beyond hydrostatic pressure at $312 \mathrm{~m}$ bgs indicating likely gas migration at this depth and to presumably through the uncemented borehole to the depth of surface casing. Two cement squeezes were performed on 1/25/2005 and 1/27/2005 to remediate casing.

On 2/8/2005, 121 barrels of slickwater and $\mathrm{CO}_{2}$ foam in a $6 \% \mathrm{KCl}$ solution was used for hydraulic fracturing at $814 \mathrm{~m} \mathrm{bgs} \mathrm{using,} \mathrm{"slickwater...121-bbls} 6 \% \mathrm{KCl}, 28.5$ tons of $\mathrm{CO} 2$ " at a maximum stimulation pressure of $38.2 \mathrm{MPa}$ (5546 psig). On 2/12/2005, hydraulic fracturing was conducted at a depth of $631 \mathrm{~m}$ bgs using "Frac slickwater, 104 bbls." at a maximum stimulation pressure of $39.4 \mathrm{MPa}$

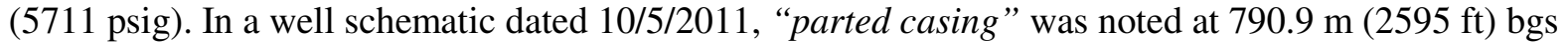
with an assigned date of 12/21/2006. Parted casing, apparently induced during hydraulic fracturing, indicates loss of zonal isolation at $790.9 \mathrm{~m}$ bgs during hydraulic fracturing. There is no record of casing repair at this depth. In a sundry notice dated 11/7/2011, tubing, casing, and Bradenhead pressure were recorded as 0 psig. In a sundry notice dated 3/6/2012, a mechanical integrity test (MIT) conducted with a cast iron bridge plug (CIBP) at $616.9 \mathrm{~m}$ bgs (above parted casing) at $2.07 \mathrm{MPa}$ (300 psig) indicated no bleed off. 
USA Tribal 258 21-15(Tribal 21-15)

USA Tribal 258 21-15 (Tribal 21-15) was completed in December 1979. The base of surface casing is at $188.7 \mathrm{~m}$ bgs. According to the well completion report, a CBL/VDL was conducted. However, the CBL/VDL is not in the well file. Hence, depth to primary cement and length of uncemented production casing below surface casing is unknown. In September 1982 during recompletion, a packer was set at $1004 \mathrm{~m}$ bgs and 1,000 gallons of a $15 \% \mathrm{HCl}$ solution was used for acid stimulation at $799 \mathrm{~m}$ bgs. In October 1982, a cement squeeze was performed above the depth of acid stimulation at $472 \mathrm{~m}$ $(1550 \mathrm{ft}) \mathrm{bgs}$. The reason for a cement squeeze following stimulation was not provided.

On 3/7/2012, a sundry notice indicated that a MIT was conducted at Tribal 21-15 with "failed casing...from 735? -1,105?” [ft]. Failed casing was at a depth (224 m bgs) lacking primary cement. It is unclear when casing failed. On 8/14/2012, the WDEQ requested information from the Bureau of Land Management (BLM) on stimulation practices at Tribal 21-15 to evaluate the potential release of stimulation fluid at $224 \mathrm{~m}$ bgs (within depths of domestic ground water use). In a response dated $8 / 23 / 2012$, BLM reiterated previously publicly available information on well completion at this production well thereby providing no additional insight on the potential for release of stimulation fluid through parted casing. The status of casing remediation at this production well is unknown. Bradenhead testing at this production well was either not conducted or documented.

$\underline{\text { Unit } 41 \mathrm{X}-10}$

Unit 41X-10 was completed in January 1973. The base of surface casing is at $188.7 \mathrm{~m} \mathrm{bgs.} \mathrm{A}$ CBL/VDL conducted on 11/22/1972 indicated top of primary cement at approximately $427 \mathrm{~m}$ bgs. On 2/7/1973, hydraulic fracturing was conducted at a depth of $551.4 \mathrm{~m}$ bgs. From the well completion report, "Frac treated... as follows...9\# KCl wtr containing 5\# J-133/1000 gal...2500 gal 15\% HCl containing 5\# J-133/1000 gal...9120 gal 9\# KCl wtr containing 40\# J-133, 20\# J-110 and 1 gal F-63/1000 gal, 1 \# J-134 and $400 \mathrm{scf} / \mathrm{bbl}$ nitrogen...1460 gal 9\# KCl wtr containing 560\# J-133, 300\# J-110, 15 gal F-63, 2 gal J34 and 139,000 scf nitrogen...flushed w/9\# KCl wtr containing 5\# J-133/1000 gal...Well flowed back approx. 600 BLW since opened to pit...considerable amt of wtr."

On 2/8/1979, hydraulic fracturing was conducted at a depth of $1232.6 \mathrm{~m}$ bgs. While perforating in the Fort Union Formation, "Flowed well to pits continuously...Controlled well w/4\% KCl wtr w/clay stabilizer \& surfactant. Lost 80 bbls to form while doing so...acdz'd gross perf 4044-4450 w/4000 gals $15 \% \mathrm{HCl}$ acid... Opened well to pit \& cont'd to flw to pit overnight to cleanup." "Foam frac treated with 90,000 gals to qualify foam, 1,950,000 SCF nitrogen \& 645 bbls gelled KCl wtr for sd carrying agent. Total load to rec 645 bls." 
In correspondence dated 5/27/1980, 41X-10 was recommended for plugging and abandonment (P\&A) because of "problems with water production and casing failure." P\&A occurred in 1981 with cement plugs. An updated well schematic for Unit 41X-10 appeared in the WOGCC Well Integrity Report $^{31}$. This well schematic did not indicate casing failure. If well casing failure occurred during well stimulation above primary cement, release up through up the uncemented borehole could have occurred at or below the depth of surface casing.

Tribal Pavillion 42-10

Tribal Pavillion 42-10 was competed in June 1994 with acid stimulation at depths of 1448, 1620, 1643, and $1669 \mathrm{~m}$ bgs. Tribal Pavillion was recompleted in June 2001 with hydraulic fracturing in 7 stages between 1005 and $1380 \mathrm{~m}$ bgs. A CBL/VDL conducted on 12/15/2004 indicated top of primary cement at approximately $792 \mathrm{~m}$ bgs. The base of surface casing is at $190.8 \mathrm{~m}$ bgs. Hence, top of primary cement was $601 \mathrm{~m}$ below surface casing.

On 12/15/2004, hydraulic fracturing, (“frac down casing”) was conducted at a depth of $807.1 \mathrm{~m}$ only 15 m below top of primary cement. The well completion report indicated use of "122 bbls slickwater." A stimulation report, one of only three for the entire field, posted on 8/9/2013, indicated use of “4351 gal 70Q CO2 WF12” consisting of “4351 gal water and 34 lb J216.” A Material Safety Data Sheet for "J216" was not provided to EPA. Thus, the chemical composition of this product is unknown.

A cement squeeze was performed on 12/17/2004 (two days after hydraulic fracturing) for "casing remediation" at $774.2 \mathrm{~m}$ bgs (18 m above top of primary cement). If well casing integrity failure occurred during well stimulation, upward migration of stimulation fluid could have occurred above the top of primary cement to some undetermined depth below surface casing.

On 12/20/2004, another CBL/VDL was conducted at 3.45 MPa (500 psig). The CBL/VDL indicated high amplitude readings above $741 \mathrm{~m}$ bgs with chevrons (pipe connections) visible at $655 \mathrm{~m}$ bgs indicating poor casing to cement bonding. On 12/20/2004, 86 barrels of "fluid" was used for hydraulic fracturing at $763 \mathrm{~m}$ bgs ( $22 \mathrm{~m}$ below poor cement).

\section{$\underline{\text { Tribal Pavillion 43-10B }}$}

Tribal Pavillion 43-10B was completed in January 2005. The base of surface casing is at $193.9 \mathrm{~m}$ bgs. A CBL/VDL conducted on 11/8/2004 indicated top of primary cement at $357 \mathrm{~m}$ bgs (163 m below surface casing). In November 2005, hydraulic fracturing was conducted over 13 intervals from $552 \mathrm{~m}$ to $1667 \mathrm{~m}$ bgs using 80,000 gallons of slickwater with $\mathrm{CO}_{2}$ assist and $\mathrm{CO}_{2}$ foam using "WF-12" having a polymer loading of $25 \mathrm{lb} / 1000$ gallons. A cement squeeze was performed subsequent to hydraulic 
fracturing on 1/14/2005 at $326.1 \mathrm{~m}$ bgs (31 m above the top of primary cement) to repair a "casing leak." If the "casing leak" occurred during well stimulation, upward migration of stimulation fluid could have occurred above the top of primary cement to some undetermined depth below surface casing. The last stimulation stage was on 11/11/2004. Hence, gas was free to migrate through casing to $357 \mathrm{~m}$ bgs prior to the cement squeeze more than two months later. Casing and tubing pressure are not provided at the time of well completion.

\section{Cement Squeezes}

Cement squeezes were performed above primary cement after, often days after, hydraulic fracturing without explanation at six production wells. In the absence of an explanation, potential migration of stimulation fluid above primary cement cannot be discounted.

\section{$\underline{\text { Pavillion Fee 12-11B }}$}

Pavillion Fee 12-11B was completed in January 2005 with the base of surface casing at $167.9 \mathrm{~m}$ bgs. A CBL/VDL conducted on 12/29/2004, indicated top of primary cement at $313 \mathrm{~m}$ bgs. On 12/29/2004, 110 barrels of “ $\mathrm{CO}_{2}$ assisted gelled water" was used for hydraulic fracturing at $429 \mathrm{~m}$ bgs. On 1/7/2005, a cement squeeze was performed above primary cement at $283 \mathrm{~m}$ bgs.

\section{$\underline{\text { Pavillion Fee 21-10B }}$}

Pavillion Fee 21-10B was completed in February 2005 with the base of surface casing at $193.2 \mathrm{~m}$ bgs. On 1/6/2005 a CBL/VDL indicated top of primary cement at $\sim 1265 \mathrm{~m}$ bgs (1072 $\mathrm{m}$ below surface casing). Good bonding ("GOBO”) was indicated starting at $1277 \mathrm{~m}$ bgs. On 1/12/2005, 168 barrels of "clean fluid" was used for hydraulic fracturing at $1292 \mathrm{~m}$ bgs - only $15 \mathrm{~m}$ below good bonding. On $1 / 17 / 2005$ and 1/19/2005, days after hydraulic fracturing, cement squeezes were performed above top of primary cement at $1216 \mathrm{~m}$ and $1125 \mathrm{~m}$ bgs, respectively. On 1/24/2005, 247 barrels of "clean fluid" was used for hydraulic fracturing at $1049 \mathrm{~m}$ and $1079 \mathrm{~m}$ bgs. Again, days after hydraulic fracturing, cement squeezes were performed on 1/25/2005 and 1/27/2005 at $928 \mathrm{~m}$ and $244 \mathrm{~m}$ bgs, respectively.

\section{$\underline{\text { Pavillon Fee 34-3B }}$}

Pavillion Fee 34-3B was completed in October 2004 with the base of surface casing at $196.6 \mathrm{~m}$ bgs. In October 2004, a CBL/VDL indicated top of primary cement at $838 \mathrm{~m}$ bgs (641 m below surface casing). In November 2004, “clean fluid" and $\mathrm{CO}_{2}$ foam was used for hydraulic fracturing between 1039 $\mathrm{m}$ and $1637 \mathrm{~m}$ bgs. On 4/3/2005, a cement squeeze was performed at $823 \mathrm{~m}$ bgs. In this instance, a cement squeeze was apparently performed to enable hydraulic fracturing above primary cement. On 
4/19/2005, 139 barrels of slickwater was used for hydraulic fracturing at $951 \mathrm{~m}$ bgs. On 4/21/2005, two days after hydraulic fracturing, another cement squeeze was performed at $256 \mathrm{~m}$ bgs.

\section{$\underline{\text { Pavillion Fee 31-10B }}$}

Pavillion Fee 31-10B was completed in January 2005 with the base of surface casing at $192.0 \mathrm{~m}$ bgs. On 12/29/2004 a CBL/VDL indicated top of primary cement at $411 \mathrm{~m}$ bgs (219 m below surface casing) with high amplitude readings and a "railroad-like" pattern on the VDL extending to $488 \mathrm{~m}$ bgs. On 12/30/2004, 36,000 gallons of slickwater with $\mathrm{CO}_{2}$ assist was used for hydraulic fracturing between $519 \mathrm{~m}$ and $1508 \mathrm{~m}$ bgs. Shortly after hydraulic fracturing, cement squeezes were performed on 1/8/2005, $1 / 13 / 2005$, and 1/18/2005 above primary cement at 381m, 305m, $213 \mathrm{~m}$ bgs, respectively.

Tribal Pavillion 12-12

Tribal Pavillion 12-12 was completed in March 2005 with the base of surface casing at $193.5 \mathrm{~m}$ bgs. A CBL/VDL conducted on 2/28/2005 indicated top of primary cement at $290 \mathrm{~m}$ bgs (97 m below surface casing). On 3/3/2005, 387 barrels of slickwater was used for hydraulic fracturing from $599 \mathrm{~m}$ to $1177 \mathrm{~m}$ bgs. On 3/9/2005, cement squeeze performed above primary cement at $259 \mathrm{~m}$ bgs.

Pavillion Fee 11-11B

Pavillion Fee 11-11B was completed in February 2002 with production casing cemented to the surface. However, a CBL/VDL conducted on 2/8/2002 indicated poor or spotty cement from 320 to 457 $\mathrm{m}$ bgs and production casing without cement between $777 \mathrm{~m}-960 \mathrm{~m}$ bgs. This extensive interval uncemented production casing without cement was noted in the well completion report. On 2/18/2005, 232 barrels of "clean fluid" was used for hydraulic fracturing at $964 \mathrm{~m}$ bgs - only $4 \mathrm{~m}$ below this interval. A maximum stimulation pressure of only 1.3 MPa (190 psig) was noted indicating little resistance to flow and likely upward flow of stimulation fluid into the uncemented region.

\section{D.2 Omissions to Bradenhead Testing}

Bradenhead testing was not conducted at John K. Coolidge 1-4, Fike Tribal A-1, Tribal NP 3111X, Tribal Pavillion 44-3, Tribal Pavillion 15-21X, and Tribal 21-14.

Pressure build-up following detection of brandenhead pressure was not measured at Ora Wells 14-12, Tribal Pavillion 24X-3, Tribal Pavillion 11-10, Tribal Pavillion 23-11, Pavillion Fee 42X-9, Tribal Pavillion 43-2, Pavillion Fee 34-11, Pavillion Fee 34-11, Pavillion Fee 21-10W, Pavillion Fee 11-3, Pavillion Fee 34-3B, and Tribal Pavillion 24-2. 
Gas samples at well exhibing bradenhead pressure were not collected at Tribal Pavillion 31-15, IND 14-20-258-41X-2, Tribal Pavillion 33-2, Tribal Pavillion 44-1, Pavillion Fee 44-3C, Tribal Pavillion 32-10, Tribal Pavillion 44-1, and Pavillion Fee 21-10.

D.3 Summary of Bradenhead Testing with Water Flow

Water flow to the surface during Bradenhead testing occurred at 4 production wells. A summary of events, including information from well completion reports, cement bond/variable density logs (CBL/VDLs), and Sundry Notices is provided below.

\section{$\underline{\text { Pavillion Fee 12-11W }}$}

Pavillion Fee 12-11W was completed in May 2001 with the base of surface casing at $131.1 \mathrm{~m}$ bgs. A CBL/VDL conducted on 4/21/2001 at Pavillion Fee 12-11W indicated top of primary cement at 594 m bgs (463 m below surface casing). During Bradenhead testing in July 2012, 4,303 barrels ( 180,700 gallons) of water flowed to the surface over a 15-day period at Pavillion 12-11W. Bradenhead pressures after 24-hour and 7-day shut-in periods were 85 and $979 \mathrm{kPa}$ (142 psig), respectively. On 10/3/2012, a mechanical integrity test (MIT) was conducted above $764 \mathrm{~m} \mathrm{bgs}$ (top perforation at $771 \mathrm{~m}$ bgs) at 3.4 MPa (500 psig) for 30 minutes - holding pressure without loss, verifying integrity of production well casing. Hence, the source of water was aqueous flow outside production casing at one or more depths. On 5/17/2013, cement squeezes were performed at $146 \mathrm{~m}$ and $442 \mathrm{~m}$ bgs.

\section{$\underline{\text { Tribal Pavillion 13-2 }}$}

Tribal Pavillion 13-2 was completed in March 2001 with the base of surface at $123.1 \mathrm{~m}$ bgs. A CBL/VDL conducted on 2/23/2001 at Tribal Pavillion 13-2, completed in March 2001, indicated top of primary cement at $198 \mathrm{~m}$ bgs (75 m below surface casing). During Bradenhead testing in January 2012, pressure after a 7-day shut-in period was $1.45 \mathrm{MPa}$ (210 psig). During testing in July 2012, 3,785 barrels ( 159,000 gallons) of water flowed to the surface over a 15-day period. Bradenhead pressures during a 7day shut-in period was $0.965 \mathrm{MPa}(140 \mathrm{psig})$.

$\underline{\text { Tribal Unit 44-10 }}$

Tribal Unit 44-10 was completed in December 1979 with the base of surface casing at $184.4 \mathrm{~m}$ bgs. A CBL/VDL conducted on 7/23/1979 at Tribal Unit 44-10, completed in December 1979, indicated top of primary cement at $\sim 585 \mathrm{~m}$ bgs (401 m below surface casing). During Bradenhead testing in May 2012, 410 barrels (17,200 gallons) of water flowed to the surface during the first 8 hours of a 15-day test period. Bradenhead pressure during subsequent a subsequent 7-day shut-in period was $0.724 \mathrm{MPa}$ (105 psig). 


\section{Tribal Pavillion 13-1}

Tribal Pavillion 13-1 was completed in June 2001 with the base of surface casing at $163.1 \mathrm{~m}$ bgs. A CBL/VDL was not conducted. Thus of primary cement is unkown. During Bradenhead testing in July 2012, 6 barrels ( 250 gallons) of water flowed to surface during the first hour of the 15-day testing period. Subsequent Bradenhead pressure after a 7-day shut-in period was $0.689 \mathrm{MPa}$ (100 psig). 


\section{D.4 Summary of Major Ion Concentrations in Produced and Bradenhead Water}

Table SI D1. Major Ions in Produced Water. Data obtained from sundry notices. NA for 33X-10 denotes not analyzed. Bradenhead samples denoted with (B) suffix

\begin{tabular}{|c|c|c|c|c|c|c|c|c|c|c|c|c|c|c|c|c|}
\hline $\begin{array}{l}\text { Well } \\
\text { Abbrev }\end{array}$ & Stimulation Dates & Stimulation Fluids & $\begin{array}{l}\text { Prod } \\
\text { Water } \\
\text { (bbls) }\end{array}$ & $\begin{array}{l}\text { Sample } \\
\text { Date }\end{array}$ & $\mathrm{pH}$ & $\begin{array}{l}\mathrm{SC} \\
(\mu \mathrm{S} / \mathrm{cm})\end{array}$ & $\begin{array}{l}\mathrm{Na} \\
(\mathrm{mg} / \mathrm{l})\end{array}$ & $\begin{array}{l}\mathbf{K} \\
(\mathrm{mg} / \mathrm{l})\end{array}$ & $\begin{array}{l}\mathrm{Ca} \\
(\mathrm{mg} / \mathrm{l})\end{array}$ & $\begin{array}{l}\mathrm{Mg} \\
(\mathrm{mg} / \mathrm{l})\end{array}$ & $\begin{array}{l}\text { Cl } \\
(\mathbf{m g} / \mathbf{l})\end{array}$ & $\begin{array}{l}\text { SO4 } \\
(\mathrm{mg} / \mathrm{l})\end{array}$ & $\begin{array}{l}\mathrm{Fe} \\
(\mathrm{mg} / \mathrm{l})\end{array}$ & $\begin{array}{l}\mathrm{CO3} \\
(\mathrm{mg} / \mathrm{l})\end{array}$ & $\begin{array}{l}\mathrm{HCO3} \\
(\mathrm{mg} / \mathrm{l})\end{array}$ & $\begin{array}{l}\text { TDS } \\
(\mathrm{mg} / \mathrm{l})\end{array}$ \\
\hline $12-1$ & Nov 2001 & $\mathrm{KCl}$ solution and $\mathrm{CO} 2$ & 653 & $8 / 8 / 2007$ & 6.86 & 5,019 & 1323 & 270 & 71 & 30 & 1,753 & 34 & 48.45 & 0 & 903 & 4,260 \\
\hline $14-1$ & $\begin{array}{l}\text { Oct 1964, Apr 1993, } \\
\text { Dec 1999 }\end{array}$ & $\begin{array}{l}\text { Undiluted diesel fuel, } \mathrm{CO} 2 \text { with methanol, } 15 \% \mathrm{HCl} \\
\text { solution }\end{array}$ & 2,735 & $8 / 14 / 2007$ & 7.15 & 3,996 & 785 & 160 & 205 & 29 & 956 & 49 & 190.60 & 0 & 902 & 3,325 \\
\hline $22-1$ & Nov 2001 & $\mathrm{CO} 2$ foam & 892 & $8 / 8 / 2007$ & 7.71 & 3,389 & 945 & 34 & 44 & 11 & 882 & 9 & 12.07 & 0 & 1,001 & 2,771 \\
\hline $24-1$ & Mar 2001 & No information on stimulation & 817 & $8 / 8 / 2007$ & 8.71 & 3,186 & 970 & 112 & 12 & 7 & 985 & 7 & 4.81 & 68 & 978 & 2,585 \\
\hline $33-1$ & Sep 2001 & No information on stimulation & 3,176 & $8 / 8 / 2007$ & 7.70 & 3,684 & 916 & 78 & 46 & 13 & 821 & 12 & 8.67 & 0 & 907 & 3,040 \\
\hline $43-1$ & Aug 2001 & $\mathrm{WF} 125$ in $6 \% \mathrm{KCl}$ solution and $\mathrm{CO} 2$ foam & 8,500 & $8 / 8 / 2007$ & 7.73 & 2,322 & 667 & 21 & 15 & 4 & 503 & 5 & 1.97 & 10 & 919 & 1,796 \\
\hline $44-1$ & Mar 2001 & No information on stimulation & 1375 & $8 / 8 / 2007$ & 7.51 & 3,934 & 1051 & 94 & 39 & 13 & 1,263 & 8 & 13.87 & 0 & 737 & 3,269 \\
\hline $24-2$ & Oct 2004 & "clean fluid" and $\mathrm{CO} 2$ & 174 & $8 / 13 / 2007$ & 8.28 & 4,940 & 1,479 & 202 & 29 & 14 & 1,774 & 7 & 0.25 & 7 & 1,235 & 4,188 \\
\hline $32-2$ & Mar 2006 & $6 \% \mathrm{KCl}$ solution, $\mathrm{CO} 2$, surfactant & 4450 & $8 / 13 / 2007$ & 6.01 & 3,823 & 768 & 268 & 67 & 14 & 817 & 11 & 57.90 & 0 & 807 & 3,167 \\
\hline $33-2$ & Oct 1994, Oct 2000 & $15 \% \mathrm{HCl}$ solution, "clean fluid", N2 & 1,263 & $8 / 13 / 2007$ & 8.63 & 2,703 & 769 & 57 & 18 & 8 & 853 & 6 & 31.58 & 20 & 614 & 2,144 \\
\hline $11-3$ & Dec 2001 & $6 \% \mathrm{KCl}$ solution, $\mathrm{CO} 2$ foam, gel & 1,895 & $8 / 13 / 2007$ & 8.35 & 2,107 & 596 & 45 & 10 & 3 & 366 & 8 & 2.63 & 14 & 963 & 1,600 \\
\hline $13-3 \mathrm{~W}$ & Apr 2005 & slickwater & 1340 & $8 / 20 / 2007$ & 6.61 & 5,702 & 1060 & 233 & 25 & 21 & 1,315 & 485 & 165.05 & 0 & 899 & 4,884 \\
\hline $14-3 \mathrm{~W}$ & Apr 2002 & $6 \% \mathrm{KCl}$ solution, $\mathrm{CO} 2$, gel & 5,695 & $8 / 14 / 2007$ & 8.20 & 4,813 & 1538 & 88 & 7 & 7 & 1,089 & 8 & 4.11 & 12 & 2,243 & 4,072 \\
\hline $24 \mathrm{X}-3$ & Nov 1965, Jun 1966 & "P-12 solvent" & 9653 & $8 / 14 / 2007$ & 8.26 & 5,159 & 1572 & 47 & 2 & 3 & 1,094 & 17 & 5.95 & 11 & 1,094 & 4,388 \\
\hline $31 \mathrm{X}-3$ & Aug 2001 & unknown & 1,215 & $8 / 13 / 2007$ & 8.09 & 2,512 & 647 & 153 & 13 & 6 & 595 & 34 & 0.00 & 0 & 802 & 1,970 \\
\hline $32-3$ & May 2005 & $6 \% \mathrm{KCl}$ solution, $\mathrm{CO} 2$ & 50,290 & $8 / 13 / 2007$ & 8.13 & 3,706 & 1264 & 17 & 13 & 3 & 1,194 & 4 & 4.82 & 35 & 1,072 & 3,060 \\
\hline $33-3$ & May 1999 & No information on stimulation & 337 & $8 / 13 / 2007$ & 8.99 & 3,270 & 1072 & 53 & 7 & 4 & 574 & 43 & 5.00 & 159 & 1,496 & 2,662 \\
\hline $44-3$ & Apr 1999 & $15 \% \mathrm{HCl}$ solution, $2 \% \mathrm{KCl}$ solution & 1,663 & $8 / 13 / 2007$ & 8.34 & 2,364 & 697 & 20 & 19 & 4 & 387 & 5 & 4.48 & 11 & 1,113 & 1,834 \\
\hline $1-4$ & May 1982 & $\begin{array}{l}15 \% \mathrm{HCl} \text { soln, } 3 \% \mathrm{HF} \text { soln, } \mathrm{NH} 4 \mathrm{Cl} \text { solution, foam. } \\
\text { Possible recompletion in } 2005\end{array}$ & 10,417 & $8 / 20 / 2007$ & 8.10 & 2,438 & 608 & 81 & 22 & 6 & 585 & 135 & 2.00 & 0 & 788 & 1,902 \\
\hline $42-4 \mathrm{~B}$ & Apr 2005 & $6 \% \mathrm{KCl}$ solution, $\mathrm{CO} 2$, "clean fluid" & 8,857 & $8 / 20 / 2007$ & 8.13 & 2,252 & 615 & 34 & 25 & 7 & 603 & 17 & 1.75 & 0 & 763 & 1,732 \\
\hline $43-4$ & Mar 2005 & $70 \mathrm{Q}$ cross-linked gel & 19,681 & $8 / 20 / 2007$ & 8.30 & 3,343 & 1044 & 59 & 12 & 4 & 915 & 16 & 6.57 & 0 & 1,402 & 2,729 \\
\hline $12-5$ & May 2001 & $\begin{array}{l}\text { WF125 in } 6 \% \mathrm{KCl} \text { and } 10 \% \text { methanol solution, } \mathrm{CO} 2 \\
\text { foam }\end{array}$ & 3402 & $8 / 8 / 2007$ & 8.88 & 2,874 & 903 & 87 & 3 & 3 & 797 & 2 & 1.17 & 100 & 1,067 & 2,300 \\
\hline $12-7$ & Sep 2001 & No information on stimulation & 118 & $8 / 8 / 2007$ & 5.93 & 10,022 & 2379 & 462 & 284 & 102 & 4,365 & 22 & 46.00 & 0 & 432 & 8,830 \\
\hline $41-9$ & Aug 1979, Oct 2004 & $\begin{array}{l}6 \% \mathrm{KCl} \text { solution, } 70 \mathrm{Q} \mathrm{CO} 2 \mathrm{WF} 125,7 \text { 1/2\% } \mathrm{HCl} \\
\text { solutions }\end{array}$ & 15,593 & $8 / 20 / 2007$ & 8.29 & 2,342 & 654 & 30 & 18 & 5 & 693 & 6 & 3.94 & 17 & 615 & 1,814 \\
\hline $42 \mathrm{X}-9$ & Feb 1999 & No information on stimulation & 10,083 & $8 / 20 / 2007$ & 8.15 & 7,739 & 2247 & 106 & 9 & 7 & 793 & 13 & 13.72 & 0 & 4,301 & 6,745 \\
\hline $12-10$ & Oct 2001 & WF125, $6 \% \mathrm{KCl}$ solution, $\mathrm{CO} 2$ foam & 1,465 & $8 / 20 / 2007$ & 8.08 & 2,876 & 827 & 90 & 10 & 6 & 808 & 9 & 7.52 & 29 & 1,012 & 2,302 \\
\hline $14-10$ & Apr 2001 & $\begin{array}{l}6 \% \mathrm{KCl} \text { and } 10 \% \text { methanol solution with additives } \\
\text { and } \mathrm{CO} 2\end{array}$ & 3,225 & 9/19/2007 & 6.30 & 6,661 & 1770 & 116 & 284 & 41 & 1,202 & 1,960 & 150.90 & 0 & 1,739 & 5,760 \\
\hline $23-10 \mathrm{~W}$ & Dec 2002 & $\mathrm{KCl}$ solution, $\mathrm{CO} 2$ & 5,892 & $8 / 20 / 2007$ & 8.00 & 3,742 & 1054 & 86 & 12 & 5 & 694 & 46 & 4.46 & 0 & 1,783 & 3,093 \\
\hline $31-10$ & Jun 1994, Jan 2002 & $6 \% \mathrm{KCl}$ solution, $\mathrm{CO} 2$ foam, $71 \frac{1}{2} \mathrm{HCl}$ solution & 15,504 & $8 / 20 / 2007$ & 8.26 & 4,639 & 1497 & 25 & 13 & 6 & 1,044 & 6 & 14.39 & 12 & 2,025 & 3,913 \\
\hline $32-10 \mathrm{~B}$ & Mar 2002 & $6 \% \mathrm{KCl}$ solution, $\mathrm{CO} 2$ & 4,727 & $8 / 14 / 2007$ & 8.21 & 5,063 & 1593 & 17 & 10 & 6 & 1,022 & 4 & 3.36 & 10 & 2,152 & 4,300 \\
\hline $32-10 \mathrm{C}$ & Feb 2005 & WF125 with $\mathrm{CO} 2$ & 7,099 & $8 / 20 / 2007$ & 8.05 & 1,779 & 421 & 15 & 15 & 3 & 131 & 175 & 1.82 & 0 & 630 & 1,300 \\
\hline $33 \mathrm{X}-10$ & Feb 1965 & "MCA", $15 \% \mathrm{HCl}$ solution & ? & $3 / 29 / 1965$ & NA & NA & 1298 & NA & 11 & 3 & 940 & 305 & NA & 72 & 1,342 & 3,971 \\
\hline $33-10$ & Apr 1999 & $6 \% \mathrm{KCl}$ solution, $\mathrm{CO} 2$, linear gel, $15 \% \mathrm{HCl}$ solution & 2,547 & $8 / 20 / 2007$ & 7.62 & 4,409 & 1220 & 218 & 12 & 6 & 894 & 46 & 5.03 & 0 & 2,254 & 3,703 \\
\hline $41-10$ & Jan 2002 & $6 \% \mathrm{KCl}$ solution, $\mathrm{CO} 2$ foam & 157 & $8 / 14 / 2007$ & 5.90 & 21,177 & 2499 & 1,911 & 1,771 & 131 & 5,945 & 2,063 & 70.60 & 0 & 3,358 & 19,020 \\
\hline $43-10$ & Sep 1994, Feb 2002 & $6 \% \mathrm{KCl}$ solution, $\mathrm{CO} 2$ foam, gel & 28,467 & $8 / 28 / 2007$ & 6.96 & 5,064 & 1656 & 57 & 6 & 3 & 1,280 & 292 & 71.95 & 0 & 1,873 & 4,301 \\
\hline $11-11$ & May 2001 & $\begin{array}{l}\text { WF125 in } 6 \% \mathrm{KCl} \text { and } 10 \% \text { methanol with } \mathrm{CO} 2 \text { foam } \\
\text { and additives }\end{array}$ & 9,051 & $8 / 13 / 2007$ & 7.08 & 2477 & 656 & 68 & 30 & 5 & 432 & 12 & 59.80 & 0 & 1,048 & 1,938 \\
\hline $12-11$ & Oct 1993, Nov 2004 & $2 \% \mathrm{KCl}$ solution, $\mathrm{CO} 2$ foam & 8,451 & $8 / 14 / 2007$ & 7.11 & 2,576 & 753 & 120 & 22 & 6 & 411 & 135 & 50.25 & 0 & 1,062 & 2,028 \\
\hline $12-11 \mathrm{~B}$ & Dec 2004 & $\mathrm{CO} 2$ with gelled water & 731 & $8 / 14 / 2007$ & 8.45 & 2,941 & 816 & 194 & 11 & 9 & 633 & 7 & 5.66 & 31 & 1,296 & 2,362 \\
\hline $21-11$ & Mar 1979 & $\begin{array}{l}2,3 \text { and } 71 / 2 \% \mathrm{HCl} \text { solutions with additives, } \\
\text { "YE4P5D fluid" }\end{array}$ & 335 & $8 / 13 / 2007$ & 7.61 & 3,304 & 938 & 123 & 40 & 11 & 1,079 & 9 & 27.45 & 0 & 762 & 2,693 \\
\hline $44-11$ & Feb 2002 & $6 \% \mathrm{KCl}$ solution with $\mathrm{CO} 2$ foam and gel & 1,643 & $8 / 28 / 2007$ & 5.59 & 2,140 & 510 & 29 & 39 & 3 & 363 & 255 & 109.20 & 0 & 647 & 1,630 \\
\hline $33-12$ & Mar 2006 & $6 \% \mathrm{KCl}$ solution, $\mathrm{CO} 2$, "clean fluid" & 5520 & $8 / 14 / 2007$ & 7.98 & 2,718 & 745 & 84 & 18 & 8 & 611 & 43 & 4.47 & 0 & 993 & 2,158 \\
\hline $42 \mathrm{X}-12$ & Jan 1974 & $15 \% \mathrm{HCl}$ solution, Possible recompletion in 2004 & 295 & $8 / 14 / 2007$ & 7.20 & 1,619 & 303 & 144 & 23 & 3 & 545 & 14 & 13.30 & 0 & 90 & 1,154 \\
\hline $21-14$ & Dec 2004 & Slickwater with $\mathrm{CO} 2$ & 1,886 & $8 / 28 / 2007$ & 7.53 & 5,242 & 1361 & 253 & 55 & 15 & 1,522 & 7 & 4.80 & 0 & 1,431 & 4,464 \\
\hline $13-1$ (B) & & & & $4 / 26 / 2012$ & 10.86 & 33196 & 2700 & 6000 & 39 & 1.6 & 88 & 2100 & 1.67 & 17000 & $<2$ & 30000 \\
\hline $13-2(\mathbf{B})$ & & & & $4 / 24 / 2012$ & 9.57 & 1341 & 230 & 3.7 & 32 & 2.7 & 53 & 170 & 7.36 & 110 & 290 & 900 \\
\hline $44-10$ (B) & & & & $4 / 23 / 2012$ & 8.64 & 903 & 280 & 9.6 & 15 & 2.3 & 7.1 & 16 & 6.45 & 40 & 240 & 500 \\
\hline 12-11W (B) & & & & $4 / 24 / 2012$ & 9.16 & 1056 & 180 & 3.1 & 7.4 & 2.5 & 67 & 130 & 4.88 & 37 & 210 & 640 \\
\hline
\end{tabular}




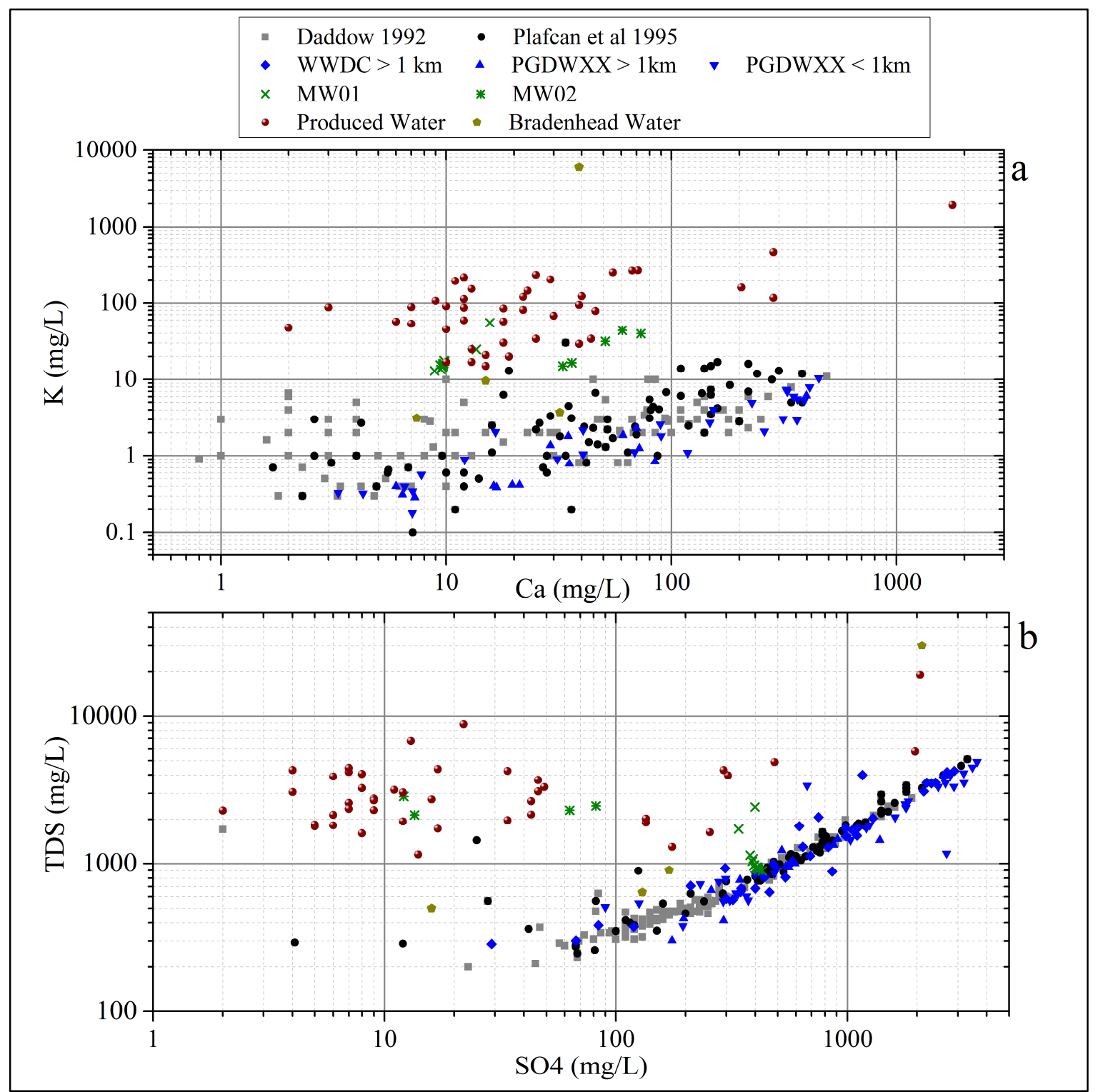

Figure SI D1. (a) Potassium concentration as a function of calcium concentration and (b) total dissolved solids (TDS) concentration as a function of sulfate concentration for the Wind River Indian Reservation (Daddow 1992) Fremont County (Plafcan et al. 1995) ${ }^{26}$, domestic wells sampled by the Wyoming Water Development Commission (WWDC) greater than $1 \mathrm{~km}$ from production wells, domestic wells (PGDWXX) less than and greater than $1 \mathrm{~km}$ from production wells, EPA monitoring wells MW01 and MW02, produced water samples, and bradenhead water samples. Mean values are represented for domestic well locations sampled more than once. Produced water and bradenhead locations were sampled once. Measurement at MW01 and MW02 represent samples collected during Phase III, IV, and V sample events to illustrate variability. TDS values for PGDWXX wells estimated from Daddow (1995) TDS $(\mathrm{mg} / \mathrm{L})=0.785 *$ specific conductance $(\mu \mathrm{S} / \mathrm{cm})-130\left(\mathrm{n}=151, \mathrm{r}^{2}=0.979\right)$ 


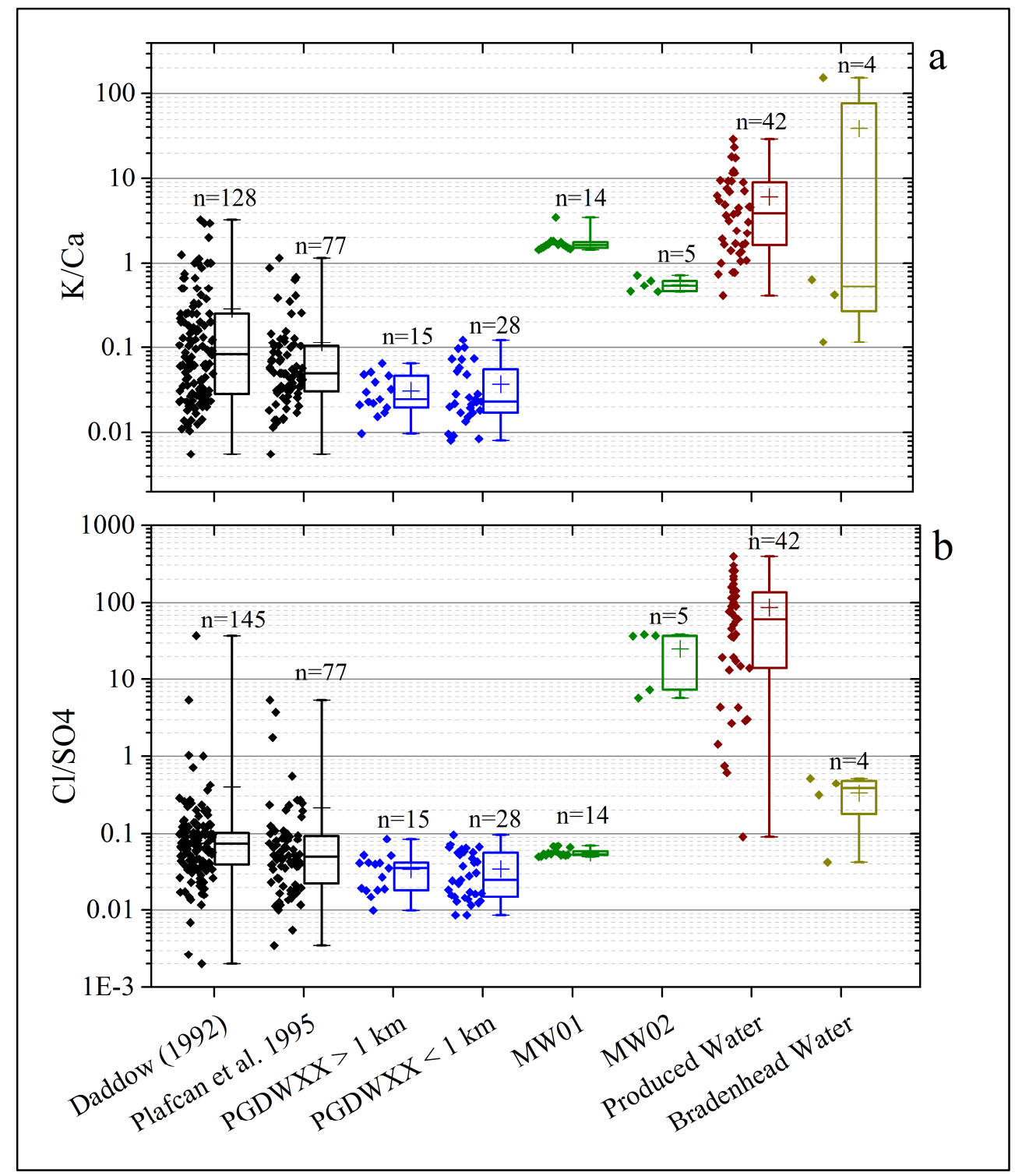

Figure SI D2. (a) Ratio of potassium to calcium and (b) chloride to sulfate concentrations for the Wind River Indian Reservation (Daddow 1992) ${ }^{5}$, Fremont County (Plafcan et al. 1995) ${ }^{26}$, domestic wells (PGDWXX) less than and greater than $1 \mathrm{~km}$ from production wells, EPA monitoring wells MW01 and MW02, produced water samples, and bradenhead water samples. Mean values are represented for domestic well locations sampled more than once.

Produced water and bradenhead locations were sampled once. Measurement at MW01 and MW02 represent samples collected during Phase III, IV, and V sample events to illustrate variability. 
D.5 Summary of Fixed Gases, Light Hydrocarbon, and BTEX Analyses in String and Bradenhead Gas

Table SI D2. String gas and bradenhead gas samples collected at production wells having sustained casing pressure. Concentrations in mole fractions $\mathrm{x}$ 100. Data obtained using API number in Table SI C1

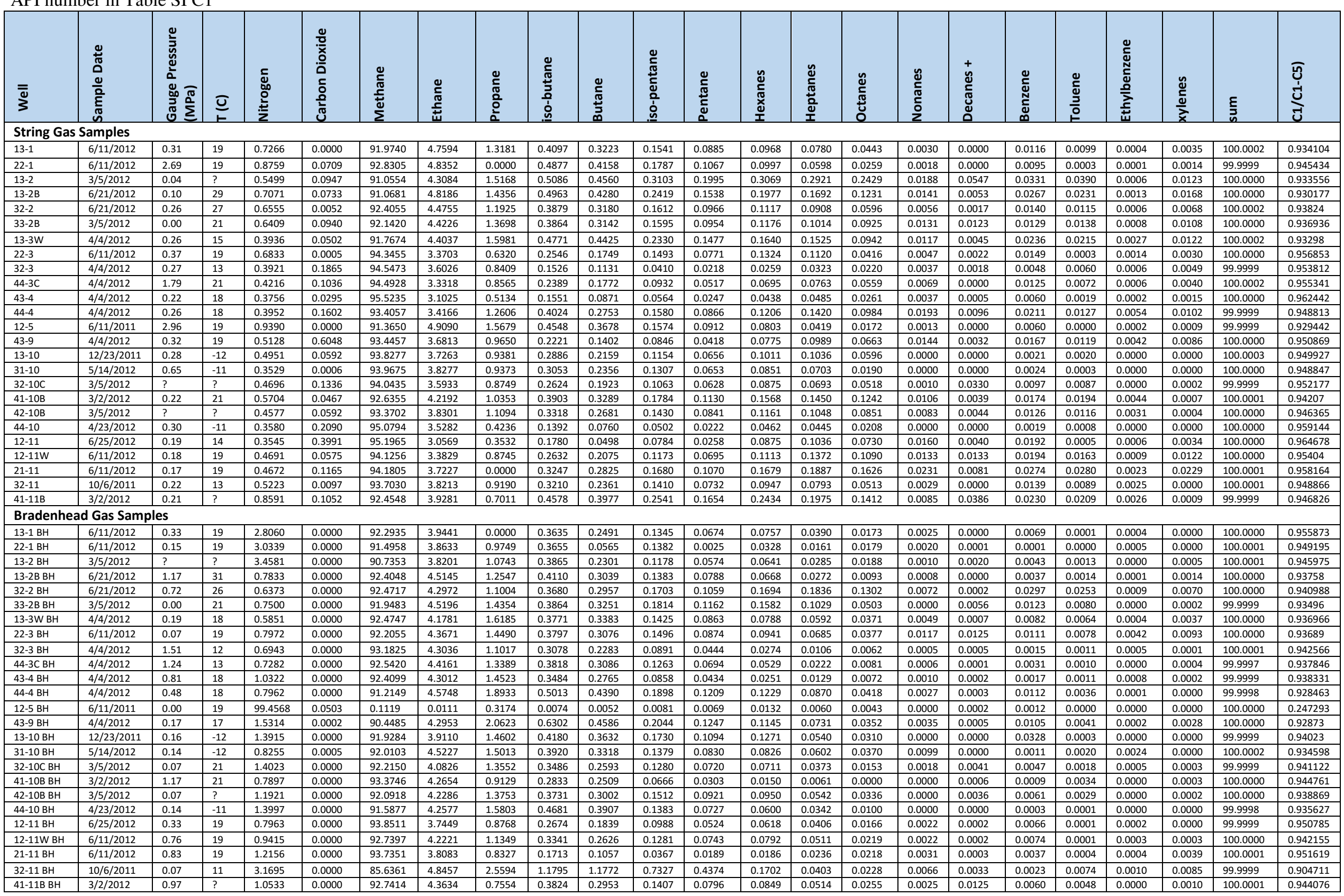




\section{D.6 Summary of Organic Compounds Detected in Produced Water Samples}

Table SI D3. Summary of organic compounds detected in produced water by EPA Region 8 and Zymax (contractor to $\left.\mathrm{EPA}^{30}\right)$. PGPPXX are EPA sample identification codes.

\begin{tabular}{|c|c|c|c|c|c|c|c|}
\hline & $\begin{array}{l}\text { Tribal } \\
\text { Pavillion } \\
\text { 14-10 } \\
\text { (PGPP01) } \\
\text { EPA R8 }\end{array}$ & $\begin{array}{l}\text { Tribal } \\
\text { Pavillion } \\
\text { 24-2 } \\
\text { (PGPP04P) } \\
\text { EPA R8 }\end{array}$ & $\begin{array}{l}\text { Tribal } \\
\text { Pavillion } \\
\text { 24-22 } \\
\text { (PGPP04W) } \\
\text { Zymax }\end{array}$ & $\begin{array}{l}\text { Tribal } \\
\text { Pavillion } \\
\text { 33-10 } \\
\text { (PGPP05) } \\
\text { EPA R8 }\end{array}$ & $\begin{array}{l}\text { Tribal } \\
\text { Pavillion } \\
\text { 33-10 } \\
\text { (PGPP05) } \\
\text { Zymax }\end{array}$ & $\begin{array}{l}\text { Tribal } \\
\text { Pavillion } \\
\text { 14-2 } \\
\text { (PGPP06) } \\
\text { EPA R8 }\end{array}$ & $\begin{array}{l}\text { Tribal } \\
\text { Pavillion } \\
\text { 14-2 } \\
\text { (PGPP06) } \\
\text { Zymax }\end{array}$ \\
\hline \multicolumn{8}{|l|}{ Volatile Analysis (mg/L) } \\
\hline Methylene chloride & $0.51 \mathrm{~J}$ & $<50 \mathrm{U}$ & NA & $<0.025 \mathrm{U}$ & NA & $<0.025 \mathrm{U}$ & NA \\
\hline Benzene & $8.02 \mathrm{~J}$ & $860 \mathrm{~J}$ & 2.053 & $0.306 \mathrm{~J}$ & 0.379 & $3.02 \mathrm{~J}$ & 1.953 \\
\hline Toluene & $97.5 \mathrm{~J}$ & $16800 \mathrm{~J}$ & 11.329 & $0.774 \mathrm{~J}$ & 1.284 & $9.07 \mathrm{~J}$ & 7.288 \\
\hline Ethylbenzene & $26.6 \mathrm{~J}$ & $4410 \mathrm{~J}$ & 0.632 & $0.476 \mathrm{~J}$ & 0.579 & $0.542 \mathrm{~J}$ & 0.262 \\
\hline m,p-Xylene & $298 \mathrm{~J}$ & $46000 \mathrm{~J}$ & 11.513 & $2.18 \mathrm{~J}$ & 3.684 & $4.76 \mathrm{~J}$ & 4.292 \\
\hline o-Xylene & $73.6 \mathrm{~J}$ & $9430 \mathrm{~J}$ & 2.64 & $0.797 \mathrm{~J}$ & 1.307 & $1.37 \mathrm{~J}$ & 1.1 \\
\hline 1,2,4-Trimethylbenzene & $31.6 \mathrm{~J}$ & $8730 \mathrm{~J}$ & 0.695 & $1.77 \mathrm{~J}$ & 2.621 & $0.765 \mathrm{~J}$ & 0.632 \\
\hline 1,3,5-Trimethylbenzene & $18.6 \mathrm{~J}$ & $6250 \mathrm{~J}$ & 0.465 & $0.818 \mathrm{~J}$ & 1.566 & $0.414 \mathrm{~J}$ & 0.366 \\
\hline n-Butyl Benzene & $1.06 \mathrm{~J}$ & $162 \mathrm{~J}$ & $<0.005$ & $0.218 \mathrm{~J}$ & 0.179 & $<0.025 \mathrm{U}$ & $<0.050$ \\
\hline sec-Butylbenzene & $0.95 \mathrm{~J}$ & $270 \mathrm{~J}$ & 0.007 & $0.243 \mathrm{~J}$ & 0.655 & $<0.025 \mathrm{U}$ & 0.062 \\
\hline tert-Butylbenzene & $0.25 \mathrm{~J}$ & $86 \mathrm{~J}$ & NA & $<0.025 \mathrm{U}$ & NA & $<0.025 \mathrm{U}$ & NA \\
\hline n-Propyl Benzene & $3.64 \mathrm{~J}$ & $1290 \mathrm{~J}$ & 0.13 & $0.198 \mathrm{~J}$ & 0.803 & $0.07 \mathrm{~J}$ & 0.11 \\
\hline Isopropylbenzene & $11.4 \mathrm{~J}$ & $948 \mathrm{~J}$ & 0.542 & $0.202 \mathrm{~J}$ & 3.427 & $0.058 \mathrm{~J}$ & 0.337 \\
\hline p-Isopropyltoluene & $1.64 \mathrm{~J}$ & $334 \mathrm{~J}$ & NA & $0.222 \mathrm{~J}$ & NA & $<0.025 \mathrm{U}$ & NA \\
\hline Naphthalene & $3.43 \mathrm{~J}$ & $<200 \mathrm{U}$ & $<0.005 \mathrm{U}$ & $2.97 \mathrm{~J}$ & $<0.050$ & $0.21 \mathrm{~J}$ & $<0.050$ \\
\hline Adamantane & $0.52 \mathrm{~J}$ & $74 \mathrm{~J}$ & NA & $0.305 \mathrm{~J}$ & NA & $<0.025 \mathrm{U}$ & NA \\
\hline 1,3-Dimethyl adamantane & $0.46 \mathrm{~J}$ & $<50 \mathrm{U}$ & NA & $0.488 \mathrm{~J}$ & NA & $<0.025 \mathrm{U}$ & NA \\
\hline \multicolumn{8}{|c|}{ Semivolatile Analysis (mg/L) } \\
\hline Phenol & NA & $<2.0 \mathrm{U}$ & NA & $<2.0 \mathrm{U}$ & NA & $6.96 \mathrm{~J}$ & NA \\
\hline 2-Methylphenol & NA & $<2.0 \mathrm{U}$ & NA & $<2.0 \mathrm{U}$ & NA & $7.76 \mathrm{~J}$ & NA \\
\hline 2,4-Dimethylphenol & & $<2.0 \mathrm{U}$ & NA & $<2.0 \mathrm{U}$ & NA & $5.00 \mathrm{~J}$ & NA \\
\hline 3 \& 4-Methylphenol & NA & $<2.0 \mathrm{U}$ & NA & $<2.0 \mathrm{U}$ & NA & $6.76 \mathrm{~J}$ & NA \\
\hline Naphthalene & NA & $30 \mathrm{~J}$ & NA & $37.8 \mathrm{~J}$ & $<0.050$ & $<0.40 \mathrm{U}$ & NA \\
\hline 2-Methylnaphthalene & NA & $5.4 \mathrm{~J}$ & $<0.005 \mathrm{U}$ & $110 \mathrm{~J}$ & $<0.050$ & $<0.40 \mathrm{U}$ & NA \\
\hline Adamantane & NA & $47.2 \mathrm{~J}$ & NA & $6.4 \mathrm{~J}$ & NA & $<1.12 \mathrm{U}$ & NA \\
\hline 1,3-Dimethyl adamantane & NA & $9.8 \mathrm{~J}$ & NA & $8.2 \mathrm{~J}$ & NA & $<1.12 \mathrm{U}$ & NA \\
\hline Triethylene glycol & ND & ND & NA & ND & NA & 17.8 (TIC) & NA \\
\hline
\end{tabular}

$\mathrm{J}$ - estimated concentration

$\mathrm{U}$ - below reporting limit

TIC - tentatively identified compound 


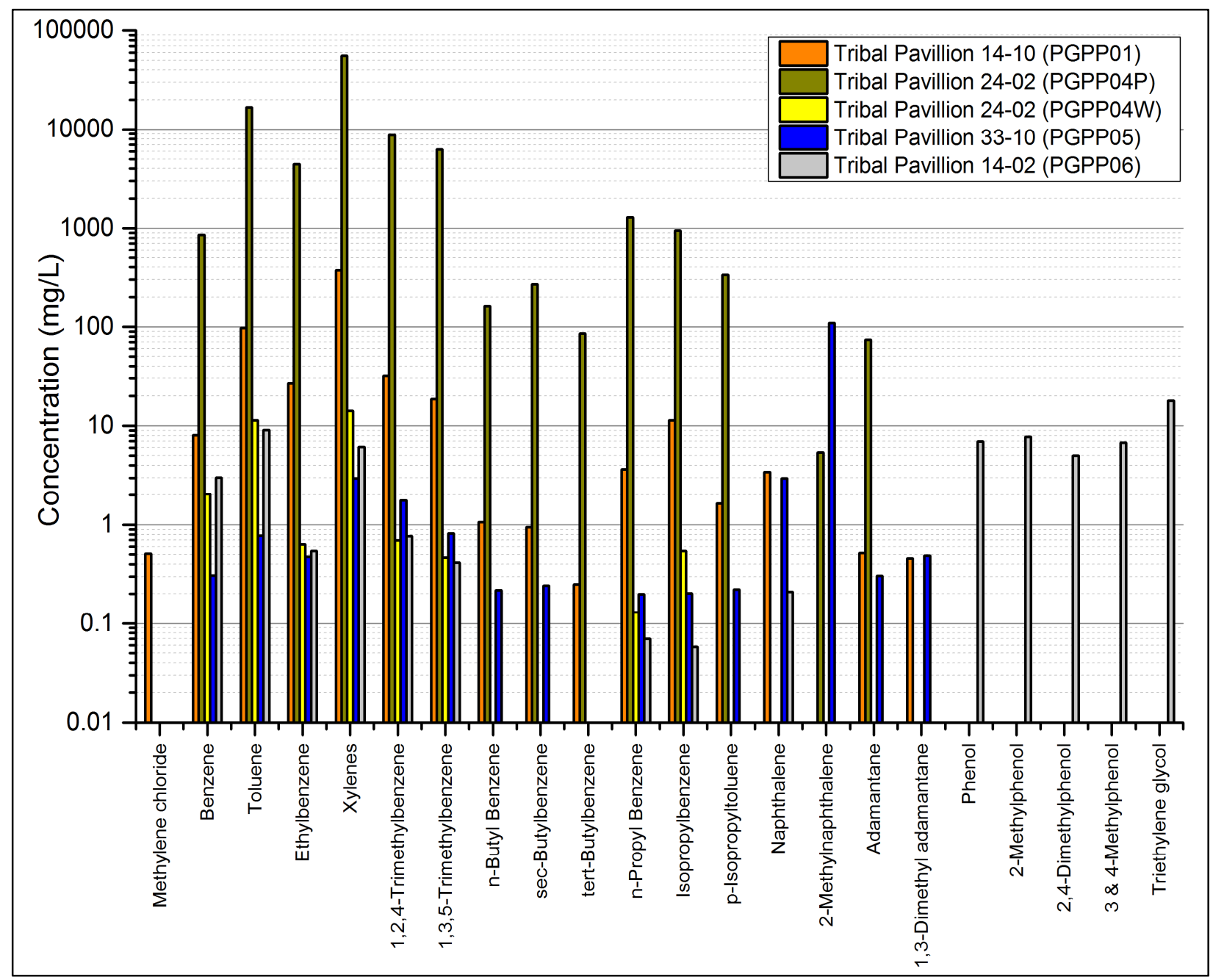

Figure SI D3. Organic compounds (volatile and semivolatile analyses) detected by EPA ${ }^{30}$ in produced water at Tribal Pavillion 14-10 (PGPP01), Tribal Pavillion 33-10 (PGPP05) and Tribal Pavillion 14-02 (PGPP06) and product present with water at Tribal Pavillion 24-02 (PGPP04P). Triethylene glycol was a tentatively identified compound (gas chromatography area at least $10 \%$ as large as the area of the nearest internal standard and a mass spectrometry match quality greater than $90 \%$ ). 


\section{E - EPA Monitoring Well Construction, Purging, and Sample Results}

\section{E.1 Monitoring Well Construction}

In June 2010, EPA installed two monitoring wells, MW01 and MW02, using mud rotary drilling. Monitoring well construction schematics for MW01 and MW02 are provided in Figures SI E1 and SI E2, respectively. We modified schematics previously illustrated in EPA's draft report ${ }^{27}$ to be more consistent with the prime contractor (Shaw Environmental) and subprime contractor (Boart-Longyear) and USGS (during redevelopment) daily logs. We typed and chronologically combined handwritten daily logs (not provided here) to gain a better understanding of activities during monitoring well construction.

\section{$\underline{\text { Water Used for Drilling Mud }}$}

Boart-Longyear used a water supply truck to transport municipal water from Riverton, WY to mix bentonite for MW01 and MW02. This truck was not used for any other purpose. Water was not stored in tanks prior to use. Municipal water was sampled by the City of Riverton on 7/26/2010 (the same time as drilling). It is unlikely that water used to mix drilling mud impacted analytical results at MW01 and MW02 for the following reasons. (1) No volatile petroleum hydrocarbons were detected. The reporting limit for benzene, toluene, ethylbenzene, o-xylene, m, p-xylenes, naphthalene, 1,2,4trimethylbenzene, and 1,3,5-trimethylbenzene was $0.50 \mu \mathrm{g} / \mathrm{L}$. (2) With the exception of chlorine disinfection products, choroform and trihalomethanes, detected at 2.3 and $2.7 \mu \mathrm{g} / \mathrm{L}$ respectively, no other volatile organic compounds were detected. Choroform and trihalomethanes were not detected in aqueous samples from MW01 and MW02 indicating loss of these compounds via volatilization and/or degradation during drilling. (3) No semivolatile organic compounds were detected. (4) No additives (e.g., biocides) were added to the water during transport and use.

\section{Additives Used for Drilling Mud}

Boart-Longyear used a number of additives (Table SI E1) in water-based drilling mud to avoid heaving of shale and a product, Aqua-Clear PFD, to facilitate removal of mud after drilling. MSDSs indicated that: (1) Quik-Gel was comprised of bentonite (60\%), crystalline silica quartz (1-5\%), crystalline silica cristobalite (0-1\%), and crystalline silica tridymite (0-1\%); (2) EZ-Mud Gold contained “no hazardous substances"; (3) Quik-Trol Gold was a cellulose derivative with a 60-100\% polysaccharide concentration; (4) dense soda ash was $100 \%$ sodium carbonate $\left(\mathrm{Na}_{2} \mathrm{CO}_{3}\right)$; (5) Penetrol contained 1-5\% of diethanolamine and 10-30\% coco diethanolamide; and (6) Aqua-Clear contained 30-60\% of an anionic polyacrylamide. 


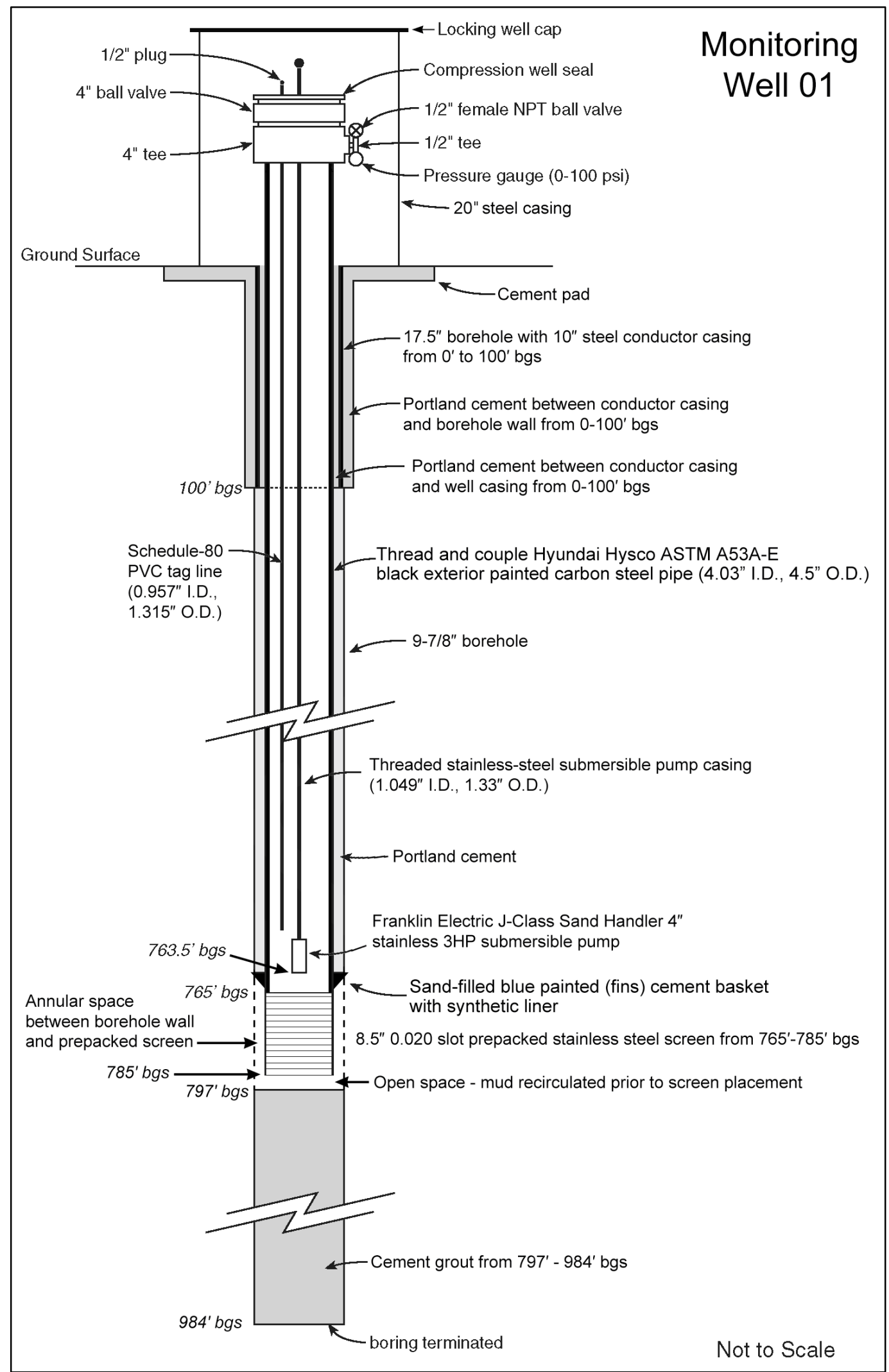

Figure SI E1. Well schematic for MW01 modified from EPA ${ }^{27}$ 


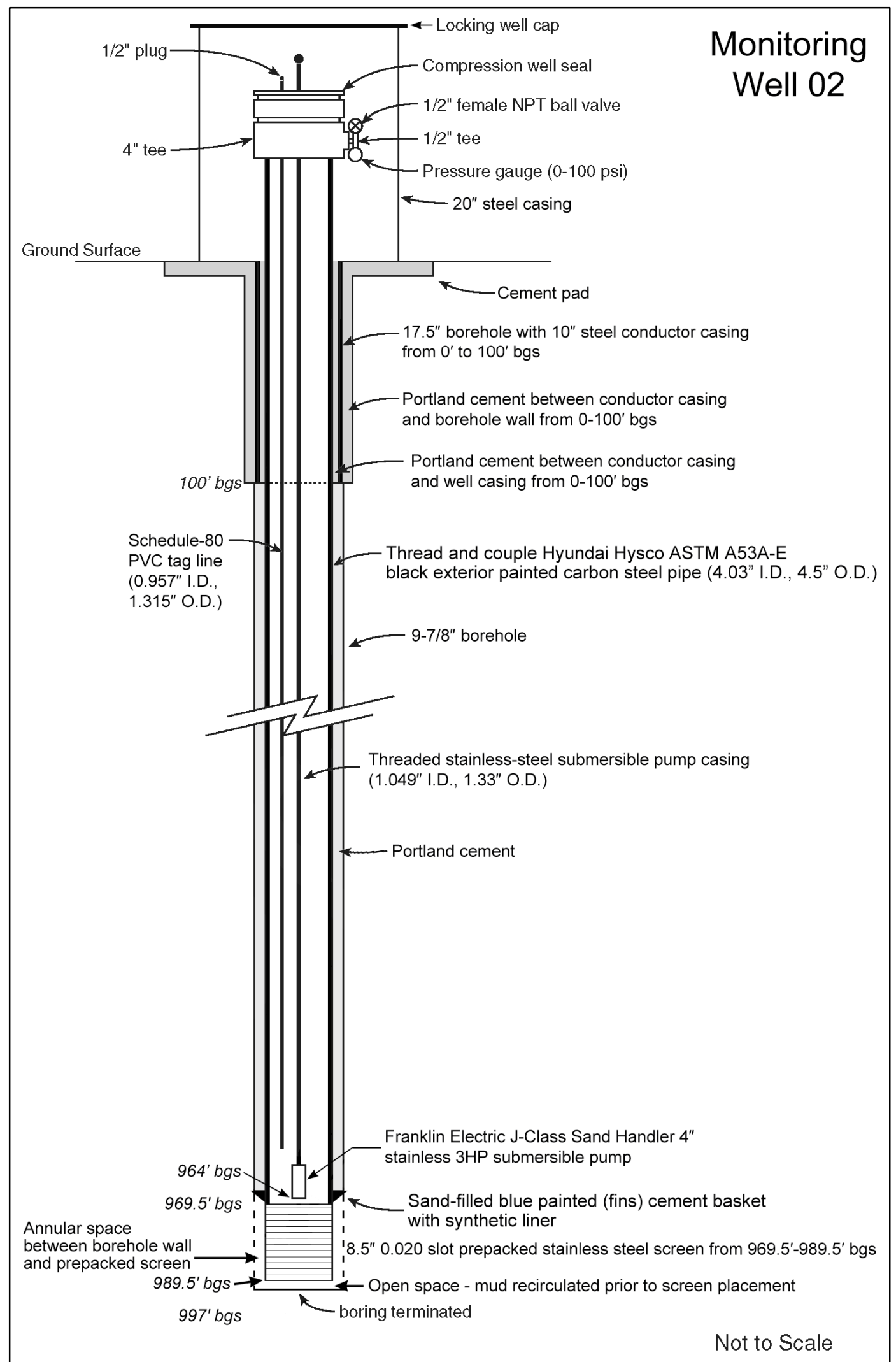

Figure SI E2. Well schematic for MW02 modified from EPA ${ }^{27}$ 
Table SI E1. Summary of products used for mud rotary drilling, mass ratios of product use, analyses of aqueous extracts of products, and maximum concentration of detection in MW01 and MW02.

\begin{tabular}{|c|c|c|c|c|c|c|c|c|}
\hline Parameter & Aqua-Clear PFD & Penetrol & $\begin{array}{c}\text { EZ-Mud } \\
\text { Gold }\end{array}$ & $\begin{array}{c}\text { Dense } \\
\text { Soda Ash }\end{array}$ & Quik-Gel & $\begin{array}{l}\text { Quik-Trol } \\
\text { Gold }\end{array}$ & MW01 & MW02 \\
\hline Manufacturer & Haliburton & Haliburton & Haliburton & $\begin{array}{c}\text { OCI } \\
\text { Chemicals }\end{array}$ & & Haliburton & ----- & ---- \\
\hline Specified use of additive & $\begin{array}{c}\text { Dispersant - assist } \\
\text { mud removal }\end{array}$ & & $\begin{array}{l}\text { shale/clay } \\
\text { stabilizer }\end{array}$ & & bentonite & clarifier & ----- & $\begin{array}{ll}---- \\
\end{array}$ \\
\hline form & liquid & liquid & solid & solid & solid & solid & ----- & ----- \\
\hline mass ratio in extract & $1: 20$ & $1: 20$ & $1: 100$ & $1: 100$ & $1: 100$ & $1: 100$ & ----- & $\begin{array}{ll}---- \\
\end{array}$ \\
\hline $\begin{array}{l}\text { mass ratio in water used for mud } \\
\text { or development at MW01 }\end{array}$ & $1: 467$ & $1: 2347$ & $1: 236$ & $1: 1211$ & $1: 36$ & $1: 634$ & ----- & ----- \\
\hline Normalization factor for MW01 & 23.4 & 117.6 & 2.36 & 12.1 & 0.36 & 6.34 & ----- & $\begin{array}{ll}---- \\
--1\end{array}$ \\
\hline $\begin{array}{l}\text { mass ratio in water used for mud } \\
\text { or development at } \mathrm{MW} 02\end{array}$ & $1: 467$ & not used & $1: 1252$ & not used & $1: 36$ & $1: 1252$ & ----- & ---- \\
\hline Normalization factor for MW02 & 23.4 & ----- & 12.5 & ----- & 0.36 & 12.5 & ----- & $\begin{array}{ll}---- \\
\end{array}$ \\
\hline \multicolumn{7}{|c|}{$\begin{array}{l}\text { Concentration in Aqueous Extract and Estimated Concentration in Aqueous Portion of Drilling Mud in Parenthesis } \\
\text { Upper and Lower sets of concentrations refer to MW01 and MW02, respectively }\end{array}$} & \multicolumn{2}{|c|}{$\begin{array}{c}\text { Maximum } \\
\text { Concentration in } \\
\text { Monitoring Wells }\end{array}$} \\
\hline $\mathrm{pH}$ of aqueous extract of product & 7.96 & 8.51 & 6.64 & 11.2 & 8.35 & NA & 11.9 & 12 \\
\hline Specific Conductance $(\mathrm{mS} / \mathrm{cm})$ & $\begin{array}{l}13.3(0.57) \\
13.3(0.57)\end{array}$ & $\begin{array}{l}0.47(0.004) \\
\text { not used }\end{array}$ & $\begin{array}{l}0.24(0.10) \\
0.24(0.02)\end{array}$ & $\begin{array}{l}15.5(1.28) \\
\text { not used }\end{array}$ & $\begin{array}{l}0.20(0.56) \\
0.20(0.56)\end{array}$ & $\begin{array}{l}\text { NA } \\
\text { NA }\end{array}$ & 3.265 & 3.812 \\
\hline $\mathrm{DOC}(\mathrm{mg} / \mathrm{L})$ & $\begin{array}{l}1640(70.2) \\
1640(70.2)\end{array}$ & $\begin{array}{c}1500(12.78) \\
\text { not used }\end{array}$ & $\begin{array}{l}388(164) \\
388(31.0)\end{array}$ & $\begin{array}{c}0.58(0.05) \\
\text { not used }\end{array}$ & $\begin{array}{l}2.11(5.86) \\
2.11(5.86)\end{array}$ & $\begin{array}{l}\text { NA } \\
\text { NA }\end{array}$ & 9.43 & 19.7 \\
\hline $\mathrm{Cl}(\mathrm{mg} / \mathrm{L})$ & $\begin{array}{l}214(9.16) \\
214(9.16)\end{array}$ & $\begin{array}{c}85(0.72) \\
\text { not used }\end{array}$ & $\begin{array}{l}2.22(0.94) \\
2.22(0.18)\end{array}$ & $\begin{array}{c}7.03(0.58) \\
\text { not used }\end{array}$ & $\begin{array}{l}\text { ND } \\
\text { ND }\end{array}$ & $\begin{array}{l}\text { NA } \\
\text { NA }\end{array}$ & 23.3 & 466 \\
\hline $\mathrm{SO}_{4}(\mathrm{mg} / \mathrm{L})$ & $\begin{array}{l}121(5.18) \\
121(5.18)\end{array}$ & $\begin{array}{c}597(5.09) \\
\text { not used }\end{array}$ & $\begin{array}{l}\mathrm{ND} \\
\mathrm{ND}\end{array}$ & $\begin{array}{l}\text { ND } \\
\text { ND }\end{array}$ & $\begin{array}{l}3.53(9.81) \\
3.53(9.81)\end{array}$ & $\begin{array}{l}\text { NA } \\
\text { NA }\end{array}$ & 428 & 81.8 \\
\hline $\mathrm{K}(\mathrm{mg} / \mathrm{L})$ & $\begin{array}{l}0.40(0.02) \\
0.40(0.02)\end{array}$ & $\begin{array}{c}0.63(0.005) \\
\text { not used }\end{array}$ & $\begin{array}{l}1.16(0.49) \\
1.16(0.09)\end{array}$ & $\begin{array}{c}0.12(0.01) \\
\text { not used }\end{array}$ & $\begin{array}{l}0.09(0.25) \\
0.09(0.25)\end{array}$ & $\begin{array}{l}\text { NA } \\
\text { NA }\end{array}$ & 54.9 & 44.0 \\
\hline acetone $(\mu \mathrm{g} / \mathrm{L})$ & $\begin{array}{l}\text { ND } \\
\text { ND }\end{array}$ & $\begin{array}{l}\text { ND } \\
\text { ND }\end{array}$ & $\begin{array}{l}\text { ND } \\
\text { ND }\end{array}$ & $\begin{array}{l}\text { NA } \\
\text { NA }\end{array}$ & $\begin{array}{l}\text { NA } \\
\text { NA }\end{array}$ & $\begin{array}{l}\text { NA } \\
\text { NA }\end{array}$ & 79.5 & 1460 \\
\hline tert-butyl alcohol $(\mu \mathrm{g} / \mathrm{L})$ & $\begin{array}{l}\text { ND } \\
\text { ND }\end{array}$ & $\begin{array}{l}\text { ND } \\
\text { ND }\end{array}$ & $\begin{array}{l}\text { ND } \\
\text { ND }\end{array}$ & $\begin{array}{l}\text { NA } \\
\text { NA }\end{array}$ & $\begin{array}{l}\text { NA } \\
\text { NA }\end{array}$ & $\begin{array}{l}\text { NA } \\
\text { NA }\end{array}$ & $<5.0$ & 6120 \\
\hline isopropanol $(\mu \mathrm{g} / \mathrm{L})$ & $\begin{array}{l}85(\mathrm{~J})(3.64) \\
85(\mathrm{~J})(3.64)\end{array}$ & $\begin{array}{c}43(\mathrm{~J})(0.37) \\
\text { not used }\end{array}$ & $\begin{array}{l}27(\mathrm{~J})(11.4) \\
27(\mathrm{~J})(2.16)\end{array}$ & $\begin{array}{l}\text { NA } \\
\text { NA }\end{array}$ & $\begin{array}{l}\text { NA } \\
\text { NA }\end{array}$ & $\begin{array}{l}\text { NA } \\
\text { NA }\end{array}$ & 212 & 862 \\
\hline ethanol $(\mu \mathrm{g} / \mathrm{L})$ & $\begin{array}{l}59(\mathrm{~J})(2.53) \\
59(\mathrm{~J})(2.53)\end{array}$ & $\begin{array}{c}58(\mathrm{~J})(0.49) \\
\text { not used }\end{array}$ & $\begin{array}{l}\text { ND } \\
\text { ND }\end{array}$ & $\begin{array}{l}\text { NA } \\
\text { NA }\end{array}$ & $\begin{array}{l}\text { NA } \\
\text { NA }\end{array}$ & $\begin{array}{l}\text { NA } \\
\text { NA }\end{array}$ & $<1.00$ & 7.49 \\
\hline benzene $(\mu \mathrm{g} / \mathrm{L})$ & $\begin{array}{l}\text { ND } \\
\text { ND }\end{array}$ & $\begin{array}{l}\text { ND } \\
\text { ND }\end{array}$ & $\begin{array}{l}\text { ND } \\
\text { ND }\end{array}$ & $\begin{array}{l}\text { NA } \\
\text { NA }\end{array}$ & $\begin{array}{l}\text { NA } \\
\text { NA }\end{array}$ & $\begin{array}{l}\text { NA } \\
\text { NA }\end{array}$ & $<0.50$ & 246 \\
\hline toluene $(\mu \mathrm{g} / \mathrm{L})$ & $\begin{array}{l}\text { ND } \\
\text { ND }\end{array}$ & $\begin{array}{l}\text { ND } \\
\text { ND }\end{array}$ & $\begin{array}{l}\text { ND } \\
\text { ND }\end{array}$ & $\begin{array}{l}\text { NA } \\
\text { NA }\end{array}$ & $\begin{array}{l}\text { NA } \\
\text { NA }\end{array}$ & $\begin{array}{l}\text { NA } \\
\text { NA }\end{array}$ & $<0.50$ & 677 \\
\hline ethylbenzene $(\mu \mathrm{g} / \mathrm{L})$ & $\begin{array}{l}\text { ND } \\
\text { ND }\end{array}$ & $\begin{array}{l}\text { ND } \\
\text { ND }\end{array}$ & $\begin{array}{l}\text { ND } \\
\text { ND }\end{array}$ & $\begin{array}{l}\text { NA } \\
\text { NA }\end{array}$ & $\begin{array}{l}\text { NA } \\
\text { NA }\end{array}$ & $\begin{array}{l}\text { NA } \\
\text { NA }\end{array}$ & $<0.50$ & 101 \\
\hline xylenes $(\mu \mathrm{g} / \mathrm{L})$ & $\begin{array}{l}\text { ND } \\
\text { ND }\end{array}$ & $\begin{array}{l}\text { ND } \\
\text { ND }\end{array}$ & $\begin{array}{l}\text { ND } \\
\text { ND }\end{array}$ & $\begin{array}{l}\text { NA } \\
\text { NA }\end{array}$ & $\begin{array}{l}\text { NA } \\
\text { NA }\end{array}$ & $\begin{array}{l}\text { NA } \\
\text { NA }\end{array}$ & $<0.50$ & 1063 \\
\hline trimethylbenzenes $(\mu \mathrm{g} / \mathrm{L})$ & $\begin{array}{l}\text { ND } \\
\text { ND }\end{array}$ & $\begin{array}{l}\text { ND } \\
\text { ND }\end{array}$ & $\begin{array}{l}\text { ND } \\
\text { ND }\end{array}$ & $\begin{array}{l}\text { NA } \\
\text { NA }\end{array}$ & $\begin{array}{l}\text { NA } \\
\text { NA }\end{array}$ & $\begin{array}{l}\text { NA } \\
\text { NA }\end{array}$ & $<1.00$ & 265 \\
\hline naphthalene & $\begin{array}{l}\text { ND } \\
\text { ND }\end{array}$ & $\begin{array}{c}2.00(0.02) \\
\text { not used }\end{array}$ & $\begin{array}{l}\text { ND } \\
\text { ND }\end{array}$ & $\begin{array}{l}\text { NA } \\
\text { NA }\end{array}$ & $\begin{array}{l}\text { NA } \\
\text { NA }\end{array}$ & $\begin{array}{l}\text { NA } \\
\text { NA }\end{array}$ & $<1.00$ & 7.2 \\
\hline ethylene glycol $(\mu \mathrm{g} / \mathrm{L})$ & $\begin{array}{l}\mathrm{ND} \\
\mathrm{ND}\end{array}$ & $\begin{array}{l}\text { ND } \\
\text { ND }\end{array}$ & $\begin{array}{l}\text { ND } \\
\text { ND }\end{array}$ & $\begin{array}{l}\text { NA } \\
\text { NA }\end{array}$ & $\begin{array}{l}\text { NA } \\
\text { NA }\end{array}$ & $\begin{array}{l}\text { NA } \\
\text { NA }\end{array}$ & $<5000$ & $<5000$ \\
\hline diethylene glycol $(\mu \mathrm{g} / \mathrm{L})$ & $\begin{array}{l}\mathrm{ND} \\
\mathrm{ND}\end{array}$ & $\begin{array}{l}\text { ND } \\
\text { ND }\end{array}$ & $\begin{array}{l}\text { ND } \\
\text { ND }\end{array}$ & $\begin{array}{l}\text { NA } \\
\text { NA }\end{array}$ & $\begin{array}{l}\text { NA } \\
\text { NA }\end{array}$ & $\begin{array}{l}\text { NA } \\
\text { NA }\end{array}$ & 60.0 & 1610 \\
\hline
\end{tabular}

ND - not detected

NA - not analyzed

$\mathrm{J}$ - estimated (below the level of quantification)

EPA stored samples of products used for drilling in glass mason jars containing lids, transported products with a chain of custody form by vehicle to its Office of Research and Development (ORD) laboratory in Ada, $\mathrm{OK}$, and archived products in a refrigerator at $4^{\circ} \mathrm{C}$ prior to extraction with water. Analysis for organic compounds were not conducted on water extracts of Quik Gel and Quik-Trol Gold due to the gel nature of extracts and concern of analytical equipment damage. The dissolved organic 
carbon of dense soda ash was only $0.58 \mathrm{mg} / \mathrm{L}$ and based on MSDS, was not expected to be a source of organic compounds. EPA's analysis of extracts is noteworthy in that we could find no other published studies in which an organization collected and analyzed samples of products used for drilling mud during installation of monitoring wells.

With the exception of dense soda ash, the $\mathrm{pH}$ of extracts were well below that maximum $\mathrm{pH}$ levels ( 12) observed in MW01 and MW02. Dense Soda Ash was not used at MW02. Alkalinity of soda ash is dominated by carbonate not hydroxide as observed in MW01. The $\mathrm{pH}$ of drilling mud in which dense soda ash was mixed in MW01 varied between 8 and 9 standard units. Thus, additives had no effect on $\mathrm{pH}$ measurements during sampling at monitoring wells.

To estimate expected concentrations of compounds in additives in water used for drilling mud, we normalized aqueous extraction concentrations by a normalization factor defined by

\section{Normalization Factor $=\frac{\text { mass ratio in water used for drilling mud or well development }}{\text { mass ratio in aqueous extract }}$}

For instance, the mass ratio of the water extract of Aqua-Clear PFD was 1:20 while use in water used for well development was 1:467 resulting in a normalization factor of $1 / 20$ divided by $1 / 467$ or 23.4 . Results are provided in Table SI E1.

At MW02, Boart-Longyear used 21 sacks or 1,050 pounds of Quik-Gel for drilling. The initial mass of bentonite used at MW01 was not provided in the logs. However, the mass of bentonite used at MW01 is expected to be similar to MW02 given that the target depth (almost $305 \mathrm{~m}$ or 1,000 feet) was the same at both wells. A log entry on 6/11/10 at 10:20 from MW02 indicates that 17,000 L (4,500 gallons) of water was used to mix mud for drilling at this well. The volume of water used to mix mud at MW01 was not specified in the drilling logs. However, both wells were drilled to the same depth under the same subsurface conditions so the volume of water used at MW01 would be expected to be similar to that used at MW02.

Concentrations of potassium in monitoring wells were well below concentrations and normalized concentrations in extracts. The extract and normalized concentration of chloride in Aqua-Clear PFD was lower and far lower than detected in MW02. During the Phase V sampling event, acrylamide was added to the target list of analytes and was not detected in any samples from MW01 and MW02 $(<10 \mu \mathrm{g} / \mathrm{L})^{33}$ indicating that any compounds associated with Aqua-Clear PFD were removed during well development. With the exception of naphthalene detected at $2.0 \mu \mathrm{g} / \mathrm{L}$ in a Penetrol extract (normalized concentration of $0.02 \mu \mathrm{g} / \mathrm{L}$ in MW01), petroleum hydrocarbons were not detected in extracts. The maximum 
concentrations and normalized concentration of isopropanol in extracts 85 was $11.4 \mu \mathrm{g} / \mathrm{L}$, respectively compared to 212 and $862 \mu \mathrm{g} / \mathrm{L}$ at MW01 and MW02, respectively. BTEX compounds (benzene, toluene, ethylbenzene, and xylenes) were not detected in extracts but were detected at MW02.

EPA collected composite samples of cuttings and sent them with a chain of custody form to TestAmerica Laboratories in Denver, Colorado for Toxic Characteristic Leaching Procedure (TCLP). Samples were analyzed for TCLP volatile organic compounds using gas chromatography-mass spectrometry (GC-MS) in accordance with EPA SW-846 Methods 1311/8260B, for TCLP semivolatile organic compounds (GC-MS) in accordance with EPA SW-846 Methods 1311/8270C, for TCLP metals in accordance with EPA SW-846 Methods 1311/6010B, and for TCLP mercury in accordance with EPA SW-846 Methods 1311/7470A. Acetone, toluene, and m \& p-xylene were detected in one sample at 6.9, 0.63 , and $1.0 \mu \mathrm{g} / \mathrm{L}$, respectively, concentrations far lower than that detected in MW02. Considering all these factors, it is unlikely that additives used for drilling mud impacted sample results in MW01 and MW02.

\section{$\underline{\text { Well Development }}$}

Well development criteria stated in EPA's Monitoring Well Installation were as follows: water samples $<10$ Nephelometric Turbidity Units (NTUs), or 3 purge volumes if $<10$ NTU prior to attainment of 3 purge volumes, or 5 purge volumes if $<10$ NTU is not achieved after three purge volumes. Daily $\operatorname{logs}$ indicate that $33,320 \mathrm{~L}$ (8815 gallons) or 24.5 purge volumes of water was removed from MW01 prior to the first round of sampling in October 2010 in which turbidity of sampled water was 7.5 NTUs. Hence, well development criteria were met at MW01. MW02 was a low yield well. Daily logs indicate that 5,760 L (1524 gallons) or 3.2 purge volumes of water was removed prior to the first sampling event in October 2010 in which the turbidity of sampled water was 24 NTUs. Elevated turbidity was caused by gas flow and pump cavitation due to gas flow. For instance, during the Phase V sampling event, during which removal of 5 purge volumes had been attained, turbidity was measured at 4.5 NTUs prior to pump cavitation and 15.7 NTUs after pump cavitation. Thus, well development criteria was ultimately achieved for both monitoring wells. Turbidity is not expected to have any impact on analysis of organic compounds nor on inorganic analyses since samples were filtered.

\section{$\underline{\text { Steel Well Casing }}$}

Boart-Longyear used thread and couple Hyundai HYSCO ASTM A53A-E steel pipe for well casing at both MW01 and MW02. A $6.1 \mathrm{~m}$ (20 ft) stainless-steel pre-packed screen was attached to casing via flush threads. EPA specified the use of metal casing to ensure sufficient tensile strength, especially at 
joints during well placement. The tensile strength of steel and stainless-steel joints is over an order of magnitude greater than polyvinyl chloride (PVC) joints. The WYDEQ (Well Construction Standards, Chapter 11, Part G) explicitly allows for the use of ASTM A53 steel casing for well construction.

Materials used for well casing and screens can impart both negative and positive bias in detection and quantification of target analytes. Steel is an alloy consisting primarily of iron (Fe). The maximum allowable concentrations of manganese $(\mathrm{Mn})$, copper $(\mathrm{Cu})$, nickel $(\mathrm{Ni})$, chromium $(\mathrm{Cr})$, molybdenum (Mo), and vanadium (V) in ASTM A53A- $\mathrm{E}^{38}$, steel pipe are $0.95,0.40,0.40,0.15$, and $0.08 \%$ by weight, respectively. Houghton and Berger ${ }^{39}$ noted a slight enrichment of $\mathrm{Fe}$ and $\mathrm{Mn}$ in water from wells constructed of steel compared to wells constructed from PVC and acrylonitrile-butadiene-styrene (ABS).

Interaction can also occur with stainless steel. Hewitt ${ }^{40}$ conducted static and dynamic (flushing) experiments on PVC, PTFE, stainless steel-SS-304 (SS-304), and stainless steel-316 (SS-316) casing materials in glass cylinders containing well water with low dissolved oxygen (DO) $(<0.5 \mathrm{mg} / \mathrm{L})$ and observed corrosion of both SS-304 and SS-316 during testing (pH 7.6 - 7.8). Low concentrations of $\mathrm{Cu}$, $\mathrm{Cr}$, and Ni leached from both virgin SS-304 and SS-316. However, leaching of Cr and Ni increased significantly with corrosion. Hewitt ${ }^{40}$ observed that concentrations of cadmium $(\mathrm{Cd}), \mathrm{Fe}$, and lead $(\mathrm{Pb})$ decreased indicating sorption to casing. Hewitt ${ }^{40}$ stated that reduction of Fe was consistent with precipitation of $\mathrm{Fe}(\mathrm{OH})_{2}$ and that iron oxides provided active exchange sites for sorption of these elements.

In an EPA report, Aller et al ${ }^{41}$ state that potentially corrosive conditions exist for steel in ground water having low $\mathrm{pH}(<7)$ and elevated DO $(>2 \mathrm{mg} / \mathrm{L})$, hydrogen sulfide $\left(\mathrm{H}_{2} \mathrm{~S}\right)(>1 \mathrm{mg} / \mathrm{L})$, TDS $(>1000$ $\mathrm{mg} / \mathrm{L}), \mathrm{CO}_{2}(>50 \mathrm{mg} / \mathrm{L})$, and $\mathrm{Cl}(>500 \mathrm{mg} / \mathrm{L})$ and that use of steel is not considered prudent in most natural geochemical environments. Subsequent to well development, purged water from MW01 and MW02 was largely devoid of dissolved oxygen during Phase III, IV, and V sampling events with a pH level in excess of 11 standard units.

In its Draft RCRA Ground-Water Monitoring Guidance, EPA ${ }^{42}$ states that use of carbon steel, low-carbon steel, and galvanized steel is not recommended for monitoring well construction in most natural geochemical environments and use of Type 304 and Type 316 stainless steel is unsuitable for use when monitoring for inorganic constituents. In an EPA Issue Paper, Pohlmann and Alduino ${ }^{43}$ state that when corrosion is a concern, use of stainless-steel casing is not appropriate. In another EPA Issue Paper, Llopis ${ }^{44}$ states that if samples are to be analyzed for metals, metal casing of any type should not be used. Thus, when installing monitoring wells to sample both metals and organic compounds, there are no consistent regulatory guidelines on use of materials for well casing. 
During the week of April 30th, 2012 (after cessation of Phase V sampling), a drilling crew from USGS removed the 3 HP submersible pump in MW02, examined the casing and screen with an optical televiewer, and attempted to redevelop the well. The optical televiewer indicated that the screen and casing was intact. During re-development, sediment was removed from the base of the screen. Four subsamples of the material were sent to Gulf Coast Accutest Laboratories for analysis of metals with the following range of results: aluminum (1950-2720 mg/kg), antimony (2.5-3.4 mg/kg), arsenic (3.3-10 $\mathrm{mg} / \mathrm{kg}$ ), cadmium (<0.26-2.1 mg/kg), chromium (79.9-108 mg/kg), copper (168-719 mg/kg), lead (16.2$56.3 \mathrm{mg} / \mathrm{kg}$ ), nickel (49.4-64.9 mg/kg), selenium (<0.26 mg/kg), and titanium (52.2-80.5 mg/kg). Results of metals analysis indicated that this sediment was either scrapings from steel pipe or a combination of scrapings from steel pipe and corrosion material removed from steel casing during development and redevelopment activities. During the Phase V sampling event, dissolved concentrations of $\mathrm{Cd}, \mathrm{Pb}, \mathrm{Fe}, \mathrm{Mn}$, $\mathrm{Cu}, \mathrm{Ni}, \mathrm{Cr}, \mathrm{Mo}$, and V at MW01 and MW02 were less than reporting limits at 1.0, 1.0, 67, 14.0, 2.0, 1.0, $2.0,17$, and $10 \mu \mathrm{g} / \mathrm{L}$, respectively. Hence, the use of metal casing and presumed corrosion in areas of swabbing did not impart a positive bias on sample results for metals at MW01 and MW02.

However, corrosion can impact measured $\mathrm{pH}$. Marsh and Lloyd ${ }^{45}$ attributed elevation of $\mathrm{pH}$ in steel casing to corrosion and precipitation reactions mediated by carbonic acid and hydrogen sulfide. In both reactions, hydrogen ion is consumed and hydrogen gas is produced. During purging of capped artesian monitoring wells, Marsh and Lloyd ${ }^{45}$ observed that $\mathrm{pH}$ decreased abruptly (e.g., $\mathrm{pH} 8.8$ to $\mathrm{pH}$ 7.2) after a short period of time in wells where water flowed through the entire length of casing to the surface. However, there was little variation in $\mathrm{pH}$ in monitoring wells having sampling tubes placed within screened intervals. In contrast to observations by Marsh and Lloyd ${ }^{45}$, $\mathrm{pH}$ reduction in MW01 during purging was gradual with the trend of $\mathrm{pH}$ reduction unrelated to removal of stored casing water. Thus, corrosion was not a causative factor of elevated pH at MW01 and MW02.

Black steel ASTM A53 pipe has a lacquered outside finish as opposed to a bare or galvanized finish. Manufactures coat the outside of steel pipe with a black paint to prevent rust and corrosion. However, sampled water does not come in contact with the exterior of well casing. Photographs (DiGiulio et al. $^{27}$ ) of casing indicate no apparent mill varnish or visual signs of oil and grease prior to or after casing decontamination procedures. Thus, it is unlikely that black varnish on the exterior of metal casing was responsible for detection of organic compounds in MW01 and MW02. Also, a non-hydrocarbon based pipe dope (Jet Lube WellGuard) was used on well casing joints. Thus, well casing is not responsible for detection of hydrocarbons in monitoring wells. 
During conventional monitoring well construction, a bentonite slurry seal is typically placed on top of a primary or secondary filter or sand pack to limit the downward movement of a cement-based grout $\left(\mathrm{ASTM}^{46}, \mathrm{EPA}^{42}\right)$. As documented in the $\mathrm{draft}^{47}$ and final ${ }^{48}$ workplans for well installation, this method of well completion was planned for both MW01 and MW02. However, as documented in field logs, during drilling, Shaw and Boart-Longyear advised EPA against the use of a conventional screen and sand pack for well completion at MW01 and MW02 due potential difficulty in sand pack placement (displacement of high density drilling mud with sand) and well development (removal of mud from sand pack). Shaw and Boart-Longyear recommended the use of pre-packed screens and cement baskets (filled with sand at the surface) in lieu of conventional screens and sand packs. EPA concurred with this approach for well completion.

An additional reason for not utilizing a bentonite slurry as a sealing agent is the presence of localized upward hydraulic gradients. Localized upgradient gradients are documented in the Wind River Formation $^{26}$, was present in a domestic well during the Phase II sampling event ${ }^{27}$, and was observed at 4 production wells during Bradenhead testing conducted by Encana in 2012. A cement-bentonite grout may not set when a strong uplift is present ${ }^{49,50}$. Under these conditions, pumped bentonite grout never sets up to anything more than thick paste ${ }^{51}$. In addition, a bentonite grout backfill would not have been volumetrically stable with overlying cement application since the specific gravity of cement grout was greater than that of the bentonite slurry. A cement-based grout has the widest regulatory acceptance and is the most commonly used to seal the annular space of deep wells ${ }^{46}$ such as MW01 and MW02.

\section{$\underline{\text { Cement Baskets }}$}

Cement baskets with blue metal "fins" and a rubber or synthetic liner were threaded to stainlesssteel casing containing the stainless-steel pre-packed screens. The joint attaching stainless-steel casing to stainless-steel screen was reinforced with spot welding to increase the structural integrity of the joint. This was necessary to properly position the screen into the borehole prior to descent to target depth. McKay electrodes were used for welding. The MSDS for this product did not indicate the presence of organic compounds.

The composition of the cured (not applied in the field) blue paint on metal springs and rubber liner were not specified by EPA. Organic compounds in cured paint exist in cross-linked polymer matrix and generally considered resistant to leaching ${ }^{52}$. However, little is published on leaching of organic compounds from cured paint. Alben et al ${ }^{53}$ studied leaching of organic contaminants from epoxy-coated flat steel panels, with emphasis on the rate of leachate production and leachate composition. Methyl isobutyl ketone (MIBK), o-, m-, and p-xylene represented a major portion (51\%) of leachate from the 
epoxy coating. These findings were supported by field studies indicating the presence of MIBK and xylenes in water from the effluent from 2 of 3 storage tanks that were monitored one month after application of an epoxy coating ${ }^{53}$. The extent of leaching of organic contaminants from epoxy resin linings was found to be strongly dependent on the duration of the curing process with longer curing periods producing more stable linings.

It is unlikely that blue paint on cement baskets was a causative factor for detection of 2butoxyethanol and other organic compounds detected in MW01 and MW02 because of the following reasons. (1) MIBK and xylenes were not detected in MW01. (2) There was a low surface area for aqueous exposure $\left(<0.05 \mathrm{~m}^{2}\right)$, a low retention time in the screened interval during sampling especially at MW01 (20 minutes) during phase IV and phase V sampling events, and a large number of number of screen exchanges prior to sampling (especially at MW01 prior to the Phase V sampling event with $>200$ exchanges).

\section{Cement Placement}

High pH levels ( 12) were observed during purging and sampling at MW01 and MW02 during Phase III, IV and V sampling events. Daily logs do not indicate that the tremie pipe for cement placement was placed directly through cement baskets or that cement was pumped directly into screened intervals at MW01 and MW02. At MW01, circulation was lost on 7/26/10 while drilling through a sandstone unit from 655 to 725 feet bgs and as shown on an electric log and borehole log descriptions. The caliper log indicates that a washout occurred at 715 feet bgs. Thus, some cement likely intruded into the formation above the screened interval at 765-785 ft bgs.

At MW01 and MW02, cement was pumped to target depths using a tremie pipe and placed in lifts to avoid buildup of pressure above the basket potentially causing basket collapse. At MW01, sand was added to the sand basket prior to setting the casing and screen in the borehole. The screen was subsequently hung and a cement plug was placed from 228.0-233.2 $\mathrm{m}(748-765 \mathrm{ft}) \mathrm{bgs}$ on 8/5/10 and left to set up overnight. The cement was tagged at 748 feet. Cement placement resumed the following day with $14 \mathrm{yd}^{3}$ of Portland cement on top of the set plug.

At MW02, more cement, approximately 76-189 L (20 - 50 gallons) was utilized than expected when grouting above the cement basket. Based on a review of daily logs, the most likely explanation for this discrepancy is that the screened interval placement at MW02 was lower than reported by the driller or subcontractor. During the week of April 30th, 2012, a drilling crew from USGS removed the submersible pump in MW02, examined the casing and screen with an optical televiewer, and attempted to redevelop 
MW02. A member of the crew stated that tagging tape indicated that the base of the screen of MW02 was at $989.5 \mathrm{ft}$ not $980.0 \mathrm{ft}$ bgs as expected. This measurement was checked a second time and the measurement tape was checked for accuracy. If the screen interval was in fact $9.5 \mathrm{ft}$ lower than indicated on the driller's logs, then additional cement would have been necessary to reach the top of the cement basket overlying the screen. Threads on casing collars were visible at all joints located above the water table surface (e.g., pieces of pipe are not threaded flush at their ends). Neither cement nor mud was visible in the joints. An extra one to two inches at each couple would result in the well screen placed $4-8$ feet deeper than expected or close to $9.5 \mathrm{ft}$ as observed. Given a borehole diameter of 9.9 inches, 30 and 50 gallons of cement is equivalent to approximately 9.5 and 15.9 linear feet of cement which is close to 10.5 linear feet of distance between tagged cement at $958 \mathrm{ft}$ and the top of the screened interval at $968.5 \mathrm{ft}$ bgs.

\section{E.2 $\quad$ Purging at MW02}

MW02 is a low flow monitoring well. During the Phase III, IV, and V sampling events, purging at MW02 was repeatedly interrupted by pump cavitation. Low flow may be due to low relative aqueous permeability due to gas flow or insufficient removal of drilling mud during well development. In May 2012, USGS unsuccessfully attempted to redevelop MW02 via swabbing ${ }^{54}$.

During the Phase V sampling event, pre- and post-purge samples were collected at MW02 to assess potential reduction in dissolved gas and VOC concentrations due to gas flow in casing ${ }^{55,56}$. MW02 was repeatedly purged over a 6-day period (Figure SI E3) to remove one borehole volume (2000 L) of water and subsequently sampled when sufficient water was present to support analysis per EPA guidelines $^{42,57}$.

$\mathrm{USGS}^{58}$ has a "rule of thumb" recommendation to avoid sampling a well that has not recovered to within $90 \%$ of its static water level within a 24 -hour period and has had less than one borehole volume of water removed during purging. Based on the slow recharge rate of MW02, USGS elected not to physically participate in sampling MW02 ${ }^{54}$. Instead, EPA collected samples at MW02 and provided them to USGS for analyses at a commercial laboratory ${ }^{59}$ similar to $\mathrm{MW} 01^{60}$. Recovery occurs more rapidly in a low yield well having a low blank casing to screened interval ratio typical of shallow wells. MW02 consisted almost entirely of blank casing. Recovery to $90 \%$ of static water level required recovery of 202 $\mathrm{m}$ of water level rise in a 24-hour period. 


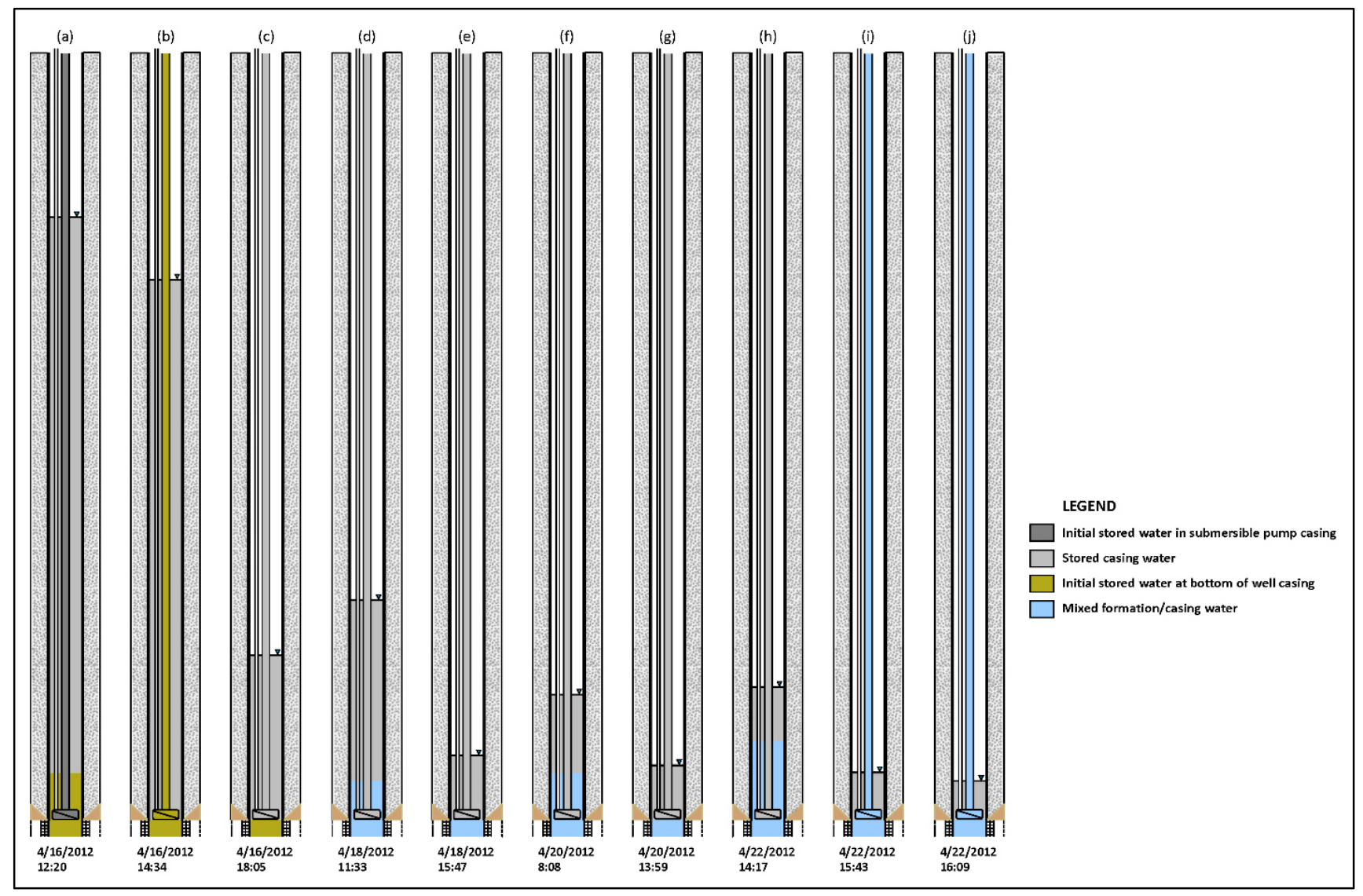

Figure SI E3. Illustration of purging and sampling sequence (date and times provided) at MW02 for Phase V sampling event. Vertical scale reflects relative lengths of screen, casing, and water levels: (a) Pre-purge condition. Dark gray color denotes water stored in submersible pump casing remaining from the Phase IV sampling event. Mustard colored water denotes water in casing from recovery following the Phase IV sampling event and targeted for removal during the first sample set. Light gray colored water denotes stored casing water from recovery during Phase III and IV sampling events. (b) Water is removed (170L or 45 gal) from the submersible pump casing, replaced with water from above the pump inlet, and sampled. (c) Water above pump inlet is removed (964 L or 255 gal). (d) Recovery occurs (132 L or 35 gallons). Light blue color indicates mixing of water initially present in the screened interval with incoming formation water. (e) Water above pump inlet removed (491 L or 130 gal) from casing. (f) Recovery occurs (167 L or 44 gallons) with further mixing of water in the screened interval. (g) Water above pump inlet removed $(215 \mathrm{~L}$ or $57 \mathrm{gal})$. (h) Recovery occurs $(189 \mathrm{~L}$ or $50 \mathrm{gal})$ with further mixing of water in the screened interval. (i) Water in submersible pump casing is removed (165 L or 45 gal) and replaced with mixed casing/formation water. (j) Mixed casing/formation water is sampled. Total volume of water removed during the Phase V sampling event prior to collection of the second sample was $2011 \mathrm{~L}$ or 532 gal equivalent to 1.04 borehole volumes.

\section{E.3 Purging and Wellbore Model Development at MW01}

In the commonly used "well volume' approach to purging, three casing volumes are typically removed prior to sampling ${ }^{58}$. It is assumed that water in blank casing is uniformly removed during purging prior to sampling. However, little or no mixing of water in blank well casing actually occurs above a pump inlet upon stabilization of drawdown ${ }^{61,62}$. Placement of a submersible pump inlet directly above a screened interval, as was done at both MW01 and MW02 (Figures SI E1 and SI E2), minimizes 
collection of purge water requiring disposal and takes advantage of flow mechanics to ensure minimal or no contact with blank well casing during sample collection.

$\mathrm{EPA}^{63}$ used a wellbore plug flow model based on flow rate and drawdown to evaluate percent collection of casing and formation water as a function of purge volume and time at MW01. To evaluate removal of dissolved solids in well casing and better evaluate collection of formation water as a function of purge volume, we modified EPA's approach to include mixing in a screened interval. Mixing in a well screen causes a slower transition from casing water to formation water than would occur from plug flow alone.

Prior to commencement of purging, we assume that the concentration of a solute in a screened interval, pre-packed screen, and annular space between the pre-packed screen and borehole wall has an initial vertically integrated initial concentration $\left(\mathrm{C}_{0}\right)$ and water in blank casing above the screened interval has an initial vertically integrated initial solute concentration $\left(\mathrm{C}_{\mathrm{C}}\right)$ (Figure SI E4a). Depending upon variation between $\mathrm{C}_{0}$ and $\mathrm{C}_{\mathrm{C}}$, differentiation of screen and casing concentrations results in complex hypothetical concentration profiles in the sampling train at the start of purging which dissipate rapidly upon removal of one casing or borehole volume (simulations not illustrated).

At the commencement of purging, water is removed from submersible pump casing. Combined downward and upward flow occurs in blank casing above and below the pump inlet respectively (Figure SI E4b) as drawdown occurs in the well. We assume that water in submersible pump and well casing undergoes unmixed plug flow. In the screened interval, radial flow occurs from the surrounding formation and mixes with water initially in the borehole and screen. The mass balance mixing model in the screened interval used is similar to that used by others ${ }^{64}$. This mixed water then undergoes upward flow in the casing to the pump inlet (Figure SI E4b). As drawdown stabilizes, all of the water entering the pump inlet is from the formation (Figure SI E4c). Sampling then occurs after water level recovery (Figure SI E4d) to ensure that all water entering the inlet and flowing to the sampling train is directly from the formation in the event of a slight change in pumping rate due to pump operation. Without allowing for some level of recovery above the pump inlet, a slight and unintentional increase in pumping rate could cause a small but measureable increase in drawdown and again induce some degree of mixing between formation and casing water. This procedure was followed by EPA at MW01 during all sampling events. 


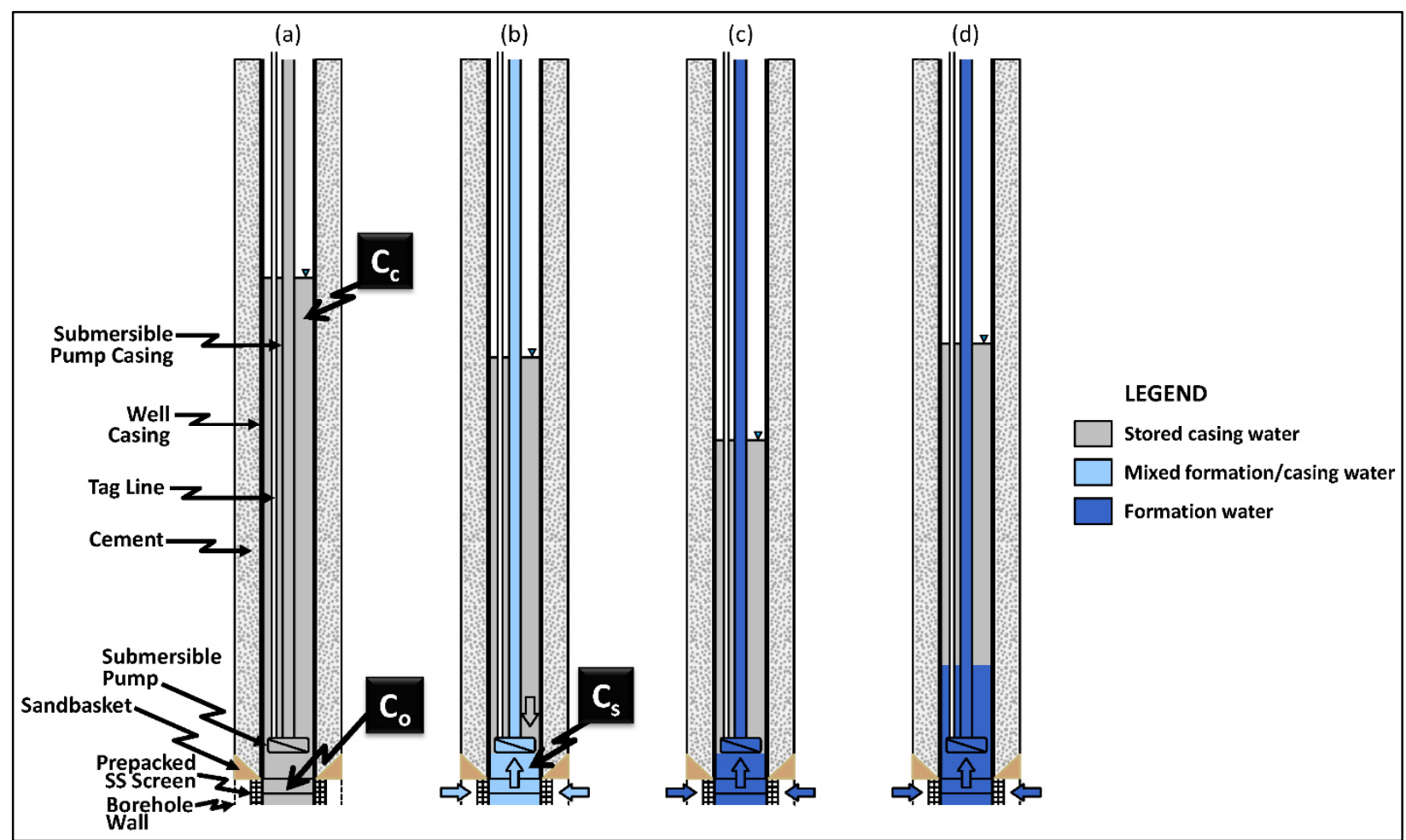

Figure SI E4. Plug flow - screen mixing model to support evaluation of purging: (a) Prior to purging, the concentration of a solute in the screened interval (and surrounding borehole) and overlying casing is equal to an initial value $\mathrm{C}_{0}$ and a constant concentration $\mathrm{C}_{\mathrm{C}}$ respectively. (b) During drawdown at commencement of purging, both downward and upward plug flow is assumed in casing above and below a pump inlet, respectively. Radial flow occurs from the surrounding formation with subsequent mixing of formation water and water initially in the borehole and screen. (c) As drawdown stabilizes, all of the water entering the pump inlet is directly from the formation. (d) Water level recovery is allowed to occur to ensure that all water entering the inlet is directly from the formation in the event of slight water level perturbation during pumping. 
Model development is summarized as follows. Casing volume $\left(\mathrm{V}_{\mathrm{C}}\right)\left[\mathrm{L}^{3}\right]$ is defined as:

$V_{C}=\frac{\pi}{4 B}\left[L_{W C} D_{I D-W C}^{2}-L_{P C}\left(D_{O D-P C}^{2}-D_{I D-P C}^{2}\right)-L_{T L}\left(D_{O D-T L}^{2}-D_{I D-T L}^{2}\right)-L_{S P} D_{O D-S P}^{2}+L_{P C^{*}} D_{I D-P C}^{2}\right]$

$\mathrm{L}_{\mathrm{wc}} \quad=$ length of water column in well (depth to base of screen - depth to static water level) [L]

$\mathrm{L}_{\mathrm{PC}} \quad=$ length of submerged pump casing (depth to top of pump - static water level) [L]

$\mathrm{L}_{\mathrm{PC}^{*}} \quad=$ length of submersible pump casing to the surface (static water level) [L]

$\mathrm{L}_{\mathrm{TL}} \quad=$ length of submerged tag line (depth of tag line - static water level) [L]

$\mathrm{L}_{\mathrm{SP}} \quad=$ length of submersible pump [L]

$\mathrm{D}_{\text {ID-wC }}=$ inside diameter of well and screen casing $(\mathrm{cm})[\mathrm{L}]$

$\mathrm{D}_{\mathrm{OD}-\mathrm{PC}}=$ outside diameter of submersible pump casing [L]

$\mathrm{D}_{\mathrm{ID}-\mathrm{PC}}=$ inside diameter of submersible pump casing [L]

D OD-TL $=$ outside diameter of tag line $[\mathrm{L}]$

$\mathrm{D}_{\mathrm{ID}-\mathrm{TL}}=$ inside diameter of tag line [L]

$\mathrm{D}_{\mathrm{SP}} \quad=$ diameter of submersible pump [L]

$\mathrm{B}=$ Dimensional constant (units of length and volume are in $\mathrm{cm}$ and liters respectively, $\mathrm{B}=1000$ ).

The term $L_{W C} D_{I D-W C}^{2}$ accounts for water storage in casing, including the screened interval, without modification for internal components. The term $L_{P C}\left(D_{O D-P C}^{2}-D_{I D-P C}^{2}\right)$ accounts for the volume of submerged submersible pump casing material. The term $L_{S P} D_{O D-S P}^{2}$ accounts for the volume of the submersible pump. The term $L_{P C} D_{I D-P C}^{2}$ accounts for water storage in the submersible pump casing above the static water level. Valves within submersible pump casing allowed water in casing to extend to land surface. Borehole volume $\mathrm{VB}\left[\mathrm{L}^{3}\right]$ is defined as:

$V_{B}=V_{C}+\frac{\pi}{4 B}\left[\phi L_{S S}\left(D_{O D-P P}^{2}-D_{O D-W C}^{2}\right)+L_{S S}\left(D_{B H}^{2}-D_{O D-P P}^{2}\right)\right]$

Lss $\quad=$ length of stainless-steel pre-packed screen [L]

DoD-wC $=$ outside diameter of steel well casing [L]

DoD-PP $=$ outside diameter of pre-packed screen [L]

$\mathrm{D}_{\mathrm{BH}} \quad=$ diameter of borehole [L]

$\varphi \quad=$ effective porosity of pre-packed screen (assumed 0.4).

The term $\phi L_{S S}\left(D_{O D-P P}^{2}-D_{O D-W C}^{2}\right)$ accounts for water storage in the pre-packed screen. The term $L_{S S}\left(D_{B H}^{2}-D_{O D-P P}^{2}\right)$ accounts for water storage in the annular space between the pre-packed screen and borehole wall. During drawdown, the relative concentration of a solute entering a sampling train at the surface can be expressed by:

$$
\left(\frac{C_{P}}{C_{F}}\right)_{i}=\frac{\frac{C_{C}}{C_{F}}\left(\frac{Q_{A P}}{Q_{T}}\right)_{i}+\left(\frac{C_{B P}}{C_{F}}\right)_{i}\left(\frac{Q_{B P}}{Q_{T}}\right)_{i}}{\left(\frac{Q_{A P}}{Q_{T}}\right)_{i}+\left(\frac{Q_{B P}}{Q_{T}}\right)_{i}}
$$

$\mathrm{C}_{\mathrm{P}}=$ concentration exiting the submersible pump casing at the surface at time increment $\mathrm{i}\left[\mathrm{ML}^{-3}\right]$

$\mathrm{C}_{\mathrm{F}}=$ constant concentration in formation $\left[\mathrm{ML}^{-3}\right]$

$\mathrm{C}_{\mathrm{C}}=$ constant storage water casing concentration $\left[\mathrm{ML}^{-3}\right]$ 
$\mathrm{C}_{\mathrm{BP}} \quad=$ concentration entering pump inlet at time increment $\mathrm{i}\left[\mathrm{ML}^{-3}\right]$

$\mathrm{Q}_{\mathrm{BP}} \quad=$ flow of water to the pump from beneath the inlet at time increment $\mathrm{i}\left[\mathrm{L}^{3} \mathrm{~T}^{-1}\right]$

$\mathrm{Q}_{\mathrm{AP}} \quad=$ flow of water to the pump from above the inlet at time increment $\mathrm{i}\left[\mathrm{L}^{3} \mathrm{~T}^{-1}\right]$

$\mathrm{Q}_{\mathrm{T}} \quad=\mathrm{Q}_{\mathrm{AP}}+\mathrm{Q}_{\mathrm{BP}}$ at time increment $\mathrm{i}\left[\mathrm{L}^{3} \mathrm{~T}^{-1}\right]$.

At the start of purging, all water originates from storage in submersible pump casing. Thus, when

$$
V_{i}<V_{P C}, \quad\left(\frac{C_{P}}{C_{F}}\right)_{i}=\left(\frac{C_{C}}{C_{F}}\right)_{i}
$$

$\mathrm{V}_{\mathrm{i}} \quad=$ cumulative volume of water extracted water volume at time $\mathrm{i}\left[\mathrm{L}^{3}\right]$

$\mathrm{V}_{\mathrm{PC}} \quad=$ volume of water in submersible casing $\left[\mathrm{L}^{3}\right]$.

At the start of purging, the initial concentration of solute below the pump inlet and above the screened interval is equivalent to $\mathrm{C}_{\mathrm{C}}$ but transitions to water from the screened interval over time. Thus, when

$$
V_{i(B P)}<V_{S P}, \quad\left(\frac{C_{B P}}{C_{F}}\right)_{i}=\left(\frac{C_{C}}{C_{F}}\right)_{i}
$$

and

$$
V_{i(B P)}>V_{S P}, \quad\left(\frac{C_{B P}}{C_{F}}\right)_{i}=\left(\frac{C_{S}}{C_{F}}\right)_{i}
$$

$V_{i(B P)}=\Delta V_{i(B P)}+\Delta V_{i-1(B P)}$.

$\mathrm{V}_{\mathrm{SP}} \quad=$ volume of water initially stored between the pump inlet and screened interval

$\mathrm{V}_{\mathrm{i}(\mathrm{BP})}=$ cumulative volume of water removed from below the pump inlet at time increment $\mathrm{i}$

$\mathrm{C}_{\mathrm{S}} \quad=$ concentration exiting the screened interval at time increment $\mathrm{i}\left[\mathrm{ML}^{-3}\right]$.

The cumulative volume of water extracted below the pump at time increment $i$ is calculated by adding the cumulative volume of water extracted below the pump at time increment i-1 to the change in extracted volume below the pump which is calculated by

$$
\Delta V_{i(B P)}=\Delta V_{i}\left(\frac{Q_{B P}}{Q_{T}}\right)_{i}
$$

and

$$
\Delta V_{i}=V_{i}-V_{i-1} \text {. }
$$

The fraction of water originating below the pump to total water extracted is calculated by:

$$
\left(\frac{Q_{B P}}{Q_{T}}\right)_{i}=1-\left(\frac{Q_{A P}}{Q_{T}}\right)_{i}
$$

and

$$
\left(\frac{Q_{A P}}{Q_{T}}\right)_{i}=\frac{\Delta D_{i}}{\Delta D_{i}^{*}}
$$




$$
\Delta D_{i}=D_{i}-D_{i-1}
$$

$\Delta D_{i}^{*}=\frac{\Delta V_{i}}{A_{C}}$

$A_{C}=\frac{\pi L_{C D}}{4 B}\left(D_{I D-W C}^{2}-D_{O D-P C}^{2}-D_{O D-T L}^{2}\right)$

$\mathrm{D}_{\mathrm{i}} \quad=$ total drawdown at time $\mathrm{i}[\mathrm{L}]$

$\Delta \mathrm{D}_{\mathrm{i}^{*}} \quad=$ the change in drawdown at time $\mathrm{i}$ if all water during drawdown comes from above the pump.

$\mathrm{A}_{\mathrm{C}} \quad=$ volume per length of casing associated with drawdown $\left[\mathrm{L}^{2}\right]$ (7.12 liters/m drawdown at MW01)

$\mathrm{L}_{\mathrm{CD}} \quad=$ characteristic drawdown length [L] (1 $\mathrm{m}$ at MW01).

During recovery,

$$
\Delta D_{i}<0 \text { and }\left(\frac{Q_{A P}}{Q_{T}}\right)_{i}=0
$$

The concentration of solute in water exiting the screened interval then is calculated using a mass balance mixing equation:

$V_{S} \frac{d C_{S}}{d t}=C_{F} Q_{B P}-C_{S} Q_{B P}$

Since $\mathrm{Q}_{\mathrm{BP}}$ is a function of time (calculated in increments using drawdown and flow data), $\mathrm{C}_{\mathrm{S}} / \mathrm{C}_{\mathrm{F}}$ varies in increments of time

$$
\left(\frac{C_{S}}{C_{F}}\right)_{i}=1-\left(1-\left(\frac{C_{S}}{C_{F}}\right)_{i-1}\right) \exp \left(-\Delta \alpha_{i}\right)
$$

where

$$
\begin{aligned}
& \Delta \alpha_{i}=\alpha_{i}-\alpha_{i-1} \\
& \alpha_{i}=\frac{V_{B P(i)}}{V_{S}}
\end{aligned}
$$

$\alpha_{\mathrm{i}} \quad=$ cumulative screen volume exchanges at time increment $\mathrm{i}$.

$\mathrm{V}_{\mathrm{S}} \quad=$ screen volume $($ casing + porosity of pre-packed screen + annular space between pre-packed screen and borehole wall).

At time $0, \mathrm{C}_{\mathrm{S}}$ equals initial concentration $\left(\mathrm{C}_{0}\right)$. $\mathrm{V}_{\mathrm{S}}$ is a constant calculated by:

$V_{S}=V_{B}-V_{C}+\frac{\pi}{4 B} L_{S S} D_{I D-W C}^{2}$

Input parameters to support modeling are summarized in Table SI E2. 
Table SI E2. Well design and input parameters for calculation of casing, borehole, and screen exchange volumes during Phase V sampling event

\begin{tabular}{|c|c|c|c|c|}
\hline \multirow[t]{2}{*}{ Label } & \multicolumn{2}{|c|}{ MW01 } & \multicolumn{2}{|c|}{ MW02 } \\
\hline & $\mathrm{m}$ & liters & $\mathrm{m}$ & liters \\
\hline Depth below ground surface to static head & 62.2 & & 60.5 & \\
\hline Depth below ground surface to base of screen & 239.3 & & 298.7 & \\
\hline Length of submersible pump $\left(\mathbf{L}_{\mathbf{S P}}\right)$ & 0.635 & & 0.635 & \\
\hline Depth of base of submersible pump & 232.7 & & 297.2 & \\
\hline Depth of top of submersible pump casing & 232.1 & & 296.6 & \\
\hline Depth of schedule 80 PVC tag line & 232.1 & & 296.6 & \\
\hline Length of water column in well (Lwc) & 177.1 & & 238.2 & \\
\hline Length of water in submerged pump casing (LPC) & 232.1 & & 296.6 & \\
\hline Length of submerged tag line ( $\left.\mathbf{L}_{\mathrm{TL}}\right)$ & 169.9 & & 236.0 & \\
\hline Length of stainless-steel pre-packed screen $\left(\mathbf{L}_{\mathbf{S S}}\right)$ & 6.10 & & 6.10 & \\
\hline Inside diameter of schedule 40 steel well casing (IDwc) & 0.102 & & 0.102 & \\
\hline Outside diameter of schedule-40 steel well casing (ODwc) & 0.114 & & 0.114 & \\
\hline Outside diameter of submersible pump casing (ODPC) & 0.034 & & 0.034 & \\
\hline Inside diameter of submersible pump casing (IDPC) & 0.027 & & 0.027 & \\
\hline Outside diameter of schedule 80 PVC tag line (OD & 0.033 & & 0.033 & \\
\hline Inside diameter of schedule 80 PVC tag line (ID TL $_{\text {) }}$ & 0.024 & & 0.024 & \\
\hline Outside diameter of submersible pump (ODSP) & 0.089 & & 0.089 & \\
\hline Diameter of borehole $\left(\mathbf{D}_{\mathbf{B H}}\right)$ & 0.251 & & 0.251 & \\
\hline Outside diameter of pre-packed screen (ODPP) & 0.216 & & 0.216 & \\
\hline \multicolumn{5}{|l|}{ Effective porosity of pre-packed screen $(\varphi)=0.45$} \\
\hline Length from Bottom of Pump to top of Screen (LPS) & 0.457 & & 0.0 & \\
\hline Well casing volume $\left(\mathbf{V}_{\mathbf{C}}\right)$ & & 1358 & & 1792 \\
\hline Borehole volume $\left(\mathbf{V}_{\mathbf{B}}\right)$ & & 1504 & & 1942 \\
\hline Screen plus borehole volume $\left(\mathbf{V}_{\mathbf{S}}\right)$ & & 200.6 & & 196.8 \\
\hline Volume above screen and below pump (V) & & 3.76 & & 0.0 \\
\hline Liters per meter of drawdown (Ac) & & 6.45 & & 6.45 \\
\hline Volume of water in submersible pump casing $\left(\mathbf{V}_{\mathbf{P C}}\right)$ & & 129 & & 165 \\
\hline
\end{tabular}




\section{E.4 Summary of Sample Results at MW01 and MW02}

Table SI E3a. Summary of Organic Compound Detections in MW01

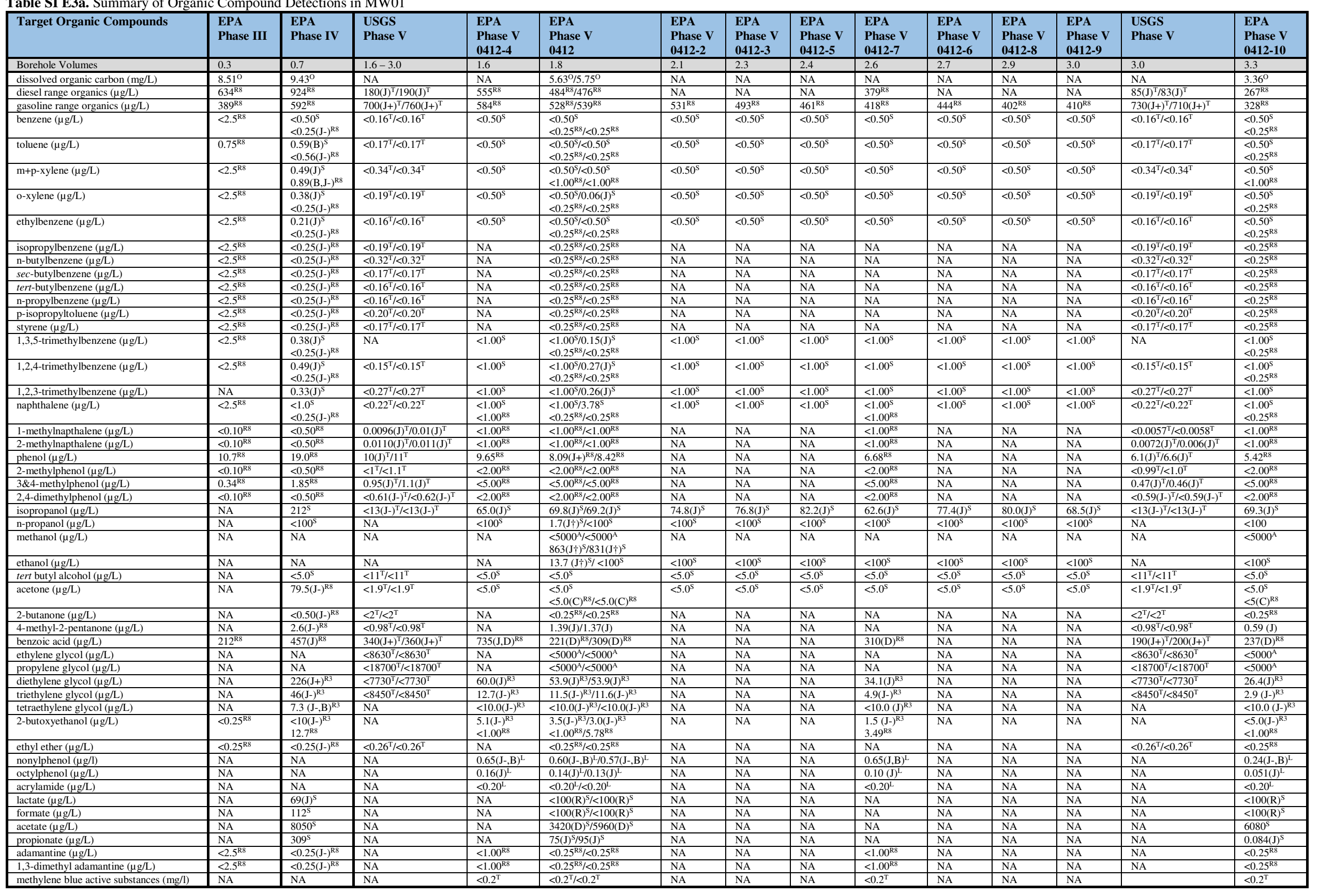


Table SI E3b. Summary of Field Parameters, Major Ions, and Dissolved Metals at MW01

\begin{tabular}{|c|c|c|c|c|c|c|c|c|c|c|c|c|c|c|}
\hline Inorganics & $\begin{array}{l}\text { EPA } \\
\text { Phase } \\
\text { III } \\
\end{array}$ & $\begin{array}{l}\text { EPA } \\
\text { Phase } \\
\text { IV } \\
\end{array}$ & $\begin{array}{l}\text { USGS } \\
\text { Phase V }\end{array}$ & $\begin{array}{l}\text { EPA } \\
\text { Phase V } \\
\text { 0412-4 } \\
\end{array}$ & $\begin{array}{l}\text { EPA } \\
\text { Phase V } \\
\text { 0412 } \\
\end{array}$ & $\begin{array}{l}\text { EPA } \\
\text { Phase V } \\
\text { 0412-2 } \\
\end{array}$ & $\begin{array}{l}\text { EPA } \\
\text { Phase V } \\
0412-3 \\
\end{array}$ & $\begin{array}{l}\text { EPA } \\
\text { Phase V } \\
0412-5 \\
\end{array}$ & $\begin{array}{l}\text { EPA } \\
\text { Phase V } \\
\text { 0412-7 } \\
\end{array}$ & $\begin{array}{l}\text { EPA } \\
\text { Phase V } \\
\text { 0412-6 } \\
\end{array}$ & $\begin{array}{l}\text { EPA } \\
\text { Phase V } \\
0412-8 \\
\end{array}$ & $\begin{array}{l}\text { EPA } \\
\text { Phase V } \\
\text { 0412-9 } \\
\end{array}$ & $\begin{array}{l}\text { USGS } \\
\text { Phase V }\end{array}$ & $\begin{array}{l}\text { EPA } \\
\text { Phase V } \\
0412-10 \\
\end{array}$ \\
\hline Borehole Volumes & 0.3 & 0.7 & $1.6-3.0$ & 1.6 & 1.8 & 2.1 & 2.3 & 2.4 & 2.6 & 2.7 & 2.9 & 3.0 & 3.0 & 3.3 \\
\hline \multicolumn{15}{|l|}{ Field Parameters } \\
\hline $\mathrm{pH}$ & 11.91 & 11.79 & 11.4 & 11.43 & $11.34 / 11.34$ & 11.20 & 11.16 & 11.08 & 11.01 & 10.94 & 10.89 & 10.80 & 10.7 & 10.71 \\
\hline specific conductance $(\mu \mathrm{S} / \mathrm{cm})$ & 3265 & 2352 & 1621 & 1539 & $1489 / 1489$ & 1413 & 1393 & 1371 & 1354 & 1336 & 1335 & 1318 & 1373 & 1307 \\
\hline dissolved oxygen (mg/l) & 0.17 & 0.03 & $<0.20$ & 0.01 & $0.00 / 0.00$ & 0.00 & 0.00 & 0.01 & 0.01 & 0.00 & 0.01 & 0.00 & $<0.20$ & 0.01 \\
\hline oxidation reduction potential $(\mathrm{mV})$ & 114 & -116 & -343.7 & -395 & $-388 /-388$ & -393 & -394 & -391 & -397 & -395 & -385 & -383 & -389.8 & -379 \\
\hline turbidity (NTU) & 7.5 & 7.9 & NM & $\mathrm{NA}$ & 2.0 & $\mathrm{NA}$ & $\mathrm{NA}$ & $\mathrm{NA}$ & $\mathrm{NA}$ & $\mathrm{NA}$ & $\mathrm{NA}$ & $\mathrm{NA}$ & NM & 2.6 \\
\hline alkalinity $(\mathrm{mg} / \mathrm{CaCO})$ & 430 & 388 & 215 & NA & $213 / 213$ & $\mathrm{NA}$ & NA & NA & NA & NA & NA & NA & $174 / 182$ & 181 \\
\hline \multicolumn{15}{|l|}{ Major Ions } \\
\hline nitrate + nitrite (mg N/l) & $0.150(\mathrm{~B})^{\mathrm{o}}$ & $<0.100^{\circ}$ & $<0.019^{\mathrm{T}} /<0.019^{\mathrm{T}}$ & NA & $0.120^{\circ} /<0.050^{\circ}$ & $\mathrm{NA}$ & NA & NA & NA & NA & NA & NA & $<0.02^{\mathrm{T}}$ & $<0.050^{\circ}$ \\
\hline ammonia (mg N/l) & $4.61^{\circ}$ & $4.25^{\circ}$ & $0.79(\mathrm{~B})^{\mathrm{T}} / 0.79(\mathrm{~B})^{\mathrm{T}}$ & $\mathrm{NA}$ & $1.81^{\circ} / 1.60^{\circ}$ & $\mathrm{NA}$ & $\mathrm{NA}$ & $\mathrm{NA}$ & $\mathrm{NA}$ & $\mathrm{NA}$ & $\mathrm{NA}$ & NA & $0.34(\mathrm{~B})^{\mathrm{T}}$ & $0.490^{\circ}$ \\
\hline SO4 $(\mathrm{mg} / \mathrm{L})$ & $398^{\circ}$ & $339^{\circ}$ & $380^{\mathrm{T}} / 380^{\mathrm{T}}$ & $391^{\circ}$ & $390^{\circ} / 388^{\circ}$ & $397^{\circ}$ & $394^{\circ}$ & $406^{\circ}$ & $407^{\circ}$ & $401^{\circ}$ & $411^{\circ}$ & $413^{\circ}$ & $410^{\mathrm{T}}$ & $428^{\circ}$ \\
\hline $\mathrm{S}(\mathrm{mg} / \mathrm{L})$ & $158(\mathrm{~J})$ & $141(\mathrm{~J})$ & NA & $187(\mathrm{~J})^{\mathrm{s}}$ & $177(\mathrm{~J})^{\mathrm{S}} / 171(\mathrm{~J})^{\mathrm{S}}$ & $202(\mathrm{~J})^{\mathrm{s}}$ & $198(\mathrm{~J})^{\mathrm{S}}$ & $210(\mathrm{~J})^{\mathrm{s}}$ & $210(\mathrm{~J})^{\mathrm{s}}$ & $208(\mathrm{~J})^{\mathrm{S}}$ & $210(\mathrm{~J})^{\mathrm{S}}$ & $214(\mathrm{~J})^{\mathrm{S}}$ & NA & $200(\mathrm{~J})^{\mathrm{S}}$ \\
\hline $\mathrm{Cl}(\mathrm{mg} / \mathrm{l})$ & $23.3^{\circ}$ & $23.1^{\circ}$ & $26^{\mathrm{T}} / 26^{\mathrm{T}}$ & $21.0^{\circ}$ & $19.4^{\circ} / 20.9^{\circ}$ & $22.0^{\circ}$ & $21.0^{\circ}$ & $21.2^{\circ}$ & $21.0^{\circ}$ & $21.5^{\circ}$ & $21.6^{\circ}$ & $20.8^{\circ}$ & $27^{\mathrm{T}}$ & $21.2^{\circ}$ \\
\hline $\mathrm{Br}(\mathrm{mg} / \mathrm{l})$ & NA & $<3.00^{\circ}$ & $0.2(\mathrm{~J})^{\mathrm{T}} / 0.2(\mathrm{~J})^{\mathrm{T}}$ & $<1.00^{\circ}$ & $<1.00^{\circ} /<1.00^{\circ}$ & $<1.00^{\circ}$ & $<1.00^{\circ}$ & $<1.00^{\circ}$ & $<1.00^{\circ}$ & $<1.00^{\circ}$ & $<1.00^{\circ}$ & $<1.00^{\circ}$ & $0.2(\mathrm{~J})^{\mathrm{T}}$ & $<1.00^{\circ}$ \\
\hline $\mathrm{F}(\mathrm{mg} / \mathrm{l})$ & $1.55^{\circ}$ & $1.88^{\circ}$ & $3^{\mathrm{T} / 3^{\mathrm{T}}}$ & $2.24^{\circ}$ & $2.29^{\circ} / 2.33^{\circ}$ & $2.37^{\circ}$ & $2.11^{\circ}$ & $2.29^{\circ}$ & $2.01^{\circ}$ & $2.33^{\circ}$ & $2.13^{\circ}$ & $2.06^{\circ}$ & $3^{\mathrm{T}}$ & $2.01^{\circ}$ \\
\hline $\mathrm{K}(\mathrm{mg} / \mathrm{l})$ & $54.9^{\mathrm{S}}$ & $24.7^{\mathrm{s}}$ & \begin{tabular}{|l}
$15.0^{\mathrm{T}} / 16.0^{\mathrm{T}}$ \\
\end{tabular} & $17.8^{\mathrm{s}}$ & $17.3^{\mathrm{s} / 17.2^{\mathrm{S}}}$ & $16.2^{\mathrm{s}}$ & $16.0^{\mathrm{s}}$ & $15.7^{\mathrm{s}}$ & $15.3^{\mathrm{s}}$ & $15.0^{\mathrm{s}}$ & $14.5^{\mathrm{s}}$ & $14.4^{\mathrm{S}}$ & $13.0^{\mathrm{T}}$ & $13.6^{\mathrm{s}}$ \\
\hline $\mathrm{Na}(\mathrm{mg} / \mathrm{l})$ & $334^{\mathrm{s}}$ & $304^{\mathrm{S}}$ & $270^{\mathrm{T}} / 280^{\mathrm{T}}$ & $273^{S}$ & $276^{\mathrm{S} / 277^{\mathrm{s}}}$ & $271^{\mathrm{S}}$ & $264^{\mathrm{S}}$ & $265^{\mathrm{s}}$ & $265^{\mathrm{s}}$ & $264^{5}$ & $264^{\mathrm{S}}$ & $268^{\mathrm{s}}$ & $280^{\mathrm{T}}$ & $264^{\mathrm{S}}$ \\
\hline $\mathrm{Ca}(\mathrm{mg} / \mathrm{l})$ & $15.6^{\mathrm{S}}$ & $13.6^{\mathrm{s}}$ & $9.4^{\mathrm{T}} / 9.4^{\mathrm{T}}$ & $9.80^{\mathrm{s}}$ & $9.87 / 9.91^{\mathrm{s}}$ & $9.75^{\mathrm{s}}$ & $9.67^{\mathrm{s}}$ & $9.69^{\mathrm{s}}$ & $9.67^{\mathrm{s}}$ & $9.66^{\mathrm{s}}$ & $9.61^{\mathrm{s}}$ & $9.65^{\mathrm{s}}$ & $8.90^{\mathrm{T}}$ & $9.47^{\mathrm{s}}$ \\
\hline $\mathrm{Mg}(\mathrm{mg} / \mathrm{l})$ & $0.05(\mathrm{~B})^{\mathrm{S}}$ & $0.12^{\mathrm{s}}$ & $0.14(\mathrm{~J})^{\mathrm{T}} / 0.15(\mathrm{~J})^{\mathrm{T}}$ & $0.13^{\mathrm{s}}$ & $0.14^{\mathrm{s}} / 0.15^{\mathrm{s}}$ & $0.14^{\mathrm{s}}$ & $0.15^{\mathrm{s}}$ & $0.15^{\mathrm{s}}$ & $0.16^{\mathrm{S}}$ & $0.16^{\mathrm{s}}$ & $0.16^{\mathrm{s}}$ & $0.17^{\mathrm{s}}$ & $0.17(\mathrm{~J})^{\mathrm{T}}$ & $0.17^{\mathrm{s}}$ \\
\hline $\mathrm{Si}(\mathrm{mg} / \mathrm{L})$ & $8.02(\mathrm{~J})^{\mathrm{s}}$ & $10.2(\mathrm{~J})^{\mathrm{s}}$ & $9.0^{\mathrm{T} / 8.7^{\mathrm{T}}}$ & $12.00(\mathrm{~J}+)^{\mathrm{S}}$ & $10.50(\mathrm{~J}+)^{\mathrm{S}} / 10.50(\mathrm{~J}+)^{\mathrm{s}}$ & $10.10(\mathrm{~J}+)^{\mathrm{S}}$ & $9.95(\mathrm{~J}+)^{\mathrm{S}}$ & $9.54(\mathrm{~J}+)^{\mathrm{S}}$ & $9.09(\mathrm{~J}+)^{\mathrm{S}}$ & $8.81(\mathrm{~J}+)^{\mathrm{S}}$ & $8.43(\mathrm{~J}+)^{\mathrm{S}}$ & $8.18(\mathrm{~J}+)^{\mathrm{S}}$ & $6.4^{\mathrm{T}}$ & $7.69(\mathrm{~J}+)^{\mathrm{S}}$ \\
\hline \multicolumn{15}{|l|}{ Dissolved Metals } \\
\hline $\mathrm{Ag}(\mu \mathrm{g} / \mathrm{L})$ & $<51^{\mathrm{s}}$ & $<51^{\mathrm{s}}$ & $<0.033^{\mathrm{T}}<0.033^{\mathrm{T}}$ & $<14^{\mathrm{s}}$ & $<14^{\mathrm{S} /}<14^{\mathrm{S}}$ & $<14^{\mathrm{S}}$ & $<14^{\mathrm{s}}$ & $<14^{\mathrm{S}}$ & $<14^{\mathrm{S}}$ & $<14^{5}$ & $<14^{\mathrm{s}}$ & $<14^{\mathrm{s}}$ & $<0.033^{\mathrm{T}}$ & $<14^{\mathrm{S}}$ \\
\hline $\mathrm{Al}(\mu \mathrm{g} / \mathrm{L})$ & $422(\mathrm{~J})^{\mathrm{S}}$ & $382(\mathrm{~J})^{\mathrm{S}}$ & $170^{\mathrm{T} / 170^{\mathrm{T}}}$ & $205(\mathrm{~J})^{\mathrm{S}}$ & $234(\mathrm{~J})^{\mathrm{s}} / 245(\mathrm{~J})^{\mathrm{s}}$ & $211(\mathrm{~J})^{\mathrm{S}}$ & $<494^{\mathrm{s}}$ & $<494^{\mathrm{S}}$ & $<494^{\mathrm{s}}$ & $<494^{\mathrm{S}}$ & $<494^{\mathrm{s}}$ & $<494^{5}$ & $100^{\mathrm{T}}$ & $<494^{\mathrm{s}}$ \\
\hline As $(\mu \mathrm{g} / \mathrm{L})$ & $<51^{\mathrm{s}}$ & $<51^{\mathrm{s}}$ & $0.62(\mathrm{~J})^{\mathrm{T}} /<0.33^{\mathrm{T}}$ & $0.40(\mathrm{~J})^{\mathrm{C}}$ & $0.49(\mathrm{~J})^{\mathrm{C}} / 0.71(\mathrm{~J})^{\mathrm{C}}$ & $<1.00^{\mathrm{C}}$ & $0.53(\mathrm{~J})^{\mathrm{C}}$ & $0.28(\mathrm{~J})^{\mathrm{C}}$ & $0.82(\mathrm{~J})^{\mathrm{C}}$ & $0.47(\mathrm{~J})^{\mathrm{C}}$ & $0.43(\mathrm{~J})^{\mathrm{C}}$ & $0.59(\mathrm{~J})^{\mathrm{C}}$ & $<0.33^{\mathrm{T}}$ & $<1.00^{\mathrm{C}}$ \\
\hline $\mathrm{B}(\mu \mathrm{g} / \mathrm{L})$ & $154(\mathrm{~J})^{\mathrm{S}}$ & $117\left(J^{\mathrm{s}}\right.$ & $130^{\mathrm{T}} / 130^{\mathrm{T}}$ & $142(\mathrm{~J})^{\mathrm{S}}$ & $136(\mathrm{~J})^{\mathrm{S} / 136(J)^{\mathrm{S}}}$ & $131(\mathrm{~J})^{\mathrm{s}}$ & $130\left(J^{5}\right)^{s}$ & $128(\mathrm{~J})^{\mathrm{S}}$ & $126(J)^{5}$ & $125(\mathrm{~J})^{\mathrm{S}}$ & $123\left(J^{5}\right)^{s}$ & $121\left(J^{\mathrm{S}}\right.$ & $120^{\mathrm{T}}$ & $119(\mathrm{~J})^{\mathrm{s}}$ \\
\hline $\mathrm{Ba}(\mu \mathrm{g} / \mathrm{L})$ & $41(\mathrm{~J})^{\mathrm{s}}$ & $26(\mathrm{~J})$ & $23^{\mathrm{T}} / 20^{\mathrm{T}}$ & $21\left(J^{\mathrm{s}}\right.$ & $21(\mathrm{~J})^{\mathrm{S}} / 21(\mathrm{~J})^{\mathrm{s}}$ & $20\left(J^{\mathrm{s}}\right.$ & $20(\mathrm{~J})^{\mathrm{s}}$ & $20(\mathrm{~J})^{\mathrm{s}}$ & 20()$^{s}$ & 20()$^{S}$ & $20\left(J^{\mathrm{s}}\right.$ & 20()$^{s}$ & $21^{\mathrm{T}}$ & $19\left(J^{\mathrm{s}}\right.$ \\
\hline $\operatorname{Be}(\mu \mathrm{g} / \mathrm{L})$ & $<4^{\mathrm{s}}$ & $<4^{\mathrm{s}}$ & $<0.08^{\mathrm{T}} /<0.08^{\mathrm{T}}$ & $<10^{\mathrm{s}}$ & $<10^{\mathrm{s} /}<10^{\mathrm{s}}$ & $<10^{\mathrm{s}}$ & $<10^{\mathrm{s}}$ & $<10^{5}$ & $<10^{\mathrm{s}}$ & $<10^{\mathrm{s}}$ & $<10^{\mathrm{s}}$ & $<10^{5}$ & $<0.08^{\mathrm{T}}$ & $<10^{\mathrm{s}}$ \\
\hline $\mathrm{Cd}(\mu \mathrm{g} / \mathrm{L})$ & $<4$ & $<4^{5}$ & $<0.1^{\mathrm{T}} /<0.1^{\mathrm{T}}$ & $<1.00^{\mathrm{s}}$ & $<1.00^{\mathrm{S}} /<1.00^{\mathrm{S}}$ & $<1.00^{\mathrm{S}}$ & $<1.00^{\mathrm{s}}$ & $<1.00^{\mathrm{s}}$ & $<1.00^{\mathrm{s}}$ & $<1.00^{\mathrm{s}}$ & $<1.00^{\mathrm{s}}$ & $<1.00^{\mathrm{s}}$ & $<0.1^{\mathrm{T}}$ & $<1.00^{\mathrm{s}}$ \\
\hline Co $(\mu \mathrm{g} / \mathrm{L})$ & $<4^{\mathrm{s}}$ & $<4^{5}$ & $<0.054^{\mathrm{T}} /<0.054^{\mathrm{T}}$ & $<4^{\mathrm{s}}$ & $<4^{\mathrm{S} /<4^{\mathrm{S}}}$ & $<4^{\mathrm{S}}$ & $<4^{\mathrm{S}}$ & $<4^{\mathrm{s}}$ & $<4^{\mathrm{s}}$ & $<4^{\mathrm{s}}$ & $<4^{5}$ & $<4^{5}$ & $<0.004^{\mathrm{T}}$ & $<4^{\mathrm{s}}$ \\
\hline $\operatorname{Cr}(\mu \mathrm{g} / \mathrm{L})$ & $<4^{\mathrm{s}}$ & $<4^{\mathrm{s}}$ & $<0.5^{\mathrm{T}} /<0.5^{\mathrm{T}}$ & $<2.00^{\mathrm{s}}$ & $2.2^{\mathrm{S}} /<2.00^{\mathrm{S}}$ & $<2.00^{\mathrm{s}}$ & $<2.00^{\mathrm{s}}$ & $<2.00^{\mathrm{s}}$ & $<2.00^{\mathrm{s}}$ & $<2.00^{\mathrm{s}}$ & $<2.00^{\mathrm{S}}$ & $<2.00^{\mathrm{s}}$ & $<0.5^{\mathrm{T}}$ & $<2.00^{\mathrm{S}}$ \\
\hline $\mathrm{Cu}(\mu \mathrm{g} / \mathrm{L})$ & $<9^{\mathrm{S}}$ & $<9^{\mathrm{s}}$ & $<0.56^{\mathrm{T}} /<0.56^{\mathrm{T}}$ & $<2.00^{\mathrm{s}}$ & $<2.00^{\mathrm{s}} /<2.00^{\mathrm{S}}$ & $<2.00^{\mathrm{S}}$ & $<2.00^{\mathrm{s}}$ & $<2.00^{\mathrm{s}}$ & $<2.00^{\mathrm{s}}$ & $<2.00^{\mathrm{s}}$ & $<2.00^{\mathrm{S}}$ & $<2.00^{\mathrm{s}}$ & $<0.56^{\mathrm{T}}$ & $<2.00^{\mathrm{S}}$ \\
\hline $\mathrm{Fe}(\mu \mathrm{g} / \mathrm{L})$ & $<63^{\mathrm{S}}$ & $>63^{5}$ & $<22^{\mathrm{T}} /<22^{\mathrm{T}}$ & $<67^{\mathrm{s}}$ & $<67^{\mathrm{S} /<67^{\mathrm{S}}}$ & $<67^{\mathrm{S}}$ & $<67^{\mathrm{S}}$ & $<67^{\mathrm{S}}$ & $<67^{\mathrm{S}}$ & $<67^{\mathrm{S}}$ & $<67^{\mathrm{s}}$ & $<67^{\mathrm{s}}$ & $<22^{\mathrm{T}}$ & $<67^{5}$ \\
\hline $\mathrm{Hg}(\mu \mathrm{g} / \mathrm{L})$ & NA & NA & $<0.027^{\mathrm{T} /}<0.027^{\mathrm{T}}$ & $\mathrm{NA}$ & NA & NA & $\mathrm{NA}$ & NA & NA & NA & $\mathrm{NA}$ & NA & $<0.027^{\mathrm{T}}$ & NA \\
\hline $\operatorname{Li}(\mu \mathrm{g} / \mathrm{L})$ & $\mathrm{NA}$ & $\mathrm{NA}$ & $44^{\mathrm{T}} / 45^{\mathrm{T}}$ & $\mathrm{NA}$ & $\mathrm{NA}$ & $\mathrm{NA}$ & $\mathrm{NA}$ & NA & $\mathrm{NA}$ & NA & $\mathrm{NA}$ & $\mathrm{NA}$ & $33^{\mathrm{T}}$ & $\mathrm{NA}$ \\
\hline $\operatorname{Mn}(\mu \mathrm{g} / \mathrm{L})$ & $<4^{\mathrm{S}}$ & $<4^{\mathrm{s}}$ & $<0.31^{\mathrm{T} / 1^{\mathrm{T}}}$ & $<14^{\mathrm{s}}$ & $<14^{\mathrm{S} /}<14^{\mathrm{S}}$ & $<14^{\mathrm{s}}$ & $<14^{\mathrm{s}}$ & $<14^{\mathrm{s}}$ & $<14^{\mathrm{s}}$ & $<14^{\mathrm{S}}$ & $<14^{\mathrm{s}}$ & $<14^{\mathrm{S}}$ & $0.42(\mathrm{~J})^{\mathrm{T}}$ & $<14^{\mathrm{S}}$ \\
\hline Mo $(\mu \mathrm{g} / \mathrm{L})$ & $20^{\mathrm{s}}$ & $<7^{\mathrm{S}}$ & $10^{\mathrm{T} / 9.7^{\mathrm{T}}}$ & $7(\mathrm{~J})^{\mathrm{S}}$ & $7(\mathrm{~J})^{\mathrm{S} / 8(8)^{\mathrm{S}}}$ & $5(\mathrm{~J})^{\mathrm{S}}$ & $<17^{\mathrm{s}}$ & $<17^{\mathrm{s}}$ & $<17^{5}$ & $<17^{\mathrm{s}}$ & $<17^{\mathrm{s}}$ & $<17^{\mathrm{s}}$ & $7.6^{\mathrm{T}}$ & $<17^{\mathrm{s}}$ \\
\hline $\mathrm{Ni}(\mu \mathrm{g} / \mathrm{L})$ & $1(\mathrm{~J})^{\mathrm{S}}$ & $3(\mathrm{~J})^{\mathrm{s}}$ & $<0.3^{\mathrm{T}} /<0.3^{\mathrm{T}}$ & $<1.00^{\mathrm{s}}$ & $<1.00^{\mathrm{s}} / 0.39(\mathrm{~J})^{\mathrm{s}}$ & $<1.00^{\mathrm{s}}$ & $0.23(\mathrm{~J})^{\mathrm{S}}$ & $<1.00^{\mathrm{s}}$ & $<1.00^{\mathrm{s}}$ & $<1.00^{\mathrm{s}}$ & $<1.00^{\mathrm{s}}$ & $<1.00^{\mathrm{s}}$ & $<0.3^{\mathrm{T}}$ & $<1.00^{\mathrm{s}}$ \\
\hline $\mathrm{P}(\mu \mathrm{g} / \mathrm{L})$ & $<0.014^{\mathrm{S}}$ & $<0.014^{\mathrm{s}}$ & \begin{tabular}{|l}
$57^{\mathrm{T}} / 89^{\mathrm{T}}$ \\
\end{tabular} & $<0.06^{\mathrm{S}}$ & $<0.06^{\mathrm{S} /<0.06^{\mathrm{S}}}$ & $<0.06^{\mathrm{s}}$ & $<0.06^{5}$ & $<0.06^{\mathrm{s}}$ & $<0.06^{\mathrm{s}}$ & $<0.06^{\mathrm{S}}$ & $<0.06^{\mathrm{s}}$ & $<0.06^{\mathrm{s}}$ & $61^{\mathrm{T}}$ & $<0.06^{\mathrm{S}}$ \\
\hline $\mathrm{Pb}(\mu \mathrm{g} / \mathrm{L})$ & $<11^{\mathrm{s}}$ & $<11^{\mathrm{s}}$ & $<0.18^{\mathrm{T}} /<0.18^{\mathrm{T}}$ & $<1.00^{\mathrm{S}}$ & $<1.00^{\mathrm{S} /} / 1.00^{\mathrm{S}}$ & $<1.00^{\mathrm{S}}$ & $<1.00^{\mathrm{S}}$ & $<1.00^{\mathrm{s}}$ & $<1.00^{\mathrm{s}}$ & $<1.00^{\mathrm{S}}$ & $<1.00^{\mathrm{S}}$ & $<1.00^{\mathrm{s}}$ & $<0.18^{\mathrm{T}}$ & $<1.00^{\mathrm{S}}$ \\
\hline $\mathrm{Sb}(\mu \mathrm{g} / \mathrm{L})$ & $\mathrm{R}^{\mathrm{S}}$ & $\mathrm{R}^{\mathrm{S}}$ & $<0.4^{\mathrm{T}} / 0.54(\mathrm{~B})^{\mathrm{T}}$ & $<2.00^{\mathrm{S}}$ & $<2.00^{\mathrm{S}} /<2.00^{\mathrm{S}}$ & $<2.00^{\mathrm{S}}$ & $<2.00^{\mathrm{s}}$ & $<2.00^{\mathrm{S}}$ & $<2.00^{\mathrm{S}}$ & $<2.00^{\mathrm{S}}$ & $<2.00^{\mathrm{s}}$ & $<2.00^{\mathrm{s}}$ & $<0.4^{\mathrm{T}}$ & $<2.00^{\mathrm{S}}$ \\
\hline Se $(\mu \mathrm{g} / \mathrm{L})$ & $<16^{\mathrm{s}}$ & $<16^{\mathrm{s}}$ & $<0.7 /<0.7$ & $<5.00^{\mathrm{s}}$ & $<5.00^{\mathrm{s} /} /<5.00^{\mathrm{S}}$ & $<5.00^{\mathrm{S}}$ & $<5.00^{\mathrm{s}}$ & $<5.00^{\mathrm{s}}$ & $<5.00^{\mathrm{s}}$ & $<5.00^{\mathrm{s}}$ & $<5.00^{\mathrm{s}}$ & $<5.00^{\mathrm{s}}$ & $<0.7^{\mathrm{T}}$ & $<5.00^{\mathrm{S}}$ \\
\hline $\operatorname{Sr}(\mu \mathrm{g} / \mathrm{L})$ & $801^{\mathrm{s}}$ & $441^{\mathrm{S}}$ & $300^{\mathrm{T}} / 310^{\mathrm{T}}$ & $328^{\mathrm{S}}$ & $315^{\mathrm{s}} / 314^{\mathrm{S}}$ & $312^{\mathrm{S}}$ & $309^{5}$ & $307^{5}$ & $304^{5}$ & $301^{\mathrm{s}}$ & $298^{\mathrm{S}}$ & $301^{\mathrm{S}}$ & $280^{\mathrm{T}}$ & $291^{S}$ \\
\hline $\operatorname{Ti}(\mu \mathrm{g} / \mathrm{L})$ & $<4^{\mathrm{s}}$ & $<4^{\mathrm{s}}$ & $<0.6^{\mathrm{T}} /<0.6^{\mathrm{T}}$ & $<7^{\mathrm{s}}$ & $<7^{5 /<7^{5}}$ & $<7^{\mathrm{s}}$ & $<7^{5}$ & $<7^{\mathrm{s}}$ & $<7^{\mathrm{s}}$ & $<7^{\mathrm{s}}$ & $<7^{\mathrm{s}}$ & $<7^{\mathrm{s}}$ & $<0.6^{\mathrm{T}}$ & $<7^{8}$ \\
\hline $\mathrm{Tl}(\mu \mathrm{g} / \mathrm{L})$ & $<37^{5}$ & $\mathrm{R}^{\mathrm{S}}$ & $<0.05^{\mathrm{T}} / 00.05^{\mathrm{T}}$ & $<1.00^{\mathrm{s}}$ & $<1.00^{\mathrm{S} /} /<1.00^{\mathrm{S}}$ & $<1.00^{\mathrm{s}}$ & $<1.00^{\mathrm{S}}$ & $<1.00^{\mathrm{S}}$ & $<1.00^{\mathrm{S}}$ & $<1.00^{\mathrm{s}}$ & $<1.00^{\mathrm{s}}$ & $<1.00^{\mathrm{s}}$ & $<0.05^{\mathrm{T}}$ & $<1.00^{\mathrm{S}}$ \\
\hline $\mathrm{U}(\mu \mathrm{g} / \mathrm{L})$ & $\mathrm{NA}$ & $\mathrm{NA}$ & $<0.05^{\mathrm{T}} /<0.05^{\mathrm{T}}$ & NA & NA & NA & NA & NA & NA & NA & $\mathrm{NA}$ & NA & $<0.05^{\mathrm{T}}$ & NA \\
\hline $\mathrm{V}(\mu \mathrm{g} / \mathrm{L})$ & $4(\mathrm{~J})^{\mathrm{S}}$ & NA & $0.6(\mathrm{~J})^{\mathrm{T}} /<0.5^{\mathrm{T}}$ & $<10^{\mathrm{S}}$ & $<10^{\mathrm{S} /}<10^{\mathrm{S}}$ & $<10^{\mathrm{S}}$ & $<10^{\mathrm{S}}$ & $<10^{\mathrm{s}}$ & $<10^{\mathrm{S}}$ & $<10^{\mathrm{s}}$ & $<10^{\mathrm{s}}$ & $<10^{\mathrm{S}}$ & $<0.5^{\mathrm{T}}$ & $<10^{\mathrm{s}}$ \\
\hline $\mathrm{Zn}(\mu \mathrm{g} / \mathrm{L})$ & $<24^{s}$ & $<24^{5}$ & $<2^{\mathrm{T}} /<2^{\mathrm{T}}$ & $<50^{\mathrm{S}}$ & $<50^{\mathrm{s} /}<50^{\mathrm{s}}$ & $<50^{\mathrm{s}}$ & $<50^{\mathrm{s}}$ & $<50^{\mathrm{s}}$ & $<50^{\mathrm{s}}$ & $<50^{\mathrm{s}}$ & $<50^{\mathrm{s}}$ & $<50^{\mathrm{s}}$ & $<2^{\mathrm{T}}$ & $<50^{\mathrm{S}}$ \\
\hline
\end{tabular}

Laboratories (Superscript)

$\begin{array}{ll}\text { S } & \text { Shaw Environmental, Ada, OK (Contro } \\ \text { R8 } & \text { EPA Region 8 Laboratory, Golden, CO }\end{array}$

Data Qualifiers

Chemtech Consulting (contractor to EPA)

Not analyzed

Estimated (non-attainment of quality control criteria or below reporting limit. R3 and ORD Las Vegas analysis are estimated because of method development)

Estimated (bised high due to $>130 \%$ m

Estimated (method development on alcohols) ) $^{6,66}$

Analyte in sample $<10 \mathrm{X}$ and $<5 \mathrm{X}$ concentration detected in a blank for EPA and USGS data respectively

Relative percent difference outside acceptance criteria
Sample result rejected due to serious deficiencies in

Acetone result in Phase V corrected from EPA (2012)

\section{O $\quad$ EPA ORD Ada, OK}

atory, Fort Meade, MD

EPA ORD, Las Vegas, NV

Test America Laboratory (Contractor to USGS and EPA) 
Table SI E4a. Summary of Organic Compound Detections in MW02

\begin{tabular}{|c|c|c|c|c|c|}
\hline Compound & $\begin{array}{l}\text { EPA } \\
\text { Phase III }\end{array}$ & $\begin{array}{l}\text { EPA } \\
\text { Phase IV }\end{array}$ & $\begin{array}{l}\text { EPA } \\
\text { Phase V } \\
\text { 0412-1 }\end{array}$ & $\begin{array}{l}\text { EPA } \\
\text { Phase V } \\
\text { 0412-2 }\end{array}$ & $\begin{array}{l}\text { USGS } \\
\text { Phase V } \\
\text { 0412-2 }\end{array}$ \\
\hline Borehole Volumes & 0.1 & 0.6 & 0.0 & 1.0 & 1.0 \\
\hline dissolved organic carbon $(\mathrm{mg} / \mathrm{L})$ & $14.5^{\circ}$ & $19.7^{\circ}$ & $19.4^{\mathrm{O}}$ & $15.5^{\circ}$ & $13^{\mathrm{T}}$ \\
\hline diesel range organics $(\mu \mathrm{g} / \mathrm{l})$ & $1440(\mathrm{~J})^{\mathrm{R} 8}$ & $4050(\mathrm{D})^{\mathrm{R} 8} / 4200(\mathrm{D})^{\mathrm{R} 8}$ & $4150^{\mathrm{R} 8}$ & $2100^{\mathrm{R} 8}$ & $670^{\mathrm{T}}$ \\
\hline gasoline range organics $(\mu \mathrm{g} / \mathrm{l})$ & $3710^{\mathrm{R} 8}$ & $2800^{\mathrm{R} 8} / 3200^{\mathrm{R} 8}$ & $4500^{\mathrm{R} 8}$ & $5290^{\mathrm{R} 8}$ & $6800^{\mathrm{T}} / 7700^{\mathrm{T}}$ \\
\hline benzene $(\mu \mathrm{g} / \mathrm{l})$ & $246^{\mathrm{R} 8}$ & $\begin{array}{l}183^{\mathrm{S}} / 191^{\mathrm{S}} \\
139(\mathrm{~J}-)^{\mathrm{R} 8} / 164(\mathrm{~J}-)^{\mathrm{R} 8}\end{array}$ & $\begin{array}{l}166^{\mathrm{S}} \\
175(\mathrm{~J})^{\mathrm{R} 8}\end{array}$ & $\begin{array}{l}232^{\mathrm{S}} \\
247(\mathrm{~J})^{\mathrm{R} 8}\end{array}$ & $250^{\mathrm{T}} / 260^{\mathrm{T}}$ \\
\hline toluene $(\mu \mathrm{g} / \mathrm{l})$ & $617^{\mathrm{R} 8}$ & $\begin{array}{l}482^{\mathrm{S}} / 464^{\mathrm{S}} \\
336(\mathrm{~J}-)^{\mathrm{R} 8} / 424(\mathrm{~J}-)^{\mathrm{R} 8}\end{array}$ & $\begin{array}{l}402^{\mathrm{S}} \\
437(\mathrm{~J})^{\mathrm{R} 8}\end{array}$ & $\begin{array}{l}607^{\mathrm{S}} \\
677(\mathrm{~J})^{\mathrm{R} 8}\end{array}$ & $690^{\mathrm{T}} / 710^{\mathrm{T}}$ \\
\hline ethylbenzene $(\mu \mathrm{g} / \mathrm{l})$ & $67.0^{\mathrm{R} 8}$ & $\begin{array}{l}68.7^{\mathrm{S}} / 62.0^{\mathrm{S}} \\
21.5(\mathrm{~J}-)^{\mathrm{R} 8} / 27.0(\mathrm{~J}-)^{\mathrm{R} 8}\end{array}$ & $\begin{array}{l}61.1^{\mathrm{S}} \\
57.0(\mathrm{~J})^{\mathrm{R} 8}\end{array}$ & $\begin{array}{l}101^{\mathrm{S}} \\
89.6(\mathrm{~J})^{\mathrm{R} 8}\end{array}$ & $100^{\mathrm{T}} / 100^{\mathrm{T}}$ \\
\hline $\mathrm{m}, \mathrm{p}$ - xylenes $(\mu \mathrm{g} / \mathrm{l})$ & $572^{\mathrm{R} 8}$ & \begin{tabular}{|l|}
$630^{\mathrm{S}} / 554^{\mathrm{S}}$ \\
$280(\mathrm{~J}-)^{\mathrm{R} 8} / 354(\mathrm{~J}-)^{\mathrm{R} 8}$
\end{tabular} & $\begin{array}{l}549^{\mathrm{S}} \\
578(\mathrm{~J})^{\mathrm{R} 8}\end{array}$ & $\begin{array}{l}894^{\mathrm{S}} \\
973(\mathrm{~J})^{\mathrm{R} 8}\end{array}$ & $1000^{\mathrm{T}} / 1000^{\mathrm{T}}$ \\
\hline o-xylene $(\mu \mathrm{g} / \mathrm{l})$ & $178^{\mathrm{R} 8}$ & $\begin{array}{l}175^{\mathrm{S}} / 160^{\mathrm{S}} \\
81.6(\mathrm{~J}-)^{\mathrm{R} 8} / 102(\mathrm{~J}-)^{\mathrm{R} 8}\end{array}$ & $\begin{array}{l}161^{\mathrm{S}} \\
164(\mathrm{~J})^{\mathrm{R} 8}\end{array}$ & $\begin{array}{l}245^{\mathrm{S}} \\
253(\mathrm{~J})^{\mathrm{R} 8}\end{array}$ & $260^{\mathrm{T}} / 250^{\mathrm{T}}$ \\
\hline isopropylbenzene $(\mu \mathrm{g} / \mathrm{l})$ & $11.0^{\mathrm{R} 8}$ & $<12.5(\mathrm{~J}-)^{\mathrm{R} 8} /<12.5(\mathrm{~J}-)^{\mathrm{R} 8}$ & $9.03(J)^{R 8}$ & $9.40(J)^{\mathrm{R} 8}$ & $11(\mathrm{~J})^{\mathrm{T}} / 12(\mathrm{~J})^{\mathrm{T}}$ \\
\hline n-butylbenzene $(\mu \mathrm{g} / \mathrm{l})$ & $<6.25^{\mathrm{R} 8}$ & $<12.5(\mathrm{~J}-)^{\mathrm{R} 8} /<12.5(\mathrm{~J}-)^{\mathrm{R} 8}$ & $0.38(\mathrm{~J})^{\mathrm{R} 8}$ & $<5.0^{\mathrm{R} 8}$ & $<6.4^{\mathrm{T}} /<6.4^{\mathrm{T}}$ \\
\hline sec-butylbenzene $(\mu \mathrm{g} / \mathrm{l})$ & $<6.25^{\mathrm{R} 8}$ & $<12.5(\mathrm{~J}-)^{\mathrm{R} 8} /<12.5(\mathrm{~J}-)^{\mathrm{R} 8}$ & $1.05(\mathrm{~J})^{\mathrm{R} 8}$ & $<5.0^{\mathrm{R} 8}$ & $<3.4^{\mathrm{T}} /<3.4^{\mathrm{T}}$ \\
\hline tert-butylbenzene $(\mu \mathrm{g} / \mathrm{l})$ & $<6.25^{\mathrm{R} 8}$ & $<12.5(\mathrm{~J}-)^{\mathrm{R} 8} /<12.5(\mathrm{~J}-)^{\mathrm{R} 8}$ & $0.83(\mathrm{~J})^{\mathrm{R} 8}$ & $<5.0^{\mathrm{R} 8}$ & $<3.2^{\mathrm{T}} /<3.2^{\mathrm{T}}$ \\
\hline n-propylbenzene $(\mu \mathrm{g} / \mathrm{l})$ & $5.75(\mathrm{~J})^{\mathrm{R} 8}$ & $<12.5(\mathrm{~J}-)^{\mathrm{R} 8} /<12.5(\mathrm{~J}-)^{\mathrm{R} 8}$ & $9.94(J)^{\mathrm{R} 8}$ & $11.8(\mathrm{~J})^{\mathrm{R} 8}$ & $13(\mathrm{~J})^{\mathrm{T}} / 12(\mathrm{~J})^{\mathrm{T}}$ \\
\hline p-isopropyltoluene $(\mu \mathrm{g} / \mathrm{l})$ & $<6.25^{\mathrm{R} 8}$ & $<12.5(\mathrm{~J}-)^{\mathrm{R} 8} /<12.5(\mathrm{~J}-)^{\mathrm{R} 8}$ & $1.36(\mathrm{~J})^{\mathrm{R} 8}$ & $<5.0^{\mathrm{R} 8}$ & $<4.0^{\mathrm{T}} /<4.0^{\mathrm{T}}$ \\
\hline styrene $(\mu \mathrm{g} / \mathrm{l})$ & $<6.25^{\mathrm{R} 8}$ & $<12.5(\mathrm{~J}-)^{\mathrm{R} 8} /<12.5(\mathrm{~J}-)^{\mathrm{R} 8}$ & $0.43(\mathrm{~J})^{\mathrm{R} 8}$ & $<5.0^{\mathrm{R} 8}$ & $<3.4^{\mathrm{T}} /<3.4^{\mathrm{T}}$ \\
\hline 1,3,5-trimethylbenzene $(\mu \mathrm{g} / \mathrm{l})$ & $35.5^{\mathrm{R} 8}$ & $43.6^{\mathrm{S}} / 35.0^{\mathrm{S}}$ & $39.5^{\mathrm{S}}$ & $71.4^{\mathrm{S}}$ & $86^{\mathrm{T}} / 91^{\mathrm{T}}$ \\
\hline 1,2,4-trimethylbenzene $(\mu \mathrm{g} / \mathrm{l})$ & $69.2^{\mathrm{R} 8}$ & $\begin{array}{l}84.1^{\mathrm{S}} / 67.2^{\mathrm{S}} \\
18.5(\mathrm{~J}-)^{\mathrm{R} 8} / 23.0(\mathrm{~J}-)^{\mathrm{R} 8}\end{array}$ & $77.0^{\mathrm{S}}$ & $148^{\mathrm{S}}$ & $140^{\mathrm{T}} / 150^{\mathrm{T}}$ \\
\hline 1,2,3-trimethylbenzene $(\mu \mathrm{g} / \mathrm{l})$ & NA & $28.8^{\mathrm{S}} / 23.8^{\mathrm{S}}$ & $27.6^{\mathrm{S}}$ & $45.5^{\mathrm{S}}$ & $45^{\mathrm{T}} / 46^{\mathrm{T}}$ \\
\hline naphthalene $(\mu \mathrm{g} / \mathrm{l})$ & $1.41^{\mathrm{R} 8} / 4.25(\mathrm{~J})^{\mathrm{R} 8}$ & \begin{tabular}{|l|}
$4.61^{\mathrm{S}} / 3.87^{\mathrm{S}}$ \\
$3.32^{\mathrm{R} 8} /<5.0^{\mathrm{R} 8}$ \\
$<12.5(\mathrm{~J}-)^{\mathrm{R} 8} /<12.5(\mathrm{~J}-)^{\mathrm{R} 8}$ \\
\end{tabular} & $\begin{array}{l}4.89^{\mathrm{S}} \\
7.19(\mathrm{~J})^{\mathrm{R} 8} / 4.29^{\mathrm{R} 8}\end{array}$ & $\begin{array}{l}7.49^{\mathrm{S}} \\
7.20(\mathrm{~J})^{\mathrm{R} 8} / 4.78^{\mathrm{R} 8}\end{array}$ & $7.2(\mathrm{~J})^{\mathrm{T}} / 7.9(\mathrm{~J})^{\mathrm{T}}$ \\
\hline 1-methylnapthalene $(\mu \mathrm{g} / \mathrm{l})$ & $0.66^{\mathrm{R} 8}$ & $1.03^{\mathrm{R} 8} /<5.00^{\mathrm{R} 8}$ & $2.23^{\mathrm{R} 8}$ & $2.85^{\mathrm{R} 8}$ & $3.5^{\mathrm{T}}$ \\
\hline 2-methylnapthalene $(\mu \mathrm{g} / \mathrm{l})$ & $1.15^{\mathrm{R} 8}$ & $1.75^{\mathrm{R} 8} /<5.0^{\mathrm{R} 8}$ & $4.08^{\mathrm{R} 8}$ & $5.52^{\mathrm{R} 8}$ & $6.7^{\mathrm{T}}$ \\
\hline phenol $(\mu \mathrm{g} / \mathrm{l})$ & $13.7^{\mathrm{R} 8}$ & $14.5^{\mathrm{R} 8} / 29.2^{\mathrm{R} 8}$ & $32.7(\mathrm{~J}-)^{\mathrm{R} 8}$ & $16.0^{\mathrm{R} 8}$ & $23^{\mathrm{T}}$ \\
\hline 2-methylphenol $(\mu \mathrm{g} / \mathrm{l})$ & $13.8^{\mathrm{R} 8}$ & $10.3^{\mathrm{R} 8} / 20.9^{\mathrm{R} 8}$ & $22.2^{\mathrm{R} 8}$ & $20.8(J)^{\mathrm{R} 8}$ & $25^{\mathrm{T}}$ \\
\hline 3\&4-methylphenol $(\mu \mathrm{g} / \mathrm{l})$ & $26.2^{\mathrm{R} 8}$ & $16.9(\mathrm{D})^{\mathrm{R} 8} / 34.6(\mathrm{D})^{\mathrm{R} 8}$ & $39.8^{\mathrm{R} 8}$ & $33.5^{\mathrm{R} 8}$ & $45^{\mathrm{T}}$ \\
\hline 2,4-dimethylphenol $(\mu \mathrm{g} / \mathrm{l})$ & $28.6^{\mathrm{R} 8}$ & $23.2(\mathrm{D})^{\mathrm{R} 8} / 46.3(\mathrm{D})^{\mathrm{R} 8}$ & $36.6(\mathrm{~J}+)^{\mathrm{R} 8}$ & $32.0^{\mathrm{R} 8}$ & $67^{\mathrm{T}}$ \\
\hline isopropanol $(\mu \mathrm{g} / \mathrm{l})$ & NA & $581^{\mathrm{S}} / 583^{\mathrm{S}}$ & $862^{\mathrm{S}}$ & $802^{\mathrm{S}}$ & $<260^{\mathrm{T}}$ \\
\hline n-propanol $(\mu \mathrm{g} / \mathrm{l})$ & NA & $<100^{\mathrm{S}} /<100^{\mathrm{S}}$ & $63(\mathrm{~J}-)^{\mathrm{S}}$ & $11.8(\mathrm{~J} \dagger)^{\mathrm{S}} /<100(\mathrm{~J}-)^{\mathrm{S}}$ & NA \\
\hline methanol $(\mu \mathrm{g} / \mathrm{l})$ & NA & NA & $<5000^{\mathrm{A}}$ & $\begin{array}{l}<5000^{\mathrm{A}} \\
592(\mathrm{~J} \dagger)^{\mathrm{S}}\end{array}$ & NA \\
\hline tert-butyl alcohol $(\mu \mathrm{g} / \mathrm{l})$ & NM & $4470^{\mathrm{S}} / 4580^{\mathrm{S}}$ & $5910^{\mathrm{S}}$ & $5395(\dagger)^{\mathrm{S}} / 6120^{\mathrm{S}}$ & $6300^{\mathrm{T}} / 6300^{\mathrm{T}}$ \\
\hline acetone $(\mu \mathrm{g} / \mathrm{l})$ & NA & $641(\mathrm{~J}-)^{\mathrm{R} 8} / 616(\mathrm{~J}-)^{\mathrm{R} 8}$ & $\begin{array}{l}1460^{\mathrm{S}} \\
982(\mathrm{~J})^{\mathrm{R} 8}\end{array}$ & $\begin{array}{l}231^{\mathrm{S}} \\
157(\mathrm{~J})^{\mathrm{R} 8}\end{array}$ & $350^{\mathrm{T}} / 450^{\mathrm{T}}$ \\
\hline 2-butanone (MEK) $(\mu \mathrm{g} / \mathrm{l})$ & NA & $120(\mathrm{~J}-)^{\mathrm{R} 8} / 118(\mathrm{~J}-)^{\mathrm{R} 8}$ & $208(J)^{\mathrm{R} 8}$ & $86.2(J)^{\mathrm{R} 8}$ & $<120^{\mathrm{T}} /<40^{\mathrm{T}}$ \\
\hline 4-methyl-2-pentanone (MIBK) $(\mu \mathrm{g} / \mathrm{l})$ & NA & $12.5(\mathrm{~J}-)^{\mathrm{R} 8} /<12.5(\mathrm{~J}-)^{\mathrm{R} 8}$ & $<0.25^{\mathrm{R} 8}$ & $<5.0^{\mathrm{R} 8}$ & $<20^{\mathrm{T}}$ \\
\hline benzoic acid $(\mu \mathrm{g} / \mathrm{l})$ & $244^{\mathrm{R} 8}$ & $209(\mathrm{D})^{\mathrm{R} 8} / 364(\mathrm{D})^{\mathrm{R} 8}$ & $513^{\mathrm{R} 8}$ & $110(\mathrm{D})^{\mathrm{R} 8}$ & $190^{\mathrm{T}}$ \\
\hline ethylene glycol $(\mu \mathrm{g} / \mathrm{l})$ & NA & $\mathrm{NA}$ & $<5000^{\mathrm{A}}$ & $<5000^{\mathrm{A}}$ & $<8630^{\mathrm{T}}$ \\
\hline propylene glycol $(\mu \mathrm{g} / \mathrm{l})$ & NA & $\mathrm{NA}$ & $<5000^{\mathrm{A}}$ & $<5000^{\mathrm{A}}$ & $<18700^{\mathrm{T}}$ \\
\hline diethylene glycol (R3) $(\mu \mathrm{g} / \mathrm{l})$ & NA & $1570(\mathrm{~J}+)^{\mathrm{R3}} / 1610(\mathrm{~J}+)^{\mathrm{R3}}$ & $1260(\mathrm{~J}-)^{\mathrm{R3}}$ & $378(\mathrm{~J})^{\mathrm{R} 3}$ & $<7730^{\mathrm{T}}$ \\
\hline triethylene glycol $(\mathrm{R} 3)(\mu \mathrm{g} / \mathrm{l})$ & NA & $310(\mathrm{~J}-)^{\mathrm{R} 3} / 293(\mathrm{~J}-)^{\mathrm{R} 3}$ & $262(\mathrm{~J}-)^{\mathrm{R3}}$ & $72.3(\mathrm{~J})^{\mathrm{R3}}$ & $<8450^{\mathrm{T}}$ \\
\hline tetraethylene glycol $(\mathrm{R} 3)(\mu \mathrm{g} / \mathrm{l})$ & NA & $27.2(\mathrm{~J}, \mathrm{~B})^{\mathrm{R} 3} / 29.0(\mathrm{~J}-, \mathrm{B})^{\mathrm{R} 3}$ & $22.6(\mathrm{~J}-)^{\mathrm{R3}}$ & $3.6(\mathrm{~J})^{\mathrm{R3}}$ & NA \\
\hline 2-butoxyethanol $(\mu \mathrm{g} / \mathrm{l})$ & NA & $\begin{array}{l}<0.10(\mathrm{~J}-)^{\mathrm{R} 3} /<0.10(\mathrm{~J}-)^{\mathrm{R} 3} \\
<0.10(\mathrm{~J}-)^{\mathrm{R} 8} /<0.10(\mathrm{~J}-)^{\mathrm{R} 8}\end{array}$ & $\begin{array}{l}6.8(\mathrm{~J}-)^{\mathrm{R} 3} \\
<1.0^{\mathrm{R} 8}\end{array}$ & $\begin{array}{l}<5.0^{\mathrm{R} 3} \\
<1.0^{\mathrm{R} 8}\end{array}$ & $\mathrm{TIC}^{\mathrm{T}}$ \\
\hline ethyl ether $(\mu \mathrm{g} / \mathrm{l})$ & $<6.25^{\mathrm{R} 8}$ & $12.5(\mathrm{~J}-)^{\mathrm{R} 8} /<12.5(\mathrm{~J}-)^{\mathrm{R} 8}$ & $1.94(\mathrm{~J})^{\mathrm{R} 8}$ & $<5.0^{\mathrm{R} 8}$ & $<5.2^{\mathrm{T}}$ \\
\hline nonylphenol $(\mu \mathrm{g} / \mathrm{l})$ & NA & NA & $28(\mathrm{~J}-)^{\mathrm{L}}$ & $7.4-7.9(\mathrm{~J}-)^{\mathrm{L}}$ & NA \\
\hline octylphenol $(\mu \mathrm{g} / \mathrm{l})$ & NA & NA & $2.9(\mathrm{~J}-)^{\mathrm{L}}$ & $0.5-0.7(\mathrm{~J})^{\mathrm{L}}$ & NA \\
\hline acrylamide $(\mu \mathrm{g} / \mathrm{L})$ & NA & NA & $<0.20^{\mathrm{L}}$ & $<0.20^{\mathrm{L}}$ & NA \\
\hline lactate $(\mu \mathrm{g} / \mathrm{L})$ & NA & $213^{\mathrm{S}} / 253^{\mathrm{S}}$ & $250^{\mathrm{S}}$ & $<100^{\mathrm{S}}$ & NA \\
\hline formate $(\mu \mathrm{g} / \mathrm{L})$ & NA & $558^{\mathrm{S}} / 584^{\mathrm{S}}$ & $\mathrm{R}$ & $\mathrm{R}$ & NA \\
\hline acetate $(\mu \mathrm{g} / \mathrm{L})$ & NA & $4310^{\mathrm{S}} / 4200^{\mathrm{S}}$ & $4800^{\mathrm{S}}$ & $2840(\mathrm{~J})^{\mathrm{S}}$ & NA \\
\hline propionate $(\mu \mathrm{g} / \mathrm{L})$ & NA & $808^{\mathrm{S}} / 687^{\mathrm{S}}$ & $844^{\mathrm{S}}$ & $687(\mathrm{~J})^{\mathrm{S}}$ & NA \\
\hline adamantine $(\mu \mathrm{g} / \mathrm{L})$ & $<6.25^{\mathrm{R} 8}$ & $<12.5(\mathrm{~J}-)^{\mathrm{R} 8} /<12.5(\mathrm{~J}-)^{\mathrm{R} 8}$ & $<0.25^{\mathrm{R} 8}$ & $<5.0^{\mathrm{R} 8}$ & NA \\
\hline 1,3-dimethyl adamantine $(\mu \mathrm{g} / \mathrm{L})$ & $>0.20^{\mathrm{R} 8} /<6.25^{\mathrm{R} 8}$ & $<0.10(\mathrm{~J}-)^{\mathrm{R} 8} /<5.0(\mathrm{~J}-)^{\mathrm{R} 8}$ & $<0.25^{\mathrm{R} 8}$ & $<5.0^{\mathrm{R} 8}$ & NA \\
\hline $\begin{array}{l}\text { methylene blue active substances } \\
(\mathrm{mg} / \mathrm{l})\end{array}$ & NA & NA & $<0.20^{\mathrm{T}}$ & $<0.20^{\mathrm{T}}$ & $0.12(\mathrm{~J})^{\mathrm{T}}$ \\
\hline
\end{tabular}


Table SI E4b. Summary of Field Parameters, Major Ions, and Dissolved Metals at MW02

\begin{tabular}{|c|c|c|c|c|c|c|}
\hline \multicolumn{2}{|c|}{ Compound } & $\begin{array}{l}\text { EPA } \\
\text { Phase III }\end{array}$ & $\begin{array}{l}\text { EPA } \\
\text { Phase IV }\end{array}$ & $\begin{array}{l}\text { EPA } \\
\text { Phase V } \\
\text { 0412-1 }\end{array}$ & $\begin{array}{l}\text { EPA } \\
\text { Phase V } \\
\text { 0412-2 }\end{array}$ & $\begin{array}{l}\text { USGS } \\
\text { Phase V } \\
\text { 0412-2 }\end{array}$ \\
\hline \multicolumn{2}{|c|}{ Borehole Volumes } & 0.1 & 0.6 & 0.0 & 1.0 & 1.0 \\
\hline \multicolumn{7}{|c|}{ Field Parameters } \\
\hline \multicolumn{2}{|l|}{$\mathrm{pH}$} & 12.01 & 11.78 & 11.96 & 11.81 & NM \\
\hline \multicolumn{2}{|c|}{ specific conductance $(\mu \mathrm{S} / \mathrm{cm})$} & 3812 & 3099 & 3313 & $2888(\mathrm{~J})$ & NM \\
\hline \multicolumn{2}{|c|}{ dissolved oxygen $(\mathrm{mg} / \mathrm{l})$} & 0.0 & 0.02 & 0.02 & 0.01 & NM \\
\hline \multicolumn{2}{|c|}{ oxidation reduction potential $(\mathrm{mV})$} & 121 & -108 & -154 & -148 & NM \\
\hline \multicolumn{2}{|c|}{ turbidity (NTU) } & 28.8 & 24.0 & 4.5 & 15.7 & NM \\
\hline \multicolumn{2}{|c|}{ alkalinity (mg/l CaCO3) } & 456 & 482 & 390 & 254 & NM \\
\hline \multicolumn{7}{|c|}{ Major Ions } \\
\hline \multicolumn{2}{|c|}{ nitrate + nitrite $(\mathrm{mg} \mathrm{N} / \mathrm{L})(\mathrm{mg} / \mathrm{L})$} & $0.379(\mathrm{~B})^{\mathrm{O}}$ & $<0.100^{\circ} /<0.100^{\circ}$ & $0.056^{\mathrm{O}}$ & $0.095^{\circ}$ & $<0.019$ \\
\hline \multicolumn{2}{|c|}{ ammonia (mg N/L) } & $1.95^{\circ}$ & $2.88^{\mathrm{O}} / 2.82^{\mathrm{O}}$ & $2.61^{\mathrm{O}}$ & $1.23^{\mathrm{O}}$ & $0.81(\mathrm{~B})$ \\
\hline \multicolumn{2}{|c|}{ SO4 (mg/l) } & $12.1^{\mathrm{O}}$ & $62.6^{\circ} / 62.5^{\circ}$ & $81.8^{\circ}$ & $13.5^{\mathrm{O}}$ & 14 \\
\hline \multicolumn{2}{|c|}{$\mathrm{S}(\mathrm{mg} / \mathrm{L})$} & $6.8(\mathrm{~B})$ & $25.1(\mathrm{~J})^{\mathrm{S}} / 25.0(\mathrm{~J})^{\mathrm{S}}$ & $32.1(\mathrm{~J})^{\mathrm{S}}$ & $7.38(\mathrm{~J})^{\mathrm{S}}$ & NA \\
\hline $\mathrm{Cl}(\mathrm{n}$ & & $466^{\circ}$ & $457^{\circ} / 456^{\mathrm{O}}$ & $469^{\circ}$ & $495^{\circ}$ & 520 \\
\hline $\mathrm{Br}(\mathrm{r}$ & & NA & $<3.00^{\circ} /<3.00^{\circ}$ & $<1.00^{\circ}$ & $<1.00^{\circ}$ & 1.9 \\
\hline $\mathrm{F}(\mathrm{m}$ & & $1.01^{\mathrm{O}}$ & $1.54(\mathrm{~J})^{\mathrm{O}} / 1.49(\mathrm{~J})^{\mathrm{O}}$ & $1.50^{\mathrm{O}}$ & $1.16^{\mathrm{O}}$ & 1.6 \\
\hline $\mathrm{K}(\mathrm{m}$ & & $39.5(\mathrm{~J})^{\mathrm{S}}$ & $43.6(\mathrm{~J})^{\mathrm{S}} / 44.0(\mathrm{~J})^{\mathrm{S}}$ & $31.4(\mathrm{~J})^{\mathrm{S}}$ & $16.6(\mathrm{~J})^{\mathrm{S}}$ & 15.0 \\
\hline $\mathrm{Na}(1$ & & $420(\mathrm{~J})^{\mathrm{S}}$ & $448(J)^{\mathrm{S}} / 449(\mathrm{~J})^{\mathrm{S}}$ & $429(\mathrm{~J})^{\mathrm{S}}$ & $379(\mathrm{~J})^{\mathrm{S}}$ & 400(B) \\
\hline $\mathrm{Ca}(1$ & & $73.3^{\mathrm{S}}$ & $60.5^{\mathrm{S}} / 60.6^{\mathrm{S}}$ & $50.8^{\mathrm{S}}$ & $36.1^{\mathrm{s}}$ & 33.0 \\
\hline $\mathrm{Mg}$ & & $<0.05^{\mathrm{S}}$ & $0.03(\mathrm{~J})^{\mathrm{S}} / 0.02(\mathrm{~J})^{\mathrm{S}}$ & $<0.10^{\mathrm{S}}$ & $<0.10^{\mathrm{S}}$ & $49(\mathrm{~J})$ \\
\hline $\mathrm{Si}(\mathrm{n}$ & & $3.00(\mathrm{~J})^{\mathrm{S}}$ & $2.94(\mathrm{~J})^{\mathrm{S}} / 2.93(\mathrm{~J})^{\mathrm{S}}$ & $4.89(\mathrm{~J}+)^{\mathrm{S}}$ & $5.00(\mathrm{~J}+)^{\mathrm{S}}$ & 15.0 \\
\hline Diss & Metals & & & & & \\
\hline $\mathrm{Ag}($ & & $<51^{\mathrm{S}}$ & $<51^{\mathrm{S}}$ & $<14^{\mathrm{S}}$ & $<14^{\mathrm{S}}$ & $<0.033$ \\
\hline $\mathrm{Al}(\mu$ & & $577(\mathrm{~J})^{\mathrm{S}}$ & $684(\mathrm{~J})^{\mathrm{S}} / 736(\mathrm{~J})^{\mathrm{S}}$ & $660(J)^{S}$ & $816(\mathrm{~J})^{\mathrm{S}}$ & 700 \\
\hline As $(1$ & & $<51^{\mathrm{S}}$ & $<51^{\mathrm{S}} /<51^{\mathrm{S}}$ & $3.1^{\mathrm{S}}$ & $3.4^{\mathrm{S}}$ & $3.1(\mathrm{~J})$ \\
\hline $\mathrm{B}(\mu$ & & $103(J)^{S}$ & $109(\mathrm{~J})^{\mathrm{S}} / 108(\mathrm{~J})^{\mathrm{S}}$ & $114(\mathrm{~J})^{\mathrm{S}}$ & $108(J)^{\mathrm{S}}$ & 110 \\
\hline $\mathrm{Ba}($ & & $210(\mathrm{~J})^{\mathrm{S}}$ & $93(\mathrm{~J})^{\mathrm{S}} / 93(\mathrm{~J})^{\mathrm{S}}$ & $95(J)^{S}$ & $147(J)^{\mathrm{S}}$ & 150 \\
\hline $\mathrm{Be}($ & & $<4^{\mathrm{S}}$ & $<4^{\mathrm{S}} /<4^{\mathrm{S}}$ & $<10^{\mathrm{S}}$ & $<10^{\mathrm{S}}$ & $<0.08$ \\
\hline $\mathrm{Cd}($ & & $<4^{\mathrm{S}}$ & $<4^{\mathrm{S}} /<4^{\mathrm{S}}$ & $<1.00^{\mathrm{S}}$ & $<1.00^{\mathrm{S}}$ & $<0.1$ \\
\hline Co ( & & $<4^{\mathrm{S}}$ & $<4^{\mathrm{S}} /<4$ & $<4^{\mathrm{S}}$ & $<4^{\mathrm{S}}$ & 0.085 \\
\hline $\mathrm{Cr}(\mu$ & & $<4^{\mathrm{S}}$ & $<4^{\mathrm{S}} /<4^{\mathrm{S}}$ & $<2.00^{\mathrm{S}}$ & $<2.00^{\mathrm{S}}$ & 4.5 \\
\hline $\mathrm{Cu}($ & & $<9^{\mathrm{S}}$ & $<9^{\mathrm{S}} /<9^{\mathrm{S}}$ & $<2.00^{\mathrm{S}}$ & $4.4^{\mathrm{S}}$ & 3.7 \\
\hline $\mathrm{Fe}(\mu$ & & $<63^{\mathrm{S}}$ & $19(\mathrm{~J})^{\mathrm{S}} /<63^{\mathrm{S}}$ & $<67^{\mathrm{S}}$ & $151^{\mathrm{S}}$ & 400 \\
\hline $\mathrm{Hg}($ & & NA & NA & NA & NA & $<0.027$ \\
\hline $\mathrm{Li}(\mu$ & & NA & NA & NA & NA & 25 \\
\hline $\mathrm{Mn}$ & & $<4^{\mathrm{S}}$ & $<4^{\mathrm{S}} /<4^{\mathrm{S}}$ & $<14^{\mathrm{S}}$ & $<14^{\mathrm{S}}$ & 5.6 \\
\hline Mo & & $14^{\mathrm{S}}$ & $13^{\mathrm{S}} / 14^{\mathrm{S}}$ & $6(J)^{S}$ & $<14^{\mathrm{S}}$ & 2.1 \\
\hline $\mathrm{Ni}(\mu$ & & $4^{\mathrm{S}}$ & $2^{\mathrm{S}} / 2(\mathrm{~J})^{\mathrm{S}}$ & $4.3^{\mathrm{S}}$ & $2.6^{\mathrm{S}}$ & 6 \\
\hline $\mathrm{P}(\mu$ & & $<0.014^{\mathrm{S}}$ & $0.014(\mathrm{~B})^{\mathrm{S}} / 0.024(\mathrm{~B})^{\mathrm{S}}$ & $<0.06^{\mathrm{S}}$ & $<0.06^{\mathrm{S}}$ & 65 \\
\hline $\mathrm{Pb}(1$ & & $<11^{\mathrm{S}}$ & $<11^{\mathrm{S}} /<11^{\mathrm{S}}$ & $<1.00^{\mathrm{S}}$ & $<1.00^{\mathrm{S}}$ & $<0.018$ \\
\hline $\mathrm{Sb}(\mu$ & & $\mathrm{R}^{\mathrm{S}}$ & $\mathrm{R}^{\mathrm{S}} / \mathrm{R}^{\mathrm{S}}$ & $<2.00^{\mathrm{S}}$ & $<2.00^{\mathrm{S}}$ & $1.3(\mathrm{~B})$ \\
\hline $\operatorname{Se}(\mu$ & & $14(J)^{S}$ & $<16^{\mathrm{S}} / 9(\mathrm{~J})^{\mathrm{S}}$ & $4.7(\mathrm{~J})^{\mathrm{S}}$ & $4.9(\mathrm{~J})^{\mathrm{S}}$ & $<0.7$ \\
\hline $\mathrm{Sr}(\mu$ & & $2020^{\mathrm{S}}$ & $1780^{\mathrm{S}} / 1790^{\mathrm{S}}$ & $1260^{\mathrm{S}}$ & $806^{\mathrm{S}}$ & 780 \\
\hline $\operatorname{Ti}(\mu$ & & $<4^{\mathrm{S}}$ & $<4^{\mathrm{S}} /<4^{\mathrm{S}}$ & $<7^{\mathrm{S}}$ & $<7^{\mathrm{S}}$ & $2.7(\mathrm{~J})$ \\
\hline $\mathrm{Tl}(\mu$ & & $<37^{\mathrm{S}}$ & $\mathrm{R}^{\mathrm{S}} / \mathrm{R}^{\mathrm{S}}$ & $<1.00^{\mathrm{S}}$ & $<1.00^{\mathrm{S}}$ & $<0.05$ \\
\hline $\mathrm{U}(\mu$ & & NA & NA & NA & NA & $<0.05$ \\
\hline $\mathrm{V}(\mu$ & & $<7^{\mathrm{S}}$ & $<7^{\mathrm{S}} /<7^{\mathrm{S}}$ & $<10^{\mathrm{S}}$ & $<10^{\mathrm{S}}$ & $2.1(\mathrm{~J})$ \\
\hline $\mathrm{Zn}($ & & $<24^{\mathrm{S}}$ & $32^{\mathrm{S}} / 24^{\mathrm{S}}$ & $<50^{\mathrm{S}}$ & $<50^{\mathrm{S}}$ & 49 \\
\hline Labor & (Superscript) & & & & & \\
\hline & Shaw Environmental, Ada, & ctor to EPA) & $\mathrm{O}$ & RD Ada, & & \\
\hline $\mathrm{L}$ & EPA ORD, Las Vegas, NV & & R8 & Region $8 \mathrm{Lal}$ & len, $\mathrm{CO}$ & \\
\hline R3 & EPA Region 3 Laboratory, F & MD & Tes & America Lab & ractor to US & (d EPA) \\
\hline $\mathrm{U}$ & USGS Laboratory & & $\mathrm{AL}$ & Environment & to EPA) & \\
\hline $\mathrm{C}$ & Chemtech Consulting (contr & & & & & \\
\hline Data & & & & & & \\
\hline NA & Not analyzed & & & & & \\
\hline J & $\begin{array}{l}\text { Estimated because of non-at } \\
\text { estimated because of method }\end{array}$ & $\begin{array}{l}\text { certain quali } \\
\text { ent. }\end{array}$ & control criteria or concent & tion below $\mathrm{q}$ & nit. R3 and & Las Vegas analysis are \\
\hline J- & $\begin{array}{l}\text { Result is estimated because } \\
\text { samples, or other quality ass }\end{array}$ & $\begin{array}{l}\text { biased low d } \\
\text { ors. }\end{array}$ & $<70 \%$ matrix spike recove & $y$ in USGS s: & dance of ho & ime in R8 Phase IV \\
\hline J+ & Result is estimated but may & igh due to $>1$ & $\%$ matrix spike recovery $\mathrm{i}$ & USGS samp & lality assura & ctors. \\
\hline $\mathrm{J}(\dagger)$ & Estimated because of metho & ent on alcohs & (Shaw 2012,,b) & & & \\
\hline B & Analyte in sample $<10 \mathrm{X}$ an & centration det & ted in a blank for EPA an & USGS data $\mathrm{r}$ & & \\
\hline D & Relative percent difference 0 & ptance criter & & & & \\
\hline $\mathrm{R}$ & Sample result rejected due to & ficiencies in & is & & & \\
\hline
\end{tabular}


Table SI E5. Light Hydrocarbon and Isotope Analysis of Production Wells and EPA Monitoring Wells

\begin{tabular}{|c|c|c|c|c|c|c|c|c|c|c|c|c|c|c|}
\hline & $\begin{array}{l}\text { BH } \\
\text { vol }\end{array}$ & $\begin{array}{l}\mathrm{C}_{1} \\
(\mathrm{mg} / \mathrm{L})\end{array}$ & $\begin{array}{l}\mathrm{C}_{2} \\
(\mathrm{mg} / \mathrm{L})\end{array}$ & $\begin{array}{l}\mathrm{C}_{3} \\
(\mathrm{mg} / \mathrm{L})\end{array}$ & $\begin{array}{l}\mathrm{C}_{4} \\
(\mathrm{mg} / \mathrm{L})\end{array}$ & $\begin{array}{l}{ }^{14} \mathrm{C}_{1} \\
(\% \\
\text { pmc) }\end{array}$ & $\begin{array}{l}\delta^{13} \mathbf{C}_{1} \\
(\%))\end{array}$ & $\begin{array}{l}\delta D_{1} \\
(\% o)\end{array}$ & $\begin{array}{l}\delta^{13} \mathrm{C}_{2} \\
(\% o)\end{array}$ & $\begin{array}{l}\delta \mathrm{DC}_{2} \\
(\%)\end{array}$ & $\begin{array}{l}\delta^{13} \mathrm{C}_{3} \\
(\%)\end{array}$ & $\begin{array}{l}\delta \mathrm{DC}_{3} \\
(\%)\end{array}$ & $\begin{array}{l}\delta^{13} \mathrm{iC}_{4} \\
(\% o)\end{array}$ & $\begin{array}{l}\delta^{13} \mathrm{nC}_{4} \\
(\% o)\end{array}$ \\
\hline \multicolumn{15}{|l|}{ Johnson and Rice (1993) } \\
\hline Tribal Pavillion 14-6 (WR) (g) & ב---- & ----- & $\begin{array}{l}---- \\
--1\end{array}$ & ----- & ----- & NA & -39.24 & NA & NA & $\mathrm{NA}$ & NA & NA & NA & NA \\
\hline Govt 21-5 (WR) (g) & ----- & \begin{tabular}{|c|}
----- \\
\end{tabular} & ----- & -.-- & --.-- & NA & -40.2 & NA & NA & NA & NA & NA & NA & NA \\
\hline Tribal Pavillion 41-9 (FU) (g) & ----- & $-----{ }_{-}$ & ------ & 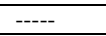 & $----{ }_{-}$ & NA & -38.04 & NA & NA & NA & NA & NA & NA & NA \\
\hline Tribal Pavillion 14-11 (FU) (g) & ----- & ----- & ב----- & 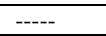 & ----- & NA & -38.4 & NA & NA & NA & NA & NA & NA & NA \\
\hline Blankenship Fee 4-8 (FU) (g) & ----- & ----- & ----- & ----- & ----- & NA & -38.08 & NA & $\mathrm{NA}$ & $\mathrm{NA}$ & $\mathrm{NA}$ & NA & $\mathrm{NA}$ & $\mathrm{NA}$ \\
\hline \multicolumn{15}{|l|}{ Phase II } \\
\hline $\begin{array}{l}\text { Tribal Pavillion 14-10 (WR) } \\
\text { (PGPP01) }\end{array}$ & ---- & & & & & NA & $-38.75^{1}$ & $-203.4^{1}$ & $-26.93^{\mathrm{I}}$ & $-162.5^{\mathrm{I}}$ & $-24.93^{1}$ & $-147.2^{1}$ & $-25.83^{1}$ & $-25.26^{1}$ \\
\hline $\begin{array}{l}\text { Tribal Pavillion 43-10 (FU) } \\
\text { (PGPP02) }\end{array}$ & ----- & & & & & NA & $-39.07^{1}$ & $-212.99^{1}$ & $-25.99^{1}$ & $-157.5^{1}$ & $-19.40^{1}$ & NA & NA & $-23.87^{1}$ \\
\hline $\begin{array}{l}\text { Tribal Pavillion 24-2 (WR) } \\
\text { (PGPP04) }\end{array}$ & ----- & & & & & NA & $-39.26^{\mathrm{I}}$ & $-204.9^{I}$ & $-26.79^{\mathrm{I}}$ & $-166.2^{1}$ & $-25.33^{1}$ & $-148.0^{1}$ & $-25.66^{\mathrm{I}}$ & $-25.05^{\mathrm{I}}$ \\
\hline $\begin{array}{l}\text { Tribal Pavillion 33-10 (FU) } \\
\text { (PGPP05) }\end{array}$ & $\begin{array}{ll}---- \\
\end{array}$ & & & & & NA & $-39.05^{1}$ & \begin{tabular}{|l|l|}
$-207.3^{1}$ \\
\end{tabular} & $-26.21^{1}$ & $-161.1^{\mathrm{I}}$ & $-18.46^{1}$ & $-101.7^{1}$ & $-23.96^{1}$ & $-23.64^{\mathrm{I}}$ \\
\hline $\begin{array}{l}\text { Tribal Pavillion 14-2 (FU) } \\
\text { (PGPP06) }\end{array}$ & ---- & & & & & NA & $-39.28^{1}$ & $-215.3^{1}$ & $-26.42^{1}$ & $-162.3^{1}$ & $-24.01^{1}$ & $-145.2^{1}$ & $-25.33^{1}$ & $-24.87^{1}$ \\
\hline \multicolumn{15}{|l|}{ MW01 } \\
\hline EPA Phase III & 0.3 & $16.0^{\mathrm{S}}$ & $2.23^{\mathrm{S}}$ & $0.79^{\mathrm{S}}$ & $0.158^{\mathrm{S}}$ & $\mathrm{NA}$ & $-38.89^{\mathrm{I}}$ & $-191.3^{1}$ & -26.55 & $\mathrm{NA}$ & $-23.85^{\mathrm{I}}$ & NA & NA & NA \\
\hline EPA Phase III(g) & ---- & \begin{tabular}{|l|}
$-\cdots--$ \\
-1
\end{tabular} & $\begin{array}{l}-\cdots \\
-\cdots\end{array}$ & +--- & ב--- & $<0.2^{1}$ & $-39.44^{1}$ & $-209.1^{1}$ & -26.63 & $-165.0^{1}$ & $-23.76^{1}$ & $-143.7^{1}$ & NA & NA \\
\hline EPA Phase IV & 0.7 & $17.93^{\mathrm{S}}$ & $2.95^{\mathrm{S}}$ & $1.25^{\mathrm{s}}$ & $0.172(\mathrm{~J})^{\mathrm{S}}$ & NA & $-38.88^{\mathrm{I}}$ & $-211.6^{1}$ & -26.70 & NA & $-24.40^{\mathrm{I}}$ & NA & $-25.3^{\mathrm{I}}$ & $-24.4^{\mathrm{I}}$ \\
\hline EPA Phase IV(g) & ----- & $\begin{array}{ll}----- \\
\end{array}$ & ----- & $\begin{array}{l}---- \\
\end{array}$ & $\begin{array}{ll}---- \\
\end{array}$ & NA & $\begin{array}{l}-39.25^{1 /} \\
-39.28^{1} \\
\end{array}$ & $\begin{array}{l}-211.2^{1 /} \\
-210.1^{1} \\
\end{array}$ & $\begin{array}{l}-26.67^{1 /} \\
-26.67^{1} \\
\end{array}$ & $\begin{array}{l}-166.8^{1 / 1} \\
-167.4^{I} \\
\end{array}$ & $\begin{array}{l}-23.74^{1 /} \\
-23.91^{1}\end{array}$ & $\begin{array}{l}-146.1^{1 / 1} \\
-146.6^{1}\end{array}$ & NA & NA \\
\hline USGS Phase V & $1.6-3.0$ & $\begin{array}{l}27.5^{\mathrm{T}} / 30.5^{\mathrm{T}} \\
26^{\mathrm{UC}} / 26^{\mathrm{UC}} \\
\end{array}$ & $\begin{array}{l}3.6(\mathrm{~J}+)^{\mathrm{T} /} \\
4.0(\mathrm{~J}+)^{\mathrm{T}} \\
\end{array}$ & $\begin{array}{l}1.4^{\mathrm{T} /} \\
1.3^{\mathrm{T}} \\
\end{array}$ & NA & $2.22^{\mathrm{W}}$ & NA & NA & NA & NA & NA & NA & NA & NA \\
\hline EPA Phase V 0412-4 & 1.6 & NA & NA & NA & NA & NA & NA & NA & NA & NA & NA & NA & NA & NA \\
\hline EPA Phase V 0412 & 1.8 & $17.3^{\mathrm{S} / 17.3^{\mathrm{S}}}$ & $\begin{array}{l}2.38^{\mathrm{S} /} \\
2.21^{\mathrm{S}} \\
\end{array}$ & $\begin{array}{l}0.763^{\mathrm{s} /} \\
0.663^{\mathrm{s}} \\
\end{array}$ & \begin{tabular}{|l|l|}
$0.199^{\mathrm{S} /}$ \\
$0.169^{\mathrm{s}}$ \\
\end{tabular} & NA & $-38.2^{\mathrm{I}}$ & $-205.3^{1}$ & $-26.5^{\mathrm{I}}$ & NA & $\mathrm{NA}$ & NA & NA & NA \\
\hline EPA Phase V 0412-2 & 2.1 & NA & NA & NA & NA & NA & NA & NA & NA & NA & NA & NA & NA & NA \\
\hline EPA Phase V 0412-3 & 2.3 & NA & NA & NA & NA & NA & NA & NA & NA & NA & NA & NA & NA & NA \\
\hline EPA Phase V 0412-5 & 2.4 & NA & NA & NA & NA & NA & NA & NA & $\mathrm{NA}$ & NA & NA & NA & $\mathrm{NA}$ & NA \\
\hline EPA Phase V 0412-7 & 2.6 & NA & NA & NA & NA & NA & $\mathrm{NA}$ & NA & NA & NA & NA & $\mathrm{NA}$ & NA & $\mathrm{NA}$ \\
\hline EPA Phase V 0412-6 & 2.7 & NA & NA & NA & NA & NA & $\mathrm{NA}$ & $\mathrm{NA}$ & $\mathrm{NA}$ & NA & $\mathrm{NA}$ & $\mathrm{NA}$ & NA & $\mathrm{NA}$ \\
\hline EPA Phase V 0412-8 & 2.9 & NA & NA & NA & NA & NA & NA & $\mathrm{NA}$ & NA & NA & NA & NA & NA & NA \\
\hline EPA Phase V 0412-9 & 3.0 & NA & NA & NA & NA & NA & NA & NA & NA & NA & NA & NA & NA & NA \\
\hline USGS Phase V & 3.0 & $\begin{array}{l}25.5^{\mathrm{T}} / 27.0^{\mathrm{T}} \\
28^{\mathrm{UC}} \\
\end{array}$ & $\begin{array}{l}3.2(\mathrm{~J}+)^{\mathrm{T} / \mathrm{I}} \\
3.3(\mathrm{~J}+)^{\mathrm{T}} \\
\end{array}$ & $\begin{array}{l}1.1^{\mathrm{T} /} \\
1.0^{\mathrm{T}} \\
\end{array}$ & NA & $1.53^{\mathrm{W}}$ & $-38.54^{\mathrm{I}}$ & $-208.0^{1}$ & NA & NA & NA & NA & NA & NA \\
\hline EPA Phase V 0412-10 & 3.3 & $18.8^{\mathrm{S}}$ & $18.8^{\mathrm{S}}$ & $0.715^{\mathrm{S}}$ & $0.184^{\mathrm{S}}$ & NA & $-38.5^{\mathrm{I}}$ & $-205.9^{I}$ & $-26.6^{\mathrm{I}}$ & NA & NA & NA & NA & NA \\
\hline \multicolumn{15}{|l|}{ MW02 } \\
\hline EPA Phase III & 0.1 & $18.99^{\mathrm{S}}$ & $3.20^{\mathrm{S}}$ & $1.82^{\mathrm{S}}$ & NA & NA & $-41.83^{\mathrm{I}}$ & -203.8 & $-26.4^{\mathrm{I}}$ & NA & $-24.28^{I}$ & NA & NA & NA \\
\hline EPA Phase III(g) & ----- & ----- & ----- & ---- & ---- & $\begin{array}{l}<0.2^{1 /} \\
<0.2^{1}\end{array}$ & $\begin{array}{l}-41.85^{\mathrm{I} /} \\
-41.72^{\mathrm{I}} \\
\end{array}$ & \begin{tabular}{|l}
$-209.4^{1 /-}$ \\
$209.2^{1}$ \\
\end{tabular} & NA & NA & NA & NA & NA & NA \\
\hline EPA Phase IV & 0.6 & $18.82^{\mathrm{S}}$ & $2.55^{\mathrm{S}}$ & $2.26^{\mathrm{S}}$ & & NA & $\begin{array}{l}-41.30^{1 /} \\
-41.37^{1} \\
\end{array}$ & \begin{tabular}{|l|}
$-210.7^{1 /}$ \\
$-208.2^{1}$ \\
\end{tabular} & $\begin{array}{l}-26.25^{\mathrm{I} /} \\
-26.28^{\mathrm{I}} \\
\end{array}$ & NA & $\begin{array}{l}-24.29^{\mathrm{I} /} \\
-24.28^{\mathrm{I}} \\
\end{array}$ & NA & $\begin{array}{l}-25.3^{1 / 1} \\
-25.3^{1} \\
\end{array}$ & $\begin{array}{l}-24.3^{\mathrm{I}} \\
-24.5^{\mathrm{I}} \\
\end{array}$ \\
\hline EPA Phase IV(g) & ----- & ---- & +---- & +---- & ---- & NA & $\begin{array}{l}-41.05^{1 / 1} \\
-41.01^{1} \\
\end{array}$ & \begin{tabular}{|l|}
$-208.9^{1 /}$ \\
$-210.8^{1}$ \\
\end{tabular} & $\begin{array}{l}-26.10^{1 /} \\
-26.09^{1} \\
\end{array}$ & $\begin{array}{l}-170.5^{\mathrm{I} /} \\
-171.4^{\mathrm{I}} \\
\end{array}$ & $\begin{array}{l}-24.05^{1 /} \\
-24.06^{1} \\
\end{array}$ & NA & NA & NA \\
\hline EPA Phase V 0412-1 & 0.0 & $19.10^{\mathrm{S}}$ & $3.06^{\mathrm{S}}$ & $1.58^{\mathrm{S}}$ & $0.380^{\mathrm{S}}$ & NA & $-41.2^{\mathrm{I}}$ & $-209.1^{1}$ & $-26.2^{1}$ & NA & NA & NA & NA & NA \\
\hline EPA Phase V 0412-2 & 1.0 & $22.00^{\mathrm{S}}$ & $3.07^{\mathrm{S}}$ & $1.78^{\mathrm{S}}$ & $0.517^{\mathrm{S}}$ & NA & $-41.2^{1}$ & $-199.6^{1}$ & $-26.3^{\mathrm{I}}$ & NA & NA & NA & NA & NA \\
\hline USGS Phase V 0412-2 & 1.0 & $32.00^{\mathrm{S}}$ & $4.90^{\mathrm{S}}$ & $2.20^{\mathrm{S}}$ & NA & NA & NA & NA & NA & NA & NA & NA & NA & NA \\
\hline
\end{tabular}

USGS Phase V 0412-2

$\mathrm{S}$ - Shaw Environmental

T - Test America

Chlorofluorocarbon Laboratory

W - Woods Hole Oceanogrpahic Institute 
Table SI E6. Dissolved Inorganic Carbon, Water Isotopes, Tritium, SF6, and He in MW01 and MW02

\begin{tabular}{|c|c|c|c|c|c|c|c|c|c|c|c|}
\hline & $\begin{array}{l}\text { Borehole } \\
\text { Volumes }\end{array}$ & $\begin{array}{l}\text { DIC } \\
(\mathrm{mg} / \mathrm{L})\end{array}$ & $\begin{array}{l}\delta^{13} \mathrm{DIC} \\
(\% \circ)\end{array}$ & $\begin{array}{l}\delta^{2} \mathbf{H} \\
(\% o)\end{array}$ & $\begin{array}{l}\delta^{18} \mathrm{O} \\
(\% \circ)\end{array}$ & $\begin{array}{l}\text { Tritium } \\
(\mathbf{p C i} / \mathrm{L})\end{array}$ & $\begin{array}{l}S_{\text {SF }} \\
\text { (fg/kg) }\end{array}$ & $\begin{array}{l}\mathrm{He} \\
\left(10^{-9} \mathrm{~cm}^{3} / \mathrm{g}\right. \\
\text { water })\end{array}$ & $\begin{array}{l}\text { Rn-222 } \\
(\mathrm{pCi} / \mathrm{L})\end{array}$ & $\begin{array}{l}\text { Rn-226 } \\
(p C i / L)\end{array}$ & $\begin{array}{l}\text { Rn-228 } \\
(\text { pCi/L) }\end{array}$ \\
\hline \multicolumn{12}{|l|}{ MW01 } \\
\hline EPA Phase III & 0.3 & $26.9^{\mathrm{O}}$ & $-12.18^{\mathrm{I}}$ & $-113.8^{\mathrm{I}}$ & $-13.8^{\mathrm{I}}$ & NA & NA & NA & NA & NA & NA \\
\hline EPA Phase IV & 0.7 & $12.7^{\mathrm{O}}$ & $-12.01 \mathrm{I}$ & $-109.5^{\mathrm{I}}$ & $-13.3 \mathrm{I}$ & NA & NA & NA & NA & NA & NA \\
\hline USGS Phase V & $1.6-3.0$ & $20^{\mathrm{T}} / 19^{\mathrm{T}}$ & $-14.39^{\mathrm{W}}$ & $\begin{array}{l}-113^{\mathrm{US} /} \\
-113^{\mathrm{US}}\end{array}$ & $\begin{array}{l}-13.32^{\mathrm{US} /} \\
-13.32^{\mathrm{US}}\end{array}$ & $0.60^{\mathrm{UM}}$ & $<1.00^{\mathrm{UC}}$ & $\begin{array}{l}1170^{\mathrm{UC} /} \\
1190^{\mathrm{UC}}\end{array}$ & $1060^{\mathrm{U}}$ & $0.087^{\mathrm{E}}$ & $0.16(\mathrm{R})^{\mathrm{B}}$ \\
\hline EPA Phase V 0412-4 & 1.6 & NA & NA & $-112.9^{I}$ & $-13.3^{\mathrm{I}}$ & NA & NA & NA & NA & NA & NA \\
\hline EPA Phase V 0412 & 1.8 & $\begin{array}{l}15.2^{\mathrm{O}} \\
15.2^{\mathrm{O}}\end{array}$ & $\begin{array}{l}-11.70^{\mathrm{I}} / \\
12.13^{\mathrm{I}} \\
\end{array}$ & $\begin{array}{l}-113.1^{\mathrm{I}} \\
-113.0^{\mathrm{I}} \\
\end{array}$ & $\begin{array}{l}-13.2^{\mathrm{I} /} \\
-13.3^{\mathrm{I}} \\
\end{array}$ & $\begin{array}{l}<0.80^{\mathrm{I}} / \\
<0.80^{\mathrm{I}}\end{array}$ & NA & NA & NA & NA & NA \\
\hline EPA Phase V 0412-2 & 2.1 & NA & NA & $-113.0^{\mathrm{I}}$ & $-13.3^{\mathrm{I}}$ & NA & NA & NA & NA & NA & NA \\
\hline EPA Phase V 0412-3 & 2.3 & NA & NA & $-113.0^{\mathrm{I}}$ & $-13.3^{I}$ & NA & NA & NA & NA & NA & NA \\
\hline EPA Phase V 0412-5 & 2.4 & NA & NA & $-113.1^{\mathrm{I}}$ & $-13.3^{I}$ & NA & NA & NA & NA & NA & NA \\
\hline EPA Phase V 0412-7 & 2.6 & NA & NA & $-113.2^{\mathrm{I}}$ & $-13.3^{\mathrm{I}}$ & NA & NA & NA & NA & NA & NA \\
\hline EPA Phase V 0412-6 & 2.7 & NA & NA & $-113.2^{\mathrm{I}}$ & $-13.4^{\mathrm{I}}$ & NA & NA & NA & NA & NA & NA \\
\hline EPA Phase V 0412-8 & 2.9 & NA & NA & $-113.3^{1}$ & $-13.4^{\mathrm{I}}$ & NA & NA & NA & NA & NA & NA \\
\hline EPA Phase V 0412-9 & 3.0 & NA & NA & -113.4 & $-13.3^{\mathrm{I}}$ & NA & NA & NA & NA & NA & NA \\
\hline USGS Phase V & 3.0 & $21^{\mathrm{T}}$ & $-14.11^{\mathrm{W}}$ & $-113^{\mathrm{US}}$ & $-13.39^{\mathrm{US}}$ & $0.30^{\mathrm{UM}}$ & $<1.00^{\mathrm{UC}}$ & $2940^{\mathrm{UC}}$ & NA & $0.100^{\mathrm{E}}$ & $0.23^{\mathrm{E}}$ \\
\hline EPA Phase V 0412-10 & 3.3 & $19.1^{\mathrm{O}}$ & $-11.94^{\mathrm{I}}$ & $-113.6^{\mathrm{I}}$ & $-13.3^{\mathrm{I}}$ & $<0.80^{\mathrm{I}}$ & NA & NA & NA & NA & NA \\
\hline \multicolumn{12}{|l|}{ MW02 } \\
\hline EPA Phase III & 0.1 & $20.4^{\mathrm{O}}$ & NA & $-117.4^{\mathrm{I}}$ & $-14.6^{\mathrm{I}}$ & NA & NA & NA & NA & NA & NA \\
\hline EPA Phase IV & 0.6 & $\begin{array}{l}1.40^{\circ} / \\
1.39^{\circ} \\
\end{array}$ & $\begin{array}{l}\text { DIC too } \\
\text { low }\end{array}$ & $\begin{array}{l}-113.4^{\mathrm{I} /} \\
-113.5^{\mathrm{I}}\end{array}$ & $\begin{array}{l}-14.2^{\mathrm{I}} / \\
-14.3^{\mathrm{I}} \\
\end{array}$ & NA & NA & NA & NA & NA & NA \\
\hline EPA Phase V 0412-1 & 0.0 & $1.25^{\mathrm{O}}$ & $\begin{array}{l}\text { DIC too } \\
\text { low }\end{array}$ & $-116.3^{\mathrm{I}}$ & $-14.2^{I}$ & $<0.80^{\mathrm{I}}$ & NA & NA & NA & NA & NA \\
\hline EPA Phase V 0412-2 & 1.0 & $2.26^{\circ}$ & $\begin{array}{l}\text { DIC too } \\
\text { low }\end{array}$ & $-116.8^{\mathrm{I}}$ & $-14.2^{I}$ & $<0.80^{\mathrm{I}}$ & NA & NA & NA & NA & NA \\
\hline USGS Phase V 0412-2 & 1.0 & $4.6^{\mathrm{T}}$ & NA & NA & NA & NA & NA & NA & NA & NA & NA \\
\hline
\end{tabular}

E - Eberline Laboratory (contractor to USGS)

I - Isotech

U - U.S. Geological Survey National Water Quality Laboratory

UC - USGS Restn Chlorofluorocarbon Laboratory

UM - USGS Menlo Park Tritium Laboratory

US - USGS Reston Stable Isotope Laboratory

W - Woods Hole Oceanogrpahic Institute

\section{E.5 Discussion of Potential Cement-pH Interaction}

Elevated $\mathrm{pH}$ levels (>11 standard units) were observed during purging and sampling at MW01

and MW02 during Phase III, IV and V sampling events (Figure SI E5). During a period of extensive purging at MW01 (over $5000 \mathrm{~L}$ ) during the Phase V sampling event, specific conductance decreased from 3.93 to $1.31 \mathrm{mS} / \mathrm{cm}$ before stabilizing and $\mathrm{pH}$ decreased from 12.14 to 10.70 .

Simulations were conducted to evaluate whether trends in $\mathrm{pH}$ and specific conductance were due to insufficient purging. Observed specific conductance and $\mathrm{pH}$ as a function of time during the Phase $\mathrm{V}$ sampling event; calculated casing, borehole, and screen exchange volumes; estimated fraction of stored casing water in the sampling train; and simulated sample train/formation concentration ratios $\left(\mathrm{C}_{\mathrm{P}} / \mathrm{C}_{\mathrm{F}}\right)$ as a function of hypothetical initial screen/formation concentration ratios $\left(\mathrm{C}_{0} / \mathrm{C}_{\mathrm{F}}\right)$ and initial casing/formation concentration ratios $\left(\mathrm{C}_{\mathrm{C}} / \mathrm{C}_{\mathrm{F}}\right)$ are illustrated in Figure SI E6a. In all calculations, the initial concentration in the screen $\left(\mathrm{C}_{0}\right)$ was set equal to casing concentration $\left(\mathrm{C}_{\mathrm{C}}\right)$ or $\mathrm{C}_{0} / \mathrm{C}_{\mathrm{F}}=\mathrm{C}_{\mathrm{C}} / \mathrm{C}_{\mathrm{F}}$. Pumping rate and observed drawdown are illustrated in Figure SI E6b.

Simulations indicated that the fraction of casing to formation water entering the sampling train at MW01 during the Phase V sampling event fell below $0.1 \%$ at 0.81 borehole volumes and was at $0.003 \%$ during the time of first EPA sample collection during the Phase V sampling event. During purging, there 
were 26 screen exchange volumes (casing volume of screen plus annular space outside screen). Regardless of the initial hypothetical condition, when $\mathrm{C}_{0} / \mathrm{C}_{\mathrm{F}}$ and $\mathrm{C}_{\mathrm{C}} / \mathrm{C}_{\mathrm{F}}$ were less than unity (i.e., initial concentration in casing and well screen less than surrounding formation), sampling train concentration reached $99 \%$ of formation concentration by 0.81 borehole exchanges. When initial hypothetical values of $\mathrm{C}_{0} / \mathrm{C}_{\mathrm{F}}$ and $\mathrm{C}_{\mathrm{C}} / \mathrm{C}_{\mathrm{F}}$ were set at values greater than unity (initial concentration in casing and well screen greater than the surrounding formation due to potential well construction effects), sample train concentration reached $101 \%$ constant formation concentration by $1.26,1.48,1.65$, and 1.84 borehole volumes for $C_{0} / C_{F}$ and $C_{C} / C_{F}$ values of $1.5,3,10$, and 100 , respectively. When $C_{0} / C_{F}$ and $C_{C} / C_{F}$ was set to an extreme value of 1000 , sample train concentration reached $101 \%$ constant formation concentration by 2.27 borehole volumes. Thus, trends in $\mathrm{pH}$ observed during purging are due to interaction with formation media, not the wellbore.

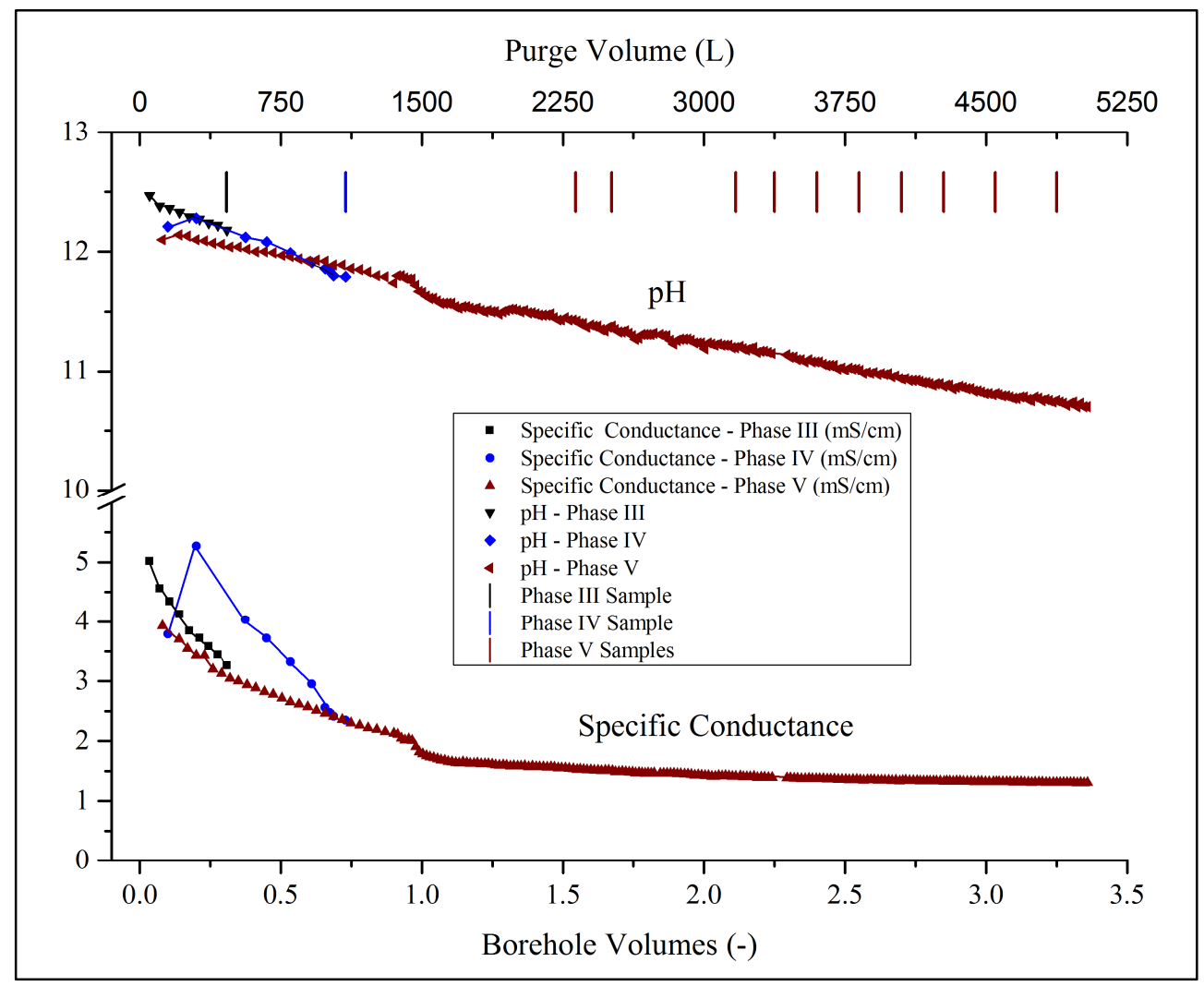

Figure SI E5. Trends in $\mathrm{pH}$ and specific conductance during purging at MW01 during Phase III, IV, and V sampling events as a function of purge volume and borehole volumes ( 1 borehole volume $\sim 1500 \mathrm{~L}$ ). Times of sample collection illustrated by straight bars

$\mathrm{pH}$ declined during purging during all three sampling events in an approximately linear fashion (Figures SI E6). Specific conductance declined rapidly and then stabilized after approximately one borehole volume. If reduction in $\mathrm{pH}$ and specific conductance were due to dissolved solids remaining in well casing as a result of drilling and/or well construction materials, this would correspond to $\mathrm{C}_{0} / \mathrm{C}_{\mathrm{F}}=$ 
$\mathrm{C}_{\mathrm{C}} / \mathrm{C}_{\mathrm{F}}$ values between $10-100$ for $\mathrm{pH}$ and 3 for specific conductance. Reduction in $\mathrm{pH}$ and specific conductance and $\mathrm{pH}$ during purging occurred much more slowly than would be expected if dissolved solids remained in well casing as a result of well construction effects.

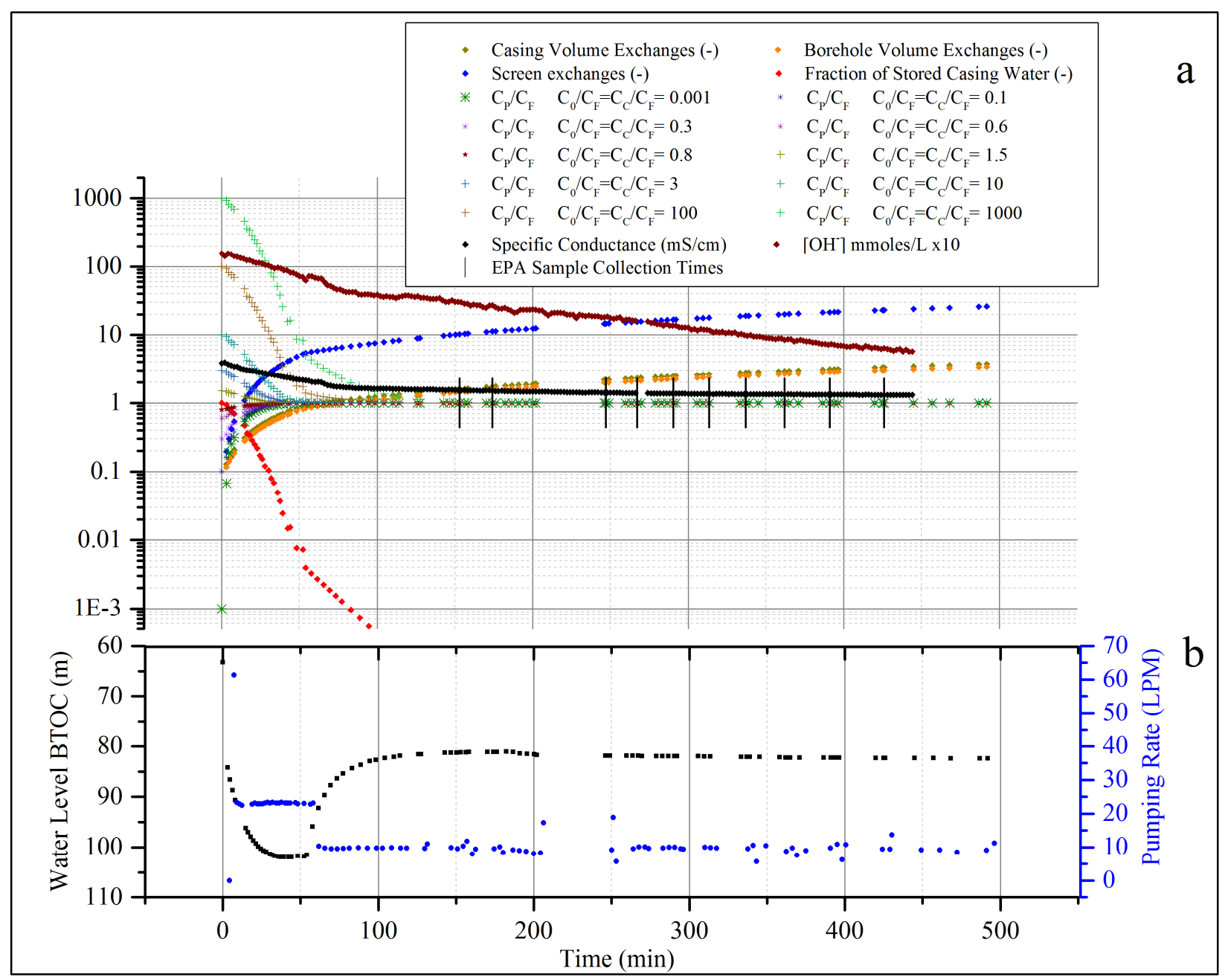

Figure SI E6. (a) Observed decrease in $\mathrm{pH}$ (in mmoles/L) and specific conductance during purging as a function of time during the Phase V sampling event in MW01. Increase in casing volumes (up to 3.8), borehole volumes (up to 3.4 ), and screen exchanges (up to 26), and EPA sample collection times (approximately 30 minutes in duration) illustrated. Simulation of fraction of store casing water in sample train $(0.003 \%$ at first sample collection), sample train $\left(\mathrm{C}_{\mathrm{P}}\right)$ / formation $\left(\mathrm{C}_{\mathrm{F}}\right)$ concentration ratios as a function of initial screen $\mathrm{C}_{0}$ and casing $\mathrm{C}_{\mathrm{C}}$ /formation $\left(\mathrm{C}_{\mathrm{F}}\right)$ concentration ratios using a casing plug flow - screen mixing wellbore process model illustrated. (b) Rise in water level in well casing as a result of reduced pumping rate in MW01 during Phase V sampling.

The $\mathrm{pH}$ of produced water samples varied from 5.6 to 9.0 standard units (Figure SI E7a). However, $\mathrm{pH}$ measurements from produced water samples were primarily from production wells where $\mathrm{CO}_{2}$ foam was used for hydraulic fracturing. There was substantial variation in hydraulic fracturing practices over time and throughout the field. Potassium hydroxide $(\mathrm{KOH})$ was used for hydraulic fracturing. In ground water having sodium - sulfate $\left(\mathrm{Na}-\mathrm{SO}_{4}\right)$ type composition, small quantities of $\mathrm{KOH}$ addition could result in $\mathrm{pH}$ approaching 12 units $^{27}$. Water flowing to the surface at Tribal Pavillion 13-1 
during Bradenhead testing had a $\mathrm{pH}$ of 10.86 and nearly all Bradenhead gas samples were devoid of $\mathrm{CO}_{2}$ (Table SI D3) suggesting elevated $\mathrm{pH}$ above intervals of stimulation. There was also an anomalous trend of increasing $\mathrm{pH}$ with depth in domestic wells (Figure SI E7b).

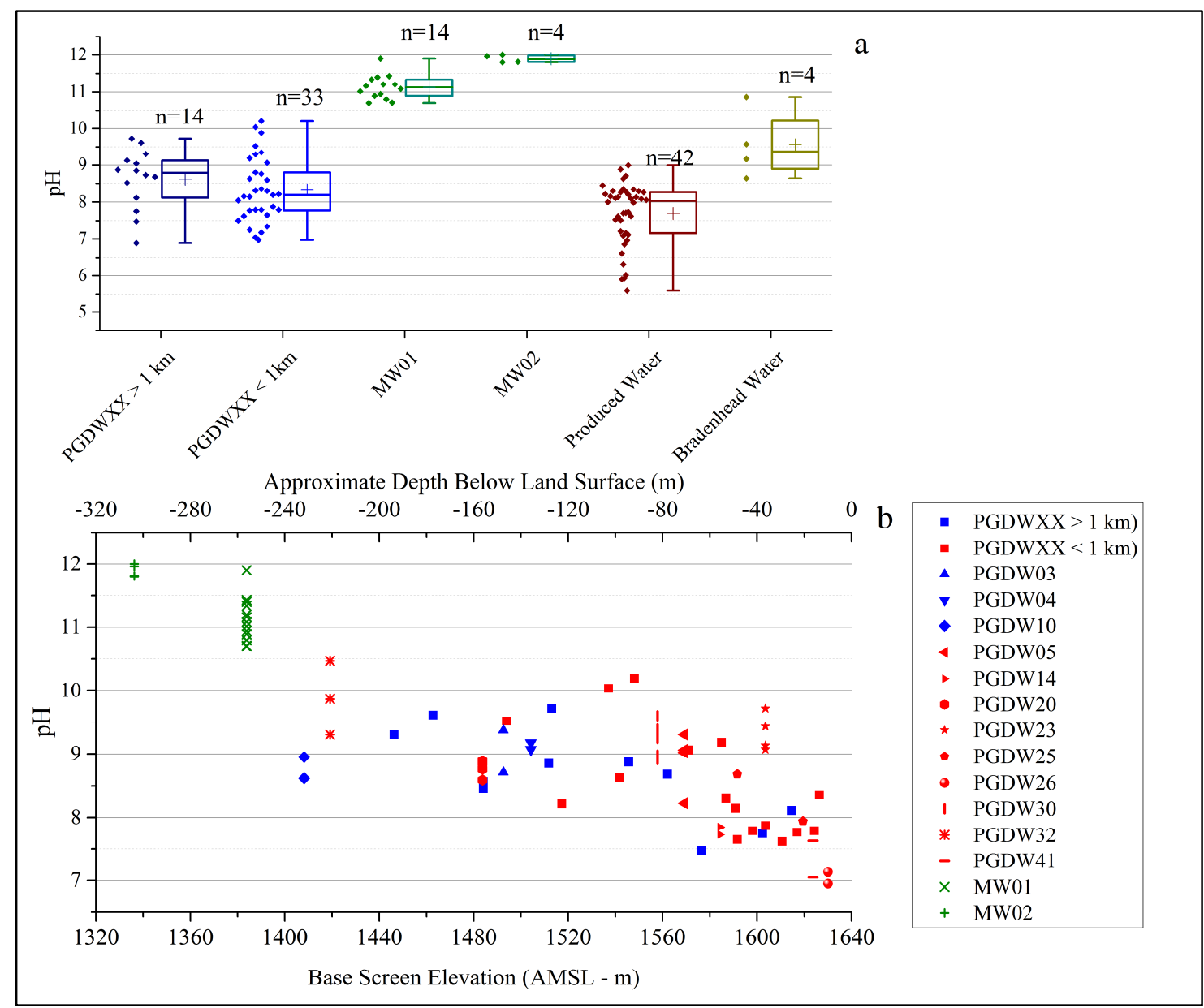

Figure SI E7. (a) Box and whisker plots of minimum, quartiles, median (line), mean (cross), and maximum values of $\mathrm{pH}$ of domestic wells (PGDWXX) greater than $1 \mathrm{~km}$ from a production well, domestic wells less than $1 \mathrm{~km}$ from a production wells, MW01, MW02, produced water, and Bradenhead water samples. Mean values are represented for domestic well locations sampled more than once. Produced water and bradenhead locations were sampled once. Measurement at MW01 and MW02 represent samples collected during Phase III, IV, and V sample events to illustrate variability. (b) $\mathrm{pH}$ levels in domestic wells (PGDWXX) less than and greater than $1 \mathrm{~km}$ of a production well (red and blue respectively) and monitoring wells as a function of absolute mean seal level (AMSL). All data points are illustrated for locations sampled more than once.

Water from MW01 and MW02 was highly undersaturated with respect to portlandite $\left(\mathrm{Ca}(\mathrm{OH})_{2}\right)$ at -4.3 and -2.3 during the Phase V sampling event, respectively. Water in contact with hydrating cement is saturated or oversaturated (saturation index greater than 0 ) to portlandite ${ }^{67-69}$ and remains oversaturated prior to degradation (e.g. carbonation) $)^{70-73}$. Calcium concentrations in MW01 and MW02 were typical of domestic wells (Figure SI E8a) displaying random scatter with depth (Figure SI E8b). Calcium concentrations should be significantly elevated in the presence of cement interaction ${ }^{74}$. 

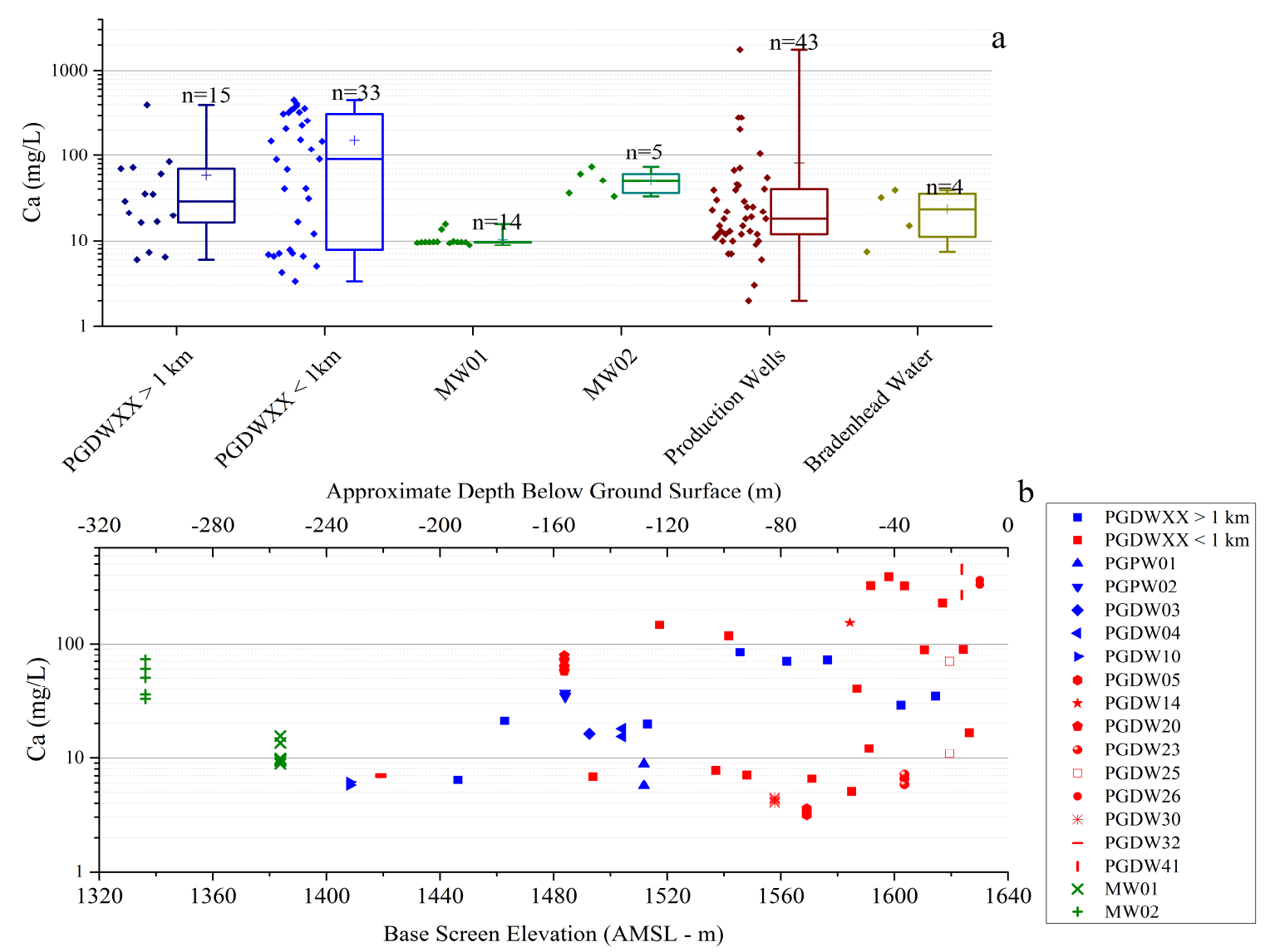

Figure SI E8. (a) Box and whisker plots of minimum, quartiles, median (line), mean (cross), and maximum values of Ca of domestic wells (PGDWXX) greater than $1 \mathrm{~km}$ from a production well, domestic wells less than $1 \mathrm{~km}$ from a production wells, MW01, MW02, produced water, and Bradenhead water samples. Mean values are represented for domestic well locations sampled more than once. Produced water and bradenhead locations were sampled once. Measurement at MW01 and MW02 represent samples collected during Phase III, IV, and V sample events to illustrate variability. (b) Ca levels in domestic wells (PGDWXX) less than and greater than $1 \mathrm{~km}$ of a production well (red and blue respectively) and monitoring wells as a function of absolute mean seal level (AMSL). All data points are illustrated for locations sampled more than once.

Another explanation of elevated $\mathrm{pH}$ in MW01 at the start of purging with decrease during purging could be progressive intake of formation water more distal from the screen that has undergone less degassing. Water underwent vigorous degassing and foaming during purging at MW01 and MW02 indicating total dissolved gas pressure (TDGP) significantly above atmospheric pressure (Figure SI E9). During the Phase IV sampling event, pump cavitation occurred at MW02 after removal of $1287 \mathrm{~L}$ of water with $40 \mathrm{~m}$ of hydrostatic head from the base of the borehole indicating 4.7 atm or $0.48 \mathrm{MPa}$ TDGP (atmospheric pressure $=0.86 \mathrm{~atm}$ ). Degassing in the immediate vicinity of the borehole could result in removal of carbon dioxide and conversion of bicarbonate/carbonate to non-carbonate alkalinity:

$$
\begin{aligned}
& \mathrm{HCO}_{3}^{-} \rightarrow \mathrm{CO}_{2(g)}+\mathrm{OH}^{-} \\
& \mathrm{CO}_{3}^{2-}+\mathrm{H}_{2} \mathrm{O} \rightarrow \mathrm{CO}_{2(g)}+2 \mathrm{OH}^{-}
\end{aligned}
$$


During purging at MW01 in the Phase V sampling event, DIC increased from 15.2 to $19.1 \mathrm{mg} / \mathrm{L}$ as pH decreased from 11.34 to 10.71. During development of MW01 in August 2010, prior to gas entry, documented by measurement of lower explosive levels in well casing, $\mathrm{pH}$ varied from 8.72 to 9.06 standard units during removal of 33,300 $\mathrm{L}$ of water.

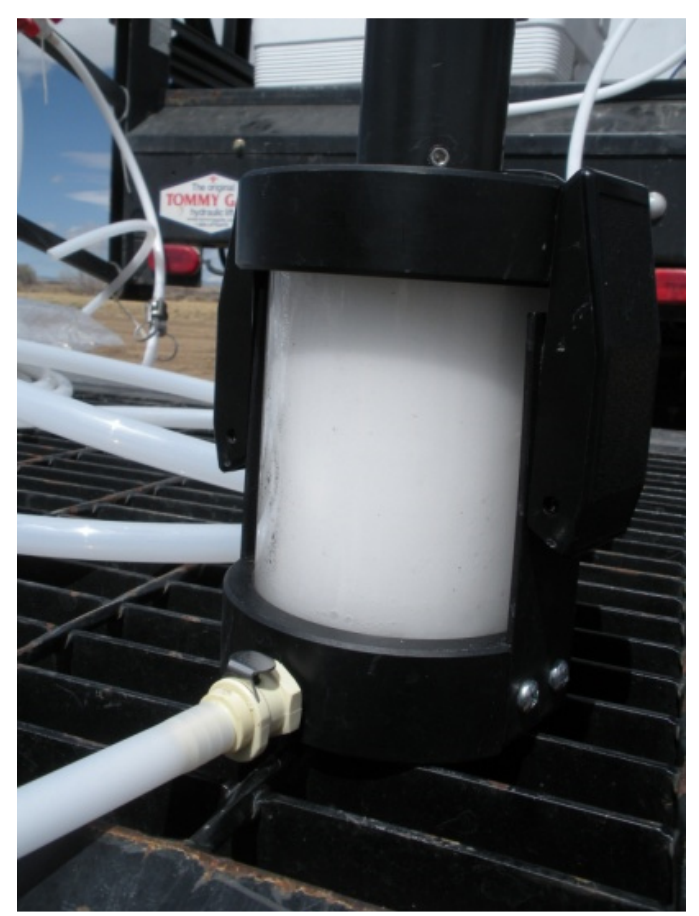

Figure SI E9. Photograph of foam in YSI flow cell during purging at MW01

\section{E.6 Discussion of Potential Cement-Potassium Interaction}

Similar to $\mathrm{pH}$, elevated potassium may be the result of interaction with cement. Alkalis in cement are present as readily soluble sulfates $\left(\mathrm{K}_{2} \mathrm{SO}_{4}\right.$ and $\left.\mathrm{Na}_{2} \mathrm{SO}_{4}\right)$ and less soluble oxides $\left(\mathrm{K}_{2} \mathrm{O}\right.$ and $\left.\mathrm{Na}_{2} \mathrm{O}\right)$ in the major clinker minerals ${ }^{70-72,75}$ leading to high alkali and sulfate concentrations during the first hours of hydration $^{76}$.

Potassium and calcium concentrations were positively correlated in domestic well and monitoring wells with apparent random scatter in bradenhead and produced water samples (Figure SI D1). With exception of EPA monitoring wells, potassium concentrations generally decreased with depth (Figure SI 10a) which was reduced to random scatter when potassium was normalized by calcium concentrations (Figure SI E10b).

Potassium concentrations decreased during purging at MW01 during the Phase V sampling event while calcium, silicon, chloride, and fluoride remained fairly constant or decreased at a slower rate (Figure SI E11a). Concentrations of sodium and strontium decreased (Figures SI E12b, c) while sulfate increased during purging (Figures SI E11b). Given that soluble sulfates are associated with curing of 
cement, sulfate concentrations should decrease rather than increase during purging. Unlike potassium but similar to calcium, strontium did not decrease during purging during the Phase V sampling event but decreased compared to Phase III and Phase IV sampling events (Figure SI 11c) potentially indicating temporal variability rather than interaction with cement.

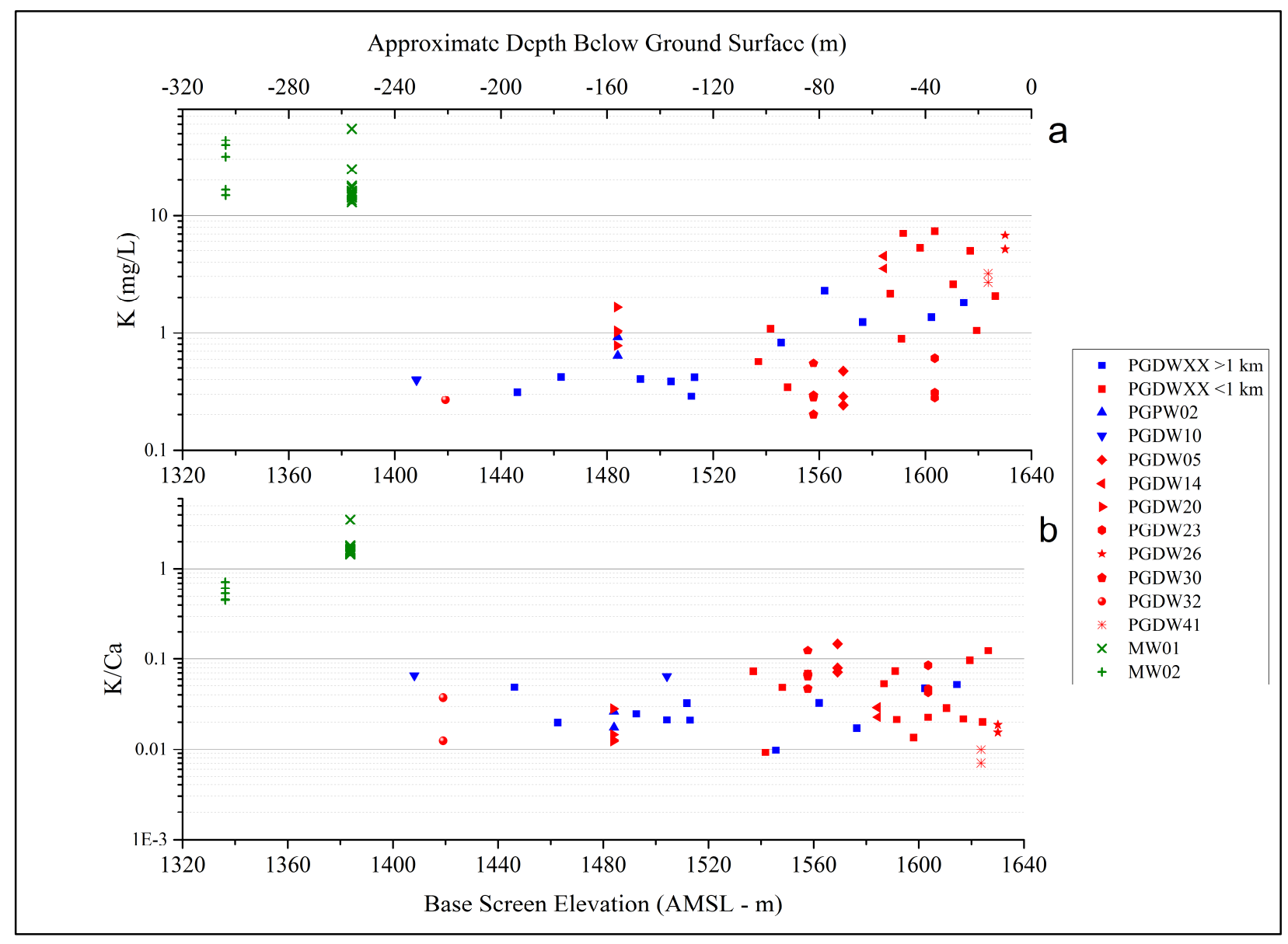

Figure SI E10. (a) Potassium concentration and (b) potassium/calcium concentration ratios for domestic wells (PGDWXX) less than and greater than $1 \mathrm{~km}$ of a production well, monitoring wells MW01 and MW02, production wells, and bradenhead samples as a function of absolute mean sea level (AMSL). Domestic wells are identified when sampled more than once.

\section{E.7 Discussion of Potential Cement-Glycol Interaction}

Polar organic compounds, including diethylene glycol (DEG) are used to reduce the energy required to grind clinker material for cement ${ }^{77-80}$. Thus, it is plausible that detection of glycols in monitoring wells is due to interaction with cement. Smith et al ${ }^{81}$ determined bulk concentrations of glycols and 2-butoxyethanol (2-BE), and a number of selected organic compounds in 5 Type I/II Portland cement samples and conducted a 5-day leaching study on the cured cement sample having the highest bulk concentrations of glycols. DEG, triethylene glycol (TEG), and tetraethylene glycol (TREG) were 
detected after the last aqueous exchange at concentrations of 97, 250, and $52 \mu \mathrm{g} / 1$, respectively. 2-BE was not detected.
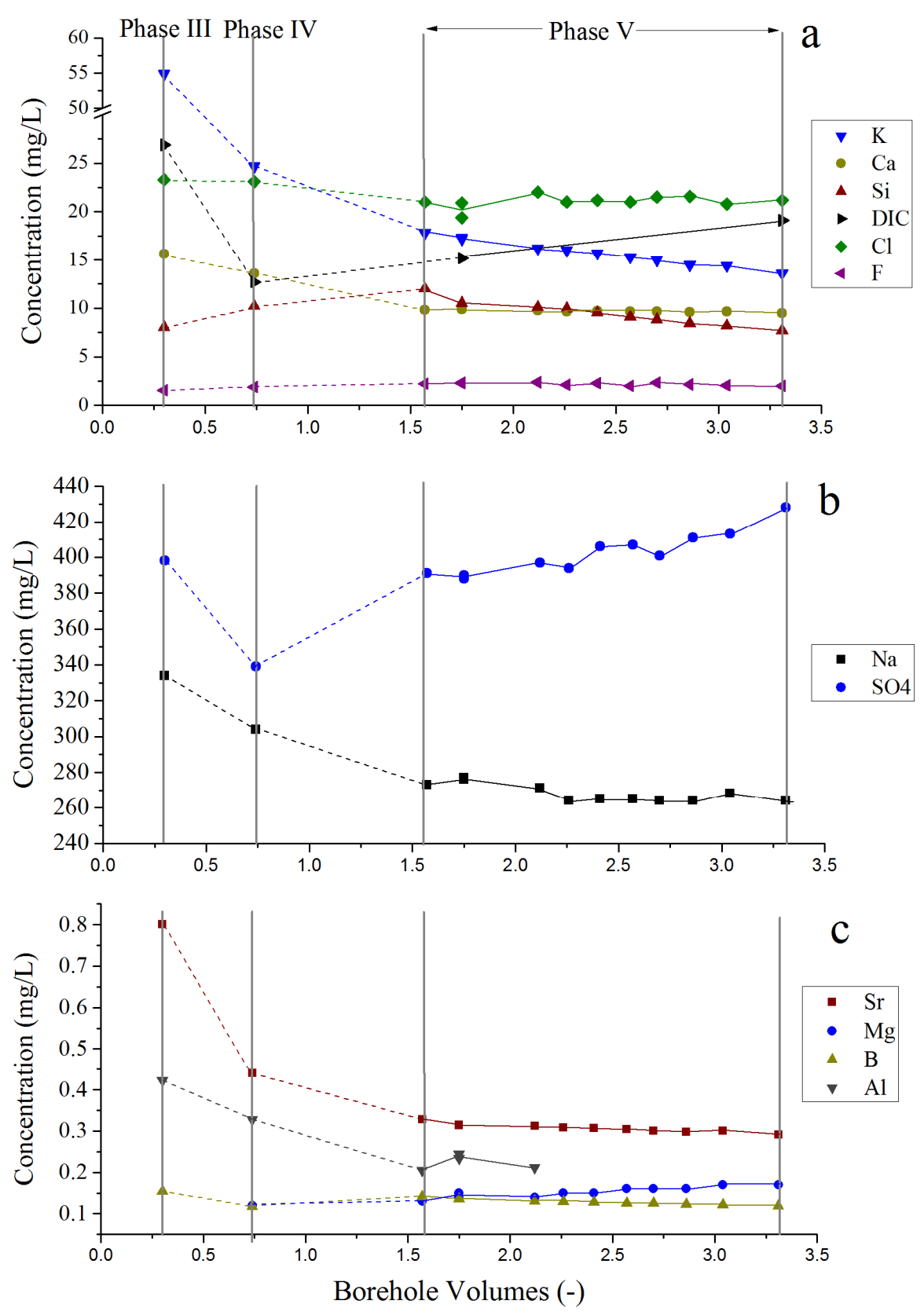

Figure SI E11. Concentration variation of a) potassium (K), calcium (Ca), silicon (Si), dissolved inorganic carbon (DIC), chloride ( $\mathrm{Cl}$ ), and fluoride (F); (b) sodium (Na) and sulfate $\left(\mathrm{SO}_{4}\right)$; and (c) Strontium ( $\mathrm{Sr}$ ), magnesium $(\mathrm{Mg})$, boron (B), and aluminum ( $\mathrm{Al}$ ) during purging at MW01 as a function of borehole volume during Phase III, IV, and $\mathrm{V}$ sampling events.

During its national study on hydraulic fracturing, EPA conducted analysis of glycols using high performance liquid chromatography with dual mass spectrometry (HPLC-MS/MS) at five retrospective 
study areas with non-detection at 83 domestic wells sampled ${ }^{82-86}$. Glycols were only detected in a produced water sample ${ }^{86}$. Using HPLC-MS/MS analysis, glycols were detected at domestic wells sampled in the Pavillion investigation. The discrepancy in detection of glycols in leachate and domestic well samples may be due variation in use of cement for domestic well construction, product variability, and exposure factors (i.e. dilution, cross-sectional exposure area, etc.) commonly used to evaluate impact of construction materials on water quality.

In EPA Method $1315^{87}$, a solution to diffusive mass transfer through a semi-infinite media $\left(\mathrm{Crank}^{88}\right)$ is utilized to evaluate mass flux from monolithic samples:

$$
\begin{array}{ll}
\text { Flux }= & D \rho\left(\frac{\partial C}{\partial x}\right)_{x=0}=\frac{D \rho C_{0}^{*}}{\sqrt{\pi D t}} \\
\text { Flux } & =\text { diffusive flux }\left(\mu \mathrm{g} / \mathrm{m}^{2} \mathrm{~s}\right) \\
C_{0}^{*} \quad=\text { initial concentration in cement }(\mu \mathrm{g} / \mathrm{kg}) & \\
\rho & =\text { density of cement }\left(\mathrm{kg} / \mathrm{m}^{3}\right) \\
\mathrm{D} & =\text { diffusion coefficient of diffusing species }\left(\mathrm{m}^{2} / \mathrm{s}\right) \\
\mathrm{t} & =\text { time }(\mathrm{s}) .
\end{array}
$$

Using this approach, concentration in a well during purging $\left(\mathrm{C}_{\text {well }}\right)(\mu \mathrm{g} / \mathrm{L})$ can then be estimated by

$$
C_{\text {well }}=\frac{(\text { flux })(S A)}{\text { flow }}
$$

To evaluate mass flux from cement at MW01, an unrealistic worst-case scenario of complete encasement of cement around a $22 \mathrm{~cm}\left(8.5^{\prime \prime}\right)$ diameter pre-packed screen was assumed (surface area $=$ $4.13 \mathrm{~m}^{2}$ or $6406 \mathrm{in}^{2}$ ) with bulk DEG concentration of $37,000 \mu \mathrm{g} / \mathrm{kg}$ from Smith et al. ${ }^{81}$. Diffusivity of cured cement was set equal to $10^{-12} \mathrm{~m}^{2} / \mathrm{s}$ (EPA 2010b) with density of $1850 \mathrm{~kg} / \mathrm{m}^{3}$ (density of cement varies from $1200-2500 \mathrm{~kg} \mathrm{~m}^{-3}$ ). An average flow rate of $16 \mathrm{LPM}$ (flow from MW01 during the Phase V sampling event varied from 8 to 24 LPM) was assumed. The hypothetical concentration of DEG in MW01 decreased from $2.0 \mu \mathrm{g} / \mathrm{L}$ at day 1 to below detection at $0.083 \mu \mathrm{g} / \mathrm{L}$ on day 600 - the approximate time for Phase $\mathrm{V}$ sampling after curing of cement. Using the maximum bulk concentration determined by Smith et al. ${ }^{81}$ for TEG $(79,000 \mu \mathrm{g} / \mathrm{kg})$ a hypothetical concentration of TEG in MW01 decreased from 43.5 $\mu \mathrm{g} / \mathrm{L}$ at day 1 to below detection at $0.178 \mathrm{ug} / \mathrm{L}$ on day 600 . 
Glycols decreased during purging in the Phase V sampling. However, compound classes and compounds (GRO, DRO, phenols) not associated with cement also decreased during purging (Figure SI E12) suggesting alternative explanations such as aquifer physical and chemical heterogeneity.

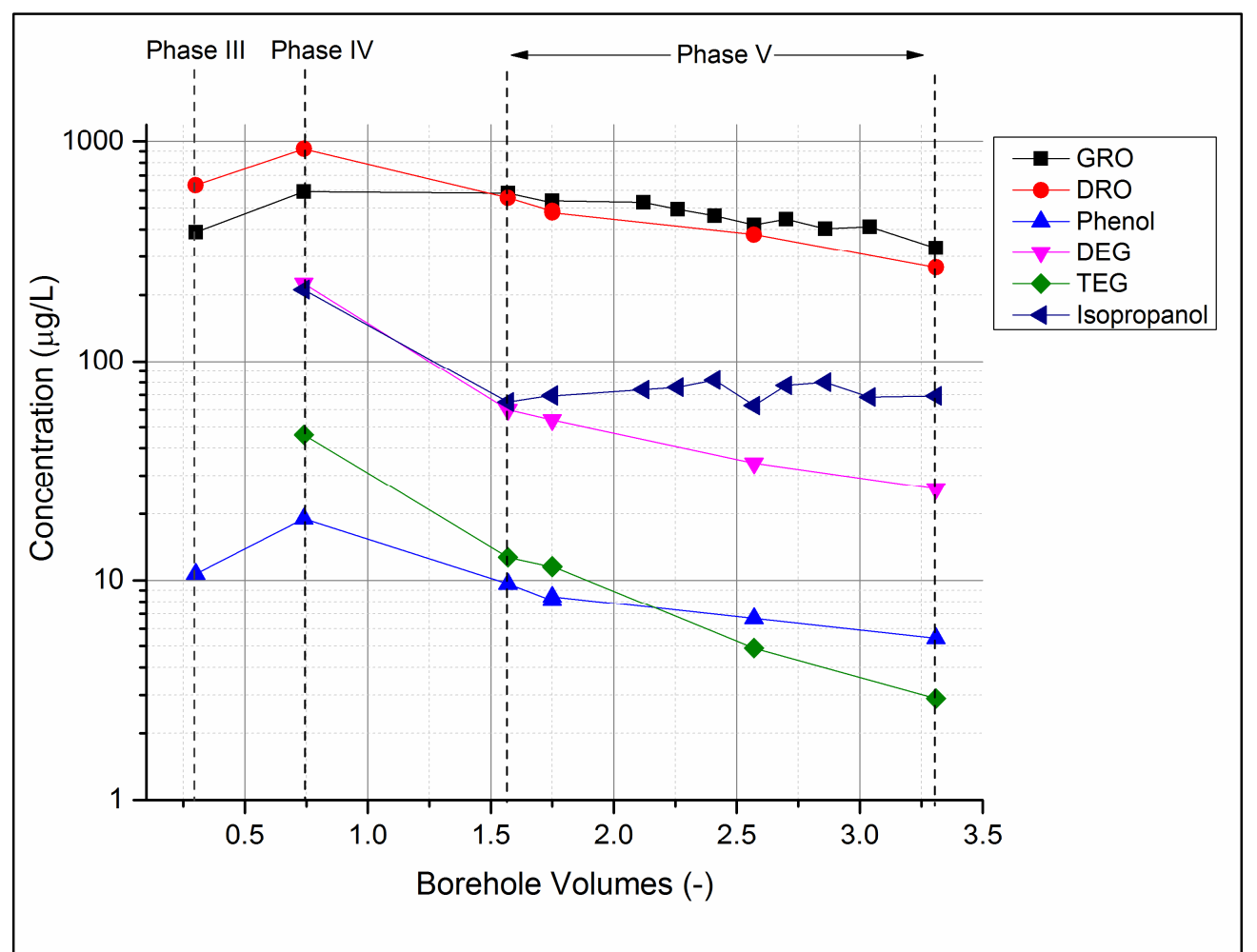

Figure SI E12. Concentration of diethylene glycol (DEG) and triethylene glycol (TEG), as a function of borehole volume during Phase III and IV sampling events and while purging during the Phase V sampling event.

Concentrations of gasoline range organics (GRO), diesel range organics (DRO), phenol, and isopropanol included for comparison.

A number of investigators have documented non-uniform concentration trends (increasing or decreasing trends as opposed to erratic variation) during purging ${ }^{89-94}$. Keith et al.$^{89}$ identified a number of potential causative factors for observation of solute concentration trends during purging: physical heterogeneity, chemical heterogeneity, screening of wells over multiple water bearing units. Analytical and numerical modeling indicate that concentration trends during purging could be a function of convergent flow ${ }^{95}$, lithologic layers of varying hydraulic conductivity ${ }^{96-98}$, anisotropy ${ }^{98}$, the ratio of hydraulic conductivity of the sandpack (in this case open borehole around pre-packed screen) to surrounding formation ${ }^{96}$, well design ${ }^{98}$, and boundary conditions ${ }^{98}$.

Concentration variation during purging could also be due to vertical ambient borehole flow prior to purging. Vertical ambient flow in long-screened monitoring wells such as MW01 and MW02 is well documented $^{99-103}$. A monitoring well is an open conduit requiring little head differential to induce substantial cumulative flow over an extended period of time between sampling events ${ }^{94,100,104-106}$. 
Significant solute redistribution can occur at head differentials below the sensitivity currently available instrumentation $^{104}$.

\section{Section F - Potential Impact of Unlined Pits on Domestic Wells}

Table SI F1. Summary of disposal of drilling mud and production fluids in from production wells

\begin{tabular}{|l|l|l|l|l|l|l|l|l|}
\hline & $\begin{array}{l}\text { Unlined Pits } \\
\text { Used to } \\
\text { Dispose } \\
\text { Invert Mud }\end{array}$ & $\begin{array}{l}\text { Unlined Pits } \\
\text { Used to } \\
\text { Dispose } \\
\text { WBM }\end{array}$ & $\begin{array}{l}\text { Unknown } \\
\text { Disposal } \\
\text { of WBM }\end{array}$ & $\begin{array}{l}\text { Disposal of } \\
\text { WBM at } \\
\text { Lozier } \\
\text { Area }\end{array}$ & $\begin{array}{l}\text { WBM } \\
\text { Used on } \\
\text { Location } \\
+\end{array}$ & $\begin{array}{l}\text { WBM } \\
\text { Disposed } \\
\text { in Lined } \\
\text { Pits }\end{array}$ & $\begin{array}{l}\text { Offsite } \\
\text { Disposal } \\
\text { of WBM }\end{array}$ & Totals \\
\hline $\begin{array}{l}\text { Pits Likely Used } \\
\text { for Disposal of } \\
\text { Production } \\
\text { Fluids }\end{array}$ & 41 & 3 & 0 & 0 & 0 & 0 & 0 & 44 \\
\hline $\begin{array}{l}\text { No Production } \\
\text { Fluids }\end{array}$ & 16 & 4 & 0 & 0 & 0 & 7 & 0 & 27 \\
\hline $\begin{array}{l}\text { Offsite Disposal } \\
\text { of Production } \\
\text { Fluids }\end{array}$ & 0 & 0 & 10 & 8 & 19 & 26 & 47 & 110 \\
\hline Totals & 57 & 7 & 10 & 8 & 19 & 33 & 47 & 181 \\
\hline
\end{tabular}

WBM - water based mud

$\dagger$ - used to construct berms or spread out on site 
Table SI F2. Summary of disposal of drilling mud and production fluids in pits, results of soil and surficial ground-water sampling, volumes of soil excavation, distance and direction of domestic wells with $600 \mathrm{~m}$ of pits, review of completion and stimulation record by WOGCC ${ }^{31}$ of production wells associated with unlined pits, and recommendations by WOGCC ${ }^{32}$ for further or no further investigation.

\begin{tabular}{|c|c|c|c|c|c|c|c|c|c|c|c|c|c|c|c|c|}
\hline 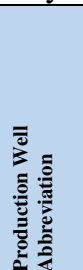 & 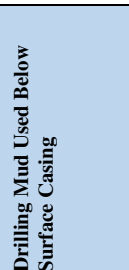 & 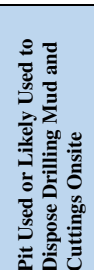 & 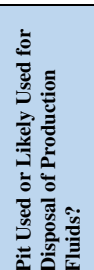 & 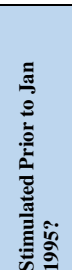 & 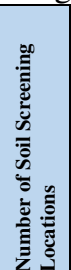 & 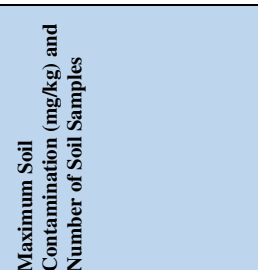 & 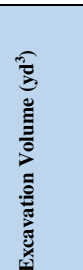 & 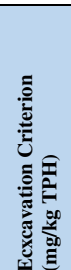 & 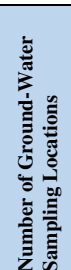 & 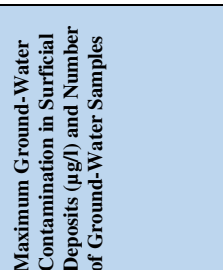 & 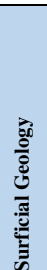 & 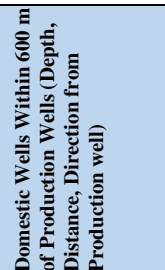 & 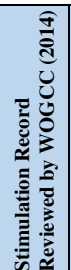 & 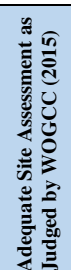 & 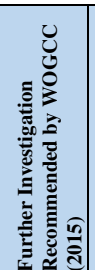 & हैं \\
\hline MHR 1 & "Chem Gel" & $\mathrm{Yes}^{\mathrm{U}}$ & No & No & $\frac{2}{0}=$ & Not sampled & 0 & & $\frac{2}{0}$ & Not sampled & Qa & $\begin{array}{l}\text { PGDW41 } \\
(31 \mathrm{~m}, 314 \mathrm{~m}, \mathrm{NW})\end{array}$ & Yes & NI & No & $\begin{array}{l}\text { Completed amd P\&A in 1953. No production or } \\
\text { stimulation. }\end{array}$ \\
\hline $14-12$ & Invert & $\mathrm{Yes}^{\mathrm{U}}$ & Yes & Yes & 20 & $\begin{array}{l}\text { GRO: } 340(\mathrm{n}=23) \\
\text { DRO:4300 }(\mathrm{n}=23) \\
\text { Benzene: } \mathrm{ND}(\mathrm{n}=20) \\
\text { Toluene: } \mathrm{ND}(\mathrm{n}=20) \\
\text { Ethylbenzene: } 0.066(\mathrm{n}=20) \\
\text { Xylenes: } 0.085(\mathrm{n}=20)\end{array}$ & $\sim 1,306$ & 1,000 & 7 & $\begin{array}{l}\text { GRO: ND }(\mathrm{n}=7) \\
\text { DRO } 1,300(\mathrm{n}=7) \\
\text { Benzene: } \mathrm{ND}(\mathrm{n}=7) \\
\text { Toluene: } \mathrm{ND}(\mathrm{n}=7) \\
\text { Ethylbenzene: } \mathrm{ND}(\mathrm{n}=7) \\
\text { Xylenes: } \mathrm{ND}(\mathrm{n}=7)\end{array}$ & Qa & $\begin{array}{l}\text { PGDW22 } \\
(? \mathrm{~m}, 261 \mathrm{~m}, \mathrm{SW}) \\
\text { PGDW32 } \\
\text { (2066,563m,SE) } \\
\text { P22660.0P } \\
\text { (53m,407m,SE) } \\
\text { P22661.0P } \\
(15 \mathrm{~m}, 535 \mathrm{~m} \text { SE) }\end{array}$ & No & VRP & VRP & $\begin{array}{l}\text { Acid stimulation in 1960. Hydraulic fracturing in } 1964 \\
\text { with "salt water." Well flowed "diesel and load water." } \\
\text { Acid stimulation with additives in } 1980 . \text { No } \\
\text { description of where flowback and other production } \\
\text { fluids were disposed. No produced water from } 1978 \\
\text { (records start) through } 1983 \text { (shut-in). Sundry notice in } \\
\text { Jan } 1998 \text { for offsite disposal of production fluids. } \\
\text { Encana waiting approval from WYDEQ for closure. }\end{array}$ \\
\hline $23-7$ & Q-Broxin Gel & $\mathrm{Yes}^{\mathrm{U}}$ & No & No & 0 & Not sampled & 0 & & 0 & Not sampled & Qa & $\begin{array}{l}\text { CR UW09/250 } \\
(?, 361 \mathrm{~m}, \mathrm{NE})\end{array}$ & No & NI & Yes & $\begin{array}{l}\text { Well completeded and P\&A in } 1961 \text {. Q-BROXIN Gel } \\
\text { used. Well history missing from well completion } \\
\text { report. Production and stimulation unlikely. }\end{array}$ \\
\hline W 1 & "Gel" & $\mathrm{Yes}^{\mathrm{U}}$ & No & No & 0 & Not sampled & 0 & & 0 & Not sampled & ? & None & No & $\mathrm{NI}$ & No & $\begin{array}{l}\text { Well completed and P\&A in 1961. Production and } \\
\text { stimulation unlikely. }\end{array}$ \\
\hline $23-2$ & Invert & $\mathrm{Yes}^{\mathrm{U}}$ & Yes & Yes & 10 & TPH: ND (n=2) & 0 & 1,000 & 0 & Not sampled & Qa & None & No & No & Yes & $\begin{array}{l}\text { Well completed in 1962. Hydraulic fracturing in } 1965 \\
\text { - no description. No description of where flowback } \\
\text { and other production fluids were disposed. Production } \\
\text { records from 1978. No produced water until } 2004 \text {. } \\
\text { Sundry notice for offsite disposal of production fluids } \\
\text { in Jan 1998. Two separate pit locations. }\end{array}$ \\
\hline $22-35$ & Invert & $\mathrm{Yes}^{\mathrm{u}}$ & No & No & 0 & Not sampled & 0 & & 0 & Not sampled & $?$ & None & No & NI & No & Completed \& P\&A in 1963. Stimulation unlikely. \\
\hline GOL 1 & Invert & Yes $^{u}$ & No & No & 0 & Not sampled & 0 & & 0 & Not sampled & Qa & None & No & $\mathrm{NI}$ & No & $\begin{array}{l}\text { Well completed in 1963. P\&A in 1974. No information } \\
\text { available on production or stimulation. }\end{array}$ \\
\hline 14-1 & Invert & $\mathrm{Yes}^{\mathrm{U}}$ & Yes & Yes & 5 & $\begin{array}{l}\text { TPH: } 3790(\mathrm{n}=5) \\
\text { (Confirmation sample) }\end{array}$ & $\sim 1,400$ & $\begin{array}{l}2,500 \\
\text { or } \\
4,000\end{array}$ & 0 & Not sampled & Qa & $\begin{array}{l}\text { PGDW36 } \\
(31 \mathrm{~m}, 296 \mathrm{~m}, \mathrm{SW})\end{array}$ & No & No & Yes & $\begin{array}{l}\text { Well completed in 1963. Hydraulic fracturing with } \\
\text { undiluted diesel fuel in } 1964 \text {. In } 1993 \text {, acid stimulation. } \\
\text { No description of where flowback and other } \\
\text { production fluids were disposed. Production records } \\
\text { from } 1978 \text { with } 789 \text { bbls produced water prior to } 1993 . \\
\text { Sundry notice in } 1993 \text { to plug water bearing } \\
\text { perforation. Sundry notice in Jan } 1998 \text { for offsite } \\
\text { disposal of production fluids. Post excavation soil TPH } \\
\text { exceeded } 4000 \mathrm{mg} / \mathrm{kg} \text {. }\end{array}$ \\
\hline $21-8$ & Invert & $\mathrm{Yes}^{\mathrm{U}}$ & Yes & $?$ & 0 & Not sampled & 0 & & 0 & Not sampled & Qa & None & No & $\mathrm{NI}$ & No & $\begin{array}{l}\text { Well completed in 1963. P\&A date unknown. Well } \\
\text { history missing from well completion report. }\end{array}$ \\
\hline $21-5$ & Invert & Yes $^{\mathrm{U}}$ & Yes & $?$ & 13 & $\begin{array}{l}\text { TPH: } 327(\mathrm{n}=8) \\
\text { (Confirmation sample) }\end{array}$ & $\begin{array}{l}\sim 60 \\
\end{array}$ & 7,000 & 0 & Not sampled & Qa & None & No & Yes & No & $\begin{array}{l}\text { Well completed in } 1963 \text {. No documented stimulation } \\
\text { until 1999. Production records from 1978. No } \\
\text { produced water until 1995. Sundry notice for offsite } \\
\text { disposal of production fluids in Jan } 1998 \text {. }\end{array}$ \\
\hline U 13-13 & Invert & $\mathrm{Yes}^{\mathrm{U}}$ & Yes & $?$ & 5 & TPH: $16(\mathrm{n}=5)$ & 0 & 1,000 & 0 & Not sampled & $?$ & $\begin{array}{l}\mathrm{P} 60032.0 \mathrm{~W} \\
(26 \mathrm{~m}, 99 \mathrm{~m}, \mathrm{~W})\end{array}$ & No & No & Yes & $\begin{array}{l}\text { Well completed in 1963. Information on completion } \\
\text { missing from well completion report. Production } \\
\text { records from 1978. No produced water until } 2007 \text {. } \\
\text { Sundry notice for offsite disposal of production fluids } \\
\text { in Jan } 1998 \text {. }\end{array}$ \\
\hline $14-6$ & Invert & $\mathrm{Yes}^{\mathrm{U}}$ & Yes & $?$ & 5 & $\begin{array}{l}\text { TPH: } 1298(\mathrm{n}=5) \\
\text { (Confirmation sample) }\end{array}$ & 120 & 2,500 & 0 & Not sampled & & $?$ & No & $?$ & $?$ & $\begin{array}{l}\text { Well completion in 1963. Information on completion } \\
\text { missing from well completion report. Production } \\
\text { records from 1978. No produced water until } 2005 \text {. } \\
\text { Further investigation by WOGCC dependent on } \\
\text { potential presence of nearby domestic well. }\end{array}$ \\
\hline $32-4$ & Invert & $\mathrm{Yes}^{\mathrm{u}}$ & No & No & 0 & Not sampled & 0 & & 0 & Not sampled & Qa & None & No & NI & No & Completed \& P\&A in 1963. Stimulation unlikely. \\
\hline $24-14$ & Invert & Yes $^{U}$ & Yes & $?$ & 11 & TPH: $3910(\mathrm{n}=4)$ & 0 & 4,000 & 0 & Not sampled & Qa & $\begin{array}{l}\text { P59499.0W } \\
(34 \mathrm{~m}, 330 \mathrm{~m}, \mathrm{NW}) \\
\text { P24502.0P } \\
(55 \mathrm{~m}, 360 \mathrm{~m}, \mathrm{E}) \\
\text { PGDW34 } \\
(31 \mathrm{~m}, 474 \mathrm{~m}, \mathrm{NE})\end{array}$ & No & No & Yes & $\begin{array}{l}\text { Well completed in 1963. No documented stimulation } \\
\text { but completion record not reviewed by WOGCC } \\
\text { (2014). Production records from 1978. No produced } \\
\text { water until } 2004 \text { but sundry notice for offsite disposal } \\
\text { of production fluids in Jan } 1998 \text {. }\end{array}$ \\
\hline
\end{tabular}




\begin{tabular}{|c|c|c|c|c|c|c|c|c|c|c|c|c|c|c|c|c|}
\hline 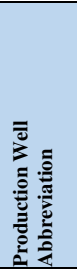 & 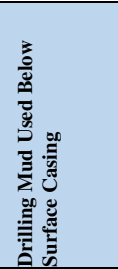 & 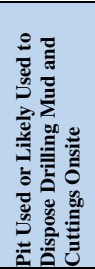 & 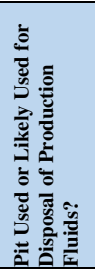 & 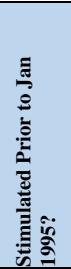 & 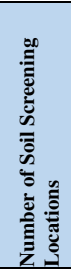 & 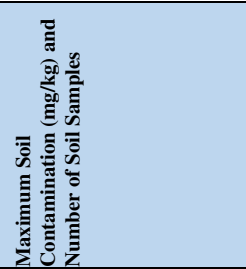 & 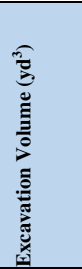 & 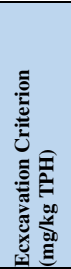 & 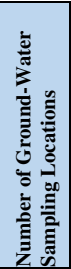 & 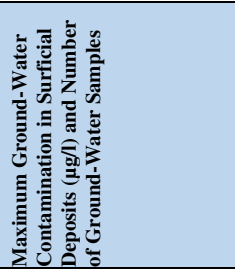 & 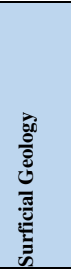 & 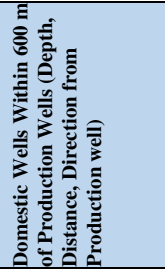 & 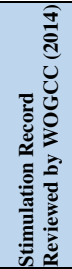 & 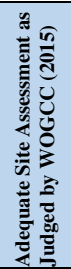 & 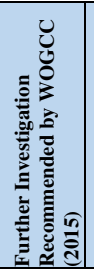 & 蒫 \\
\hline $44-20$ & Invert & Yes $^{U}$ & No & No & 0 & Not sampled & 0 & & 0 & Not sampled & Twdr & $\begin{array}{l}\text { P9334.0P } \\
(6 \mathrm{~m}, 430 \mathrm{~m}, \mathrm{SW})\end{array}$ & No & NI & Yes & $\begin{array}{l}\text { Well completed and P\&A in 1964. Stimulation or } \\
\text { production unlikely. Further investigation by WOGCC } \\
\text { dependent on confirmation of nearby domestic well. }\end{array}$ \\
\hline $23 \mathrm{X}-24$ & Invert & Yes $^{\mathrm{U}}$ & No & No & 0 & Not sampled & 0 & & 0 & Not sampled & Qa & None & No & $\mathrm{NI}$ & No & $\begin{array}{l}\text { Well completed and P\&A in 1964. Stimulation or } \\
\text { production unlikely. }\end{array}$ \\
\hline $44-17$ & Invert & $\mathrm{Yes}^{\mathrm{U}}$ & No & No & 0 & Not sampled & 0 & & 0 & Not sampled & Qa & $\begin{array}{l}\text { PGDW03 } \\
(137 \mathrm{~m}, 265 \mathrm{~m}, \mathrm{SE}) \\
\text { PGDW04 } \\
(134 \mathrm{~m}, 351 \mathrm{~m}, \mathrm{SE}) \\
\text { P120203.0W } \\
(137 \mathrm{~m}, 255 \mathrm{~m}, \mathrm{~W}) \\
\text { P23056.0P } \\
(116 \mathrm{~m}, 255 \mathrm{~m}, \mathrm{~W}) \\
\end{array}$ & No & $\mathrm{NI}$ & Yes & $\begin{array}{l}\text { Well completed and P\&A in 1964. Stimulation or } \\
\text { production unlikely. No investigation recommended by } \\
\text { WOGCC if no nearby domestic wells. }\end{array}$ \\
\hline $34-33$ & Invert & Yes $^{\mathrm{U}}$ & No & $?$ & 0 & Not sampled & 0 & & 0 & Not sampled & $?$ & None & No & NI & No & $\begin{array}{l}\text { Well completed in 1964. P\&A unknown. No } \\
\text { information on stimulation or production. }\end{array}$ \\
\hline $33 \mathrm{X}-10$ & Invert & Yes $^{\mathrm{U}}$ & Yes & Yes & $?$ & Sample data not available & $\sim 560$ & 1,000 & 0 & Not sampled & Qa & $\begin{array}{l}\text { PGDW14 } \\
(58 \mathrm{~m}, 224 \mathrm{~m}, \mathrm{NW}) \\
\text { PGDWW23 } \\
(53 \mathrm{~m}, 172 \mathrm{~m}, \mathrm{SE}) \\
\text { (PGDW44 } \\
\text { 229m,102m,NW) } \\
\text { P24508.0P } \\
(53 \mathrm{~m}, 365 \mathrm{~m}, \mathrm{SE}) \\
\end{array}$ & Yes & No & Yes & $\begin{array}{l}\text { Acid stimulation and well completion in } 1964 . \\
\text { Hydraulic fracturing in } 1965 \text { with "salt water...flow to } \\
\text { pit.” P\&A in 1983. Invert mud up to 10\% oil. } \\
\text { Production records from 1978. No produced water to } \\
1983 \text {. }\end{array}$ \\
\hline $24 \mathrm{X}-3$ & Invert & Yes $^{\mathrm{U}}$ & Yes & Yes & 9 & $\begin{array}{l}\text { GRO: } 9,200(\mathrm{n}=15) \\
\text { DRO: } 3,300(\mathrm{n}=15) \\
\text { Benzene: } 4.2(\mathrm{n}=15) \\
\text { Toluene: } \mathrm{ND}(\mathrm{n}=15) \\
\text { Ethylbenzene: } 110(\mathrm{n}=15) \\
\text { Total Xylenes: } 750(\mathrm{n}=15) \\
\text { Naphthalene: } 8.6(\mathrm{n}=11)\end{array}$ & $\begin{array}{c}1,000 \\
\sim\end{array}$ & 1,000 & 11 & $\begin{array}{l}\text { GRO: } 45,200(\mathrm{n}=38) \\
\text { DRO: } 59,000(\mathrm{n}=38) \\
\text { Benzene: } 1,960(\mathrm{n}=38) \\
\text { Toluene: } 0.17(\mathrm{n}=38) \\
\text { Ethylbenzene: } 950(\mathrm{n}=38) \\
\text { Xylenes: } 4,200(\mathrm{n}=38) \\
\text { Naphthalene: } 267(\mathrm{n}=10)\end{array}$ & Qa & $\begin{array}{l}\mathrm{P} 66345.0 \mathrm{~W} \\
(21 \mathrm{~m}, 258 \mathrm{~m}, \mathrm{NW})\end{array}$ & No & VRP & VRP & $\begin{array}{l}\text { Well completed in } 1965 \text {. Acid stimulation with solvent } \\
\text { in } 1966 \text {. No description of where flowback and other } \\
\text { production fluids were disposed. Production records } \\
\text { from } 1978 \text { with } 9 \text { blls produced water in } 1986 \text { and } \\
\text { increasing to } 9,653 \text { bbls after } 1995 \text {. Invert mud up to } \\
55 \% \text { oil. Sundry notice for offsite disposal of } \\
\text { production fluids in Jan } 1998 \text {. Ground water } \\
\text { monitoring ongoing. A remedial alternatives } \\
\text { evaluation report is being drafted by Encana. }\end{array}$ \\
\hline 14-11 & Invert & $\mathrm{Yes}^{\mathrm{U}}$ & Yes & $?$ & 12 & $\begin{array}{l}\text { GRO: } 7,400(\mathrm{n}=13) \\
\text { DRO: } 7,800(\mathrm{n}=13) \\
\text { Benzene: } \mathrm{ND}(\mathrm{n}=4) \\
\text { Toluene: } \mathrm{ND}(\mathrm{n}=4) \\
\text { Ethylbenzene: } 5.1(\mathrm{n}=4) \\
\text { Total Xylenes: } 5.1(\mathrm{n}=4) \\
\text { Naphthalene: } 15(\mathrm{n}=6) \\
\end{array}$ & $\sim 940$ & 1,000 & 8 & $\begin{array}{l}\text { GRO: } 91,100(\mathrm{n}=79) \\
\text { DRO: } 78,000(\mathrm{n}=79) \\
\text { Benzene: } 476(\mathrm{n}=79) \\
\text { Toluene } 15(\mathrm{n}=79) \\
\text { Ethylbenzene: } 60(\mathrm{n}=79) \\
\text { Xylenes: } 68(\mathrm{n}=79) \\
\text { Naphthalene: } 486(\mathrm{n}=46)\end{array}$ & Qa & $\begin{array}{l}\text { PGDW46 } \\
(15 \mathrm{~m}, 119 \mathrm{~m}, \mathrm{~W}) \\
\text { P31805.0W } \\
(31 \mathrm{~m}, 132 \mathrm{~m}, \mathrm{SE}) \\
\text { P69549.0W } \\
\text { (31m,132m,SE) } \\
\text { (Same well?) } \\
\end{array}$ & No & VRP & VRP & $\begin{array}{l}\text { Well completed in } 1965 \text {. Well completion report and } \\
\text { information on stimulation not available. Production } \\
\text { records from } 1978 \text {. No produced water until } 2005 \text { but } \\
\text { sundry notice for offsite disposal of production fluids } \\
\text { in Jan } 1998 \text {. Remedial Agreement submitted to } \\
\text { WOGCC entailing limitited additional soil excavation } \\
\text { and ground water monitoring by Encana. }\end{array}$ \\
\hline $13-13$ & "Chem Gel" & $\mathrm{Yes}^{\mathrm{U}}$ & Yes & Yes & 0 & Not sampled & 0 & & 0 & Not sampled & $?$ & None & No & NI & No & $\begin{array}{l}\text { Well completed in 1966. P\&A date unknown. } \\
\text { Hydraulic fracturing with undiluted diesel fuel, } 15 \% \\
\text { HCl, and } 4 \% \text { "salt water" in } 1965 \text {. No description of } \\
\text { where flowback and other production fluids were } \\
\text { disposed. }\end{array}$ \\
\hline $31-15$ & Invert & Yes $^{\mathrm{U}}$ & Yes & $?$ & 12 & $\begin{array}{l}\text { TPH: } 70(\mathrm{n}=6) \\
\text { (Confirmation sample) }\end{array}$ & $\begin{array}{c}1,500 \\
\sim\end{array}$ & 5,500 & 0 & Not sampled & Qa & $\begin{array}{l}\text { PGDW11 } \\
(107 \mathrm{~m}, 230 \mathrm{~m}, \mathrm{NE})\end{array}$ & No & ? & $?$ & $\begin{array}{l}\text { Well completed in 1968. Information on completion } \\
\text { missing. Production records from } 1978 \text { with } 8 \text { bbls } \\
\text { produced water in } 1988 \text { and increasing to } 1,493 \text { bbls } \\
\text { after 2004. No description of where produced water } \\
\text { was disposed. Sundry notice for offsite disposal of } \\
\text { production fluids in Jan } 1999 \text {. Recommendation of } \\
\text { further investigation pending - no soil sample in area } \\
\text { with highest PID reading (WOGCC 2015) }\end{array}$ \\
\hline $32-9$ & Invert & Yes $^{\mathrm{U}}$ & No & No & 0 & Not sampled & 0 & & 0 & Not sampled & Qa & $\begin{array}{l}\text { PGDW42 } \\
(61 \mathrm{~m}, 375 \mathrm{~m}, \mathrm{NW})\end{array}$ & Yes & NI & Yes & $\begin{array}{l}\text { Well completion and P\&A after in } 1968 \text {. No apparent } \\
\text { stimulation or production. Investigation recommended } \\
\text { by WOGCC because of proximity to a domestic well. }\end{array}$ \\
\hline
\end{tabular}




\begin{tabular}{|c|c|c|c|c|c|c|c|c|c|c|c|c|c|c|c|c|}
\hline 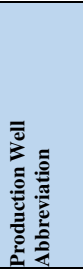 & 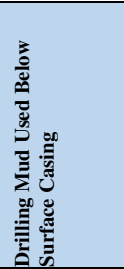 & 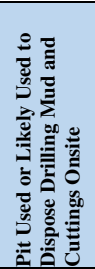 & 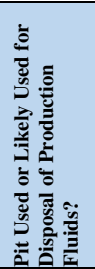 & 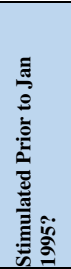 & 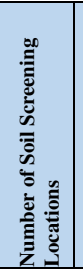 & 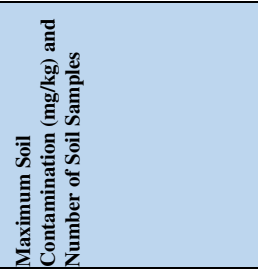 & 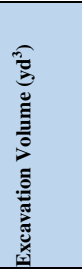 & 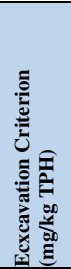 & 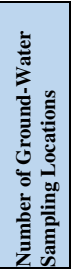 & 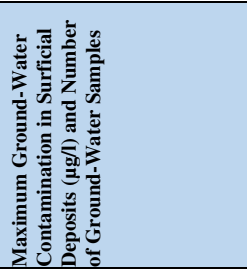 & 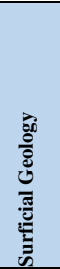 & 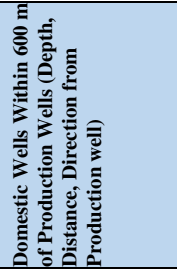 & 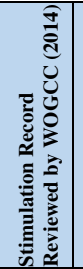 & 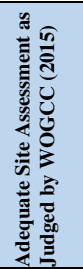 & 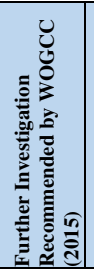 & 嶽 \\
\hline WEL & "Chem Gel" & Yes $^{\mathrm{U}}$ & Yes & Yes & 8 & $\begin{array}{l}\text { GRO: ND (n=8) } \\
\text { DRO: } 390(\mathrm{n}=8) \\
\text { BTEX: not analyed } \\
\text { Naphthalene: } 0.22(\mathrm{n}=1)\end{array}$ & 0 & 1,000 & 2 & $\begin{array}{l}\text { GRO: }<0.1 \mathrm{mg} / \mathrm{l}(\mathrm{n}=2) \\
\text { DRO: } 0.32 \mathrm{mg} /(\mathrm{n}=2) \\
\text { BTEX: }<1-<5 \mu \mathrm{g} / \mathrm{l}(\mathrm{n}=2) \\
\text { Naphthalene: }<1 \mu \mathrm{g} / \mathrm{l}(\mathrm{n}=2)\end{array}$ & Qa & $\begin{array}{l}\text { PGDW30 } \\
\text { (79m,361m,SE) } \\
\text { PGDW40 } \\
\text { (67.1m,470m,NE) }\end{array}$ & Yes & Yes & No & $\begin{array}{l}\text { Well completed in 1972. P\&A in 1981. Acidized in } \\
\text { Frontier formation with HCl and KCl solutions in } \\
\text { 1971. Potential wellhead leak and discharge to "pits' } \\
\text { discussed in memorandum in } 1980 . \text { Encana (2015) } \\
\text { stated that a pit could not be identified. }\end{array}$ \\
\hline $41 \mathrm{X}-10$ & Invert & $\mathrm{Yes}^{\mathrm{U}}$ & Yes & Yes & 7 & $\begin{array}{l}\text { GRO: ND (n=1) } \\
\text { DRO: ND }(\mathrm{n}=1) \\
\text { Sample data not in well file }\end{array}$ & 0 & & 2 & $\begin{array}{l}\text { GRO: } \mathrm{ND}(\mathrm{n}=2) \\
\text { DRO: } \mathrm{ND}(\mathrm{n}=2) \\
\text { BTEX: } \mathrm{ND}(\mathrm{n}=2) \\
\text { Sample data not in well file }\end{array}$ & Qa & $\begin{array}{l}\text { PGDW30 } \\
(79 \mathrm{~m}, 273 \mathrm{~m}, \mathrm{~N}) \\
\text { PGDW49 } \\
(15 \mathrm{~m}, 335 \mathrm{~m}, \mathrm{E})\end{array}$ & Yes & No & Yes & $\begin{array}{l}\text { Well completed in 1973. P\&A in 1981. Invert mud } \\
\text { contained up to } 78 \% \text { oil while drilling. In } 1973 \text { and } \\
1979, \text { hydraulic fracturing with } \mathrm{KCl} \text { water, gelled } \mathrm{KCl}, \\
\text { solvents, surfactants, and } \mathrm{N}_{2} \text { foam. No description of } \\
\text { where flowback and production fluids were disposed. } \\
\text { P\&A due to parted casing and water production. No } \\
\text { production record. }\end{array}$ \\
\hline $41 \mathrm{X}-2$ & Invert & Yes $^{U}$ & Yes & $?$ & 6 & TPH: $1190(\mathrm{n}=2)$ & 0 & 2,500 & 0 & Not Sampled & Qa & None & No & Yes & No & $\begin{array}{l}\text { Invert mud up to } 76 \% \text { oil. No documented stimulation } \\
\text { or gas production. Sundry notice for offsite disposal of } \\
\text { production fluids in Jan } 1998 \text {. }\end{array}$ \\
\hline $31 \mathrm{X}-3$ & Invert & $\mathrm{Yes}^{\mathrm{U}}$ & Yes & $?$ & 17 & $\begin{array}{l}\text { GRO: } 66(\mathrm{n}=24) \\
\text { DRO: } 1200(\mathrm{n}=24) \\
\text { Benzene: } \mathrm{ND}(\mathrm{n}=18) \\
\text { Toluene: } \mathrm{ND}(\mathrm{n}=18) \\
\text { Ethylbenzene: } \mathrm{ND}(\mathrm{n}=18) \\
\text { Xylenes: } \mathrm{ND}(\mathrm{n}=18) \\
\text { Naphthalene: } 0.34(\mathrm{n}=1)\end{array}$ & $\sim 4,392$ & 1,000 & 5 & $\begin{array}{l}\text { GRO: } 300(\mathrm{n}=5) \\
\text { DRO: } 2,700(\mathrm{n}=5) \\
\text { Benzene: } \mathrm{ND}(\mathrm{n}=5) \\
\text { Toluene: } \mathrm{ND}(\mathrm{n}=5) \\
\text { Ethylbenzene: } 2.4(\mathrm{n}=5) \\
\text { Xylenes: } \mathrm{ND}(\mathrm{n}=5) \\
\text { Naphthalene: } \mathrm{ND}(\mathrm{n}=1) \\
\end{array}$ & Qa & $\begin{array}{l}\text { P197335.0W } \\
(?, 301 \mathrm{~m}, \mathrm{NW}) \\
\text { P19736.0W } \\
(?, 304 \mathrm{~m}, \mathrm{NW})\end{array}$ & No & Yes & No & $\begin{array}{l}\text { Well completed in } 1973 \text {. No documented stimulation } \\
\text { until } 2001 \text {. Production record from } 1983 \text { with } 29 \text { bbls } \\
\text { in } 1986 \text { and } 1987 \text {. No description of where produced } \\
\text { water was disposed. Increased to } 1,215 \text { bbls after } 2004 \text {. } \\
\text { Sundry notice for offsite disposal of production fluids } \\
\text { in Jan } 1998 \text {. }\end{array}$ \\
\hline $42 \mathrm{X}-11$ & Invert & Yes $^{\mathrm{U}}$ & Yes & $?$ & 8 & $\begin{array}{l}\text { GRO: } 580(\mathrm{n}=11) \\
\text { DRO: } 450(\mathrm{n}=11) \\
\text { Benzene: } \mathrm{ND}(\mathrm{n}=11) \\
\text { Ethylbenzene: } 0.52(\mathrm{n}=11) \\
\text { Toluene: } \mathrm{ND}(\mathrm{n}=11) \\
\text { Xylenes: } 1.1(\mathrm{n}=11) \\
\text { Naphthalene: } 0.58(\mathrm{n}=2)\end{array}$ & $\sim 900$ & 1,000 & 7 & $\begin{array}{l}\text { GRO: } 50,000(\mathrm{n}=59) \\
\text { DRO: } 31,000(\mathrm{n}=59) \\
\text { Benzene: } 240(\mathrm{n}=55) \\
\text { Toluene: } 25(\mathrm{n}=55) \\
\text { Ethylbenzene: } 240(\mathrm{n}=55) \\
\text { Xylenes: } 620(\mathrm{n}=55) \\
\text { Naphthalene: } 236(\mathrm{n}=32)\end{array}$ & Qa & None & No & VRP & VRP & $\begin{array}{l}\text { Well completed in 1974. No documented stimulation } \\
\text { but completion record not reviewed by WOGCC } \\
\text { (2014). Production record from } 1983 \text { with } 9 \text { bbls } \\
\text { produced water in } 1986 \text {. No description of where } \\
\text { produced water was disposed. Increased to } 222 \text { bbls } \\
\text { after 2004. Sundry notice for offsite disposal of } \\
\text { production fluids in Jan } 1998 \text {. Encana evaluating } \\
\text { alternatives for final remedy and ground water } \\
\text { monitoring. }\end{array}$ \\
\hline $42 \mathrm{X}-12$ & Invert & $\mathrm{Yes}^{\mathrm{U}}$ & Yes & Yes & 5 & $\begin{array}{l}\text { GRO: ND }(\mathrm{n}=5) \\
\text { DRO: } 1,100(\mathrm{n}=5) \\
\text { BTEX: ND }(\mathrm{n}=1)\end{array}$ & $\sim 280$ & 2,500 & 0 & Not sampled & Qa & None & No & Yes & No & $\begin{array}{l}\text { Completed in 1974. Acid stimulation in 1974. No } \\
\text { description of where flowback and production fluids } \\
\text { were disposed. Production record from 1983. No } \\
\text { produced water until } 2004 \text { after which } 295 \text { bbls } \\
\text { produced. Sundry notice for of ofsite disposal of } \\
\text { production fluids in Jan } 1998 \text {. }\end{array}$ \\
\hline $31 \mathrm{X}-14$ & Invert & Yes $^{\mathrm{U}}$ & Yes & $?$ & 6 & $\begin{array}{l}\text { TPH: ND (n=5) } \\
\text { (confirmation sample) }\end{array}$ & $\sim 260$ & 2,500 & 0 & Not sampled & Qa & $\begin{array}{l}\text { PGDW34 } \\
(31 \mathrm{~m}, 518 \mathrm{~m}, \mathrm{~S}) \\
\text { P44255.0W } \\
(69 \mathrm{~m}, 148 \mathrm{~m}, \mathrm{~N}) \\
\text { P41320.0W } \\
(31 \mathrm{~m}, 148 \mathrm{~m}, \mathrm{~N}) \\
\text { P99671.0W } \\
(17 \mathrm{~m}, 148, \mathrm{~N}) \\
\end{array}$ & No & $?$ & ? & $\begin{array}{l}\text { Well completion in } 1974 \text {. No documented stimulation. } \\
\text { Production records from } 1983 \text { with } 6,546 \text { bbls water in } \\
\text { Feb } 1985,4 \text { bbls in } 1986 \text { and } 4 \text { bbls in } 1988 \text {. No } \\
\text { description of where produced water was disposed. } \\
\text { After } 2007,7,691 \text { cumulative bbls water. Sundry } \\
\text { notice for offsite disposal of production fluids in Jan } \\
1998 \text {. Further investigation recommend by WOGCC } \\
\text { pending review of pre-excavation samples. }\end{array}$ \\
\hline CCD & Invert & $\mathrm{Yes}^{\mathrm{U}}$ & No & No & 0 & Not sampled & 0 & & 0 & Not sampled & Qa & $\begin{array}{l}\text { Converted to } \\
9441.0 \mathrm{P} \\
(177 \mathrm{~m}, 45 \mathrm{~m}, \mathrm{~W}) \\
\end{array}$ & No & $\mathrm{NI}$ & No & $\begin{array}{l}\text { Well completion and P\&A after completion in } 1974 . \\
\text { Stimulation or production unlikely. No evidence of } \\
\text { converted well sampled. }\end{array}$ \\
\hline TR1-22 & Invert & Yes $^{\mathrm{U}}$ & Yes & Yes & 0 & Not sampled & 0 & & 0 & Not sampled & Qa & $\begin{array}{l}\text { PGDW48 } \\
(116 \mathrm{~m}, 397 \mathrm{~m}, \mathrm{NW}) \\
\text { PGDW35 } \\
(88 \mathrm{~m}, 449 \mathrm{~m}, \mathrm{NW})\end{array}$ & No & $\mathrm{NI}$ & Yes & $\begin{array}{l}\text { Well completion in 1976. Acid stimulation and } \\
\text { hydraulic fracturing in 1980. No description of where } \\
\text { flowback and production fluids were disposed. P\&A } \\
\text { 1986. Well production file starts in } 1978 \text { with } 5,281 \\
\text { bbls produced water between } 1978 \text { and } 1986\end{array}$ \\
\hline $1-21$ & Invert & Yes $^{U}$ & No & No & 0 & Not sampled & 0 & & 0 & Not sampled & Qa & None & No & NI & No & $\begin{array}{l}\text { Well completion and P\&A in 1976. Stimulation or } \\
\text { production unlikely. Investigation recommended by } \\
\text { WOGCC pending confirmation of nearby domestic } \\
\text { wells. No evidence of former well pad - no further } \\
\text { investigation recommended (Encana 2015) }\end{array}$ \\
\hline
\end{tabular}




\begin{tabular}{|c|c|c|c|c|c|c|c|c|c|c|c|c|c|c|c|c|}
\hline 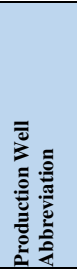 & 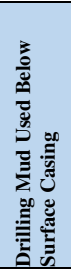 & 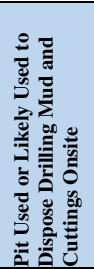 & 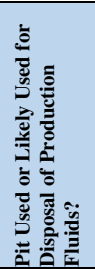 & 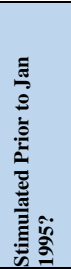 & 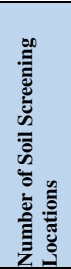 & 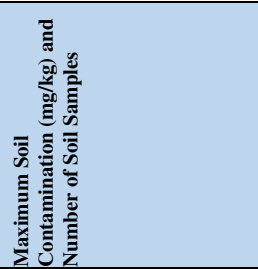 & 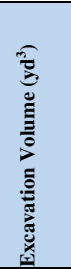 & 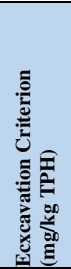 & 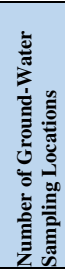 & 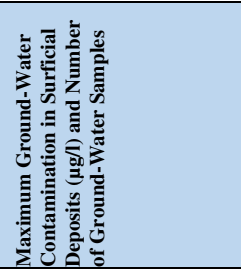 & 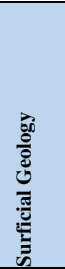 & 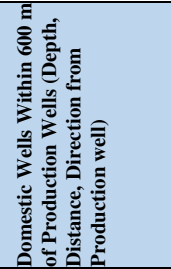 & 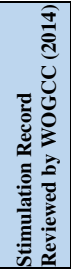 & 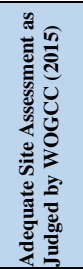 & 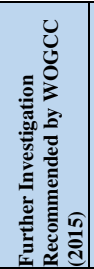 & 嶽 \\
\hline TP 1 & Invert & $\mathrm{Yes}^{\mathrm{U}}$ & Yes & $?$ & 0 & Not sampled & 0 & & 0 & Not sampled & Qa & None & No & NI & No & $\begin{array}{l}\text { Well completion in 1976. P\&A date unknown. No } \\
\text { record of stimulation. Well production file starts in } \\
1978 \text { with } 5,607 \text { bbls produced water from } 1978 \text { to } \\
1980 \text {. No description of where produced water was } \\
\text { disposed. }\end{array}$ \\
\hline $1-31$ & Invert & Yes $^{\mathrm{U}}$ & Yes & $?$ & 0 & Not sampled & 0 & & 0 & Not sampled & $?$ & $\begin{array}{l}\text { PGDW06 } \\
(116 \mathrm{~m}, 341 \mathrm{~m}, \mathrm{SE}) \\
\text { PGDW12 } \\
(116 \mathrm{~m}, 491 \mathrm{~m}, \mathrm{NW}) \\
\end{array}$ & No & NI & No & $\begin{array}{l}\text { Well completion in 1976. P\&A in 1977. Well } \\
\text { completion report not available. }\end{array}$ \\
\hline TU 1 & Invert & Yes $^{U}$ & Yes & $?$ & 0 & Not sampled & 0 & & 0 & Not sampled & Qa & None & No & NI & No & $\begin{array}{l}\text { Completed in 1976. P\&A in 1982. No record of } \\
\text { stimulation but completion record not reviewed by } \\
\text { WOGCC (2014). Well production file starts in } 1978 \\
\text { with } 220 \text { bbls produced water from } 1978 \text { to } 1982 \text {. No } \\
\text { description of where produced water was disposed. } \\
\text { Encana (2015) states that there is no evidence of } \\
\text { former well pad. }\end{array}$ \\
\hline $4-8$ & Invert & Yes $^{\mathrm{U}}$ & Yes & Yes & 6 & $\begin{array}{l}\text { GRO: } \mathrm{ND}(\mathrm{n}=6) \\
\text { DRO: } 32(\mathrm{n}=6) \\
\text { Naphthalene: } 5.3(\mathrm{n}=3)\end{array}$ & 0 & 1,000 & 2 & $\begin{array}{l}\text { GRO: } 5,200(\mathrm{n}=2) \\
\text { DRO: } 13,000(\mathrm{n}=2) \\
\text { Benzene: } 110(\mathrm{n}=2) \\
\text { Toluene: } 250(\mathrm{n}=2) \\
\text { Ethylbenzene: } 240(\mathrm{n}=2) \\
\text { Xylenes: } 1,200(\mathrm{n}=2) \\
\text { Naphthalene: } 72(\mathrm{n}=2) \\
\end{array}$ & Qa & $\begin{array}{l}\text { PGDW41 } \\
\text { (21m,421m,SE) } \\
\text { P66345.0W } \\
(21 \mathrm{~m}, 407 \mathrm{~m}, \mathrm{SE})\end{array}$ & Yes & VRP & VRP & $\begin{array}{l}\text { Well completed in Mar 1977. Hydraulic fracturing in } \\
1977 \text { with "gel water" and } \mathrm{N}_{2} \text {. No description of where } \\
\text { flowback and production fluids were disposed. } \\
\text { Production file starts in Jan } 1978 \text { with } 36,978 \text { bbls } \\
\text { water from Jan } 1978 \text { to Dec } 1993 \text {. No description of } \\
\text { where produced water was disposed. Encana (2015) } \\
\text { drafting report for site closure. }\end{array}$ \\
\hline 34-13 & Invert & Yes $^{\mathrm{U}}$ & Yes & $?$ & 0 & Not sampled & 0 & & 0 & Not sampled & $?$ & $\begin{array}{l}\text { P197335.0W } \\
(?, 632 \mathrm{~m}, \mathrm{SE}) \\
\text { P197336.0W } \\
(?, 626 \mathrm{~m}, \mathrm{SE})\end{array}$ & No & NI & No & $\begin{array}{l}\text { Well completion in } 1977 \text {. P\&A date unknown. Well } \\
\text { production file starts in } 1978 \text { with } 290 \text { bbls produced } \\
\text { water from } 1978 \text { to } 1979 \text {. No description of where } \\
\text { produced water was disposed. }\end{array}$ \\
\hline T24-11 & Invert & Yes $^{\mathrm{U}}$ & No & No & 0 & Not sampled & 0 & & 0 & Not sampled & Twdr & None & No & NI & No & $\begin{array}{l}\text { Well completed and P\&A in 1978. Stimulation or } \\
\text { production unlikely. }\end{array}$ \\
\hline $24-4$ & Invert & $\mathrm{Yes}^{\mathrm{U}}$ & No & No & 0 & Not sampled & 0 & & 0 & Not sampled & Qa & None & No & NI & No & $\begin{array}{l}\text { Well completed and P\&A in 1978. Stimulation or } \\
\text { production unlikely. }\end{array}$ \\
\hline $44-15$ & Invert & $\mathrm{Yes}^{\mathrm{U}}$ & Yes & Yes & 6 & TPH: $486(\mathrm{n}=1)$ & 0 & 5,500 & 0 & Not sampled & Qa & $\begin{array}{l}\text { PGDW48 } \\
(116 \mathrm{~m}, 329 \mathrm{~m}, \mathrm{SW}) \\
\text { PGDW35 } \\
(88 \mathrm{~m}, 374 \mathrm{~m}, \mathrm{~W}) \\
\text { P108128.0W } \\
(116 \mathrm{~m}, 115 \mathrm{~m}, \mathrm{~W}) \\
\text { P146856.0W } \\
(116 \mathrm{~m}, 115 \mathrm{~m}, \mathrm{~W}) \\
\end{array}$ & No & No & Yes & $\begin{array}{l}\text { Well completed in 1978. Acid stimulation in } 1976 \text { and } \\
1978 \text { with } \mathrm{HCl} \text { and } \mathrm{KCl} \text { solutions. No description of } \\
\text { where flowback and production fluids were disposed. } \\
\text { Production file starts in } 1983 \text {. No produced water } \\
\text { production from } 1983 \text { to } 2005 \text {. Sundry notice for } \\
\text { offsite disposal of production fluids in Jan } 1998 \text {. }\end{array}$ \\
\hline $21-11$ & Invert & Yes $^{\mathrm{U}}$ & Yes & Yes & $?$ & $\begin{array}{l}\text { Sample results not available } \\
\text { in pit report (WOGCC 2015) } \\
\text { or online. }\end{array}$ & $\sim 100$ & 1,000 & $?$ & $\begin{array}{l}\text { Sample results not } \\
\text { available in pit report } \\
\text { (WOGCC 2015) or online. }\end{array}$ & Qa & $\begin{array}{l}\text { PGDW26 } \\
(20 \mathrm{~m}, 229 \mathrm{~m}, \mathrm{~W})\end{array}$ & No & VRP & VRP & $\begin{array}{l}\text { Well completed 1n 1979. Acid stimulation in } 1979 \\
\text { with HCl solution and additives. Hydraulic fracturing } \\
\text { in } 1979 \text { with "YE4P5D" "fluid. No description of where } \\
\text { flowback and production fluids were disposed. } \\
\text { Production file starts in } 1983 \text {. No produced water until } \\
\text { 2005. Sundry notice for offsite disposal of production } \\
\text { fluids in Jan 1998. Soil and ground water sampling } \\
\text { data not available. Ground water and additional soil } \\
\text { data were collected in October 2014. Encana (2015) is } \\
\text { preparing a Supplemental Site Characterization report } \\
\text { to recommend site closure. }\end{array}$ \\
\hline
\end{tabular}




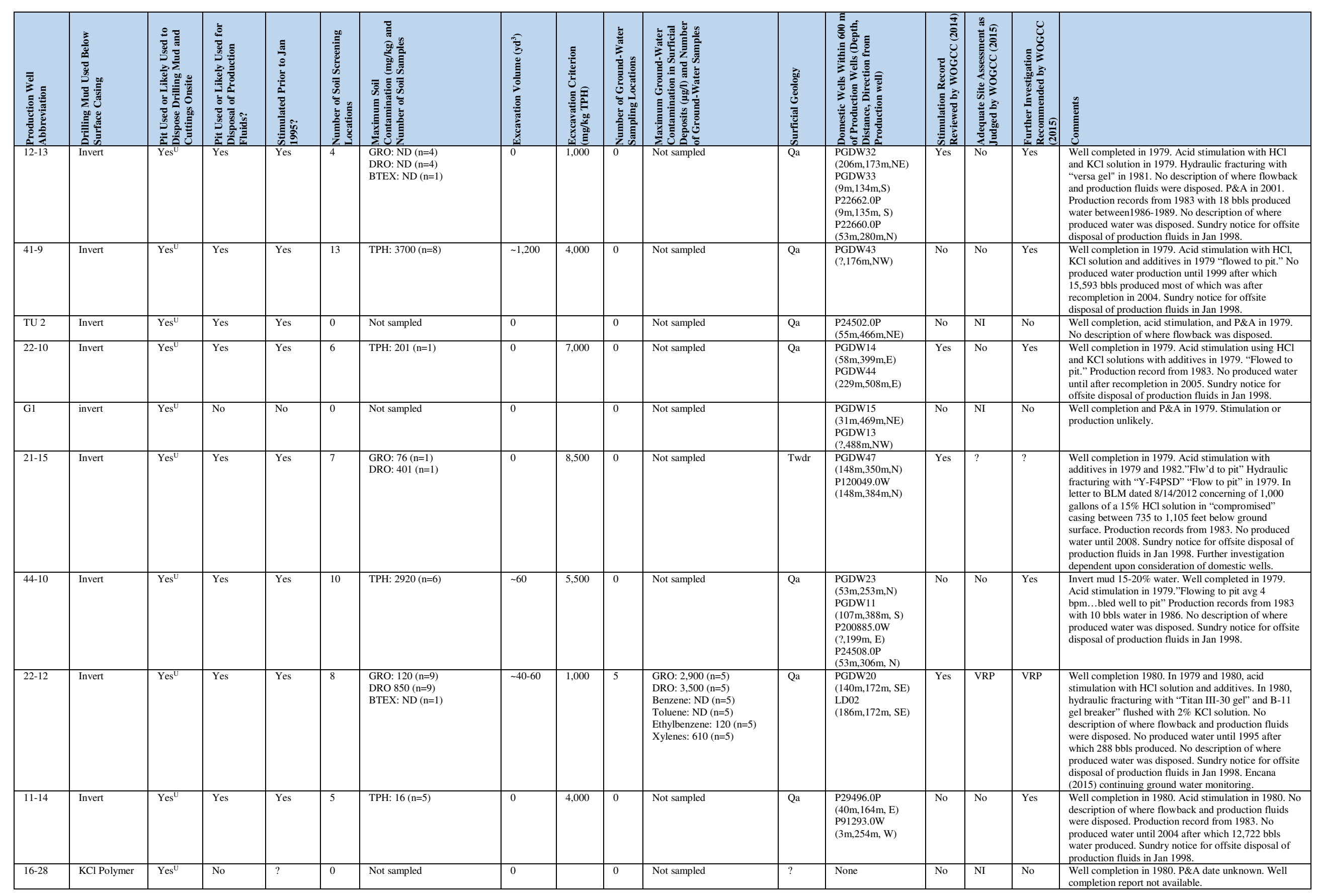




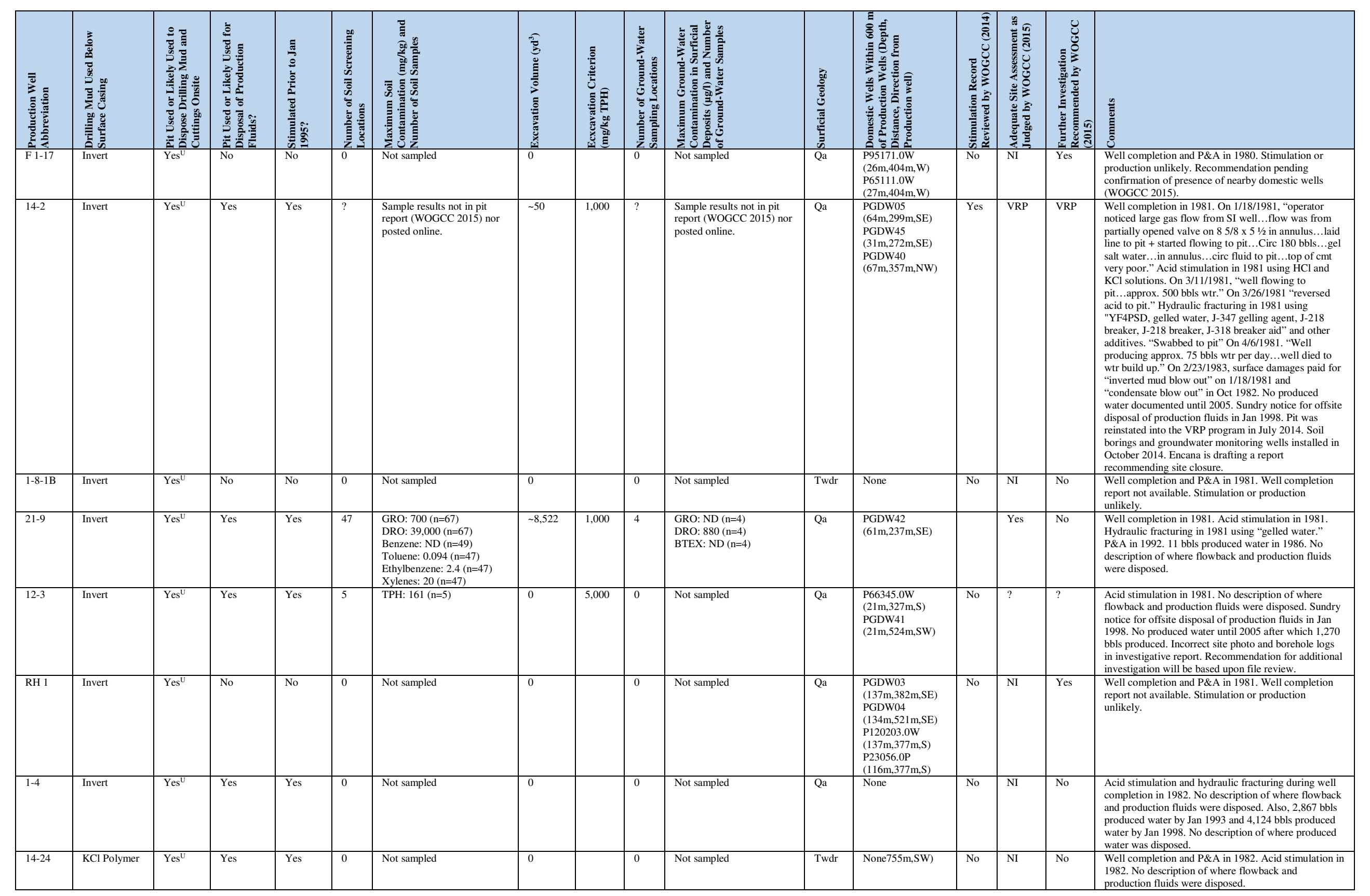




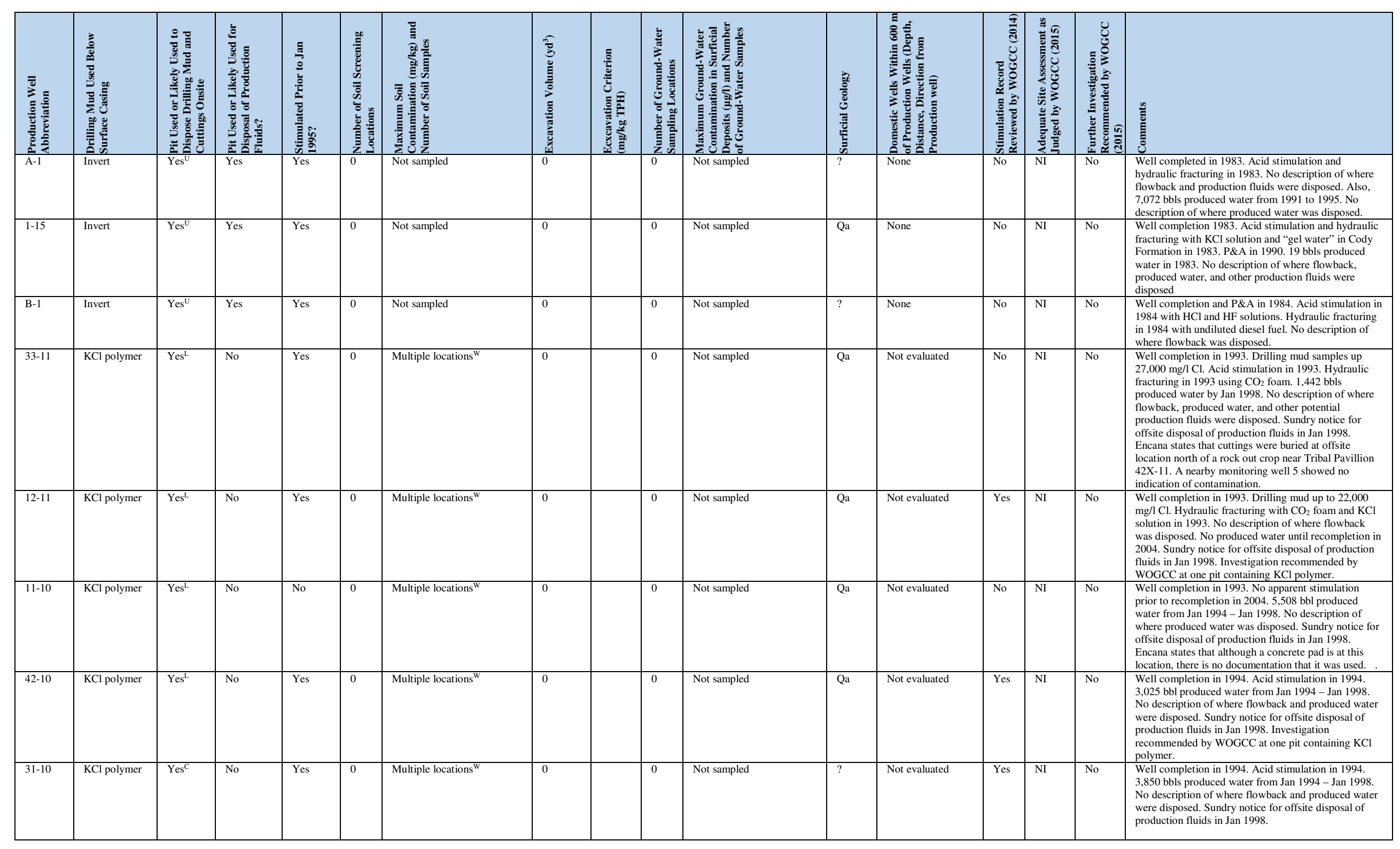




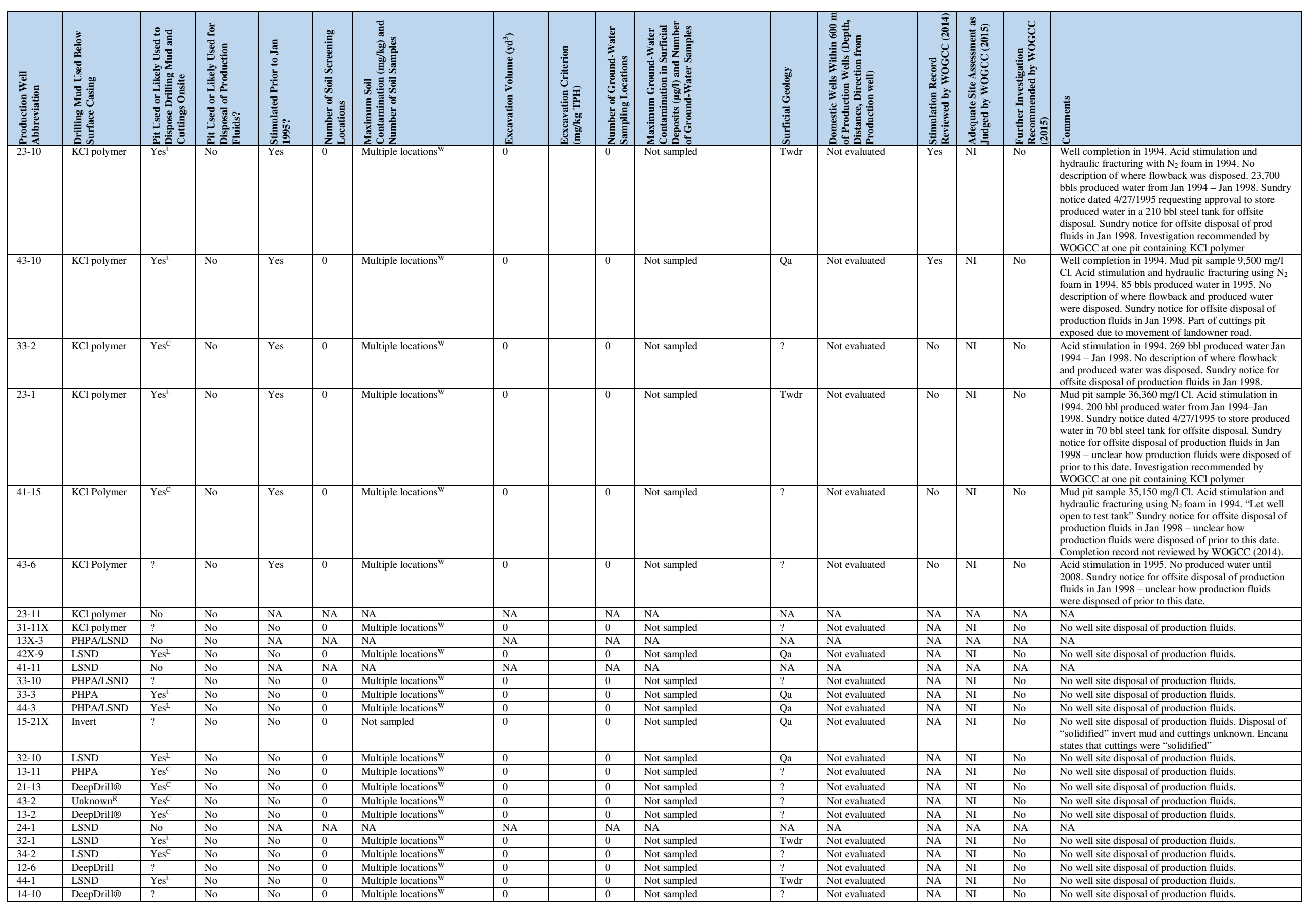




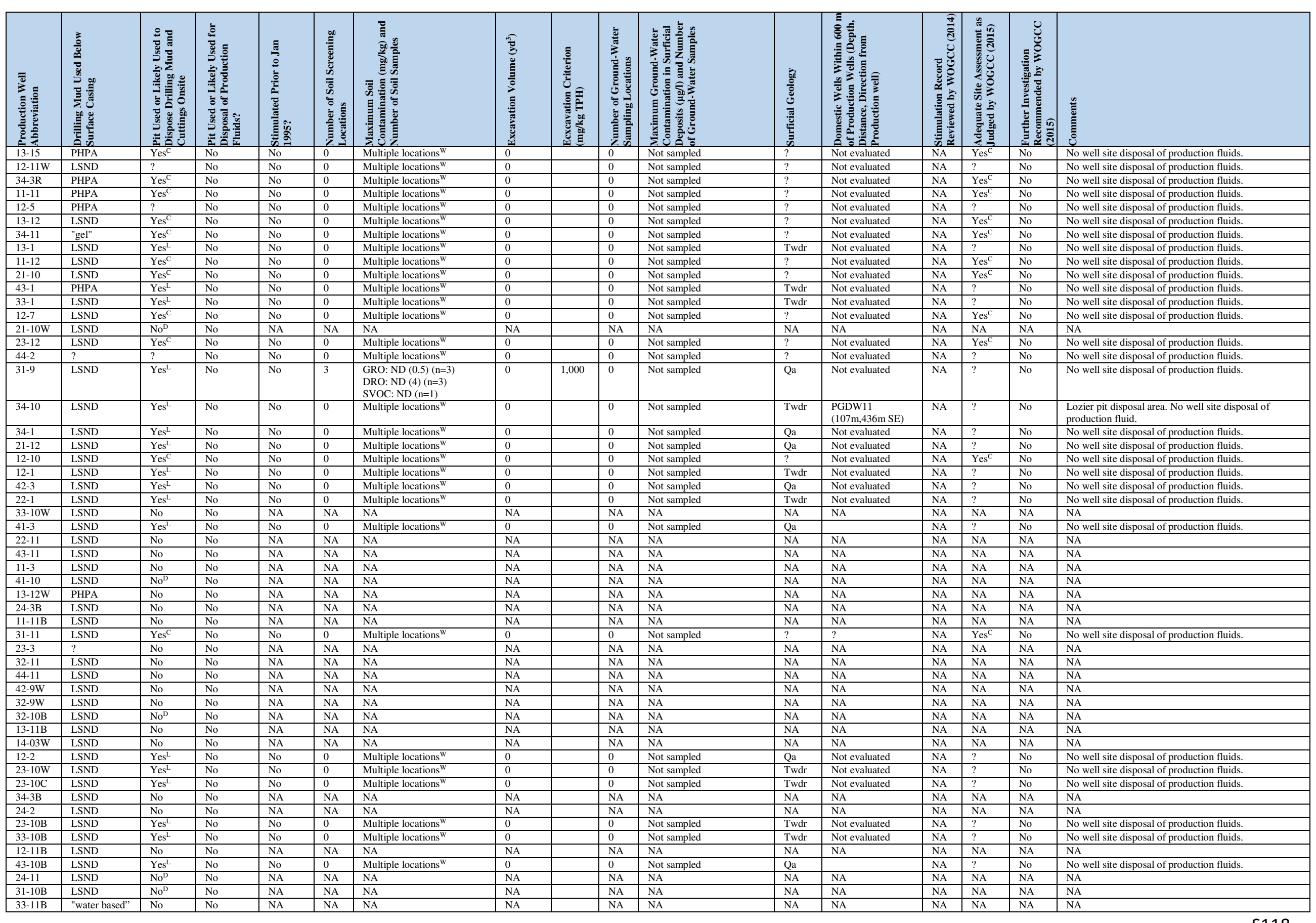




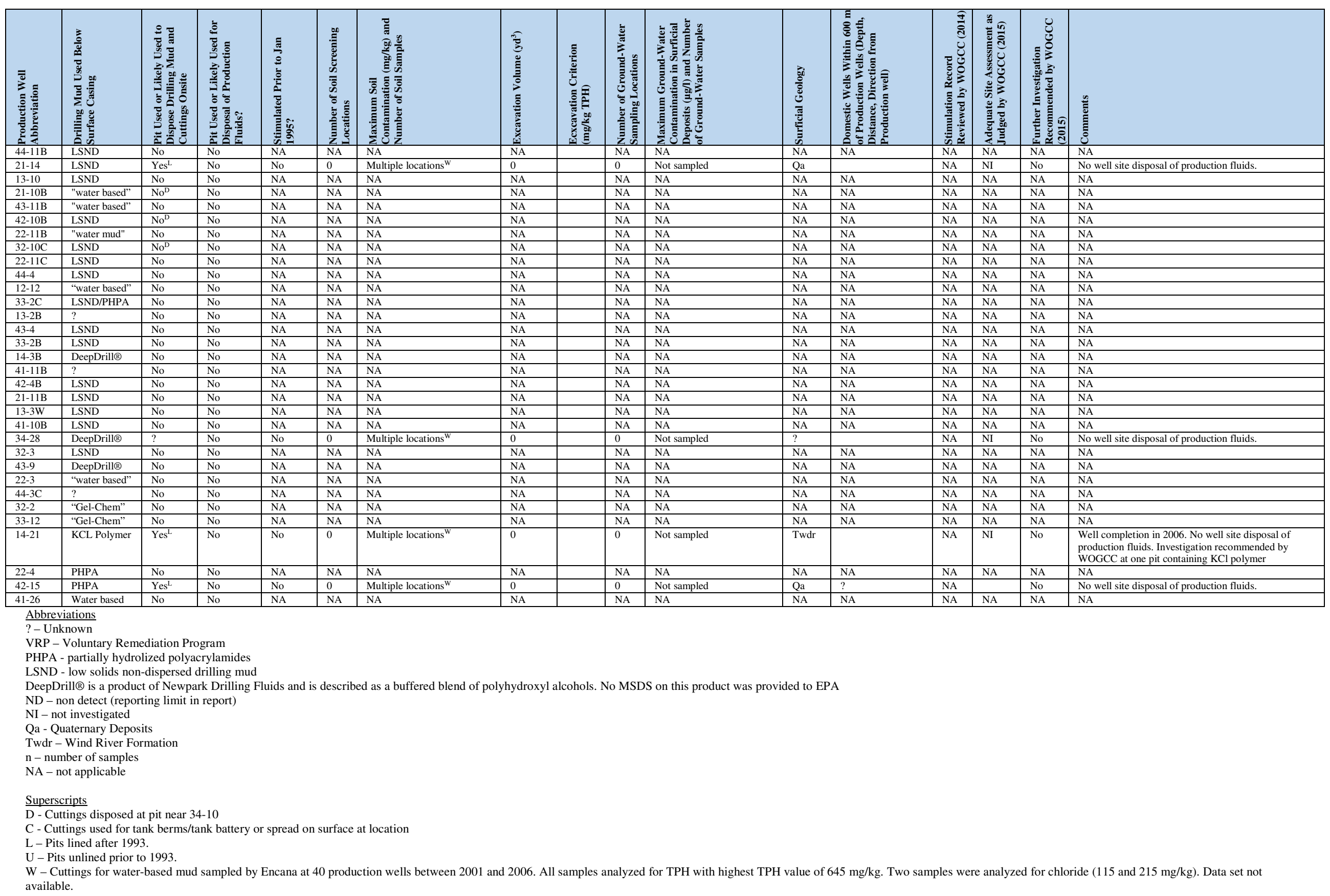

LSND - low solids non-dispersed drilling mud

DeepDrill@ is a product of Newpark Drilling
ND - non detect (reporting limit in report)

NI - not investigated
N

Qa - Quaternary Deposits

Twd - Wha River Formatio

NA - not applicable

$\underline{\text { Superscripts }}$

D - Cuttings disposed at pit near 34-10

C - Cuttings used for tank berms/tank battery or spread on surface at location

L-Pits lined after 1993.

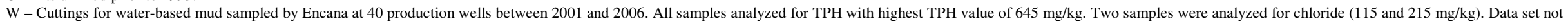
available. 
Table SI F3. Summary of detection of light hydrocarbons and organic compounds in domestic wells less than and greater than $600 \mathrm{~m}$ from unlined pits. Concentrations in $\mu \mathrm{g} / \mathrm{L}$ unless otherwise indicated.

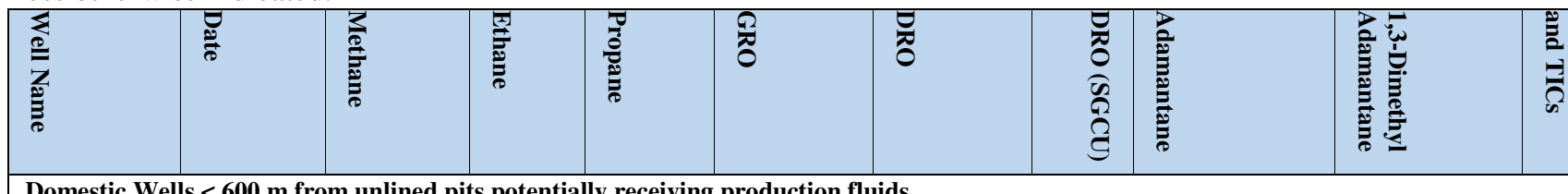

\section{Domestic Wells $<\mathbf{6 0 0} \mathbf{m}$ from unlined pits potentially receiving production fluids}

\begin{tabular}{|l|l|l|l|l|l|l|l|l}
\hline PGDW05 & Mar-09 & 16.6 & NA & NA & NA & 105 & NA & NA \\
\hline
\end{tabular}

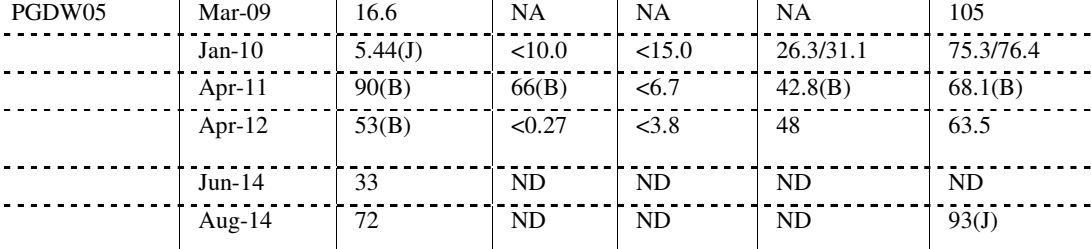

\begin{tabular}{|c|c|c|c|}
\hline NA & NA & NA & \\
\hline $\mathrm{NA}$ & $<0.20(\mathrm{~J}) /<0.20(\mathrm{~J})$ & $1.7 \overline{4}(\mathrm{~J}) / 1.71(\mathrm{~J})$ & 2-methyladamantane (TIC) $-2.5 \mu \mathrm{g} / \mathrm{L}^{-}$ \\
\hline $\mathrm{NA}$ & $0.12(\mathrm{~J})$ & $1.35(\mathrm{~J})$ & 1,3 -dimethyladamantane + isomers (TIC) $-8.6 \mu \mathrm{g} / \mathrm{L}$ \\
\hline$\overline{N A}$ & $<0 . \overline{25}$ & $2.82^{-}$ & $\begin{array}{l}\text { 2-methyladamantane (TIC) }-3.39 \mu \mathrm{g} / \mathrm{L} \\
\text { cis-1,4-dimethyladamantane (TIC) }-1.37 \mu \mathrm{g} / \mathrm{L}\end{array}$ \\
\hline $26(\mathrm{~J})$ & $\mathrm{NA}$ & $\overline{\mathrm{NA}} \overline{-}$ & isopropanol $-8.1(\mathrm{~J}) \mu \mathrm{g} / \mathrm{L}$ \\
\hline $31(\mathrm{~J})$ & $\mathrm{NA}$ & $\overline{\mathrm{NA}} \overline{-}$ & \\
\hline NA & NA & NA & \\
\hline NA & NA & NA & \\
\hline
\end{tabular}

\section{PGDW11}

\begin{tabular}{l|l|l|l} 
Mar-09 & NA \\
\hline Mar-09 -09 & NA
\end{tabular}

\begin{tabular}{l|l|} 
NA & NA \\
\hline NA & NA
\end{tabular}

\begin{tabular}{|l|l|}
\hline NA & NA \\
\hline NA & NA \\
\hline
\end{tabular}

\begin{tabular}{|c|c|c|}
\hline NA & NA & $\mathrm{N}$ \\
\hline
\end{tabular}

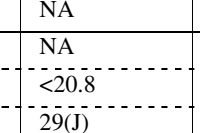

PGDW14

\begin{tabular}{|c|c|c|c|c|}
\hline Mar-09 & NA & NA & NA & NA \\
\hline Apr-11 & $23(\mathrm{~B})$ & $<4.8$ & $<6.7$ & $<20.0$ \\
\hline Jun-14 & $0 . \overline{4}(\bar{J})$ & $\mathrm{ND}$ & ND & $\mathrm{ND}$ \\
\hline
\end{tabular}

\section{PGDW20}

\begin{tabular}{l|l}
\hline Aug-14 & $0.2(\mathrm{~J})$ \\
\hline Mar-09 & 137
\end{tabular}

\begin{tabular}{|l|l|l|}
\hline $\mathrm{ND}$ & $\mathrm{ND}$ \\
\hline $\mathrm{ND}$ & $\mathrm{ND}$ \\
\hline $\mathrm{NA}$ & $\mathrm{NA}$ \\
\hline
\end{tabular}

NA

\begin{tabular}{|c|c|c|}
\hline PGDW20 & Mar-09 & 137 \\
\hline & Jan-10 & 172 \\
\hline & Oct- 10 & $190(\mathrm{~B})$ \\
\hline & Apr-11 & $137(\mathrm{~B})$ \\
\hline & Apr-12 & $111 / 108$ \\
\hline & Jun-14 & $110^{-}$ \\
\hline & Âug-14 & $130^{-}$ \\
\hline PGDW20 & Mar-09 & NA \\
\hline
\end{tabular}

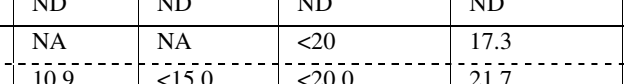

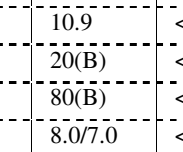

\begin{tabular}{|c|c|c|c|c|c|c|}
\hline & Apr-11 & $137(\mathrm{~B})$ & $80(B)$ & $<6.7$ & $<20.0$ & $<21.9$ \\
\hline & Apr-12- & $111 / 108$ & $8.0 / 7.0$ & $<3.8 \overline{<}<3.8$ & $<20.0 /<20.0$ & $<20.0 /<20.0$ \\
\hline & Jun-14 & 110 & 7 & $0 . \overline{9}(\mathrm{~J})$ & $\mathrm{ND}$ & $\mathrm{ND}$ \\
\hline & Augu-14 & 130 & 8 & $0.24(\mathrm{~J})$ & $\mathrm{ND}$ & $4 \overline{3}(\mathrm{~J})$ \\
\hline $\begin{array}{l}\text { PGDW20 } \\
\text { (carbon trap) }\end{array}$ & $\begin{array}{l}\text { Mar-09 } \\
\end{array}$ & NA & NA & NA & NA & 377 \\
\hline $\begin{array}{l}\text { PGDW20 } \\
\text { (RO filter) }\end{array}$ & Jan-10 & NA & NA & NA & NA & $\begin{array}{l}752,000 \\
\mu \mathrm{g} / \mathrm{kg}\end{array}$ \\
\hline
\end{tabular}

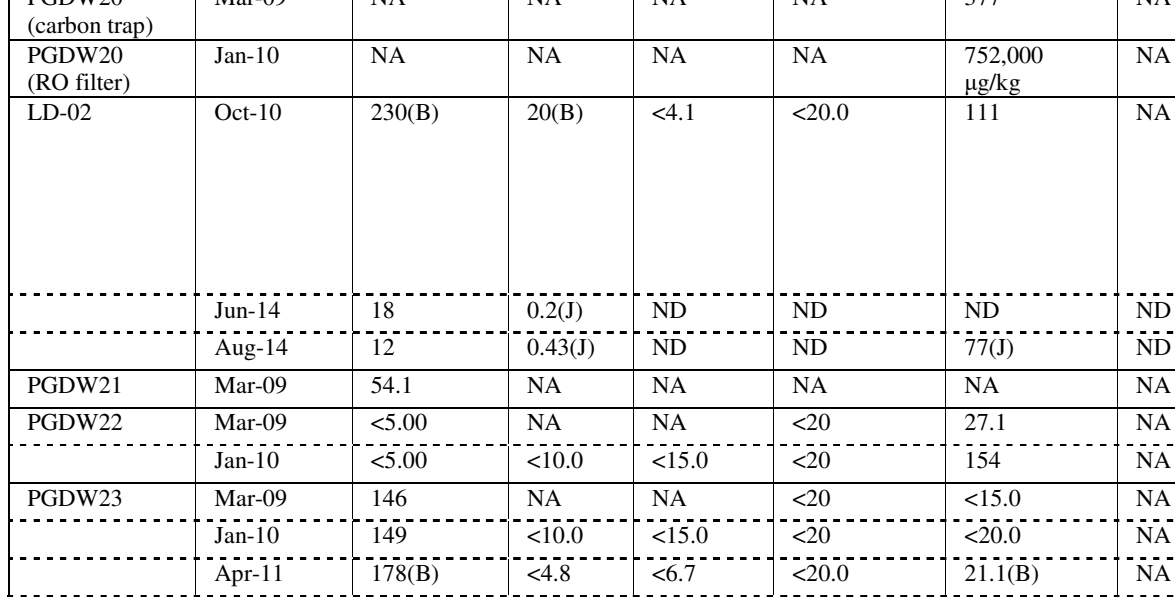

\begin{tabular}{|l|l|}
$\mathrm{NA}$ & $\mathrm{NA}$ \\
\hline$<0.10$ & $<0.10$ \\
\hline $\mathrm{NA}-0.0$
\end{tabular}

isopropanol $-6.0(\mathrm{~J}) \mu \mathrm{g} / \mathrm{L}$

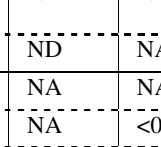

NA

$\mathrm{NA}$ naphthalene $-0.6(\mathrm{~J}) \mu \mathrm{g} / \mathrm{L}$

\begin{tabular}{|c|c|c|c|}
\hline 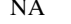 & NA & NA & \\
\hline NA- & $<0.20(\mathrm{~J})$ & $-<0.20(\mathrm{~J})$ & 2-methyladamantane (TIC) $-0.49 \mu \mathrm{g} / \mathrm{L}$ \\
\hline $\mathrm{NA}$ & $1.35(\mathrm{~J}) / 1.37(\mathrm{~J})$ & $0.14(\mathrm{~J}) / 0.14(\mathrm{~J})$ & 2 -methyladamantane (TIC) $-1.03 / 0.43 \mu \mathrm{g} / \mathrm{L}$ \\
\hline $\mathrm{NA}$ & $<0.10$ & $<0.10$ & 1 -isobutyladamantane (TIC) $-0.27 \mu \mathrm{g} / \mathrm{L}$ \\
\hline $\mathrm{NA}$ & $<-\overline{c o s}$ & $-<0.25 /<0.25$ & 2-methyladamantane (TIC) $-0.67 \mu \mathrm{g} / \mathrm{L}$ \\
\hline $\mathrm{ND}$ & $\mathrm{NA}$ & $\mathrm{NA}$ & acetone $-1.8(\mathrm{~J}) \mu \mathrm{g} / \mathrm{L}^{-}$ \\
\hline $\mathrm{ND}$ & NA- & $\mathrm{NA}$ & \\
\hline NA & NA & NA & \\
\hline NA & $420 \mu \mathrm{g} / \mathrm{kg}$ & $2960 \mu \mathrm{g} / \mathrm{kg}$ & 2-methyladamantane (TIC) - $9400 \mu \mathrm{g} / \mathrm{kg}$ \\
\hline NA & $0.510(\mathrm{~J})$ & $<0.25(\mathrm{~J})$ & $\begin{array}{l}\text { benzene }-0.060(\mathrm{~J}) \mu \mathrm{g} / \mathrm{L} \\
\text { ethylbenzene }-0.240(\mathrm{~J}) \mu \mathrm{g} / \mathrm{L} \\
\text { o-xylene }-0.260(\mathrm{~J}) \mu \mathrm{g} / \mathrm{L} \\
1,2,4 \text {-trimethylbenzene }-0.200(\mathrm{~J}) \mu \mathrm{g} / \mathrm{L} \\
1,3,5 \text {-trimethylbenzene }-0.210(\mathrm{~J}) \mu \mathrm{g} / \mathrm{L} \\
\text { isopropylbenzene }-0.350(\mathrm{~J}) \mu \mathrm{g} / \mathrm{L} \\
\text { methyl tert-butyl ether }-0.140(\mathrm{~J}) \mu \mathrm{g} / \mathrm{L} \text {. }\end{array}$ \\
\hline $\mathrm{ND}$ & $\mathrm{NA}$ & $\mathrm{NA}$ & \\
\hline $\mathrm{ND}$ & NA- & $-\overline{N A}$ & pyruvic Acid $-2060 \mu \mathrm{g} / \mathrm{L}$ \\
\hline NA & NA & NA & \\
\hline NA & $<0.20(\mathrm{~J})$ & $<0.20(\mathrm{~J})$ & \\
\hline $\mathrm{NA}$ & $<0.10$ & $<0.10$ & \\
\hline NA & NA & NA & \\
\hline $\mathrm{NA}$ & $<0.20(\mathrm{~J})$ & $-80.20(\mathrm{~J})$ & \\
\hline 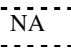 & $<0.10^{-}$ & $<0.10$ & \\
\hline
\end{tabular}

\begin{tabular}{|c|}
\hline $\mathrm{EPA}^{29}$ \\
\hline EPA $^{30}$ \\
\hline EPA 27 \\
\hline $\mathrm{EPA}^{33}$ \\
\hline WYDEQ $^{34}$ \\
\hline WYDEQ $^{34}$ \\
\hline $\mathrm{EPA}^{29}$ \\
\hline $\mathrm{EPA}^{29}$ \\
\hline $\mathrm{EPA}^{29}$ \\
\hline $\mathrm{EPA}^{27}$ \\
\hline WYDEQ $^{34}$ \\
\hline WYDEQ $^{34}$ \\
\hline EPA $^{29}$ \\
\hline $\mathrm{EPA}^{30^{-}}$ \\
\hline $\mathrm{EPA}^{27}$ \\
\hline $\mathrm{EPA}^{27}$ \\
\hline $\mathrm{EPA}^{3}$ \\
\hline WYDEQ $^{34}$ \\
\hline WYDEQ $^{34}$ \\
\hline $\mathrm{EPA}^{29}$ \\
\hline $\mathrm{EPA}^{30}$ \\
\hline $\mathrm{EPA}^{27}$ \\
\hline WYDEQ $^{34}$ \\
\hline WYDEQ $^{34}$ \\
\hline $\mathrm{EPA}^{27}$ \\
\hline $\mathrm{EPA}^{29}$ \\
\hline $\mathrm{EPA}^{30^{-}}$ \\
\hline $\mathrm{EPA}^{29}$ \\
\hline $\mathrm{EPA}^{30}$ \\
\hline $\mathrm{EPA}^{27^{-}}$ \\
\hline
\end{tabular}




\begin{tabular}{|c|c|c|c|c|c|c|c|c|c|c|c|}
\hline 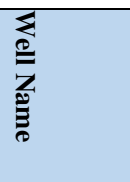 & $\begin{array}{l}\overline{\text { Fे }} \\
\text { है }\end{array}$ & 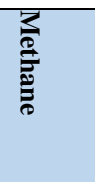 & 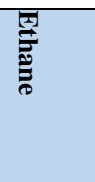 & 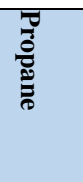 & $\stackrel{\Omega}{\tilde{0}}$ & 㱐 & 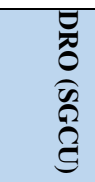 & 苞 & 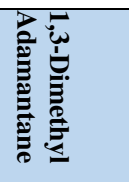 & 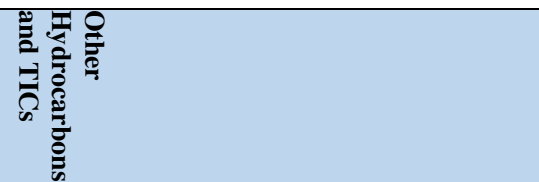 & 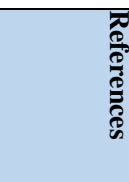 \\
\hline & Apr-12 & 226 & 19 & 11.4 & $<20.0$ & $<20.0$ & NA & $<0.25$ & $<0.25$ & & $\mathrm{EPA}^{33}$ \\
\hline & Jun-14 & 98 & $0.76(\mathrm{~J})$ & $\mathrm{ND}$ & ND & $21(\mathrm{~J})$ & $\mathrm{ND}$ & $\mathrm{NA}$ & $\mathrm{NA}$ & beta-BHC-0.06 (J) $\mu \mathrm{g} / \mathrm{L}$ & WYDEQ $^{34}$ \\
\hline & Āug-14 & 210 & $0.77(\mathrm{~J})$ & $\mathrm{ND}$ & ND & $\mathrm{ND}$ & ND & $\mathrm{NA}$ & $\mathrm{NA}$ & & WYDEQ $^{34}$ \\
\hline \multirow{2}{*}{ PGDW26 } & Mar-09 & $<5.00$ & NA & NA & NA & NA & NA & NA & NA & & $\mathrm{EPA}^{29}$ \\
\hline & Äpr-11 & $27(\mathrm{~B})$ & $<4.8$ & $<6.7^{--}$ & $<20.0$ & $47.2^{-}$ & $\mathrm{NA}^{-}$ & $<0.10$ & $<0.10^{--}$ & & $\mathrm{EPA}^{27^{-1}}$ \\
\hline \multirow[t]{7}{*}{ PGDW30 } & Mar-09 & 558 & NA & NA & NA & 46.8 & NA & NA & NA & & $\mathrm{EPA}^{29}$ \\
\hline & Jan-10 & 808 & $<10.0$ & $<15.0^{-}$ & $<20.0$ & 35.0 & $\mathrm{NA}$ & $<0.20(\mathrm{~J})$ & $1.81(\mathrm{~J})$ & $1,3,5$-trimethyladamantane (TIC) $-0.29 \mathrm{ug} / \mathrm{L}$ & $\mathrm{EPA}^{30^{-}}$ \\
\hline & Oct-10 - & $760(\mathrm{~B})$ & $<3.0$ & $<4.1^{--}$ & 29.4 & 32.7 & $\mathrm{NA}$ & $<0.25$ & 2.48 & & $\mathrm{EPA}^{27}$ \\
\hline & Apr-11 & 644 & $76(\mathrm{~B})$ & $-86.7^{-}$ & $2 \overline{1} \cdot \overline{6}(\mathrm{~B})$ & 37.0 & NA- & $<0.10$ & $0 . \overline{98}$ & 1,3 -dimethyladamantane + isomers (TIC) $-3.96 \mu \mathrm{g} / \mathrm{L}$ & $\mathrm{EPA}^{2}$ \\
\hline & Âr-12 12 & 384 & $3^{--}$ & $<3.8^{--}$ & $27.3^{-1}$ & $4 \overline{3} .8$ & $\mathrm{NA}^{--}$ & $<0.25$ & $2.50^{-}$ & 1,4 -dimethyladamantane (TIC) $-2.33 \mu \mathrm{g} / \mathrm{L}^{-}$ & $\mathrm{EPA}^{33}$ \\
\hline & Jun-14-- & 720 & $0.39(\mathrm{~J})$ & $\mathrm{ND}$ & ND & $34(\mathrm{~J})$ & $-21(\mathrm{~J})$ & $\mathrm{NA}$ & $\mathrm{NA}-$ & & WYDEQ $^{34}$ \\
\hline & Äug-14-- & 1100 & $0.61(\mathrm{~J})$ & $\mathrm{ND}$ & ND & $74(\mathrm{~J})$ & $\mathrm{ND}$ & $\mathrm{NA}$ & $\overline{N A}-$ & & WYDEQ $^{34}$ \\
\hline \multirow[t]{5}{*}{$\begin{array}{ll}\text { PGDW32 } \\
.\end{array}$} & Mar-09 & 21.4 & NA & NA & NA & 19.2 & NA & NA & NA & & $\mathrm{EPA}^{29}$ \\
\hline & Jan-10 & 36.3 & $<10.0$ & $<15.0^{-}$ & 22.6 & $<20.0$ & $\mathrm{NA}^{--}$ & 0.30 & $<0.20$ & 1-ethyl-4-methyl benzene (TIC) $-0.17 \mathrm{ug} / \mathrm{L}$ & $\mathrm{EPA}^{29^{-\cdots}}$ \\
\hline & Äpr-1i & $67(\mathrm{~B})$ & $<4.8$ & $<6.7^{--}$ & $22.4(\mathrm{~B})$ & $<20.9 /<22$ & $\mathrm{NA}^{--}$ & $0.12(\mathrm{~J}) / 0.12(\mathrm{~J})$ & $<0.10 /<0.10$ & & $\mathrm{EPA}^{27} \cdots$ \\
\hline & Jun-14-- & $33^{-\cdots}$ & $0.2(\mathrm{~J})$ & $0.2(\mathrm{~J})$ & ND & $\mathrm{ND}$ & $\mathrm{ND}$ & $\mathrm{NA}-1-1-1$ & NA & $\begin{array}{l}\text { sopropanol- } 24(\mathrm{~J}) \mu \mathrm{g} / \mathrm{L} \\
\text { isopropybenzene }-0.1 \mathrm{~J}) \mu \mathrm{g} / \mathrm{L} \\
\text { tert-butyl alcohol }-2.0 \mathrm{~J}) \mu \mathrm{g} / \mathrm{L} \\
\text { gamma BHC (lindane) }-0.08(\mathrm{~J}) \mu \mathrm{g} / \mathrm{L}\end{array}$ & WYDEQ $^{34}$ \\
\hline & Āug-14 & 68 & ND & $\mathrm{ND}$ & ND & $\mathrm{ND}$ & $\mathrm{ND}$ & $\mathrm{NA}$ & $\mathrm{NA}$ & isopropylbenzene $-0.1(\mathrm{~J}) \mu \mathrm{g} / \mathrm{L}$ & $\mathrm{WYDEQ}^{34}$ \\
\hline \multirow[t]{3}{*}{ PGDW33 } & Mar-09 & NA & NA & NA & NA & NA & NA & NA & NA & & $\mathrm{EPA}^{29}$ \\
\hline & Jun-14 & $0 . \overline{4}(\mathrm{~J})$ & ND & $\mathrm{ND}$ & ND & $\mathrm{ND}$ & $\mathrm{ND}^{--}$ & $\mathrm{NA}$ & $\mathrm{NA}$ & $\begin{array}{l}\text {-butoxyethanol }-3300 \mathrm{~J} / \mathrm{J}) \mu \mathrm{g} / \mathrm{L} \\
\text { sopropanol }-3.5(\mathrm{~J}) \mu \mathrm{g} / \mathrm{L} \\
\text { endosulfan II }-0.1(\mathrm{~J}) \mu \mathrm{g} / \mathrm{L} \\
\text { gamma BHC (lindane) }-0.1(\mathrm{~J}) \mu \mathrm{g} / \mathrm{L}\end{array}$ & WYDEQ $^{34}$ \\
\hline & Āug-14 & $0.3(\mathrm{~J})$ & ND & $\mathrm{ND}$ & $\mathrm{ND}$ & $\mathrm{ND}$ & ND & $\mathrm{NA}$ & $\mathrm{NA}$ & & WYDEQ $^{34}$ \\
\hline PGDW34 & Mar-09 & NA & NA & NA & NA & NA & NA & NA & NA & & $\mathrm{EPA}^{29}$ \\
\hline PGDW35 & Mar-09 & 21.6 & NA & NA & NA & 17.9 & NA & NA & NA & & $\mathrm{EPA}^{29}$ \\
\hline PGDW36 & Mar-09 & NA & NA & NA & NA & NA & NA & NA & NA & & $\mathrm{EPA}^{29}$ \\
\hline PGDW40 & Jan-10 & 98.9 & $<10.0$ & $<15.0$ & $<20.0$ & 32.6 & NA & $<0.20$ & 0.36 & & $\mathrm{EPA}^{30}$ \\
\hline PGDW41A & Jun-14 & ND & ND & ND & ND & $150(\mathrm{~J})$ & ND & NA & NA & methylene chloride $-0.2(\mathrm{~J}) \mu \mathrm{g} / \mathrm{L}$ & WYDEQ $^{34}$ \\
\hline \multirow[t]{4}{*}{ PGDW41 } & Jan-10 & $<5.00$ & $<10.0$ & $<15.0$ & $<20.0$ & 479 & NA & $<0.20$ & $<0.20$ & & $\mathrm{EPA}^{30}$ \\
\hline & Âpr-1̄i & 385 & $\overline{1} \overline{4} \overline{2}(\bar{B})$ & $<6.7^{--}$ & $<20.0$ & $132^{-}$ & $\mathrm{NA}^{--}$ & $<0.25$ & $<0 . \overline{2} 5$ & & $\mathrm{EPA}^{27^{--}}$ \\
\hline & Jun-14 & $1.6(\mathrm{~J})$ & $0.2(\mathrm{~J})$ & $\mathrm{ND}$ & ND & $170(\mathrm{~J})$ & $180(\mathrm{~J})$ & $\mathrm{NA}$ & $\mathrm{NA}$ & & WYDEQ $^{34}$ \\
\hline & Äug-14 & $2.0(\mathrm{~J})$ & ND & $\mathrm{ND}$ & ND & $250(\mathrm{~J})$ & $230(\mathrm{~J})$ & $\mathrm{NA}$ & $\mathrm{NA}$ & & WYDEQ $^{34}$ \\
\hline PGDW42 & Jan-10 & 60.0 & $<10.0$ & $<15.0$ & $<20.0$ & 21.6 & NA & $<0.20(\mathrm{~J})$ & $<0.20(\mathrm{~J})$ & & $\mathrm{EPA}^{30}$ \\
\hline PGDW43 & Jan-10 & $<5.00$ & $<10.0$ & $<15.0$ & $<20.0$ & 49.7 & NA & $<0.20$ & $<0.20$ & $\begin{array}{l}\text { benzene }-0.540 \mu \mathrm{g} / \mathrm{L} \\
\text { acenaphthylene }-0.210 \mu \mathrm{g} / \mathrm{L} \\
\text { naphthalene }-0.300 \mu \mathrm{g} / \mathrm{L} \\
\text { phenol }-0.170 \mu \mathrm{g} / \mathrm{L}\end{array}$ & $\mathrm{EPA}^{30}$ \\
\hline PGDW44 & Jan-10 & $<5.00$ & $<10.0$ & $<15.0$ & $<20.0$ & 44.3 & NA & $<0.20(\mathrm{~J})$ & $<0.20(\mathrm{~J})$ & $\begin{array}{l}\text { 2-methylnaphthalene - 0.370(J) } \mu \mathrm{g} / \mathrm{L} \\
\text { fluorene - 0.150(J) } \mu \mathrm{g} / \mathrm{L} \\
\text { 1-methyl naphthalene (TIC) - } 0.33 \mu \mathrm{g} / \mathrm{L} \\
\text { 1,6-dimethyl naphthalene (TIC) }-0.42 \mu \mathrm{g} / \mathrm{L} \\
\text { 1,7-dimethyl naphthalene (TIC) }-0.48 \mu \mathrm{g} / \mathrm{L} \\
\text { 2,7-dimethyl naphthalene (TIC) }-0.25 \mu \mathrm{g} / \mathrm{L} \text {. }\end{array}$ & $\mathrm{EPA}^{30}$ \\
\hline
\end{tabular}




\begin{tabular}{|c|c|c|c|c|c|c|c|c|c|c|c|}
\hline 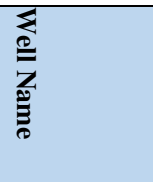 & $\begin{array}{l}\text { एें } \\
\text { है }\end{array}$ & 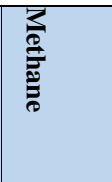 & $\overrightarrow{\vec{F}}$ & 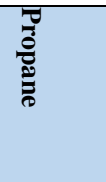 & $\frac{2}{0}$ & రृ & 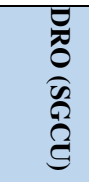 & 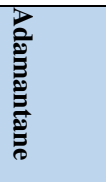 & 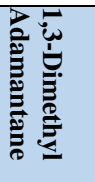 & 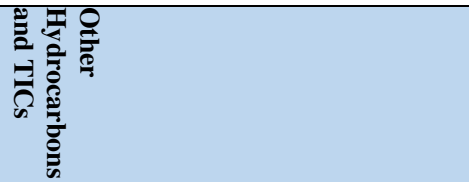 & 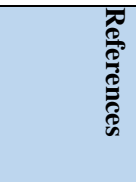 \\
\hline & Apr-11 & $24(\mathrm{~B})$ & $<4.8$ & $<6.7$ & $<20.0$ & $60.5(\mathrm{~B})$ & NA & $<0.25$ & $<0.25$ & & $\mathrm{EPA}^{27}$ \\
\hline & Jun-14 & $1.3(\mathrm{JP})$ & $0.37(\mathrm{~J})$ & $0.51(\mathrm{~J})$ & $\mathrm{ND}^{---}$ & $3 \overline{1}(\mathrm{~J})$ & $\mathrm{ND}$ & $\mathrm{NA}^{--}$ & $\mathrm{NA}^{--}$ & $\begin{array}{l}\text { isopropanol - 11(J) } \\
\text { gamma BHC (lindane) }-0.02(\mathrm{~J}) \\
\text { naphthalene }-0.2(\mathrm{~J})\end{array}$ & WYDEQ $^{34}$ \\
\hline & Âug-14 & $1.4(\mathrm{~J})$ & $0.43(\mathrm{~J})$ & $0.59(\mathrm{~J})$ & & & & $\mathrm{NA}$ & $\mathrm{NA}^{--}$ & & $\mathrm{WYDEQ}^{34}$ \\
\hline PGDW45 & Jan-10 & $<5.00$ & $<10.0$ & $<15.0$ & $<20.0$ & 41.3 & NA & $<0.20(\mathrm{~J})$ & $<0.20(\mathrm{~J})$ & 1,3-dimethyladamantane + isomers $-9.5 \mu \mathrm{g} / \mathrm{L}$ & $\mathrm{EPA}^{30}$ \\
\hline & Âpr-11 & $24(\bar{B})^{-}$ & $64(\mathrm{~B})$ & $<6.7^{-}$ & $<20.0$ & $32.1^{-1}$ & $\mathrm{NA}^{--}$ & $<0.10$ & $1.25^{-1}$ & & $\mathrm{EPA}^{27}$ \\
\hline & Jun-14 & $1(\mathrm{~J})$ & $\mathrm{ND}$ & $\mathrm{ND}$ & $-\overline{25}(\mathrm{~J})--$ & $\mathrm{ND}^{-\cdot-}$ & $33(\mathrm{~J})$ & $\mathrm{NA}^{-\cdots-}$ & $\mathrm{NA}$ & $\begin{array}{l}\text { isopropanol - } 15(\mathrm{~J}) \mu \mathrm{g} / \mathrm{L} \\
\text { gamma BHC (lindane) - 0.06(J) } \mu \mathrm{g} / \mathrm{L}\end{array}$ & WYDEQ $^{34}$ \\
\hline PGDW46 & Jan-10 & $<5.00$ & $<10.0$ & $<15.0$ & $<20.0$ & 25.5 & NA & $<0.20$ & $<0.20$ & & $\mathrm{EPA}^{30}$ \\
\hline PGDW47 & Jan-10 & $<5.00$ & $<10.0$ & $<15.0$ & $<20.0$ & 26.6 & $\mathrm{NA}$ & $<0.20(\mathrm{~J})$ & $<0.20(\mathrm{~J})$ & & $\mathrm{EPA}^{30}$ \\
\hline PGDW48 & Jan-10 & $<5.00$ & $<10.0$ & $<15.0$ & $<20.0$ & $<20.0$ & NA & $<0.20$ & $<0.20$ & & $\mathrm{EPA}^{30}$ \\
\hline PGDW49 & Jan-10 & $<5.00$ & $<10.0$ & $<15.0$ & $<20.0$ & 130 & NA & $<0.20$ & $<0.20$ & & $\mathrm{EPA}^{30}$ \\
\hline & Apr-11 & $24(\bar{B})$ & $62(\mathrm{~B})$ & $<6.7^{-}$ & $<20.0$ & $51.9^{--}$ & $\mathrm{NA}$ & $<0.10$ & $<0.10^{-}$ & & $\mathrm{EPA}^{27^{-1}}$ \\
\hline & Jun-14"- & $0.2(\mathrm{JP})$ & $\mathrm{ND}$ & $\mathrm{ND}$ & $\mathrm{ND}$ & $110(\mathrm{~J})^{-\cdot}$ & $\mathrm{ND}$ & $\mathrm{NA}^{--1}$ & $\mathrm{NA}^{--}$ & endosufan II $-0.07(\mathrm{~J}) \mu \mathrm{g} / \mathrm{L}$ & WYDEQ $^{34}$ \\
\hline & Âug-14 & $0.3(\mathrm{JP})$ & $\mathrm{ND}^{--1}$ & $\mathrm{ND}$ & $\mathrm{ND}$ & $38(\mathrm{~J})$ & $\mathrm{ND}$ & $\mathrm{NA}^{-}$ & $\mathrm{NA}^{---}$ & & WYDEQ $^{34}$ \\
\hline P108128.0W & NS & & & & & & & & & & \\
\hline P124049.0W & NS & & & & & & & & & & \\
\hline P146856.0W & NS & & & & & & & & & & \\
\hline P197335.0W & NS & & & & & & & & & & \\
\hline P197336.0W & NS & & & & & & & & & & \\
\hline P200885.0W & NS & & & & & & & & & & \\
\hline $\mathrm{P} 22660.0 \mathrm{P}$ & NS & & & & & & & & & & \\
\hline P22661.0P & NS & & & & & & & & & & \\
\hline P22662.0P & NS & & & & & & & & & & \\
\hline P24502.0P & NS & & & & & & & & & & \\
\hline P24508.0P & NS & & & & & & & & & & \\
\hline P29496.0P & NS & & & & & & & & & & \\
\hline P31805.0W & NS & & & & & & & & & & \\
\hline P41320.0W & NS & & & & & & & & & & \\
\hline P41517.0W & NS & & & & & & & & & & \\
\hline P44255.0W & NS & & & & & & & & & & \\
\hline P59499.0W & NS & & & & & & & & & & \\
\hline P60032.0W & NS & & & & & & & & & & \\
\hline P66345.0W & NS & & & & & & & & & & \\
\hline P69549.0W & NS & & & & & & & & & & \\
\hline P91293.0W & NS & & & & & & & & & & \\
\hline P99671.0W & NS & & & & & & & & & & \\
\hline \multicolumn{12}{|c|}{ Domestic Wells $<600 \mathrm{~m}$ from Unlined Pits Not Receiving Production Fluids } \\
\hline \multirow[t]{2}{*}{ PGDW03 } & Mar-09 & NA & NA & NA & NA & NA & NA & $\mathrm{NA}$ & NA & & $\mathrm{EPA}^{29}$ \\
\hline & Jan-10 & $<5.00^{-}$ & $<10.0$ & $<15.0^{-}$ & $<20.0$ & $<20.0^{-\cdots}$ & $\mathrm{NA}^{-1}$ & $<0.20^{\circ}$ & $<0.20^{-}$ & & $\mathrm{EPA}^{30^{-}}$ \\
\hline \multirow[t]{2}{*}{ PGDW04 } & Mar-09 & $<5.00$ & NA & NA & $<20.0$ & $<15.0$ & $\mathrm{NA}$ & $\mathrm{NA}$ & NA & & $\mathrm{EPA}^{29}$ \\
\hline & Jan-10 & $<5.00^{-}$ & $<10.0$ & $<15.0^{-}$ & $<20.0$ & $<20.0$ & $\mathrm{NA}^{-}$ & $<0.20$ & $<.020^{-}$ & & $\mathrm{EPA}^{30^{-}}$ \\
\hline PGDW13 & Mar-09 & NA & NA & NA & NA & NA & NA & $\mathrm{NA}$ & NA & & EPA $^{29}$ \\
\hline PGDW15 & Mar-09 & NA & NA & NA & NA & $\mathrm{NA}$ & $\mathrm{NA}$ & $\mathrm{NA}$ & $\mathrm{NA}$ & & EPA $^{29}$ \\
\hline P120203.0W & NS & & & & & & & & & & \\
\hline $\mathrm{P} 23056.0 \mathrm{P}$ & NS & & & & & & & & & & \\
\hline
\end{tabular}




\begin{tabular}{|c|c|c|c|c|c|c|c|c|c|c|c|}
\hline 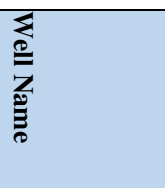 & $\begin{array}{l}\text { F्ञ } \\
\text { हैं }\end{array}$ & \begin{tabular}{l}
$\frac{3}{2}$ \\
\multirow{2}{*}{}
\end{tabular} & 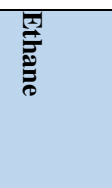 & 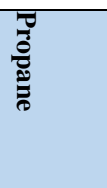 & Q & 鴊 & 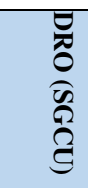 & 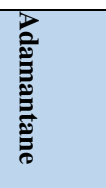 & 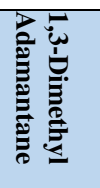 & 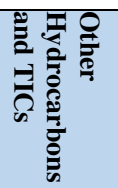 & $\begin{array}{l}2 \\
0 \\
0 \\
0 \\
0 \\
0 \\
0 \\
0 \\
0\end{array}$ \\
\hline P65111.0W & NS & & & & & & & & & & \\
\hline P95171.0W & NS & & & & & & & & & & \\
\hline P9334.0P & NS & & & & & & & & & & \\
\hline P9441.0P & NS & & & & & & & & & & \\
\hline CR UW09/250 & NS & & & & & & & & & & \\
\hline \multicolumn{12}{|c|}{ Domestic Wells > 600m From an Unlined Pits } \\
\hline \multirow{2}{*}{ PGPW01 } & Mar-09 & $<5.00$ & NA & NA & NA & 17.1 & NA & NA & NA & & $\mathrm{EPA}^{29}$ \\
\hline & Jan-10 & $\mathrm{NA}^{--}$ & $\mathrm{NA}^{--}$ & $\mathrm{NA}$ & $\mathrm{NA}^{-1}$ & $<20.0^{-1}$ & $\mathrm{NA}$ & $<0.20$ & $<0.20^{--}$ & & $\mathrm{EPA}^{30^{-}}$ \\
\hline \multirow[t]{3}{*}{ PGPW02 } & Mar-09 & NA & NA & NA & NA & NA & NA & NA & NA & & $\mathrm{EPA}^{29}$ \\
\hline & Jan-10 & $\mathrm{NA}$ & $\mathrm{NA}^{--}$ & $\mathrm{NA}$ & $\mathrm{NA}^{--}$ & $<20.0$ & $\mathrm{NA}$ & $<0.20$ & $<0.20$ & & $\mathrm{EPA}^{30}$ \\
\hline & Äpr-12 & $8(\mathrm{~B})$ & $<2.7$ & $<3.8$ & $<20.0$ & $<20.0$ & $\mathrm{NA}$ & $<0.25$ & $<0.25$ & & $\mathrm{EPA}^{33}$ \\
\hline PGDW01 & Mar-09 & NA & NA & NA & NA & NA & NA & NA & NA & & $\mathrm{EPA}^{29}$ \\
\hline PGDW02 & Mar-09 & NA & $\mathrm{NA}$ & NA & NA & NA & NA & NA & NA & & $\mathrm{EPA}^{29}$ \\
\hline PGDW09 & Mar-09 & NA & NA & NA & NA & NA & NA & NA & NA & & $\mathrm{EPA}^{29}$ \\
\hline \multirow[t]{2}{*}{ PGDW10 } & Mar-09 & $<5.00$ & NA & NA & $<20$ & 23.1 & NA & $<0.20(\mathrm{~J})$ & $<0.20(\mathrm{~J})$ & & $\mathrm{EPA}^{29}$ \\
\hline & Jan-10 & $<5.00(\mathrm{~J})$ & $<10.0(\mathrm{~J})$ & $<15.0(\mathrm{~J})$ & $<20$ & $<20.0$ & $\mathrm{NA}$ & $<0.25$ & $<0.25$ & & $\mathrm{EPA}^{30^{-}}$ \\
\hline PGDW12 & Mar-09 & NA & NA & NA & NA & NA & $\mathrm{NA}$ & NA & NA & & $\mathrm{EPA}^{29}$ \\
\hline PGDW16 & Mar-09 & NA & $\mathrm{NA}$ & NA & NA & NA & $\mathrm{NA}$ & NA & NA & & $\mathrm{EPA}^{29}$ \\
\hline PGDW17 & Mar-09 & 10.6 & NA & NA & NA & 17.5 & NA & NA & NA & & EPA $^{29}$ \\
\hline PGDW18 & Mar-09 & NA & NA & NA & NA & NA & NA & NA & NA & & $\mathrm{EPA}^{29}$ \\
\hline PGDW19 & Mar-09 & NA & NA & NA & NA & NA & NA & NA & NA & & $\mathrm{EPA}^{29}$ \\
\hline PGDW24 & Mar-09 & NA & NA & NA & NA & NA & NA & NA & NA & & $\mathrm{EPA}^{29}$ \\
\hline \multirow[t]{2}{*}{ PGDW25 } & Mar-09 & NA & $\mathrm{NA}$ & NA & NA & NA & NA & NA & NA & & $\mathrm{EPA}^{29}$ \\
\hline & Jan-10 & $<5.00^{-}$ & $<10.0$ & $<15.0^{-}$ & $<20^{-}$ & 27.8 & $\mathrm{NA}$ & $<0.20$ & $<0.20^{-}$ & & EPA $^{30-}$ \\
\hline PGDW28 & Mar-09 & NA & NA & NA & NA & NA & NA & NA & NA & & $\mathrm{EPA}^{29}$ \\
\hline PGDW29 & Mar-09 & $<5.00$ & NA & NA & NA & 16.2 & NA & NA & NA & & $\mathrm{EPA}^{29}$ \\
\hline PGDW31 & Mar-09 & NA & NA & NA & NA & NA & NA & NA & NA & & $\mathrm{EPA}^{29}$ \\
\hline PGDW37 & Mar-09 & NA & $\mathrm{NA}$ & NA & NA & NA & NA & NA & NA & & $\mathrm{EPA}^{29}$ \\
\hline PGDW38 & Mar-09 & $<5.00$ & NA & NA & NA & 19.7 & NA & NA & NA & & $\mathrm{EPA}^{29}$ \\
\hline PGDW39 & Jan-10 & $<5.00$ & $<10.0$ & $<15.0$ & $<20.0$ & 30.0 & NA & $<0.20(\mathrm{~J})$ & $<0.20(\mathrm{~J})$ & & $\mathrm{EPA}^{30}$ \\
\hline PGDW50 & Apr-12 & $<1.3$ & $<2.7$ & $<3.8$ & $<20.0$ & $<20.0$ & NA & $<0.25$ & $<0.25$ & & $\mathrm{EPA}^{33}$ \\
\hline
\end{tabular}

Data (tables) from WYDEQ obtained from the Powder River Basin Resource Council in April 2015.

NS - Not sampled

NA - Not analyzed

ND - Not detected. Method detection and reporting limits not known

TICs - Tentatively identified compounds

$\mathrm{J}$ - Estimated value

$\mathrm{B}$ - Compound detection in blank. For EPA data, detection less than 10X value in blank.

SGCU - Silica gel cleanup performed before analysis 

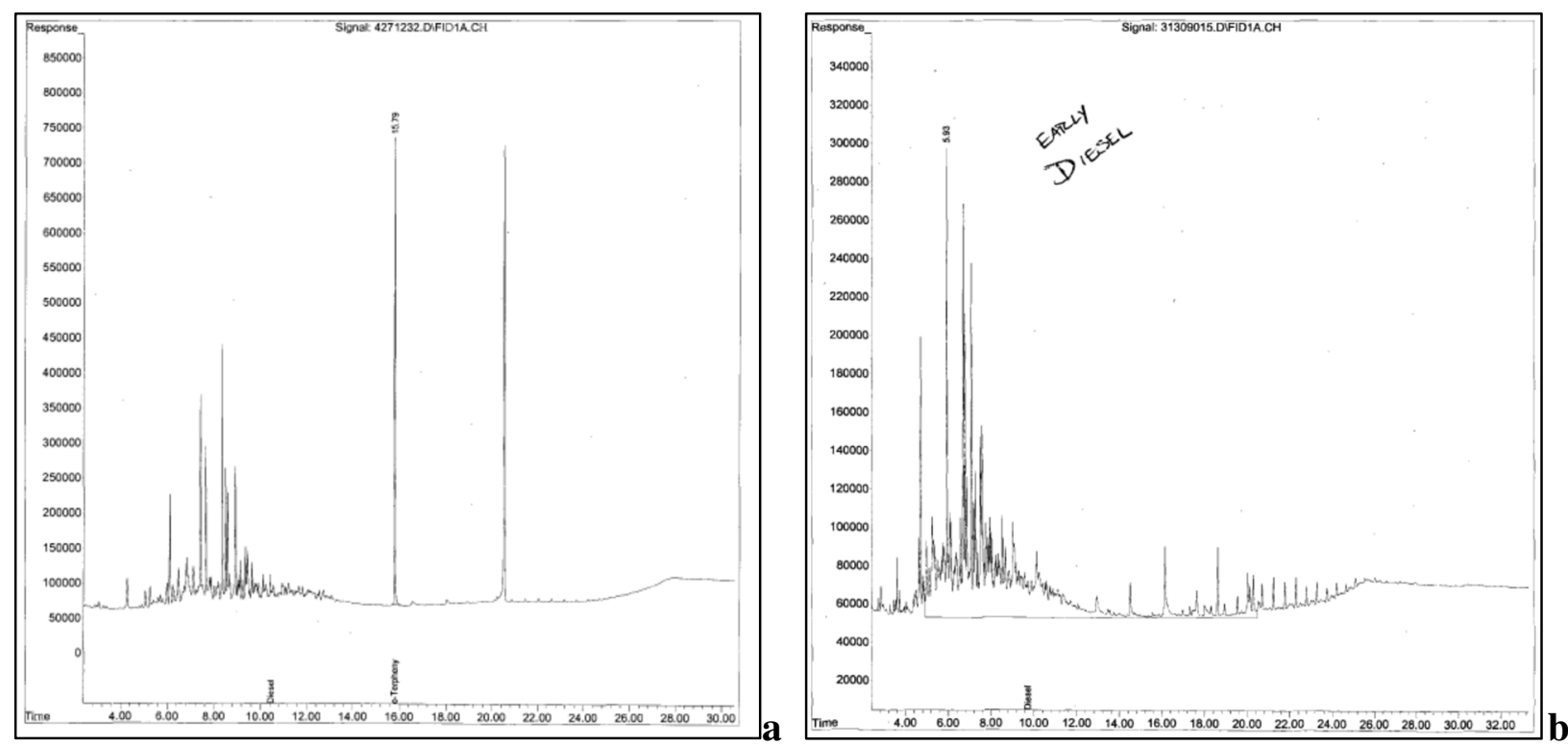

Figure SI F1. Chromatograms of DRO analysis at (a) PGDW05 during Phase V sampling event, and (b) PGDW30 during Phase I sampling event. Handwritten note, "Early Diesel”, by EPA Region 8 chemist.
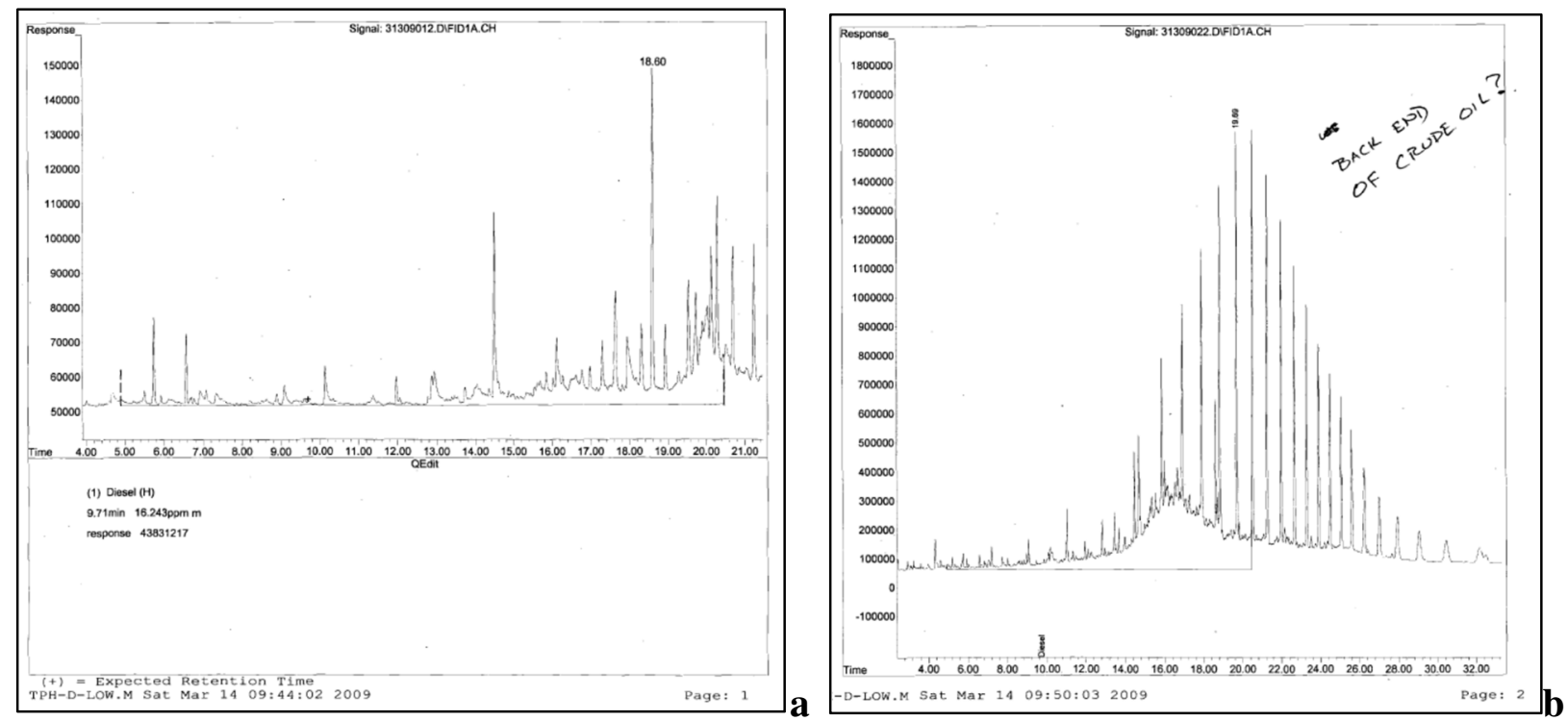

Figure SI F2. Chromatograms of DRO analysis at PGDW20 during Phase I sampling event (a) aqueous sample (b) carbon trap sample. Handwritten note, "Back End of Crude Oil" by EPA Region 8 analytical chemist. During the Phase (I) sampling event, water was circulated through a carbon trap for 24 hours (cumulative water approximately $2,950 \mathrm{~L}$ ) with detection of DRO at $377 \mu \mathrm{g} / \mathrm{L}$ (EPA 2009). 


\section{G. Drilling Fluids Remaining in Wellbores}

A review of well completion reports, drilling reports, and wireline geophysical logs indicates that invert mud consisted up to 79\% oil (Unit 41X-10). At Govt 23-7, Q-BROXIN Gel, a ferrochrome lignosulfonate based ge ${ }^{107}$ was used which contains high concentrations of chromium (e.g., > 16,000 $\mathrm{mg} / \mathrm{kg})^{108}$. After 1993, water-based mud systems consisted of $\mathrm{KCl}$ polymer, partially hydrolized polyacrylamides (PHPA), low solids non-dispersed drilling mud (LSND), and "DeepDrill” drilling systems (Table SI C1). MSDSs indicate that "NewPHPA" contained 24\% hydrotreated light petroleum distillate.

The contact length of drilling mud to the Wind River and Fort Union Formations was estimated by the difference in depth to surface casing and depth to top of primary cement outside production casing based on available information from cement bond logs (CBLs) and temperature logs. Production casing was not used for 16 "dry" exploration wells (no cement below surface casing with exception of plugs for abandonment) 14 of which were drilled using invert mud. CBLs were unavailable at 42 of 165 (25\%) of production wells. Top of cement could not be discerned at 5 wells due to high and erratic amplitude readings characteristic of free pipe. The extent of drilling mud below casing could be estimated at 128 of $181(71 \%)$ total production wells and at 36 of $58(62 \%)$ of production wells where invert mud was used (Table SI C1). The elevation of surface casing and top of cement are illustrated in Figure SI F1.

Boreholes retaining drilling mud outside production casing represent vertical "line" source terms for mass flux of contaminants into a surrounding formation. However, a more complicated geometry occurs when mud is lost to a formation. For example at Tribal 1-21, invert mud was lost to the Wind River Formation at two depths $\left(9.5\right.$ and $\left.12.7 \mathrm{~m}^{3}\right)$, with the latter "likely lost to a fractured zone." At USA Tribal $25822-10,39.7 \mathrm{~m}^{3}$ of invert mud was lost to the Fort Union Formation at two depths. Given the composition of invert mud, impact to ground water is expected but the distribution of impact would be expected to be a function of linear extent of mud below surface casing, surface area of exposure, dispersion, biodegradation, and distance (both vertical and lateral) from boreholes, and local hydraulic gradients. No monitoring wells exist in the immediate of boreholes, thus assessment of impact is not possible.

Ground water contamination from invert mud retained in boreholes would be expected to be primarily manifested by detection of elevated levels of diesel range organics (DRO). Fourteen domestic wells in the Pavillion Field were identified as having sufficient depth or proximity to production wells to be potentially impacted by invert mud retained in boreholes (Table SI G1). Twelve of these wells were sampled by EPA of which 9 were analyzed for DRO. Given distance to production well, depths of surface 
casing and domestic wells, expected direction of ground water flow (east), and detection of elevated DRO, LD-02 appears to have the highest probability of impact with detection of DRO at $111 \mu \mathrm{g} / \mathrm{L}$.

However, if DRO detection is due to an anthropogenic source term, differentiation from other source terms (unlined pits and stimulation) is not possible with currently available data.

Table SI G1. Domestic wells greater than intermediate depth and distance to nearest production well with invert mud

\begin{tabular}{|c|c|c|c|c|c|c|c|c|c|}
\hline $\begin{array}{l}\text { EPA/ WY SEO } \\
\text { Well } \\
\text { Identification }\end{array}$ & $\begin{array}{l}\text { Depth of } \\
\text { Domestic } \\
\text { Well (m } \\
\text { bgs) }\end{array}$ & $\begin{array}{l}\text { Nearby } \\
\text { Production } \\
\text { Well with } \\
\text { Invert } \\
\text { Mud } \\
\end{array}$ & $\begin{array}{l}\text { Direction from } \\
\text { Production } \\
\text { Well to } \\
\text { Domestic Well }\end{array}$ & $\begin{array}{l}\text { Distance } \\
\text { (m) }\end{array}$ & $\begin{array}{l}\text { Depth of } \\
\text { Surface } \\
\text { Casing (m } \\
\text { bgs) }\end{array}$ & $\begin{array}{l}\text { Depth of } \\
\text { Surface } \\
\text { Casing - } \\
\text { Domestic } \\
\text { Well (m) } \\
\end{array}$ & $\begin{array}{l}\text { Depth to Top of } \\
\text { Primary } \\
\text { Cement or Base } \\
\text { of Borehole (m } \\
\text { bgs) }\end{array}$ & $\begin{array}{l}\text { Length of } \\
\text { Invert Mud } \\
\text { Below } \\
\text { Surface } \\
\text { Casing (m) } \\
\end{array}$ & $\begin{array}{l}\text { DRO } \\
(\mu \mathrm{g} / \mathrm{L}) \text { except } \\
\text { PGFM20 and } \\
\text { EPa Phase }\end{array}$ \\
\hline PGDW03 & 137.2 & $\begin{array}{l}\text { 44-17 } \\
\text { RH } 1 \\
\end{array}$ & $\begin{array}{l}\text { SE } \\
\text { SE }\end{array}$ & $\begin{array}{l}427 \\
382 \\
\end{array}$ & $\begin{array}{l}183.8 \\
185.9 \\
\end{array}$ & $\begin{array}{l}46.6 \\
48.7 \\
\end{array}$ & $\begin{array}{l}\text { None } \\
\text { None } \\
\end{array}$ & $\begin{array}{l}1108 \\
1035 \\
\end{array}$ & $<20.0$ (II) \\
\hline PGDW04 & 134.1 & $\begin{array}{l}44-17 \\
\text { RH } 1 \\
\end{array}$ & $\begin{array}{l}\text { SE } \\
\text { SE }\end{array}$ & $\begin{array}{l}565 \\
521 \\
\end{array}$ & $\begin{array}{l}183.8 \\
185.9 \\
\end{array}$ & $\begin{array}{l}49.7 \\
51.8 \\
\end{array}$ & $\begin{array}{l}\text { None } \\
\text { None }\end{array}$ & $\begin{array}{l}1108 \\
1035 \\
\end{array}$ & $\begin{array}{l}<15.0 \text { (I) },<20.0 \\
\text { (II) }\end{array}$ \\
\hline PGDW06 & 115.8 & $1-31$ & SE & 341 & 125.3 & 9.5 & Unknown & Unknown & NA \\
\hline PGDW11 & unknown & $\begin{array}{l}44-10 \\
31-15 \\
\end{array}$ & $\begin{array}{l}\text { NE } \\
\text { SW }\end{array}$ & $\begin{array}{l}388 \\
230 \\
\end{array}$ & $\begin{array}{l}184.4 \\
205.7 \\
\end{array}$ & $\begin{array}{l}\text { Unknown } \\
\text { Unknown }\end{array}$ & $\begin{array}{l}585 \\
\text { Unknown }\end{array}$ & $\begin{array}{l}585 \\
\text { Unknown }\end{array}$ & NA \\
\hline PGDW12 & 115.8 & $1-31$ & NW & 491 & 125.3 & 9.5 & Unknown & Unknown & NA \\
\hline PGDW20 & 140.2 & $22-12$ & SE & 172 & 178.6 & 38.4 & Unknown† & Unknown & $\begin{array}{l}17.3 \text { (I), } 21.7 \text { (II), } \\
<20 /<20 \text { (III), } \\
<21.9 \text { (IV), } \\
<20 /<20 \text { (V) }\end{array}$ \\
\hline $\begin{array}{l}\text { PGDW20 } \\
\text { (Carbon Trap) }\end{array}$ & 140.2 & $22-12$ & $\mathrm{SE}$ & 172 & 178.6 & 38.4 & Unknown† & Unknown & 377 (I) \\
\hline $\begin{array}{l}\text { PGFM20 (RO } \\
\text { Filter sample } \\
\text { from PGDW20) }\end{array}$ & 140.2 & $22-12$ & $\mathrm{SE}$ & 172 & 178.6 & 38.4 & Unknown† & Unknown & $\begin{array}{l}752,000(\mu \mathrm{g} / \mathrm{kg}) \\
\text { (II) }\end{array}$ \\
\hline PGDW21/LD-02 & 185.9 & $22-12$ & SE & 172 & 178.6 & -7.3 & Unknown $\dagger$ & Unknown & 111 (III) \\
\hline PGDW22 & unknown & 14-12 & SW & 261 & 190.1 & Unknown & 191 & 0.9 & 27.1 (I), 154 (II) \\
\hline PGDW32 & 205.7 & $12-13$ & $\mathrm{NE}$ & 173 & 175.6 & -30.1 & 290 & 114 & $\begin{array}{l}19.2(\mathrm{I}), \\
<20.9 /<22.0(\mathrm{IV})\end{array}$ \\
\hline PGDW43 & unknown & $41-9$ & NW & 176 & 184.1 & Unknown & 274 & 90 & 49.7 (II) \\
\hline PGDW47 & 147.5 & $21-15$ & $\mathrm{~N}$ & 350 & 188.7 & 41.2 & Unknown & Unknown & 26.6 (II) \\
\hline PGDW48 & 115.8 & $44-15$ & SW & 329 & 189.9 & 74.1 & 198 & 8 & $<20.0 \mathrm{~J}$ (II) \\
\hline P108128.0W & 115.5 & $44-15$ & $\mathrm{~W}$ & 115 & 189.9 & 74.4 & 198 & 8 & NA \\
\hline P124049.0W & 147.5 & $21-15$ & $\mathrm{~N}$ & 384 & 188.7 & 41.2 & Unknown & Unknown & NA \\
\hline
\end{tabular}

$\dagger$ Well schematic indicates drilling mud below surface casing. 


\section{H - Summary of Analytical Data Sources}

Table SI H1. Major reports summarizing data and/or data quality

\begin{tabular}{|c|c|c|c|}
\hline Agency & Report Title & Date & Source (accessed 9/1/2015) \\
\hline EPA & $\begin{array}{l}\text { Site Inspection - Analytical Results Report Pavillion Area } \\
\text { Groundwater Investigation Site, Pavillion, Fremont County, } \\
\text { Wyoming }\end{array}$ & Aug 2009 & http://www2.epa.gov/sites/production/files/documents/Pavillion_GWInvestigationARRTextAndMaps.pdf \\
\hline EPA & $\begin{array}{l}\text { U.S. Environmental Protection Agency (EPA 2010a). Expanded } \\
\text { Site Investigation - Analytical Results Report Pavillion Area } \\
\text { Groundwater Investigation, Fremont County, Wyoming }\end{array}$ & Aug 2010 & http://www2.epa.gov/sites/production/files/documents/PavillionAnalyticalResultsReport.pdf \\
\hline EPA & Pavillion Quality Assurance Project Plans (5 revisions) & Feb 2010 & ftp://ftp.epa.gov/r8/pavilliondocs/QA Documents/QAPPs/ \\
\hline EPA & Audits of Data Quality (ADQ) October 2010 Sampling Event & Apr 2011 & ftp://ftp.epa.gov/r8/pavilliondocs/QA_Documents/Audits_Of_Data_Quality_Lab_Results/Phase3/ \\
\hline EPA & Audits of Data Quality (ADQ) January 2010 Sampling Event & Aug 2011 & 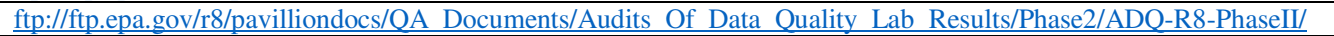 \\
\hline EPA & Audits of Data Quality (ADQ) March 2009 Sampling Event & Dec 2011 & ftp://ftp.epa.gov/r8/pavilliondocs/QA_Documents/Audits Of Data_Quality_Lab_Results/Phase1/ADQ-R8-PhaseI/ \\
\hline EPA & Audits of Data Quality (ADQ) April 2011 Sampling Event & Aug 2011 & ftp://ftp.epa.gov/r8/pavilliondocs/QA_Documents/Audits_Of_Data_Quality_Lab_Results/Phase4/ \\
\hline EPA & Investigation of Ground Water Contamination near Pavillion, WY & Dec 2011 & http://www2.epa.gov/region8/draft-investigation-ground-water-contamination-near-pavillion-wyoming \\
\hline EPA & $\begin{array}{l}\text { Investigation of Ground Water Contamination near Pavillion, } \\
\text { Wyoming Phase V Sampling Event Summary of Methods and } \\
\text { Results }\end{array}$ & Sep 2012 & ftp://ftp.epa.gov/r8/pavilliondocs/phase5/PavillionSeptember2012Narrative.pdf \\
\hline EPA & $\begin{array}{l}\text { Groundwater Sampling Results at Locations near Pavillion, WY } \\
\text { Pavillion Phase V (April 2012) Groundwater Quality Results and - } \\
\text { Control (QC) Data }\end{array}$ & Sep 2012 & ftp://ftp.epa.gov/r8/pavilliondocs/phase5/PavillionSeptember2012Appendices.pdf \\
\hline USGS & $\begin{array}{l}\text { Transmittal of Contract Laboratory Results and Evaluation of } \\
\text { Laboratory-Specific Quality Control Measures, U.S. Environmental } \\
\text { Protection Agency Monitoring Well MW02, Pavillion Wyoming } \\
\text { 2012, Administrative Report Prepared for the U.S. Environmental } \\
\text { Protection Agency. Director Approved August 30, } 2012\end{array}$ & Aug 2012 & ftp://ftp.epa.gov/r8/pavilliondocs/phase5/USGS_MW02_AdministrativeReportSep2012.pdf \\
\hline USGS & $\begin{array}{l}\text { Sampling and Analysis Plan for the Characterization of } \\
\text { Groundwater Quality in Two Monitoring Wells near Pavillion, WY }\end{array}$ & Sep 2012 & http://pubs.usgs.gov/of/2012/1197/ \\
\hline USGS & $\begin{array}{l}\text { Groundwater-Quality and Quality-Control Data for Two } \\
\text { Monitoring Wells near Pavillion, Wyoming, April and May } 2012\end{array}$ & Sep 2012 & http://pubs.usgs.gov/ds/718/ \\
\hline USGS & $\begin{array}{l}\text { Analytical Report: Job Number: 280-28076-1 Job Description: EPA } \\
\text { - Pavillion Fracking }\end{array}$ & Sep 2012 & ftp://ftp.epa.gov/r8/pavilliondocs/phase5/Appendix9_J28076-1_EPA_Std_Tal_L4_Package_MiniFinalReport.pdf \\
\hline EPA & Pavillion Gas Well Integrity Evaluation & Jul 232013 & $\begin{array}{l}\text { ftp://ftp.epa.gov/r8/pavilliondocs/OtherDocuments/WellAndFieldPitsEvaluationJuly2013/GasWellIntegrityEvaluation } \\
\text { 25July2013Final.pdf }\end{array}$ \\
\hline EPA & Pavillion Oil \& Gas Field Pits Evaluation & Jul 252013 & $\begin{array}{l}\text { ftp://ftp.epa.gov/r8/pavilliondocs/OtherDocuments/WellAndFieldPitsEvaluationJuly2013/25July2013PavillionPitsRep } \\
\text { ort4pmFinal.pdf }\end{array}$ \\
\hline WOGCC & Pavillion Field Well Integrity Review & Oct 2014 & http://wogcc.state.wy.us/pavillionworkinggrp/PAVILLION_REPORT_1082014_Final_Report.pdf \\
\hline WOGCC & Pavillion Field Pit Review & Jun 2015 & http://wogcc.state.wy.us/pavillionworkinggrp/PAVILLION_REPORT_1082014_Final_Report.pdf \\
\hline WDEQ & $\begin{array}{l}\text { Pavillion, Wyoming Area Domestic Water Wells Draft Final Report } \\
\text { and Palatability Study }\end{array}$ & Dec 2015 & http://deq.wyoming.gov/wqd/pavillion-investigation/ \\
\hline
\end{tabular}


Table SI H2. Summary of analytical methods used and sources of data and associated information on quality control and assurance.

\begin{tabular}{|c|c|c|c|c|c|c|}
\hline Parameters & Phase & $\begin{array}{l}\text { Medi } \\
\text { a }\end{array}$ & Methods & & Lab & Sources (links) of Analytical and Associated Information on Quality Assurance and Control ) accessed 9/1/2015) \\
\hline \multirow{6}{*}{$\begin{array}{l}\text { Major ions, } \\
\text { alkalinity }\end{array}$} & I & W & EPA Methods 300.0 and 310.1 & EPA & R8 & ftp://ftp.epa.gov/r8/pavilliondocs/RawLabData/Phase1/R8_Lab/85621LSR_Report_Alkalinity_Anions_DRO.pdf \\
\hline & II & $\mathrm{W}^{-}$ & EPA Methods 300.0 and 310.1 & EPA- & $\mathrm{R} 8$ & http://www2.epa.gov/sites/production/files/documents/PavillionAnalyticalResultsReport.pdf (data summary only) \\
\hline & IIII & $\mathrm{W}^{-}$ & $\begin{array}{l}\text { RSKSOPS } 276 v 3 \text { and } 214 v 5 . \text { EPA } \\
\text { Methods SW-846 6500, 350.1, and } 353.2\end{array}$ & $\mathrm{EPA}$ & Àda & ftp://ftp.epa.gov/r8/pavilliondocs/RawLabData/Phase3/ORD_GP_Lab_Analysis_for_Phase_III/ \\
\hline & IV & $\mathrm{W}^{-}$ & $\begin{array}{l}\text { RSKSOPs } 276 v 3 \text { and } 214 v 5 . \text { EPA } \\
\text { Methods SW-846 6500, 350.1, and } 353.2\end{array}$ & $\mathrm{EPA}$ & Âda & $\begin{array}{l}\text { ftp///ftpepa.gov/r8/pavilliondocs/RawLabData/Phase4/ORD_GP_Lab_TOC_DIC_Analysis_Phase_IV// } \\
\text { ftp://ttpepa.gov/r8/pavilliondocs/RawLabData/Phase4/ORD_GP_Lab_TOC_DIC_Analysis_Phase_IV/DICrawdata.pdf } \\
\text { ftp://ftp.epa.gov/r8/pavilliondocs/RawLabData/Phase4/ORD_GP_Lab_TOC_DIC_Analysis_Phase_IV/EPAGP256rev. } \\
\text { 1_SS\%236030_6032_R.Wilkin_Pavillion_Groundwater.pdf } \\
\text { ftp//ftp.epa.gov/r8/pavilliondocs/RawLabData/Phase4/ORD_GP_Lab_TOC_DIC_Analysis_Phase_IV/NPDOC_rawdat } \\
\text { a.pdf }\end{array}$ \\
\hline & $\mathrm{V}$ & $\mathrm{W}^{-}$ & $\begin{array}{l}\text { RSKSOPs } 276 v 3 \text { and } 214 v 5 \text {. EPA } \\
\text { Methods SW-846 6500, 350.1, and } 353.2\end{array}$ & $\mathrm{EPA}$ & Äda & ftp://ftp.epa.gov/r8/pavilliondocs/phase5/PavillionSeptember 2012 Appendices.pdf (data summary only) \\
\hline & & $\mathrm{W}^{-}$ & EPA Methods $300.0,310.1,9056,9060^{-\cdots}$ & USGS'-1 & $\mathrm{TA}$ & 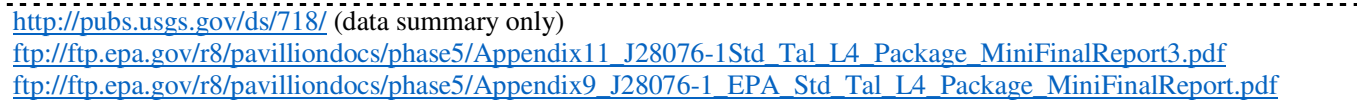 \\
\hline \multirow[t]{6}{*}{$\begin{array}{l}\text { Dissolved } \\
\text { Metals. }\end{array}$} & I & $\mathrm{W}$ & EPA Methods 6010B, 6020, 7470A & EPA & $\mathrm{K}$ & $\begin{array}{l}\text { http://www2.epa.gov/sites/production/files/documents/Pavillion_GWInvestigationARRTextAndMaps.pdf(data } \\
\text { summary_only) }\end{array}$ \\
\hline & $\bar{I}$ & $\mathrm{~W}$ & EPA Methods $6010 \mathrm{~B}, 6020,7470 \mathrm{~A}$ & $\mathrm{EPA}$ & $\mathrm{L}$ & $\begin{array}{l}\text { http://www2.epa.gov/sites/production/files/documents/Pavillion_GWInvestigationARRTextAndMaps.pdf (data } \\
\text { summary only) }\end{array}$ \\
\hline & IIII & $\mathrm{W}$ & $\begin{array}{l}\text { RSKSOP } 213 \mathrm{v} 4 \text { and } 257 \mathrm{v} 2 \text {, or } 332 \mathrm{~V} 0 \\
\text { and EPA Methods } 200.7 \text { and } 6020 .\end{array}$ & $\mathrm{EPA}$ & $\mathrm{S}$ & $\begin{array}{l}\text { ftp://ftp.epa.gov/r8/pavilliondocs/RawLabData/Phase3/Shaw_Metals_Analysis_Phase_III/ } \\
\text { ftp:/ftp.epa.gov/r8/pavilliondocs/RawLabData/Phase3/Shaw_Metals_Analysis_Phase_III/Shaw_Metals_Analysis_Phas } \\
\text { e_III-ICP Data/ } \\
\text { ftp://ftp.epa.gov/r8/pavilliondocs/RawLabData/Phase3/Shaw_Metals_Analysis_Phase_III/Shaw_Metals_Analysis_Phas } \\
\text { e_III-ICP_Data/ICP_Data_1 of_2 101110.pdf } \\
\text { ftp://ftp.epa.gov/r8/pavilliondocs/RawLabData/Phase3/Shaw_Metals_Analysis_Phase_III/Shaw_Metals_Analysis_Phas } \\
\text { e_III-ICP Data/ICP_Data2 of 2 101110.pdf } \\
\text { ftp://ftp.epa.gov/r8/pavilliondocs/RawLabData/Phase3/Shaw_Metals_Analysis_Phase_III/Shaw_Metals_Analysis_Phas } \\
\text { e III-ICP Data/Pavillion_ICP-MS_Data.pdf }\end{array}$ \\
\hline & IV & $\mathrm{W}^{-}$ & $\begin{array}{l}\text { RSKSOP } 213 \mathrm{v} 4 \text { and } 257 \mathrm{v} 2 \text {, or } 332 \mathrm{~V} 0 \\
\text { and EPA Methods } 200.7 \text { and } 6020 .\end{array}$ & $\mathrm{EPA}$ & $\bar{S}$ & 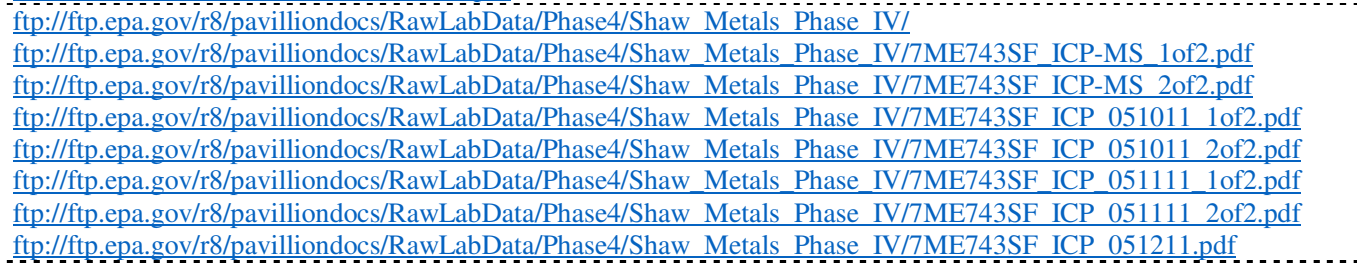 \\
\hline & $\overline{\mathrm{V}}$ & $\overline{\mathrm{W}}$ & $\begin{array}{l}\text { RSKSOP } 213 \mathrm{v} 4 \text { and } 257 \mathrm{v} 2 \text {, or } 332 \mathrm{~V} 0 \\
\text { and EPA Methods } 200.7 \text { and } 6020\end{array}$ & $\mathrm{EPA}$ & $\bar{S}$ & ftp://ftp.epa.gov/r8/pavilliondocs/phase5/PavillionSeptember2012Appendices.pdf (data summary only) \\
\hline & $\mathrm{V}$ & $\mathrm{W}$ & EPA Methods $6010 \mathrm{~B}, 6020,7470 \mathrm{~A}$ & $\mathrm{EPA}$ & $\mathrm{C}$ & 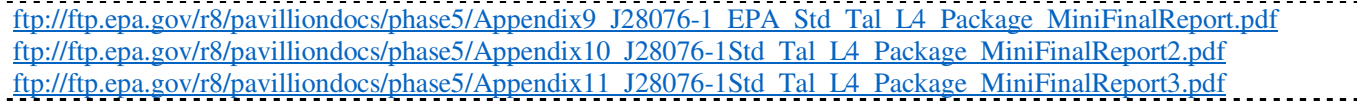 \\
\hline \multirow[t]{2}{*}{$\begin{array}{l}\text { Fixed Gases, } \\
\text { C1-C4 }\end{array}$} & I & $\mathrm{W}$ & ORGM-004 & EPA & R8 & ftp://ftp.epa.gov/r8/pavilliondocs/RawLabData/Phase1/R8_Lab/85622Headspace8270.pdf \\
\hline & & & ORGM-004 & EPA- & $\mathrm{R} 8$ & production/files/doct \\
\hline
\end{tabular}




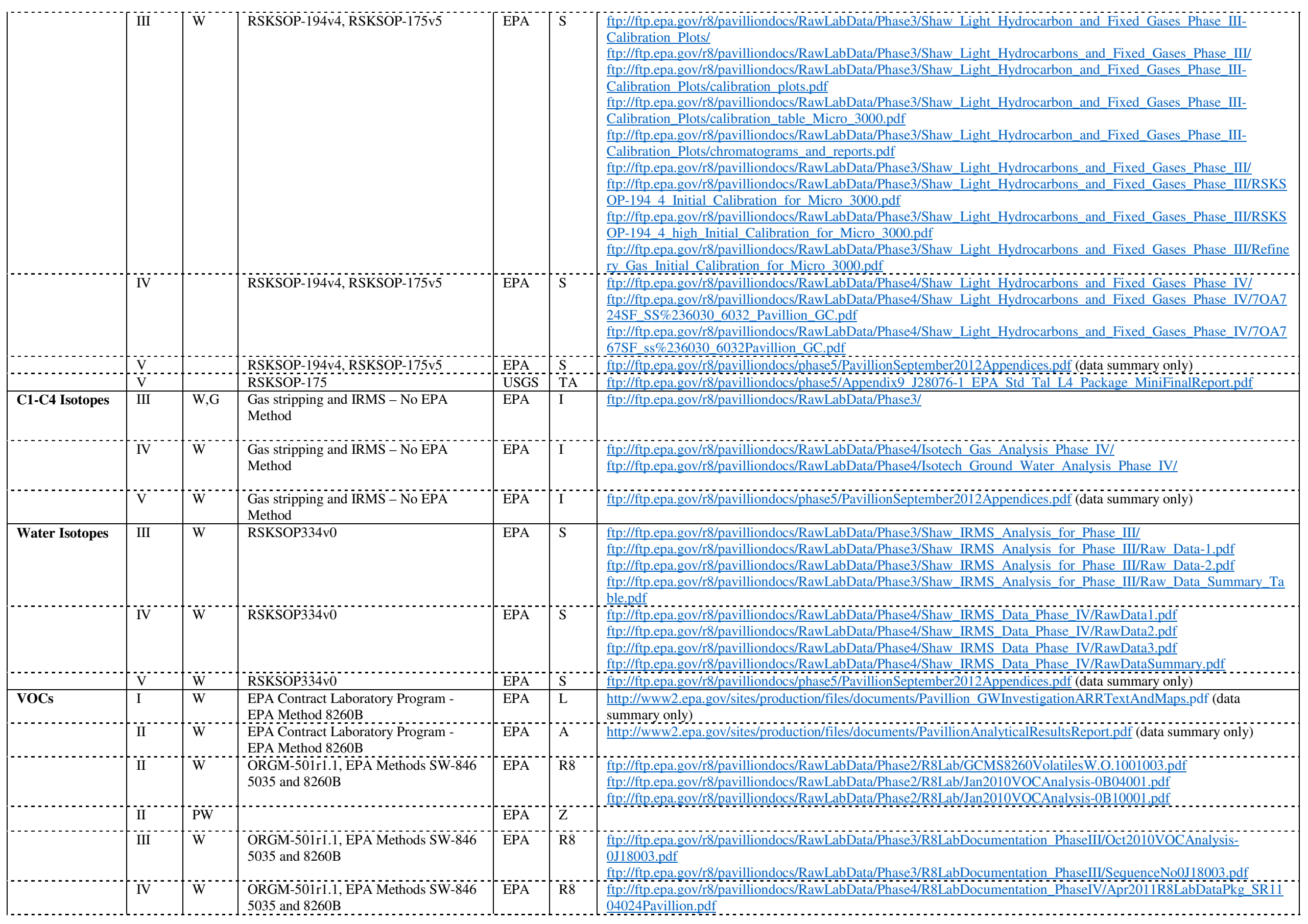




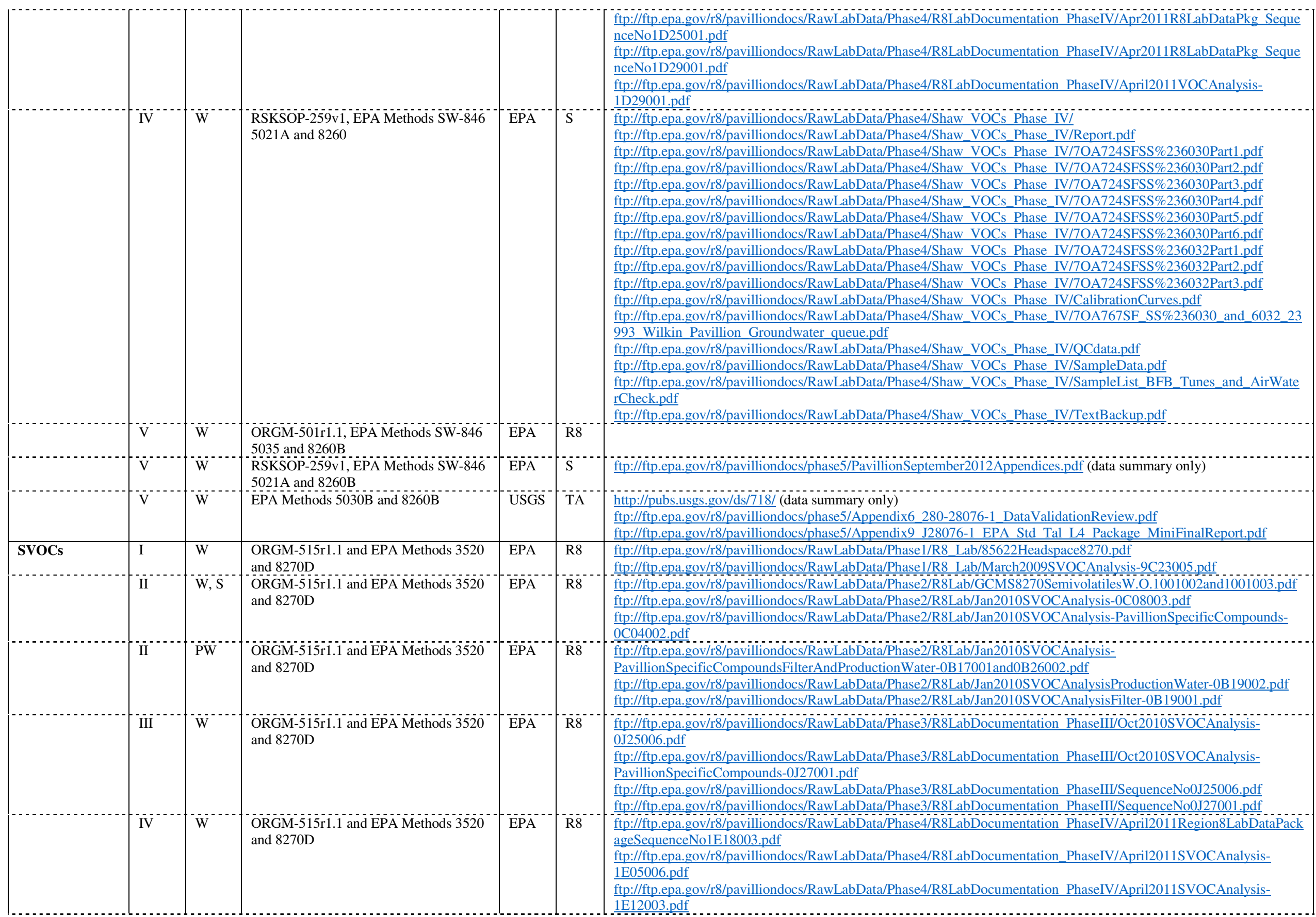




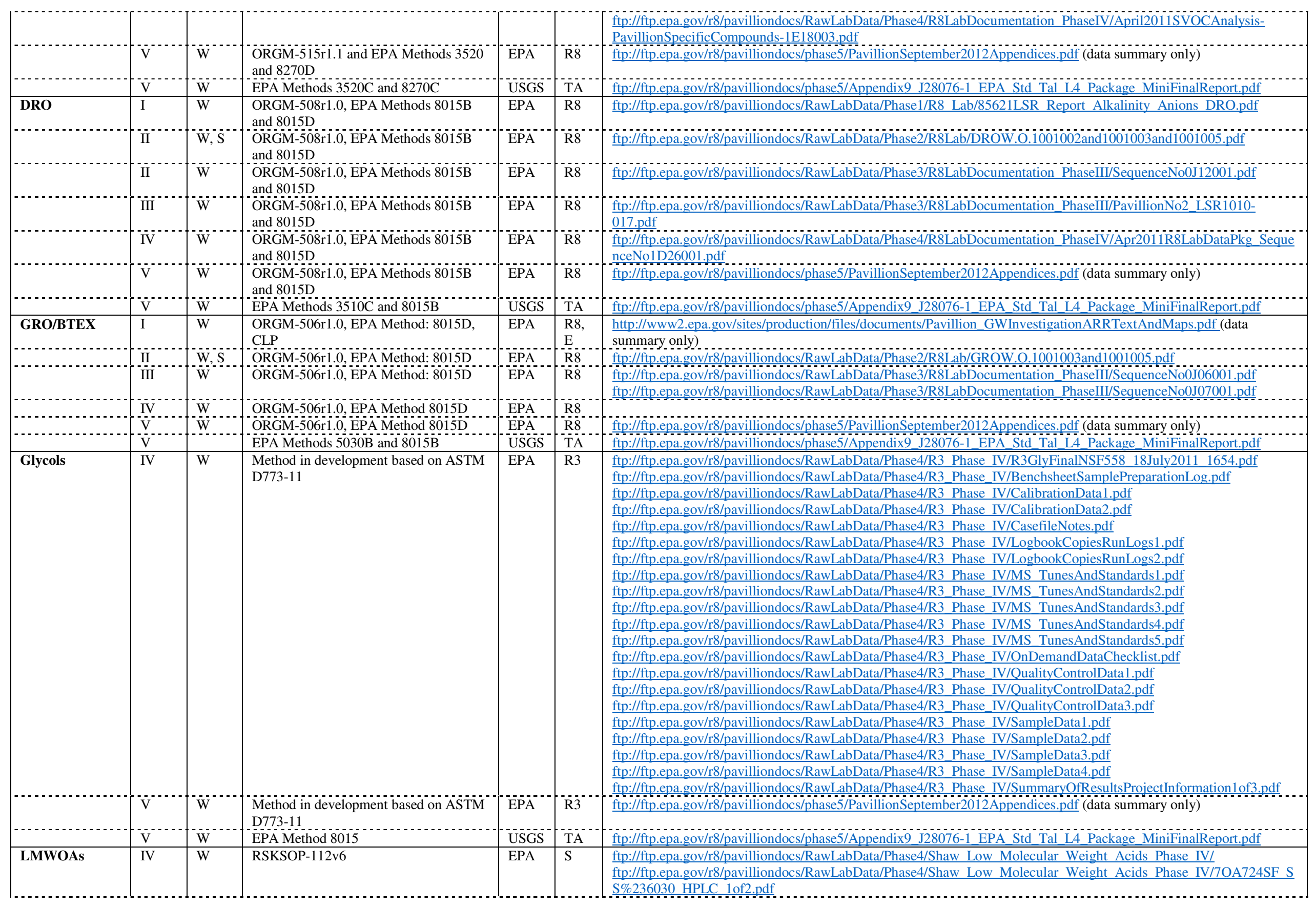




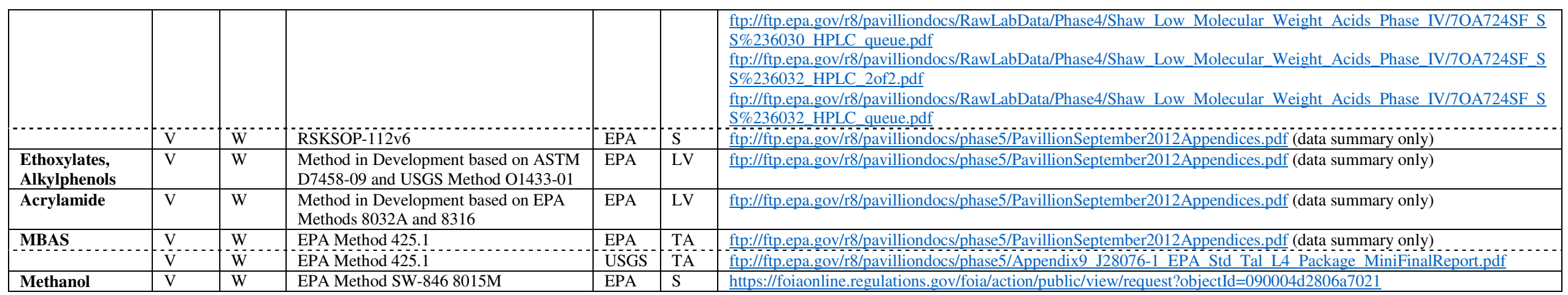

\section{Abbreviations}

C1-C4 - methane, ethane, propane, and butane

VOCs - volatile organic compounds

SVOCs -semi-volatile organic compounds

LMWOAs - low molecular weight organic acids

MBAS -

GRO - gasoline range organic compounds

DRO - diesel range organic compounds

$\mathrm{W}$ - water

$\mathrm{G}$ - gas

$\mathrm{S}$ - solid

\section{Laboratories}

A - ALS Laboratory Group, Salt Lake City, UT

A4 - A4 Scientific, The Woodlands, TX

Ada - EPA Office of Research and Development, Ada, OK

C - Chemtech

E - Energy Laboratories Inc., Billings, MT

I - Isotech Laboratories, Champaign, IL

K - KAP Laboratories, Vancouver, WA.

L - Liberty Analytical, Salt Lake City, UT

LV - EPA Office of Research and Development, Las Vegas, NV

R3 - EPA Region 3 Laboratory, Fort Meade, MD

R8 - EPA Region 8 Laboratory, Golden, CO.

$S$ - Shaw Environmental, Ada, OK

TA - Test America, Denver, CO

Z - Zymax

\section{Analytical Methods}

ORGM-501r1.1 EPA Region 8 Standard Operating Procedure used with EPA Method 8260 for VOC analysis

ORGM-506 r1.0 - EPA Region 8 Standard Operating Procedure used with EPA Method 8015 for GRO analysis

ORGM-508 r1.0 - EPA Region 8 standard operating procedure used with EPA Method 8015D for DRO analysis

ORGM-515 r1.1 - EPA Region 8 standard operating procedure used with EPA Methods 3520 and 8270D for SVOC analysis

RSKSOP-112v6 - EPA Standard Operating Procedure for Quantitative Analysis of Low Molecular Weight Acids in Aqueous Samples by HPLC

RSKSOP-175v5 - Sample Preparation and Calculations for Dissolved Gas Analysis in Water Samples Using a GC Headspace Equilibration Technique, 16 p.

RSKSOP-194v4 - Gas Analysis by Micro Gas Chromatographs (Agilent MIcro 3000), 13 p.

RSKSOP-213v4 - Standard operating procedure for operation of Perkin Elmer Optima 3300 DV ICP-OES, 21 p. 
RSKSOP-214v5 - Quality control procedures for general parameters analysis using Lachat Flow Injection analysis (FIA), $10 \mathrm{p}$.

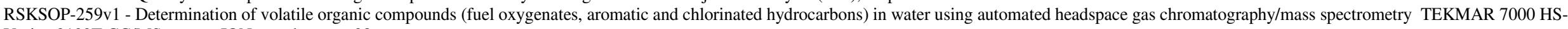

Varian 2100T GC/MS system-ION trap detector, $28 \mathrm{p}$.

RSKSOP-257v2 - Standard operating procedure for elemental analysis by ICP-MS, $16 \mathrm{p}$.

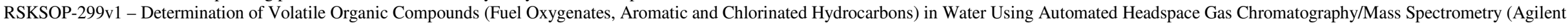
6890/5973 Quadruple GC/MS System), 25 p.

RSKSOP-276v3 - Determination of major anions in aqueous samples using capillary ion electrophoresis with indirect UV detection and Empower 2 software, $11 \mathrm{p}$.

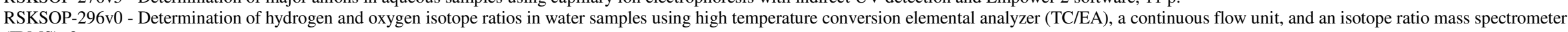
(IRMS), $8 \mathrm{p}$.

RSKSOP-297v1 - Metals Speciation Determination by LC/ICP-MS, $21 \mathrm{p}$.

RSKSOP-298v1 - Arsenic Speciation Determination by LC/ICP-MS with Anion Suppression and NaOH Mobile Phase, 21 p.

RSKSOP-313v1 - Determination of R-123 using the H25-IR Infrared Refrigerant Gas Leak Detector, 12 p.

RSKSOP-314v1 - Determination of Fixed Gases using the GEM2000 and GEM2000 Plus Gas Analyzers \& Extraction Monitors, 13 p.

RSKSOP-320v1 - Determination of Organic and Inorganic Vapors Using the TVA-1000B Toxic Vapor Analyzer, $18 \mathrm{p}$.

RSKSOP-330v0 - Determination of Various Fractions of Carbon in Aqueous Samples Using the Shimadzu TOC-VCPH Analyzer, $16 \mathrm{p}$.

EPA Method 200.7 - Determination of Metals and Trace Elements in Water and Wastes by Inductively Coupled Plasma-Atomic Spectrometry, Rev. 5, Jan 2001.

EPA Method 300.0 - Determination of Inorganic Anions by Ion Chromatography, Rev. 2.1, Aug. 1993.

EPA method 310.1 - Alkalinity (Titrimetric, pH 4.5), Rev. 1978.

EPA Method 350.1 - Determination of Ammonia Nitrogen by Semi-Automated Colorimetry, Rev. 2, Aug. 1993.

U.S. EPA Method 5021A - Volatile Organic Compounds in Various Sample Matrices Using Equilibrium Headspace Analysis, Rev. 1, June 2003.

U.S. EPA Method 6020 - Inductively Coupled Plasma-Mass Spectrometry, Rev. 1, Feb. 2007.

U.S. EPA Method 6500 - Dissolved Inorganic Anions in Aqueous Matrices by Capillary Electrophoresis, Rev. 0, Feb. 2007.

U.S. EPA Method 8260C - Volatile Organic Compounds by Gas Chromatography/Mass Spectrometry (GC/MS), Rev. 3, Aug. 2006.

EPA Method 8015B - Determination of Nonhalogenated Organics Using GC/FID, Rev. 2, Dec. 1996.

U.S. EPA Method 8015D - Nonhalogenated Organics Using GC/FID, Rev. 4, May 2003.

U.S. EPA Method 8270D - Determination of Semivolatile Organic Compounds by Gas Chromatography/Mass Spectrometry (GC/MS), Rev. 4, Feb. 2007.

U.S. EPA Method 8000C - Determinative Chromatographic Separations, Rev. 3, Mar. 2003.

U.S. EPA Method 8260C - Volatile Organic Compounds by Gas Chromatography/Mass Spectrometry (GC/MS), Rev. 3, Aug. 2006.

U.S. EPA Method 8270D - Semivolatile Organic Compounds by Gas Chromatography/Mass Spectrometry (GC/MS), Rev. 4, Feb. 2007.

U.S. EPA Method 9060A - Total Organic Carbon, Rev. 1, Nov. 2004 


\section{References}

(1) Finn, T.M. Source Rock Potential of Upper Cretaceous Marine Shale in the Wind River Basin, Wyoming in USGS Wind River Province Assessment Team, Petroleum systems and geologic assessment of oil and gas in the Wind River Basin Province, Wyoming: U.S. Geological Survey Digital Data Series DDS-69J, ch 8, 24 p. 2007.

(2) Finn, T.M. Subsurface Stratigraphic Cross Sections of Cretaceous and Lower Tertiary Rocks in the Wind River Basin, Central Wyoming, in USGS Wind River Province Assessment Team, Petroleum systems and geologic assessment of oil and gas in the Wind River Basin Province, Wyoming: U.S. Geological Survey Digital Data Series DDS-69J, ch 9, 28p. 2007.

(3) Johnson, R.C.; Finn, T.M.; Kirschbaum, M.A.; Roberts, S.B.; Roberts, L.R.; Cook, T.; Taylor, D.J. The Cretaceous-Lower Tertiary Composite Total Petroleum System, Wind River Basin, Wyoming, in USGS Wind River Province Assessment Team, Petroleum systems and geologic assessment of oil and gas in the Wind River Basin Province, Wyoming: U.S. Geological Survey Digital Data Series DDS-69J, Chapter 4, 2007.

(4) Roberts, L.N.R., Finn, T.M., Lewan, M.D. Kirschbaum, M.A. Burial History, thermal Maturity, and Oil and Gas Generation History of Petroleum Systems in the Wind River Basin Province, Central Wyoming, in USGS Wind River Province Assessment Team, Petroleum systems and geologic assessment of oil and gas in the Wind River Basin Province, Wyoming: U.S. Geological Survey Digital Data Series DDS-69J, Chapter 6, 2007.

(5) Daddow, R.L. Water resources of the Wind River Indian Reservation, Wyoming. U.S. Geological Survey WaterResources Investigation Report 95-4223, 1996

(6) McGreevy, L.J.; Hodson, W.G.; Rucker IV, S.J. Ground-Water Resources of the Wind River Indian Reservation Wyoming, Geological Survey Water-Supply Paper 1576-1, 1969

(7) Morris, D.A.; Hackett, O.M.; Vanlier, K.E.; Moulder, E.A.; Durum, W.H. Ground-Water Resources of Riverton Irrigation Project Area, Wyoming, Geological Survey Water-Supply Paper 1375, 1959.

(8) Nelson, P.H.; Kibler, J.E. Distribution of Fluids and Pressures in the Wind River Basin, Wyoming, In USGS Wind River Province Assessment Team, Petroleum systems and geologic assessment of oil and gas in the Wind River Basin Province, Wyoming: U.S. Geological Survey Digital Data Series DDS-69J, Chapter 7, 2007.

(9) Single, E.L. Pavillion Field, Fremont County, Wyoming. In Wyoming Geological Association 21st Field Conference Guidebook, 1969, 101-103.

(10) Bjorklund, T.K. Pavillion Gas Field, Resources of the Wind River Basin, 30th Annual Field Conference, Wyoming Geological Association Guidebook, 1978

(11) Roberts, S., Roberts, L.N.R., Cook, T. Geologic Assessment of Undiscovered Petroleum Resources in the Waltman Shale Total Petroleum System, Wind River Basin Province, Wyoming, in USGS Wind River Province Assessment Team, Petroleum systems and geologic assessment of oil and gas in the Wind River Basin Province, Wyoming: U.S. Geological Survey Digital Data Series DDS-69J, Chapter 5, 2007.

(12) Johnson, R.C.; Keighin, W.C. Origins of natural gases from upper Cretaceous reservoirs, Bighorn Basin, Wyoming and Montana, and comparison with gases from the Wind River Basin, Wyoming: In Keefer, W.R. and Goolsby, J.E. eds. Cretaceous and Lower Tertiary Rocks of the Bighorn Basin, Wyoming and Montana: Wyoming Geological Association Forty-Ninth Guidebook, 1998, 223- 249.

(13) Johnson, R.C.; Rice, D.D. Variations in composition and origins of gases from coal bed and conventional reservoirs, Wind River Basin, Wyoming, in Keefer, W.R, Metzger, W.J. and Godwin, L.H. eds., Oil and Gas and Other Resources of the Wind River Basin, Wyoming: Wyoming Geological Association Special Symposium, 1993, 319-335. 
(14) Lillis, P.G.; Johnson, R.C. Characterization of the natural gas systems of the Wind River Basin, Wyoming [abs.]: American Association of Petroleum Geologist. Rocky Mountain Section Meeting, Jackson Hole, Wyoming, Sept 24-26, 2005.

(15) Schelling, D.D.; Wavrek, D.A. Structural Geology and Petroleum Systems of the Northwestern Wind River Basin, Wyoming [abs.]: American Association of Petroleum Geologists Bulletin. 2001, 85 (13)

(16) Keefer, W.R.; Johnson R.C. Stratigraphy and oil and gas resources in uppermost Cretaceous and Paleocene rocks, Wind River Reservation, Wyoming, in W.R. Keefer, W.J. Metzger and L.H. Godwin, eds., Oil and Gas and Other Resources of the Wind River Basin, Wyoming: Wyoming Geological Association Special Symposium, 1993, 71-86.

(17) Courdin, J.L.; Hubert, J.F. Sedimentology and Mineralogic Differentiation of Sandstones in the Fort Union Formation (Paleocene), Wind River Basin, Wyoming, in Twenty-First Annual field Conference, Wyoming Geological Association Guidebook, 1969, 29-37.

(18) Flores, R.M.; Keighin, C.W. (1993). Reservoir Anisotropy and Facies Stratigraphic Framework in the Paleocene Front Union Formation, Western Wind River Basin, Wyoming, in W.R. Keefer, W.J. Metzger and L.H. Godwin, eds., Oil and Gas and Other Resources of the Wind River Basin, Wyoming: Wyoming Geological Association Special Symposium, 1993, 121-141.

(19) Keefer, W.R. General Stratigraphy and Depositional History of the Fort Union, Indian Meadows, and Wind River Formations, Wind River Basing, Wyoming, in Twenty-First Annual Field Conference, Wyoming Geological Association Guidebook, 1969, 19-28.

(20) Keefer, W.R. Stratigraphy and Geologic History of the Uppermost Cretaceous, Paleocene, and Lower Eocene Rocks in the Wind River Basin, Wyoming. Geological Survey Professional Paper 495-A, United States Geological Survey, 1965

(21) Seeland, D. Sedimentology and Stratigraphy of the Lower Eocene Wind River Formation, Central Wyoming. Thirtiest Annual Field Conference, Wyoming Geological Association Guidebook, 1978, 181-198.

(22) Osiensky, J.L.; Winter, G.V.; Williams, R.E. (1984). Monitoring and mathematical modeling of contaminated ground-water plumes in fluvial environments. Groundwater, 1984, 22, 298-306.

(23) Stephens, D.B. Analysis of the groundwater monitoring controversy at the Pavillion, Wyoming natural gas field. Groundwater 2015, 53(1), 29-37.

(24) Itasca Denver, Inc. (Sterrett, R.J.). Review of the United States Environmental Protection Agency DRAFT Report Entitled "Investigation of Ground Water Contamination near Pavillion, Wyoming." Prepared for: Encana Oil \& Gas (USA) Inc., Denver CO, March 2012. EPA Docket ID No. EPA-HQ-ORD-2011-0895.

(25) Gores and Associates. Pavillion Area Water Supply I Study, Final Report for the Wyoming Water Development Commission, October 2011

http://www.jamesgoresandassociates.com/DocFiles/Pavillion_Area_Water_Supply_Level_I_Study__Final_Report. pdf

(26) Plafcan, M., Eddy-Miller, C.A., Ritz, G.F., and Holland, J.P.R. Water Resources of Fremont County, Wyoming. U.S. Geological Survey, Water-Resources Investigations Report 95-4095, 1995

(27) DiGiulio, D.C., Wilkin, R.T., Miller C., Oberley, G. Investigation of Ground Water Contamination near Pavillion, Wyoming - Draft Report. U.S. Environmental Protection Agency, Office of Research and Development, National Risk Management Research Laboratory, Ada, OK and Region 8, Denver CO, December 2011 
(28) Robinson, J.W., McCabea, P.J. Sandstone-body and shale-body dimensions in a braided fluvial system: Salt wash sandstone member (Morrison formation), Garfield County, Utah. American Association of Petroleum Geologists Bulletin, 1997, 81, 1267-1291

(29) U.S. Environmental Protection Agency. Site Inspection - Analytical Results Report Pavillion Area Groundwater Investigation Site, Pavillion, Fremont County, Wyoming, CERCLIS ID\# WYN000802735, URS Operating Services, Inc., START 3, EPA Region 8, Contract No. EP-W-05-050, August 2009. http://www2.epa.gov/sites/production/files/documents/Pavillion_GWInvestigationARRTextAndMaps.pdf

(30) U.S. Environmental Protection Agency. Expanded Site Investigation - Analytical Results Report Pavillion Area Groundwater Investigation, Fremont County, Wyoming, Superfund Technical Assessment and Response Team, START 3, EPA Region 8, Contract No. EP-W-05-050, August 30, 2010. http://www2.epa.gov/sites/production/files/documents/PavillionAnalyticalResultsReport.pdf

(31) Wyoming Oil and Gas Conservation Commission (WOGCC). Pavillion Field Well Integrity Review, October 8, 2014 http://wogcc.state.wy.us/pavillionworkinggrp/PAVILLION_REPORT_1082014_Final_Report.pdf

(32) Wyoming Oil and Gas Conservation Commission (WOGCC). Pavillion Field Pit Review, November 24, 2014 http://wogcc.state.wy.us/pavillionworkinggrp/Draft\%20Pavillion\%20Field\%20Pits\%20Review_11242014.pdf

(33) U.S. Environmental Protection Agency. Groundwater Sampling Results at Locations near Pavillion, WY, Pavillion Phase V (April 2012) Groundwater Quality Results and Quality-Control (QC) Data. Office of Research and Development, National Risk Management Research Laboratory and Region 8, Denver, CO. 2012.

(34) Wyoming Department of Environmental Quality. Pavillion, Wyoming Domestic Water Wells Draft Final Report and Palatability Study. Prepared by Acton Michelson Environmental, Inc. El Dorado Hills, CA, December 14, 2015. Accessed on 3/1/2016 at http://deq.wyoming.gov/wqd/pavillion-investigation/

(35) EnCana Oil \& Gas (USA) Inc. (Encana 2009) Submittal of Material Safety and Data Sheets and Letter to U.S. EPA, Region 8, Denver, CO, October 19, 2009, 3p.

(36) Wyoming Department of Environmental Quality (WYDEQ), Ref: Tribal 21-15, Letter dated 8/14/2012 from John Wagner to Jon Kaminski of BLM Lander District.

(37) U.S. Department of the Interior, Bureau of Land Management (BLM), Wind River/Bighorn Basin District, Lander Field Office, Lander Wyoming. Ref: Tribal 21-15. Letter dated 8/23/2012 from Jon Kaminski to John Wagner of Wyoming Department of Environmental Quality.

(38) American Society for Testing and Materials (ASTM). ASTM A53 / A53M - 12 Standard Specification for Pipe, Steel, Black and Hot-Dipped, Zinc-Coated, Welded and Seamless Active Standard ASTM A53 / A53M I Developed by Subcommittee: A01.09 Book of Standards Volume: 01.01 (accessed 2013)

(39) Houghton, R.L. and Berger, M.L. Effects of well-casing composition and sampling method on apparent quality of ground water. In Proceedings of the Fourth National Symposium on Aquifer Restoration and Ground Water Monitoring, May 23-25, 1984 National Water Well Association.

(40) Hewitt, A.D. Dynamic study of common well screen materials. Ground Water Monitoring Review, 1994, 87-94.

(41) Aller, L., Bennett, T.W., Hackett, G., Petty, R.J., Lehr, J.H., Sedoris, H., Nielsen, D.M., and Denne, J.E. Handbook of Suggested Practices for the Design and Installation of Ground-Water Monitoring Wells, Environmental Monitoring Systems Laboratory, Office of Research and Development, U.S. Environmental Protection Agency, Las Vegas, Nevada. EPA 160014-891034, March 1991

(42) U.S. Environmental Protection Agency. Draft RCRA Ground-Water Monitoring Technical Enforcement Guidance Document. Office of Solid Waste and Emergency Response. November 1992 
(43) Pohlmann, K.F. and Alduino. Potential Sources of Error in Ground-Water Sampling at Hazardous Waste Sites, Ground-Water Issue, U.S. Environmental Protection Agency, Office of Research and Development and Office of Solid Waste and Emergency Response, EPA/540/S-92/019, 1992

(44) Llopis, J.L. The Effects of Well Casing Material on Ground Water-Quality. Ground-Water Issue, U.S. Environmental Protection Agency, Office of Research and Development and Office of Solid Waste and Emergency Response, EPA/540/4-91/005, 1991.

(45) Marsh, J.M. and Lloyd, J.W. Details of hydrochemical variations in flowing wells. Groundwater, 1980, 18 (4), 366-373.

(46) American Society for Testing and Materials (ASTM). Standard Practice for Design and Installation of Ground Water Monitoring Wells D5092-04 (reapproved 2010). Philadelphia, PA.

(47) Shaw Environmental Services. Draft Work Plan. Borehole Drilling, Well Installation, Well Development, and Other Support Services Groundwater Investigation in Pavillion, Wyoming. Attachment 1 - Monitoring Well Installation Work Plan Narrative. Contract No. EP-C-08-034. Prepared by Shaw Environmental and Infrastructure, Inc., 312 Directors Drive, Knoxville, TN. Prepared for U.S. Environmental Protection Agency, National Risk Management Research Laboratory, Ground Water and Ecosystem Restoration Division, Ada, OK. Project No. 135976, April 2010.

(48) Shaw Environmental Services. Final Monitoring Well Installation Work Plan Pvillion, Wyoming. Contract No. EP-C-08-034. Prepared by Shaw Environmental and Infrastructure, Inc., 312 Directors Drive, Knoxville, TN. Prepared for U.S. Environmental Protection Agency, National Risk Management Research Laboratory, Ground Water and Ecosystem Restoration Division, Ada, OK. Project No. 135976, May 2010.

(49) U.S. Army Corps of Engineers (USACE). Monitoring Well Design, Installation, and Documentation at Hazardous, Toxic, and Radioactive Waste Sites. EM 1110-1-4000. Washington, DC 20314-1000, Nov 1998.

(50) Colangelo, R.V. Inert Annular Space Materials, the Acid Test. Ground Water Monitoring Review, 1988, Spring

(51) Mikkelsen, P.E. Cement-bentonite grout backfill for borehole instruments. Geotechnical News, 2002, December, pp. 38-42

(52) U.S. Environmental Protection Agency. Permeation and Leaching. Office of Water (4601M), Office of Ground Water and Drinking Water, Distribution System Issue Paper, August 15, 2002

(53) Alben, K., Bruchet, A., and Shpirt, E. Leachate from Organic Coating Materials Used in Potable Water Distribution Systems. Denver, CO, American Water Works Association, Jan 1989

(54) Wright, P.R., McMahon, P.B. Sampling and Analysis Plan for the Characterization of Groundwater Quality in Two Monitoring Wells near Pavillion, Wyoming: U.S. Geological Survey Open-File Report 2012-1197, 2012

(55) McAlary T.A. and Barker J.F. Volatilization losses of organics during ground water sampling from low permeability materials. Ground Water Monitoring Review, 1987, 7(4), 63-68.

(56) Roy J.W.; Ryan M.C. In-well degassing issues for measurements of dissolved gases in groundwater. Ground Water, 2010, 48(6), 869-877.

(57) Yeskis, D. and B. Zavala. Ground-Water Sampling Guidelines for Superfund and RCRA Project Managers, Ground Water Issue Paper, U.S. Environmental Protection Agency, Office of Solid Waste and Emergency Response, EPA 542-S-02-001, 2002

(58) U.S. Geological Survey (USGS). National Field Manual for the Collection of Water-Quality Data. Chapter A4. Collection of Water Samples. Revised 2006. 
(59) Cottrell, G. L. and Myers, D.N. U.S. Geological Service (USGS). Transmittal of Contract Laboratory Results and Evaluation of Laboratory-Specific Quality Control Measures, U.S. Environmental Protection Agency Monitoring Well MW02, Pavillion Wyoming 2012, Administrative Report Prepared for the U.S. Environmental Protection Agency. Director Approved August 30, 2012.

(60) Wright, P.R., McMahon, P.B., Mueller, D.K., Clark, M.L. Groundwater-Quality and Quality-Control Data for Two Monitoring Wells near Pavillion, Wyoming, April and May 2012: U.S. Geological Survey Data Series 718, 2012

(61) Humenick, M.J., Turk L.J., and Colchin M.P. (1980). Methodology for monitoring ground water at uranium solution mines. Ground Water 1980, 18(3), 262-273.

(62) Robin M.J.L.; Gillham R.W. Field evaluation of well purging procedures. Ground Water Monitoring Review 1987, 7(4), 85-93.

(63) U.S. Environmental Protection Agency. Investigation of Ground Water Contamination near Pavillion, Wyoming Phase V Sampling Event, Summary of Methods and Results. Office of Research and Development, National Risk Management Research Laboratory and Region 8, Denver, CO. September 2012.

(64) Robbins G.A. and Martin-Hayden, J.M. (1991). Mass balance evaluation of monitoring well purging. Part I:

Theoretical models and implications for representative sampling. Journal of Contaminant Hydrology, 1991, 8, 203224.

(65) Shaw Environmental and Infrastructure, Inc. (Shaw 2012a). Method Development-Alcohol Analysis by P\&T GC FID. Contract No. EP-C-08-034, Subject: 8OA865CS, May 29, 2012. https://foiaonline.regulations.gov/foia/action/public/view/request?objectId=090004d2806a7021

(66) Shaw Environmental and Infrastructure, Inc. (Shaw 2012b). Method Development-Alcohol Analysis by P\&T GC FID. Contract No. EP-C-08-034, Subject: 8OA878DW, May 30, 2012. https://foiaonline.regulations.gov/foia/action/public/view/request?objectId=090004d2806a7021

(67) Andersson, K.; Allard, B.; Bengtsson, M.; Magnusson, B. Chemical composition of cement pore solutions. Cement and Concrete Research, 1979, 19, 327-332.

(68) Thomas, J.J.; Rothstein, D.; Jennings, H.M.; and Christensen, B.J. Effect of hydration temperature on the solubility behavior of Ca-, S-, Al-, and Si-bearing solid phases in Portland cement pastes. Cement and Concrete Research, 2003, 33, 2037-2047.

(69) van Eijk, R.J.; Brouwers, H.J.J. Prediction of hydroxyl concentrations in cement pore water using a numerical cement hydration model. Cement and Concrete Research, 2000, 20, 1801-1806.

(70) Lothenbach, B., Le Saout, G., Gallucci, E., and Scrivener, K. Influence of limestone on the hydration of Portland cements. Cement and Concrete Research, 2008, 38, 848-860.

(71) Lothenbach, B., Matschei, T., Möschner, G., and Glasser, F.P. Thermodynamic modeling of the effect of temperature on the hydration and porosity of Portland cement. Cement and Concrete Research, 2008, 28, 1-18.

(72) Lothenbach, B.; Winnefeld, F. Thermodynamic modeling of the hydration of Portland cement. Cement and Concrete Research, 2006, 36 (2), 209-226.

(73) Rothstein, D.; Thomas, J.J.; Christensen, B.J.; Jennings, H.M. Solubility behavior of Ca-, S-, Al-, and Sibearing solid phases in Portland cement pore solutions as a function of hydration time. Cement and Concrete Research, 2002, 32, 1663-1671.

(74) Barcelona, M.J. and Helfrich, J.A. (1986). Well construction and purging effects on ground-water samples. Environmental Science and Technology 1986, 20, 1179-1184. 
(75) Brouwers, H.J.H and van Eijk. Alkali concentrations of pore solution in hydrating OPC. Cement and Concrete Research, 2003, 33, 191-196.

(76) Leemann, A.; Lothenbach, B. The influence of potassium-sodium ratio in cement and concrete expansion due to alkai-aggregate reaction. Cement and Concrete Research, 2008, 38, 1162-1168.

(77) Engelsen, C.J. Quality Improvers in Cement Making - State of the Art. COIN Project P1 Advanced Cementing Materials. SP 1.1F Reduced CO2 missions. COIN Project report $2-2008$.

http://www.sintef.no/upload/Byggforsk/Publikasjoner/coin-no2.pdf

(78) Ervanne, H., and Hakanen, M. Analysis of Cement Superplasticizers and Grinding Aids, A Literature Survey, Working Report, Posiva, 2007

(79) Jeknavorian, A.A., Barry, E.F., Serafin, F. Determination of grinding aids in Portland cement by pyrolysis gas chromatography-mass spectrometry. Cement and Concrete Research, 1998, 28(9), 1335-1345.

(80) Teoreanu, I.; Guslicov, G. Mechanisms and effects of additives from the dihydroxy-compound class on Portland cement grinding. Cement and Concrete Research, 1999, 29, 9-15.

(81) Smith, B., Siegel, D., Neslund, C. and Carter, C. Organic contaminants in Portland cement used in monitoring well construction. Groundwater Monitoring \& Remediation, 2014, 34(4), 102-111.

(82) Beak, D.G; Oberley, G.G.; Ruybal, C.J.; Acree, S.D.; Ross; R.R. Retrospective Case Study in Killdeer, North Dakota: Study of the Potential Impacts of Hydraulic Fracturing on Drinking Water Resources, U.S. Environmental Protection Agency, Office of Research and Development, Washington, DC, EPA/600/R-14/103, May 2015a

(83) Beak, D.G.; Overbay, M.D.; Mravik, S.C. Retrospective Case Study in Wise County, Texas Study of the Potential Impacts of Hydraulic Fracturing on Drinking Water Resources, U.S. Environmental Protection Agency, Office of Research and Development, Washington, DC, EPA/600/R-14/090, May 2015b

(84) Ludwig, R.D.; Beak, D.G.; Wilkin, R.T.; Ruybal, C.J.; Rectenwald, D.J. Retrospective Case Study in Northeastern Pennsylvania Study of the Potential Impacts of Hydraulic Fracturing on Drinking Water Resources, U.S. Environmental Protection Agency, Office of Research and Development, Washington, DC, EPA/600/R-14/088, May 2015

(85) Wilkin, R.T.; Lee, T.R.; Ruybal, C.J.; Rectenwald, D.J. Retrospective Case Study in Southwestern Pennsylvania Study of the Potential Impacts of Hydraulic Fracturing on Drinking Water Resources, U.S. Environmental Protection Agency, Office of Research and Development, Washington, DC, EPA/600/R-14/084, May 2015

(86) Wolfe, A.L; Wilkin, R.T; Lee, T.R.; Ruybal, C.J.; Oberley, G.G. Retrospective Case Study in the Raton Basin, Colorado Study of the Potential Impacts of Hydraulic Fracturing on Drinking Water Resources U.S. Environmental Protection Agency Office of Research and Development Washington, DC, EPA/600/R-14/091, May 2015

(87) U.S. Environmental Protection Agency (EPA 2010b). Background Information for the Leaching Environmental Assessment Framework (LEAF) Test Methods, Appendix C - Preliminary Version of Method 1315, EPA/600/R10/170, November 2010, Office of Research and Development, National Risk Management Research Laboratory, Research Triangle Park, NC

(88) Crank, J. The Mathematics of Diffusion, Second Edition, Oxford University Press, New York, 1975, 414p.

(89) Keith, S.J.; Wilson, L.G.; Fitch, H.R.; Esposito, D.M. Sources of spatial-temporal variability in ground-water quality data and methods of control. Ground Water Monitoring and Remediation 1983, Spring, 21-32. 
(90) Wilson, L.C.; Rouse, J.V. Variations in water quality during initial pumping of monitoring wells. Ground Water Monitoring and Remediation 1983, 3 (1), 103-109.

(91) Rivett, M.O.; Lerner, D.N.; Lloyd, J.W. Temporal variations of chlorinated solvents in abstraction wells. Ground Water Monitoring and Remediation 1990, 10 (4), 127-133.

(92) Gibs J.; Imbrigiotta T.E. Well-purging criteria for sampling purgeable organic compounds. Ground Water 1990, $28(1), 68-78$.

(93) Reilly, T.E.; Gibs, J. Effects of physical and chemical heterogeneity on water-quality samples obtained from wells. Ground Water 1993, 31(5), 805-813.

(94) Reilly, T.E.; LeBlanc, D.R. Experimental evaluation of factors affecting temporal variability of water samples obtained from long-screened wells. Ground Water 1998, 36 (4), 566-576.

(95) Cosler, D. J. Ground-water sampling and time-series evaluation techniques to determine vertical concentration distributions. Ground Water 1997, 35 (5), 825-841.

(96) Cohen, R.M.; Rabold, R.R. Simulation of sampling and hydraulic tests to assess a hybrid monitoring well design. Ground Water Monitoring and Remediation 1988, 8 (1), 51-59.

(97) Robbins G.A.; Martin-Hayden, J.M. Mass balance evaluation of monitoring well purging. Part I: Theoretical models and implications for representative sampling. Journal of Contaminant Hydrology 1991, 8, 203-224.

(98) Varljen, M.D.; Barcelona, M.J.; Obereiner, J.; Kaminski, D. Numerical simulations to assess the monitoring zone achieved during low-flow purging and sampling. Ground Water Monitoring and Remediation 2006, 26 (1), 4452.

(99) Molz, F.J.; Boman, G.K.; Young, S.C.; Waldrop, W.R. Borehole flowmeters - Field application and data analysis. Journal of Hydrology 1994, 163, 347 - 371.

(100) Church, P.E.; Granato, G.E. Effects of well design and sampling methods on bias of water-quality samples. Ground Water 1996, 34 (2), 262-273.

(101) Boman, G.K.; Molz, F.J.; Boone, K.D. Borehole flowmeter application in fluvial sediments: Methodology, results, and assessment. Ground Water 1997, 35 (3), 443-450.

(102) Hutchins, S.R.; Acree, S.D. Shallow water sampling Bias observed in shallow, conventional wells. Ground Water Monitoring and Remediation 2000, 20 (1), 86 - 93.

(103) Crisman, S.A.; Molz, F.J.; Dunn, D.L.; Sappington, F.C. Application procedures for the electromagnetic flowmeter in shallow unconfined Aquifers. Ground Water Monitoring and Remediation 2001, 21 (4), 96 - 100.

(104) Reilly, T.E.; Franke, O.L.; and Bennett, G.D. Bias in ground water samples caused by wellbore flow. Journal of Hydraulic Engineering 1989, 115 (2), 270-276.

(105) Elci, A.; Molz III, F.J.; Waldrop, W.R. Implications of observed and simulated ambient flow in monitoring wells. Ground Water 2001, 39 (6), 853-862.

(106) Corcho Alvarado, J.A.; Barbecot, F.; Purtschert, R. Ambient vertical flow in long-screen wells: A case study in the Fontainebleu Sands Aquifer (France). Hydrogeology Journal 2009, 17, 425 - 431.

(107) Caenn, R.; Darley, H.C.H; Gray, G.R. Composition and Properties of Drilling and Completion Fluids, Sixth Edition, ISBN: 978-0-12-383858-2, Gulf Professional Publishing, Waltham, MA, 2011

(108) Neff, J.M. Fate and Biological Effects of Oil Well Drilling Fluids in the Marine Environment: A Literature Review, U.S. Environmental Protection Agency, Environmental Research Laboratory, Gulf Breeze, FL, Sept 9, 1981, Report No 15077, 17 